\title{
BIOLOGY AND MEDICINE DIVISION ANNUAL REPORT 1985
}

LBL- -20345

DE86 012736

\author{
Lawrence Berkeley Laboratory \\ University of California \\ Berkeley, California 94720
}

\section{DISCLAIMER}

\begin{abstract}
This report was prepared at an sccount of work sponsored by an asency of the United States Government. Neither the United States Government aur any asency thereof, nor any of their employece, makes any warranty, expres or implied, or asumes any lefal liability of responsibility for the socuracy, completenem, of unefulnow of any information, apparatus, product, or proces disclosed, of represents that its use would not infringe privately owned rights. Refercace berein to any apocific commercial product, procen, or service by trade name, trademark. manufacturer, of otherwive does not necensarily constitute or imply its endorsement, reocmmeadation, or favories by the Usited Stutes Governmeat or any seacy thereot. The view and opinion of autions expresed berein do not necenarily atale or refloct those of the United States Goverament or any apeacy theroof.
\end{abstract}

This work was supported by the Office of Health \& Environmenlal Research of the United States Department of Energy under Contract DE-ACO3765F00098. Portions of this work were also supported by the National instilutes of Heallh. Department of Health and Human Services; the National Aeronautics and Space Administration; the Nuclear Regulatony Commission, the Veterans Administration. International Business Machines Corporation (IBMi Instrumenis), and the Elleciric Power Research Institule. Coniracts and grants ate listed in Appendix $A$. 


\section{CONTENTS}

\section{INTRODUCTION}

Edward L. Alpen

\section{RESEARCH MEDICINE}

INTRODUCTION

EVALUATION AND USE OF IODINE-122 BRAIN BLOOD FLOW

RADIOPHARMACEUTICALS

Chester A. Mathis, Thornton Sargent III, Thomas F. Budinger,

Yukio Yano, William J. Jagust, Alexander Shuigin,

Natalia Kusubov, and Kathleen $M$. Brennan

DYNAMIC PET STUDIES OF GLUCOSE UPTAKE IN ALZHEIMER'S DISEASE

William J. Jagust, Robert P. Friedland, Ronald $\mathrm{H}$. Huesman,

Bernard M. Mazoyer, Yukio Yano, Chester A. Mathis,

Kathleen M. Brennan, Brian Knittel, and Thomas F. Budinger

HUMAN MYOCARDIAL STUDIES WITH ${ }^{82}$ Rb AND PET

Thomas F. Budinger, Yukio Yano, Julia A. Twitchell,

Kathleen M. Brennan, Stephen E. Derenzo, and Ronald H. Huesman

8

POSITRON EMISSION TOMOGRAPHY OF FDG IN SCHIZOPHRENIA

Thornton Sargent III and Natalia Kusubov

GALLIUM-68 LABELED PLATELETS AND POSITRON EMISSION TOMOGRAPHY FOR

DETECTING VASCULAR LFSIONS

Yukio Yano, Kathleen M. Brennan, Mohindar Singh,

Julia A. Twitchell, Dorothy A. Carpenter, Elizabeth M. Mazoyer,

Shirley N. Ebbe, Kanu Dalal, Ronaid H. Huesman,

Chester A. Mathis, and Thomas F. Budinger

ABNORMALITIES OF BLOOD PLATELETS IN RABBITS WITH DIETARY

HYPERCHOLESTEROLEMIA AND ATHEROSCLEROSIS

Elisabeth Mazoyer, Kanu Dalal, Dorothy Carpenter, Kathleen Brennan,

Tamlyn Yee, Bernard Mazoyer, Robert Leven, and Shirley Ebbe

IN VITRO MATURATION OF BONE MARROW MEGAKARYOCYTES

Robert M. Leven, Marilyn Yee, and Cheryl Lynn Tanguilig

RELATIONSHIP BETWEEN MEGAKARYOCYTE SIZE ANO PLOIDY IN MICE DURING

RECOVERY FRO I ACUTE IMMUNOTHROMBOSYTOPENIA

Shirley Ebbe, Tamlyn Yee, Dorothy Carpentet and Elizabelh Phalen

THROMBOCYTOPO'ETIC RESPONSE TO MMMUNOTHROMBOCYTOPENA

IN NUDE MIICE

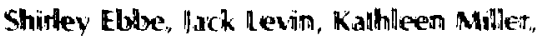

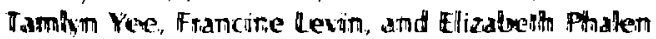


DEVELOPMENT OF RADIONUCLIDES AND RADIOPHARMACEUTICALS FOR EMISSION COMPUTED TOMOGRAPHY

Yukio Yano, Chester A. Mathis, Stephen M. Moerlein,

Mohindar Singh, Reese Jones, and Thomas F. Budinger

NEW INSTRUMENTATION FOR HIGH RESOLUTION, DYNAMIC,

THREE DIMENSIONAL IMAGING OF POSITRON LABELED COMPOUNDS

IN THE HUMAN BODY

Stephen E. Derenzo, John L. Cahoon, Ronald H. Huesman,

Tony Vuletich, and Thomas F. Budinger

STATISTICAL PROPERTIES OF COMPARTMENTAL MODEL PARAMETERS

EXTRACTED FROM DYNAMIC POSITRON EMISSION TOMOGRAPHY EXPERIMENTS

Bernard M. Mazoyer, Ronald H. Huesman, Thomas F. Budinger,

and Brian L. Knittel

DEAD TIME CORRECTION AND COUNTING STATISTICS FOR POSITRON

TOMOGRAPHY

Bernard M. Mazoyer, Mark S. Roos, Ronald H. Huesman,

and Thomas F. Budinger

BIOLOGICAL EFFECTS OF STATIC MAGNETIC FIELDS

Thomas F. Budinger, Kay S. Bristol, Dorothy A. Carpenter,

Patricia A. Garbutt, Priscilla D.C. Wong, and Chi-Kwan Yen

2. DONNER PAVILION

INTRODUCTION

STEREOTACTIC HEAVY ION BRAGG PEAK RADIOSURGERY

Jacob I. Fabrikant, John T. Lyman, Kenneth A. Frankel,

Mark H. Phillips, Edward L. Alpen, Neela B. Manley,

Richard P. Levy, Myrtle L. Foster, Frederick W. Yeater,

George J. Hampton, Maureen H. Morford, and Barbara Modlinski

\section{PHYSIOLOGY}

INTRODUCTION

HEMOPOIESIS AND MALARIA

Paul H. Silverman, John C. Schooley, and Lynn I. Mahlmann

MALARIAL DYSERYTHROPOIESIS: A POSSIBLE ROLE FOR INTERLEUKIN I?

John C. Schooley and Birgitta Kuilgren

\section{SEEDING OF SINGLE HEMOPOIETIC STEM CELLS AND SELF RENEWAL} OF COMMITTEO STEM CELLS

George Brecher

CHARACTERIZATION OF ANTISERA TO ERYTHROPOAETHN AND IMAUNOHOGICAL DIFFERENCES BETWEEN MZS LABELED HUMAN URINARY AND

RECOMBNNANT ERYTHROPOUETIN 
PERIPHERAL CHANGES OF THYROID HORMONE BINDING TO SERUM PROTEINS AFTER SHORT TERM OZONE EXPOSURE

Sherry L. Fitzsimmons and Gisela K. Clemons

BIOLOGICAL EFFECTS OF MAGNETIC FIELDS

Thomas S. Tenforde, Cornelius T. Gaffey, Robert P. Liburdy, and Lynette Levy

MICROWAVE STIMULATED DRUG RELEASE FROM LIPOSOMES

Robert P. Liburdy 68

NEW SEQUESTERING AGENTS FOR THE ACTINIDES: EFFECTIVENESS FOR REMOVAL OF PU FROM MICE OF HYDROXAMIC ACID DERIVATIVES OF DTPA, EDTA AND DESFERRIOXAMINE AND AN N-CENTERED TRIS(CATECHOYLAMIDE) LIGAND; GRADED DOSAGES OF DESFERRIOXAMINE PYRIDINONE OXIDE (desferriHOPOCAM); PROTRACTED ADMINISTRATION OF 3,4,3-LICAM(C)

Patricia W. Durbin, Nylan Jeung, Kenneth N. Raymond,

David L. White, Steven J. Rodgers and Petra Turowski

\section{RADIATION BIOPHYSICS}

INTRODUCTION

Physics of Interactions between Fast Charged Particles and Matter

HIGH ENERGY HEAVY ION BEAMS USED IN BIOLOGY AND MEDICINE: MEASUREMENT OF PARTICLE DISTRIBUTIONS IN THE FRAGMENTATION TAIL OF A 670 A NieV NEON BEAM STOPPING IN WATER

Mervyn Wong, Walter Schimmerling, Marwin Rapkin, and Jerry Howard

MULTIPLE COULOMB SCATTERING OF HEAVY IONS Mervyn Wong, Mark Phillips, Walter Schimmerling, Don L. Murphy, and Cornelius A. Tobias

${ }^{16} \mathrm{O}$ AND ${ }^{56} \mathrm{Fe}$ EXCITATION FUNCTIONS

John P. Wefel, T. Gregory Guzik, Henry J. Crawford, Walter Schimmerling,

Peter I. L.indstrom, Douglas E. Greiner, and T. James M. Symons

BERKLET UPGRADE: IMPROVED DETECTION OF LOW LET PARTICLES IN HIGHLY FRAGMENTED HEAVY ION BEAMS

Inrge Llacer, Julius ]. Almasi, and Cornelius A. Tobias

\section{CARBON PROTON BEAM COMPARISONS}

Cornelius A. Tobias, Eleanor A. Blakely, Willian T. Chu,

Bernhard A. Ludewiga, and Julius I. Almasi

Molecular Studies

DEIECTION OF RADIATION INDUCEO LESIONS AT THE MOLECULAR LEVEL

Ruth B. Roots, Gianiranco Grossi, and Comelius A. Tobias 
PROUUCTION OF STRAND BREAKS IN DNA BY WATER RADICALS:

A THEORETICAL STUDY

Aloke Chatterjee, John L. Magee, Patrice Koehl, and William Holley

A STEREOCHEMICAL MODEL FOR THE REACTIONS OF DNA WITH THE HYDROXYL RADICAL

William R. Holley, Patrice A. Koehl, Aloke Chatterjee,

and John L. Magee

Cellular and Tumor Radiobiology

NEOPLASTIC CELL TRANSFORMATION BY HEAVY ION RADIATION

Tracy Chui-hsu Yang, Laurie $M$. Craise, Mantong Mei, and Cornelius A. Tobias 96

CHEMICAL MODIFICATION OF NEOPLASTIC CELL TRANSFORMATION BY HEAVY ION RADIATION

Tracy Chui-hsu Yang, Laurie M. Craise, Mantong Mei, and Cornelius A. Tobias .......

CELL AGE DEPENDENT VARIATIONS IN OXIDATIVE PROTECTIVE ENZYMES

Eleanor A. Blakely, Polly Y. Chang, Leora Lommel,

and Cornelius A. Tobias

RESPONSE OF AEROBIC AND HYPOXIC HUMAN LUNG SQUAMOUS CARCINOMA CELLS TO NEON IONS

Eleanor A. Blakely, Hiroshi Ohara, Polly Y. Chang, and Leora Lommel

MULTIPLE CHROMATIN BREAKS PRODUCED BY NEON IONS

Edwin H. Goodwin, Eleanor A. Blakely, and Cornelius A. Tobias

EFFECT OF INHIBITION OF PROTEIN SYNTHESIS ON THE DEVELOPMENT

OF THERMOTOLERANCE

Polly Y. Chang, Eleanor A. Blakely, and Ileana Gonzalez-Flores

CELL CYCLE KINETICS AND IN VIVO MICRONUCLEI INDUCTION IN RAT

RHABDOMYOSARCOMA TUMORS USING A MONOCLONAL ANTIBODY TO BrdUrd AND CELL SORTING

Michael Nüsse, S.M. Javed Afzal, Betsy C. Carr,

Kristina S. Kavanau, Thomas S. Tenforde, and Stanley B. Curtis

106

TUMOR RADIOBIOLOGY STUDIES WITH HEAVY CHARGED PARTICLE BEAMS

Stanley B. Curtis, Thomas S. Tenforde, and S.M. Javed Afzal

110

COMPARATIVE ANALYSIS OF MODELS DESCRIBING GLUCOSE UPTAKE

IN THE BRAIN

Hugo A. Massaldi

115

HOLISTIC BIOPHYSICS OF RED BLOOD CELL MEMBRANE SYSTEMS

Howard C. Mel, Gary V. Richien, Hugo A. Massaldi, and Robett Bridwell

\section{Trume Ethects of Hewny Conned Paticte Dewn}

\section{LATE RADIAIION DAMAGE IN THE MOUSE KIDNEY}

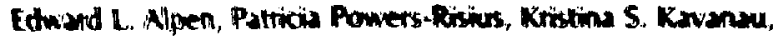

and Randy I. DeGuaman 
HELIUM IRRADIATION OF THE RAT SPINAL CORD

Adrian Rodriguez, Edward L. Alpen,

Randy J. DeGuzman, and John C. Prioleau

INACTIVATION OF MOUSE MARROW STEM CELLS BY HEAVY CHARGED PARTICLES

E. John Ainsworth, Lynn J. Mahlmann, and John C. Prioleau

ACUTE RADIATION LETHALITY IN MICE EXPOSED TO HEAVY CHARGED

PARTICLES

E. John Ainsworth, John C. Prioleau, and Lynn J. Mahlmann

SKYHOOK PROJECT: PROGRESS REPORT

E. John Ainsworth, John C. Prioleau, and Lynn J. Mahlmann

Heavy lon Therapy and Related Physics and Biology

HEAVY CHARGED PARTICLE RADIOTHERAPY TRIAL

Joseph R. Castro, Theodore L. Phillips, David E. Linstadt,

1. Michael Collier, Samuel Pitluck, William T. Chu,

Sheri D. Denderson, Tim R. Renner, Robert E. Walton,

Jacquelyn I. Iler, Marilyn A. Fowler, Marc L. Kessler,

and Monica M. Reimers

ESTIMATING RADIATION THERAPY COMPLICATION PROBABILITIES

John T. Lyman

CLINICAL AND CELLULAR RADIOBIOLOGICAL STUDIES OF SILICON ION BEAMS

Eleanor A. Blakely, Joseph R. Castro, Mary M. Austin-Seymour,

George T.Y. Chen, Leora Lommel, Michael Yezzi,

Polly Y. Chang, and Cornelius A. Tobias

PLANNING CANCER TREATMENT WITH RADIOACTIVE BEAMS

Aloke Chatterjee, Joseph R. Castro, Edward L. Alpen,

Jorge Llacer, George T.Y. Chen, and William T. Chu

ISOSURVIVAL TESTING OF BAR RIDGE FILTERS FOR WOBBLED NEON ION BEAMS Stanley B. Curtis, Adrian Rodriguez, Tracy C.h. Yang,

and Eleanor A. Blakely

RADIOBIOLOGICAL STUDIES FOR HELIUM ION THERAPY OF UVEAL MELANOMA Eleanor A. Blakely, John T. Lyman, George T.Y. Chen, loseph R. Castro. Polly Y. Chang, Leora Lommel. Frederick Yealer, George I. Hamplon, Nina C. Wong, and Shari-Lyn K. Baba

\section{LIPOPROTEIN AND STRUCTURAL BIOLOGY}




\section{Lipoproteins}

THE AI-MILANO HDL PARTICLES

Alex V. Nichols, Guido Franceschini, Cesare R. Sirtori, and Elaine L. Gong

GENETIC STUDIES OF LDL SUBCLASSES

Melissa A. Austin and Ronald M. Krauss

INTERCORRELATIONS OF SUBCLASSES OF LDL AND HDL BY GRADIENT GEL

ELECTROPHORESIS (GGE) AND ANALYTIC ULTRACENTRIFUGATION (ANUC)

Frank T. Lindgren, Alex V. Nichols, Peter D. Wood,

Gerald L. Adamson, Melissa A. Austin, Laura A. Glines,

Vera Martin, and Ronald M. Krauss

PARTIAL SPECIFIC VOLUME AND PREFERENTIAL HYDRATION OF LOW DENSITY LIPOPROTEIN SUBFRACTIONS

Talwinder S. Kahlon, Gerald L. Adamson, Laura A. Glines,

Joseph R. Orr, and Frank T. Lindgren

SERUM LIPID AND LIPOPROTE!N CONCENTRATIONS FOLLOWING

EXPOSURE TO OZONE

William J. Vaughan, Gerald L. Adamson, Frank T. Lindgren,

and John C. Schooley

APOLIPOPROTEIN (APO) E LEVELS AND DISTRIBUTION IN HUMAN CORD BLOOD

Trudy M. Forte, Paul A. Davis, and Conrad B. Blum

FORMATION OF PHOSPHOLIPID-RICH HDL WITH UNUSUAL PHYSICAL

PROPERTIES: POSSIBLE MODEL FOR INTERSTITIAL FLUID HDL

Trudy M. Forte, Robert W. Nordhausen,

C. Luming Ren, and Alex $V$. Nichols

\section{Structural Biology}

DIFFERENTIAL POLARIZATION IMAGING MICROSCOPY Marcos F. Maestre, William Mickols, Ignacio Tinoco, Jr., and Stephen H. Embury

ABSORPTION FLATTENING IN THE CIRCULAR DICHROISM SPECTRA OF

SMALL MEMBRANE FRAGMENTS

Robert M. Glaeser and Bing K. Jap

CONTRAST IMPROVEMENT WITH SMALL SPOT ILLUMINATION FOR

HIGH RESOLUTION ELECTRON MICROSCOPY OF BEAM SENSITIVE SPECIMENS

Kenneth $H$. Downing and Robert $M$. Glaeser

SECONDARY STRUCTURE OF HALORHODOSPIN

Bing $K$. lap and Seok-Hwan Kong

STRUCTURAL ANALYSIS OF PhOE PORIN, AN OUTER MEMBRANE PROTEIN FROM ESCHERICHA COII

Bing K. lap 


\section{LOW TEMPERATURE SCANNING ELECTRON MICROSCOPY OF FROZEN HYDRATED LUNG}

Jacob Bastacky, Gregory R. Hook, Gregory L. Finch, and Thomas L. Hayes

IMMUNE RECOGNITION OF MEMBRANES

Aaron B. Kantor and John C. Owicki

\section{CELLULAR AND MOLECULAR BIOLOGY}

INTRODUCTION

BACTERIOPHAGE T4 GENE 32 PROTEIN AFFINITY COLUMN CHROMATOGRAPHY Junko Hosoda, Herbert W. Moise, Maren Bell, and Midori Hosobuchi

FRACTIONATION OF DNA METABOLIC PROTEINS OF SACCHAROMYCES CEREVISIAE BY DNA CELLULOSE CHROMATOGRAPHY: SSB-1, SS-DNA DEPENDENT ATPase,

DNA POLYMERASE, DNA PRIMASE, TOPOISOMERASE I, AND RESOLVASE Junko Hosoda, Libby L. Holbrook, Herbert W. Moise, Kathleen A. Bjornstad, Dimitrios T. Maleas, Midori Hosobuchi, Maren Bell, and Michael S. Esposito

IN VITRO RESOLUTION OF HOLLIDAY JUNCTIONS BY CELL-FREE PROTEIN EXTRACTS OF SACCHAROMYCES CEREVISIAE: RESOLVASE ACTIVITY OF HAPLOID AND DIPLOID CELLS

Libby Litzenberger Holbrook, Kathleen A. Bjornstad,

Dimitrios T. Maleas, and Michael S. Esposito

THE REC46 GENE OF SACCHAROMYCES CEREVISIAE CONTROLS MITOTIC CHROMOSOMAL STABILITY, RECOMBINATION AND SPORULATION: CELL TYPE AND LIFE CYCLE STAGE SPECIFIC EXPRESSION OF THE rec46-1 MUTATION

Dimitrios T. Maleas, Katheleen A. Bjornstad, Libby L. Holbrook, and Nichael S. Esposito

THE GENETIC MAP OF YEAST AND THE GENEALOGY OF LABORATORY STRAINS Robert K. Mortimer, David Schild, John R. Johnston, and Rebecca Contopoulou

ISOLATION AND MOLECULAR CHARAETERIZATION OF YEAST DNA REPAIR

GENES AND THE USE OF A NEW GEL SYSTEM TO SIUDY DNA REPAIR

Robert Mortimer, John Game, David Schild, Mari Aker, Gary Cole,

Rebecca Contopoulou, Vincent Cook, Minh Dang, Leslie Kay.

Susan Lovett, and Karen Sitney

ISOLATION OF DNA FRAGMENTS CONTAINING REPLICATION FORKS BY

TWO DIMENSIONAL AGAROSE GEL ELECTROPHORESIS

Priscilla $K$. Cooper and Vincent ling

ALKYLATION OF POLYNUCLEOTIDES IN VITRO AND IN VIVO

Bea A Singer, Sylvia 月. Spengler, Frank Chavez,

and Heinz Fraenket-Conrat 


\section{CARCINOGENIC POTENCY}

Lois Swirsky Gold, Bruce N. Ames, Renae I. Magaw,

Catherine Wright, Joan Schwalbe, Georganne Backman,

Mark Blumential, and Thomas $\mathrm{H}$. Slone

TEMPORAL EXPRESSION OF ROUS SARCOMA VIRUS IN MICROINJECTED EMBRYONIC CHICK LIMBS

Anthony R. Howlett, Betsey Cullen, and Mina J. Bissell

TUMOR PROMOTERS CAUSE RSV MEDIATED TUMORS ONLY WITH

CONCOMITANT LOCAL IRRITATION

Mark D. Hertle, David S. Dolberg, Robert E. Hollingsworth,

and Mina J. Bissell

ROLE OF EXTRACELLULAR MATRIX AND HORINONES IN MODULATION OF

TISSUE-SPECIFIC FUNCTION IN COMMA-1-D, A MOUSE MAMMARY EPITHELIAL

CELL LINE

Ming-Liang Li, Li-How Chen, and Mina !, Bissell

CLONING OF MOUSE TRANSFERRIN CDNA

Li-How Chen, Ming-Liang Li, and Mina J. Bissell

PROCOLLAGEN SECRETION-A CRITICAL STEP IN THE REGULATION OF PROCOLLAGEN SYNTHESIS

Nancy Owens and Richard I. Schwarz

AN ANALYSIS OF THE ANTIGENIC STRUCTURE AND MODE OF REGULATION OF A DIFFERENTIATION ANTIGEN ON THE SURFACE OF NORMAL AND

MALIGNANT HUMAN MAMMARY EPITHELIAL CELLS

Lenny Moss, Betsey Cullen, and Gordon Parry

THE ROLE OF TIGHT JUNCTIONS AND CELL SUBSTRATUM INTERACTIONS IN CONTROLLING MEMBRANE POLARITY IN CULTURED HUMAN MAMMARY

EPITHELIAL CELLS

Gordon Parry, Lenny Moss, and Betsey Cullen

GROWTH CONTROL IN HUMAN MAMMARY EPITHELIAL CELLS

Martha Stampfer and lack Bartley

EXPRESSION AND MODULATION OF DIFFERENTIATION IN HUMAN MAMMARY EPITHELIAL CELLS

Jack Bartley, Gerri Levine, and Martha Stampfer

EVALUATION OF NORMAL AND TRANSFORMED HUMAN MAMMARY EPITHELIAL CELLS WITH MONOCLONAL ANTIBODIES TO CELL SURFACE ANTIGENS

Martha Stampier, Gordon Parny, and lack Bartley

USE OF DNA RESTRICTION FRAGMENT LENGTH POLYMORPHISMS FOR THE IDENTIFICATION OF HONEYBEE RACES

H. Glenn Hail 


\section{INTRODUCTION}

In looking back at the introduction to last year's report, I can only wistfully say that at that time I had hoped that the financial stresses of a shrinking budget would be behind us before now. Unfortunately that has not proved to be the case. This has been the year of the "deficit crisis" and Gramm-Rudman. As a result, the federal funding of research has continued to be a serious problem. Nevertheless, we have managed to hold our own and to contribute in a very respectable fashion to the world of science. Let me express my heartfelt thanks and appreciation to all our staff. You have weathered significant tribulations while maintaining your sense of proportion and commitment to good science.

During the past year the Donner Pavilion program on the treatment of arteriovenous malformations in the brain has chalked up very significant successes. The disease control rate has been high and objective measures of success using cerebral angiography have been established. This program along with the Research Medicine program on brain disease have received very wide public exposure, including a successful appearence before a House Committee hearing. The new high resolution positron emitting tomographic imager has been demonstrated to operate successfully.

The NMR imaging program is now in iull swing, with projections for development of magnets with fields up to 10 tesla as a goal for the future. This imaging program, particularly the high-field aspects, is coordinating nicely with the laboratory program in magnetic field effects on animals.

In the Radiation Biophysics program, the availability of higher mass ions up to uranium has allowed us to carry out cell and tissue studies in a radiation domain that is entirely new. Using uranium beams, investigators have already made new and exciting findings that are described in the body of the report.

To increase the effectiveness of our mutagenesis-carcinogenesis group, now formally named "Cellular and Molecular Biology," we have consolidated nearly all of the investigators concerned in one building-Building 934, which is more or less affectionately called the "Dymo Building" after its former occupants.

Our Division staff continues to provide outstanding service to the investigators, particularly at a time when our volume of new proposal submissions has increased dramatically. I wish to extend to all of tiem my particular thanks.

Also, "welcome aboarci" to our new staff members. I believe you will enjoy your participation in our programs and contribute to our continuing high-quality science. 


\section{SECTION 1. RESEARCH MEDICINE}

\section{INTRODUCTION}

The Research Medicine Group has continued to develop and apply new instruments and procedures for evaluation of the physiological state of normal and pathological processes of major diseases such as aging and atherosclerosis. Cancer, of course, is a continuing challenge, but in this area we focus on the use of noninvasive methods to aid the heavyion radiotherapy program. The two major tools we are developing in this quest to understand disease mechanisms are emission tomography and nuclear magnetic resonance (NMR). Emission tomography involves the injection of radioactive isotopes bound to chemicals that go to specific regions of the body, depending on the normal or abnormal metabolic activity in the region of interest. NMR is used to learn about physical and chemical composition of body tissues as well as enzyme activities that might be specific for disease.

Our approach is to make pictures of normal and abnormal body function. An example of this comparison appears in the Alzheimer's disease study reported in this section.

We are strongly focused on noninvasive methods for mapping body metabolism because basic principles of science dictate that energy changes must precede anatomical changes. Thus, we believe metabolic images are more sensitive than anatomic images for evaluation of aisease problems.

Accomplishments of the Fesearch Medicine Group over the last year are:

1. Establishment of a new method for brainblood-flow :neasurements in Alzheimer's disease and stroke.

2. Development of a method for labeling human blood platelets so that early signs of stroke ran be detected.

3. Development of a method for evaiuation of coronary artery disease using positron tomography and 7 rubidium- 82 generator device developed at LBL.

4. Installation of tivo new medical rejearch NiMR magnets for in-vivo studies of human metabolism in normal subjects and patier:ts with early aging or atherosclerosis.

5. Discovery of a rernarkably abnormal behavior of platelets when in blood with high. cholesterol.

Reseurch Medic ine (at a glance).
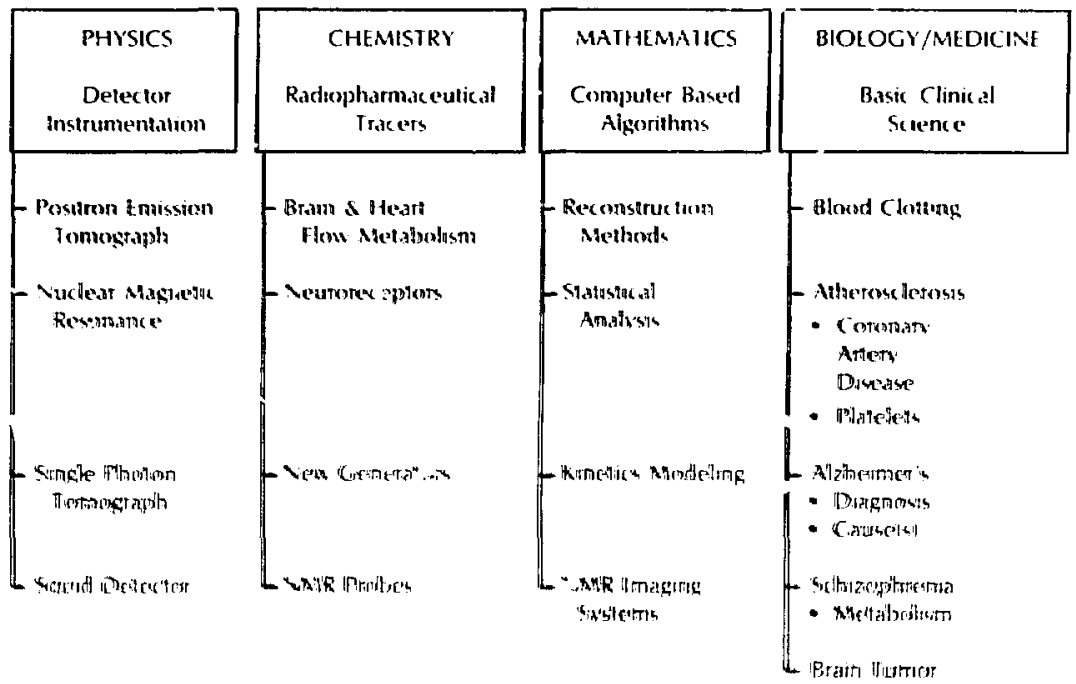
EYAUATION AND USE OF IODNE-122 EAAN LLOD FOW RADIOMHARMCEUTICALS

\author{
Chester A. Mathis, Thoonton Sargent III, Thomas F. Budinger, Yukio Yano, \\ William I. Jagust, Alexander Shulgin, Natalia Kusubov, and Kathleen M. Brennan
}

The development of positron emission tomography (PET) has provided a quantitative brain imaging device that can help correlate such physiological parameters as brain blood flow, blood volume, oxygen metabolism, glucose metabolism, rotein incorporation, and receptor site densities 1 affinities. The accurate measursment of brain blood flow would be of considerable value in the understanding of the pathophysiology of various brain diseases, including Alzheimer's disease, stroke, and radiation necrosis. When brain blood flow is measured in concert with one or more of the other cerebral function $\mathrm{a}^{\prime}$ indicators, therapeutic decisions concerning the medical treatment of patients with brain disorders can be made on the basis of more precise diagnosis.

The radiopharmaceuticals presently used to assess brain blood flow must be produced at the PET facility because of the short half-lives of their radionuclides. We have investigated the possibility of using the ${ }^{122} \mathrm{Xe} /{ }^{122}$ I generator system to provide a radionuclide that can be produced at a regional cyclotron and shipped to PET imaging centers without on-site cyclotrons. The 20-hour half-life of the ${ }^{122}$ Xe parent makes this generator system attractive for widespread use, and the 3.6-min half-life of ${ }^{i 22}$ I results in a low radiation dose to the patient. In addition, repeat studies can be performed under changed physiological conditions because the injected activity will have disappeared in $20 \mathrm{~min}$. We have previously described a generator that we load with about $200 \mathrm{mCi}$ of ${ }^{122} \mathrm{Xe}$ and from which we are able to remove $25-100 \mathrm{niCi}$ of ${ }^{122}$ l every 20 min for 2 days.'

We have investigated several compounds that can be labeled with ${ }^{122} 1$ and have evaluated their potential usefulness as brain blood-flow agents. One class of compounds is the iodo-dimethoxy$\mathrm{N}, \mathrm{N}$-dimelhylamphelamines, and we have studied several $122 /$-labelef amphetamine derivatives in animals.,3 Another compound is a substituled diamine (termed HIPCA1) that previously has been labeled with 1231 and used in single-pholon ganuma camera sludies of brain blood now. Clinic:" trials wish 123/-labeied HIPDM have demonstraled the usefulness of this agent to delect hlow defects somelimes not seen with x-tíy computed tomography. We are interested in PEI application of 122 IHIPDM because single pholon imaging devices have decreased resolution compared to PET syslems and the 13-hr half-life of ${ }^{123}$ / limits repeat studies with ${ }^{123}$ I-HIPDM.

In collaboration with Dr. Hank F. Kung of the Veteran's Administration Medical Center, Buffalo, N.Y., the rapid labeling of HIPDM with ${ }^{122}$ I (Fig. 1) has been investigated. We have labeled HIPDM with ${ }^{122} /$ rapidly $(2 \mathrm{~min})$ and in high yield $(70 \%)$. The radiopharmaceutical was ready for injection about 4 min after remicval of ${ }^{122}$ from the generator, and preliminary studies in animals indicated its potential usefulness for human brain blood flow studies.

The results of our first patient study with ${ }^{122} 1$ HIPDM are shown in Fig. 2. The patient had suffered a stroke three years previously and $x$-ray $C T$ scans had shown some deficits in the frontal area of the brain. As seen in Fig. 2, ${ }^{122}$ |-HIPDM uptake in the left frontal region (right side of the photo) is markedly diminished. Later PET images were taken following the injection of a radiolabeled sugar compound, ${ }^{18}$ F-FDG, which showed decreased metabolic function in the same brain region (Fig. 2). The dynamic uptake and retention of ${ }^{122}$-HIPOM and ${ }^{18} \mathrm{~F}$-FDG in different brain regions were determined and the data are sinown (Fig. 2). It can be seen that the ${ }^{18} \mathrm{~F}$-FDG uptake in the stroke area was about $50 \%$ that of the normal contralateral side; the uptake and retention of ${ }^{122}$ I-HIPDM in the stroke area was about $40 \%$ that of the unaffected side. Quantitative measurements of brain blood flow, metabolism, and blood volume in stroke<smiles>Cc1cc(I)cc(CN(C)CCN(C)C)c1O</smiles><smiles></smiles>

F. 1. Exchange dabeling of HIPOM with 722 j-iodide to produce DESAHIPDM.

( $\left.K B 1+5 t^{\prime}-8\right)+9$ ) 

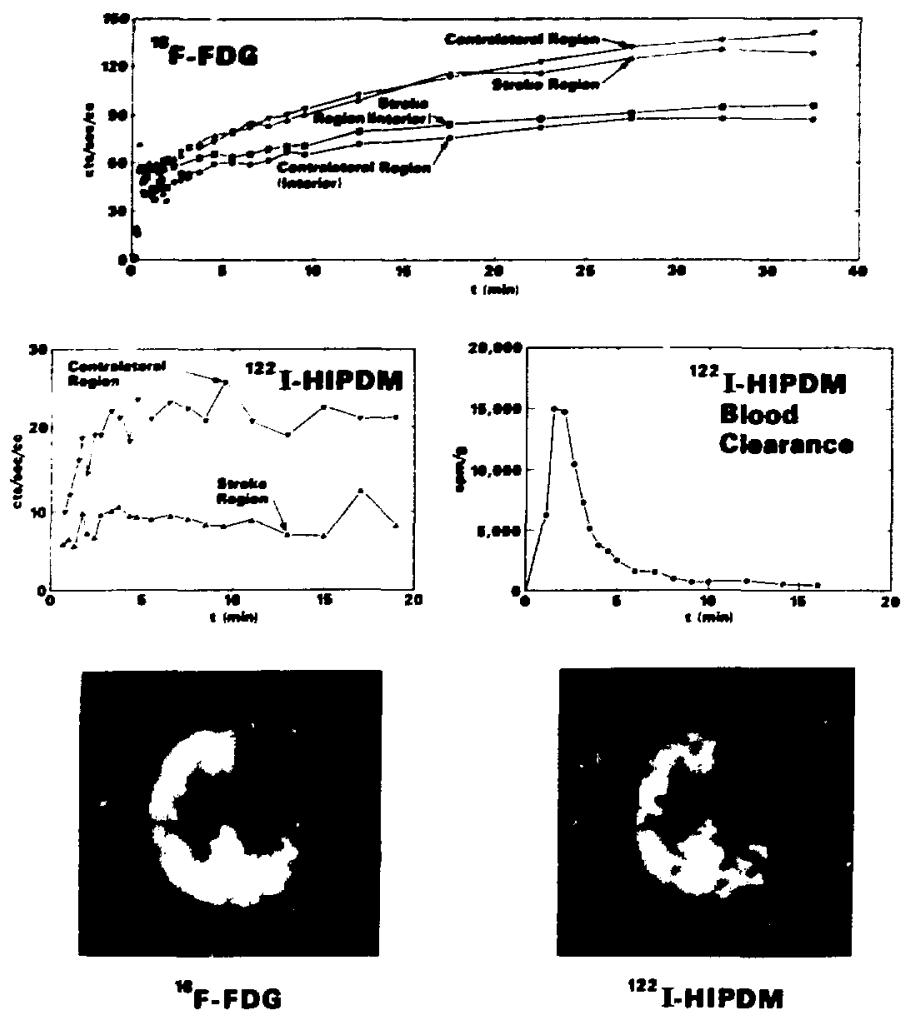

Fig. 2. ${ }^{122} /$-HIPDM and ${ }^{18} F-F D G$ in a patient with a left frontal stroke three years previously. PET images are as labeled at bottom. Dynamic PF7 data for ${ }^{18} \mathrm{~F}-F D G$ are plotted in the top panel and ${ }^{12}$ I-HIPDM data from the cortical areas only are shown in the middle left panel. ${ }^{122}$ / blood clearance data are shown in the middle right panel.

patients such as the one shown in Fig. 2 will help guide therapeutic interventions such as hyperbaric oxygen treatment and cerebral bypass surgery. We are collaborating with Dr. Philip Weinstein of the VA Hospital, San Francisco and the Neurosurgery Department. UCSF in following stroke patients before and after medical treatment to assess their progress.

Preliminary studies such as the one shown in Fig. 2 demonstrate the potential usefulness of the

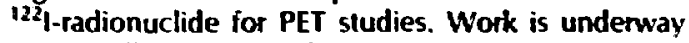
to critically evaluate characteristics of HIPOM and the iododimethoxyamphetamines, which are important in their ability to accurately measure brain Now. These include the extent of melaboiic breakdown during the uptake sludy, and the fraction that is extracted into the brain. When these comparative studies have been compleled, we will choose the best agent for development as the brain blood-flow radiopharmaceutical to be used in our research on brain dysfunction and for clinical procedures. We are: also investibating the application of the 1221radiolabel to other compounds such as albumin i.? measure brain blood volume and to fatty acid derivatives to assess heart funclion. The short half-life of the 1221 -radionuclide permits extraction from the generator every 20-30 $\mathrm{min}$, so that it can be used to label different compounds. These radiopharmaceuticals may be used in concert is measure several functional parameters in the same patient.

\section{REFERENCES}

I. Yano, Y., Budinger, T.F., Mathis, C.A., Singlh, M., Moore, $D_{\text {., }}$ and Jones, $R$. Development of radionuclides and radioptharmaceuticats for 
positron emission tomography. Biology and Medicire Division Annual Report 1983-1984, LBL-18393 (1985).

2. Mathis, C.A., Sargent III, T., Shulgin, A.T., Yano, Y., Budinger, T.F., and Lagunas-Solar, $M$. Synthesis and evaluation of metasubstituted 1-122-labeled dimethoxy-N,Ndimethylamphetamines for brain imaging. $f$. Nucl. Med. 26, P69 (1985).
3. Mathis, C.A., Sargent III, T., and Shulgin, A.T. lodine-i22-labeled amphetamine derivative with potential for PET brain blood-flow studies. I. Nucl. Med. 26, 1295 (1985).

4. Kung, H.F., Tramposch, K.M., and Blau, M. A new brain perfusion agent: [t-123] HIPOM: $N, N, N^{\prime}$-trimethyl-N'|2-hydroxy-3-melhyl-5iodobenzyl|-1,3-propanediamine. J. Nucl. Med. 24, 66 (1983).

\section{DYNAMIC PET STUDIES OF GLUCOSE UPTAKE IN ALZHEIMER'S DISEASE}

William ). Jagust, Robert P. Friedland, Ronald H. Huesman, Bernard M. Mazoyer, Yukio Yano, Chester A. Mathis, Kathleen M. Brennan, Brian Knittel, and Thomas F. Budinger

Studies utilizing positron emission tomography (PET) with $/{ }^{18}$ F/fluorodenxyglucose (FDG) in the investigation of cerebral glucose metabolism in Alzheimer's disease (AD) have been underway at the Donner Laboratory for several years. Alzheimer's disease is the most common degenerative brain disease of adulthood, and as such is responsible for 50 to $60 \%$ of all cases of progressive mental deterioration, or dementia, in adult life. We have previously found that Alzheimer's disease is characterized by glucose hypometabolism in the temporal and parietal cortex.' These findings are consistent in all the $A D$ patients we have studied, and the pattern of regional cerebral hypometabolism is now found to be useful in the clinical evaluation and diagnosis of elderly patients with impaired cognition. In fact, we have evaluated several patients with communicating hydrocephalus, a potentially reversible dementia caused by accumulation of cerebrospinal fluid in the cerebral ventricles, which is rreatable by a surgical shunting procedure. This diagnosis, however, is often difficult to confirm because patients with hydrocephalus frequently cannot be distinguished from patients with AD. We found that patients who improved following surgery did nol demonstrate the typical $A D$ paltern of temporoparietal hypometabolism, thus suggesting that PET's ability to exclude AD may be useful in selecting patients for surgen.?

Recent work has involved the correlation of these metabolic abnomalities with clinical features of AD. Thus, we have obsenved differences in metabolism in leit and right hemispheres that conteWhe wath behuviomal features of the disease and age at onset. ${ }^{3,4}$ In addition, we have found that the new methods of NMR and high-resolution $x$-ray technioues cannot detect the disease. ${ }^{5}$

PET, however, is not limited to the evaluation of regional rates of glucose metabolism, but can also be used to investigate more basic physiologic processes through the use of tracer techniques with mathematical modeling. The kinetics of glucose metabolism can be represented by a threecompartment model (Fig. 1, top) with three rate constants ${ }^{6} ; k 1$ represents transport of glucose from blood to brain, $k 2$ is the reverse transport, and $k 3$ represents the phosphorylation of glucose by the enzyme hexokinase. Because dephosphorylation occurs very slowly in brain, if at all, its rate constant, $k 4$, is not modeled.

The tracer $~^{18} \mathbf{F}$ ffluorodeoxyglucose (FDG) is utilized in these studies. Between 5 and $10 \mathrm{mCi}$ of activity are injected in the patient. Subsequently, blood is collected at frequent intervals from a hand vein for $\mathbf{6 0}$ minutes in order to derive a description of the variation of blood activity with time. Concurrenily with this, the Donner 280-crystal tomograph acquires data from the brain. Because of the high temporal resolution of the instrument we are able 10 acquire data rapidly, thus providing a good description of the time-course of brain activity. Through the use of a nonlinear iterative least squares filting procedure, we are able to fil the brain and blood time-activity cunves to the threecompartment model in ordet to derive the rate constanis for gilucose transport and phosphonylation.

Results in II patients with Nzheimer's disease and 6 healthy elderly control subjects are presented 

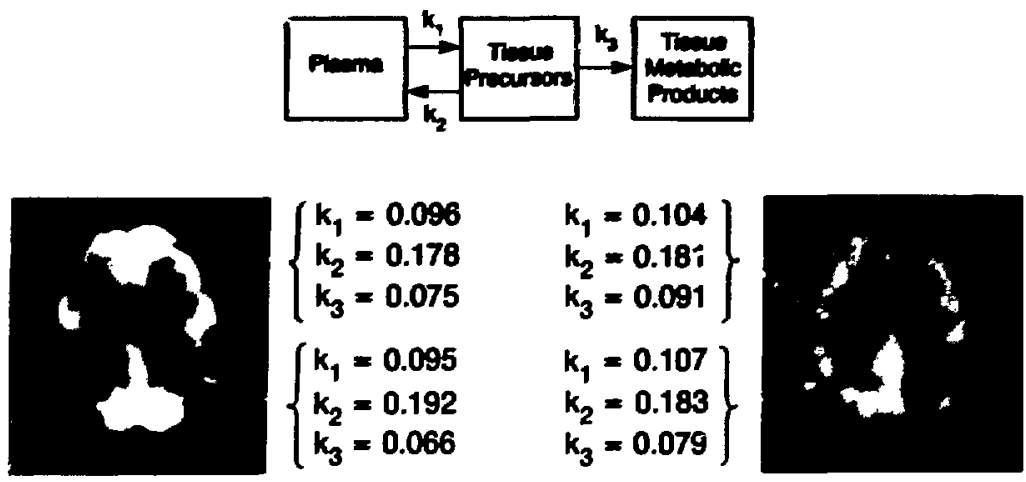

Fig. 1. Top: The three-compartment model representing forward and reverse transport ( $k_{1}$ and $k_{2}$ ) and phosphorylation $\left(k_{3}\right)$ of glucose. Bottom: Rate constants from Alzheimer patients (left) and control subjects (right). Data represent regional values for frontal and temporoparietal cortex of each group.

(XBB 850-10104)

in Fig. 1, bottom. Rate constants have been calculated for regions of frontal, temporoparietal, occipital, and entire cortex. Values shown here are those for the mean of left and right frontal and temporoparietal cortex for Alzheimer and control subjects. These results are not significantly different from each other because of the large variability of the populations. When ratios are constructed if the individual rate constants in frontal temporoparietal, and occipital cortices divided by the same rate constant in entire cortex, this variability is reduced. We then find that the ratio of $k 3$ for temporoparietal cortex to $\mathrm{k} 3$ for entire cortex is smaller in the Alzheimer patients than the controls $(p<0.02)$.

We can alsc calculate metabolic rates using the kinetic data. The local cerebral metabolic rate for glucose (LCMRglc) is given by the steady-state equation $(k 1 \times k 3) /(k 2+k 3)$ times the plasma glucose concentration. Using this method, we find that LCMRglc is significantly lower $(p<0.05)$ in temporoparietal cortex in the Alzheimer patients.

These results, in addition to demonstrating that the temporoparietal cortex is most severely involved in AD, provide new evidence that helps to localize the site of the physiological lesion. The findings of normal $k 1$ and $k 2$ in all cortical regions suggest that glucose transport is normal in the illness. The lower $k 3$, which is the rate constant for the hexokinase reaction, implicates the phosphorylation process as the factor limiting glucose metabolism in $A D$. II remains to be determined whether this occurs because of an actual abnormal. ity in the enzyme or its kinelics, or that the results reflect a normal physiologik process occurring in fewer normally funcioning cortical meurons.

\section{REFERENCES}

1. Friedland, R.P., Budinger, T.F., Ganz, E., et al. Regional cerebral metabolic alterations in dementia of the Alzheimer type: positron emission tomography with $1^{18} \mathrm{~F}$ fluorodeoxyglucose. /. Comput. Assist. Tomogr. 7, 590-598 (1983).

2. Jagust, W.J., Friedland, R.P., Budinger, T.F. Positron emission tomography with ${ }^{18}$ F|fluorodeoxyglucose differentiates normal pressure hydrocephalus from Alzheimer-type dementia. J. Neurol. Neurosurg. Psychiat. 48, 1091-1096 (1985).

3. Friedland, R.P., Budinger, T.F., Koss, E., and Ober, B.A. Alzheimer's disease: anteriorposterior and lateral hemispheric alterations in cortical glucose utilization. Neurosci. Left. 53, 235-240 (1985).

4. Koss, E., Friedland, R.P., Ober, B.A., Jagust, W.j. Latera! hemispheric asymmetries of glucose utilization are different in early and late onset Alzheimer-type dementia. Am. 1. Psychiatr. 142, 638-640 (1985).

5. Friedland, R.P., Budinger, T.F., BrantZawadzki, M., and Jagust, W.I. The diagnosis of Alzheimer-type dementia: a preliminary. comparison of positron emission tomography and prolon magnetic resonance. JAMA, 252, 2750-2752 (1984).

6. Sokołoff, L., Reivich, M., Kennedy, C., et al. The $\left.\right|^{34} \mathrm{C}$ deoxyglucose method for the meas. urement of local cerebral glucose utilization: theony, procedure, and normal values in the conscious and anesthetized albino rat. 1. Neu. rochem. 28, 897-916, (1977). 


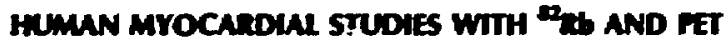

\author{
Thomas F. Encinger, Yukio Yaso, Juli A. Twitchell, Kathleen M. Brennan, Stephen \\ E. Derenzo, and Romald H. Fhesman
}

Since 1978 we have been perfecting a method for the early detection of atherosclerosis leading to heart attacks. Each day an estimated 3400 Americans, more than two each minute, suffer a heart attack. While most heart attacks are not fatal, they frequently occur in subjects who are not under physicians' care or who do not know they have a problem.

Our method employs positron emission tomography (PET) for production of pictures reflecting the integrity of blood flow to heart muscle. This procedure is believed to be far more definitive and practical than coronary angiography because the PET method shows the actual muscle capillary perfusion, whereas coronary angiography done through catheterization shows only narrowing of the coronary arteries, which is not strictly indicative of compromised heart muscle flow. After six years of development, including the improvement of the Donner 280-crystal PET device, we discovered a new procedure providing important results.

The new protocol for the examination of heart patients before and after exercise using ${ }^{82} \mathrm{Rb}$ and positron emission tomography (PET) has now been successfully completed on four patients. Shortlived ${ }^{82} \mathrm{Rb}$ (76-sec half-life) is available from a small portable generator (developed at LBL in 1968), which eliminates the need for a cyclotron.

The patient is exercised briefly on a treadmill with a cardiologist in attendance. ${ }^{82} \mathrm{Rb}$ is injected at a point of peak exercise when, in our judgement, exercise can be continued for another 60 seconds. At the end of exercise the patient is positioned in the tomograph at the appropriate transverse section, predetermined by a transmission image taken prior to exercise. A static image is acquired over the ensuing 5-minute interval. The patient is allowed to rest and another ${ }^{82} \mathrm{Rb}$ study is performed 15 or 30 minutes later.

Of the patients studied, one had an inferior posterior wall infarction and had been studied by coronary artery angiography. The rubidium study was made to ascertain if there was a margin of viable tissue in the region of this previous infarction that might benefit from angioplasty. We ascertained that such was the case because the exercise image showed a greater volume of diminished rubidium uptake than was the case during rest. Based on this study, the patient received an angioplasty.

Two patients had septal wall lesions demonstrated on thallium and angiography studies. Their ${ }^{82} \mathrm{Rb}$ studies showed a remarkable differential uptake of rubidium in the septal and left ventricular wall regions before and after exercise. Results from one of these studies are shown in Figs. 1 and 2 . We observed that the compromised muscle, which has limited blood flow, receives even less blood relative to the normal muscle during exercise. However, after exercise there is a rebound flow (hyperemia) in the compromised muscle. This leads to reversal in the ratio of uptake of $\mathrm{Rb}$ between the compromised and normal muscle.

These results are new findings, which have not been observed before because no isotope studies involving separate injections before and after exercise had been done. The reason is that radionuclides previously used do not have the short hairlife of ${ }^{82} \mathrm{Rb}$ which enables the before- and afterexcercise studies to be done.

These observations have given us strong evidence that we now have a very sensitive method for detection of coronary artery disease that entails radiation doses to the patient no greater than those received from routine back $x$ rays. 


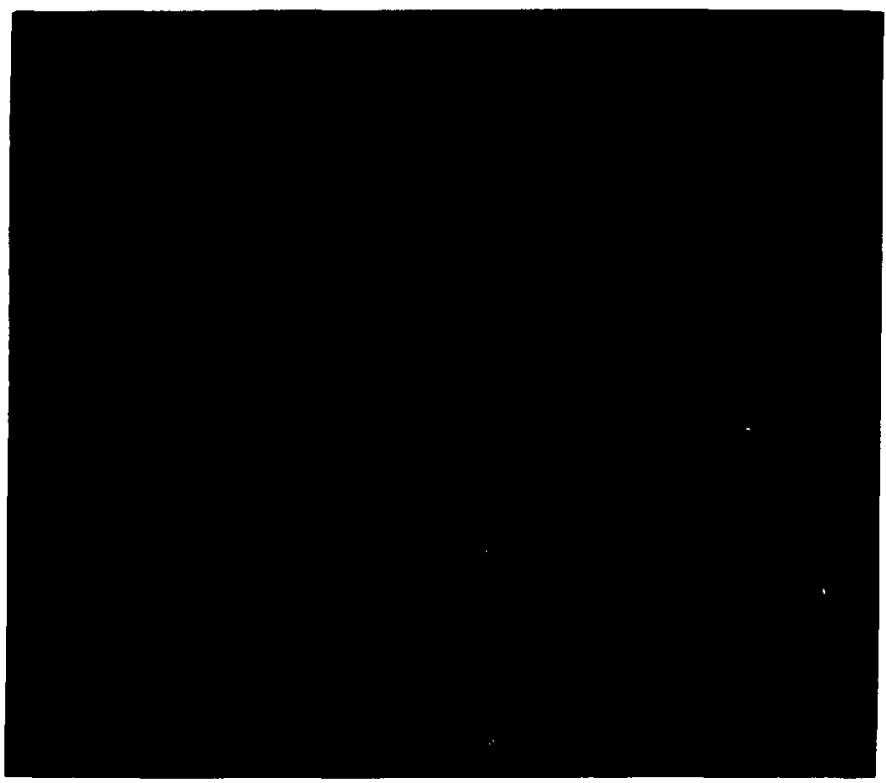

Fig. 1. Left shows decreased septal uptake during exercise. This area shows "rebound" blood flow increase affer rest. This pattern of decreased-then-increased rubidium accumulation relative to the accumulation in normal heart muscle is a new finding.

(CBB 858-6389)

30 min Resting
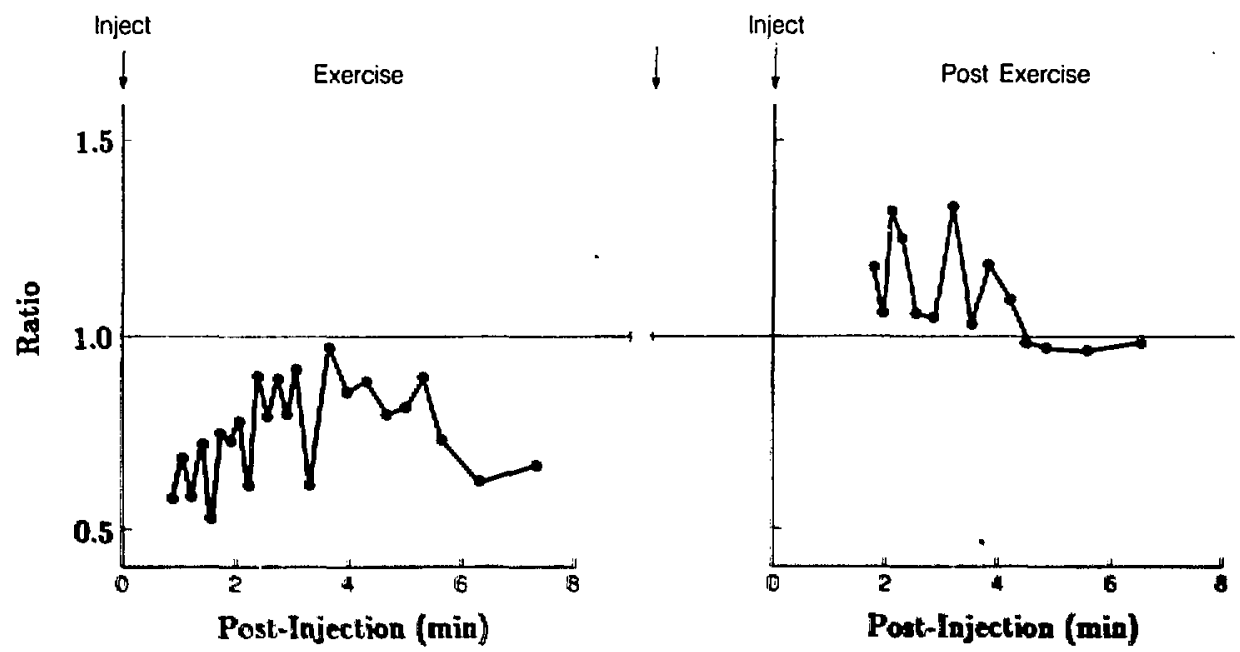

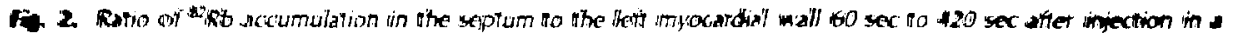

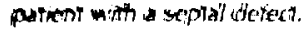




\section{FOSITRON EMISSION TOMOGRAMHY OF FDG IN SCHIZOMHRENIA}

\section{Thornton Sargent 111 and Natalia Kusubov}

Research groups studying schizophrenia at several laboratories have reported reduced frontal blood flow, reduced frontal glucose metabolism, and enlarged ventricles in the brains of patients with this disorder. We have used the Donner dynamic positron emission tomograph (PET), which has the spatial and temporal resolution to quantify metabolism of the brain more accurately than other instruments, to study fluorodeoxyglucose labeled with fluorine-18 (18-FDG) in six patients with schizophrenia and six normal controls in a collaborative study with the Palo Alto Veteran's Administration Medical Center. This radiopharmaceutica' has been widely used and studied; it is taken up by the brain and phosphorylated by the same mechanisms used for glucose metabolism, but at the step after phosphorylation the metabolism is arrested. Th: is, the quantitative localization of the positron-emitting fluorine-18 provides a quantitative image of regional glucose uptake. A mathematical model describing the metabolism has been derived by other workers, utilizing the compartment model shown in Fig. 1. Solution of the model to obtain values for $k_{1}, k_{2}$, and $k_{3}$ requires, in addition to rapid blood sampling to obtain the input function, rapid dynamic sampling of appearance of the radioactivity in the brain. The Donner PET imaging system is unique in its ability to obtain rapid s..mpling and thus obtain the $\mathrm{k}^{\prime} \mathrm{s}$ for any desired brain region in each patient. Other laboratories use the mean values from a few normal subjects to solve an operational equation that provides the local cerebral metabolic rate (ICMR) for various brain regions. In this method the static values of fluorine-18 concentration seen by PET 40 minutes after injection are used in what is known as the operational equation, a complex function incorporating many assumptions, including the average $k$ values to calculate ICMR. By use of our dynamic

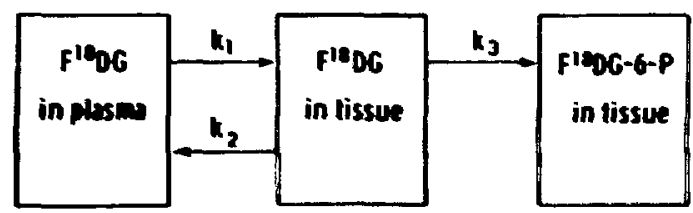

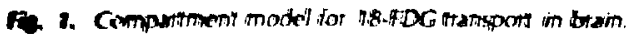

(1) $1517-4572)$
PET imaging capabilities we are able to calculate a glucose metabolic rate (GMR) from the equation

$$
G M R=\frac{k_{1} k_{3}}{k_{2}+k_{3}} C p
$$

where $C_{p}$ is the plasma glucose concentration. The dynamic brain uptake data and the blood input function are used to calculate the $k$ 's by an iterative least squares fitting program on our VAX $780 \mathrm{com}$ puter, for all regions of interest chosen in the brain.

All of the patients had been withdrawn from medications for two weeks prior to the 18-FDG study. One important difference between our study and that of other laboratories that reported reduced frontal uptake in patients with schizophrenia was that our subjects were all given an auditory vigilance task during the study. The purpose of this was to have all subjects in the same cognitive state, so that any differences seen could be attributed to underlying pathophysiogy rather than to variability of cognition. We did not observe any differences between patients and controls in relative uptake in the static 40-min FDG images in any brain region that we examined, which included the frontal lobes, the peri-sylvian, temporal-parietal and occipital cortex. However, in the four patients and three normals in whom we were able to measure the dynamic uptake data and solve the compartment model, we found differences in the values for the rate constants of Fig. 1. All three rate constants, $k_{1}, k_{2}$, and $k_{3}$ were lower on the average in the patients with schizophrenia. These differences reached statistical significance for $k_{3}$ in specific regions of the brain, mostly on the right side, as shown in Fig. 2. The 18-FDG images of patients and normal controls were not visually different; the image in Fig. 2 is one from a patient in which individual regions of the cortex, for which calculations were made, are outlined in while.

Although this is too small a group of patients on which to base conclusions, differences in $k_{3}$ were statistically significant in several brain regions; the means of $k_{1}$ and $k_{2}$ were also lower in the patients, but did not reach statistical significance. In spite of these overall lower values, the calculated GMR values were not lowered to the same degree due to the nature of the equation for GMR and the operational equation for KCMR, as shown in Table 0 .. That is, since $k_{3}$ appears in both the 


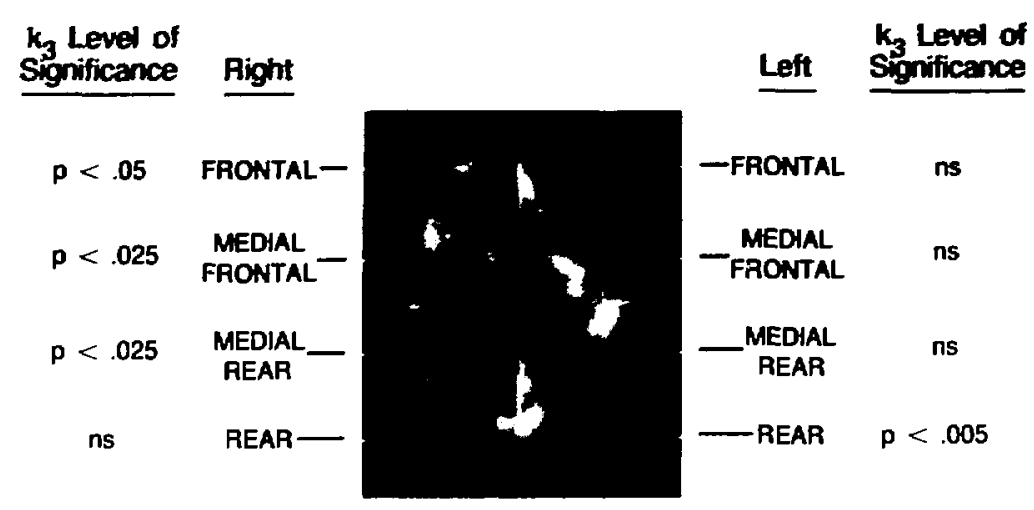

Fig. 2. ${ }^{18}$ FDG concentration (in white) in transverse section of brain of a patient with schizophrenia; normal controls were not visually different. White outlines are regions for which k's were calculated, and the level of significance (t-test) between normals $(n=3)$ and patients $(n=4)$ for each region is shown in the side columns (ns $=n o t$ significant).

(XBB 850-10176)

numerator and denominator, if it is reduced and $k_{1}$ and $k_{2}$ are also reduced, the value of GMR obtained from the equation is relatively insensitive to such changes in the $k^{\prime} s$. Thus it appears possible that there may be differences in glucose transport and metabolism in the brain that are not as apparent from simple static measurements used for ICMR calculations, or even from the GMR. The physiologic association customarily made is that $k_{1}$ represents 18-FDG uptake by the cell from circulating blood and that $k_{3}$ is the rate of phosphorylation. Our results suggest that these rate parameters may change in schizophrenia, although probably as a result of some other basic pathophysiology, and we will study further patients to provide a better statistical basis for these observations.

Table 1. Mean values of transport parameters calculated for patients and controls.

\begin{tabular}{lllr}
\hline & \multicolumn{2}{c}{ Mean values for all cortical regions } \\
\cline { 2 - 4 } & $\begin{array}{l}\text { Normal } \\
\text { controls }\end{array}$ & $\begin{array}{c}\text { Unmedicated } \\
\text { patients }\end{array}$ & $\begin{array}{c}\text { Percent } \\
\text { difference }\end{array}$ \\
\hline$k_{1}$ & 0.124 & 0.107 & $-13.7 \%$ \\
$k_{2}$ & 0.196 & 0.173 & $-11.7 \%$ \\
$k_{3}$ & 0.0996 & 0.0727 & $-27.0 \%$ \\
GMR & 3.40 & 3.32 & $-2.3 \%$ \\
ICMR & 3.84 & 3.25 & $-15.3 \%$ \\
\hline \hline
\end{tabular}

\section{GALLIUM-68 LABELED PLATELETS AND POSITRON EMISSION TOMOGRAPHY FOR DETECTING VASCULAR LESIONS}

Yukio Yano, Kathleen M. Brennan, Mohindar Singh, Julia A. Twilchell, Dorothy A. Carpenter, Elizabeth M. Mazoyer, Shirley N. Ebbe, Kanu Dalal, Ronald H. Huesman, Chester A. Mathis, and Thomas F. Eudinger

The enthusiasm for platelet accumulation studies is based on substantial evidence that platelet deposition at sites of antetial injury play a central rote in arteriothrombosis and altherogenesis. The rechnique of platelet labeling and imaging, particu- larky using high resolution positron-emission tomography, may permit the evaluation of localized pla. tetel deposition on vascular lesions. Imaging capable of assessing platelet activity and distribution would be invaluable in the evaluation of platelet 
dynamics associated with high risk for stroke and in following the kinetics of platelet accumulation noninvasively in the body for detecting clinical complications of atherosclerosis before embolization occurs.

Human and animal platelets have been labeled with ${ }^{67} \mathrm{Ga},{ }^{68} \mathrm{Ga}$, ${ }^{99 m} \mathrm{Tc}$, and ${ }^{111}$ In for imaging studies. $\quad 111$ In-labeled platelets demonstrate normal functional characteristics of the more traditional ${ }^{51} \mathrm{Cr}$ platelets. ${ }^{111}$ In-labeled autologous platelets have been used to detect carotid atherosclerosis in man, to measure thrombogenicity of arterial grafts and to identify sites of peripheral balloon angioplasty. However the use of ${ }^{11}$ In-platelet imaging is limited to a dose of only $300 \mu \mathrm{Ci}$ due to the radiation dose from the $67-\mathrm{h}$ half-life of ${ }^{111} \mathrm{In}$. Furthermore the ${ }^{111}$ In activity from platelets in the general circulation causes interference in normal single projection imaging. A solution to these problems might lie in the use of platelets labeled with generator-produced ${ }^{68} \mathrm{Ga}$, a positron emitter with a half-life of $68 \mathrm{~min}$. Using proper instrumentation for positron imaging, the sensitivity for detection of ${ }^{68} \mathrm{Ga}$-labeled platelets can be 10 or more times greater than for single-photon imaging. Of equal importance is the fact that due to the short-half life of ${ }^{68} \mathrm{Ga}$ a dose 10 or more times greater than ${ }^{111} \mathrm{In}$ can be injected. Thus ${ }^{68} \mathrm{Ga}$-labeled platelets should provide a sensitivity of 100 times more than the ${ }^{111}$ In-platelet procedure. In addition, cross-sectional positron tomographic methods are able to provide quantitative data with a resolution of $2.5 \mathrm{~mm}$, which is not available with single-photon imaging.

\section{GALLIUM-68 LABELLED PLATELETS AND ANIMAL STUDIES}

From work reported last year it was found that ${ }^{68} \mathrm{Ga}$ complexed to the lipophilic ligand mercaptopyridine- $\mathrm{N}$-oxide $\left({ }^{68} \mathrm{Ga}\right.$ MPO) gave the best platelet labeling results of $43-63 \%$ with both human and rabbit platelets.

The platelets used in these studies were separated by centrifugation from $35-45 \mathrm{ml}$ of rabbit blood collected in Squibb modified acid citrate dextrose. The platelet-rich plasma (PRP) was separated from the red cells; then the platelets were separated irom the PRP by centrifugation. The separated platelets were suspended in saline and incubated with the Ga MPO complex. After centrifugation, the free Ga was removed in the supemalant, and the "Go-labeled platelets were resuspended in saline for intravenous infusion into scraped-aonta rabbil models for PET imaing and issure distribution studies of the ca platelets.
The ${ }^{60} \mathrm{Ga}$-labeled platelets were evaluated in animal studies using New Zealand white rabbits weighing 3-4 kg. The scraped-aorta rabbit models were prepared about $10-20 \mathrm{~min}$ before intravenous injection of the ${ }^{68} \mathrm{Ga}$-labeled platelets. An embolectomy catheter was introduced intrafemorally and pushed to the aorta arch. The bulb was inflated to about the same pressure each time, and the aorta was scraped three times before the catheter was removed.

The ${ }^{68} \mathrm{Ga}$ platelets were administered intravenously, and whole body PET images (Fig. 1) were obtained with the Donner 280 bismuth germanate ( $B G O)$ detector positron tomograph. The animals were sacrificed, and the percent uptake of the injected tracer was determined for selected tissues as well as for the normal and scraped aorta. Enface autoradiography of the aortic wall was also used to demonstrate the regional uptake of the labeled platelets on the exposed media smooth muscle layer. Normally, intimal endothelium protects the medial layer from platelet adherence and degranulation. The removal of the endothelial layer from the vessel was confirmed by the uptake of Evans blue dye on the aortic wall when the dye was injected $30 \mathrm{~min}$ before sacrifice. The percent uptake of the injected dose (ID) of ${ }^{68} \mathrm{Ga}$ platelets in selected organs and aorta is seen in Table 1.

The spleen has the greatest concentration of the ${ }^{68} \mathrm{Ga}$ platelets with a range of $1.9-6.1 \% / \mathrm{g}$. Rabbit \#3 data were excluded because of acute trauma to the aorta due to over-inflation of the balloon cathether. Using the carotid arteries and the proximal aorta for normal aortic uptake ( $n=8$ ) gives $0.04 \pm 0.01 \%$ ID in normals. For the scraped aorta $(n=3)$ there was $0.15 \pm 0.06 \%$ of ID in damaged aorta. Nearly four times more uptake occurred in the damaged endothelium of the aorta compared to the normal. The lung uptake has a wide range from 0.13 to $1.30 \%$ ID. In some animals the lungs were abnormally enlarged and showed infiltration with granular clusters. It is kncwn that trauma or endotoxins can cause pulmonary platelet aggregation. The damaged aorta shewed staining wilh Evans blue dye and about four times the radioactivity compared to the normal dorta as determined by autoradiography and den, itometer measurements.

Plicielet viability, i.e., the ability to recirculate, is a stringent test of the integrity of labeled platelets. Survival of labeled platelets also gives an estimate of the permanence of the attachment of the radioisotope to platekets. Longer-lived ${ }^{67} \mathrm{Ca}\left(\mathrm{l}_{1 / 2}=\right.$ $3.2 \mathrm{~d}$ ) was treated identically to ${ }^{6} \mathrm{Ga}$ and babeted to 

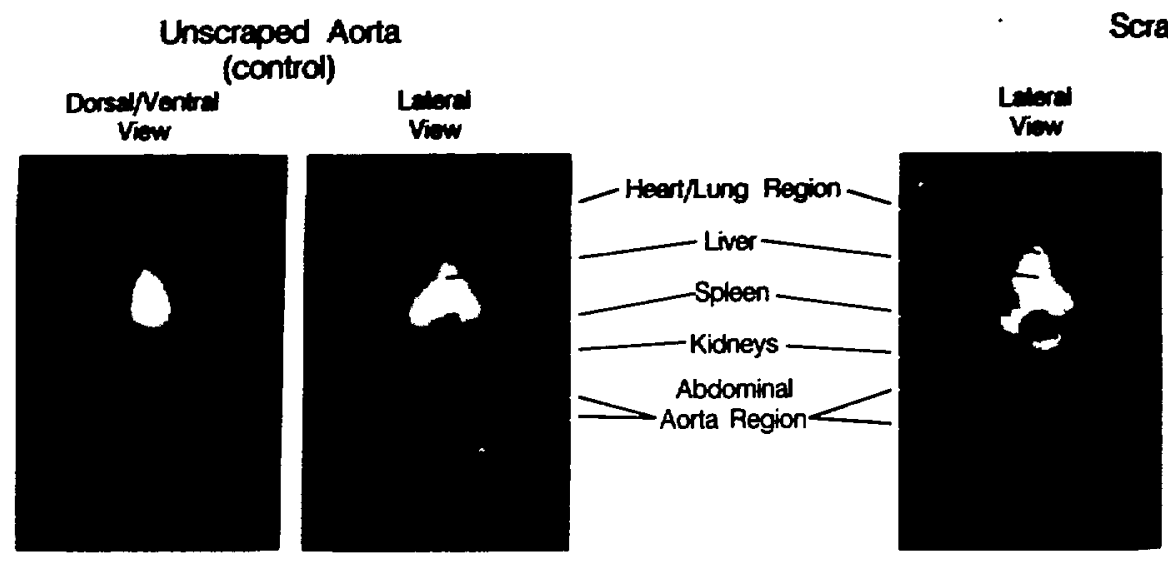

Scraped Aorta

$60-70 \%$ Labelling Efficiency of $\mathrm{Ga}^{68}$ MPO. onto Platelets

Final Platelet Suspension for injection:

$$
\begin{aligned}
& \text { 5-10 Million Platelets/cc } \\
& 4-5 \mathrm{cc} \text { Injected into Rabbit } \\
& \sim 1 \mathrm{mCi} \mathrm{Ga} a^{68} \text { activity }
\end{aligned}
$$

Fig. I. ${ }^{60} \mathrm{Ga}$ platelet imaging in scraped-aorta rabbit model by positron emission tomography whole-body scans.

\begin{tabular}{|c|c|c|c|c|c|c|c|c|c|}
\hline & $\begin{array}{l}\text { Wherı } \\
\text { aorta } \\
\text { scraped }\end{array}$ & $\begin{array}{l}\text { Carotid } \\
\text { arteries }\end{array}$ & $\begin{array}{l}\text { Proximal } \\
\text { aorta }\end{array}$ & $\begin{array}{l}\text { Distal } \\
\text { aorta }\end{array}$ & Spleen & Heart & Lung & $\begin{array}{l}\text { Kidney } \\
\text { cortex }\end{array}$ & Liver \\
\hline Rabbit $\# 1$ & $\begin{array}{l}20 \mathrm{~min} \\
\text { before inj }\end{array}$ & 0.03 & 0.03 & 0.11 & 4.65 & 0.06 & 0.13 & & 0.23 \\
\hline Rabbit 2 & $\begin{array}{l}\text { Immediately } \\
\text { before inj }\end{array}$ & 0.05 & 0.05 & 0.22 & 1.89 & 0.07 & 0.50 & 0.10 & 0.21 \\
\hline Rabbit $\# 3$ & $\begin{array}{l}\text { Immediately } \\
\text { after inj }\end{array}$ & 0.02 & 0.09 & $4.88^{b}$ & 10.24 & 0.13 & 0.44 & 0.10 & 0.28 \\
\hline Rabbit 14 & $\begin{array}{l}\text { Immediately } \\
\text { after inj }\end{array}$ & 0.02 & 0.04 & 0.13 & 3.02 & 0.03 & 0.31 & 0.05 & 0.33 \\
\hline Rabbit 15 & $\begin{array}{c}\text { Control } \\
\text { not scraped }\end{array}$ & 0.04 & 0.04 & 0.06 & 6.06 & 0.14 & 1.30 & 0.08 & 0.28 \\
\hline
\end{tabular}

Table 1. Uplake of ${ }^{60}$ Ga platelets (\% injected dose/gram fissue).

Average Uplake Ratio $\frac{\text { Scraped distal aonta }}{\text { Normal proximal zorta }}=3.77$

tDoes not include distal sorta from Rabbit $\$ 3$.

'Dislal sorta on Rubbit "3 was very damaged. 
rabbit platelets to determine the recovery and survival after intravenous injection into normal rabbits. Results were compared to ${ }^{51} \mathrm{Cr}$-labeled platelets and are presented in Table 2.

These results demonstrate the feasibility of labeling viable rabbit platelets with ${ }^{67} \mathrm{Ga}$ or ${ }^{68} \mathrm{Ga}$. Their survival in vivo is similar to that of ${ }^{57} \mathrm{Cr}$ labeled platelets.
Table 2.

\begin{tabular}{llccr}
\hline \hline Isotope & Medium & $\begin{array}{c}\text { 1-hour } \\
\text { recoveny (\%) }\end{array}$ & $\begin{array}{c}\text { Survival, days } \\
\text { (extrapolated) }\end{array}$ & n \\
\hline${ }^{51} \mathrm{Cr}$ & plasma & $50-100$ & $3-4$ & 7 \\
${ }^{51} \mathrm{Cr}$ & saline & $60-100$ & 4 & 5 \\
${ }^{67} \mathrm{Ga}$ & saline & $43-104$ & $3-4$ & 19 \\
\hline
\end{tabular}

\section{ABNORMALITIES OF BLOOD PLATELETS IN RABBITS WITH DIETARY HYPERCHOLESTEROLEMIA AND ATHEROSCLEROSIS}

Elisabeth Mazoyer, Kanu Dalal, Dorothy Carpenter, Kathleen Brennan, Tamlyn Yee, Bernard Mazoyer, Robert Leven, and Shirley Ebbe

Atherosclerosis is a common disease that accounts for extensive morbidity and mortality in human beings, but its causes and pathogenesis are not well understood. Currently available diagnostic procedures usually permit diagnosis of the disease only when it is far advanced. Because of the difficulty in detecting and studying early vascular lesions in human beings, animal models have been used for development of better diagnostic procedures and study of etiologic factors. This report will describe some aspects of our preliminary observations of rabbits that were fed a high cholesterol diet to induce atherosclerosis.

Rabbits were fed a diet consisting of $1 \%$ cholesterol and $4 \%$ peanut oil. At two-week intervals, complete blood counts, serum cholesterol, platelet volume, and platelet lipids and protein were determined. After 2 to 3 months on the diet, the survival of homologous platelets labeled with ${ }^{51} \mathrm{Cr}$ was measured. The animals were then killed, and their aortas were removed and examined grossly and microscopically.

Hypercholesterolemic rabbits developed pronounced atherosclerotic lesions of the aorta (Fig. 1). On microscupic sections, the lesions showed subendothelial infiltration with a substance that stained with the lipid-specific stain, oil red $O$. One of the purposes of this project was to develop an animal model that would be appropriate to use for testing the feasibility of noninvasively imaging vascular lesions by positron emission lomography or other techniques. The reproducibte and rapid development of vasculat lesions makes this a usetiul animal model for detection of substances that interact with lipid. Iaden vessel walls.
It is thought that blood platelets may play a role in the production of atherosclerotic lesions by interacting with the vessel wall in areas where the endothelium is damaged. However, studies by others of the aortas of monkeys that were fed an atherogenic diet indicated that platelet adherence to the vessel wall occurred late in the course of dietinduced atherosclerosis relative to the subendothelial accumulation of lipid. In addition to their proposed role in the pathogenesis of vascular lesions, the possibility that blood platelets themselves may be abnormal in hypercholesterolemia
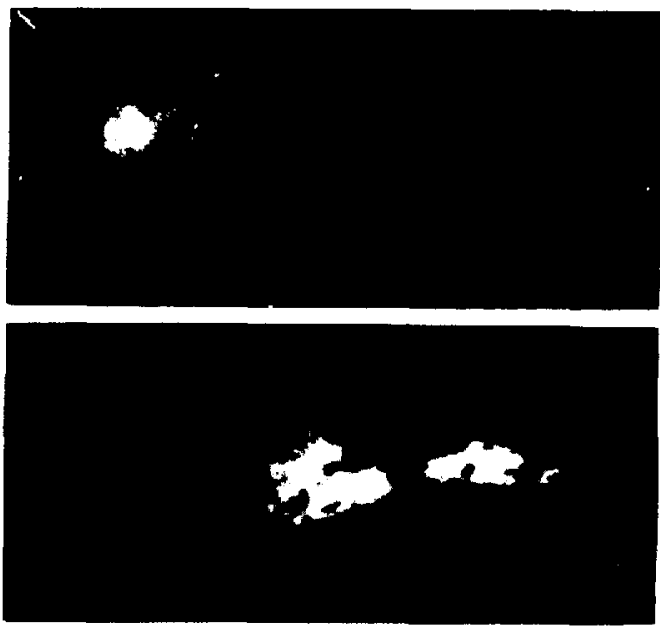

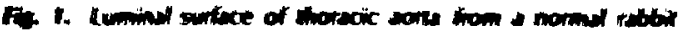

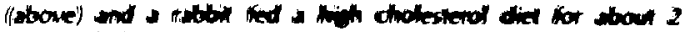

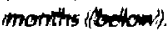


has been reported. Functional abnormalities may differ in different species, but they indicate that platelets may be hyperreactive to stimuli that induce aggregation. Platelet abnormalities do not appear to result from exchange of plasma lipids. Rather, the platelets are probably abnormal when produced by the megakaryocytes in hypercholesterolemic subjects. These reports by others support our findings that the platelets in hypercholesterolemic rabbits are not only chemically abnormal but are small in size.

Rabbits fed the high cholesterol diet rapidly developed hypercholesterolemia, with serum cholesterol increasing to about $1500 \mathrm{mg} \%$ after 8 weeks (normal $=<100 \mathrm{mg} \%$ ). After 2 weeks on the diet, platelet counts gradually increased, so that after 8 weeks, they were about $70 \%$ greater than those of control rabbits. The difference between diet and control animals was amplified by the fact that platelet counts of control animals (also bled every 2 weeks) declined during the course of the experiments; the reason for this decline is not evident. Platelet volumes were measured by three different methods: 1) platelets in samples of whole blood were electronically sized in an instrument with a hydrodynamically focused aperture tube; 2 ) platelets in platelet rich plasma were sized by Coulter counter, and the possible contribution of lipid droplets to the result was eliminated by correcting each result by "sizing" samples of platelei poor plasma; and 3) platelets separated from plasma by Stractan gradient were sized by Coulter counter. Absolute values for platelet volumes varied somewhat depending on the method, but the differences between platilets from control and diet rabbits were found with all three methods. After 2 weeks, platelets of animals fed a high cholesterol diet were consistently smaller than those of controls. The difference was due to two factors: a pronounced decrease in size of platelets in hypercholesterolemic rabbits and a moderate increase in size in controls. After 8 weeks, the average volume of platelets of diet rabbits was about $70 \%$ that of controls.

Even though the platelets were smaller than controls, they contained more lipid (1575 vs. 737 $\mu \mathrm{g} / 10^{9}$ platelets) and more protein (2330 vs. 1768 $\mu \mathrm{g} / 10^{9}$ platelets). The lipid increase was due to an increase in free cholesterol and was associated with a reduction in total phospholipid. The molar ratio of free cholesterol to total phospholipid was increased (3.5 vs. 0.5). The significance of these changes to platelet function, prostaglandin metabolism, and fatty acid composition of platelets is under investigation.

The survival of normal homologous platelets in hypercholesterolemic rabbits was normal, suggesting that the observed platelet abnormalities were not due to damage inflicted by circulation through atherosclerotic vessels. Preliminary observations suggest that the survival of the small, biochemically abnormal platelets produced by the hypercholesterolemic rabbits may be shorter than normal. Studies of the kinetics of production and destruction of platelets are continuing.

Platelet and vascular abnormalities are prominent under the experimental conditions described. However, it is clear that these conditions do not accurately mimic human atherosclerosis. Rabbiis tolerate the diet for no more than 3 months. They gradually become anemic with . canthocytosis and reticulocytosis, and develop fatty infiltration of their livers and other tissues. The possibility that the platelet abnormalities may represent an enlightening caricature of more subtle changes that have been described in other species encourages further study.

\section{IN VITRO MATURATION OF BONE MARROW MEGAKARYOCYTES}

\section{Robert M. Leven, Marilyn Yee, and Cheryl Lynn Tangusg}

The maturation of bone marrow megakaryocytes is being studied in order to understand the mechanisms by which platelet formation is controlled in normal animals and in conditions of induced thrombocytopenia. Present studies are investigating the eifiect of plasma from thrombo. cylopenic rabbits on megakary ocyte momphology. It has been shown that a fraction of plasma from thrombocyloperic rabbits can stimulate thrombopoiesis in vivo, bul no direct effect of this factor, called thrombopoietin, on malure megakaryocytes has previously been demonstrated.

For these experimenls megalkaryocyles are iso.

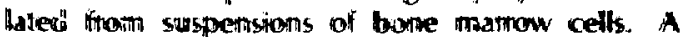


combination of density gradient centrifugation and velocity sedimentation is used to prepare megakaryocytes that are purified up to 900 -fold compared to the starting material (Fig. 1). The cells are maintained in liquid culture for up to $\mathbf{4 8}$ hours with little loss of viability.

To prepare crude thrombopoietin, citrated plasma is collected from control rabbits and from experimental rabbits 4 hours postinjection of antiplatelet antiserum. Platelet count and hematocrit are measured to ensure that the animals are thrombocytopenic without any anemia. The plasma is fractionated by ammonium sulfate precipitation. The fraction of proteins that precipitates between $60 \%$ and $80 \%$ saturation is collected, solubilized, dialyzed and concentrated. This fraction from the plasma of control animals or thrombocytopenic animals is added at one tenth volume to cultures with isolated megakaryocytes.
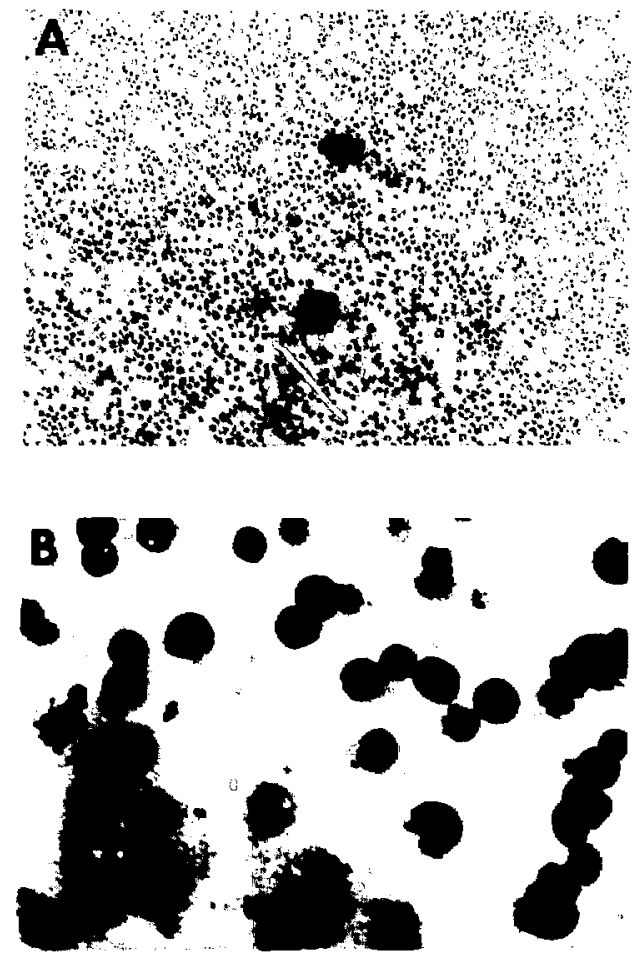

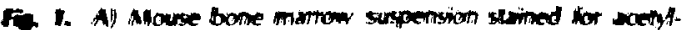

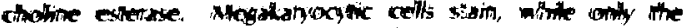

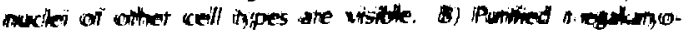

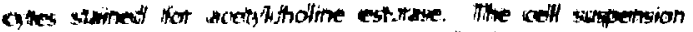

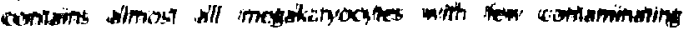
corits.

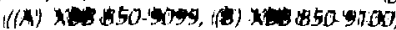

Normal megakaryocytes, when isolated in liquid culture, are spherical (Fig. 2). After 12-18 hours incubation with the thrombocytopenic plasma fraction, $5 \%-7 \%$ of the cells form many long cytoplasmic ertensions (Fig. 3). The cytoplasm becomes attenuated and may actually fragment. It has been postulated that the formation of cytoplasmic processes may be an important step leading to platelet formation, but although in our culture conditions there is some cytoplasmic fragmentation, it remains to be shown if true platelets are formed.

The cytoskeletal basis for the stimulation of megakaryocyte morphogenesis is also under investigation. Colchicine and vincristine, which disrupt microtubules, inhibit the effect of the thrombocytopenic plasma. Unexpectedly, cytochalasin B, whicn disassembles microfilaments, seerrs to mimic the effect of the thrombopoietin preparation, causing almost all of the megakaryocytes in the culture to form cytoplasmic processes (Fig. 4). Therefore it appears that intact microtubules are requires for the effect of the thrombocytopenic plasma on megakaryocyte morphology, while the role of microfilaments remains obscure.

The possible role of cyclic AMP in this stimulation is also of interest. Drugs that affect cyclic AMP metabolism have been shown to profoundly alter the morpholosy of platelets and megakaryocytes. We have iound that the phosphodiesterase inhibitor isobu'ylemethylxanthine can reverse the stimulation of cytoplasmic process formation if it has not pr'sgressed too far. The very long processes are not affected, while shorter ones retract.

These studies show that a still poorly defined factor in the plasma of thrombocytopenic rabbits,

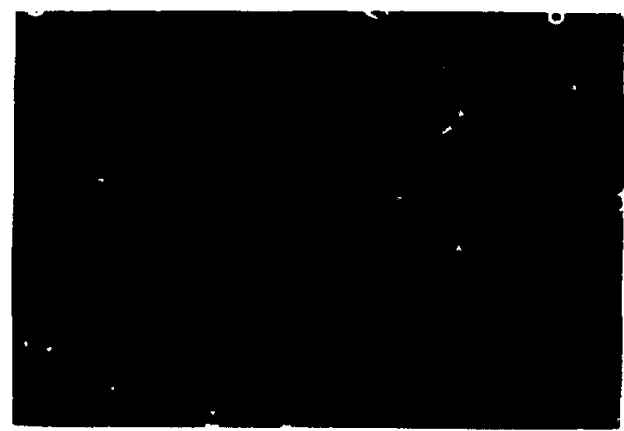

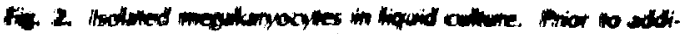

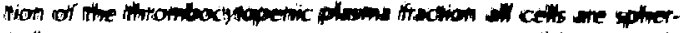
ucit! 


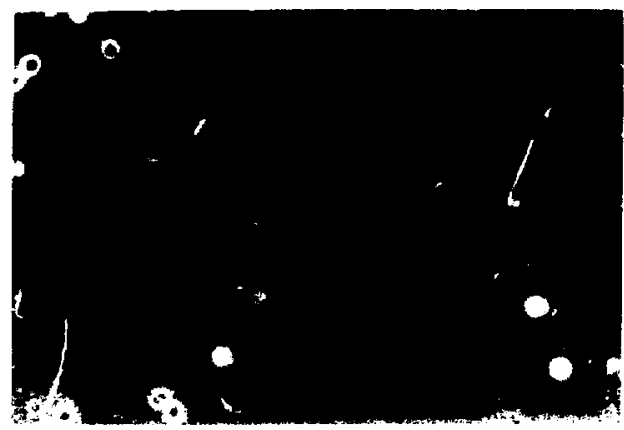

Fig. 3. Isolated megakaryocytes incubated with the thrombocyfopenic plasma fraction for 18 hours. The formation of cytoplasmic processes and the attenuation of the cytoplasm are apparent in the center call.

(XBB 850-90\%)

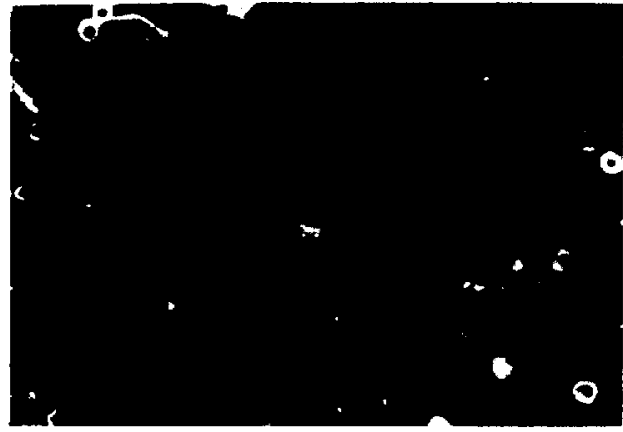

Fig. 4. Isolated megakaryocytes incubated for 18 hours in the presence of the thrombocytopenic plasma fraction and $10 \mu \mathrm{g} / \mathrm{m} /$ of cytochalasin B. Muny more cells react, and the cytoplasm begins to fragment.

(XBB 850-9u97) but not normal rabbits, is capable of stimulating morphological change in megakaryocytes thought to be similar to that which occurs during platelet formation in vivo. This implies that a possible mechanism by which animals compensate for thrombocytopenia could be through the direct action of a humoral agent on mature megakaryocytes, stimulating their platelet forming activity. This is in contrast to control of platelet formation by an increase in the number or maturaiion rate of megakaryocyte precursor cells, a very different mechanism of altering thrombopoiesis.

\title{
RELATIONSHIP BETWEEN MEGAKARYGCYTE SIZE AND PLOIDY IN MICE DLRING. RECOVERY FROM ACUTE IMMUNOTHROMBOCYTOPEN!A
}

\author{
Shirley Ebbe, Tamlyn Yee, Dorothy Carpenter, and Elizabeth Phal n
}

Megakaryocytes in the bone marrow are normally polyploid cells, and the final size of a megakaryocyte is determined by its ploidy. Siace the size of a megakaryocyte determines the cytoplasmic mass that fragments into platelets, ploidy is also a determinarit of the mass of platelets producted by a megakaryocyte. When the level of blood platelets is experimentally perturbed, megakaryocyte ploidy and size are adjusted to change the rate of platelet production in the direction of nurmalizing the platelet count; these changes eccur together with changes in th. number and maturation rate of megakaryocytes. These findings show that there is a feed-back mechanism s; which the rate of prociuction of platelets is regulated by their number in the blood.

Macronreyakaryocytosis occurs in response to depletion of blood platetets, and the ploity distribution of megakanyocytes shifts to higher values.
The present experiments were done to determine if the shift in ploidy accounts for all the macrocytosis or if size changes would occur within ploidy groups. The size are ploidy of individ"' $i$ ! mouse megakaryocytes of a defined morpholosical class were compared from 4 hours to 4 days after thrombocytopenia was produced $x y$ an injeciien of guinea pig antimouse platelet serum (APS). The areas of post-DNA syithetic mature (stage III) megakaryocytes were measured in bonc marrow smears stained with Wiright and Gien sa stains. The smears were destained, restained by the Feulgen technique, and the ploidy of the same celis was measured by two-wavelength m.arospectrophotometry. Small segmented nuclei were measured as $2 \mathrm{~N}$ reference cells.

Of 78 , nomal stage lil megakaryocytes, $0.1 \%$ were $4 \mathrm{~N}_{n} 8.7 \% 8 \mathrm{~N}_{n}, 72.1 \% 16 \mathrm{~N}, \quad 18.0 \% 32 \mathrm{~N}$, and $1.0 \% 64 \mathrm{~N}$. From 8 so $54 \mathrm{~N}$, mean siza increased by 
a factor of 1.4-1.6 between ploidy groups, but there was a considerable amount of overlap of sizes between ploidy groups. Four hours after a single injection of APS, platelet counts of the mice were less than 5\% of normal, and recovery from the thrombocytopenia occurred gradually over several days. Changes in ploidy distribution of stage III megakaryocytes occurred on days 2-3 after the injection of APS and were maximal at day 2.5 $(1.4 \% 8 \mathrm{~N}, 47.2 \% 16 \mathrm{~N}, 45.8 \% 32 \mathrm{~N}, 4.2 \% 64 \mathrm{~N}$, and $1.4 \% 128 \mathrm{~N}$ ). The mean size of megakaryocytes of all ploidy groups paralleled the proportion of $32 \mathrm{~N}$ and $64 \mathrm{~N}$ cells, indicaling that ploidy was a major determinant of overall megakaryocyte size. Mean size of all stage III megakaryocytes was maximally increased to $155 \%$ of normal on day 2.5 . In one experiment, $32 \mathrm{~N}$ megakaryocytes were $22 \%$ larger than normal $32 \mathrm{~N}$ megakaryocytes $(\mathrm{p}<0.005) 2$ days after injection of APS, showing that megakaryocyte size may increase out of proportion to ploidy in some cases. However, in thres experiments there was no change in size within ploidy groups (Fig. 1).

These results show that the main cause of macrocytosis of megakaryocytes in response to the stimulatory effect of acute thrombocytopenia is a shift to higher nuclear DNA content. Size may occasionally increase within the $32 \mathrm{~N}$ ploidy group, suggesting that this group of cells may be more responsive to the demands imposed by acute thrombocytopenia than the others. The mechanism of macrocytosis within the $32 \mathrm{~N}$ group is not known, but it may be related to the acceleration of maturation that occurs in response to platelet depletion.
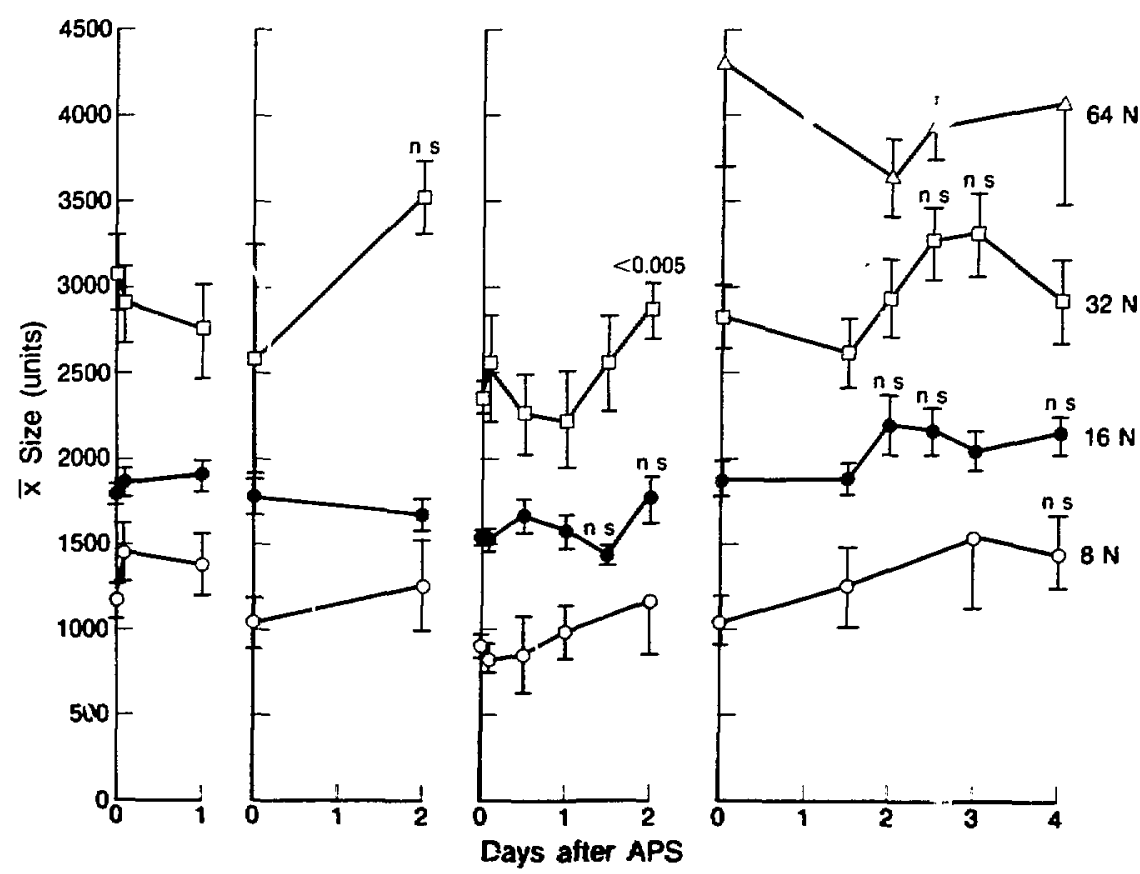

Fis. 1. The results of iour experiments in which sizes and ptoidy of individual stage III (mature, plateles-producing) megakanocites were measured atier acute thrombocyopenia was produced by a single injection of APS. The ordi. mates show the alerase sizes of megilangacyes nithin exch ploidy group; the abscissas shotv lime after injection of APS. Changes in size that mere not splistically significant (Student's rest, $p>0.05$ ) are designaled as "In" prot

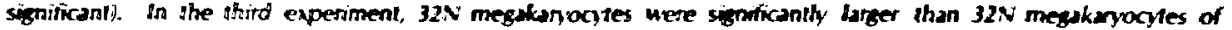
uninjected controls 2 days afier injection of APS.

(XBY 858-8440) 


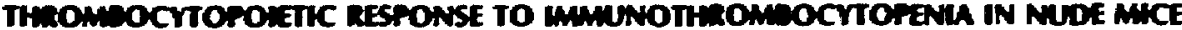

\author{
Shirley Ebe, Iack Levin, Katbleen Miller, Tanilyn Yee, Francine Levin, and \\ Elizabeth Fhalen
}

It is known that platelet production is regulated by feed-back mechanisms thit are, in turn, regulated in part by the level of circulating platelets. Thus induction of immunothrombocytopenia in normal mice by injection of heterologous antiplatelet antibodies, is followed by compensatory increases in megakaryocytopoiesis and platelet production to correct the platelet deficit. A humoral thrombopoietin appears to account for this stimulation of trrombocytopoiesis, but the cellular mechanisms responsible for determining the level of thrombopoietin are unknown.

Hemopoietic tissues contain cells that form colonies of differentiated cells when cultured with appropriate conditioning medium in vitro. The precursors of the colonies are thought to be committed stem cells for the individual types of myeloid cells, and they include those that form megakaryocytes (Meg-CFC), granulocytes and macrophages (GM-CFC), and erythrocytes (BFU-E and CFU-E). Conditioning medium for Meg-CFC is commonly prepared from mitogen stimulated $T$ lymphocytes, thus introducing the possibility that $T$ lymphocytes may have a role in meditating the regulation of platelet production in vivo. It has been further suggested that the thrombocytopoietic stimulatory effects of acute imr unothrombocytopenia could be partly mediated by $T$ cell activation by the antigenantibody reaction that follows the injection of preformed heterologous antiplatelet serum (APS). In either case, nude mice (nu/nu) should show abnormalities of thrombocytopoiesis or the response to acute platelet depletion, since, in addition to being hairless, nu/nu mice are deficient in T lymphocites. The present experiments were done to deterinine if thrombocytopoiesis would be abnormal in $\mathbf{T}$ cell deficient nu/nu mice or if they would respond to thrombocytopenia differently than normal $(\mathrm{nu} / \mathrm{+})$ coistrols.

Untreated nude mice had normal platelet and megakaryocyte counts, indicating that unperturbed platelet production was normal. They had a slight but significant anemia and significantly fewer bone marrow cells than nu/+ mice. Their spleen cells showed a normal mitogenic response, as determined by tritiated thymidine incorporation, to lipopolysaccharide (a B lymphocyte mitogen), but none to phytohemaglutinin (PHA) or concanavalin A (Con A) (both are $T$ lymphocyte mitogens), thus confirming a deficiency of $T$ cells in nu/nu mice.

Megakaryocyle and platelet responses to an injection of APS were the same in nu/nu and nu/t+ mice: acute thrombocytopenia was followed by increased megakaryocytes in the bone marrow (137-155\% of normal on day 2), macromegakaryocytosis (megaharyocyte size was $150-178 \%$ of normal on day 2), and rebound thrombocytosis (platelet counts were $168-212 \%$ of normal on days 5 and 6). These results showed that stimulation of megakaryocytopoiesis and platelet production in response to acute thrombocytopenia was normal in nude mice. Meg-CFC were increased in nude mice 4 to 5 days after APS or an injection of normal guinea pig serum (NGpS) to $3 \times$ controls in the spleen and $2 \times$ controls in the bone marrow. GMCFC showed a 6-fold increase in the splcen on days 5 and 6 after APS and no increase in the marrow. Neither CFC increased in nu/t mice. Marrow BFU-E did not change. Nude spleen cells showed no response to either $T$ cell mitogen on days 2, 4, and 5 after APS or NGpS. However, weak responses occurred sporadically at 6 days after APS to ConA, 4 hours after NGpS to ConA, and 6 days after NGpS to both ConA and PHA.

Thrombocytopoiesis was normal in undisturbed $\mathrm{nu} / \mathrm{nu}$ mice, and stimulation of platelet production in response to acute immunothrombocytopenia was the same in nu/nu and nu/+ mice. Therefore, it is unlikely that $T$ cells play an important role in these processes. Sporadic weak $T$ cell mitogenic responses in $n u / n u$ mice did not correlate with thrombocytopoietic activity. Increases in megakaryocyte number and size and platelet production occurred independenlly of changes in numbers of Meg-CFC. GM-CFC, or marrow BFU-E. 


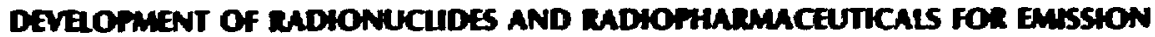 COMUUTED TOMOGAArHY
}

\author{
Yulkio Yano, Chester A. Mathis, Stephen M. Moerkin, Mohindar Singh, leese \\ lones, and Thomas F. Budinger
}

Our objective is to develop methods for the production of radionuclides and for the radiolabeling of biochemical substrates to investigate human physiology and metabolism noninvasively by emission-computed tomography.

Our approach is to use radioisotope generators or cyclotrons to obtain relatively shost-half-life positron or single-photon emitters. Generators provide a readily available supply of short-lived radionuclides at a low cost while cyclotrons provide the radionuclides of carbon, nitrogen, oxygen, fluorine, bromine, or iodine that can be incorporated into biochemical compounds or analogues.

\section{RADIOISOTOPE GENERATORS}

Generators for short-lived positron emitters such as $68-\mathrm{min}^{68} \mathrm{Ga}, 76-\mathrm{sec}^{82} \mathrm{Rb}$, and $3.6-\mathrm{min}{ }^{122}$ are listed in Table 1. The ${ }^{82} \mathrm{Sr} /{ }^{82} \mathrm{Rb}$ generator was reloaded every three months with $200 \mathrm{mCi}$ of ${ }^{82} \mathrm{Sr}$ obtained from Los Alamos National Laboratory. The generator routinely provided $20-60 \mathrm{mCi}$ doses of ${ }^{82} \mathrm{Rb}$ for PET studies of myocardial blood flow in heart patients or changes in the permeability of the blood-brain barrier in patients with brain tumor or radiation therapy necrosis. Validation studies of myocardial blood flow measurements with ${ }^{82} \mathrm{R.b}$ were done with ${ }^{15} \mathrm{O}$ water and radiolabeled microspheres.

The ${ }^{68} \mathrm{Ge} /{ }^{68} \mathrm{Ga}$ generator was used to obtain ionic ${ }^{68} \mathrm{Ga}$ for labeling platelets and low density lipoproteins (LDL) for PET.studies of thrombosis and atherosclerosis in cardiovascular disease.

${ }^{68} \mathrm{Ga}$ was attached $10 \mathrm{LDL}$ via a bifunctional chelating agent, DTPA. Low pressure gel column chromatography and HPLC were used to preparatively separate and analyze ${ }^{68} \mathrm{Ga} L \mathrm{LD}$ for uptake studies in the healing endothelium of the scraped aorta rabbil model. The biological viability and radiolabeling yield of ${ }^{60} \mathrm{Ga} \mathrm{LDL}$ were dependent upon maintaining a concentration of $15-20 \mathrm{mg}$ LDL $/ \mathrm{ml}$ of plasma.

${ }^{68} \mathrm{Ga}$ EDTA was used to measure cerebral blood volume and blood-brain barrier breakdown in Alzheimer's type dementia. ${ }^{68}$ Ga EDTA was also used to study brain tumor patients for radiation therapy necrosis.

The ${ }^{122} \mathrm{Xe} /{ }^{122}$ I generator work was continued with ${ }^{122}$-radioiodinated amphetamine analogues to measure regional cerebral flow in patient studies utilizing PET.

For broader applications in nuclear medicine, a ${ }^{99} \mathrm{Mo}\left(\mathrm{t}_{1 / 2}=2.7 \mathrm{Bd}\right) /{ }^{99 \mathrm{M}} \mathrm{TC}\left(\mathrm{t}_{1 / 2}=6 \mathrm{~h}\right)$ generator was used to obtain high specific concentration ${ }^{99 \mathrm{~m}} \mathrm{Tc}$ $(20-100 \mathrm{mCi} / \mathrm{ml}$ saline) and to utilize the ideal $140-\mathrm{keV}$ photons of ${ }^{99 \mathrm{~m}} \mathrm{Tc}$ for imaging studies with an improved single-photon emission-computed tomography (SPECT) system now in operation at Donner Laboratory.

A new complexing agent thiocaprolactam (TCL) and 8-hydroxyquinoline (oxine) were studied for ${ }^{99 \mathrm{~m}} \mathrm{Tc}$ labeling. Various concentrations of ligand $(0.400-3.0 \mathrm{mg})$ were evaluated with stannous ion or sodium borohydride $\left(\mathrm{NaBH}_{4}\right)$ reduction of TC VII. HPLC analysis and octanol/aqueous buffer partition coefficients were determined for the ${ }^{99 \mathrm{~m}} \mathrm{Tc}$ complex prior to platelet labeling studies. In concentrations of $400-500 \mu \mathrm{g}$ both oxine and TCL gave $70-100 \%$ complex formation when $\mathrm{NaBH}_{4}$ was used to reduce Tc VII to Tc V. The octanol/saline buffer partition coefficient was about 12 for ${ }^{99 m} \mathrm{Tc}$ oxine and about 6 for ${ }^{99 m} \mathrm{Tc}$ TCL.

The ${ }^{99 . \mathrm{m}} \mathrm{Tc}$ complex of oxine and thiocaprolactam was incubated with separated rabbit platelets in saline alone and in platelet-poor plasma (ppp):

Table I. Generators for positron emilters.

\begin{tabular}{|c|c|c|c|c|c|c|}
\hline Paren1 & Mali-life & $\begin{array}{c}\text { Decay mode } \\
(n \%)\end{array}$ & Daughter & Half-life & $\begin{array}{c}\text { Decay mode } \\
(\%)\end{array}$ & $\begin{array}{c}\text { Gammas (MeV) } \\
(\%)\end{array}$ \\
\hline Me & $275 d$ & ECIINO) & ${ }^{*} \mathrm{Ca}$ & $68 \mathrm{~m}$ & N(88),EC(12) & $1.083 .5)$ \\
\hline${ }^{2} 54$ & $25 d$ & ECInos) & $* \mathrm{Rb}$ & $75 \mathrm{~s}$ & $S^{*}(9), E C(4)$ & $0.73(9)$ \\
\hline "77xe & $20.11 \mathrm{~h}$ & $\operatorname{col}(000)$ & $n=z_{i}$ & $3.6 \mathrm{~m}$ & $5(B 7)$ & 0.5614 \\
\hline
\end{tabular}


saline. The platelet labeling vields with $10-\mathrm{min}$ incubation at room temperature were $10 \%$ for 9mic oxine in saline and $24 \%$ for "Mintc thiocaprolactam in satine: ppp (1:1).

We continued our studies of lipophilic compounds to serve as carriers for the single-photon emitter ${ }^{9 m} \mathrm{Tc}$. We determined complex formation by HPLC analysis and lipophilicity by octanol partition. The following ligands were studied: diethyldithiocarbamate, pyrolidinedithiocarbamate, 2-(2pyridyl) benzimidazole, 3-(2-methoxyphenyl) piperazine, and 2-mercapto-4-methyl-pyrimidine. Some of these ${ }^{99 m} \mathrm{Tc}$-labeled compounds were evaluated in animal studies to determine their usefulness as tracers for blood flow to the brain, heart, and lungs.

\section{CYCLOTRON PRODUCED RADIONUCLIDES}

The Lawrence Berkeley Laboratory 88-Inch Cyclotron was used to produce $\mathrm{Ci}$ quantities of ${ }^{11} \mathrm{C}$ $\mathrm{CO}_{2}$ and $\mathrm{CH}_{3} \mathrm{l}$ for the synthesis of palmitic acid, methionine, and choline. ${ }^{15} \mathrm{O}$-labeled water was produced in $100-\mathrm{mCi}$ batches for use as a freely diffusible tracer for blood-flow measurements in the heart and brain. We continued to explore the production of $98-\mathrm{min}{ }^{75} \mathrm{Br}$ by the proton irradiation of an isotopically enriched ${ }^{76} \mathrm{Se}$ target of $\mathrm{SeF}_{6} .{ }^{75} \mathrm{Br}$ may be a useful positron label for high specific activisy receptor ligands.
The Crocker Nuclear Laboratory cyclotron at U.C. Davis produced Iff ficorodeoxyducose on a weekly basis for studies of glucose metabolism in brain and heart. Further modifications in the chemical synthesis of ${ }^{18} \mathrm{FDC}$ using ${ }^{10} \mathrm{~F}$-acetyl hypofluorile increased the labeling yield and radiochemical purity of the product. About $80 \mathrm{mCi}$ of ${ }^{18} \mathrm{FDG}$ results from a $30 \mu h$-hr irradiation with a proton beam.

The radiochemical yields of nine N,Ndimethyl-2-(substituted phenyl) isopropylamines (amphetamine analogues) were determined following reaction with ${ }^{18} \mathrm{~F}$-acetyl hypofluorite in a $0.1 \mathrm{M}$ $\mathrm{HCl}$ solution at room temperature. The metadimethoxy substituted amphetamines gave the highest radiofluorination yields (24-32\%, at EOB). Purification of the ${ }^{18} \mathrm{~F}$-labeled amphetamines was achieved in $10-20 \mathrm{~min}$. 5- ${ }^{18} \mathrm{~F}-2,4-$ dimethoxy- $\mathrm{N}, \mathrm{N}$ dimethylamphetamine $\left(5^{18} \mathrm{~F}-2,4-D N N A\right)$ was utilized to determine brain and lung uptake in rats. Positron-emission tomography studies were conducted in a dog to determine the dynamic brain uptake and retention of this agent.

We continued to use the Medi-Physics CS-22 cyclotron to produce ${ }^{18} \mathrm{~F}$ fluoride by the irradiation with protons of an ${ }^{18} \mathrm{O}$-enriched water target. The target is filled and emptied remotely from a hot laboratory adjacent to the cyclotron vault. The enriched water target and the ${ }^{18} \mathrm{~F}$-fluoride are captured in a trap that serves as a vessel for the evaporation of the water and as a reaction vessel for incorporating ${ }^{18} \mathrm{~F}$ into radiopharmaceuticals for ligand receptor binding studies in the brain.

\section{NEW INSTRUMENTATION FOR HIGH RESOLUTION, DYNAMIC, THREE DIMENSIONAL IMAGING OF POSITRON LABELED COMPOUNDS IN THE HUMAN BODY}

Stephen E. Derenzo, John L. Cahoon, Ronald H. Huesman, Tony Vuletich, and Thomas F. Bedinger

\section{0-CRYSTAL HIGH RESOLUTION POSITRON TOMOGRAPH}

This new tomograph has 600 bismuth germanate scintillation crystals that encircle the head in a close-packed ring. The patient sits in an adjustable chair during the study (fig. 1). After administration of a positron-bbeled compound, the distribution of the tracer is measured as a function of time by recording coincidences belween detectors in opposite erougs of the tine and performing a mathematical process called computed tomography. The crystals are small $(3 \mathrm{~mm} \times 10 \mathrm{~mm})$ and the in-plane resolution is $2.5 \mathrm{~mm}$. The resolution along the patient axis can be varied from $0.5 \mathrm{~mm}$ to 5 $\mathrm{mm}$ by adjusting a lead shielding ring. The electronics employ many high-speed emitter-coupled logic (ECL) circuits for rates of $1,000,000$ transmission events per sec and 100,000 emission events per sec. In addition, the pulse height windows and timing for all 600 detectors are adjusted by com. puter control. 


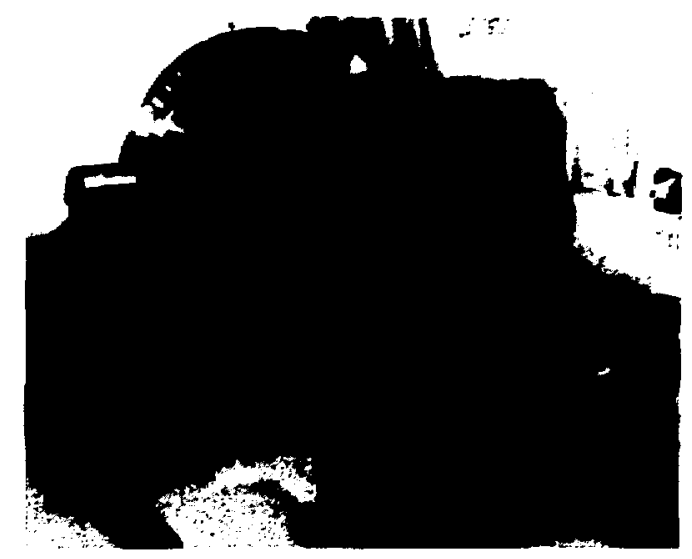

Fig. 1. Donner ultrahigh resolution 600-crystal positron tomograph with patient chair.

(CBB 852-1304)

\section{STATISTICAL PROPERTIES OF COMPARTMENTAL MODEL PARAMETERS EXTRACTED FROM DYNAMIC POSITRON EMISSION TOMOGRAPHY EXPERIMENTS}

Bernard M. Mazoyer, Ronald H. Huesman, Thomas F. Budinger, and

Brian L. Knittel

The determination of physiological properties (such as tissue blood flow, metabolic rates, receptor activity, and substrate turnover or clearance) relies on accurate measurements of the response of a tissue or organ system to an input. The input might be an injected radioactive tracer of biological activity in nuclear medicine imaging procedures, or a pulse sequence in nuclear magnetic resonance studies. Over the past years a major focus of research in physiologic studies employing tracers has been the computer implementation of mathematical methods of kinetic modeling for extracting the desired physiological parameters from tomographically derived data. We have recently completed a sludy of factors that affect the statistical properties of compartmental model paramelers extracled from dynamic positron emission tomography (PET) experiments.

\section{PARAMETER ESTIMATION USING DYNAMIC PET DATA}

A dynamic PET experiment can be generalized as follows (see Fig. 1): a single intravenous injertion of a radionuclide is pertormed, and the time course of the activity, a(t) (which we will refer to as the model residue function), in some region of interest in the tomographic slice, is recorded. The input function, $x_{1}(t)$, is usually measured by acquiring venous or arterial blood samples, but could be acquired by tomographic sampling under some conditions. A mathematical model is used to represent the physiological behavior of the tracer in the region of interest; for example

$$
a(t)=h(p) * x_{1}(t)
$$

where $h(p)$ is the impulse response of the system, which depends on a parameler vector $p$, and * denotes the convolution operation. A 3compartment model such as used in ${ }^{16} \mathrm{~F}$-fluorodeoxyglucose (FDG) experiments is the reference system in our sludy. In this model, the activity in the region of interest is

$$
\left.\left.a(t)=s_{1}^{\prime} f_{2} x_{1}(t-r)+\left(1-f_{v}\right) \mid x_{2}(t)+x_{3}(t)\right]\right\}
$$

where $s$ is a concentration-activity scaling factor, $f_{2}$ is the fractional volume of tissue comprising blood 


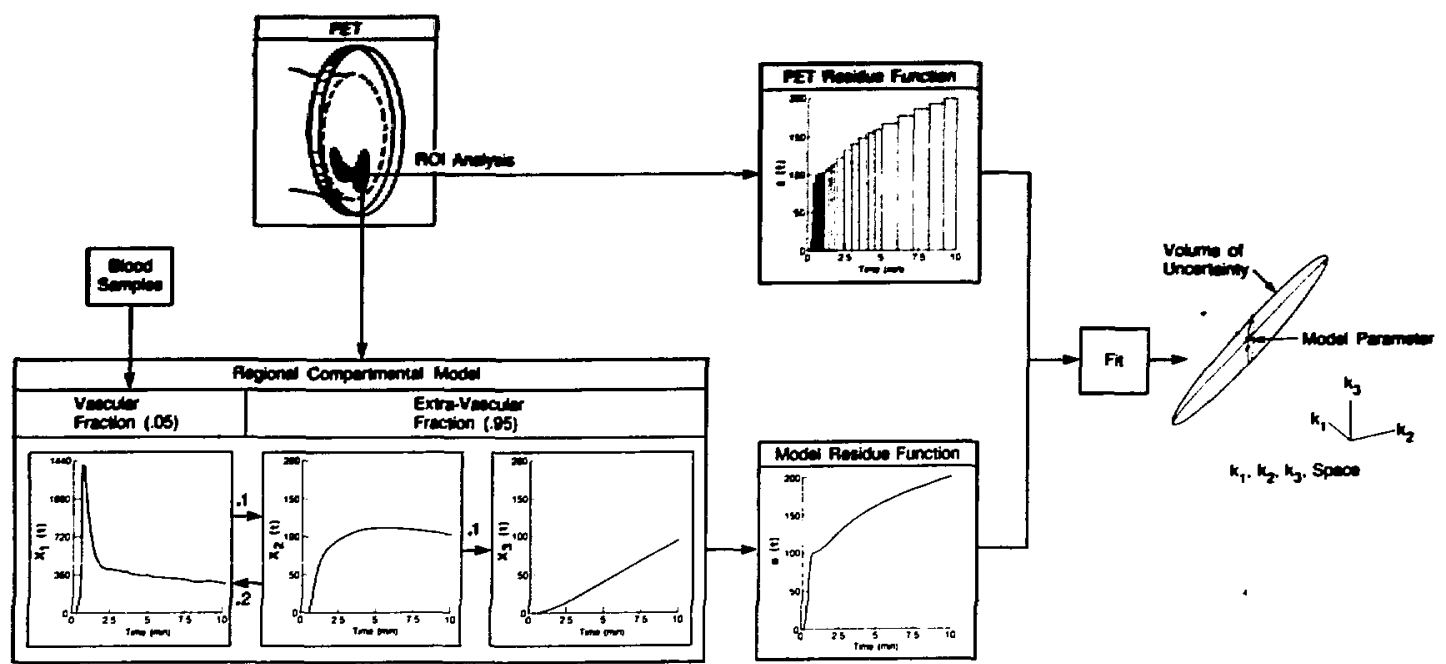

Fig. 1. Flow-chart of a dynamic FDG-PET experiment. The fit provides estimates of the compartmental model parameters and of their accuracies in terms of a covariance matrix. On the right is the uncertainiy elipsoid corresponding to the covariance matrix of $k_{1}, k_{2}$ and $k_{3}$.

(XBL 855-8292)

capillaries, and $\tau$ is the sample delay time which depends on the blood sampling site and method (e.g., the longer the catheter the larger $\tau$ ).

Actually, the tomographic device provides a series of measures of mean values of a(t) over time intervals $\left(\left[t_{i-1}, t_{i}\right), i=1, \ldots, n\right)$ which can be modeled as

$$
\begin{gathered}
z_{i}=y_{i}+e_{i} \\
y_{i}=\frac{1}{t_{j}-t_{j-1}} \int_{t_{i-1}}^{t_{1}} a(t) d t, i=1,2, \ldots, n,
\end{gathered}
$$

where the $y_{i}$ are the theoretical model values for the data, and the $z_{i}$, which we will refer to as the PET residue funclion, represent the aclual data with statistical fluctuations given by the $\mathbf{e}_{i}$.

Using any of the available procedures to fit $y$ (the vector of $y_{i}$ ) to $z$ (the vector of $z_{i}$ ) will provide a parameter vector estimate $\hat{\mathbf{p}}$. Two kinds of information are useful in order to assess the quality of this estimate: the bias in its mean,

$$
\mathbf{b}=\mathbf{E}(\hat{\mathbf{p}})-\mathbf{p} .
$$

and its covariance matrix,

$$
\left.\operatorname{COV}(\dot{\mathbf{p}})=\mathbf{E}_{i}\left(\dot{\mathbf{p}}-\mathbf{E}_{i} \dot{\mathbf{p}}\right)\right)^{2}
$$

where $E_{1}$, stands for the matinematical expectation of a random variable. The bias gives a measure of the accuracy of $\hat{p}$, and we have investigated the biases resulting from

- using a mid-point interpolation instead of a true integral model to compute the $y_{i}$ in Eq. (4),

- $\quad$ using a wrong model to describe the data, such as fixing (and not fitting) the parameter $f_{v}$ and $\tau$ to wrong constant values, and

- using venous (instead of arterial) bloodtracer data as input function.

The covariance matrix of $\hat{p}$ as defined in gives a measure of the precision of $\hat{p}$ and can be estimated as

$$
\operatorname{COV}(\hat{p})=\left(S^{\top} \Sigma^{-1} S\right)^{-1} .
$$

The matrix $S=\left(s_{i j}\right)$, called the sensitivity matrix, denotes the derivative of $y$ relative to $p$.

$$
s_{i j}=\frac{\partial y_{i}}{\partial p_{i}}, i=1,2, \ldots, n, i=1,2, \ldots, r .
$$

(r equals the number of parameters), and $\mathrm{S}$ is the data covariance matrix. Equation (7) clearly shows how the data stalistical uncertainties are propagated to the parameter eslimale uncertainties through the 
matrix S. The determinant of COV(j) is proportional to the volume of the parameter confidence region and provides a criterion for evaluating the effects of factors on parameter estimate precision. The principal parameters of interest in dynamic PET experiments are the rate constants $\left(k_{1}, k_{2}, k_{3}\right.$ for our model), because they represent the physiological process under study. The parameters $f_{v}$ and $\tau$ are necessarily included in the model because of the nature of the data acquired in PET experiments. For this reason, we shall use the determinant, $\Delta$, of the covariance matrix of only $k_{1}, k_{2}$ and $k_{3}$ as a measure of merit for the various protocols and input functions. Because $p$ also includes $f_{v}$ and $\tau$, this matrix is a submatrix of $\operatorname{COV}(\hat{\mathrm{p}})$. In subsequent figures we will plot a normalized form of this quantity denoted by $\mathscr{Z}$, and defined as:

$$
\mathscr{Z}=\left(\frac{\Delta}{\left(k_{1} k_{2} k_{3}\right)^{2}}\right)^{1 / 6} \text {. }
$$

W can be interpreted as a mean coefficient of variation for the k's. We have investigated the effects on $\mathscr{Z}$ of various experimental factors over which the experimenter has control, which are

- the tracer injection duration, which will condition the input function shape, and

- the tomographic data collection protocol, e.g., the number and time series of images.

\section{SIMULATIONS AND RESULTS}

Patient blood data were acquired using a fast venous injection into the arm and a typical sampling procedure to provide a function used for the standard or reference input data, $x_{1}(t)$. Given $x_{1}(t)$, a set of values for the parameter vector $p=\left(k_{1}, k_{2}\right.$, $\left.k_{3}, f_{y}, \tau\right)^{\top}$, and the sequence of $t_{i}$ (the sampling protocol), the exact values of the $y_{i}$ are computed using Eqs. (4) and (2). We approximate the $e_{i}$ (the random errors on the $y_{j}$ ) as $n$ uncorrelated normal distributions with zero means and variances given by:

$$
\sigma_{i}^{2}=\frac{y_{i}}{\left(t_{1}-t_{1-1}\right)} \cdot i_{i} j=1,2, \ldots . n
$$

Different sampling protocols have been tested, some of which are currently used by various groups (see Tables la and (b). Also, a set of blurred-input functions (and the corresponding residue functions) were generated by convolving the reference inpul function with unit area rectangular functions of durations 10, 30, 60 and $120 \mathrm{sec}$. These blumed-
Table 1a. Sampling soratepies with various initivi frame durations and constant study durations (2400 sec).

\begin{tabular}{ccl}
\hline $\begin{array}{c}\text { Protocol } \\
\text { mumber }\end{array}$ & $\begin{array}{c}\text { Initial frame } \\
\text { duration (sec) }\end{array}$ & \multicolumn{1}{c}{$\begin{array}{c}\text { Sampling stratezy } \\
\text { (no. of frames } \times \text { frame duration) }\end{array}$} \\
\hline 1 & 1 & $60 \times 1-6 \times 10-6 \times 30-35 \times 60$ \\
2 & 2 & $60 \times 2-4 \times 30-12 \times 180$ \\
3 & 5 & $24 \times 5-12 \times 15-10 \times 60-5 \times 300$ \\
4 & 30 & $10 \times 30-10 \times 60-5 \times 300$ \\
5 & 60 & $5 \times 60-5 \times 120-5 \times 180-2 \times 300$ \\
6 & 180 & $10 \times 180-2 \times 300$ \\
\hline
\end{tabular}

Table ib. Sampling strategies with varic is study durations. The initial frame durations are kept consta $7 t$ for each of the three groups of protocols, and correspond to the initial

\begin{tabular}{|c|c|c|}
\hline $\begin{array}{l}\text { Initial frame } \\
\text { duration (sec) }\end{array}$ & $\begin{array}{c}\text { Sampling strategy } \\
\text { (no. of frames } X \text { frame duration) }\end{array}$ & $\begin{array}{c}\text { Study } \\
\text { duration } \\
\text { (sec) }\end{array}$ \\
\hline \multirow{8}{*}{1} & $95 \times 1-12 \times 2$ & 119 \\
\hline & $60 \times 1-6 \times 10-31 \times 6$ & 306 \\
\hline & $60 \times 1-6 \times 10-6 \times 30-35 \times 9$ & 615 \\
\hline & $60 \times 1-6 \times 10-6 \times 30-35 \times 26$ & 910 \\
\hline & $60 \times 1-6 \times 10-6 \times 30-35 \times 43$ & 1505 \\
\hline & $60 \times 1-6 \times 10-6 \times 30-35 \times 60$ & 2400 \\
\hline & $60 \times 1-6 \times 10-6 \times 30-35 \times 77$ & 2995 \\
\hline & $60 \times 1-6 \times 10-6 \times 30-35 \times 94$ & 3590 \\
\hline \multirow{8}{*}{30} & $6 \times 30$ & 180 \\
\hline & $10 \times 30$ & 300 \\
\hline & $10 \times 30-5 \times 60$ & 600 \\
\hline & $10 \times 30-10 \times 60$ & 900 \\
\hline & $10 \times 30-10 \times 60-2 \times 300$ & 1500 \\
\hline & $10 \times 30-10 \times 60-5 \times 300$ & 2400 \\
\hline & $10 \times 30-10 \times 60-5 \times 300-1 \times 600$ & 3000 \\
\hline & $10 \times 30-10 \times 60-5 \times 500-2 \times 600$ & 3600 \\
\hline \multirow{8}{*}{180} & $5 \times 180$ & 900 \\
\hline & $7 \times 180$ & 1260 \\
\hline & $9 \times 180$ & 1620 \\
\hline & $10 \times 180$ & 1800 \\
\hline & $10 \times 180-1 \times 300$ & 2100 \\
\hline & $10 \times 180-2 \times 300$ & 2400 \\
\hline & $10 \times 180-1 \times 400$ & 3000 \\
\hline & $10 \times 180-6 \times 300$ & 3600 \\
\hline
\end{tabular}
sampling rates of protocols 1,4 and 6 of Table 1 .

input functions are used to evaluate the effects of prolonging the intravenous injection.

\section{SOURCES OF PARAMETER INACCURACIES}

The biases resulting from fitting values computed using a midpoint interpolation to data generated using the integral model are shown in Table 2. Notice that bias amplitude increases with the initial scan duration and injection duration. 
Table 2. Eises (in \% of the paraneter inve valce) in the mean of parameter estimates resuling fom the use of a non-integal model.

\begin{tabular}{cccccccc}
\hline \hline & \multicolumn{3}{c}{ Sharp-input function } & & \multicolumn{2}{c}{ Bhurred-input function } \\
\cline { 2 - 4 } Parameter & Prot. 1 & Prot. 4 & Prot. 6 & & Prot. 1 & Prot. 4 & Prot. 6 \\
\hline$k_{1}$ & 0.02 & 1.7 & 9.9 & 0.002 & 5.7 & 7.3 \\
$k_{2}$ & 0.002 & 2.8 & 11.4 & 0.003 & 9.3 & 10. \\
$k_{3}$ & 0.005 & 0.32 & 7. & 0.04 & 1.7 & 9.3 \\
$f_{v}$ & 0.6 & 1.3 & 62. & 0.01 & 34. & 94. \\
$t_{0}$ & 0.06 & 50. & 100. & 0.08 & 67. & 170. \\
\hline \hline
\end{tabular}

Values were obtained by fitting the non-integral model using two different input functions (the sharp reference and a blurred function obtained by convolving the reference with a unit area rectangular function of 60-sec duration), and three PET data protocols (1, 4 and 6 of Table 1a with initial frame duration 1, 30 and $180 \mathrm{sec}$ ). Notice that since a one-second time scale was used to generate the data, no significant biases are observed for protocol 1 (sampling rate $=$ $1 / \mathrm{sec})$.

Table 3 shows that significant biases are generated for the fit parameters when wrong values are used for the parameters not fitted. The bias values can be predicted using a first order expansion of the fitting criterion, and Table 3 gives the first order derivatives of the parameters $k_{1}, k_{2}, k_{3}$ considered as functions of the parameters $f_{v}$ and $\tau$. Notice that $k_{1}$ and $k_{2}$ are more sensitive than $k_{3}$ because they are more correlated with $f_{v}$ and $\tau$, and that biases are independant of the data collection protocol.

Assuming that circulation can be roughly modeled as a convolution by a square function, we fit the model with the 10-second input function to the data generated with the sharp reference input. Table 4 shows that significant biases are generated. This effect is more important for protocols with faster initial data collection rates, and may be explained by the fact that the model is sensitive to inaccuracies in the input function measurements at early times through the blood contribution |represented in Eq. (2) by $f_{v} x_{1}(t-\tau)$. Protocols with lower initial sampling rates are less sensitive to this contribution, because the averaging of the blood contribution over a relatively long time interval makes this average value more stable with respect to the shape of the input function.

\section{EFFECTS OF EXPERIMENTAL DESIGN ON PARAMETER PRECISION}

Effects of various sampling rates at early times and various injection duralions on parameter esti-

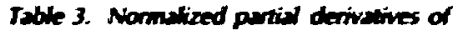
if paraneters $\left(k_{1}, k_{2} k_{2}\right)$ ndetive so constent paraneter $f_{4}$ and $s$.

Sharp-inpul function A

Protocol 1 Protocol 4 Protocol 6

\begin{tabular}{lrrr}
\hline$\frac{f_{v}}{k_{1}} \partial k_{1} / \partial f_{v}$ & -0.33 & -0.34 & -0.38 \\
$\frac{\tau}{k_{1}} \partial k_{1} / \partial \tau$ & 0.059 & 0.067 & 0.12
\end{tabular}

$\begin{array}{lrrr}\frac{f_{v}}{k_{2}} \partial k_{2} / \partial f_{v} & -0.61 & -0.64 & -0.7 \\ \frac{\tau}{k_{2}} \partial k_{2} / \partial \tau & 0.11 & 0.13 & 0.25\end{array}$

$\begin{array}{lrrr}\frac{f_{v}}{k_{3}} \partial k_{3} / \partial f_{v} & -0.065 & -0.07 & -0.85 \\ \frac{\tau}{k_{3}} \partial k_{3} / \partial \tau & 0.028 & 0.036 & 0.076\end{array}$

Normalized derivatives (in relative value) are given for protocols 1,4 , and 6 with initial frame durations $1,30,180 \mathrm{sec}$ respectively (see Table 1a) and the sharp reference input function. These derivatives can be used to predict the basis of fit parameter estimates when using wrong constant values for other parameters. For example setting $f_{y}$ to 0.04 instead of 0.05 and $\tau$ to 11 instead of $10 \mathrm{sec}$ will generate a value of $k_{\mathbf{i}}$ overestimated by $7.2 \%$ $(-20 \% \times-0.33+10 \% \times 0.059)$ for protocol 1 .

Table 4. Biases (in 96 of the true parameter value) in the mean of parameter estimates due to arm venous blood sampling instead of true capillary sampling.

\begin{tabular}{cccccc}
\hline & \multicolumn{2}{c}{ Blurred-input function A } & \multicolumn{2}{c}{ Blurred-input function B } \\
\cline { 2 - 3 } Parameter & Protocol 3 & Protocol 4 & & Protocol 3 & Prolocol 4 \\
\hline$k_{1}$ & 4.3 & 0.6 & 9.7 & 3.3 \\
$k_{2}$ & 8.1 & 1.0 & 18. & 5.5 \\
$k_{3}$ & 0.2 & 0.1 & 0.4 & 0.5 \\
$f_{4}$ & 9.4 & 3.0 & 49. & 16. \\
r & 21. & 47. & 40. & 118. \\
\hline \hline
\end{tabular}

Venous sampling was simulaled by convolving the shap refer. ence input function (assumed to represent the true brain capil. In tracer concentitation) with unit-area rectangalar functions of (A) 10. and (B) 30-sec durations. The PET das were peneraied with the reference residue cunc sompled with probool 3 and 4 (inimial frame duration 5 and 30 sec respectincty) of Table $1 \mathrm{a}$ 
mate precision are shown in Fig. 2. Note that only small improvement is gained by using initial frame durations smaller than $\mathbf{3 0}$ seconds, and that use of fast injection provides only small gains in parameter precision. Notice that for each injection duration, the fastest sampling rate always provides the more precise parameter estimate but that an optimal input function seems to exist for the protocols with an initial sampling of 30 seconds or larger.

Figure 3 decribes the effects of the total study duration for three different protocols. For each protocol, the initial frame duration is kept constant while additional information is added using later images. Only small improvements in $\mathscr{D}$ as well as in $k_{3}$ precision are obtained by setting the total study duration to values larger than 40 minutes for the set of rate constants encountered in FDG experiments. The precision of $k_{1}$ and $k_{2}$ do not change significantly.

\section{CONCLUSION}

We have shown that it is important to include the parameters $f_{y}$ and $\tau$ in the model and to account for the integration process inherent in emission tomography data when fast data collection is impossible. We have also found that higher rates of injection and higher rates of data collection lead to nore precise parameter e'stimates. However, for the range of rate constants encoutered in PET studies with ${ }^{18} \mathrm{FDG}$, insignificant differences were found between protocols with initial scan durations less than 30 seconds. Moreover, we have shown that in the absence of arterial blood data, the use

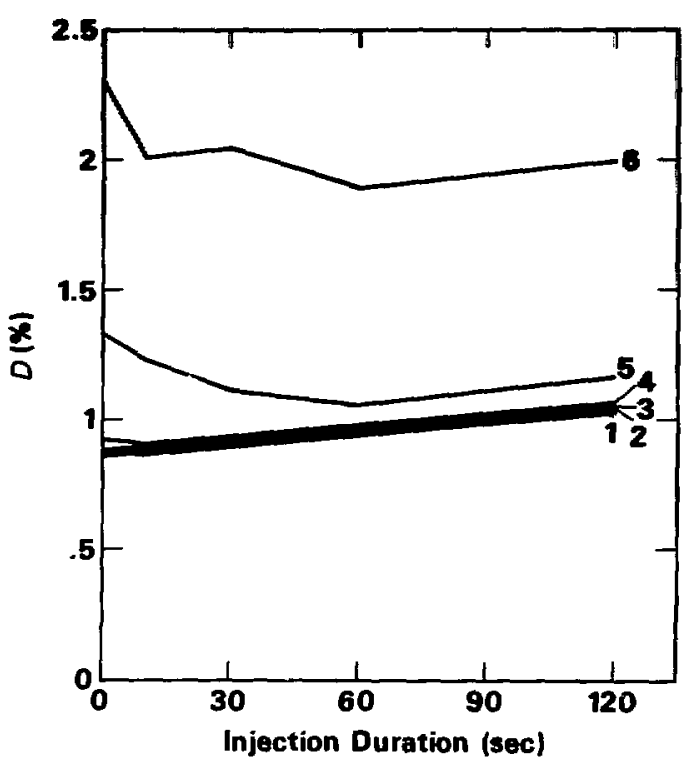

Fig. 2. Comparison of experiments using the six different sampling protocols of Table la as a function of the injection duration: normalized determinant of $k_{2}, k_{2}, k_{3}$ covariance matrix (S).

(XBL 855-8288)

of venous blood data as an input function leads to biases for fast data collection protocols. We are currently developing methods for retrieving arterial tracer time activity functions from venous blood data. 

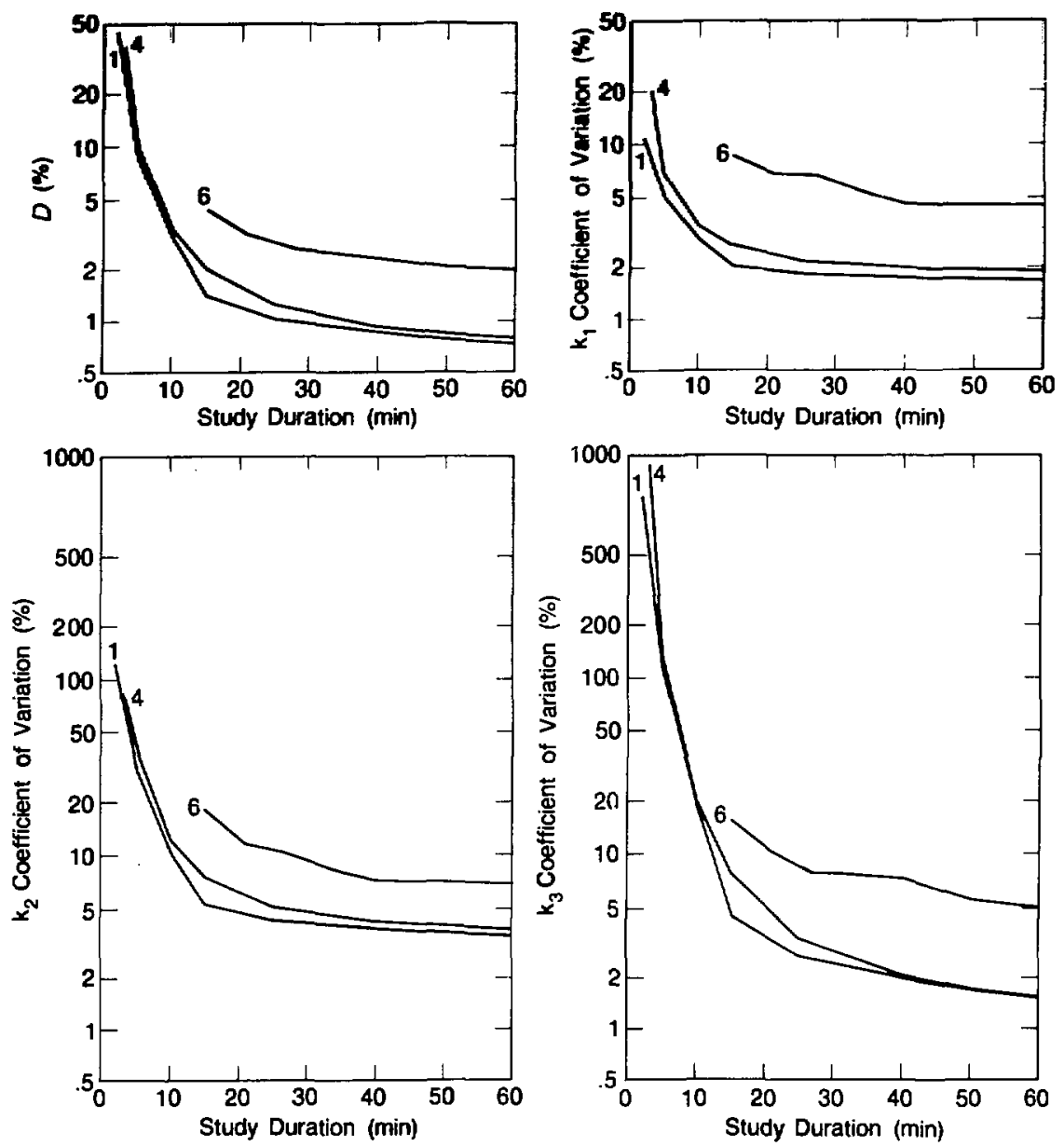

Fig. 3. Comparison of experiments as a function of the study duration using the protocols of Table $1 b$; $a$ : normalized determinant of $k_{1}, k_{2}, k_{3}$ covariance matrix $(\mathscr{Z}) ; b, c, d$ : coefficient of variation of $k_{1}, k_{2}$ and $k_{3}$ respectively.

\section{DEAD TIME CORRECTION AND COUNTING STATISTICS FOR POSITRON TOMOGRAPHY}

\section{Bernard M. Ma:zyer, Mark S. Roos, Ronald H. Huesman, and Thomas F. Budinger}

A correction for loss of events due to dead time in dynamic positron emission tomography has been studied. The model employs a paralyzing dead time to describe the behavior of a tomograph over the range of event rates normally encountered in patient studies (up to 200,000 events) sec/detector layer).
The Donner 280-Crystal Positron Tomograph is characlerized by a dead time of $1.8 \mu \mathrm{sec} / \mathrm{event}$ for observed count rates of less than 200,000 events/sec. The dead time correction factor is 1.8 at 180,000 events $/ \mathrm{sec}$.

The correction is applied to projection data and to region-of-interest analysis of dynamic PET studies 
and formulae for the covariances between corrected projection data and between counts in regions of interest in different images from the same dynamic study are established. At 180,000 events/sec, the variance of the actual (corrected) number of events in a region containing $3.34 \times$ $10^{5}$ actual events is predicted from the model to be $3.86 \times 10^{6}$ events $^{2}$, which is more than 10 times the variance that would be expected from a naive assumption of Poisson statistics (Fig. 1). These statistical results are verified experimentally.
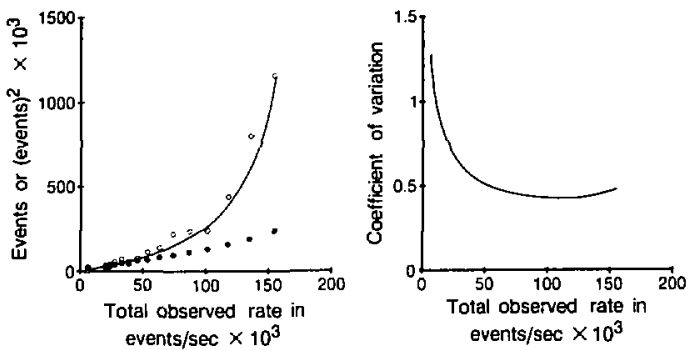

Fig. 1. Statistics for corrected number of events as a function of the total observed count rate. a) Sample mean ( $\bullet$ ) and variance (O); predicted variance (solid line) using propagation of errors in the dead time model. b) Predicted coefficient of variation.

(XBL 846-7197A)
The necessity and importance of dead-time correction in dynamic PET is shown by an example of an observed error of $25 \%$ in myocardial flow if dead-time compensation is not applied (Fig. 2).

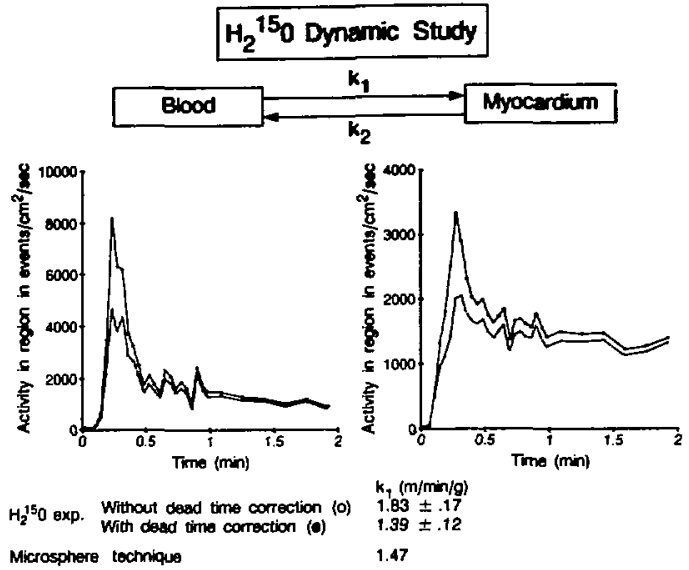

Fig. 2. Input (left) and residue (right) curves from an $\mathrm{H}_{2}^{15} \mathrm{O}$ dynamic study with ( $)$ and without (O) correction for dead time. Using the microsphere trchnique, the flow value ( $\mathrm{ml} / \mathrm{min} / \mathrm{g}$ tissue) was measured to be 1.47 ; the flow values derived from the $\mathrm{H}_{2}^{15} \mathrm{O}$ experiment were 1.83 before and 1.39 after correction for dead time. The correction factor at the time of the peak of the input curve was 1.8 for a 180,000 events/sec total observed count rate.

(XBL 846-7195A)

\section{BIOLOGICAL EFFECTS OF STATIC MAGNETIC FIELDS}

Thomas F. Budinger, Kay S. Bristol, Dorothy A. Carpenter, Patricia A. Garbutt, Priscilla D.C. Wong, and Chi-Kwan Yen

A retrospective epidemiological study on the health effects, if any, of stationary and alternating magnetic fields produced by man-made devices such as cyclotrons, bubble chambers, and proton synchrotrons was started in 1977 and is now completed. The magnetic fields to which the workers were exposed are as high as 2 tesla $\left(2 \times 10^{4}\right.$ gauss) for a few hours and as low as a few times the natural earth magnetic field ( 5 gauss) lor long durations.

Two quite different exposure environments can be distinguished: the alternating held and the static field environments. This particulai study is locused on the eviects of steady magnetic fields and low- frequency alternating fields. The induced-current effects of alternating fields on the human body with a conductivity of approximately $0.25 \mathrm{~S} / \mathrm{m}$ are reasonably well known, or at least there is a biophysical basis for analysis and prediction. On the other hand, the influences of static or very low frequency fields on human subjects exposed over long time periods are neither known nor theoretically predictable.

The objectives were the acquisition of lowexposure data that can $b:$ used to evaluate any risks to the population incidentally exposed to environmental increases in magnetic fields as well as the acquisition of high-exposute data to be used 
in determining allowable exposure standards for the technical personnel working at magnetic facilities.

The study involves 792 scientists and technician who have been occupationally exposed to fields of at least $5 \mathrm{G}$ in strength for $\mathbf{8 0 0 0} \mathrm{G}$-hr during their working careers. The criterion is based on a typical exposure of a cyclotron worker for about a year and a half. The study includes acquisition of medical data from 792 exposed subjects as well as from 792 controls matched for sex, age, race and socioeconomic status from seven national laboratories in North America. Listed below are the institutions that participated and the number of subjects studied, which are matched by the number of controls.

1. Lawrence Berkeley Laboratory (LBL), 354

2. Brookhaven National Laboratory (BNL), 159

3. National Magnet Laboratory at MIT (NML), 6 (combined with FNAL)

4. Argonne National Laboratory (ANL), 66

5. Fermi National Accelerator Laboratory (FNAL), 71

6. Stanford Linear Accelerator Center (SLAC), 101

7. Oak Ridge National Laboratory (ORNL), 41
The studies of the latent effects from acute and very high exposures involve subjects who have worked in fields greater then $\mathbf{4 0 0} \mathrm{G}$ at facilities such as the calutrons at Berkeley (California) and Oak Ridge (Tennessee) during World War II. In addition, individuals involved in calibrating large magnets and individuals exposed to very high fields around bubble chambers are included in this highfield exposure population.

The number of individuals with diseaes from $\mathbf{2 0}$ catagories are shown for exposed and control populations in Fig 1 . No significant increase or decrease in the prevalence of diseases was found. Of the 792 subjects, 198 had $0.3 \mathrm{~T}$ or higher exposures for 1 hour or longer, and no difference was found between this group and the remainder of the exposed populations or the matched controls. There were no trends in the data suggestive of a dose-response (Fig. 2). Major advances made over the last two years involve a quality check on the data, including chi-square analysis of different portions of the population in a search for biases. A sound test of the data completed recently was an evaluation of whether there is a greater prevalence of respiratory conditions in those individuals who Were smokers vs. the nonsmokers. Evaluations relative to subject age, etc., have also been completed. The report on this work should be published by January 1986. 

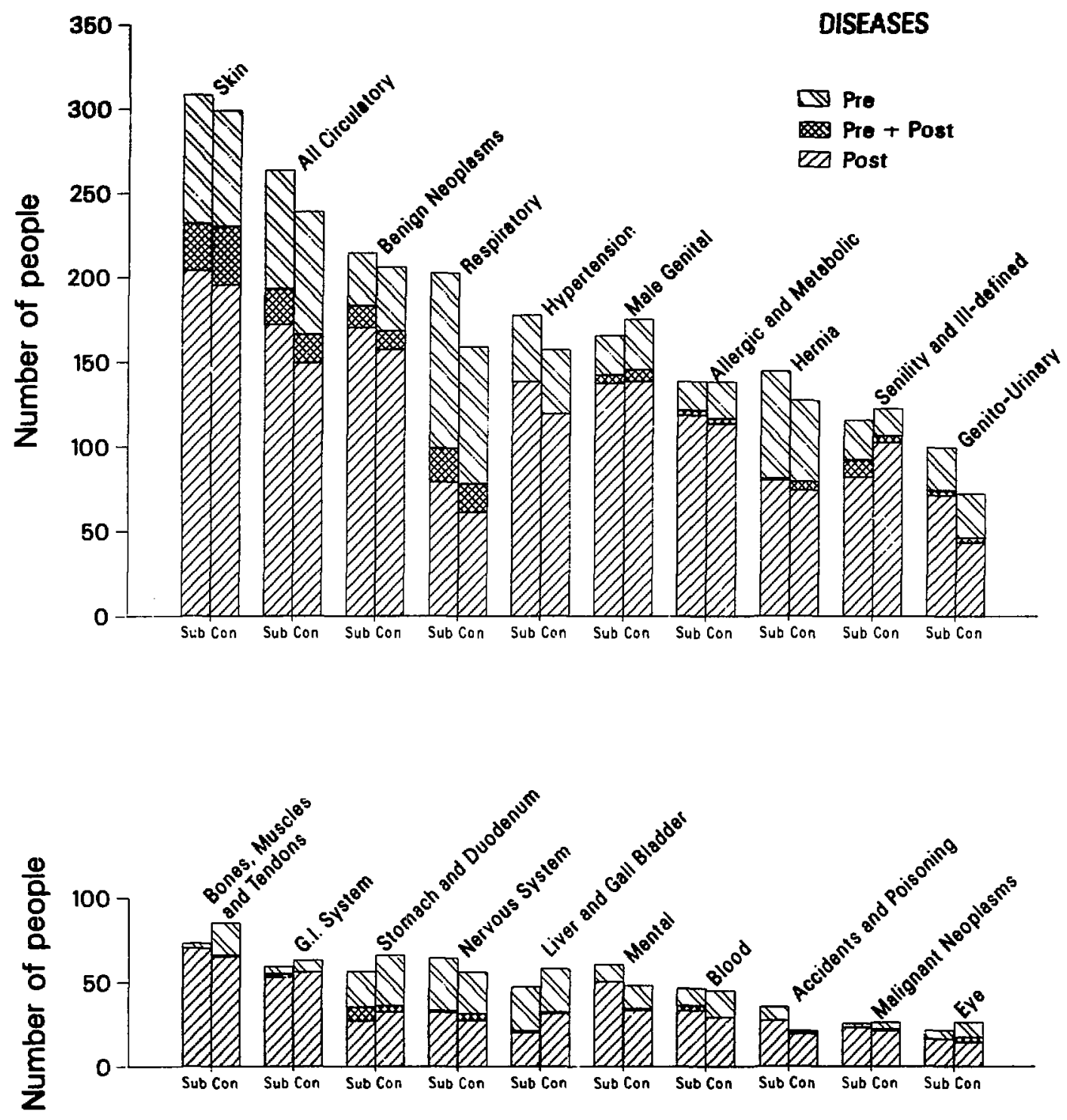

Fit. I. Disease frequency for exposed subjects (sub) and controls (con). Bars are oivided for disease occurrences pre., pre- and post-, and post-emplosment in the exposure or control environments.

(XCG 858-384) 
No. Subjects / No. Total with Disease $(S / T)$

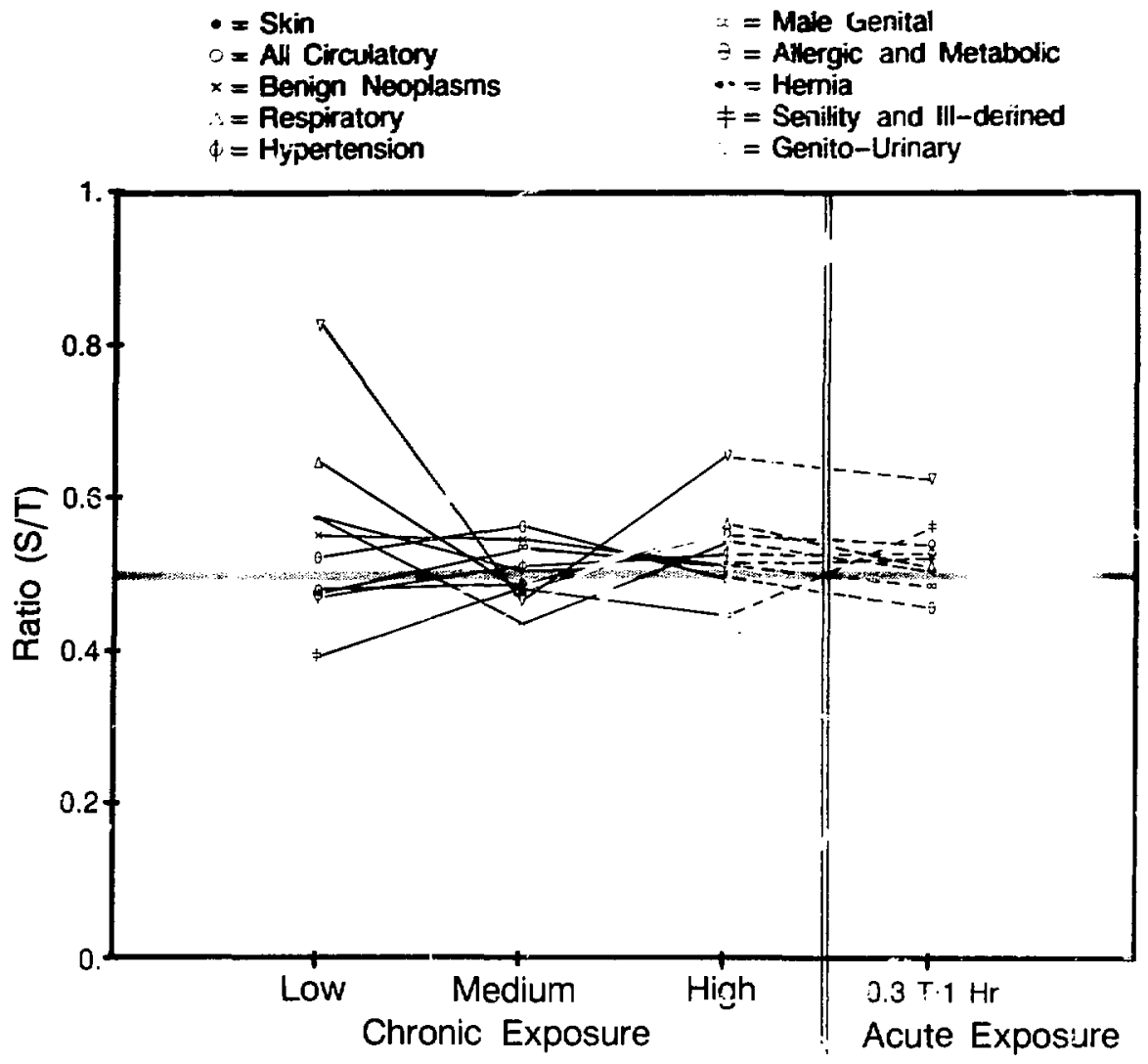

Fig. 2. Post-employment disease ratios as a function of exposure.

$(X B \backslash 847-7746)$ 


\section{Arrendices}

Appendix A. List of Contacts and Grants Supporting

Portions of Work Presented in this Annual Report

Appendix 8. 1985 Publications

238

Appendix C. Biology and Medicine Division Staff

Septsmber 30, 1985

249 


\section{Section 2. DONNER PAVILION}

\section{INTRODUCTION}

The biomedical program at Donner Pavilion continues to apply nuclear medicine research to the diagnosis and radiosurgical treatment of lifethrealening central nervous system (CNS) disorders, for example, intracranial arteriovenous malformations, which affect approximately one million Americans. In the clinical research program, stereotactic Bragg-peak radiosurgery, using narrow beams of heavy charged particles, demonstrate superior biological and physical characteristics in brain over $x$ rays, and $\gamma$ rays, and protons, namely, improved dose distribution in the Bragg peak, sharp lateral and distal borders, and less multiple scattering and range straggling for the same residual range in CNS tissues. CNS tissue response and alteration of cerebral blood-flow dynamics related to heavy-ion Bragg-peak radiosurgery is being investigated using three-dimensional treatment planning and quantitative neuroradiological imaging, including stereotactic cerebral angiography, x-ray computerized tomography $(C T)$, magnetic resonance imaging (MRI), cine$C T$, xenon $x$-ray $C T$ and positron emission tomography (PET). Based on the evaluation and treatment with stereotactically-directed narrow beams of heavy charged particles in over 160 clinical patients, using extensive clinical and neuroradiological follow-up, it appears that stereotactic heavy charged-particle radiosurgery can obliterate intracranial arteriovenous malformations (AVMS) or protect against recurrent hemorrhage with reduced morbidity and mortality. The clinical research program continues in collaboration with Stanford University and the Universily of California San Francisco.

Also under study are the physical properties of narrow heavy-ion beams for improving methods of dose defivery and dose distribution and for establishing clinical RBE/LET and dose-response relationships for human CNS lissues. The biophysics research program continues to examine the cellular basis of radiation-induced injury-cellular response and cell proliferation kinetics-in defined cell populations of the mammalian brain. Studies include the hierarchical organization and glial cell kinetics and the radiation response to cytotoxic insult, primarily the proliferative kinetics of the subependymal plate and the pathogenesis of early and late delayed radiation injury in brain, and CNS repair and regeneration after exposure to narrow beams of charged particles. The proliferative capacity of the mature glial cells and their ability to modulate responses to injury are of importance in CNS disease and healing processes. Neuroglial cells may enhance or decrease the regenerative capacity of neurons and the reformation of their interconnections with appropriate target cells. Furthermore, these altered capacities of neuroglial cells may also affect myelin renewal and remyelination processes following heavy charged-particle radiation injury. 


\title{
STEAOTACTIC HEAYY LON BAAGG FEAK RADNOSURCEXY
}

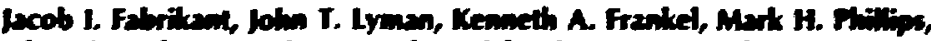 \\ Elward L Apen, Neela B. Manley, Richard P. Levy, Myrile L. Foster, \\ Frederick W. Yeater, George 1. Hampton, Maureen H. Mortord, and \\ Cabara Modlinski
}

\section{CLINICAL RESEARCH}

To date, the Donner Pavilion program has evaluated and treated over 160 neurological patients with intracranial vascular disorders, using stereotactic heavy charged-particle Bragg peak radiosurgery. Most lesions have been deep, surgically inaccessible arteriovenous malformations in the brain. Patient selection and management are based on defined human subject protocols; patients are placed into one of three radiosurgical treatment groups: stereotactic heavy charged-particle radiosurgery, flow-directed embolization of accessible feeder vessels followed by stereotactic radiosurgery, or ligation or excision of accessible feeder vessels followed by stereotactic radiosurgery. The aim of flow-directed embolization and/or surgical ligation of feeder vessels is to decrease the size and to shape the AVM for stereotactic radiosurgery using narrow heavy-ion beams. More than half of our patients are treated solely with stereotactic radiosurgery; more than one-third undergo surgical ligation of accessible feeder-shunts prior to radiosurgery; and a small number undergo prior embolization procedures.

All patients are followed frequently, both clinically and radiologically, after radiosurgical treatment; about two-thirds have now had 24-month follow-up and extended follow-up to 5 years is now done on a regular basis. Clinical objectives are to achieve changes in the intracerebral hemodynamic condition, resulting in elimination of subarachnoid or parenchymatous brain hemorrhage and a decrease in progressive or fixed neurological deficils, in frequency of seizures, and in subjective complaints, including frequency and intensity of headaches. Preliminary observations in the first 96 patients thus far treated to the end of 1984 indicate that these objectives are being achieved. The first 66 patients have been evaluated clinically to the end of 1984: 45 patients have improved neurologically, 16 patients demonstrated no change, and 5 have worsened (Table 1). The follorr-up periods for the remaining patients are too short 10 draw firm conclusions al this time, but the general pattem of clinicall response continues. There have
Table 1. Sfereotactic adiosuren cerebral arferiovenous matfomwions: Results.

\begin{tabular}{|c|c|c|c|c|c|c|c|c|}
\hline \multirow[b]{2}{*}{ Year } & \multirow[b]{2}{*}{ Pis } & \multicolumn{4}{|c|}{ Symptoms } & \multicolumn{3}{|c|}{ Angopraphy } \\
\hline & & Imp & $N C$ & Prog & SAH & $\operatorname{Comp}$ & Part & $N C$ \\
\hline 1980 & 2 & $2 / 2$ & $0 / 2$ & $0 / 2$ & $0 / 2$ & $0 / 1$ & $1 / 1$ & $0 / 1$ \\
\hline 1981 & 5 & $3 / 5$ & $1 / 5$ & $1 / 5$ & $0 / 5$ & $0 / 4$ & $2 / 4$ & $2 / 4$ \\
\hline 1982 & 16 & $12 / 16$ & $4 / 16$ & $0 / 16$ & $1 / 16$ & $1 / 12$ & $7 / 12$ & $4 / 12$ \\
\hline 1983 & 30 & $18 / 28$ & $8 / 28$ & $2 / 28$ & $2 / 28$ & $5 / 16$ & $10 / 16$ & $1 / 16$ \\
\hline 1984 & 43 & $10 / 15$ & $3 / 15$ & $2 / 15$ & $1 / 15$ & & & \\
\hline Totals & 96 & $45 / 66$ & $16 / 66$ & $5 / 66$ & $4 / 66$ & $6 / 33$ & $20 / 33$ & $7 / 33$ \\
\hline
\end{tabular}

Pts = patients; Imp = improved; NC = no change; Prog $=$ progressively worsened; $\mathrm{SAH}=$ subarachnoid hemorrhage; Comp = complete obliteration; Part $=$ partial obliteration; $\mathrm{NC}=$ no change.

been four cases of intracranial hemorrhage occurring since radiosurgery; two of these patients were cured of their AVM and two died as a result of the hemorrhage. This complication rate falls well below the probability of hemorrhaging in a population of untreated deep AVM patients. There have been no patients with known late delayed radiation injury in the brain.

Hemodynamic and anatomic changes in cerebral vessels are examined by cerebral angiography. The hemodynamic changes are manifested by a decrease in blood flow through the pathologic cluster of abnormal blood vessels with a decrease in size of the feeding arteries, shunts and draining veins; anatomic changes include progressive decrease in the size of the AVM until stabilization, or lotal disappearance. The hemodynamic changes occur successively and are usually observed before the morphologic changes. The results of the yearly follow-up cerebral angiographic studies in the initial patients examined to date demonstrate complete obliteration of the AVM has occurred in about $20 \%$ of the patients; partial obliteration has occurred in iwo-thirds of the patients; no change has occurred in the remaining patients. Hemodynamic changes with attendant decrease in blood flow through the AVM has been seen to occur in about b0\% of patients thus far investigated. 


\section{PHYSKCS RESEARCH}

The new beamline configuration for stereotactic cereioral imadiation with the Bragg jonization peak of the helium-ion beam ( $230 \mathrm{MeV} / \mathrm{u}$ ) has been developed at the 184-Inch Synchroirclotron. The modified beam has a $14.7-\mathrm{cm}$ range in water to the Bragg peak with very sharply delimited lateral and distal borders. The practical limits on beam diameter range from $6 \mathrm{~mm}$ to $40 \mathrm{~mm}$. The unmodulated Bragg peak maximum dose is greater than three times the entrance dose and ihe width of the peak at $80 \%$ of the maximum is $8 \mathrm{~mm}$. The range can be modulated by a rotaling acrylic variablethickness absorber to increase the width of the high-dose region to $40 \mathrm{~mm}$. The maximum range of the helium-ion beam $(230 \mathrm{MeV} / \mathrm{u})$ is greater than that of the Harvard and Uppsala proton beams, and more energy degradation is necessary to obtain the same residual range. Since the nuclear charge and mass of helium are greater than those of the proton, the multiple scattering and the range straggling can be less for the same residual range in tissues. Studies are in progress at the Bevalac to develop beam characteristics of heavier charged particles, st.ch as carbon ions and neon ions, for stereotactic Bragg-peak radiosurgery in the brain; such parallel beams of monoenergetic heavy ions with small uniform transverse profile and modified Bragg peak have physical characteristics with unique advantages for achieving much improved dose distribution and dose focalization with multiple port delivery.

Our present procedures use the modulated or unmodulated helium-ion beam at the 184-Inch Synchrocyclotron. We are currently planning stereotactic heavy-charged-particle radiosurgery at the Bevalac to take advantage of the heavier chargedparticle beams, namely, carbon-12 (308 MeV/u), and neon-20 (425 MeV/u) and a new helium beam line. The advantages of these heavier ions over helium ions and protons include narrow beams with less range straggling and less inultiple scattering for the same residual range in tissues, and improved dose distribution in the Bragg peak, with very sharp lateral and distal borders, and with greater sparing of adjacent critical struclures in the brain.

Chatterjee and his colleagues are exploring the use of radioactive beams, e.g., carbon-11 and neon-19. at the Bevalac for localizing the region where heavy-ion beams are deposited in tissue. If the majority of the deposited beam particles in tissues do nol leave the region of deposition, then imaging the distribulion of radioactivily can serve to measure the stopping power of brain tissue and to check the adequacy of beam delivery in radiosurBery. A special coincidence camera has been builh at LBL to image the distribution of the shon-lived positron-emitling beam particles in human tissues. The stopping region of a small radioactive beam can be delermined wilhin a few seconds with an accuracy better than $1 \mathrm{~mm}$ when the dose delivered by the beam is about $0.02 \mathrm{~Gy}$. The application of this new nuclear medicine procedure to stereotactic radiosurgery of intracranial vascular disorders is presently being explored.

\section{Heavy Charged-Particle Treatment Planning}

Narrow beams of heavy charged particles provide improved physical dose distributions due to the relatively small amount of multiple scattering and by the rapid fall-off of dose with depth beyond the end of the Bragg peak. The same physical requirements make stringent demands on the accurate assessment and compensation of inhomogeneities without which precision radiosurgery with focal charged-particle beams cannot be accomplished. Our method for treatment planning for stereotactic charged-particle radiosurgery continues to develop, and involves a sequence of stages using stereotactic neuroradiological procedures (namely stereotactic cerebral angiography, stereotactic computerized tomography (CT) and magnetic resonance imaging), AVM-target contouring, reconstruction of $C T$ data and angiographic mapping, conversion of $C T$ data to relative stopping power values, threedimensional reconstruction of isoeffective centralaxis depth distributions for dose-distribution calculations and aperture-compensator fabrication. Cerebral angiographic and $C T$ data used for target contouring and conversion to relative stopping power values are transferred to the VAX-11/78O computer system; multiple-entry angles and beam ports are chosen to confine the high-dose Bragg ionization peak to the contoured target of the AVM and the arterial feeders/shunts, while carefully protecting adjacent critical structures in the brain (Fig. 1). The development of pixel-by-pixel treatment planning for heavy charged-particle radiosurgery permits design of collimator aperlures for entry portals and selection of the appropriate range and modulation of the heavy-ion beam, thereby providing isoeffect and physical dose-distributions on reconstruction CT images (Fig. 2).

\section{Noninvasive Imaging Research}

Neuroradiological and nuclear medicine pro. cedures are used to investigate three areas of brain 


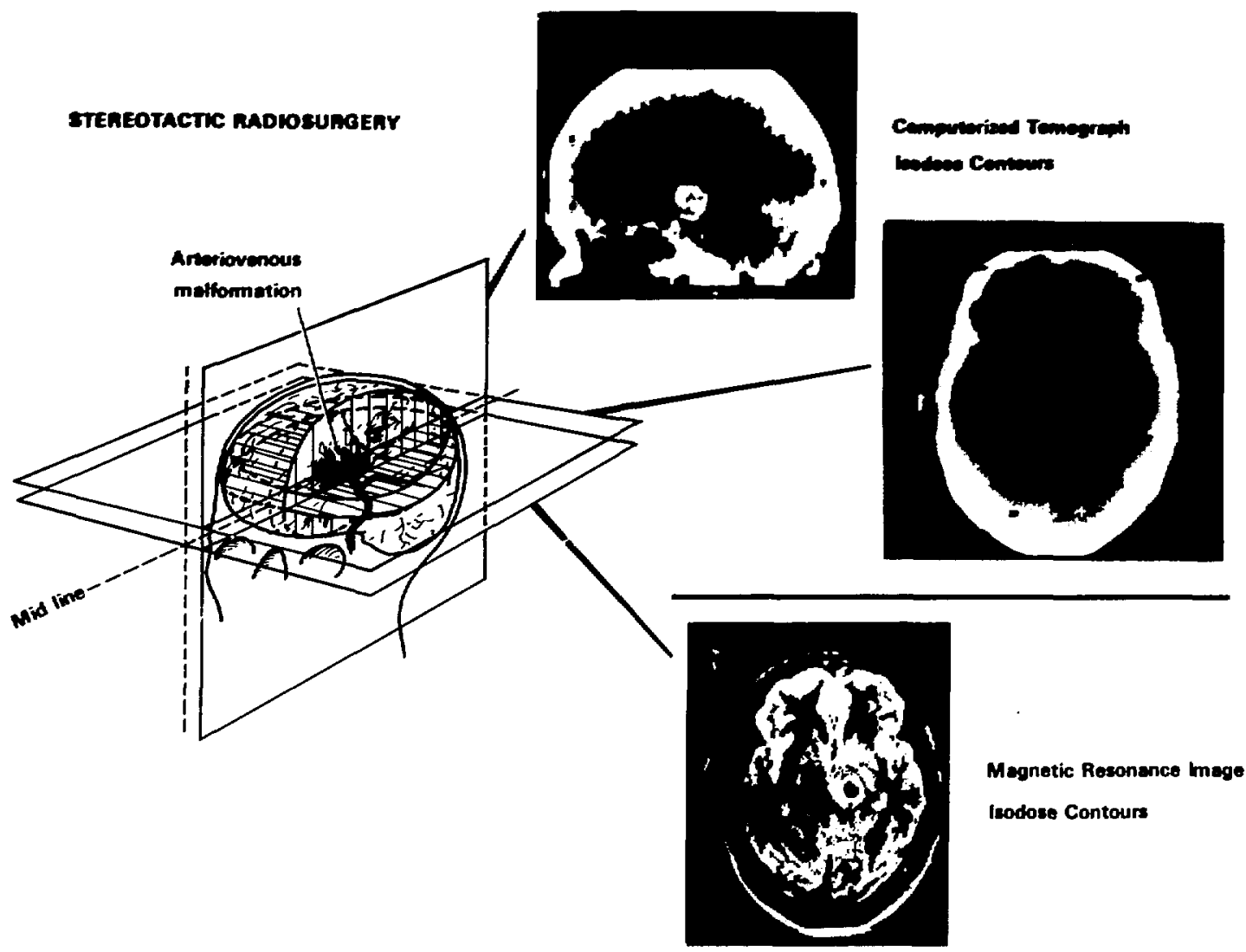

Fig. 1. The physical basis for stereotactic heavy-charged-parlicle Bragg-peak radiosungery trealment planning for intracranial vascular disorders integrates information from the stereotactic cerebral angiogram (left), the stereotacic $C T$ scans (upper right, right) and the magnetic resonance image (lower right). The data are used for target contouring and conversion to relative stopping power values. Multiple-erivy angles and beam ports are chosen to confine the high-dose Bragg ionization peak to the contoured target of 't artericsenous malformation, while protecting adjacent critical structures in the brain.

(CBB 843-1469)

structure and function and the effects of focal irradiation with heavy-ion beams: hemodynamic function and cerebral blood flow, alteration of structure and function and homeostatic control, myelin maintenance, and injury and repair in irradiated brain tissue.

Cerebral Angiography. Stereotactic cerebral angiography is carried out before stereotactic radiosurgery in order to determine the precise size, shape and location of the treatment target volume for heavy charged-parlicle Brags-peak radiosurgery. Following irradiation, cerebral angiography is done at 12-month intervals on all patients, up to a period of 5 vears. The arteriographic changes being exam- ined include hemodynamic changes, e.g., decrease in cerebral blood flow, in the size of arterial feeders/shunts and in draining veins, and morphologic changes, for example progressive decrease in size of AVM until stabilization or total disappearance (Fig. 3).

The hemodynamic changes occur successively and are frequently observed before the morphologic alterations. In a few cases, the effects of the irradiation appear to occur early; nevertheless, in the majority of patients who have had angiograms within 6 months of radiosurgery, no changes are visible. In some patients, particularly those with veny large cerebral AVMs, no visible changes have occurred affer $I$ years. In several patients, before 


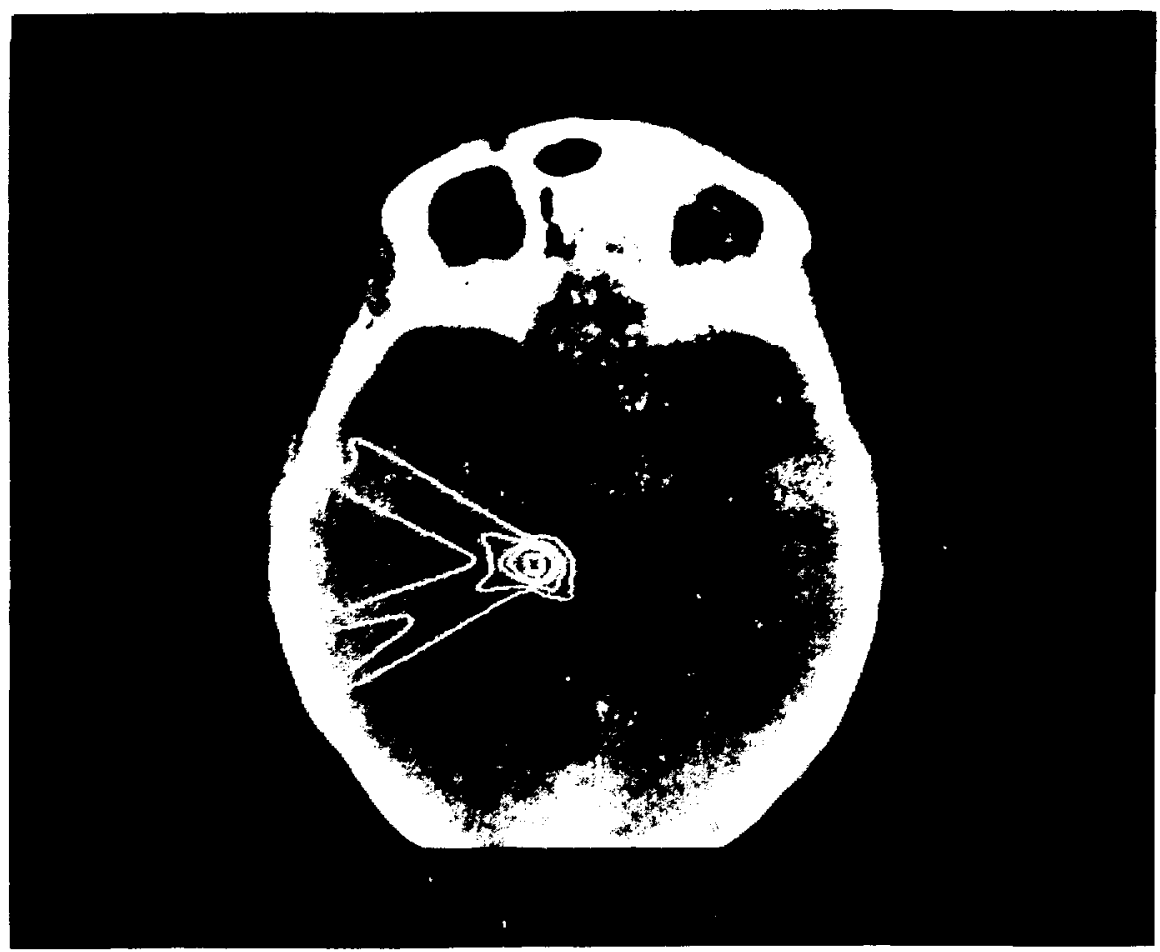

Fig. 2. Isodose contour, stereotactic heavy charged-particle Bragg-peak radiosurgery of a brain stem AVMi in a 42-vear-old female. The helitum-ion beam was collimated by an $8-\mathrm{mm}$ circular aperture. Treatment was carried out using four coplaniw ports in 1 day to a volume of $250 \mathrm{~mm}^{3}$ within the brain stem: trea'ment dose. $45 G \mathrm{~F}$.

(XBB 853-1740)

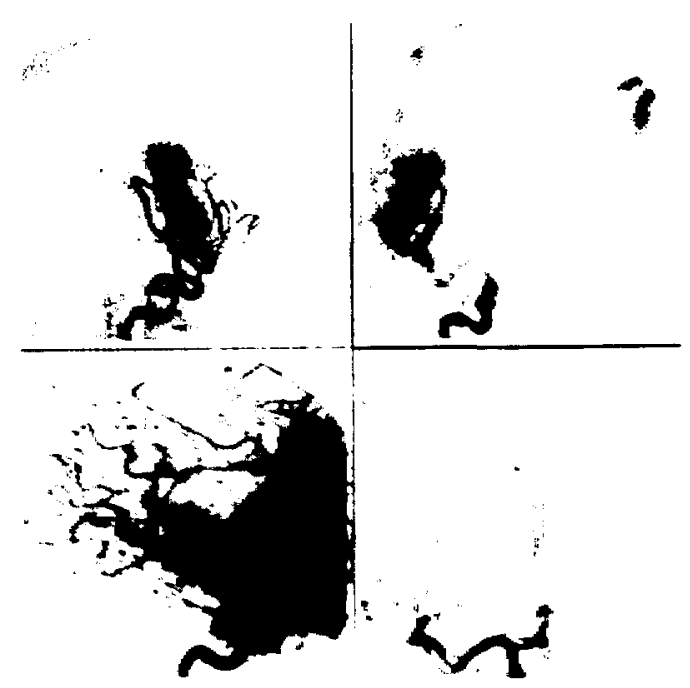

gross morphologic changes in the AVM can be determined, hemodynamic changes are noted: the cerebral blood flow rate through the malformation appears to decrease with time, with concomitant decrease in the size of the feeding arteries and outflow veins. In most patients, where response has occurred, the angiograms initially show a marked decrease in the size of the AVM due to partial obli-

Fit. 3. Palient is a 39-year-old female with recurrent seizures and progressive motor and sensory changes due to a steal phenomenon resulting from a right cerebral (parietal) AVM. Upper: Cerebral angiogram lat. and AP/ demonstrates the size, shape, and location of the AVM and the feeding vessels arising from the branches of the middle cerebral attery. The anterior cerebral arteny and its branches do not fill. Lower: Cerebral angiogtams itaf. and API I year affer sleseotactic helium-ion Brage peak radiosurgen tolose, 45 Gyll. There has been complete ablitetation of the AVM with redistriburtion of the normat biood tilow. There is mow filling of the anjerior and posterior crenterll arieries and their ounches.

(x85 851-2094) 
Ieration of the malfomation within the first year; some stabilize, and progression to complete oblileration may or may nof occur. Total obliteration, in cases where it is documented angiographically, usually occurred within 1 to 2 years after treatment. Since early filling of a small vein may be the only indication of a persistent shunt, complete obliteration of the AVM can be confirmed only by angiography.

X-Ray Computerized Tomography. Stereotactic CT scanning has become the basis for pretherapeutic diagnostic confirmation, for assessing AVM contour information, for transferring the angiographic data into three-dimensional format for treatment planning, and for conversion of CT data to relative stopping power, i.e., relating $\mathrm{CT}$ numbers on a pixelby-pixel basis and water-equivalent pathlength per pixel. This provides the basis for heavy chargedparticle radiosurgery treatment planning. The highresolution scans are necessary for establishing the relationship of the AVM to critical brain structures, such as the brain stem, by relating the $C T$ sections corresponding to the coordinates obtained on the stereotactic angiogram. Stereotactic CT is essential for evaluating and treating cryptic AVMs; these are small vascular malformations that are not detectable by means of routine cerebral angiography, but that are identified by pathological examination as a cause of spontaneous ceretral hemorrhage. CT may reveal possible delayed radiation necrosis, hemorrhage or infarct as iell. Initially, serial CT scans were obtained on ali patients every 3 to 6 months to assess response to radiosurgery and potential focal radiation injury to the brain resulting from charged-particle bean intadiation. However, no early or bie changes in the brain were seen during the first 2 years of scanning in any patients. Accordingly, because such frequent scanning was considered clinically unnecessary and expensive, we have now limited serial scanning to yearly intervals for the first 5 years unless more frequent examination is clinically warranted.

Cine-Computerized Tomography. Cine-CT scanning has been carried out on five AVM patients (in a collaborative program with the Division of Neuroradiology, University of California San Francisco). We are examining the extent to which cine-CT scanning can determine cerebral blood flow through vascular disorders. In cine-CT scanning, iodinated contrast agent is injected and then two or four contiguous slices with subsecond rate exposures are obtained. Following the scan sequence, cerebral blood flow dynamics can be computed through a region of interest as a function of time (Fig. 4). By scanning through various regions of the body, it is possible to compute such quantities as the percentage of cardiac output that the arteriovenous malformation receives. Such information should prove useful in measuring the temporal and morphologic responses of cerebral blood flow through brain tissue following stereotactic radiosurgery.

Magnetic Resonance Imaging. We have begun MRI studies before and following radiosurgical treatment. Currently, all nonsurgical patients at Stanford
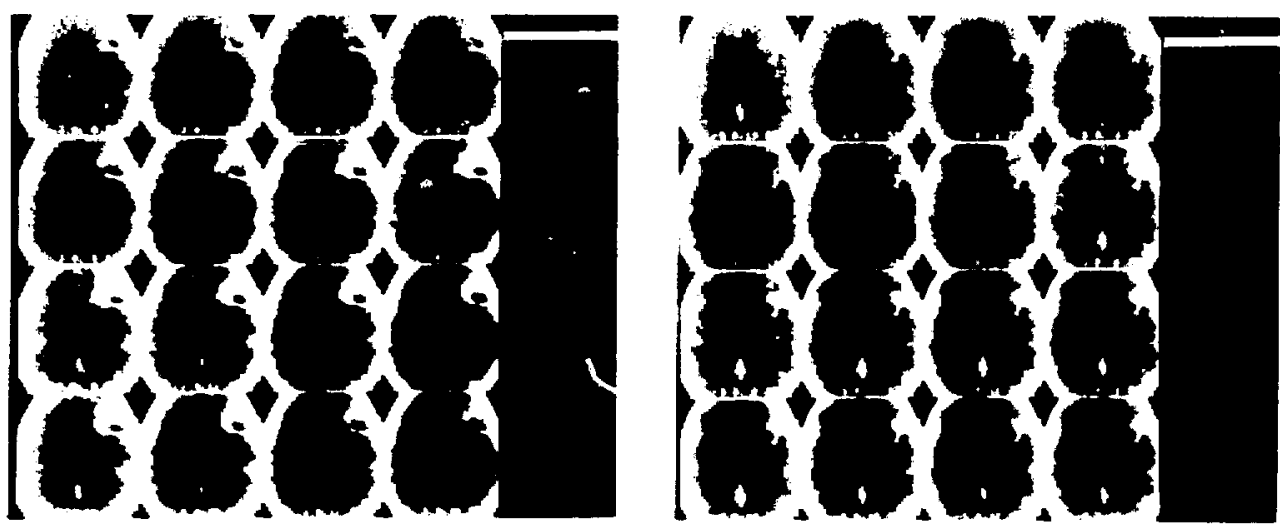

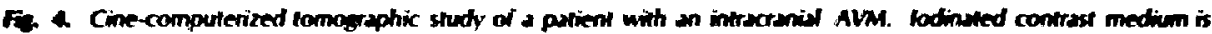

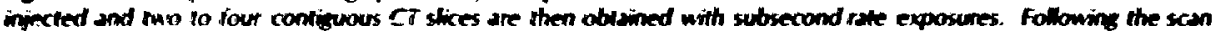
sequence, cerebral blood ikow dinumics can be compuned through a region of inierest as a function of lime. 
University Medical Center hrve been selected for study, primarity on the basis of location and size of lesion. Changes of edema and scarring surrounding the AVM have been noted as eary as 5 months following radiosurgical treatment, with a concomitant decrease in cerebral blood flow through the pathologic cluster of vessels. These MRI studies are aimed at determining and evaluating brain tissue tolerance, the mechanisms of radiation injury and repair, the examination of cerebral blood flow (Fig. 5), and the processes of myelination and myelin renewal, in relation to dose, dose-fractionation, heavy charged particle, and temporal patterns of radiation response. Furthermore, we are exploring the application of MRI to stereotactic heavy charged-particle treatment planning; the early results appear promising.

Xenon Computerized Tomography. Blood shunting through the AVMs in the brain, due to highvolume, high-flow changes, can cause focal or generalized neurological deficits by the vascular steal phenomenon where tissue perfusion in the adjacent brain is decreased. The obliteration of the AVM may be associated with decrease in neurological deficits and recovery. There is evidence that such deficits can regress, and that improved perfusion can, at times, reverse the cerebrovascular steal, However, little is known about the pathophysiology and cerebral blood flow dynamics of AVMs and the effect of this altered brain tissue perfusion. We are presently studying patients with AVMs who are undergoing stereotactic radiosurgery (collaborative program with Stanford University Medical Center Departments of Radiology and Surgery) using stable xenon computerized tomography to quantitate the temporal pattern of tissue response and cerebral blood flow following heavy-ion radiosurgery. The results of xenon-CT studies provide a detailed mapping for quantifying altered cerebral perfusion in brain tissue adjacent to and remote from the AVM (Fig. 6). Reduction in cerebral blood flow occurs in both cerebral hemispheres and is most severe when the AVM is large or has a major intracranial arterial supply, and is most pronounced in brain tissue adjacent to the AVM. It has been demonstrated that, following partial or complete vascular obliteration of an AVM, cerebral blood flow is decreased, whereas blood flow in adjacent and remote brain tissue increases. Neurological improvement may occur as a result of reduction in the vascular steal phenomenon because of obliteration of the pathologic cluster of shunts following stereotactic radiosurgery.

\section{BIOPHYSICS RESEARCH}

Symthesis and Maintenance of Myelin. One of the major problems in the neurosciences is the extreme cell heterogeneity of the brain. The CNS tissue is composed largely of three types of highly characteristic cells-neurons, astrocytes, and oligodendroglia - that are recognized by their size and distinctive morphology. The architecture of the brain and
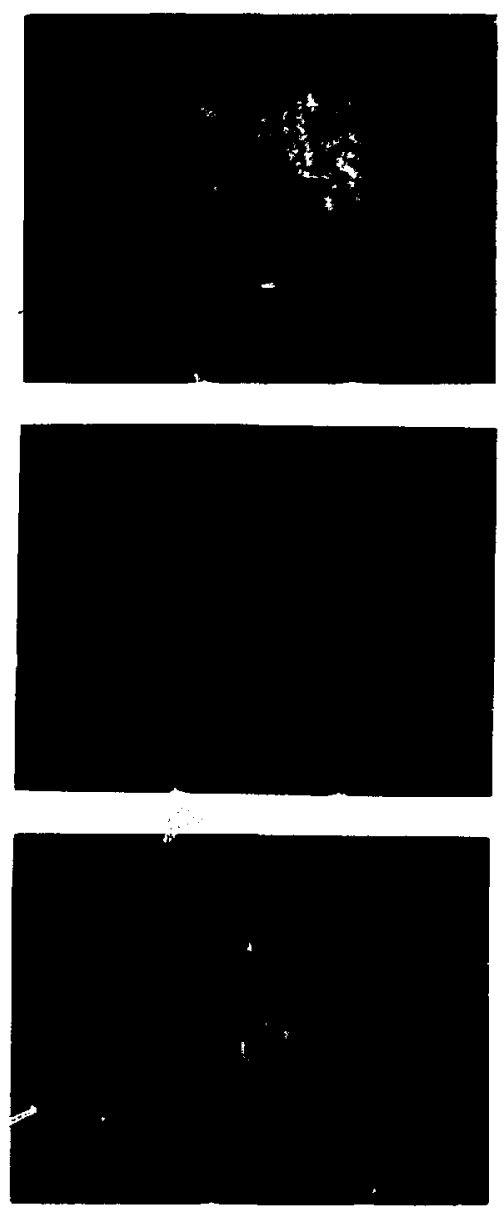

F. 5. Magnetic resonince imare demonstrating regional perfusion kinetios and brain blood-fiow ansit in a palient with a bage brain stem arteriovenous malformation. The vascular region of interest selected is defined (upper panel) and charackerized (midtie panel), and blood how sherations ine quentiuned as a finction of time through the offending vascular lesion (bomer pinel). An colliborstion with Or. Peter Vall, Rexeunch Medicine Laboratony.) 


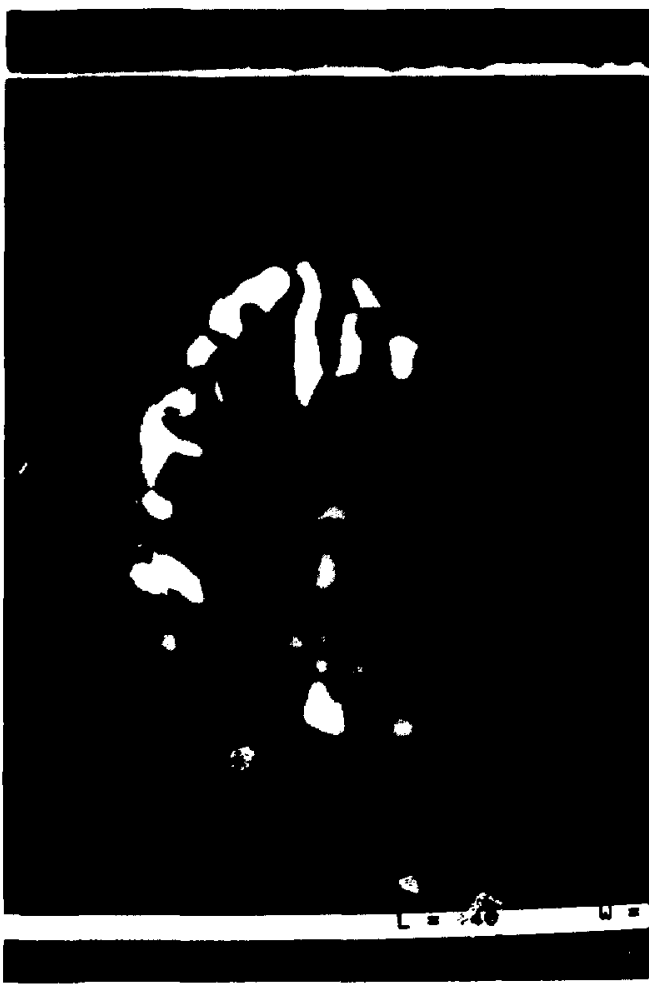

Fis. 6. Xenon-computerized tomographic imaging of an intracranial AVM in a 49-year-old male with significant steal phenomenon involving the left cerebral (parieto-occipital)cortex. The scan demonstrates cerebral blood flow dynamics and alterations of brain tissue perfusion involving the immediate area of the AVM and diminished perfusion in the entire ipsilateral cere. bral cortex.

(XBB 852-1629)

its hierarchical structure are such that all three cell populations, together with the vascular structures, intertwine to form a highly integrated tissue. This complex tissue also contains populations of cells with different biochemical and metabolic characteristics; this has made biochemical studies on defined cell types in intact normal brain tissue virtually impossible, despite the distinct functions of these cells. The problem of cellular heterogeneity in the brain is accentuated when the brain is abnormal. The abnormalities may include changes in the size, shape, or architeclure of the cells or the cell populations so that their characteristic morphology or metabolic function may be altered, for example, when brain cells undergo radiation injuny leading to early and late delayed elfects. in order to overcome the difificulties in delining changes in cells or their membranes in the highty integrated brain tissue, we have begun to develop techniques to isolate and maintain defined brain cell populations is short-term tissue culture in order to provide information about the specific abnormalities and to assess potential cell-specific propenties for use as indicators of cell damage. We have begun studying specific membrane components in isolated purified populations of defined cell types from the brain. We are particularly interested in myelin, the membrane essential for normal functioning of the nervous system, with special emphasis on oligodendrogial function and synthesis of myelin and its breakdown, and its response to charged-particle irradiation.

\section{Induction and Repair of DNA Damage}

Capillary Endothelium. The late delayed radiation effects in the brain are considered vascular in origin and associated with vascular endothelial injury and functional disturbances, leading to ischemia, cell death and necrosis. Late radiation effects on the capillary endothelium in irradiated mammalian brain have been described and have been studied with respect to dose and temporal patterns of change; however, the mechanisms leading to these changes are not known. To clarify the sequence of events in the mammalian brain following charged-particle irradiation, we are examining the endothelial cells early after irradiation with low and moderate doses. The induction of and in-vivo repair of DNA strand breaks, a very sensitive index of early radiation effects, is being studied in rat and mouse brain cerebral and cerebellar capillary endothelial cells after helium-ion $(230 \mathrm{MeV} / \mathrm{u})$ and neon-ion (425 $\mathrm{MeV} / \mathrm{u}$ ) irradiation in vivo (doses: 2-15 Gy). A microscope photometer method that measures the unwinding of DNA is used. Single endothelial cell nuclei are stained with the fluorescent dye acridine orange and the intensity of the red fluorescence (from single-stranded DNA) relative to the green fluorescence (from double-stranded DNA) is used as a measure of DNA strand breaks. Initial observalions demonstrate that most DNA strand breaks in cerebral and cerebellar capillary endothelial cells are repaired within 30 minules post-irradiation with low-LET radiation, with a linear dose-effect relationship. This appears to obtain, as well, for DNA strand breaks in single endothelial cells in cerebrum and cerebellum exposed to high-LET chargedparticle beams (Fig. 7). We are continuing to investigate dose-effect relationships for helium-ion, catbon-ion, and neon-ion irradiation, the radiation 

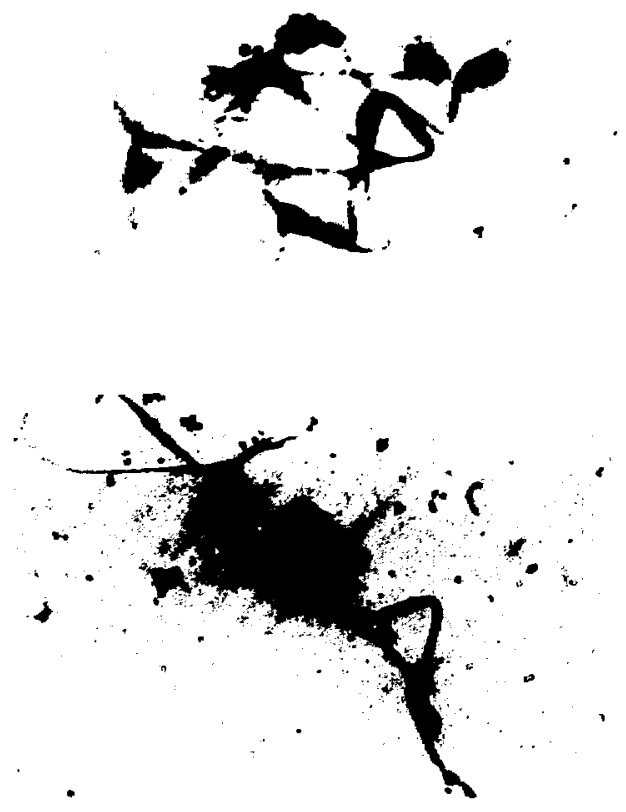

Fig. 7. DNA strand breaks in capillary endothelium in the irradiated mouse brain. The microscope photometer method used measures the unwinding of DNA; the fluorescent dye technique provides a measure of single-stranded and double-stranded DNA lesions reflecting the damage to the genome of these target cells of the brain. L'poer: unirradiated; Lower: irradiated. (In collaboration with Dr. Kirsten Rosander, Swedish National Defense Research institute.)

(XBB 8S1-796-A)

responise (DNA strand breaks) in vivo, the response vs. time after irradiation, and the kinetics of shortterm and long-term repair of DNA strand breaks (in collaboration with Dr. Kirsten Rosander, Swedish National Defense Research Institute).

DNA Damage and Biological Dosimetry. The repair kinetics of radiation-induced DNA strand breaks demonstrate three components: an initial fast rate of repair, followed by a slow rate of repair, and delayed or long-term repair associated with residual and irrepairable breaks. We are developing a method of assaying for DNA strand breaks in the Simion virus $\mathbf{4 0}$ genome, irradiated intracellularly, and are attempting to apply the technique to biological dosimeiry in vivo for study of narrow heavy-charged particle beams. The method uses an electro-optical effect to measure conformation changes in phage DNA, and the number of strand breaks is thereby obtained (Fig 8). For a radiation of a given quality. this information yields the dose applied, or it can be used to compare the RBE of different radiation fields. This will prove valuable as we examine heavier ion beams for the treatment of brain disorders. Furthermore since the target volume of viral DNA is very small, and because it is difficult to assay DNA lesions in the large mam. malian genome, dose distribution can also be mapped in order to understand biological effects and tolerances. As we move to the in-vivo isolation and maintenance of brain cell populations, the method may be adapted for understanding radiation response of different brain struclures in vivo, with applications to improved treatment and to evaluate the potential of heavier charged particle beams and beam delivery systems in clinical research.

Brain Cell Population Kinetics. In the young adult CNS, the subependymal plate of the lateral ventricles in the brain appears to be the only tissue that is actively proliferating. The relatively high rate of proliferation may be a continuous source of progenitor or stem cells for new glial cells, both for glial cell renewal in normal conditions or during regeneration after cytoxic injury. Based on experimental observations of quantitative analysis of changes in time of counts of various cell types, a model of glial cell renewal and differentiation can be prcposed, based on original cytologic criteria established by Leblond: four characteristic nuclear patterns-small dark, small light, large dark, and large light nuclei-define stages of cell proliferation and differentiation and migration patterns of glial

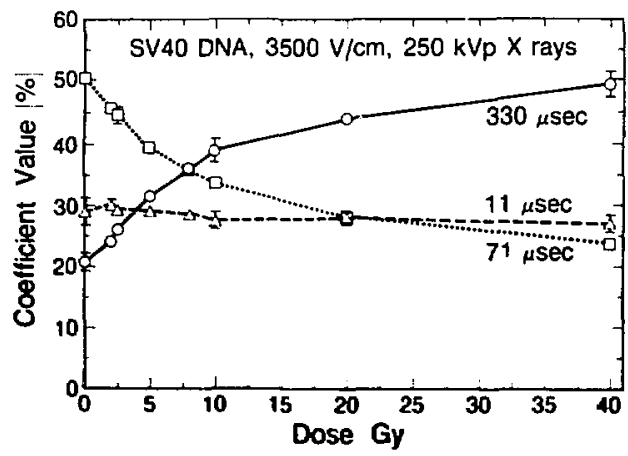

fit. a Decay carne coefficienss as a function of radiation dose in Gy) tor SW+O DNA strand breaks $(3500 \mathrm{~V} / \mathrm{(m})$ for low-LET radiation $\left(250-k V_{p} \times\right.$ rays $)$. The meythod has proven to be rellable and reproducible in the dose tanges of clinicall importance in sterectactic adiosurgen. 
cell precursors and their progemy. Following injection of tritiated thymidine, initially only lange dark nuclei are labeled, these apparently divide promptly to become small dark cells. Small dark cells represent a stem cell pool in the subependymal plate; they appear to be glial cell precursors and young oligodendrocytes. These cells transform into cells with small light nuclei, which are glial ceil precursors that migrate in the brain. About $20 \%$ of the small light cells transform to large light nuclear cells, as precursors of astrocytes.

The subependymal plate cells undergo prompt radiation-induced injury. The cellularity of the plate is depressed following exposure to low-LET radiation, and the mitotic activity is strongly reduced for doses up to $45 \mathrm{~Gy}$, but recovery appears to be dose dependent, e.g., after 40 Gy no recovery occurs and cell population counts are depressed, whereas after lesser doses, control levels are reached after a few months. It has been observed that following irradiation the dark cells disappear rapidly and the light cells do not; this suggests that the light cells remaining represent a glial stem cell pool with relatively slow turnover times, whereas the dark cells comprise a rapidly proliferating stem cell population. This observation is consistent with the view that the dark cells represent a stem cell population in the subependymal plate and is responsible for recovery after high-LET exposure (Fig. 9).

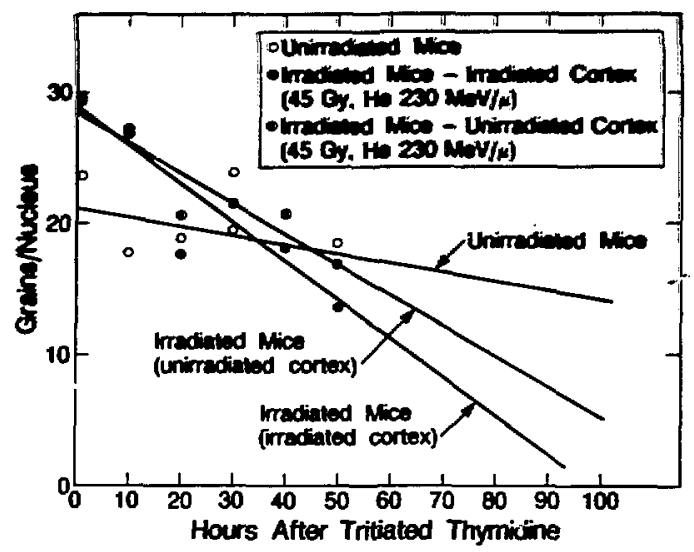

fig. 9. The nuclear grain counts in sube,sendymal plate cells in the mouse brain following $45 \mathrm{~Gy}$ (helium ions, $230 \mathrm{MeV} / \mathrm{u}$ ) are plotted as a function of time after injections of tritiated thymidine $66 \mathrm{hr}$ after exposure. Linear regression analysis demonstrates a profound shortening of the population doubling times compared with the unirradiated controls; the unirradiated half of the cerebral cortex in irradiated mouse, however, shows the same temporal pattern of cellular response. Population doubling times of subependymal cells are: irradiated mice (imadiated cortex), $\sim 44 \mathrm{hr}$; irradiated mice (unirradiated cortex), $\sim 56$ hr; unirradiated mice, $\sim 139 \mathrm{hr}$.

(XBL 8511-8597) 


\section{SECTION 3. PHYSIOLOGY}

\section{INTRODUCTION}

During the past year the name of the Environmental Physiology Group was changed to Physiology Group in order to reflect more accurately the nature of its programs. Limited research on environmental issues such as ozone toxicity is still being performed by scientists within the Physiology Group. However, the primary research emphasis is on elucidating cellular and tissue regulatory mechanisms, and on assessing the perturbations of biological control mechanisms that occur in response to pathogens, toxic chemicals, and radiation.

This year's annual report for the Physiology Group contains a discussion of research progress in programs related to the endocrine and hematopoietic systems, hematozoan infections, nonionizing radiation bioeffects, and transuranium element clearance techniques. It includes summaries of the research programs of two new investigators, Paul $\mathrm{H}$. Silverman and Robert P. Liburdy, who joined the Group in the latter half of 1984. Silverman is a specialist in the field of parasitology and Liburdy in the field of microwave radiation bioeffects. The following is a summary of highlights of the Physiology Group programs described in this year's annual report.

Two of the major effects of advanced malarial infections are a severe anemia and immunosuppression. The research reported by Silverman et al. suggests that both syndromes may be due to the depletion of hematopoietic stem cells in response to malarial infection. Studies with three species of murine malaria have shown that the marrow content of pluripotential stem cells measured in a spleen-colony assay (CFU-S) was significantly reduced during advanced stages of parasitemia in mice. The interesting finding was made that the level of biologically active serum erythropoietin (Ep) increases in the infected hosts in parallel with the onset of severe anemia. This observation suggests that the decreased level of CFU-S may lead to a deptetion of exythroid progenilor cells that can respond to Ep. Along with the decrease in CFU-S, a marked decrease in marrow cellularity and a significant splenomegaly were observed. The Latter finding indicates that the spleen alone is not able to satisfy the hematopoietic requirements of malariainfected mice. Another interesting finding by Silverman et al. was the observation that nonspecific stem cell stimulation by endotoxin conferred some degree of protection in mice against the lethal effects of malarial infection.

Additional new information on the molecular basis of the dyserythropoiesis that accompanies malarial infection is described by Schooley and Kullgren. They have compared the rate of DNA synthesis following exposure to Ep of spleen cells from mice made anemic by malarial infection and by injection of phenylhydrazine, a drug that destroys mature erythrocytes. The spleen cells from phenylhydrazine-treated mice exhibited a burst of DNA synthesis in response to Ep, whereas the spleen cells from malaria-infected mice did not. It was also found that the addition of conditioned medium from cultures of malaria-infected spleens to cultures of spleen cells from phenylhydrazinetreated mice inhibited the resporise of the latter to Ep. This finding indicates that a molecular inhibitor produced by cells from malaria-infected mice may prevent the normal response of erythroid precursor cells to $\mathrm{Ep}$, and thereby cause the anemia associated with this disease.

Using two sublines of CBA mice whose cells can be distinguished by the presence of two district alloenzymes of phosphoglycerate kinase (PGK-A and PGK-B), Brecher has been studying the fate of transfused bone marrow cells. The research described in this year's report was designed to answer the specific question of whether pluripotential bone marrow stem cells divide symmetrically to form a clone of mature cells. In order to maintain a pool of pluripotential stem cells, the symmetric division of a commilted slem cell inlo differentiated daughter cells would require its replacement by a new uncommilled :tem cell. This process has been termed the clonal succession of pluripotential stem cells. By transfusing small inocula of matrow cells containing PCik alloenzyme markers, Brecher was able to demonistrate that the clonal succession process does not occur in the simple manner 
described above. Instead, his data indicate that each single stem cell must produce a pool of pluripotential stem cells before it loses its capacity for self-renewal through the production of differentiated daught $\lrcorner$ r cells.

Two papers describing endocrine research by Clemons and her colleagues also appear in this year's report. In the first paper, she describes tests on antisera Lsed for the radioimmunoassay (RIA) of Ep. In order to be useful for Ep RIA, it was found that an antis rum must also exhibit binding affinity for $\mathrm{Ep}$ in a slatively insensitive in vivo assay technique using plethoric mice. However, among different antisera with this property, there was no correlation $b$ atween Ep neutralizing capacity in vivo and the binding affinity for ${ }^{125}$-labeled $\mathrm{Ep}$ in the in vitro RIA. Various antisera prepared against human urinary Ep were also found to have differing affinities for Ep from rats, mice and sheep. In another series of exp sriments, the RIA procedure was used to compare the binding affinities of antisera for two samples of purified human urinary Ep and one sample of human Ep prepared by recombinant DNA techniques. Jne of the chemically purified human Ep samples had the highest binding affinity for antiserum, but when the three different Ep samples were heated briefly at $\mathrm{pH} 5.5$, their binding to antiserum was identical and higher than that observed for any of the unheated samples. This finding suggests that heat treatment may unmask Ep immunologically reactive sites, possibly through ari effect on the carbohydrate moiety of Ep.

Previous studies by Clemons demonstrated that the exposure of rats for one day to an atmosphere containing $1 \mathrm{ppm}$ of ozone leads to a significant decrease in the levels of circulating thyroid hormones. Because high levels of thyroxine serve to potentiate the toxic effects of ozone, it was hypothesized that the decrease in Ihyroid hormone levels may represent an adaptive mechanism in the exposed animals. In this year's report, Fitzsimmons and Clemons describe the results of polyacrylamide gel electrop'orelic studies designed to test the binding of i yroxine to serum proteins in ozoneexposed rats It was found that a one-day exposure to $1 \mathrm{prm}$ of ozone increases the num'ier of binding sites : - thyroxine on pre-albumin molecules by about $30 \%$. This change in prealbumin binding capacily cortelates well with the previously determined decrease in the thevel of circulating thyroxine in rats exposed to I pom of ozone.

An anallysis of the biological eiffects of magnetic finekts and tadiofrequency radiatiom form an innegral part of the Physiology Group's research program. Studies on cellular, tissue, and animal responses to high-intensity magnetic fialds are described in this year's report by Tenforde el al. A variety of sensitive electrical recording techniques have been used in this program to detect magnetically-induced alterations in physiological functions that involve ionic conduction prucesses, such as those in the cardiovascular, nervous, and visual systems. A description is given in this year's report of studies on visual processes in the retinas of cats and monkeys exposed to high-intensity stationary magnetic fields. By recording the electrical activity oi the retina following photon absorption, it was demonstrated that no significant visual alterations occurred in six cats and three monkeys during exposures to magnetic fields with graded intensities up to $\mathbf{1 . 5}$ tesla. Several studies of physiological parameters and their circadian variations in rodents exposed to a 1.5-tesla magnetic field are also described in this year's report. Using an exposure system with an array of noninvasive monitoring devices, it was demonstrated that none of seven simultaneously measured circadiaı. variables was significantly perturbed by a high-intensity magnetic field. New research findings with a superconducting magnet are also described in the report by Tenforde et al. This magnet achieves an ultrahigh field level of 9 tesla, and it is being used to study mechanisms of magnetic field interactions that cannot be analyzed using conventional electronıagnets that reach a maximum field strengtt; of approximately 2 tesla. Of particular interest ire the responses of molecular systems such as biological membranes that have a high degree of diamagnetic anisotropy. A new phenomenon discovered during initial experiments with the 9-tesla magnet is a magneticaily-induced change in the permeability properties of model liposome membranes. A: temperatures close to the thermal phase transition point, liposome unilamellar membranes exhibit a significant increase in solute permeability in the presence of an applied magnetic field. This new finding has important implications for magnetic field interaclions with cellular membranes.

In another series of experiments with liposomes, liburdy has investigated the use of microwaves to stimulate the release of a chemolhetapeulic drug encapsulated in unilamellar liposome 4. siches. As iescribed in this yesiin"s repont, the release of tritialed cytosine arabinofuranoside $\left({ }^{3} \mathrm{H}\right.$ ARA-C) can be triggered by microvave exposure., thus indicaling the feasibility of using wis technique

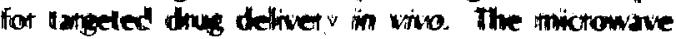


stimulation of ${ }^{3} \mathrm{H}$-ARA-C release is not a result of liposome disruption, since radioisotopically labeled phospholipid molecules are not lost from the liposome niembrane in response to microwave exposure. In other experiments, Liburdy has characterized the effects of temperature and the chemical environment of liposomes in modifying the microwave-stimulated release of encapsulated solutes. He has observed that components of blood plasma ard a high oxygen tension potentiate the microwave effect on liposome permeability. In contrast, an inhibitory effect on microwave-induced solute release is exerted by ascritit acid, mercaptoethanol, or an anaerobic environment.

Durbin and collaborators f: $3 \mathrm{~m}$ the LBL Materials and Molecular Research Division and the UC Berkeley Department of Chemistry have been studying several classes of chelating agents with appropriate molecular dimensions for the efficient binding of Pu(IV). The long-range goal of this pro- gram is to develop a set of chelating agents that are effective in accelerating the elimination of actinides and chemically similar metals from the body, while simultaneously having a low biological toxicity. In this year's report, progress is described in the testing of four newly synthesized ligands for their ability to promote the excretion of Pu(IV) from mice. Two of these agents are promising as Pu(IV) cheleting agents when administered by the oral route. In addition, the results of dose-effect studies are described for the hydroxypyridinone oxide derivative of desferrioxamine, which is the most effective $\mathrm{Pu}$ (IV) chelating agent developed thus far. Another chelating agent, a tetrameric carboxy(catechoylamide) ligand, was studied for its effectiveness in removing $\mathrm{Pu}(\mathrm{IV})$ that is recirculated from the liver and other soft tissues. It was shown that multiple daily injections of this ligand can promote the long-term clearance of residual Pu(IV) by directing the recirculated plutonium to an excretion pathway.

\section{HEMOPOIESIS AND MALARIA}

Paul H. Silverman, John C. Schooley, and Lynn I. Mahlmann

The World Health Organization has viewed with increasing concern the continued spread of malaria since failure of the eradication campaigns of the post World War II era. These campaigns were based heavily on the use of insecticides to control the mosquito vector and on antimalarial drugs to treat the infection (Fig. 1). Unfortunately, development of resistance to insecticides by the mosquitos and resistance to the antimalarials by the parasite combined with administrative instability in the affected countries led to a breakdown in malaria control. It is estimated that $\mathbf{1 . 8}$ billion people are now in need of some form of protection from the disease. Children are particularly at risk. In Africa alone, more than a million deaths in children are attributed to malaria annually because of the direct and indirect effects of this disease. Even a slight parasitenia induces immunosuppression and creates an increased susceptibility to other infectious organisms. Recent optimism about the development of a malaria vaccine presupposes that the target population is immunologically intact. Howvevet, evidence firom clinicat sludies on the use of mellestiablished

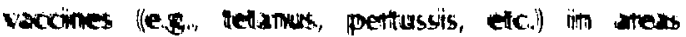

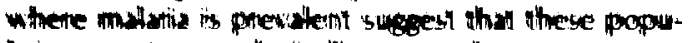
Hations ate inmumologically rimpaned.
The work reported here suggests that the anemia and immunosuppression characteristic of malaria may be due to the depletion of hemopoietic stem cell populations. The hematopoietic stem cell is the ultimate source of all red blood cells, white cells, and immune lymphocytes (Fig. 2). A decrease in stem cells might result in perturbations of all clonal progeny. This work then suggests the basis for new strategies to achieve protection from and treatment of malaria and other related protozoan parasite diseases.

Anemia is a major complication of malaria and contributes to the morbidity and mortality effects of the disease. The causes of anemia have been variously ascribed (u 1) destruction of red cells by the parasites, 2) autoimmune phenomena that result in enhanced monsperific enthrophagontosis, and 3! gross dyserythropoinsis leading to impaited red cell pryduction that continues for several weeks, even afiet the parasilemia has been chemotherapeutically conitnollect.

Numerous studies over the past 20 years have indicalled that mechanisms of med cell destruction and sutoimmunc nesponses are, by themsetwes,

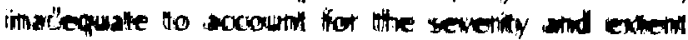

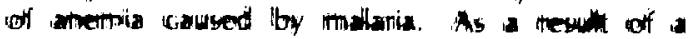




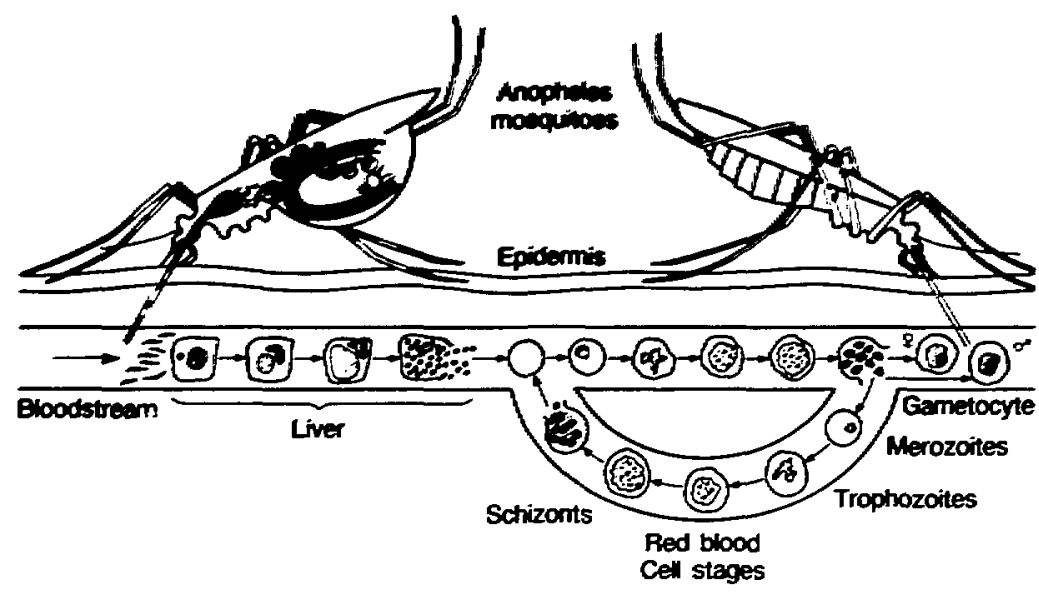

Mammalian host

Fig. 1. Malaria lifecycle.

number of human studies, Weatherall and his coworkers" concluded that, "It is becoming apparent that severe dyserythropoiesis with minimal hemolysis plays a major role in the anemias of Plasmodium falciparum infection....".

Another aspect of malaria infection concomitant with anemia is immunosuppression. Malaria has been observed to induce a state of immunological hyporesponsiveness to homologous (malaria) and heterologous organisms (measles, gastroenteritis,

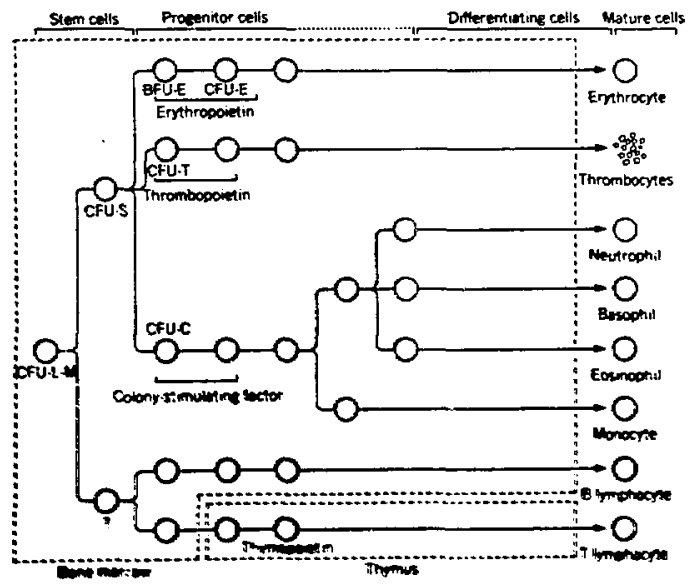

F2 2. Scheme of hemwopoictic cell diterestiotion. BFL", bers-forming unit; $C R$, colont-forming wit: $C$. chlore; $t$.

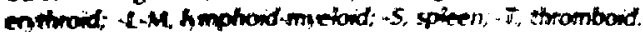

(x) - 152-7+27)

$(X B L 8510-8505)$

and respiratory infections). Immunological hyporesponsiveness is associated with both acute and chronic human $P$. falciparum malaria.

Efforts to elucidate the mechanisms of malariainduced immunosuppression have been largely limited to clinical observations and studies that have focused on peripheral blood effector cells. The studies have focused, in particular, on T and B lymphocytes, monocytes, and macrophages, and have tended to suggest that splenic macrophages play a central role in regulating immune hyporesponsiveness. However, it is clear that the regulatory mechanism of immunosuppression cannot be compartmentalized to the spleen because immune responses to antigens administered peripherally are also blunted.

Some workers have suggested that immunosuppression is due either to impairment of the progenitor cells of granulocytes/macrophages or an imbalance of the competition between erythropoietic and immune cell lineages, which are all derived from a common pluripotent stem cell (CFU-s).

Although there is much that is unknown about the processes involved in the renewal and differen tiation of hemopoietic stem cells, there is general agreement that the sequence of events leads unidirectionally from a pluripotent cell to a committed mature cell with specialized function anci eventual death. It is also apparent that the pluripotent slem cell must renew itself, achieving an effective state of immortality. Several models have been proposed for the regulation of stem cell renewal and 
differentiation. The preponderance of data favor a stochastic model in which the decision to setfrenew or to differentiate into a committed progenitor is a random process, not under the influence of exiemal factors. The process might be positionally affected and may lead to committed lines or to the random loss of lineage potentials.

In unstressed homeostatic animals or humans. the great majority (90\%) of hemopoietic stem cells are in a quiescent state. Normally only a few stem cells are active in the hemopoietic system, generating between 5 and 15 cell clones. Each progenitor cell line is capable of proliferation in response to systemic needs. For example, hypoxia stimulates the production of the hormone erythropoietin, which acts on erythroid progenitor cells to give rise to a population of erythrocytes. Other clones give rise to megakaryocytes, polymorphonuclear leukocytes, and lymphocytes under the influence of specific hormones. Although no specific activator or regulator for pluripotent stem cells has been identified, a number of nonspecific substances have been shown to increase the number of CFU-s undergoing proliferation.

Schooley and $\mathrm{Lin}^{2}$ observed that an acute and continuing demand for erythrocytes may result in a depleted population of bone marrow stem cells. During stress, the proliferation of CFU-s and the intermediate populations of unipotent cells derived from CFU-s are increased. However, the resulting stem celis may have a limited capacity to renew or to develop certain cell lineages. In the context of these interacting and interdependent cell regulatory mechanisms, there exists a possible explanation for the concommitant anemia and immunosuppression associated with malaria infection.

\section{ERYTHROPOIETIN PRODUCTION DURING MALARIA}

The effects of infection with three species of murine malaria (Plasmodium berghei, $P$. chabaudi adami, and $P$. vinckei vinckei) were determined on the plasma levels of erylhropoietin (Ep), the hormone responsible for regulating erythrocyte production. A polycythemic mouse bioassay and a radioimmunoassay were used to follow plasma titers of Ep during the course of infection. The potential capacity of Ep production was assessed by exposing infected mice for 5 hours in a decompression chamber equivalent to a 22,000 -fool altitude $\mathbf{1 6 7 0 6}$ $\mathrm{m})\left(\mathrm{pO}_{2}=67.1\right.$ torr)

As exemplified by the results for $P$. berghei (Fig. 3). Ep production increases as the infection progresses until maximum capacity is reached dur- ing peak parasiternia. The Ep plasma concentration, which is increased in the early styes of malaria infection, is increased even more when the mouse is exposed to hypoxia, indicating that the lissues producing the hormone are not maximally stimulated. During the later stages of infection, the Ep plasma levels are not further increased by an hypoxic exposure, suggesting that the Ep generating system is stimulated maximally. It is possible that the sites of produclion of Ep are different at later stages of infection with extrarenal sites, such as the liver, becoming involved.

The temporal pattern of Ep production and increase in production capacity in response to infection with $P$. berghei, $P$. vinckei and $P$. cha-

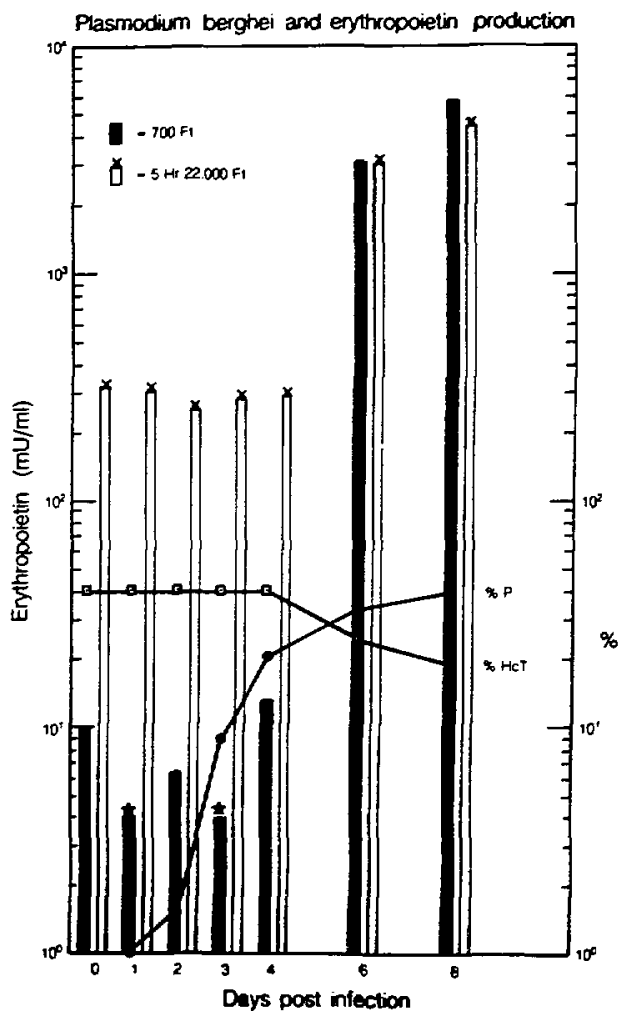

Fi2. 3. Parasitemia (P). hemafocril (HCT) and erythropoietin (Ep) levels duning the first 8 days of inoculation of female Swiss Webster mice with Plasmodium berghei. On day 0 each mouse was injected with $\times 1 \sigma^{\circ} P$ benghei iv. Plasma samples were colfected from 8 to 15 mice per day for subsequent Ep determi. nation by RIA. Bas graphs represent Ep titers before ol and afier (D, hipoxic stimulus. It Ep leuels on days I and 3 mere estmuled because the rifers mere betow the threshold of sensiIritin' of the RIA nfinch is sboul $5 \mathrm{mU} / \mathrm{ml}$.

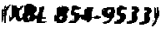


baudi were similar. The results, which are based on a bioassay method that assesses the biological function of Ep, confirm that an amount of active Ep is present in the vascular circulation adequate to stimulate the differentiation and proliferation of erythroid committed progenilor cells. The data suggest that the lack of an adequate erythropoietic response during malaria infection must be due to a failure of the erythroid progenitor cells (BFU-e, CFU-e) to respond to Ep, or a decreased production of erythroid progenitors, and/or a diminished production of CFU-s.

\section{BONE MARROW STEM CELL KINETICS DURING MALARIA}

Bone marrow cells from donor mice when transplanted into lethally irradiated syngeneic recipients will form macroscopic cell colonies in the spleen of the recipient mouse. After 7 days, the number and type of the newly formed colonies (as determined by histological examination) provide qualitative and quantitative data on the pluripotent stem cells derived from the donor. Since the spleen colony assay was introduced over 25 years ago, several workers have demonstrated that each of the spleen colonies in the recipient mouse is derived from a single pluripotent cell. Experiments in this laboratory did not reveal any statistical difference between spleen colony assays counted on days 8,9 or 10 after transplantation. The spleen colony assay results reported here are based on the widely accepted standard of counts made on cay-8 posttransplantation.

The status of CFU-s in mice infected with murine malaria was examined at peak parasinemias as well as during the course of infection. The dat presented here are representative of the results obtained (Table 1). A minimum of four but usually five infected donor mice were used as sources of bone marrow cells, aliquots of which were transplanted into each of 10 lethally irradiated recipient mice for spleen colony assays.

\section{FEMORAL CELLULARITY}

Determinations of the total number of nucleated cells in the femurs of mice infected with $P$. berghei and $P$. vinckei indicated, consistently, that a marked depletion (40-60\%) occurs in the later stages of the disease (Table 1). It is highly probable that the results reported here are minimal estimations of the actual proportional reduction because of the evidence of osteoclasia that was apparent in the femurs of the infected mice. Femoral bones from infected mice were very brittle.

\section{STEM CELL KINETICS}

Changes in CFU-s populations were measured as a proportion of the nucleated bone marrow cells and in relation to the total cellularity of the femoral contents. Observations made during the early phases of infection with $P$. berghei and $P$. vinckei

Table I. Results of the spleen colony assay using BALB/c female mice. Depending upon the time postinoculation and level of parasitemia, $0.5-1.0 \times 10^{5}$ nucleated bone marrow cells pooled from the femurs of each donor mouse was injected i.v. into 10 syngeneic recipients. Spleen colonies were observed on dap 8 after transplantation. The mean spleen colony counts $( \pm$ SEM) represent $n=40$ for

$P$. berghei days 5 and 11 . $n=50$ for $P$. berghei day 14, $P$. vinckei day 7 and $n=70$ for uninfected controls.

Femoral cellularity was determined by Coulter Counter (Hieleah. Florida) on suspensions of bone marrow cells which were freed of erthrocytes by antiserum trealment (Zap-Oglobulin II, Coulter Diagnostics)

\begin{tabular}{|c|c|c|c|c|c|c|c|c|c|c|}
\hline Donor & $\begin{array}{c}\text { Post- } \\
\text { Inoculation } \\
\text { (Day) }\end{array}$ & $\begin{array}{c}\text { Parasilemia } \\
\qquad(0, t))^{4}\end{array}$ & $\begin{array}{l}\text { Cells/ } \\
\text { femur } \\
\left(\times 10^{6}\right)^{\circ}\end{array}$ & $\begin{array}{c}\text { CFU.s } \\
\left(\times 10^{-6} \mathrm{BMC}\right)^{4}\end{array}$ & $\begin{array}{l}\text { CFU-s/ } \\
\text { Femur }\end{array}$ & $\begin{array}{c}\text { Femoral } \\
\text { cellularity } \\
\text { (\$ Normal) }\end{array}$ & $\begin{array}{c}\text { Femoral } \\
\text { CFU-s } \\
\text { (\% Normal) }\end{array}$ & $\begin{array}{l}\text { Hematocrit } \\
(\%)^{d}\end{array}$ & $\begin{array}{c}\text { Body } \\
\text { weight } \\
\text { (g) }\end{array}$ & $\begin{array}{c}\text { Spleen } \\
\text { weight } \\
(g)^{\prime}\end{array}$ \\
\hline
\end{tabular}

Uninfected controls

\begin{tabular}{|c|c|c|c|c|c|c|c|c|c|c|}
\hline 7 & - & & $14.2 \pm 090$ & $275=10$ & $3870=140$ & - & - & $47 \pm 0.04$ & $20.7 \pm 0.19$ & $0.10 \pm 0.01$ \\
\hline \multicolumn{11}{|c|}{ Tlasmodium berghe } \\
\hline 4 & 5 & $8.3=26$ & $99=0.76$ & $\$ 73=20$ & $4590=190$ & $70=6.9$ & $119=6.4$ & $48 \pm 0.65$ & $20.2=0.55$ & $0.25 \div 0.01$ \\
\hline 4 & 11 & $32=31$ & $64=0.99$ & $149=70$ & $990=73$ & $45=75$ & $26 \div 2.1$ & $20 \pm 3.0$ & $15.8 \div 0.92$ & $0.74 \div 0.12$ \\
\hline 5 & 14 & $55=56$ & $B G=0.63$ & $105=70$ & $697 \div 63$ & $61=5.9$ & $23=1.8$ & $24 \pm 1.0$ & $13.8=0.23$ & $0.65 \pm 0.08$ \\
\hline \multicolumn{11}{|c|}{ Lasmodium vincher ancks: } \\
\hline 5 & 7 & $65=33$ & $61=05 i$ & $312 \pm 10$ & $1580=94$ & $+3=4 B$ & $49=3.0$ & $24=0.92$ & $18.5=0.21$ & $0.64 \pm 0.02$ \\
\hline
\end{tabular}

\footnotetext{
- $\overline{\mathrm{n}}$ - SEM

BMC - bone andaron cells

CrU-s - ohrowem slem crith
} 
indicaled early entuanced proliferation of stem cell populatinns. However, in the bate stages of infection with murine malaria, stem cell depletion dropped to less than $25 \%$ of the normal (Fig 4). It is important to note that the degree of CFU-s depletion during late stages of infection is greater than the depletion observed for the total femoral cellularity. The differences in the response of the two cell populations suggest that factors affecting stem cell renewal and differentiation may be different, or are affecting different target cells in the bone marrow.

Whether the parasite-induced factors are acting on one or more constituents of the bone marrow cell population is difficult to assess from these experiments. However, the net effect of a depleted hemopoietic stem cell population is of considerable importance to the potential outcome of the infection. Stem cell suppression might result in reduced capacity to generate blood cells to meet physiologic needs and for survival itself. All of the main disease characteristics of malaria could result from, or be exacerbated by, stem cell depletion. Erythrokinetic studies indicate that an unstressed human requires more than $2.0 \times 10^{6}$ new red cells each second to replace senescent erythrocytes. The effects of suppressed production of new erythrocytes by CFU-s inhibition might theoretically account for half the anemia observed in the mouse on day 14 postinoculation with $P$. berghei. Whatever the degree of contribution to anemia caused by CFU-s depletion, it is apparent that perturbation of progenitor cell production will enhance the anemia caused by parasite induced red cell destruction and delay recovery of erythropoiesis after cure has been achieved chemotherapeutically. Because of the random nature of the pluripotential stem cell renewal and differentiation, it is probable that suppression of stem cell production of one cell linnage is accompanied by depression of all cell lineages. Thus, an inevitable outcome of stem cell depletion will be reduced production of megakaryocyles, granulocytes, and lymphocytes, resulting in thrombocytopenia, leuckocytopenia, and immunodepression, all of which occur during malaria and infection with other hematazoa (e.g., trypanosomes, Babesia, and Anaplasma).

\section{NONSPECIFIC IMMUNITY .}

Support for the concept that parasite-induced perturbation of stem cell renewal and progenitor cell production is a characteristic of hematozoan infection is provided by observations tha! a wide variely of known slem cell stimulators induce resis-

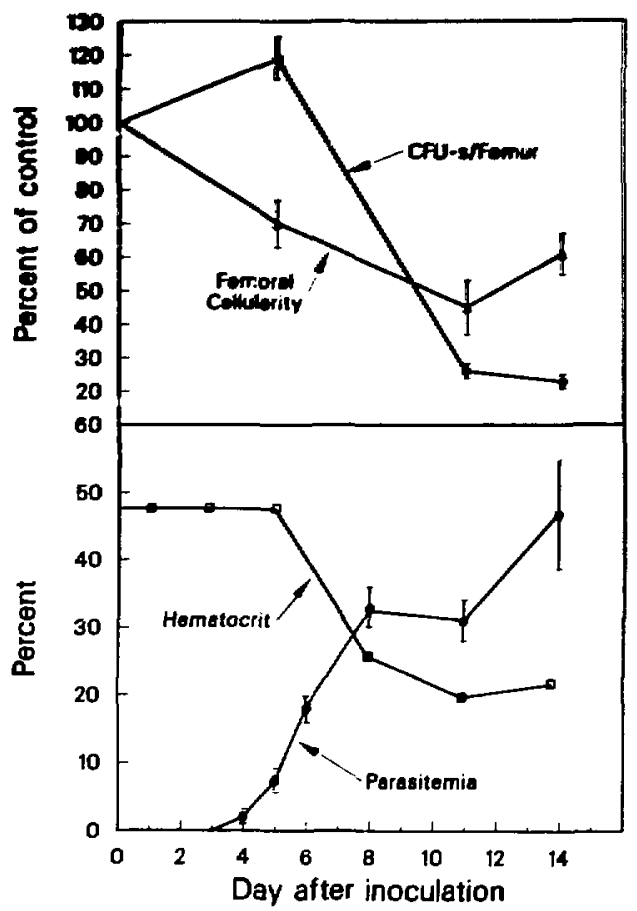

Fig. 4. Changes in femoral cellularity and bone marrow stem cell (CFU-s) populations following inoculation of Balb/c female mice with $1 \times 10^{5}$ Plasmodium berghei injected i.v.

(XCG 858-377)

tance to hematozoa infections. If stem cell depression is an important factor in malaria pathogenesis, then stem cell stimulation should ameliorate the virulence of malaria infection. This prediction is borne out by reports that various intact bacteria (Cornybacterium, Brucella, Listeria, and Salmonella) and bacterial cell wall components such as endotoxins (lipopolysaccharides), when injected prior to infection, induce a degree of nonspecific protection that varies from reduction in parasitemia to prevention of lethal effects. It is well established that when endotoxins from bacterial cell walls are injected into mice there is a rise in the number of pluripotent hemopoietic stem cells and progenitor cells in both the bone marrow and spleen. This rise peaks approximately 5 to 7 days after injection of the endotoxin.

We tested the concepl of stem cell and progenitor cell stimulation as an effective indicer of resistance to the lethal effects of murine malaria. A series of injections of 5, 10, and 15 48 E. coli lipopolysaccharide- $W$ were interspersed with mild 
Impoxia to stimulaie stem cells and enthroid proaenitor cells for 5 days prior to infection with a virulent inoculum of $P$. vinckei (Fiz 5). The results indicate that the combination of stem cell and erythroid progenitor cell stimubtion confers significant protection. Indeed, after all controls and unprotected mice had died by day 15, the prolected survivors resolved the infection by day 22 , and 2 weeks later, when challenged with another infective inoculum, showed either no or only a transient parasitemia.

\section{THE ROLE OF THE SPLEEN}

Splenomegaly has long been recognized as a characteristic response to malaria and other parasitic infections. As our results indicate, the size of the spleen may increase 7 times over normal during the course of the infection (Table 1). The increase in weight of the spleen may be due to the splenic migration of cells from the bone marrow as well as prolifercition of cells located in the spleen. As impressive as is the remarkable increase in spleen cellularity, it remains to be demonstrated whether it is capable of replacing the hemopoietic functions of the bone marrow when it is depleted of a substantial portion of pluripotent stem cells. The obvious fact that the enlarged spleen does not meet the hemopoietic needs of the malaria-infected mouse suggests that it is not functionally equivalent to the bone marrow.

The discovery that murine malaria induces substantial depletion of bone marrow pluripotent stem cells is of practical and theoretical importance. It suggests that through appropriaie use of stem cell stimulators, alone or in conjunction with specific antigens, the immunological status of affected populations might be significantly improved. This approach might enhance immune responses to vaccines in endemic malarial areas as well as improve the general nonspecific immune response in malaria-infected persons to heterologous infections.

The theoretical importance of a stem cell suppressor mechanism is considerable. Since malaria organisms are not known to invade

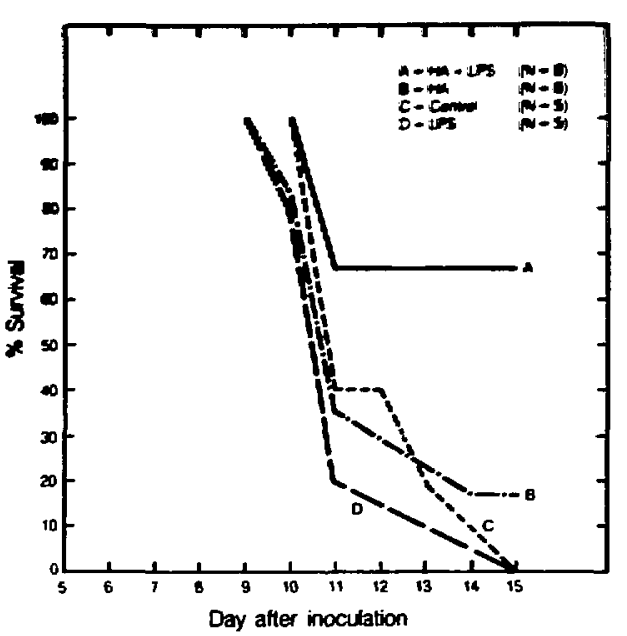

Fi. 5. Cumulative sunvival of female Swiss Webster mice that were infected with $1 \times 10^{5}$ Plasmodium vinckei inoculated i.v. after various treatments: (A) i.p. injection on days 1, 4, and 6 with 5, 10, and $15 \mu \mathrm{g} E$. coli lipopolysaccharide-W and heid in a decompression chamber equivalent to high altitude (HA) of $22,000 \mathrm{ft}$ for 5 hours on days 0,3 , and 5 . (B) . Held in a decompression chamber equivalent to 22,000 ft for 5 hours on days 0,3 , and 5. (C) Control group. (D) I.p. injection on days 1, 4, 6 with 5, 10, and $15 \mu g$ lipopolysaccharide-W (E. coli 0127: B., Difco Labs.).

(XBL 854-9534)

nucleated bone marrow cells in vivo, it is probable that the stem cell suppression is accomplished by a diffusable product of the infection process. Elucidation of how the stem cell is affected by malaria, directly or indirectly, may lead to new insights in the physiology of stem cell renewal and differentiation.

\section{REFERENCES}

1. Weatherall, D.J., Abdalla, S., and Pipparo, M.J. Malaria and the Red Cell. Ciba Symposium, Pittman, London, p. 74-97 (1983).

2. Schooley, J.C., and Lin, D.H.Y. Regulation of Enthropoiesis. A.S. Gordon, M. Condorelli, C. Peschle, Eds., II Ponte, Milan, pp. 52-66 (1972). 


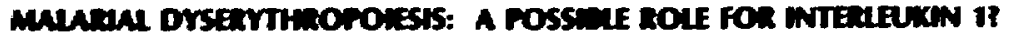

fenc C. Schooley and eirgitta kenteren

Malaria is a mosquito-borne parasitic disease that has afflicted mankind since antiquity. Various methods for eradication of the mosquito and new drugs to treat infected individuals raised hopes that ultimate victory against this dreaded disease was near. Yet these hopes have not been realized. Mosquitos have developed resistance against various pesticides, and the malarial parasite has developed resistance against many of the new drugs. In $196 i$ only 50,000 cases of malaria were reported in India, but by 1977 slightly more than 30 million individuals had the disease. Today, the disease afflicts $200-400$ million persons in tropical and subtropical areas, and it is the major cause of early childhood mortality. Persons surviving the disease are often anemic, and this contributes to the debilitating aspects of the disease.

Anemia - the lack of an adequate mass of functioning erythrocytes in the circulating blood-may result from numerous causes related to an increased destruction or a decreased production of erythrocytes. The malarial parasite invades the erythrocytes and reproduces within them during one stage of its life cycle, during which considerable destruction of erythrocytes occurs. Infected individuals, however, are often anemic even when the parasite is no longer present in their erythrocyles, implying that erythrocyte production must also be abnormal in this case.

In healthy individuals erythrocytes live 120 days. Therefore $1 / 120$ th of the total circulating erythrocyte mass must be produced each day if a steady state is to be maintained. It can be calculated that about 1 to 2 million erythrocytes are made per second in a healthy individual. The regulation of this day-to-day production, as we have previously demonstrated, is achieved by the hormone erythropoietin, which is a glycoprotein produced in the kidney and other tissues. It appeared possible that the anemia in malaria infection might relate to inadequate erythropoietin production.

Similar malaria-like organisms also infect rodents, birds, reptiles, and amphibians. We have studied the effect of a number of different rodent malarias that mimic various clinical aspects of human malaria. Within a few days after infection with Plasmodium berghei, $P$. vinckei, or $P$. chabaudi, the tlood erythrocytes contain the malarial parasite, a pronounced splenomegaly develops, and the animal becomes severety anemic. The severity of the disease and its mortality depend upon the infecting organism.

In collaboration with Paul Silverman and Lynn Mahlmann, we determined the serum concentration of erythropoietin during infection. In addition, we determined whether the serum erythropoietin levels were increased if a hypoxic exposure was imposed on the infected mouse. It is well-known that hypoxia stimulates erythropoielin production. These data have been presented elsewhere in this report, and we will only emphasize our conclusion that the anemia of malaria is not due to a lack of erythropoietin production. Indeed, the erythropoietin-generating tissues are apparently working maximally soon after the anemia is evident. These results suggested that the anemia might result from the failure of the infected animal to produce within its hematopoietic tissues the appropriate cell populations that respond to erythropoietin.

During the last 30 years a large body of experimental evidence indicates that all of the blood cells-the erythrocytes, platelets and various types of granulocytes and lymphocytes-are derived from a pluripotential "stem cell." This pluripotential cell, which is a slowly proliferating cell, gives rise to more rapidly proliferating progenitor cell populations that are unipotential or possibly bipotential. The agents regulating differentiation, such as erythropoietin, thrombopoietin, and CSF (the regulator of granulocyte production), interact with these unipotential precursor populations and produce the morphologically recognizable blood cells. The scheme of this hierarchical concept of the development of blood cells is shown in Fig. 2 of the preceding report by Silverman, Schooley and Mahlmann.

Consideration of this proposed scheme for the production and interrelationships of the various types of hematopoietic progenitor cells clearly indicates that the lack of mature blood cells of a particular cell line may result from numerous changes. For example, the humoral agents regulating the production of one cell line may stimulate the pluripotential progenitor to produce more of the direct precursor of this line, and therefore fewer CFU-S are available to produce the early progenitors of other cell lines, and marked increases in lymphocytopoiesis may inhibit the production of erythroid cell progenitors. As mentioned, our collabotative experiments indicate that the CFU-5 population is 
depressed in malaria-infected mice. We do not know as yet, however, whether these CFU-S are proliferating more rapidly than usual and producing increased numbers of the unipotential progenitor populations or whether the surviving CFU-S maintain their normal slow proliferative rate. Some preliminary observations suggest the former possibility is correct; that is, the CFU-S are proliferating more rapidly than in the normal mouse.

If a single-cell suspension is prepared of the spleen of mice made severely anemic by injections of phenylhydrazine-a drug that destroys mature red cells-and incubated with various concentrations of erythropoietin for 22 hours, the subsequent 2-hour uptake of ${ }^{3} \mathrm{H}$-thymidine into cells synthesizing DNA is increased as shown in the upper curve of Fig. 1 (Control). A log-dose response to the hormone is observed. In contrast, if a spleen cell suspension is prepared from spleens taken from mice infected with malaria but with an anemia equivalent to that induced by phenylhydrazine injection, the addition of erythropoietin does not induce a significant increase in DNA synthesis. This suggests that erythropoietin-responding cells are decreased in the spleen of the malaria-infected mouse. Conditioned media ( $\mathrm{CM}$ ) from cultures of malaria-infected spleens or from cultures of normal spleens to which the malaria organism has been added are collected after 24 hours and the cells and parasites removed. These $C M$ were then added to cultures of spleen cells obtained from phenylhydrazine-injected mice and the ability of Ep to induce DNA synthesis determined. The addition of both of these CM almost completely inhibited the action of erythropoietin on cells normally responsive to the hormone (bottom two curves of Fig. 1). Those erythropoietin-responsive cells that are induced to synthesize DNA by addition of erythropoietin are composed of BFU-E and CFU-E precursor populations of erythroid cells. We conclude that an inhibition of the early stages of erythropoiesis contributes to the dyserythropoiesis of malaria infection. The nature of this soluble hormonal agent is unknown, and its characterization forms the basis for future work. We believe that the unknown substance may be interleukin I (IL-1) since we know from other experiments that IL-1, a product of activated macrophages, and interleukin 2 (IL-2), a product of T-lymphocytes, also inhibit the action of enythropoietin in this system. We therefore advance as a working hypothesis the scheme shown in Fig. 2 to account for our observations.

The malaria organism of the parasitized erythro. cyte or its products interacl with macrophages

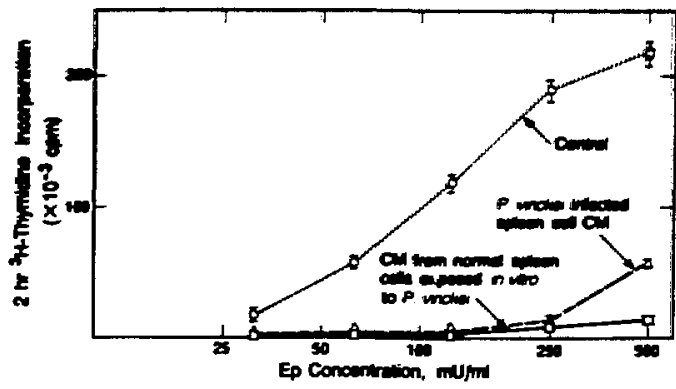

Fit. 1. Inhibition of the erythropoietin-induced stimulation of DNA synthesis of splenic cells, measured by the 2-hour incorporation of ${ }^{3} \mathrm{H}$-thymidiric, by conditioned media (CM) from $\mathrm{Cu}$ tures of normal spleen cells incubated with $P$. vinckei for 24 hours or cultures of spleen cells infected with $P$. vinckei.

(XBL $8510-8519)$

inducing the production of IL-1. IL-1 stimulates Tcells to produce $\mathrm{IL}-2$. IL-1 and IL-2 inhibit the action of erythropoietin on erythropoietinresponsive cells (BFU-E and CFU-E). Activated macrophages also produce tumor necrotic factor (TNF), and evidence indicates that TNF inhibits the development of adipocytes. Adipocytes of the

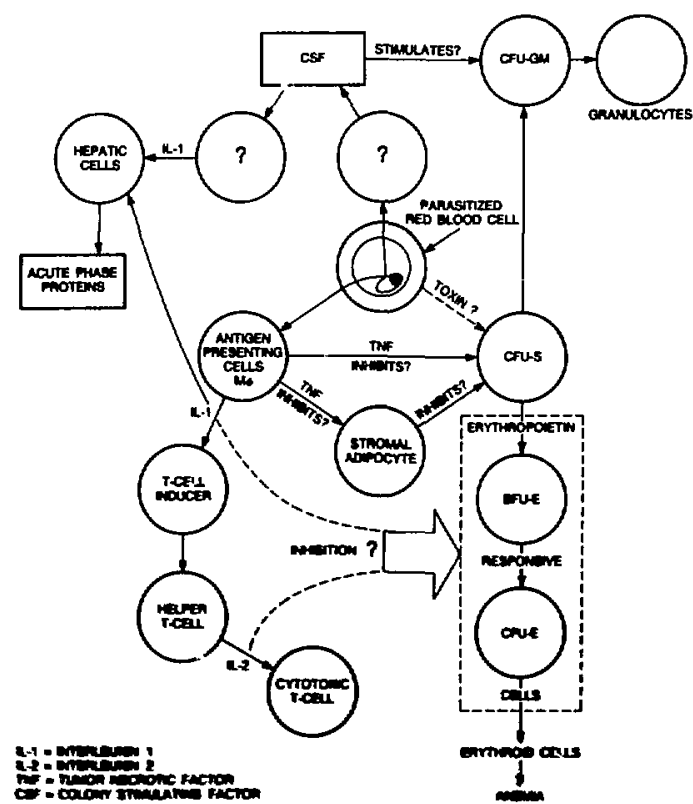

Fi. 2 Schematic presentation of the proposed role of $\| l-1$ in the denelopment of the dysenthopoiesis of mularia infection.

(rat 859-453A) 
bone marrow stromal population are necessary for bone marrow growth and development, and their absence would inhibit ultimately the growth of enthroid cells. Experiments in collaboration with Paral Silverman are in progress to explore this aspect of the role of TNF in malaria. It is also possible that the malaria parasite generates some agent toxic to CFU-S. The malaria parasite may trigger some mononuclear cell to produce CSF via an endotoxin-like action, and this increased CSF may also stimulate macrophages and other cells to produce IL-1. We have found that CSF is markedly increased in the serum of mice infected with malaria. Lastly, IL-1 stimulates hepatic cells and initiates the acute-phase response.

The acute-phase response is a systemic reaction to inflammation or tissue injury that is characterized by complex changes that include fever, inc. ease in white blood cells, increased breakdown of muscle, altered carbohydrate and trace metal metabolism, and a pronounced change in hepatic protein synthesis. The hepatic production of some blood proteins is increased, and the production of others is decreased. Humans with malaria infection often have some of these acuite-phase responses; however, a more thorough study of the kinetics of these changes during experimental malaria infection is desirable. Evidence has been accumulating that these changes in hepatic protein synthesis are mediated by IL-1.

Very recent work indicating that IL-1 can stimulate hepatic cells pretranslationally to increase the production of acute-phase proteins (serum amyloid $A$, etc.) and inhibit albumin synthesis. Thus, the possibility that $: L-1$ directly inhibits erythropoiesis appears reasonable. Whether IL-1 inhibits erythropoietin-receptor synthesis or the synthesis of some key protein of the earliest proteins involved in erythroid differentiation or masks the erythropoietin-receptor is the subject of future work. This preliminary work provides some exciting insights into possible mechanism responsible for the anemia of malaria. The possibility that IL-1 and/or IL-2 may be involved in other diseases that exhibit an elevated serum level of erythropoietin with an anemia, such as aplastic anemia, opens some very exciting new approaches to investigate.

\section{SEEDING OF SINGLE HEMOPOIETIC STEM CELLS AND SELF RENEWAL OF COMMITTED STEM CELLS}

\section{George Brecher}

The fate of transfused bone marrow cells can now be followed more readily and in greater detail than with the older cytogenetic method. This is due to the availability of a subline of CBA mice with two alloenzymes of phosphoglycerate kinase (PGK $A$ and B). By using mice that have only the A enzyme as donors in B hosts (or vice versa), cells of donor origin can be identified in the recipient. Because PGK is present in the cytoplasm of all body cells, one can determine the percentages of cells of donor origin in red cells, white cells, and platelets of the peripheral blood. (The cytogenetic method could only identify the donor origin of dividing cells in the marrow.)

The new methodology is available in our laboratory thanks to a Director's grant in 1981 that enabled us to obtain the new strain of mice and to acquire the necessary experience in the electrephoretic quantitation of the enzymes. Recently we have used the new methodology to explore the question of clonal succession, an unsolved problem of stem-cell kinetics. There is extensive evidence that the cell in transfused marrow that seeds in the recipient and is responsible for repopulation of an irradiated marrow is a pluripotential stem cell. However, the initial steps of the development of s!em cells committed to production of a single celi line, e.g., red blood cells, is not known. When a pluripotential stem cell divides and produces two daughter cells committed to a single cell line, it necessarily loses its ability to function as a pluripotential stem cell. It is possible that a pluripotential stem cell may undergo an asymmetric division and produce one pluripotential stem cell to replace itself and one committed stem cell, thus maintaining a stable number of pluripotential stem cells. It has been argued that this type of differentiation would require an excess number of divisions and that each pluripotential stem cell divides symmetrically. producing a clone of mature cells. Having 
done so, it must be replaced by a new pluripotential stem cell, hence the notion of clonal succession.

In collaboration with H.S. Micklem of the University of Edinburgh, we have succeeded in transfusing both single cells and two to five proliferating cells into animals whose own stem cells had been killed by irradialion. The PGK enzyme is sex linked. Hence female mice heterozygous for PGK $A$ and $B$ contain either $A$ or $B$ enzymes in any individual cell. When we gave a small inoculum of 50,000 AB marrow cells, only 4 of 20 recipients survived, but all 4 had only PGK $A$ enzyme in their peripheral blood cells. (The fact that the single enzyme in the host was $A$ is explained by the normal ratio of PGKA/PGKB in CBA mice being 70:30. A single cell sceding is thus much more likely to be A than B.) The results indicate that the survivors received a single pluripotential stem cell capable of proliferating. The chances of two or three cell seedings all being $A$ (or $B$ ) can be shown to be less than $10 \%$. The loss of 16 out of 20 animals that received 50,000 cells confirms that the average of cells seeded and proliferating was 1 or fewer, resulting in a high proportion of recipients receiving no pluripotential stem cells. The survivors among the recipients of 50,000-cell inocula have now been observed for many months and show no deterioriation in their blood picture. It must be concluded that there is no clonal succession in the simple form originally proposed. At a minimum, the single stem cell must have laid down a reservoir of pluripotential stem cells before losing its capacity for self-renewal through differentiation and maturation.

After transfusion of $100.000 \mathrm{AB}$ marrow cells, all of 20 lethally irradiated recipients survived. There were two animals with only PGK A enzyme in the peripheral blood cells, suggesting that the average number of seeded stem cells was about two, when the expectation of all seeded cells being $A$ is of the order of $10 \%$. There was a striking variation between animals in the ratios of $A: B$ enzymes in the RBC and platelets of the recipients of 100,000 cells compared with the similarity of the ratios after 10,000,000 cells were given (Fig. 1.) In addition, the A/B ratios of red blood cells and platelets differed more markedly over time in the recipients of 100,000 cells than in those that had been given $10,000,000$ cells. Similar observations have been made on the $A / B$ ratios of granulocytes as well as $T$ and $B$ lymphocyles by Micklem. The
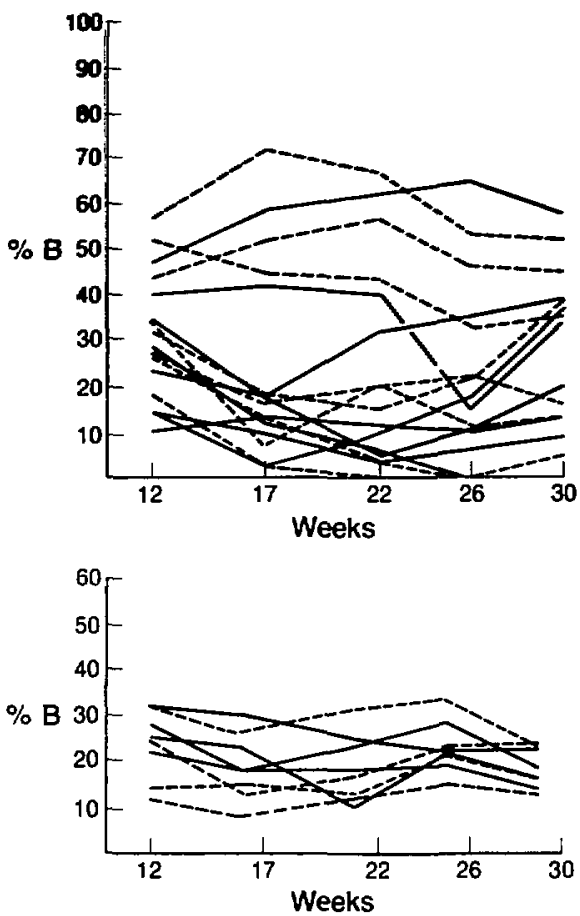

Fig. 1. Percent of B enzyme in the red blood cells (___ and platelets (-.-.-) following transfusions of $A B$ marrow cells. Note the great variation among curves following transfusions of 100,000 cells (upper graph) compared with the marked similarity among curves following transfusions of $10,000,000 \mathrm{ce} / \mathrm{ls}$ (lower graph).

(XBL 8511-8582)

most likely explanation for this observation is the production of committed stem cells that have significant self-renewal. The alternative, that pluripotential stem cells produce committed stem cells of different lineages at different rates and that these rates vary appreciably over time appears unlikely. Apart from the complexity of such a scheme, it sho:ld be recalled that many of the animals that received 100,000 cells started out with only two pluripotential stem cells and that there is evidence that the $A: B$ ratio of pluripotential stem cells is maintained during self-renewal.

The findings are of interest. because continued self-renewal of committed stem cells has not been demonstrated previously. The implications for our views of stem cell kinetics are being studied. 


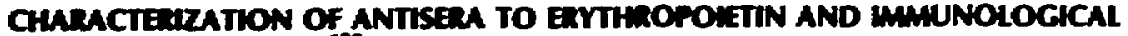 DIFEAENCES EETWEEN ${ }^{123} 1$ LARELD HUMAN URNARY AND RECOMANANT EMTHLOPOLTIN}

\section{Civela K. Clewons}

The maintenance of a normal number of circulating red blood cells and hemoglobin that is optimal for oxygen transport to body tissues is dependent on the hormone erythropoietin (Ep). Erythropoietin assays comprise bioassays that measure the hormone effect in the whole animal or the in-vitro action on tissues or whole cells and radioimmunoassays (RIA) that determine the interactions with antibodies raised against the hormone. The advantage of the bioassay in the whole animal is that it measures the biological activity of the hormone. However, these assays usually have a low sensitivity. The sensitivity of bioassays has treen improved recently thanks to the introduction of in-vitro methods. These use signals generated by erythropoietin in whole cells, such as fetal mouse liver cells or spleen and bone marrow cells derived from phenylhydrazine-treated mice and rabbits. While these assays have the sensitivity required, they are still subject to effects of nonspecific factors in serum, plasma, or crude Ep, a feature of all bioassays from which the RIA appears to be free.

The essential requirements for the development of a radioimmunoassay are specific antibodies to the hormone, the availability of the pure hormone for labeling, a standard reference preparation, and a technique to separate the antibody-bound and nonantibody-bound hormone after a given incubation period.

The first requirement is the preparation of antisera to Ep that ase sensitive and specific for this purpose. In order for a polyclonal antiserum to be useful, it must be specific for the hormone of interest and have a reasonable titer, i.e., a high dilution at which the labeled antigen binds such that antibodies to other substances cause no interference. The biological and immunological properties of some antisera are shown in Table 1. These antisera were obtained by immunization with crude urinary Ep extract with an activity in units (U)] of $20 \mathrm{U} / \mathrm{mg}$ protein. Boosters were either the same preparation or purified Ep with activities of either 8,000 or $29,000 \mathrm{U} / \mathrm{mg}$. For the initial immunization, each rabbit received $200 \mathrm{U} \mathrm{Ep}$, and booster shots were given at $100 \mathrm{U}$ per animal.

The neutralizing capacities of these antisera were tested in the polycythemic mouse assay, and they appear to be proportional to the purity of the antigen used for immunization, ranging from 25 $\mathrm{U} / \mathrm{ml}$ with only crude human urinary erythropoietin (HUE) to greater than $1,000 \mathrm{U} / \mathrm{ml}$ with highly purified Ep. However, this cannot be generalized because Rabbit $\# 139$ was also a contributor to Pool $I$, and the neutralizing capacity of Pool I may be lower because it was diluted with antisera from other rabbits not having this high quality. No correlation was found between the purity of the antigen used, the neutralizing capacily in vivo, and the possible use for binding of ${ }^{125}$ I-Ep in an immunological assay. But in order for an antiserum to be useful for the development of an RIA, it apparently has to have some neutralizing capability, as tested in the in-vivo assay, because we have found that nonneutralizing antisera do not bind labeled Ep.

Based on litration curves, a radioimmunoassay is dependent upon the competition between labeled and unlabeled antigen for binding sites on

Table 1. Biological and immunological propenties of enthropoietin antisera.

\begin{tabular}{|c|c|c|c|c|}
\hline $\begin{array}{l}\text { Specific activily of } \\
\text { antigen used for } \\
\text { immunization } \\
(\mathrm{U} / \mathrm{mg})\end{array}$ & $\begin{array}{l}\text { Boosters } \\
\text { (U/mg }\end{array}$ & I.U. & $\begin{array}{c}\text { Neulralizing } \\
\text { copability in tino } \\
\text { (U// } \mathrm{ml})\end{array}$ & $\begin{array}{c}\text { Final } \\
\text { dilution } \\
\text { lo bind } \\
40 \%{ }^{223} \mathbf{I - E p} \\
\end{array}$ \\
\hline 20 & 20 & Pool I & 25 & $: 5,000$ \\
\hline 20 & 8000 & 139.78 & 300 & $1: 50,000$ \\
\hline 20 & 29,000 & $3136-310$ & 500 & $\pi: 50,000$ \\
\hline 20 & 29.000 & $378-6-100$ & 100 & 17: 50,000 \\
\hline 20 & 29,1000 & $233-6-30$ & 700 & 1): 50,000 \\
\hline 20 & 29,000 & $3 \pi 46-30$ & 7,0000 & $\pi: 40,000$ \\
\hline
\end{tabular}


the antibodies. Dilutions of antisera were chosen to bind $40 \%$ of the label. Use of excess antibody results in loss of sensitivity because all binding sites must be occupied before unlabeled Ep can compete with the tracer. The suitability and sensitivity of each antiserum for radioimmunoassay use was determined at three dose levels of unlabeled human, rat, mouse, and sheep Ep (Fig. 1). This RIA established that only two of the antisera tested had any significant competition between labeled and unlabeled Ep, AS 139-18, and Pool I. In addition, these two antisera also neutralize Ep's from several species.

Some competition could be obtained at the highest dose of human Ep $(100 \mathrm{mU} / \mathrm{ml})$ using two antisera made with highly purified antigen, but the possible "sensitivity" of the assays would lie considerably above that of the polycythemic mouse assay, and very little, if any, competition with animal Ep's could be established. Since only antisera with the steepest slopes are suitable for sensitive assays, it appears from these data that for obtaining good polyclonal antisera for radioimmunoassay purposes the use of highly purified erythropoietin does not seem to be necessary, and may be even undesirable.

The second requirement for the radioimmunoassay is the availability of the hormone in the pure form and its ability to accept a radioactive label. Erythropoietin was purified to a specific activity of $70,000 \mathrm{U} / \mathrm{mg}$ in Dr. Eugene Goldwasser's laboratory, and he made some of it available for distribution through the National Institutes of Health. This material was obtained from urine of anemic pratients. Recently two other pure Ep preparations have become available for labeling. One was also purified from human urine by Dr. Gerald Krystal (Terry Fox Laboratory, Vancouver,
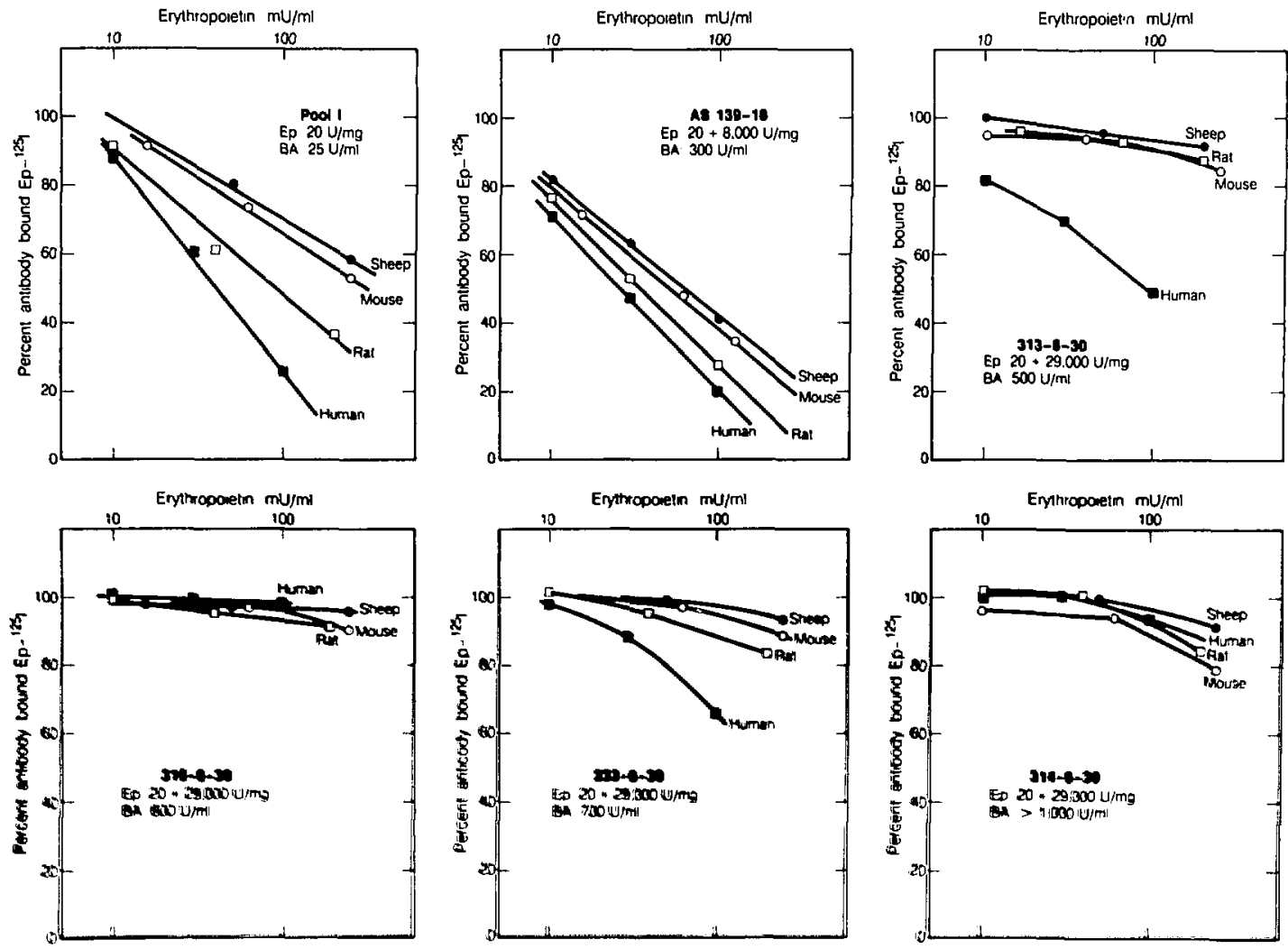

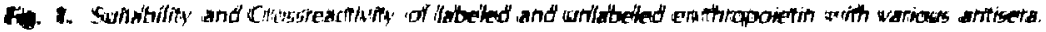


Canada), and the other is recombinant Ep distributed by Amgen Company, Thousand Oaks, California.

The three preparations were iodinated in our laboratory, using an identical procedure for all, and the labels were tested in the RIA as described by the late Joseph Garcia (LBL). The specific activities of the labeled erythropoietins were comparable (Table 2). Four standard curves of each, human and mouse Ep, and internal standards were tested in quadruplicate. The second International Reference Preparation for human Ep was obtained from the National Institute of Biological Standards, Mill Hill, England, and the mouse standard was a previously bioassayed serum preparation from animals exposed to 24 hours of hypoxia. As can be seen from Table 2, the highest binding to the antibody was obtained with Goldwasser's Ep, and the bindings for the other two preparations were significantly lower. As can be seen from Fig. 2 there was no immunological difference between the two labeled Ep's that were purified from urine $(G$ and K). However, the label obtained with A-Ep differed such that it appears to have a slightly higher affinity to the antibody than the respective unlabeled standard preparations. This resulted in a shift of the curves to the right and reduced the sensitivity slightly. However, the determinations of the inter-

Table 2. Comparison of RIA quality controls using three highly purified Ep preparations.

\begin{tabular}{|c|c|c|c|}
\hline & $G^{2}$ & $K^{2}$ & $A^{a}$ \\
\hline Specific activity of ${ }^{125} \mathrm{I}-\mathrm{Ep}(\mu \mathrm{Ci} / \mu \mathrm{g})$ & 195 & 170 & 200 \\
\hline Total binding to antibody $(\%)$ & 35 & 21 & 22 \\
\hline \multicolumn{4}{|l|}{$50 \%$ Total Binding $(\mathrm{mU} / \mathrm{ml})$; } \\
\hline Human & 30 & 30 & 52 \\
\hline Mouse & 54 & 54 & 82 \\
\hline \multicolumn{4}{|l|}{ Internal Standards $\left(\mathrm{mu}, \mathbf{m}^{\prime} \mathrm{ml}\right)$ : } \\
\hline \multicolumn{4}{|l|}{ Human: } \\
\hline Normal human serum pool & 22 & 20 & 16 \\
\hline Anephric pool & 12 & 12 & 12 \\
\hline Iron-deviziern pool & 130 & 130 & 130 \\
\hline \multicolumn{4}{|l|}{ Ntouse: } \\
\hline loxi poo' & 26 & 23 & 23 \\
\hline Hingtopont & 120 & 020 & 120 \\
\hline 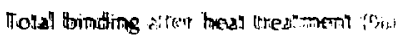 & $N T^{b}$ & B2 & 52 \\
\hline
\end{tabular}

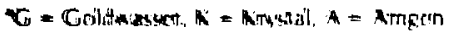

TWT = mod trestred
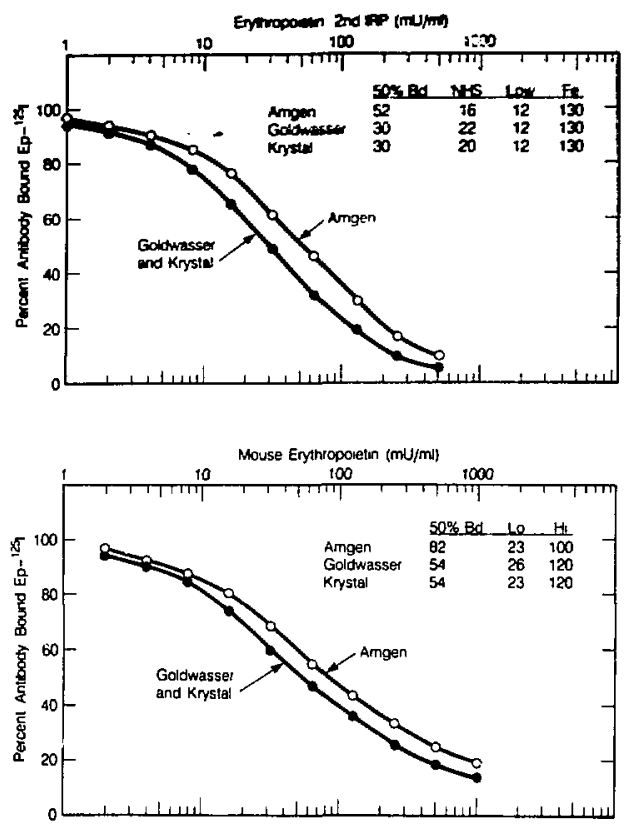

Fig. 2. Differences between recombinant $(A)$ and human urinary erythropoietin ( $G$ and $K$ ).

(XBL 855-8294A)

nal standards for each species of immunoreactive Ep were comparable among all three labeled preparations.

We then compared the effect of heat treatment on human urinary and recombinant Ep. Two fractions from the immunologically active ${ }^{125}$-Ep peak of A-Ep and K-Ep were pooled each, acidified to $\mathrm{pH} 5.5$, boiled 5 minutes, the $\mathrm{pH}$ readjusted and the labels repurified. Both preparations were tested in the RIA under conditions identica! to those before. Heat treaimeni had two significant effects: The total binding to the antibody for both labels increased significantly, and the immunological differences observed between the two urinederived Ep's and the recombinunt Ep had disappeared and the standard curves were superimposable. The higher binding an be ulilized to inctease the sensilivity of the IA juther by being able to use the antiserum at a higher ditution thas: previously.

So ffat we thave thot beem abte to detect ath

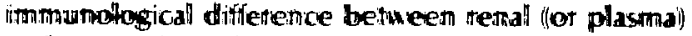

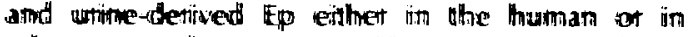
orther matrimalliam species. This ccompatison of urti- 
nary and recombinant $c p$ is the first instance in which the antiserum does discriminate between preparations. Since it has been established that the amino arid sequence in both the urinary and recombinant $\mathrm{Ep}$ is identical, the difference observed has to lie in the carbohydrate moiety. It is not presently known whether the degree of glycosylation in recombinant $\mathrm{Ep}$ is identical to native $\mathrm{Ep}$, but brief boiling appears to unmask the immunologically reactive site(s).

\section{PERIPHERAL CHANGES OF THYROID HORMONE BINDING TO SERUM PROTEINS AFTER SHORT TERM OZONE EXPOSURE}

\section{Sherry L. Fitzsimmons and Gisela K. Clemons}

Previous studies have shown a profound decrease in the levels of circulating thyroxine (T4), triiodothyronine (T3), and thyroid stimulating hormone (TSH) in serum of rats exposed to relatively low levels of ozone for a short period $(1 \mathrm{ppm} \times 24$ $\mathrm{hr}$. This decrease is thought to represent an alteration of the hypothalamic set point that regulates thyroid hormone homeostasis at the level of the central nervous system.

Ozone is an environmental pollutant, a product of photochemical smog, that causes pulmonary injury and edema. It was found that high levels of thyroxine administered to rats potentiaies the toxic effects of ozone, thus decreasing their survival. Thyroideclomized rats, however, are less susceptible to ozone toxicity. Therefore, this reduction of thyroid hormone levels in the normal animal measured after ozone exposure may be be an adaptive mechanism of the organism. Previous studies have suggested thal ozone induces a total suppression of the hypolharamic-pituitary-thyroid axis by lowering the set point at the level of the ceniral nervous system. However, this can only partially explain the reduction of thyroid hormone levels since rats administered exogenous $\mathrm{T} 4$ also showed a reduction in their circulating levels after short-term ozone exposure. Inhalation of ozone appears to also induce peripheral changes in serum that lead to the redurtion of measurable thytoid hormone levels.

Most of the circulating thyroid homones in the blocd ane bound to bindling proteins. A very small iraction of thyroxine (T4), which represents also the melabolically active thormone, tremains in the unbound or free state $(0,025 \%)$. This thee thomone is avillable for preniptwetal dewodinaliom into trivod-

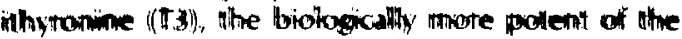
thatoind hormomes. The binding of th to serwem

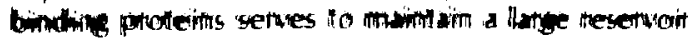

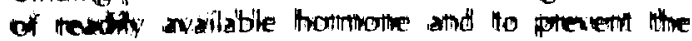

otherwise rapid excretion of thi: small hormone. In both humans and rats, there exist several binding proteins that are characterized by different affinities and binding capacities for $\mathrm{T} 4$. In humans, $75 \%$ of T4 is bound with a very high affinity and specificity to thyroid binding globulin (TBG), 15\% of circulating T4 is bound less tightly to thyroxine binding pre-albumin (TBPA), and the remaining T4 is bound with a low specific activity to the abundant protein, albumin. In the rat the majority of the circulating T4 is bound quite specifically to TBPA, with less than $10 \%$ bound to a TBG-like protein.

These three binding proteins can be separated on the basis of their size and electrophoretic mobility using native polyacrylamide gel electrophoresis. Serum samples were incubated with a tracer utuan. tity of ${ }^{125} \mathrm{I}$-T4 and electrophoresed overright, and the resultant dried gels were later autoradiographed. The autoradiogram riealed three radioaclive regions corresponding to the three $\mathrm{T} 4$ binding proteins. Since the majority of the T4 is bound to TBPA in the rat, we determined the binding characteristics of this prolein and any alterations induced by short-term ozone exposure.

The binding coistants of TBPA can be determined using polyacrylamide gel electroph resis. Varying concentrations of unlabeled T4 were incubated with serum samples containing tracer quantities of labeled hormone in order to compete for the binding sites on the TBPA molecules. From the experimental data and a scalchard plot the binding constants were determined. The affinity of TBPA for $T 4$ is given by the slope of the line, while the binding capacity of TBPA comesponds to the $x$ intencept. Since ozone is capable of oxidizing warious side chain residues of amino acids, and in has been shown to have denvituing effects on aboumin and antosine in mitho, in was hopothesized this an

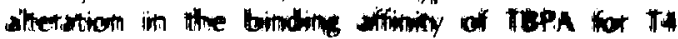


could lead to an early disappearance of the hormone from the circulation.

Control and experimental animals were housed in identical chambers for the duration of the experiments. The experimental animals were exposed to 1 ppm ozone for 24 hours, while the control animals received normal air. Serum from each group was collected and incubated with appropriate quantities of labeled and unlabeled hormone. All samples were electrophoresed under identical conditions using a slab gel apparatus and were later autoradiographed. Examination of the autoradiogram showed that the binding of T4 by rat TBG had been abolished after short-term ozone exposure, since the labeled hormone migrated oniy in two bands (Fig. 1). The Scatchard analysis for both sera is shown in Fig. 2. The affinity of TBPA for T4 is given by the slope of the line. There does not appear to be a significant difference in the affinity

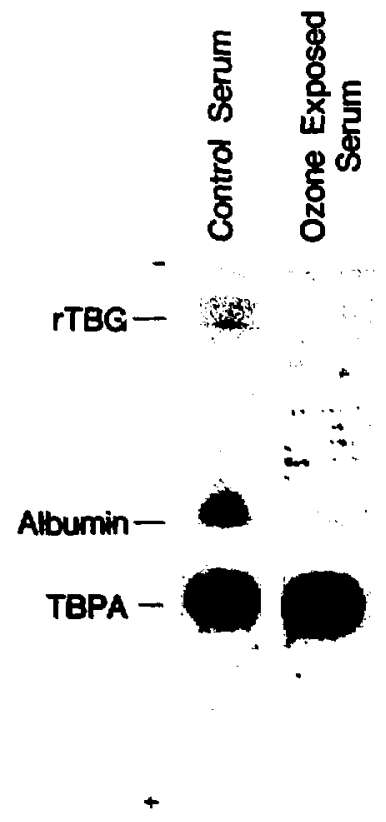

F. I. Ereat ef shot term azone exposute on the binding of

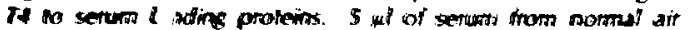
(herit) and zone exposed (thithl) were electrophatesed for 118

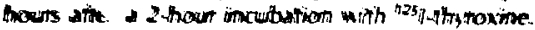

(ABB 850-9234)

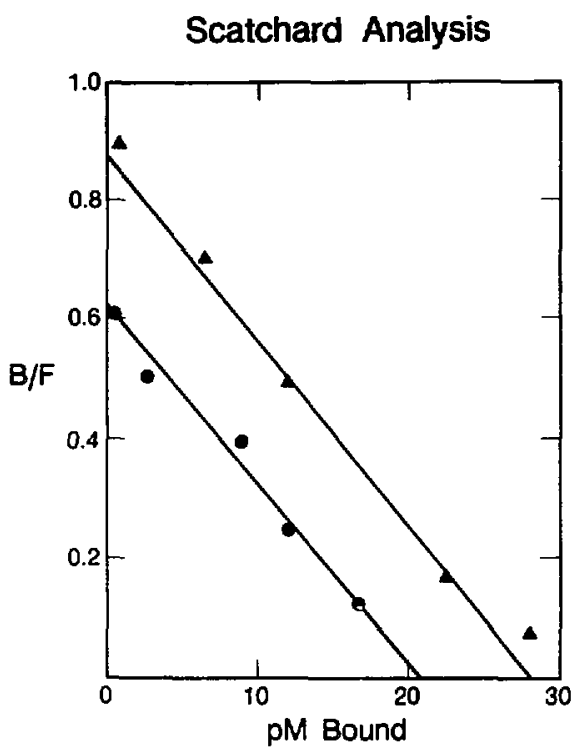

Fig. 2. Scatchard plot of ${ }^{125} /$-thyroxine binding to thyroxine binding prealbumin (TBPA). - I rat exposed to normal air and $\Delta=$ rats exposed to $1 \mathrm{ppm} \times 24$ hours ozone. Bound and free T4 were separated using polyacrylamide $8 \%$ gel electrophoresis. Values hav? been corrected for nonspecific binding.

(XBL 8510-8547)

of TBPA for T4 as a result of short-term ozone exposure. The apparent affinity constant for this protein in the presence or absence of ozone is 3.5 $\times 10^{8} \mathrm{M}$, which denotes a high degree of specificity for the hormone. Although the affinity of TBPA does not seem to be reduced in those animals previously exposed to ozone, there is a significant increase in the binding capacity of TBPA in these animals as iepresented by the $x$-axis intercept in the Scatchard plot. This suggests an increase in the number of unoccupied binding sites. This could be either the result of increased levels of circulating TBPA, or the previously bound T4 was being removed from the circulation as the result of ozone exposure, creating more available binding sites. The latter seems to be the more plausible explanation since the $30 \%$ increase in the binding capacity corresponds to the decreased levels of the circulating Tt leveis observed in animals afte: short-term exposure to ozone. 


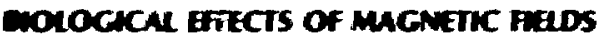

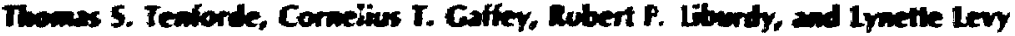

High-intensity magnetic lieids are used in several new!y devioping energy technologies liusion reactors, magnetohydrodyramic generafors, superconducting magnetic energy' storage rings). research facilities raccelerators, bubble chambers, superconducing spectrometers), industrial operafions (chemical separation processes, electrosteel production), and medicine (nuclear magnelic resonance imaging and in vivo spectroscopy). Because of the increasing number of applications of strong magnetic fields, a program is being carried out to evaluate the potential health effects of this form of nonionizing radiation. Both laboratory animals and selected cellular and tissue systems are being used to detect alterations in physiological functions during acute and chronic exposures to high-intensity magnetic fields. The initial work in this program involved studies with stationary magnetic fields produced by electromagnets at intensities up to 2 tesla ( $1 \mathrm{~T}=10^{4}$ gauss). During the past year, a new superconducting magnet was installed that is capable of producing fields up to $9 \mathrm{~T}$ for biological research with cells, tissues and small mammals. These investigations will provide essential data for assessing the potential health effects associated with the use of ultrahigh magnetic fields (in the 2 to $10 \mathrm{~T}$ range) in medical applications of nucleas magnetic resonance.

\section{ELECTROPHYSIOLOGICAL MEASUREMENTS}

A major area of research in this program has been the use of sensitive electrical recording techniques to detect functional alterations in the cardiovascular, neural, and visual systems during exposure to high-intensity magnetic fields. The physiological functions of these systems involve ionic conduction processes that are potentially sensitive to electrodynamic interactions with large stationary magnetic fields. Our investigations in this research area include 1) the use of noninvasive radiotelemetry to monitor cardiac electrical activity during the chronic exposure of rats to a 1.5-T stationary magnetic field, 2) study of blood flow characteristics in rals and dogs during exposure to magnetic ifeld intensities that exceed the threshold for producing measurable magnetohydrodynamic eifects. 3) recording the bioelectric activity of isolated neurons and the central menous syslem during exposure to fold intersities up to $1.5 \mathrm{~J}$, and th measutement of the photically-elicited electrical responses of the retina in cats and monkeys during exposure to fields of graded intensity up to $1.5 \mathrm{~T}$. During the past year, data acquisition was completed in the fourth of these research areas. ard the experimental resulls will be described briefly in this report.

The influence of strong magnetic fjelds on visual phototransduction was studied by using corneal contact electrodes to record the iransient electrical signals that originate in the retina following photon absorption. This electrical activity, known as the electroretinogram (ERG), consists of two prominent signals of opposite polarity. The first of these signals, the A wave, is a brief negative potential. The second major signal in the ERG is a large positive potential, the $B$ wave, which has a duration in excess of $100 \mathrm{msec}$. The relative magnitudes and durations of the $A$ and $B$ waves are shown in the top ERG trace in Fig. 1, which was
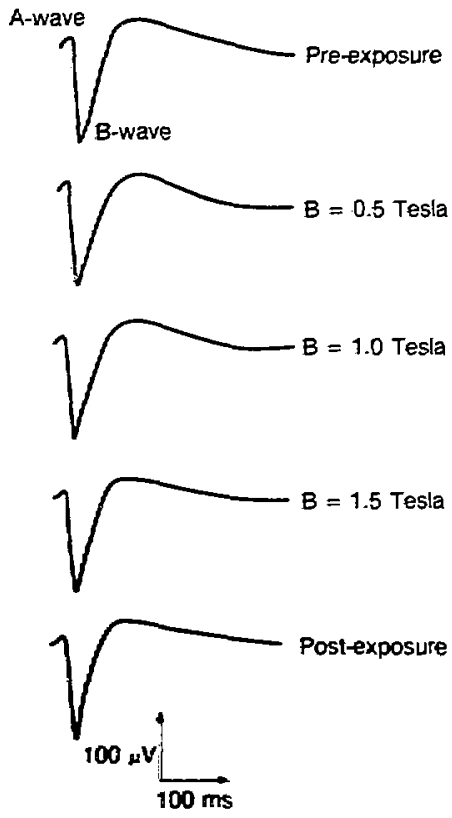

Fi2. 1. Electroretinagrato traces recosded in sino from a cat duting exposyte lo slationary magartic fieids with gaded iniensities up to $1.5 \pi$. The magnitudes and dunations of the A-wate and B-mase signals wete unafected by exposute to the lield.

(XBI 8500-6519) 
recorded in wo from a cot's eye subjected to a shon putse of white bight following a 30-min datk adaptation period. Ahtough the origins of the eye's electrical activity have not been determined previously, experimemts with intracellular microelectrodes indicate that the $A$ wave originates from the outer segments of the photoreceptor cells and the $B$ wave from the synaptic region of the bipolar cells.

A total of th:se monkeys (Macaca) and six cats (Felis domestica) were used for visual response studies during exposure to stationary magnetic fields with graded intensities up to $1.5 \mathrm{~T}$. After dark adaptation, the ERG was recorded following 10usec white light flashes with relative intensities of 1 , $4,16,48$, and 80 , where the highest intensity flash was 240 lumen $/ \mathrm{m}^{2} / \mathrm{sec}$. As demonstrated by the ERG data presented in Fig. 1 for a cat, there was no measurable change in either the $A$ or $B$ wave signals of the nine animals studied during exposure to fields up to $1.5 \mathrm{~T}$. C phthalmic examinations of all the subjects and electrical measurements of flickerfusion frequencies in five animals also indicated no effects of the magnetic field exposure.

\section{ANIMAL PHYSIOLOGY AND CIRCADIAN REGULATION}

Noninvasive techniques have been used for the continuous monitoring of physiological and behavioral variables in rodents exposed to a 1.5-T stationary magnetic field. The parameters being measured include heart rate, core body temperature, respiration, nutrient consumption, body mass, excreta, and locomotor activity. The circadian waveforms of these variables are sensitive indicators of stress, and they were therefore studied to detect physiological and behavioral alterations in rodents exposed to a 1.5-T field for periods up to 10 days.

One set of three experiments completed during the past year utilized an exposure chamber and physiological monitoring systems designed and fabricated at $L B L$ for the simultaneous recording of seven circadian variables. A schematic diagram of the exposure chamber and transducers is shown in Fig. 2. Groups of 40 female LAF-1 mice were housed in an aluminum cage with a floor area of $0.37 \mathrm{~m}^{2}$ that was placed within the homogeneous field region of an electromagnet operated at $1.5 \mathrm{~T}$. The cage was supplicd with uniform lighting at an intensity of $155 \mathrm{~cd} / \mathrm{m}^{2}$, and a steady now of conditioned air at a temperalure of $22.0 \pm 0.5^{\circ} \mathrm{C}$ and a relative humidity of $50 \%=10 \%$. Six strain gage monitors provided continuous data on the following parameters: II tolal body mass, 21 center-of-mass coordinates of the entire rodent poputation as in index of migatory locomotor activity, and 31 dimbing activity regüstered on an exercise bur. Respiratony rate was assessed by infraxed measurements of the carbon dioxide content of air emerging from the cage outlet port. The rodents were fed a complete nutrient diet in liquid form on an ad lib basis. and their nutrient consumption was measured volumetrically. Urine and feces excretion were monitored gravimetrically from the contents of a collecting tray located below the honeycomb aluminum floor of the exposure chamber. A wire mesh covering the excreta collecting tray was used to separate the feces and urine, and the urine samples were preserved in a frozen state for subsequent chemical analysis. Data from the various transducers and environmental monitoring devices were recorded continuously on magnetic tape at 5min intervals throughout experiments of 50 to 60 days duration, and the circadian waveforms of physiological and behavioral parameters were analyzed using a CDC 7600 computer.

Figure 3 shows the circadian waveforms in respired carbon dioxide, body mass, and two measures of activity based on the weight applied to a triangular exercise bar and the time rate of change of the center-of-mass coordinates of the rodent population. Circadian waveforms with large amplitudes were also measured for nutrient consumption and urine and fecal excreta. The data shown in Fig. 3 were continuously recorded at 5 -min intervals during five serial days. The animals were maintained in a free-running circadian state throughout this period by imposing constant dim illumination. Various mathematical models of circadian oscillations have been used to generate besi-fit curves for these data using a least-squares analysis. For the smoothly varying circadian parameters such as respired carbon dioxide and body mass, a cosine function provides an optimal fit based on the criterion of minimizing the sum of the squares of residuals between the measured data and the best-fit curve. For circadian variables with superimposed stochastic fluctuations, e.g., the two measures of locomotor activity shown in panels (C) and (D) of Fig. 3. alternate models such as a piecewise-linear approximation provide a fit of the data that is equally as good as the conventional cosine analysis procedure. The smooth curves shown in Fig. 3 represent the best-fil cosine functions for the four circadian variables, along with the $95 \%$ confidence intervals. It is evident from the parameters of the best-fit curves that in the free-running circadian state. i.e.. in the absence of external time cues, the periods of the circadian waveforms in physiological 


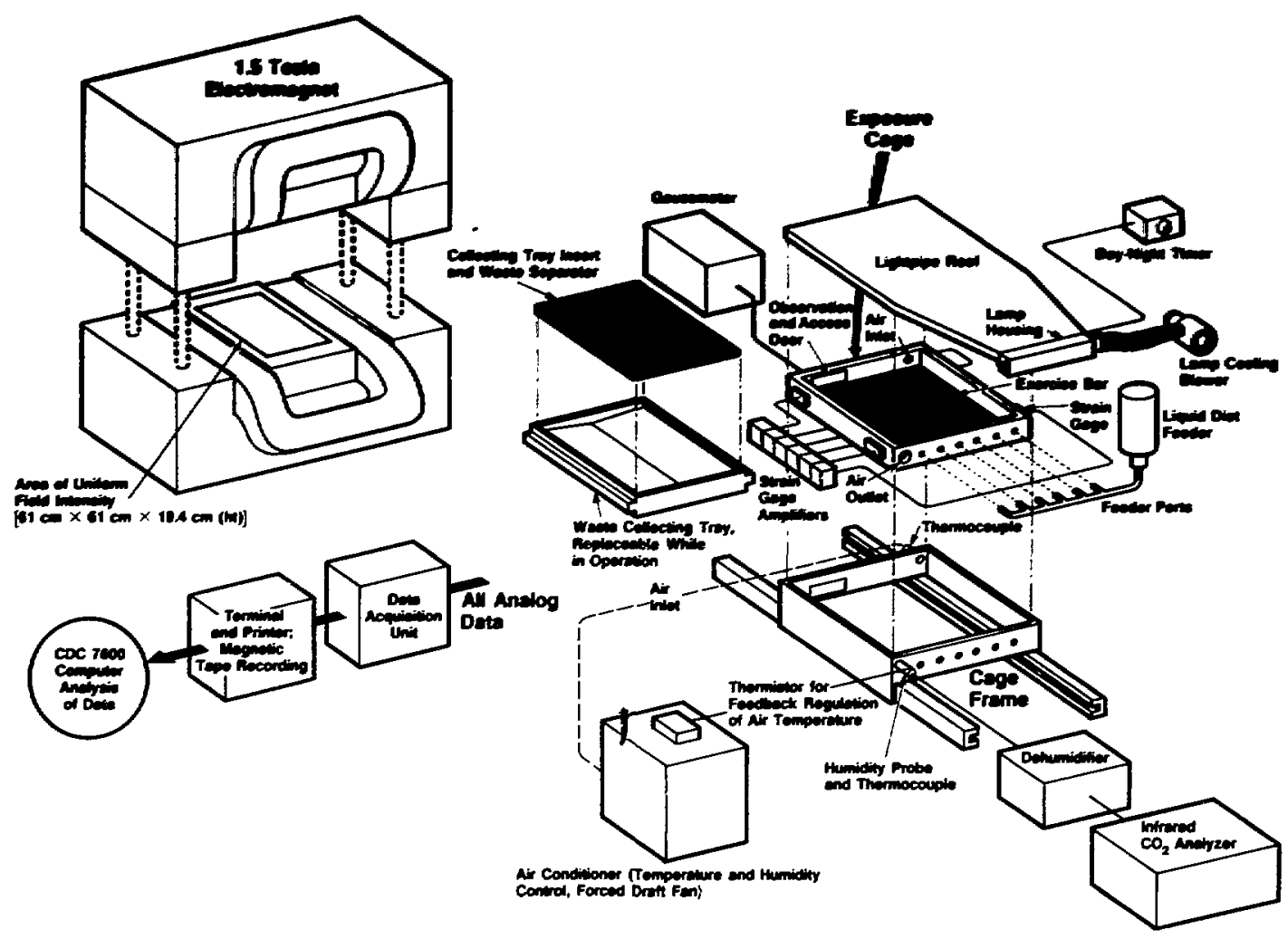

fis. 2. Schematic diagram of the exposure chamber, transducer system, and environmental monitors used in experiments to detect magnetic field effects on physiological and behavioral variables in mice.

(XBL 849-7946)

and behavioral variables range from approximately 25 to 26 hours. When a strong time cue such as a daily light/dark schedule is imposed, these circadian variables become entrained within 5 to 10 days and exhibit periods close to 24 hours.

In the first two long-term experiments designed to test for physiological and behavioral effects of a 1.5-T stationary magnetic field, the seven circadian variables under study were measured noninvasively for a continuous two-month period throughout which the rodent population was maintained in an entrained state by an imposed 12-hr-light/12-hrdark diurnal cycls Following a pre-exposure control period, the field was applied either continuously for 5 days or intermittently in an $8-\mathrm{hr}-\mathrm{on} / 16$ hr-off daily cycle for 10 consecutive days. The circadian variables were continuously monitored before, during. and atter each application of the magnetic field. In the third long-lerm experiment. rodents were maintained in a free-running circadian state by imposing constant dim illumination in the exposure chamber. Circadian waveforms were measured before, during, and after the application of a 1.5-T field for 10 consecutive days in a 8-hron/16-hr-off diurnal cycle. As a positive test for entrainment, the rodents were then subjected to a daily 8-hr-dark/16-hr-light cycle for 20 consecutive days. Both parametric and nonparametric analyses of the extensive data obtained in these three experiments indicated that exposure to a highintensity magnetic field had no effect on the circadian waveforms of the seven simultaneously measured physiological and behavioral variables. In addition, no entraining influence of the field was observed when the animals were maintained in a free-running circadian stale during the third experiment. An imposed diumal light/dark cycle was found, however, to produce a rapid entrainment of 


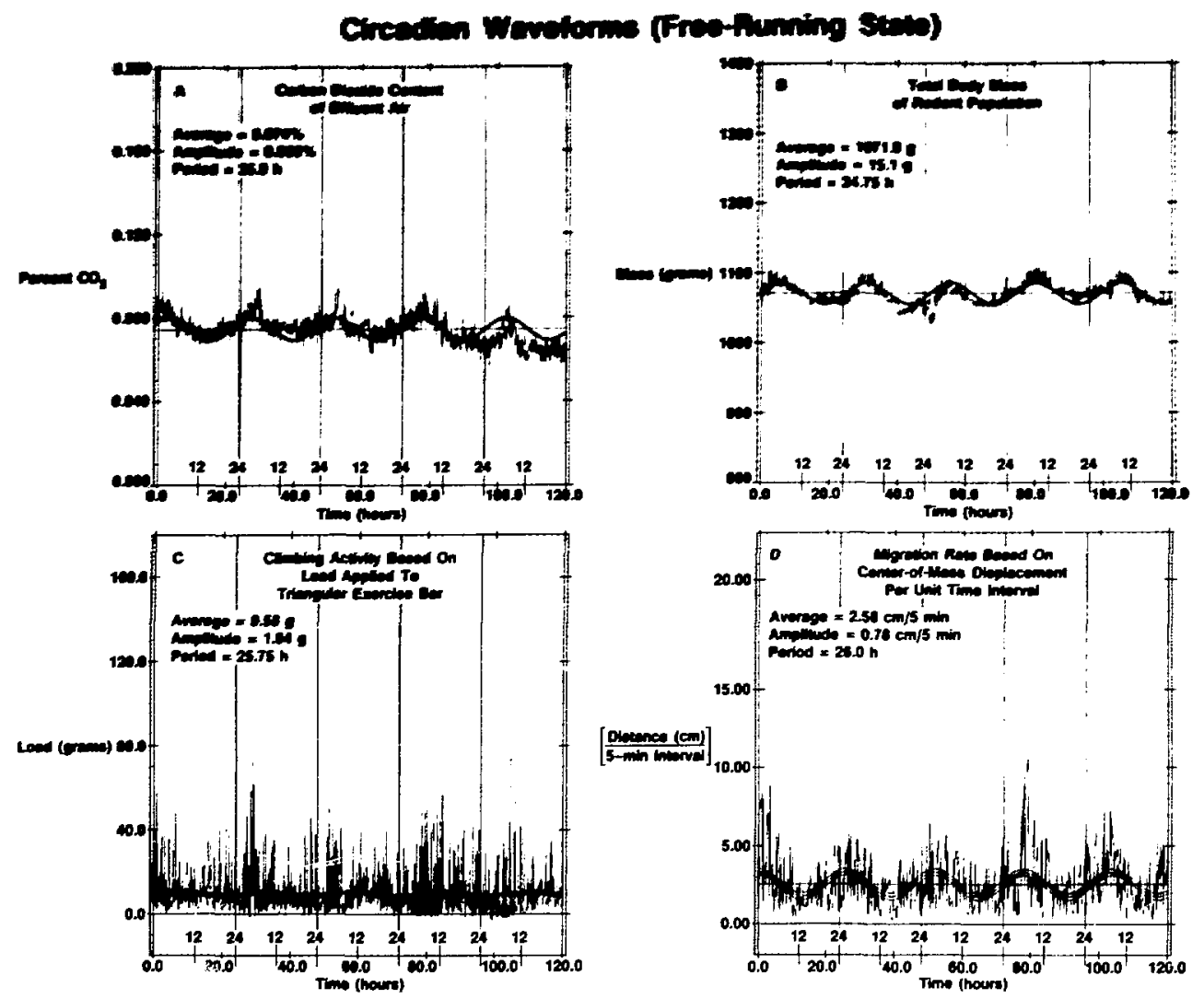

Fig. 3. Circadian waveforms in respired carbon dioxide, body mass, and two independent measures of locomotor activity measured for a population of 40 female LAF-1 mice. The mice were maintained in a free-running circadian state by imposing constant dim illumination in the exposure chamber shown in Fig. 2. The three smooth curves superimposed on each sel of circadian data represent a cosine function tit by a least-squares analysis technique, along with the upper and lower bounds of the $95 \%$ confidence interval. The amplitude and period of the best-fit cosine function are listed for each of the four circadian variables.

(XBL 848-7916)

all the circadian variables under study. Our observation that no significant circadian alterations occur in response to a high-intensity stationary magnetic field is in distincl contrast to a large number of earlier publications which reported that physiological regulation and circadian timing in rodents and other animal species are influenced by relatively weak magnetic stimuli.

\section{SUPERCONDUCTING MAGNET RESEARCH}

A new 9-T superconducting magnel from Oxford Instruments, Lid. was installed at LBL in the Fall of 1984. This magnet has a $7.25-\mathrm{cm}$ bore suilable for biological studies of cellular and tissue specimens and small laboratory animals. Following installation, the magnet was subjected to detailed performance tests and field mapping using a nuclear magnetic resonance (NMR) probe, a search coil, and an axial Hall-effect probe. All three independent methods of field detection gave consistent results when compared at a field level of 7.5 T. As shown in panel (A) of Fig. 4, the stationary magnelic field in the central $20-\mathrm{cm}$ region of the $73.6-\mathrm{cm}$ long cylindrical bore is homogeneous, and large spatial gradients up to $60 \mathrm{~T} / \mathrm{m}$ are present in the end regions of the bore. NMR measurements on a $\mathrm{D}_{2} \mathrm{O}$ sample were performed in the ceniral 6 . $\mathrm{cm}$ region of the bore, and the field homogeneily in the middle of this region was found to be belter 
(A)

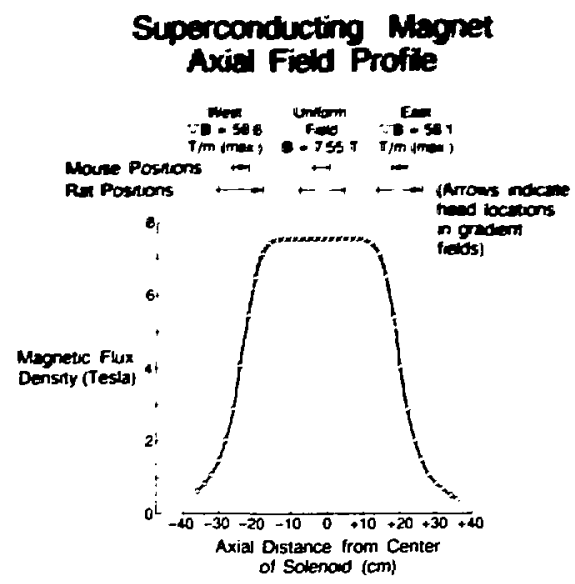

(B) Thermoregulation in Rodents During Exposure to Homogeneous (7.55 T) and Gradient $(58.1-58.6 \mathrm{~T} / \mathrm{m})$ Magnetic Fields

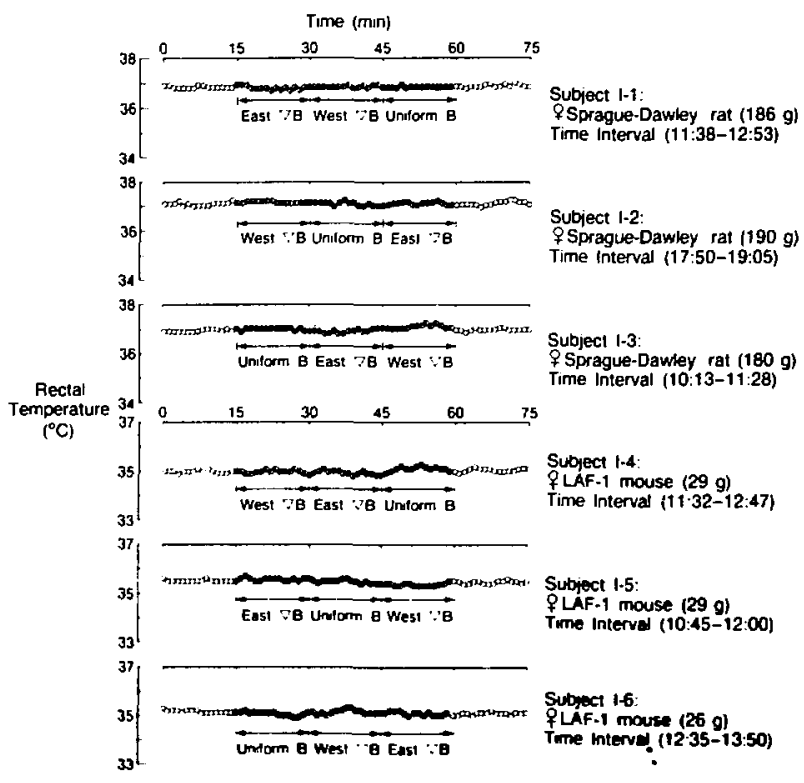

Fi. 4. (A) Magnelic field profile measured with a search coil along the central axis of the superconducting magnet being used for biological rescarch. The positions of mice and rats in the uniform central feld and in the ascending and descending gradient regions are shown bv brackets. (8) Recral temperatures recorded at 1 -min intenals are shomn for six rodents sulyected to 45 -min exposures to the unifom and gradient fields protuced by the supercon.

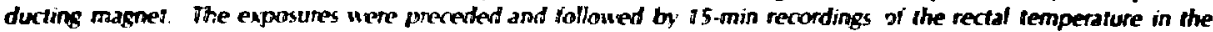
dosence of a mapnetic field. 
then one purt in 75,000. Following the completion of this detailed fiekd characterization, biological experiments were initiated 10 study the effects of utrahigh magnetic fields on ionic conduction processes in the cardiac and nervous systems, on cyclosis in plant cells, on the thermoregulatory system of rodents, and on the structural and functional properties of artificial lipid membranes. Research results in the last two of these areas will be described in this report.

Two recent reports from Germany have indicated that thermoregulation in humans and in laboratory animals is significantly influenced by uniform magnetic fields and by strong spatial gradients of magnetic fields. Gremmel et al.' reported changes up to $5^{\circ} \mathrm{C}$ in human skin temperature studied by infrared thermography during exposure to stationary fields of 0.2 to $1.5 \mathrm{~T}$ intensity. Both increases and decreases in temperature were observed, and these results were qualitatively confirmed in laboratory studies with cats. Sperber el al. ${ }^{2}$ subsequently reported similar changes in rodents exposed to stationary fields with intensities ranging from 0.4 to $8.0 \mathrm{~T}$. In the strong magnetic field gradients near the end of a superconducting magnet bore, these investigators observed both increases and decreases in skin and rectal temperatures. The temperature changes were reported to begin within $5 \mathrm{~min}$, and reached a maximum magnitude of approximately $2.4^{\circ} \mathrm{C}$ after 2 to 3 hours of exposure to a magnetic field. When the field was turned off, the skin and rectal temperatures slowly returned to their pre-exposure values within 2 hours, Sperber et al. ${ }^{2}$ proposed that orientational effects of strong magnetic fields on biologically important macromolecules may be the basis for the apparent loss of thermoregulatory capacity observed in their experiments.

Because these results, if confirmed, would have major implications for the safety of NMR imaging with human subjects, an effort was made to reproduce the reported effects in rodents subjected to large uniform magnetic fields and high magnetic field spatial gradients. Both pigmented LAF-1 mice and albino Balb/c mice were used in these experiments, the latter strain being identical to the mice used by Sperber et al. ${ }^{2}$ in their earlier experiments. In addition, albino Sprague-Dawley rats were used as test subjects in our experiments. The positions of the adult female mice and rats in the uniform field and the gradient fields are indicated in panel (A) of Fig. 4. The unanesthetized animals wete placed with minimal restraint in lucite holders, and rectal temperatures were recorded with a nonmag- netic copper/constantan thermocouple costed with plastic.

Three series of experiments were camied out to determine whether the thermoregulatory capacity of rodents was influenced by high-intensity magnetic fields. In the first experiment, shown in panel (B) of Fig. 4, three Sprague-Dawley rats and three LAF-1 mice were exposed for conseculive $15 \mathrm{~min}$ intervals to the uniform central field and the ascending and descending gradient fields. The order of presentation of the uniform and gradient fields was randomized for the six animals. The interval of $15 \mathrm{~min}$ was longer than the time reported by Gremmel et al. ${ }^{1}$ and Sperber et al. ${ }^{2}$ to produce a measurable rectal or skin temperature change. An analysis of variance of the data obtained in these experiments showed that no statistically significant change occurred in rectal temperature in response to magnetic field exposure. This result was independent of the field uniformity or the time of day during which the exposures were made. Exposures 10 a strong magnetic field gradient for periods of 1 to 3 hours were also carried out with two Sprague-Dawley rats, two LAF-1 mice, and six Balb/c mice. The results of these experiments are presented in Fig. 5. An analysis of variance again showed no significant change in the rectal temperature of any of the ten rodents during the magnetic field exposure. This result was also independent of the time of day during which the animals were exposed to the field. These studies with a total of 16 animals clearly indicate the absence of effects of a high-intensity magnetic field on the thermoregulatory capacity of laboratory animals, in distinct contrast to the earlier results obtained by two other groups of investigators.

In another area of superconducting magnet research, the influence of ultrahigh magnetic fields on the structure and permeability properties of lipid membranes was studied using liposomes with a well defined molecular composition. Jiamagnetically anisotropic assemblies of molecules such as membrane phospholipids experience an orientational torque in strong magnetic fields and adopt a spatial configuration that minimizes their free energy state in the field. For thin nematic liquid crystals, stationary fields in the range of 0.1 to $1.0 \mathrm{~T}$ have been shown to significantly alter the rate of solute transport as a consequence of magnetoorientation of the lipoidal coristituents. In our experiments, 200- $\mu \mathrm{m}$ liposome vesicles bounded by a phospholipid bilayer containing saturated fatty acyl chains were used as test specimens. The dipalmiloylphosphatidyl choline (DPPC) moieties in 


\section{Thermoregulation in Rodents During Exposure to a $58.6 \mathrm{~J} / \mathrm{m}$ Magnetic Field Gradiont [o Control periods; o field exposure period]}

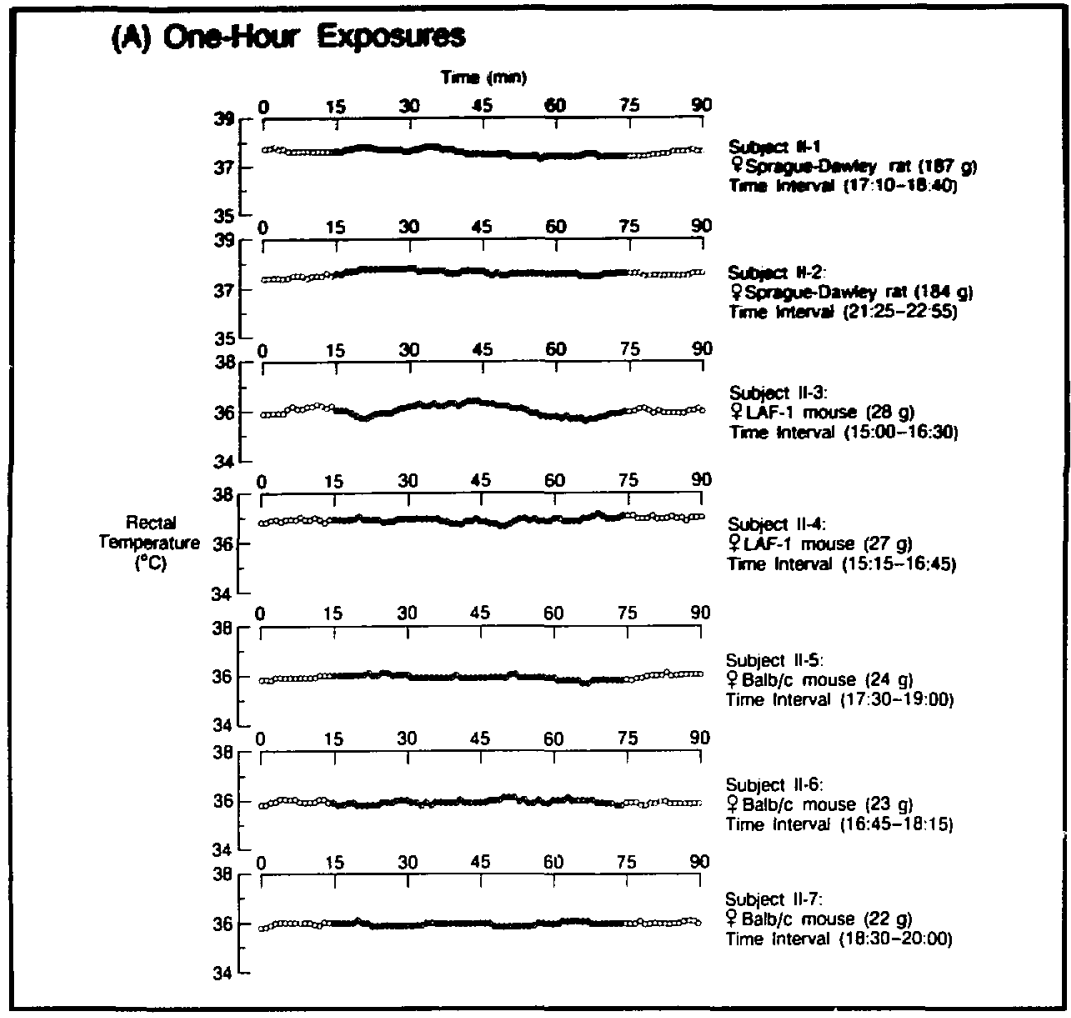

(B) Three-Hour Exposures

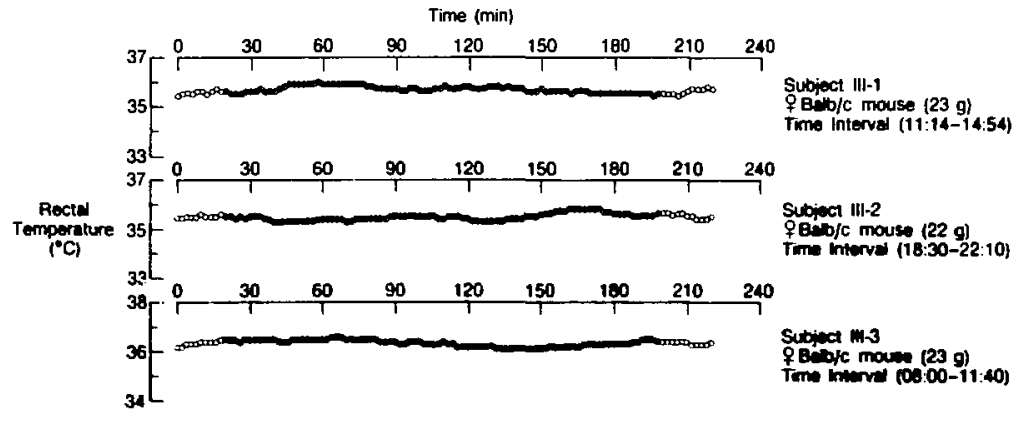

Fi. 5. (A) Reclal lemperalure necondings are shoun for seven rodenls exposed to a $58.6 \mathrm{I} / \mathrm{m}$ magnefic field gradient for $1 \mathrm{hr}$. The median field strength at the midpoist of the exposed subjects was $+16 \mathrm{~T}$. The continuous $\mathrm{I}$.hr exposure was preceded and tolkowed by $15-\mathrm{min}$ recordings of the tectal temperature in the absence of a manetic

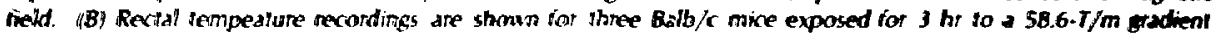
inetd.

(X6L 85ta363) 
the liposomal membranes were radiolabeled with ${ }^{14} \mathrm{C}$, and the inner compartment of the vesicles contained ${ }^{3} \mathrm{H}$-labeled cytosine arabinoside $\left({ }^{3} \mathrm{H}\right.$ ARA-C: M.W. = 243). The telease of ${ }^{3} \mathrm{H}$-ARA-C from these liposomes has recently been shown to be stimulaled by $2450-\mathrm{MHz}$ microwaves, as described by R.P. Liburdy in this annual report. In experiments with the new superconducting magnet, the release of ${ }^{3} \mathrm{H}-\mathrm{ARA}-\mathrm{C}$ and ${ }^{14} \mathrm{C}-\mathrm{DPPC}$ was measured following exposures that ranged in duration from $1 \mathrm{~min}$ to $18 \mathrm{hr}$ at graded field intensities from 0.001 to $7.5 \mathrm{I}$. At each field level, the release of trapped ${ }^{3} \mathrm{H}$-ARA-C solute and ${ }^{14} \mathrm{C}$-DPPC membrane phospholipids was determined over a temperature range of $4^{\circ} \mathrm{C}$ that bracketed the thermal phase transition which occurs at $40.5^{\circ} \mathrm{C}$. The temperature of the liposome samples was maintained with an accuracy of $0.08^{\circ} \mathrm{C}$ at each of the specified temperatures over this range, and control samples were assayed for release of ${ }^{3} \mathrm{H}$-ARA-C in parallel with the samples exposed to a magnetic field. At temperatures below $40^{\circ} \mathrm{C}$ and above $41^{\circ} \mathrm{C}$, the release of ${ }^{3} \mathrm{H}$-ARA-C solute was identical in the exposed and control liposome samples. However, in the prephase transition region from 40.0 to $40.5^{\circ} \mathrm{C}$, an accelerated release of trapped solute was observed in samples exposed to stationary magnetic fields greater than $0.01 \mathrm{~T}$. This magnetic field effect on liposome permeability is rapid, and a difference between the exposed and control liposome samples of $30 \%$ of the total maximal release of ${ }^{3} \mathrm{H}$-ARA-C was observed within one minute. As shown in Fig. 6 , exposures at fjeld levels between 0.001 and $7.5 \mathrm{~T}$ revealed that this permeability effect has a sigmoidal dependence on the magnetic flux density. The $50 \%$ effect level is $0.015 \mathrm{~T}$, with a $95 \%$ confidence interval of 0.0065 to $0.0349 \mathrm{~T}$. Experiments were also conducted with liposomes exposed to magnetic fields produced by samarium-cobalt permanent magnets. As shown in Fig. 6, 0.40-T fields produced by the permanent magnets and the superconducting magnet produced a comparable release of ${ }^{3} \mathrm{H}$-ARA-C at $40.5^{\circ} \mathrm{C}$. The observed change in permeability to ${ }^{3} \mathrm{H}$-ARA-C was also shown not to result from disruption of the liposomes, since there was no accelerated release of ${ }^{14} \mathrm{C}$-DPPC from the liposome membranes at any of the magnetic field strengths that were used in these studies.

These liposome studies indicate that a stationary magnetic field of $0.015 \mathrm{~T}$ or greater can signifi-

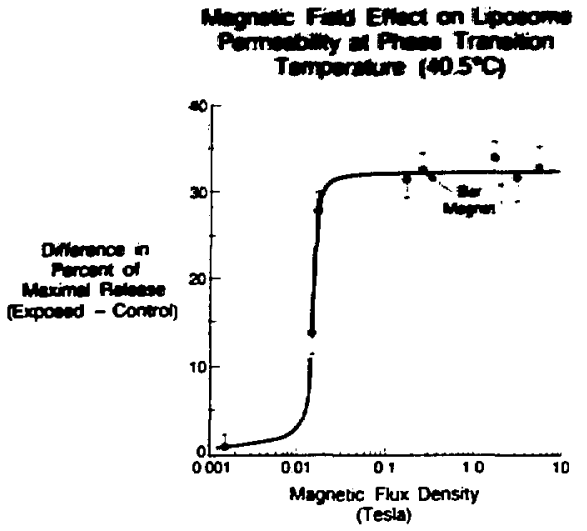

Fig. 6. The release of 'H-ARA-C from liposomes is shown as a function of magnetic field strength from 0.001 to $7.5 \mathrm{~T}$. Exposures of liposome samples were made in a superconducting magnet for $15 \mathrm{~min}$ at $40.5^{\circ} \mathrm{C}$, and the solute permeability change is expressed as the difference in percent of maximal release observed between the field-exposed and control samples. The dependence on magnetic field strength of the permeability increase is sigmoidal with a $50 \%$ effect occurring at $0.015 \mathrm{~T}$. The data point labeled as Bar Magnet corresponds to a $0.40-T$ exposure made with a pair of samarium-cobalt permanent magnets. Error bars represent 1 S.E. (XBL 859-8500)

cantly increase passive solute transport in phospholipid bilayers maintained at or near the membrane phase transition temperature. Lipid clustering which occurs at pre-phase transition temperatures may predispose membrane phospholipids to diamagnetic orientation in a magnetic field, and thereby trigger a phase transition enabling drug release. This finding represents a totally new phenomenon that potentially has important implications for magnetic field interactions with cellular membranes.

\section{REFERENCES}

1. Gremmel, H., Wendhausen, H., and Wunsch, F. Biologische Effekte statischer Magnetfelder bei NMR-Tomographie am Menschen. Wiss. Mitt. Univ. Kiel, Radiologische Klinik (1983).

2. Sperber, D., Oldenbourg, R., and Dransfeld, $K$. Magnetic freld induced temperature change in mice. Naturwiss. 71, 100-101 (1984). 


\section{Manowave STMULATED DAUG RELESE FROM LFOSOMES}

Robert P. Liburdy

Recent results from our laboratory raise the important question of whether proteins, phospholipids, or bolh are responsible for the phenomenon of microwave-induced cation permeability we observe in the erythrocyte and in the lymohocyte. $^{1-3}$ To determine if phospholipids in membranes are influenced by microwaves we have construcled liposomes devoid of proteins and examined their permeability in a microwave environment. ${ }^{4}$ These studies demonstrate that microwaves stimulate the release of an aqueous chemotherapeutic drug from liposome vesicles and indicate that liposomes should be useful model systems for the study of biological membrane responses to nonionizing radiation. In addition, this technology may prove useful as an in-vivo drug-delivery modality.

Liposome vesicles containing tritiated cytosine arabino-furanoside $\left({ }^{3} \mathrm{H}\right.$-ARA-C, mol $\left.w t=243\right)$ were prepared using the reverse phase evaporation process from two highly purified and completely saturated phospholipids, dipalmitylphosophatidylcholine (DPPC) and dipalmitylphosphotidylglycerol (DPPG); this technique forms large unilamellar vesicles $2000 \AA$ in diameter. Trace amounts of ${ }^{14} \mathrm{C}$ DPPC were used to enable monitoring of membrane disruption. Exposures to microwaves (2450 $\mathrm{MHz}, 60 \mathrm{~mW} / \mathrm{g}, 10 \mathrm{~min}$ ) were accomplished using a waveguide apparatus that controls and monitors sample temperature to $0.05^{\circ} \mathrm{C}^{1-3}$ Release of drug and membrane phospholipid in response to microwaves were determined by monitoring each radioisotope in the supernatant obtained after pelleting exposed liposomes $(170,000 \times \mathrm{g}, 1 \mathrm{~min})$.

Release of ${ }^{3} \mathrm{H}$-ARA-C and ${ }^{14} \mathrm{C}$-DPPC from liposomes as a function of exposure temperature is shown in Fig. 1. A characteristically sharp increase in drug release $\left({ }^{3} \mathrm{H}\right.$-ARA-C) occurs at $40^{\circ} \mathrm{C}$ for sham-exposed liposomes with essentially no membrane phospholipid release; this occurs at the membrane phase transition temperature. $T_{c}$. In saline a $60 \%$ maximal release occurs in contrast to $80 \%$ in plasma; thus a plasma component(s) facilitates drug release from these liposomes at $T_{c}$. Significantly. microwaves are observed to stimulate drug release at temperatures as low as $33^{\circ} \mathrm{C}$ and $25^{\circ} \mathrm{C}$ for liposomes in saline and plasma, respectively. This stimulation of drug release is remarkable because it occurs at temperatures well below $T_{c}$, where these

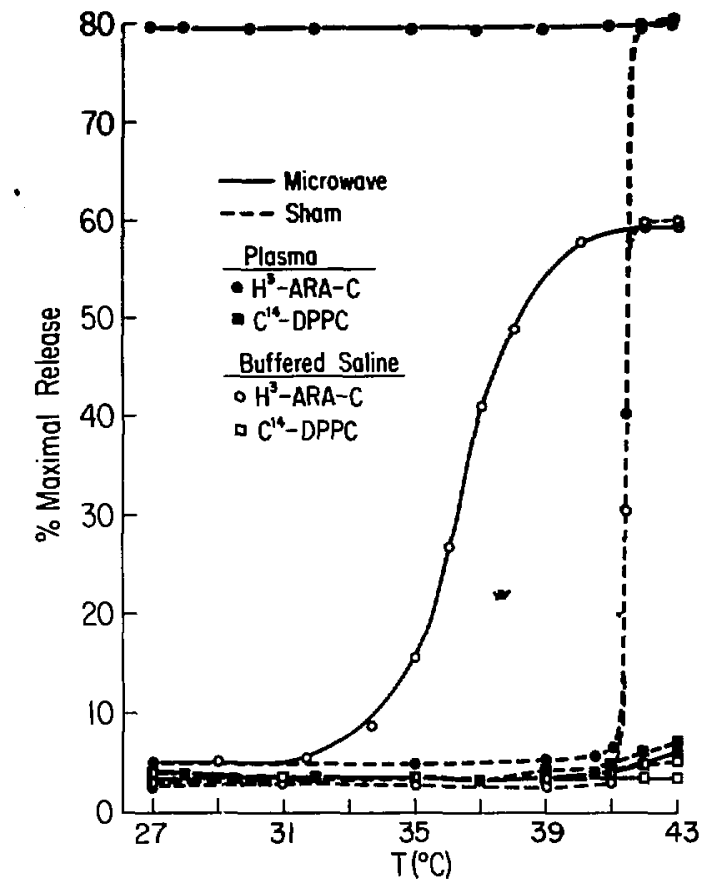

Fig. 1. Microwave-stimulated drug release from liposomes. Drug release curves for liposomes heated by microwaves 160 $\mathrm{mW} / \mathrm{g}, 10 \mathrm{~min}$ ) and by conventional means; effects in buffered saline and plasma. Tritiated cytosine arabinofuranoside $\left({ }^{3} \mathrm{H}\right)$ $A R A-C)$ was encapsulated into the aqueous compartment and $1^{14} \mathrm{Cl}$-DPPC into the lipid membrane.

(XBL 8411-8034)

liposomes are normally not leaky. In contrast, microwaves do not result in release of membrane phospholipid $\left({ }^{14} \mathrm{C}\right.$-DPPC), indicating that gross membrane structure is not altered. These data are consistent with a mechanism of action in which microwaves induce the formation of a pore, and this could occur if an apparent membrane phase Iransition is triggered during exposures.

Since $\mathrm{pO}_{2}$ varies considerably in vivo, the effect of oxygen on this liposome response to microwaves was investigated. Figure 2 shows heat release curves for exposures at $\mathrm{pO}_{2}=760 \mathrm{~mm} \mathrm{Hg}(100 \%$ oxygen), $0 \mathrm{~mm} \mathrm{Hg}(100 \%$ nilrogen), and $150 \mathrm{~mm}$ Hglatmospheric air). Oxygen is seen to markedly potentiate the microwave elfect while having no 


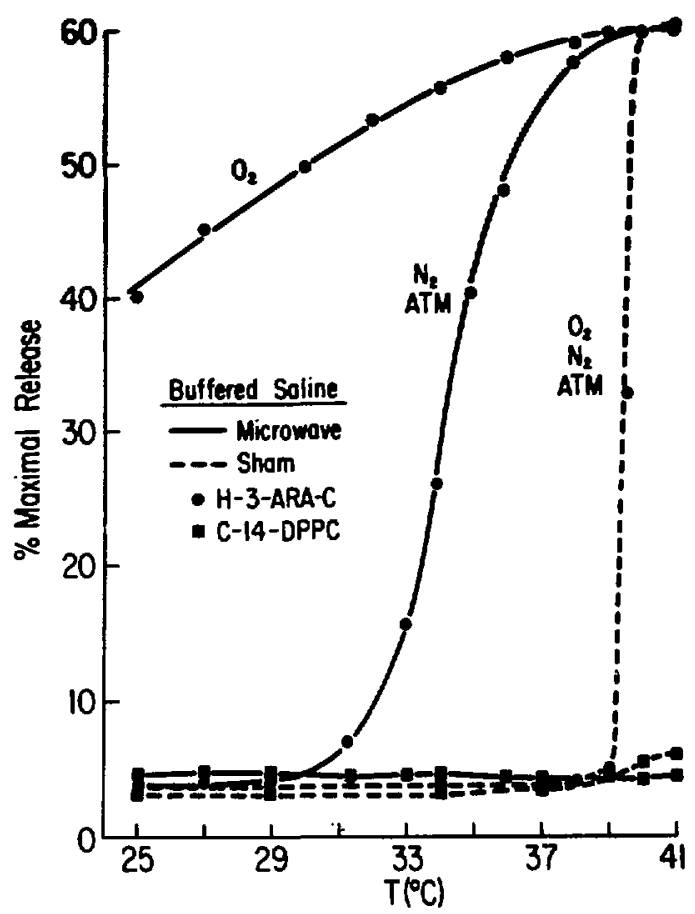

Fig. 2. Effect of axygen on microwave-stimulated drug release. Liposomes were exposed as in Fig. 1 with the exception that prefiltered $\mathrm{O}_{2}$ and $\mathrm{N}_{2}$, as well as atmospheric air (ATM), were used as vehicles to mix the sample volume and maintain $\mathrm{pO}_{2}$ during exposures.

(XBL $84 I J-8032)$

effect on drug release from sham-exposed liposomes. This indicates that oxygen significantly facilitates permeability of liposomes in the presence of microwaves. The $\mathrm{kiw}$ level of ${ }^{14} \mathrm{C}$-DPPC released from the liposome mimbrane also indicates that oxygen plus microwaves does not act to disrupt bilayer integrity. This permeability increase is therefore consistent with a pore-formation event.

Free-radical formation can lead to lipid peroxidation and an increase in liposome permeability ${ }^{3}$ : thus the influence of oxygen on microwavestimulated drug release might be mediated through free-radical formation. To lest this hypothesis liposomes were microwave-treated at $37^{\circ} \mathrm{C}$ in the presence of $100 \%$ oxygen, $100 \%$ nilrogen, or $100 \%$ הitrogen plus two different free-radical scavengers, ascorbic acid (ASC) and mercaptoethanol (ME). Figure 3 indicates that a significant reduction in drug release is obsenved for nitrogen compared to oxysen, as observed for $37^{\circ} \mathrm{C}$ in Fig. 2. Significantly, in the presence of either litee radical scavenger drus release is further redkuced. This

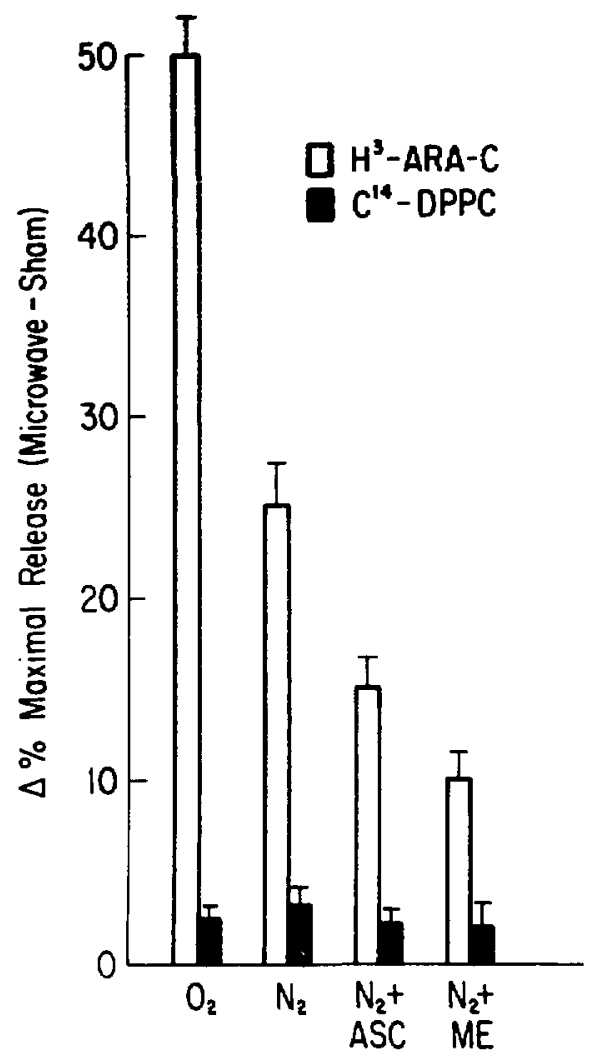

Fig. 3. Effect of antioxidants on microwave-stimulated drug release. Exposures at $37^{\circ} \mathrm{C}$ as in Fig. 2. Ascorbic acid or mercaploethanol was present at a $5 \times$ and $i \times$ mole ratio of antioxidant to lipid, respectively. Mean \pm S.D., $n=7$.

(XBL 8411.8031)

strongly suggests that free-radical formation may play a role in the oxygen effect.

The above results demonstrate that microwaves can interact wilh phospholipids in membrane bilayers to increase liposome permeability below $T_{c}$. This phenomenon occurs in the absence of membrane protein and does not involve gross bilayer disruption; this latter fact suggests that a poreformation event occurs. One way microwaves might accomplish this is by physically exciting phospholipid rotational/vibrational motion to trigger a phase Itansition": phospholipid acyl chains are reported to exhibit rotational motion with relaxation frequencies in the micnowave range during phase transitions. Although the exact molecular mectian. ism responsible for microwavestimulated dro release is at present unknown, this inceraction is 
modulated by chemical factors. Both plasma and oxygen potentiate drug release, whereas antioxidants inhibit it. This latter observation strongly suggests that free-radical formation plays a role in modulating this interaction.

\section{REFERENCES}

1. Liburdy, R.P., and Fanek, Jr., P.F. Microwaves and the cell membrane. II. Temperature, plasma, and oxygen mediate microwaveinduced membrane permeability in the erythrocyte. Radiation Research 102, 190-205 (1985).
2. Liburdy, R.P., Penn, A., Nesta, D., and Vanek, Ir., P.F. Microwaves alter cation transport and induce protein shedding in the lymphocyte. Federation Proceedings 43, 908 (1984).

3. Liburdy, R.P. and Penn, A. Microwave bioeffects in the erythrocyte are temperature and $\mathrm{pO}_{2}$ dependent: Cation permeability and protein shedding occur at the membrane phase transition. Bioelectromagnetics 5, 283-291 (1984).

4. Liburdy, R.P. and Magin, R.L. Microwavestimulated drug release from liposomes. Radiation Research 103, 266-275 (1985).

\section{NEW SEQUESTERING AGENTS FOR THE AGIINIDES: EFFECTIVENESS FOR REMOVAL OF Pu FROM MICE OF HYDROXAMIC ACID DERIVATIVES OF DTPA, EDTA AND DESFERRIOXAMINE AND AN N-CENTERED TRIS(CATECHOYLAMIDE) LIGAND; GRADED DOSAGES OF DESFERRIOXAMINE PYRIDINONE OXIDE (desferriHOPOCAM); PROTRACTED ADMINISTRATION OF 3,4,3-LICAM(C)}

Patricia W. Durbin, Nylan Jeung, Kenneth N. Raymond," "` David L. White, ${ }^{\ddagger}$ Steven J. Rodgers ${ }^{\dagger}$ and Petra Turowski ${ }^{\dagger}$

\section{INTRODUCTION}

The purpose of this program is to develop biological data needed to guide the design of safe effective chelating agents that will accelerate elimination of actinides and chemically similar metals from the body. Macromolecules have been synthesized containing multiple catechoylamide (CAM), hydroxamate $(X)$, hydroxypyridinone (HOPOCAM) and carboxyl (C) functional groups, either all the same structure le.g., the tetrameric carboxycatechoylamide, 3,4,3-LICAM(C) or of more than one structure (e.g., desferriHOPOCAM, which contains three hydroxamate and one hydroxypyridinone oxide groups). All those functional groups, in a variety of molecular arrangements, form stable complexes with Pu(IV) and Fe(III) at $\mathrm{pH} 7.4$ and promole significant excretion of circulating Pu(IV) in mice and dogs. ${ }^{1-1}$ Three-dimensional models of all the ligands designed and synthesized in this laboratony demonstrate that Pu(IV) can be fulty coordinated at the approprate bond angles and distances by the octadentate ligands le.g. desfentiHOPO.

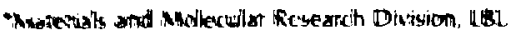

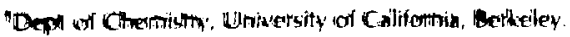

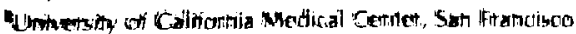

CAM, Fig. 1) and Fe(III), by the hexadentate ligands (e.g., TRENCAM, Fig. 2).

This report consists of three parts: 1) the initial screening of four new ligands for their ability to promote excretion of circulating $\mathrm{Pu}(\mathrm{IV}), 2$ ) the effectiveness of desferriHOPOCAM at reduced dosages, and 3) tests of the ability of 3,4,3LICAM(C) to remove deposited Pu(IV) from mice.

\section{METHODS}

The test of effectiveness for promoting excretion of newly injected (circulating) ${ }^{238} \mathrm{Pu}$ (IV) in mice has been described. ${ }^{1,2}$ Briefly, groups of five mice each receive an intravenous injection (i.v.) of 9250 $\mathrm{Bq} / \mathrm{kB}_{\mathrm{B}}{ }^{23 \mathrm{Pu}}(\mathrm{lV})$ in $0.2 \mathrm{ml}$ of citrale buffer. One hour later, $30 \mu \mathrm{mole} / \mathrm{kg}$ of ligand is injected intraperitoneally (i.p.) in $0.5 \mathrm{ml}$ of saline. The mice are killed $24 \mathrm{hr}$ after the $\mathrm{Pu}$ injection, frozen, and dissected afer partial thawing. The ${ }^{280} \mathrm{pu}$ in skeleton, tissues, and separated excreta is determined by counting the $2{ }^{2 J} \mathrm{U} L \mathrm{~L}$ tays.

The effect of desferritHOPOCAM dosage on its ability to promole excretion of citculating $P u$ was inturestigated by wanying the dosage in the 24 the rest 


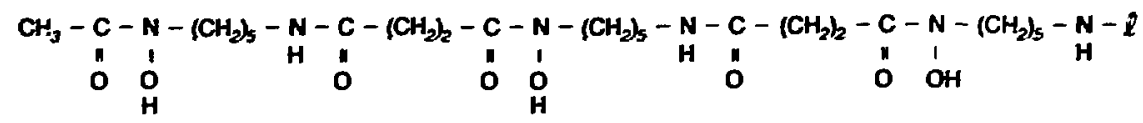

Abbreviation $\quad \ell \quad \times \quad$\begin{tabular}{l}
$\begin{array}{l}\text { Molocular formula } \\
\text { and (weight) }\end{array}$ \\
\hline
\end{tabular}

DFOM

$-\mathrm{CH}_{3}-\mathrm{SO}_{3} \mathrm{H}_{2}$

$\mathrm{C}_{26} \mathrm{H}_{52} \mathrm{~N}_{6} \mathrm{O}_{11} \mathrm{~S}$

(657)

DesferriCAM<smiles>[X]c1ccc(C(C)=O)c(O)c1O</smiles>

H $2_{32} \mathrm{H}_{52} \mathrm{~N}_{6} \mathrm{O}_{11}$ (697)

DesferriCAM(C)

Same as above

$-\mathrm{CO}_{2} \mathrm{Na}$

$\mathrm{C}_{33} \mathrm{H}_{51} \mathrm{~N}_{6} \mathrm{O}_{13} \mathrm{Na}$

DesferriHOPOCAM<smiles>COn1c(C(C)=O)cccc1=O</smiles>

$\mathrm{C}_{31} \mathrm{H}_{5}, \mathrm{~N}_{7} \mathrm{O}_{11} \mathrm{Na}$

ToluyH-Desferrix

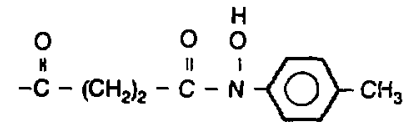

$\mathrm{C}_{36} \mathrm{H}_{59} \mathrm{~N}_{7} \mathrm{O}_{11}$ (765)

Fig. 1. Octadentate derivatives of desierrioxamine. Otficial IUPAC names ine been requested.

of ligand potency just described. ${ }^{5}$ The ability of 3,4,3-LICANI(C) to remove Pu deposited in the target organs was investigated by administering 30 $\mu$ mole $/ \mathrm{kg}$ of ligand $24 \mathrm{hr}$ after the $\mathrm{Pu}$ injection; Pu-injected controls received one saline injection; mice were killed $24 \mathrm{hr}$ later $(\mathbf{4 8} \mathrm{hr}$ after the $\mathrm{Pu}$ injection). The ability of prolonged administration of 3,4,3-LICAMIC) to promole excretion of deposited Pu was investigaled by giving 13 daily injections of $30 \mathrm{mmole} / \mathrm{kg}$ of ligand starting $24 \mathrm{hr}$ after the Pu injection; Pu-injected conltols recerved daily injections of saline; all mice wete killed 14 days afier the Pu injection.

\section{Effectiveness of New Ligands}

Desferrioxamine is a selective hexadentate ligand that forms stable Pu(IV) and Fe(III) complexes, but it is slow acting. Addition of a catechoyl or carboxycalechoyl group to DFO to fo'm desferriCAM or desferriCAM(C) (Fig. i) greatl: e ihanced both the rate of removal of Fe(III) from transfertin (TI) (Ref. 6) and the excretion of Pu from mice "see Table I and Rel. 4h. A fourn hydroxa. mate group was added to the terminal $N$ of DFO through an amide lliskage io prepare rolund. (destetrix (IFig. 1) to keatin whether enhanced kinetic 


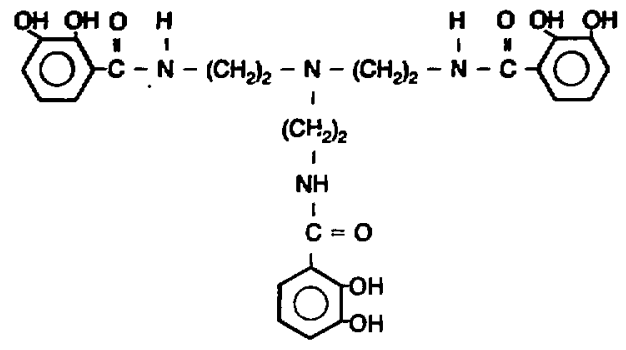

TRENCAM

\begin{tabular}{cc} 
Abbreviation $\quad \begin{array}{c}\text { Molecular formula } \\
\text { and weight }\end{array}$ \\
\hline
\end{tabular}

TRENCAM $\quad \mathrm{C}_{27} \mathrm{H}_{30} \mathrm{~N}_{4} \mathrm{O}_{9}$ (554.6)

Fig. 2. Catechoylamide trimer centered on N. Official IUPAC name has been requested.

(XEL 8511-11425) capability would also be conferred by conversion of DFO to a tetrahydroxamate ligand. The toluylhydroxamic acid DFO derivative was prepared because suitable starting materials were available. It is sparingly soluble, and the standard 30 $\mu \mathrm{mole} / \mathrm{kg}$ dosage was administered to mice as a suspension. As shown in Table 1, toluyl-desferrix promoted net $\mathrm{Pu}$ excretion in $24 \mathrm{hr}$ (treated control) of $15.2 \%$ injected dosage (ID). It was more effective than desferriCAM, which is even less soluble, but it was not as effective as an equimolar amount of unsubstituted DFOM. The partitioning of $\mathrm{Pu}$ excretion (70\% in urine and $30 \%$ in feces) resembled DFO. Acute toxicity will be examined, and $\mathrm{Pu}$ excretion will be retested at the limiting concentration of the ligand.

TRENCAM (Fig. $<$, is an enterobactin analogue consisting of three catechoyl amide groups attached to a central $\mathrm{N}$ atom. It is a sterically flexible molecule with a high affinity for Fe(III) that should also form stable $\mathrm{Pu}$ (IV) chelates. Unsubstituted TRENCAM is sparingly soluble, and the standard dosage of $30 \mu \mathrm{mole} / \mathrm{kg}$ was administered to mice as a suspension. Excess Pu excretion was $24.2 \%$

Table 1. Effect of desferrioxamine (DFO) containing a fourth hydroxamate group on distribution and excretion of ${ }^{238} \mathrm{Pu}(\mathrm{IV})$ in mice.

\begin{tabular}{|c|c|c|c|c|c|c|c|}
\hline & \multicolumn{7}{|c|}{ Percent of injected ${ }^{23 \varepsilon_{i}} u \pm$ S.D. at $24 h r^{a, b}$} \\
\hline & \multicolumn{5}{|c|}{ Tissues } & \multicolumn{2}{|c|}{ Excreta } \\
\hline & Liver & skeleton & Kidneys & $\begin{array}{c}\text { Residual } \\
\text { soft tissue }\end{array}$ & $\begin{array}{c}\text { Body } \\
\text { content }\end{array}$ & Urine & $\begin{array}{l}\text { Feces and } \\
\text { Gl contents }\end{array}$ \\
\hline \multicolumn{8}{|l|}{ Test ligand } \\
\hline Toluyl-desferrix & $39 \pm 7.5$ & $26 \pm 5.2$ & 1.6 & $8.5 \pm 2.3$ & 76 & 17 & 6.9 \\
\hline \multicolumn{8}{|l|}{ Similar ligands } \\
\hline DesferriCAM & $43 \pm 3.7$ & $31 \pm 2.3$ & 3.1 & $8.6 \pm 2.2$ & 86 & 7.5 & 6.5 \\
\hline DesferriCAM(C) & $11 \pm 2.0$ & $13 \pm 1.2$ & 0.9 & $3.0=0.4$ & 27 & 28 & 45 \\
\hline \multicolumn{8}{|l|}{ Baseline ligand } \\
\hline DFOM & $19 \pm 13$ & $20 \pm 11$ & 1.8 & $4.5 \pm 1.4$ & 46 & 40 & 15 \\
\hline \multicolumn{8}{|c|}{ 2MPu-Injocted Cantrof } \\
\hline 24-ht & $49 \pm 8.3$ & $32 \pm 7.9$ & 2.0 & $7.7 \pm 2.0$ & 92 & 4.7 & 4.1 \\
\hline I-hr & $30 \pm 7.4$ & $24 \pm 4.4$ & $2=$ & $37 \pm 7.1$ & 94 & 1.1 & 4.8 \\
\hline
\end{tabular}

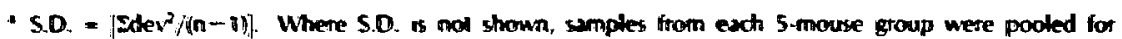

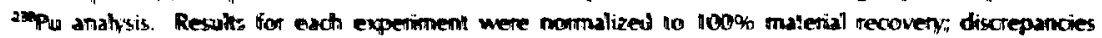
ate idve to tounding.

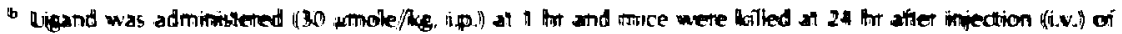

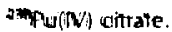

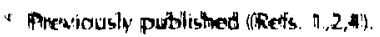


ID (Table 2), about the same as for the benzenecentered tris(catechoylamide)s, MECAM and MECAM(S). ${ }^{2}$ TRENCAM was not as effective for Pu removal as the soluble, more acidic hexadentate ligands-the sulfonated linear tris(catechoylamide), 3,4,3-LICAM(S), or the benzene-centered tris(hydroxypyridinone), HOPO-MECAM. Investigation of acute toxicity and retesting of $\mathrm{Pu}$ excretion at a reduced dosage are planned.

One of the goals of this research is development of an orally effective removal agent for Pu(IV) and (or) Fe(III). The $\mathrm{Ca}$ or $\mathrm{Zn}$ salts of DTPA (Fig. 3), which are used clinically to treat actinide contamination, are not well absorbed from the GI tract and ;.e usually administered by i.v. injection. In general, a large fraction of Pu chelated by ligands with a low net negative charge tends to be eliminated by the liver and GI tract. ${ }^{2,4}$ Such ligands are also likely to be better absorbed from the GI tract and effective when administered by mol.in. To that end, two of the four terminal acetic acid groups of DTPA or EDTA were replaced by bis(isopropyl)hydroxamic acid, which reduced the net negative charge of the ligands and increased their metal-binding capacity, Bis(isopropyl)DTPADX and bis(isopropyl)EDTA-DX (Fig. 3) were admin- istered to mice as the tri-and di-sodium salks, respectively. Because of the known ability of DTPA to bind essential $C a$ and $Z n, P u$ removal by the $C_{a}$ and $\mathrm{Zn}$ complexes of bis(isopropyl)DTPA-DX was also investizated.

Net $\mathrm{Pu}$ excretion promoted by the $\mathrm{Na}, \mathrm{Ca}$, and $\mathrm{Zn}$ forms of bis(isopropyl)DTPA-DX and by bi(isopropyl)EDTA-DX was 66.3, 66.3, 57.2, and $8.8 \%$ ID, respectively (see Table 3 ). The $\mathrm{Na}$ and Ca forms of bis(isopropyl)DTPA-DX promoted slightly more, and the $\mathrm{Zn}$ form slightly less Pu excretion than the baseline ligand, $\mathrm{CaNa}_{3}$-DTPA (net Pu excretion $61.2 \%$ ID). While nearly all of the Pu-DTPA chelate is excreted by the kidneys, 53 to $68 \%$ of the Pu excretion promoted by the dihydroxamic acid derivatives was via the GI tract. All three forms of bis(isopropyl)DTPA-DX will be tested for acute toxicity, and the $\mathrm{Zn}$ complex will be investigated for dosage effectiveness, ability to remove deposited $\mathrm{Pu}$, and oral activity.

\section{Dosage Effectiveness of desferriHOPOCAM}

To date, the most effective ligand prepared for $\mathrm{Pu}$ remuval is the hydroxypyridinone oxide derivative of desferrioxamine, desferriHOPOCAM (Fig. 1).

Table 2. Effect of an $\mathrm{N}$-centered tris(catechoylamide) ligand on distribution and excretion of ${ }^{238} \mathrm{Pu}$ in mice.

\begin{tabular}{|c|c|c|c|c|c|c|c|}
\hline & \multicolumn{7}{|c|}{ Percent of injected ${ }^{238} \mathrm{Pu} \pm$ S.D. at $24 \mathrm{hr}^{\mathrm{d} \cdot \mathrm{b}}$} \\
\hline & \multicolumn{5}{|c|}{ Tissues } & \multicolumn{2}{|c|}{ Excreta } \\
\hline & Liver & Skeleton & Kidneys & $\begin{array}{l}\text { Residual } \\
\text { soft tissue }\end{array}$ & $\begin{array}{c}\text { Body } \\
\text { content }\end{array}$ & Urine & $\begin{array}{l}\text { Feces and } \\
\text { Gl contents }\end{array}$ \\
\hline \multicolumn{8}{|l|}{ rest ligand } \\
\hline TRENACAM & $31 \pm 9.2$ & $27 \pm 12$ & 2.1 & $6.8 \pm 0.6$ & 67 & 12 & 21 \\
\hline \multicolumn{8}{|l|}{ Similar ligands } \\
\hline MECAM & $18 \pm 3.4$ & $33 \pm 3.8$ & 4.6 & $7.9 \simeq 1.3$ & 63 & 13 & 24 \\
\hline 3.4-LICAM(C) & $17 \pm 1.6$ & $17 \pm 2.8$ & 9.0 & $5.2 \pm 1.2$ & 48 & \multicolumn{2}{|r|}{52} \\
\hline 3.4,3-LICAM & $18 \pm 3.4$ & $26 \pm 5.2$ & 4.3 & $5.1 \pm 0.8$ & 53 & 9.4 & 35 \\
\hline
\end{tabular}

- S.D. = $\left.\mid \Sigma \mathrm{dev}^{2} /(n-1)\right]$ Where S.D. is not shown, samples from each 5-mouse group were pcaled for ${ }^{20} \mathrm{Pu}$ analysis. Results for each experiment were normalized to $100 \%$ material recovery; discrepancies are due to rwunding.

b ligand was administered ( 30 mmoleghe, i.p.) at I hr and mice were kifled al $24 \mathrm{hr}$ after iniecthor (i v.) of 2 pu(n) cituale.

- Previoissly published (Red. 2). 


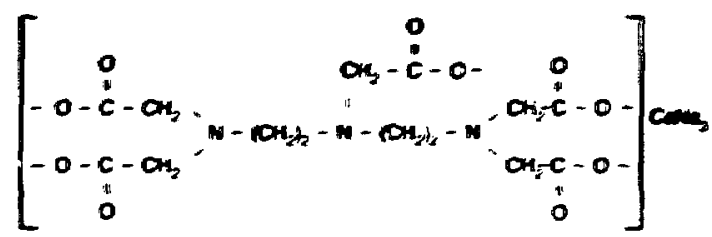

DTPA - Ca Nas

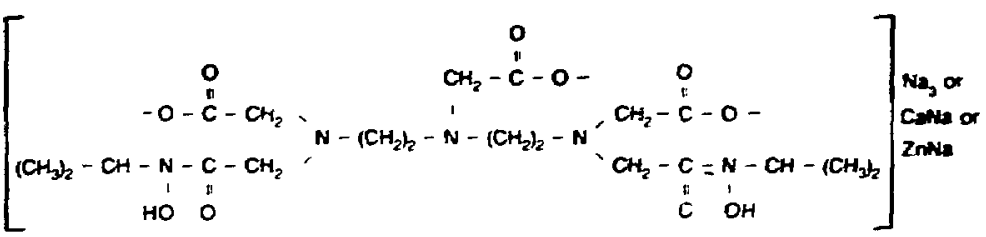

Bis(isopropy)DTPA-DX
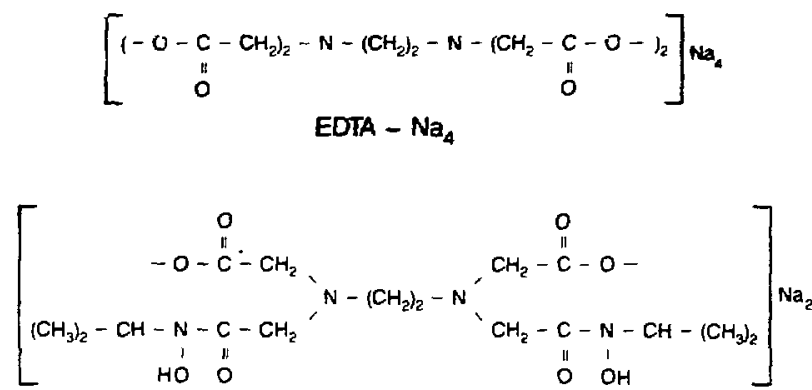

Bis(isopropyl)EDTA-DX-Na

Fig. 3.

(XBL 8511-71427)

\begin{tabular}{|c|c|c|}
\hline Abtreviation & $\begin{array}{l}\text { Molecular formula } \\
\text { and weight }\end{array}$ & IUFAC name \\
\hline DTPA-CaNa & $\begin{array}{c}\mathrm{C}_{i 4} \mathrm{H}_{18} \mathrm{~N}_{3} \mathrm{O}, \mathrm{CaNa}_{3} \\
(497,4)\end{array}$ & $\begin{array}{l}\text { Calcium disodium (diethylene- } \\
\text { trinitrilo)pentaacetate }\end{array}$ \\
\hline Bis/isopropyllDTPA-DX-Nia, & $\begin{array}{c}\mathrm{C}_{20} \mathrm{H}_{24} \mathrm{~N}_{5} \mathrm{O}_{10} \mathrm{Na}_{3} \\
(573.5)\end{array}$ & $\begin{array}{l}\text { Trisodium diethyletrinitrilo- } \\
N, N, N^{\prime \prime} \text {-(riacetic-N,N"- } \\
\text { di(isoproprlacelichydroxamate) }\end{array}$ \\
\hline Bistisopropy/IDTPA-DX-CaNa & $\begin{array}{c}\mathrm{C}_{20} \mathrm{H}_{3 a} \mathrm{~N}_{5} \mathrm{O}_{10} \mathrm{CaNa}_{2} \\
(567.6)\end{array}$ & $\begin{array}{l}\text { Calcium sodium diethylenetrinitrilo. } \\
N, N, N^{\prime} \text {-triacetic- } N, N^{\prime \prime} \text { - } \\
\text { difisopropylacetichydraxamatel }\end{array}$ \\
\hline Bislusopropl/IDTPA-DX-ZnNa & $\begin{array}{c}\mathrm{C}_{20} \mathrm{H}_{34} \mathrm{~N}_{5} \mathrm{O}_{10} \mathrm{ZnNd \textrm {Na }} \\
(592.9)\end{array}$ & $\begin{array}{l}\text { Zinc sodium diethydenelrinitrilo- } \\
N, N, N ' \text {-iriacetic-N,N"- } \\
\text { difisopropylacetichydroxamate) }\end{array}$ \\
\hline EDTA-Aa, & $\begin{array}{c}\mathrm{C}_{12} \mathrm{H}_{2,2} \mathrm{~N}_{2} \mathrm{O}_{2} \mathrm{NO}_{4} \\
(3800.21\end{array}$ & $\begin{array}{l}\text { Tetrasodium (ethylenedinitrilo)- } \\
\text { teiraacetate }\end{array}$ \\
\hline BistisopropiJeDTA-Dx-Na, & $\mathrm{C}_{34} \mathrm{H}_{\mathrm{X}} \mathrm{N}_{2} \mathrm{O}_{\mathrm{e}} \mathrm{Na}$ & $\begin{array}{l}\text { Disodium ethylenedinitrib-N. } N \text {. } \\
\text { diacetic-N,N"difisopropyt. } \\
\text { acefichydroxamste! }\end{array}$ \\
\hline
\end{tabular}




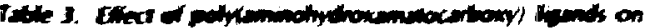

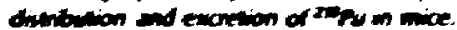

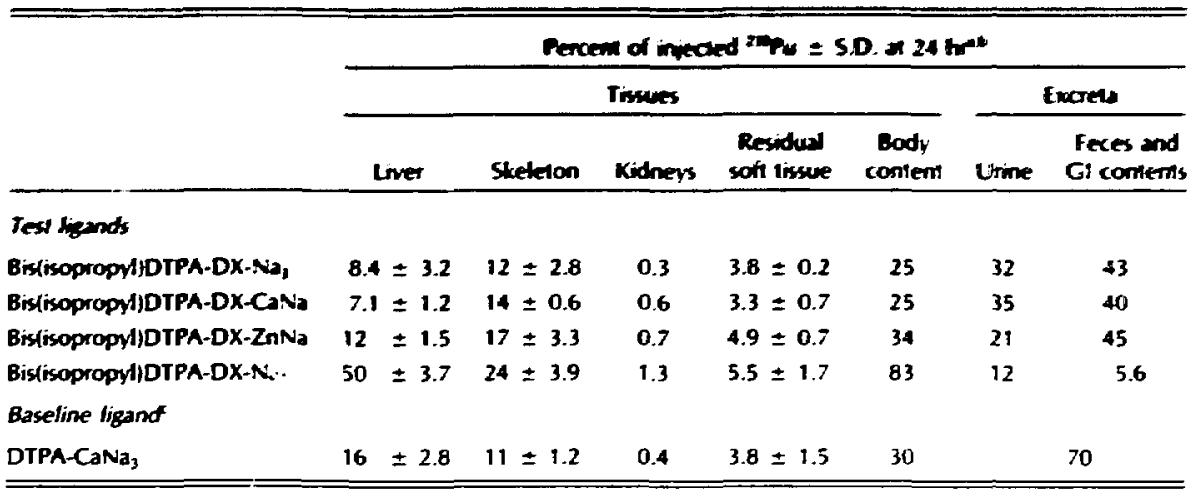

- S.D. $=\left|\Sigma \operatorname{dev}^{2} /(n-1)\right|$. Where S.D. is not shown, sie -ples from each 5-mouse group were pooled for ${ }^{238} \mathrm{Pu}$ analysis. Results for each experiment were normalized to $100 \%$ material recovery; discrepancies are due to rounding.

b Ligand was administered (30 umole/kg, i.p.) at $\mathrm{h} \mathrm{hr}$ and mice were killed at $24 \mathrm{hr}$ after injection (i.v.) of ${ }^{238} \mathrm{Pu}(\mathrm{IV})$ citrate.

- Previousily published (Rer. 1).

The net $\mathrm{Pu}$ excretion promoted in $24 \mathrm{hr}$ by the standard dosage of $30 \mu \mathrm{mole} / \mathrm{kg}$ was $77.1 \%$ ID; acute toxicity in mice appears to be low. ${ }^{4}$ The dosage effectiveness curve for this ligand is shown in Fig. 4. The discontinuous line provides comparison with the more carefully studied tetracatechoylate, 3,4,3-LICAM(C). ${ }^{5}$ Significant $\mathrm{Pu}$ removal was observed at dosages as low as 0.3

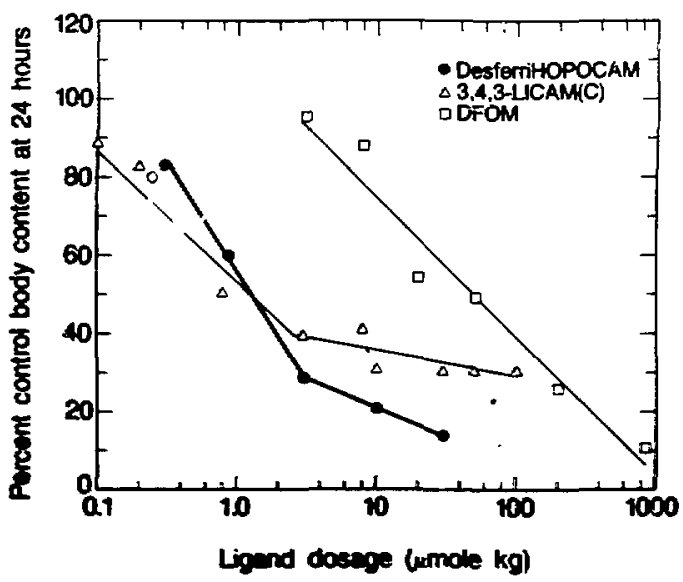

F. 4 Effect of destemitrapocas: dosage on retertion of smpu in mine. $\mu \mathrm{mole} / \mathrm{kg}$, and additional $\mathrm{Pu}$ removal probably can be achieved with dosages greater than 30 $\mu \mathrm{mola} / \mathrm{kg}$. The slope $(\mathrm{m})$ of a straight line fitted by least squares $\mid \mathrm{m}=-35.2 \% \quad \mathrm{ID}$ in body $/ \log _{10}(\mu \mathrm{mole} / \mathrm{kg}$ of ligand) $)$ is the same as that for the parent compound, DFOM $\mid \mathrm{m}=-35.9 \%$ ID in body $/ \log _{10}(\mu \mathrm{mole} / \mathrm{kg}$ of ligand)]. The curve for desferriHOPOCAM is displaced towards lower dosage, and for removal of equal fractions of circulating $\mathrm{Pu}$ from the body, desferriHOPOCAM is about 30 times more effective than DFOM. The effect of delayed administration of desferriHOPOCAM is under investigation, and study of its oral effectiveness and $\mathrm{Pu}$ excretion under protracted administration are planned. The properties of this ligand indicate that it is a good candidate for investigation in a larger animal.

\section{Removal of Deposited Pu by 3,4,3-L/CAM(C)}

Within $24 \mathrm{hr}$ after i.v. injecuion of Pu(IV) citrate in adult mice about $90 \%$ of the Pu remaining in the body is deposited in the liver and skeleton.' Elimination of the liver $\mathrm{Pu}$ begins immediately, mainly by way of bilian secretion into the GI tract; a small variable fraction is recirculated. Pu recirculated from soft tissues and liver augments skeletal Pu by about $20 \%$ during the first few days atter injection. If a ligand that reacts with metals only in the body fluids is adminislered $24 \mathrm{hr}$ after the $\mathbf{P u}$, it can be 
expected at a minimum to react with undeposited Pu remaining in the body fiuds and Pu released and recirculated from sofi lissues and liver but not yet redeposited. If the residence lime of the ligand is sufficiently long, and it is effective at low concentration, most of the recirculated Pu can be intercepted and the early skeletal increase prevented.

The tetrameric carboxy(catechoylamide) ligand, 3,4,3-LICAM(C), was shown to be effective for removal of circulating $P_{u}$ at low dosage and to be of low toxicily. ${ }^{2-4}$ The potential of 3,4,3-LICAM(C) 2's a clinically useful $P_{u}$ removal agent was explored further by investigating removal of deposited Pu by one administration or 13 doly administrations of 30 mole/ks of 3,4,3-LKCAMIC).

Single Administration 24 th affer Po. Two Eoups of five mice each were injected iv. with Pu; $24 \mathrm{hr}$ later excreta were collected and the ligand was injected: the mice were killed at $\mathbf{4 8} \mathrm{hr}$ and excreta were again collected. The pooked results are shown in Table 4. One group of five Pu-injected control mice was killed at 2 days postinjection; the distribution of $\mathrm{Pu}$ in their tissues is given in Table 4. Excreta were also collected at 1 and 2 days after

Table 4. Effect of 3,4.3-LICAMICI on ${ }^{238}$ Pu(IV) deposited in mice: single or protracted administrafion.

\begin{tabular}{|c|c|c|c|c|c|}
\hline \multirow[b]{4}{*}{ Liver } & \multicolumn{5}{|c|}{ Percent injected ${ }^{238} \mathrm{Pu}=5 . \mathrm{D}^{2,6}$} \\
\hline & \multicolumn{2}{|c|}{ 3,4,3-LICAM $(C)^{6}$} & \multicolumn{3}{|c|}{${ }^{238} \mathrm{Pu}$-injected controls } \\
\hline & - 2 day & 14-day & 24-hour & $2-d a y^{b x}$ & $14-d a y^{b}$ \\
\hline & $38.1 \pm 3.6$ & $6.3 \pm 2.4$ & \pm 8.3 & $42.7 \pm 5.2$ & $9.7 \pm 4.6$ \\
\hline Skeleton & $29.0 \pm 4.0$ & $20.4 \pm 3.9$ & $32 \pm 7.9$ & $34.5 \pm 5.1$ & $29.7 \pm 6.2$ \\
\hline Kidneys & 1.2 & 1.3 & 2.0 & 0.51 & 0.6 \\
\hline $\begin{array}{l}\text { Residual } \\
\text { soft tissue }\end{array}$ & $6.6+1.0$ & $5.6 \pm 0.6$ & $7.7 \pm 2$ & $6.2 \pm 0.7$ & $4.4 \pm 0.9$ \\
\hline Retainied in body & $74.7 \pm 4.3$ & $33.7 \pm 3.6$ & 92 & $84.0 \pm 3.5$ & $44.5 \pm 3.5$ \\
\hline Urine, day 1 & 4.0 & 5.6 & 4.7 & 5.5 & 6.0 \\
\hline 2 & 3.4 & 3.6 & & 1.0 & 0.6 \\
\hline 3 & & 1.8 & & & 0.4 \\
\hline $4.5,6$ & & 2.9 & & & 0.6 \\
\hline 7.8 & & 1.5 & & & 0.4 \\
\hline 9,10 & & 1.1 & & & 0.1 \\
\hline $11,12,13$ & & 3.0 & & & 0.3 \\
\hline 14 & & 0.7 & & & 0 \\
\hline Total & 7.4 & 18.2 & 4.7 & 6.5 & 8.4 \\
\hline Feces, Jay 1 & 2.8 & 2.9 & $4.7^{e}$ & 2.4 & 2.4 \\
\hline 2 & $15.1^{\circ}$ & 11.6 & & $7.1^{\mathrm{e}}$ & 3.4 \\
\hline 3 & & 5.8 & & & 6.5 \\
\hline $4,5,6$ & & $14 . \therefore$ & & & 15.1 \\
\hline 7.8 & & 5.5 & & & 7.8 \\
\hline 9.10 & & 4.3 & & & 5.3 \\
\hline $11,12.13$ & & 2.8 & & & 5.3 \\
\hline 14 & & $0.6^{\circ}$ & & & $1.2^{\mathrm{e}}$ \\
\hline lotal & 17.9 & 47.9 & 4.1 & 9.4 & 47.0 \\
\hline
\end{tabular}

- See vound ie a, Table 1 .

b Ligand was administered ( 30 umole/kg. i.p.) once (2-day group) or daily for 13 days (14da groupl starting at $24 \mathrm{hr}$, and mice were killed at $48 \mathrm{hr}(2$-day group) or at 14 days

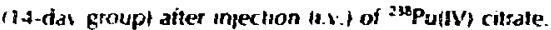

- Control excretic. data at 2 dass p.t. are averages for five 5 -mouse groups studied from 2

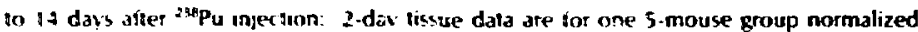
$108 \%$ to resained.

- Previously published tRets 1.21.

- Includes Gl contenis. 
Pu injection from four proups of longer-ferm Pu. minecied conturl mice; the control urine and fecal dor in Table 4 are grand means of all five sets of control excretion dita. Net Pu excretion by the mice given 3,4.3-LKCAMHCl at 24 hr was 9.4\% ID. The mean body contern of the ligand-treated group, $74.7 \pm 4.3 \%$ ID, was significantly less ip $<$ 0.01, Ref. B) than that of the Pu-injected control growp. $84.0 \pm 3.5 \%$ ID. The Pu contents of both liver and skeleton were reduced by the ligand, but the differences from the appropriate control values were not statisticaliy significant. Both urinary and fecal excretion oi $\mathrm{Pu}$ were increased by the single ligand injection, to two to three times the quantities excreted by the controls.

Protracted Administration of 3,4,3-LICAM(C). Mice tolerated 13 injections of 3,4,3-LICAM(C) well; body weight, appetite, behavior, and irritability were not different from saline-injected controls, and no grossly visible lesions were found at autopsy. The $\mathrm{Pu}$ content of the skeleton and the whole body were significantly different from the controls (see Table 4). Between days 2 and 14, daily administration of 3,4,3-LICAM(C) promoted an excess $\mathrm{Pu}$ excretion of $10.6 \%$ ID. Nearly all the excess $\mathrm{Pu}$ excretion was renal. The urinary and fecal excretion rates of the control and ligandtreated mice are shown in Fig. 5. If fecal $\mathrm{Pu}$ excretion is derived mainly from clearance of the liver via bile, then it appears that the action of 3,4,3-LICAM(C) is to slightly accelerate Pu clearance from the liver, thereafter diverting chelated $\mathrm{Pu}$ to renal excretion and precluding additional $\mathrm{Pu}$ accumulation in the liver. Under the influence of daily 3,4,3-LICAM(C) urinary excretion of $\mathrm{Pu}$ was six to seven times greater than for the control mice.

Even after 13 ligand injections, urinary $\mathrm{Pu}$ excretion was still 0.3 to $0.4 \%$ ID/aay suggesting that the more rapidly clearing (or renewing) compartments in soft tissues (other than liver) and skeleton had not been exhausted, and additional reduction might be achieved by further prolonging the ligand treatment. It has often been suggested that the main action of chelating agents that do not enter cells is to prevent redeposition by diverting recirculaled $\mathrm{Pu}$ to excretion. The parallel urinary excretion rate curves for the ligand-treated and contral mice lend support to that hypothesis. For metals like Pu that are not efficiently excreted, the physiological rates at which they are transferred out of the target organs are masked by continual redeposition of recirculated metal. If an effective Pu-binding agent like 3,4,3-1ICAM(C) were administered to Pu-injected animals continuously for an

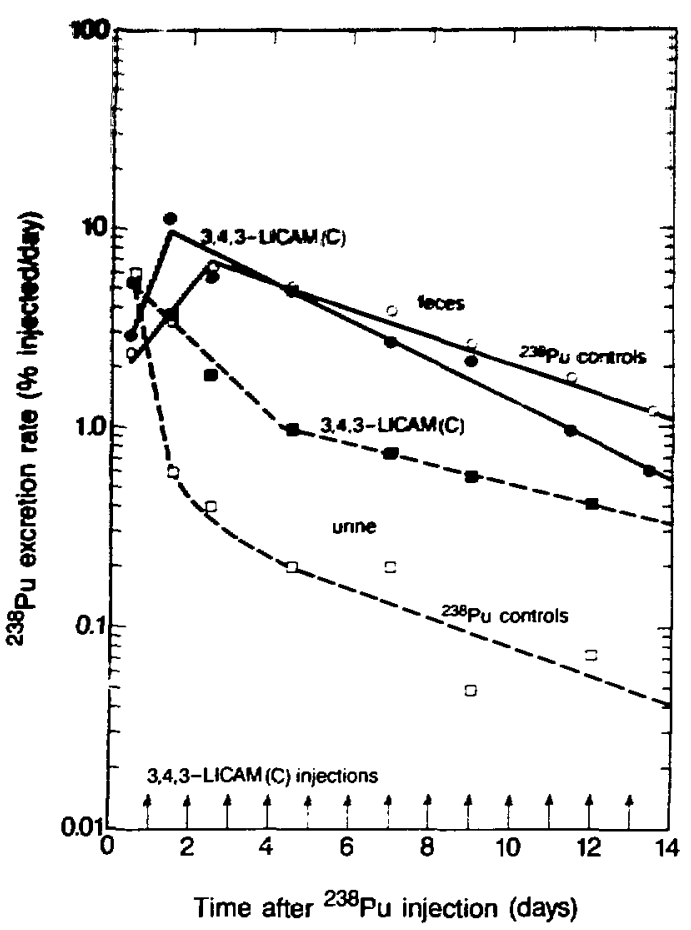

Fig. 5. Effect of protracted administration of $3,4,3-1 / C A M(C)$ on rates of ${ }^{2 B} \mathrm{Pu}_{\mathrm{u}}$ excretion in urine and feces of mice.

(XBL 8511-11424)

extended period, diverting a large fraction of recirculated $\mathrm{Pu}$ to excretion, it should be possible to measure the true turnover rates of $\mathrm{Pu}$ in the major target organs.

\section{REFERENCES}

1. Durtin, P.W., Jones, E.S., Raymond, K.N., and Weitl, F.L. Specific sequestering agents for the actinides: 4 . Removal of ${ }^{238} \mathrm{Pu}(\mathrm{IV})$ from mice by sulfonated tetrameric catechoyl amides. Radiat. Res. 81, 170-187 (1980).

2. Durbin, P.W., Jeung, N., Jones, E.S., Weitl, F.L., and Raymond, K.N. Specific sequestering agents for the actinides: 10. Enhancement of ${ }^{238} \mathrm{Pu}$ elimination from mice by poly(catechoylamide) ligands. Radiat Res. 99. 85-105 (1984).

3. Lloyd, R.D., Bruenger, F.W., Mays, C.W., Atherton, D.R., lones, C.W., Taylor, G.N., Slevens, W., Duibin, P.W., Jeung, N., Jones, E.S., Kappel, M.J., Raymond, K.N., and Weitl, F.L. Removal of Pu and Am from beagles and 
mice by 3,4,3-LCAM(C) or 3,4,3-1KCAM(S). Radiat. Res. 99, 106-128 41904 ).

4. Durtin, P.W., feung, N., Rodgers, S.J., White, D.L. and Raymond, K.N. New sequestering agents for the actinides: acute toxicity and effectiveness for removal of Pu from mice of derivatives of desfertioxamine and of poly(hydroxypyridinone) ligands and their ferric and zinc complexes. Biology Medicine Division Annual Report 1983-1984, Lawrence Berkeley Laboralory report LBL-18393, pp. 59-64 (1985).

5. Durbin, P.W., leung, N., Jones, E.S., Raymond, K.N., and Weill, F.L. Plutonium removal from mice by poly(catechoylamide) ligands, Ca-DTPA, desferrioxamine B, and Tiron: effect of ligand dosage. Biology and Medicine Divi- sion Annual Report 1982-1981. Lawrence Berkeley Laboratory repon LBL-16840, pp. 43-47 (1984).

6. Rodgers, S.I. and Raymond, K.N. Fetric ion sequestering agents. 11. Synthesis and kinetics of iron removal from iransferrin of catechoyl derivatives of desferrioxamine B. $J$. Med. Chem. 26, 439-442 (1983).

7. Durbin, P.W. and leung, N. Kinetics of plutonium deposition in the mouse. Division of Biology and Medicine Annual Report 1982-1983, Lawrence Berkeley Laboratory report LBL-16840, pp. 56-59 (1984).

8. Fisher, R.A. Statistical Methods for Research Workers. Hafner Publishing Co., New York (1954). 


\section{SECTION 4. RADIATION BIOPHYSICS}

\section{MNTLODCTION}

The Radiation Biophysics Group has an interdisciplinary approach to the understanding of the deleterious and beneficial effects of a broad array of ionizing radiations. The Bevalac accelerator is a unique source of accelerated heavy particles, from p:otons to uranium ions, and our group is the central core of a worldwide effort. In this effort, the Department of Energy supports the accelerator and some fundamental biophysical studies. Our cell, tissue, and tumor radiobiology programs al. controlled cancer therapy studies with heavy ions are sponsored by the National Cancer Institute. Since fast heavy ions are part of interplanetary space radiation, our space radiobiology program is important for achieving the goals of NASA.

Most of the biological effects of ionizing radiations are ultimately due to macromolecular changes in genetic material and consequent alterations in inherited and acquired characteristics of cells. The studies reported in this section are organized into five sections. These are: Physics of Interactions between Fast Charged Particles and Matter, Molecular Studies, Cellular and Tumor Radiobiology, Tissue Effects of Heavy Charged Particle Beams. and Heavy-lon Therapy and Related Physics and Biology.

Studies of physical interactions between fast charged particles and matter are essential for the measurement and understanding of particle fields. Walter Schimmerling and associates have made progress in quantitating the complex particle fragmentation events ad multiple elastic collisions that determine the pathways of particles in biological material, and Jorge Llacer has provided new irstrumental methodology for these purposes.

The heavy particles allow the delivery of ionizing radiation deep in the body with localization more precise than is possible with other radiations. The use of protons and of helium ions (see Castro et al.) has led to important advances in the therapy of local cancer, for example, of certain tumors of the eye and of otherwis incurable tumors near the spinal cord. The beam localization properties have also allowed rapid advances in the treatment of arteriovenous malformations in the brain. For these reasons we have initiated a new study to find the particle beams that are patticulatly suited to the delivery of bigh localized doses with maximum sparing of surrounding normal lissue. These presently appear to be carbon ions.

Other investigators are involved with studies at the molecular level. The manner in which ionizing radiations produce alterations in DNA is mediated by diffusion-controlled free radical interactions. Aloke Chatterjee, John Magee, and their coworkers are developing an important new theoretical approach to this problem. They can model the kinetics of elementary interactions that sccur using three-dimensional representations of DNA molecules immersed in water. With their approach it is possible to follow the fate of water radicals and the detailed free radical chemistry of DNA. Viral DNA is being used in vitro to study the influence of the chemical milieu on the yield of DNA lesions.

In studies of cellular and tumor radiobiology, it is shown that heavy ions are more efficient than $x$ rays in producing cell transformation as well as cell lethality. At very high linear energy transfers, the lethal effects predominate. The maximum effectiveness for cell transformation occurs at a linear energy transfer of well above $100 \mathrm{keV} / \mu \mathrm{m}$. Accelerated iron nuclei transform with peak efficiency, and these particles are also prominent in space radiations. Tracy Yang and Mantong Mei, a scientist visiting from mainland China, have devised experiments that allow one to test the effects of specific chemical and physical environments on these cell changes, and also permit one to study the effects of heavy ions on gene expression.

Eleanor Blakely et al. have a systematic program for investigating the role of antioxidant enzymes in modifying the expression of radiation damage and repair during the course of the cell division cycle. This study ir being conducted in collaboration with the Armed Forces Radiobiology Research Institute. Blakely found that the activity of these enzymes rapidly rises in the period immediately following cell division in unirradiated cells and that this rise coincides with an increased resistance to $x$-ray radiation injury. In cerlain genetically abnormal cell lines, the antioxidant enzyme levels are significantly reduced: these cell lines are correspondingly much more sensiture to radiatous injur than normal cell Dunes 
tring sivecil chromosome condensition vechniques that require cell fusion to permit visucheration of inierphase chromatin, Edwin Coodwia has experimentally demonstrated that when sindo fas heavy ions penetrate the $G_{y}$-phase cell nucieus, the particles can produce muiliple chromatin breaks. $X$ rays, electrons, or ultraviolet radiation can only rarely produce multiple chromatin lesions, and only when large doses are administered. The interactions between the multiple chromatin breaks during the course of repair (and misrepair) processes, appear to explain the high carcinogenic potency and lethality of heavy ions.

The group is collaborating with scientists from the National 1 istitute of Radiological Science at Chiba, Japan. Hiroshi Ohara and our group have investigated chromcsome aberrations produced by heavy ions in human blood cells and cell killing effects in cultured human squamous cell carcinoma cells from lung.

Synchronized mammalian cells are used in a systematic study of the role of protein synthesis in the repair of DNA injury. The use of temperaturesensitive mutants helped to establish the relationship of protein synthesis during the DNA replicating $S$ phase to delays and disturbances in cell progression. A current phase of this work is reported by Polly Chang, who has shown that inhibition of protein synthesis in exponentially growing cells can disrupt the development of thermotolerance.

Michael Nusse from Johann Wolfgang Goethe University in West Germany collaborated with Stanley Curtis in a study of the induction of micronuclei in mammalinn celis following exposure to heavy charged-particle beams. The micronuclei originate from chromatin fragments that rejoined in a stable configuration; their presence is a measure of the degree of radiation injury and in some cell strains of the degree of sensitivity to radiation.

Stan Curtis also has a program of studying tumor cell killing and kinetics after exposure to heavy particle beams. The data indicate that the rhabdomyosarcoma tumor cells repair a considerab'e amount of potentially lethal damage following both low- and high-LET radiaicon, but that this repair cannot be detected in the absence of repair inhibitors because it is so rapid. Repopulation studies after $x$-ray doses show that tumor cells accumulate in $\mathrm{G}_{1}$-phase.

Tr. $\approx$ physical properties of red blood cell membranes change in a very sensitive manner relating to the chemical and macromolecular composition of blood serven. Sach nelationships ane under continued investigations by thowand Mid and associkes.

The study of tissure effects of heavy chaned. particle beams continues in our group. The dose that can be detivered in the radiation therapy of cancer is limited by the possible coccurrence of irreparable injury to normal tissues in the path of the therapy beam. Edward Apen and group are focusing their interest on two diffetent systems: the kidney and the spinal cord. The development of late effects is of particular interest. Npen is using a system developed in collaboration with the Gray Laboratory in England for assessing kidney function in mice. He has quantitated the dose dependence of late injury occurring after fractionated doses of helium ions. With Adrian Rodriguez another set of studies is focusing on spinal cord injury.

John Ainsworth is reporting the consequences of acute heavy-ion injury to stem cells in bone marrow. Of particular interest is the finding that repair of heavy-ion injury appears to leave some permanent damage in the hemopoietic system, as evidenced by the lack of full recovery of the red corpuscle series. Ainsworth is also reporting progress on short-and long-term lethal effects caused by exposures to charged heavy-ion particles, a study undertaken in order to clarify the hazards of space radiations.

Heavy-ion therapy and related physics and biology continues as a major effort of our group. Joseph Castro is leading a group dedicated to therapeutic investigations with heavy-ion beams. In this effort he is further supported by the Radiation Oncology Department of the UCSF Medical School and by the Northern California Oncology Group. Gratifying results have been achieved in treatment of ocular tumors, particularly melanoma of the retinal choroid, and of tumors adjacent to the spinal cord. Other cancers of interest are pancreatic carcinoma, some classes of lung carcinoma, and prostate carcinoma.

John Lyman describes a quantitative system that can be used to estimate complications that might arise as a consequence of localized or generalized therapy.

Aloke Chatterjee et al. have for some years worked on the use of radioactive beams for accurately localizing the Bragg peak of beams used in therapy. This approach is uniquely possible when heavy ions are used. The first radioactive beam studies have been carried out in cancer therapy patisnts Juring the current year. This method promises to significantly improve our 
abitives to accurately localiz: therapy beams and thus to protect sensitive adjicent structures in patients.

The cell biology group has been collaborating with the therapists in order to assess the sensitivity of human skin for late radialion injury and in quantilating the radiobiological responses to beams in uveal melanoma. The group is also engaged in an ambitious program to improve therapy-beam delivery by wobbling and scanning the high-energy particte beams. Curtis is reporting the status of radiobictogical studies with such beans.

In summany, the use of heay particte heams is instrumental in leaning basic molecular and cethudar biology of the cell response and cell transfiormation processes. Application of these beams to cancer therapy has already been extremely useful in special classes of patients, and further heavy-ion studies appear to be impontant to the progress of biomedical science.

\title{
Physics of Interactions between Fast Charged Particles and Matter
}

\author{
HIGH ENERGY HEAVY ION BEAMS USED IN BIOLOGY AND MEDICINE: MEASUREMENT OF \\ PARTICLE DISTRIBUTIONS IN THE FRAGMENTATION TAIL OF A 670 A MEV NEON BEAM \\ STOPPING IN WATER
}

Mervyn Wong, Walter Schimmerling, Marwin Rapkin, and Jerry Howard

When a beam of high-energy heavy ions passes through matter, the beam fragmentation products penetrate more deeply than the original ions. As a consequence, in heavy-ion radiotherapy, vital organs located behind and in close proximity to the tumor volume to be treated receive a significant fraction of the absorbed dose. Therefore, it is important to measure the beam quality in the distal portion of the Bragg curve, where only fragments contribute. We have performed a measurement of this contribution at the Bevalac biomedical facility with a $670 \mathrm{~A} \mathrm{MeV}$ neon beam.'

Fragments emerging from a $33-\mathrm{cm}$ water absorber, which is sufficiently thick to stop the beam, were detected with a spectrometer consisting of a coaxial array of solid-state detectors. A schematic of the experimental setup is shown in Fig. 1. The beam enters from the righi of the diagram. There are two sets of ion-implanted silicon detectors. Two fully depleted silicon detectors, $T_{1}$ and $T_{2}(\sim 200 \mu \mathrm{m}$ thick and $1.9 \mathrm{~cm}$ in diameter $)$ are mounted $-1 \mathrm{~m}$ apart. These two detectors provide the time-of-flight (and hence velocity) measurement of the fragments. The energy deposition of particles traversing the spectrometer is measured in $D_{1}, D_{2}$. and $D_{1}$, each being $-3 \mathrm{~mm}$ shick. Particle identification is achieved through correlation of velocity and energy deposition measurements. A delailed description of the apparalus is given in Ref. 2.
The charge of each particle for the case where $\mathrm{dE} / \mathrm{dx}$ does not vary significantly inside the detector

DUAL T.O.F. CONFIGURATION

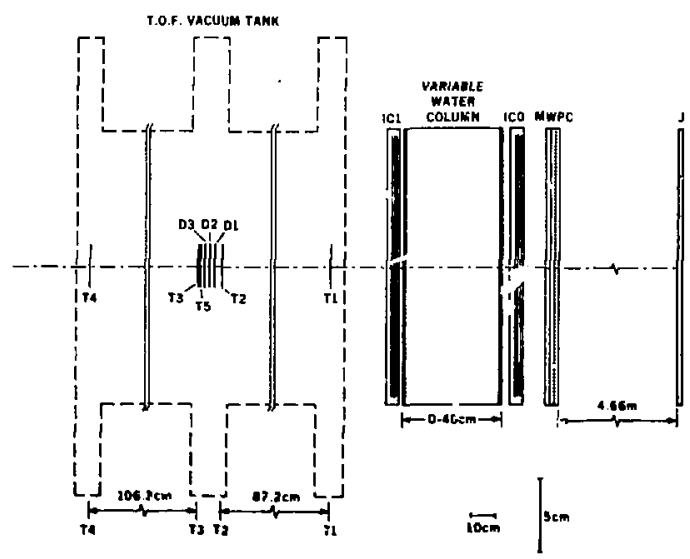

Fig. 1. Schematic layout of the experimental apparatus. There are three sets of defectors: 1$)$ ion chambers $I C_{1}, I C_{0}$ are used to measure the Bragg cunv: at aigh b.am intensity: 2) $T_{v}, C_{p}$ and

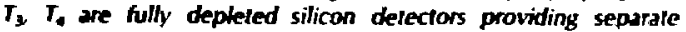
sime-of-fight (and hance velocity) measuremen's of the fragmenis; 3) $D_{11} D_{2}$ and $D_{3}$ are silicon detectors used to measure the enerby deposition of partictes traversing the spectrometer. Purticte identificstion is achieted through correlation of velociny and energy deposition mexurements. (XCh ajo.prow) 
can be cakulated using the expression

$$
z^{2}=\left(\frac{\partial E}{\Delta x}\right)_{d k x},\left(\frac{d E}{d x}\right)_{p}
$$

where $I E$ is the energy deposition in the delector and $(\mathrm{dE} / \mathbf{d x})_{0}$, the proton stopping power calculated al the measured velocity $v$. The charge spectrum obtained in this way. using the energy deposition measured in $D_{1}$, is shown in Fig. $2 a$. In the present experiment amplifier gains and discriminalor thresholds were set such that emphasis was placed on detection of the high-LET fragments. A wider range of sensitivity was obtained with detector $D_{2}$, as shown in Fig. 2b, where partial detection of helium is observed.

Equation (1) is an approximation that becomes less accurate for particles stopping in the detector, such as $O$ and $F$ (and, possibly, some residual primary beam particles). The increased asymmetry in the $Z^{2}$-distribution of these particles may be attributed to this cause. Further work is in progress to refine the analysis for the stopping particles.

The individual velocity spectrum for each fragment species can be projected out by applying cuts to the charge spectra. As an example, Fig. 3 shows the projected oxygen velocity spectrum. Given the $\mathrm{Z}^{2}$ and the measured velocity, the LET for each particle can be calculated. The water-equivalent LET spectrum for oxygen obtained in this way is shown in Fig. 4. Plots, such as are shown in Figs. 3 and 4, can be displayed on-line during data acquisition.

The dose per incident particle due to fragments emitted into the $\sim 0.8^{\circ}$ angle subtended by the spectrometei, can be calculated using:

$$
\operatorname{Dose}(Z)=\frac{1}{A} \frac{1}{n_{\text {inc }}} \int \frac{\operatorname{dn}(Z)}{d L} L d L
$$

where:

$$
\begin{aligned}
& n_{\text {inc }}=\text { number of incident beam ions, } \\
& n(Z)=\text { number of particles of charge } Z \text {, } \\
& \text { corrected for computer dead time losses, } \\
& \mathrm{dn}(\mathrm{Z}) / \mathrm{dL}=\text { differential LET spectrum } \\
& \text { (usir:3 } L \equiv L E T \text { ), and } \\
& \text { A }=\text { area of geometry-defining } D_{1} \\
& \text { detector }\left(2.84 \mathrm{~cm}^{2}\right. \text {; }
\end{aligned}
$$

Table 1 summarizes the results ior the contribution of each iragnent to the total dose. The uncertainties result irom an estimate of the os:'? between the tails of the $Z^{2}$ distribution fot .te neighboring changes.
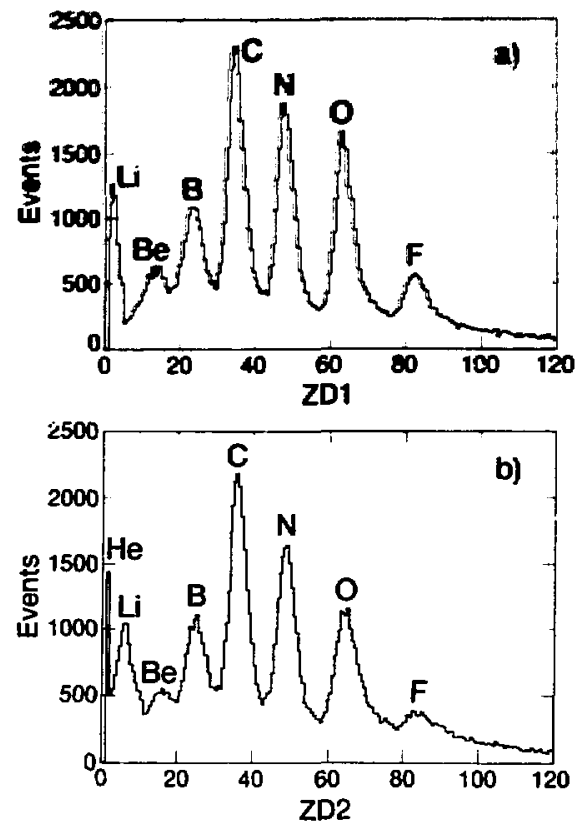

Fig. 2. Measured fragment charge-squared spectra for a 670 MeV/A neon beam incident on $33 \mathrm{~cm}$ of water. Shown are the spectra obtained for the two detectors $D_{1}$ and $D_{2}$, respectively

(XBL 8510-8536)

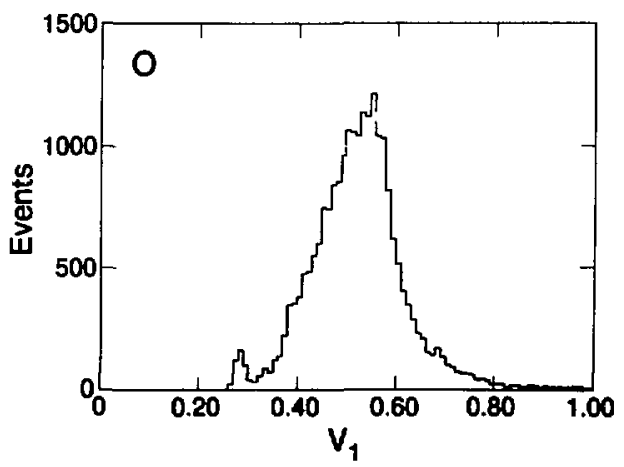

Fis. 3. Meusured velocily spisctrum of oxygen fragments. Velocily is expressed as a fraction of speed of light.

(X81 8510-8537)

The contribution to the io'al dose from the low-LET particles helium and hydiogen is expected to be small. If we assume $\sim 6 \%$ conibinat contribution from these, as suggested by transpont calculations. ${ }^{3}$ the percentage contribution to the total 


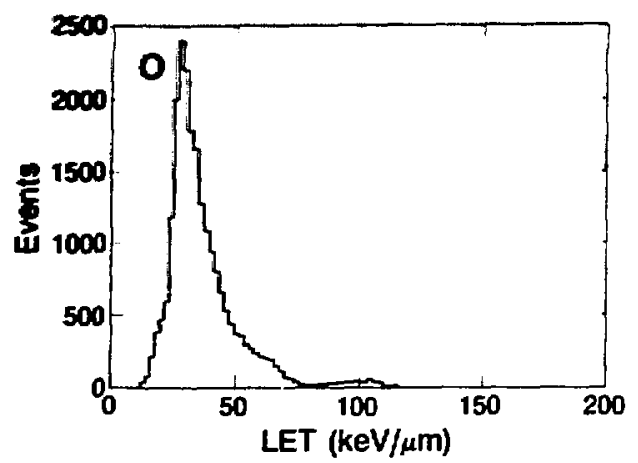

Fit. 4. The water-equivalent LET spectrum for oxygen derived from the charge and velocity measurements as discussed in the text. Such spectra can be displayed on-fine during data acquisition.

(XBL B510-8538)

Table 1. Neon 670-MeV/nucleon dose at Bragg tail $(33.07 \mathrm{~cm} \mathrm{H}, \mathrm{O})$.

\begin{tabular}{ccccc}
\hline Element & $Z$ & Dose (Gy/incident inn) & $\begin{array}{c}\text { \% of tatal } \\
\text { dase at tail }\end{array}$ & $\begin{array}{c}\text { \% of } \\
\text { peak dose }\end{array}$ \\
\hline $\mathrm{F}$ & 9 & $(1.55 \pm 0.30) \times 10^{-10}$ & $21.6 \pm 4.3 \%$ & $\sim 6.3 \%$ \\
$\mathrm{O}$ & 8 & $(2.10 \pm 0.21) \times 10^{-10}$ & $29.3 \pm 2.9$ & 8.5 \\
$\mathrm{~N}$ & 7 & $(1.29 \pm 0.13) \times 10^{-10}$ & $18.0 \pm 1.8$ & 5.2 \\
$\mathrm{C}$ & 6 & $(1.07 \pm 0.11) \times 10^{-10}$ & $14.9 \pm 1.5$ & 4.3 \\
$\mathrm{~B}$ & 5 & $(0.43 \pm 0.09) \times 10^{-10}$ & $6.1 \pm 1.2$ & 1.8 \\
$\mathrm{Be}$ & 4 & $(0.20 \pm 0.04) \times 10^{-10}$ & $2.8 \pm 0.6$ & 0.8 \\
$\mathrm{Li}$ & 3 & $(0.10 \pm 0.02) \times 10^{-10}$ & $1.4 \pm 0.3$ & 0.4 \\
$\mathrm{He}$ & 2 & & 6.0 (assumed) & 1.7 \\
$\mathrm{H}$ & 1 & & 6.0 (assumed) & $-29.0 \%$ \\
\hline
\end{tabular}

dose from each fragment is given by the second column of Table 1 . The third column expresses these results as a fraction of the dose deposited at the Brags peak. It is seen that the nuclear fragments fluorine, oxygen, nitrogen, and carbon comptetely dominate the high-LET contribution. The total dose contribution from all fragments amounts to $\sim 29 \%$ of the peak dose, which support earlier predictions made on the basis of transport theory.

\section{REFERENCES}

1. Wong, M., Schimmerling, W., Rapkin, M. Howard, 1. High-energy heavy ion beams used in biology and medicine: Measurement of particles distributions in the fragmentation taii. Radiation Research Society, 33rd Annual Scientific Meeting, Los Angeles, May 5-9, 1985 (Abstract HF-3).

2. Schimmerling, W., Rapkin, M., Wong, M., Howard, J., Kaplan, S.N., Spieler, H.G., Jarrett, B.V. and Walton, I.T. Physical Characterization of Heavy-lon Beams. Biology and Medicine Division Annual Report, Lawrence Berkeley Laboratory report LBL-16840, p. 77 (1984).

3. Wong, M., Schimmerling, W., Civello, J., Howard, J., Wilson, J.W., Townsend, L.W. and Bidasaria, H.B. Transport of High-Energy Heavy Ions through Extended Matter. Biology and Medicine Division Annual Report, Lawrence Berkeley Laboratory report LBL16840, p. $85,(1984)$.

\section{MULTIPLE COULOMB SCATTERING OF HEAVY IONS}

Mervyn Wong, Mark Phillips, Walter Schimmerling, Don L. Murphy, and Cornelius A. Tobias

Multiple scattering processes affect dosimetry, performance, effectiveness, and resolution in the use of he: $y y$-ion beams in radiotherapy (dose localization, critical structures, beam relivery systems), radiohiology (loss of particles to sample), radiological imaging (limits of resolution), and radioactive beams (optimization of target thickness). On the other hand. nardly any experimental dala on heawy-ion multiple scattering exist We have therefore initialed at this labotatory a s; stematic experimental investigation of heav'-ion multiple scaltering with a variety of beams and iargets
The experimenta! method is summarized in Fig. 1. The angle tretween the incoming particle trajeclory and the rutgoing scattered particle trajectory is measured i. ithree dimensions. The equipment cor sists of four sets of position-s: asilive silicon det actors (PSD's), each sel providing information in on'- pair of coordinates $(x, y)$. The target is located between PSO sets 2 and 3 . With the target removed, straight-through (collinear) tracks are used to measure the alignment and spatial resolution of the detectors. This experimental configutation measures the multiphe scattering in the larget only, 


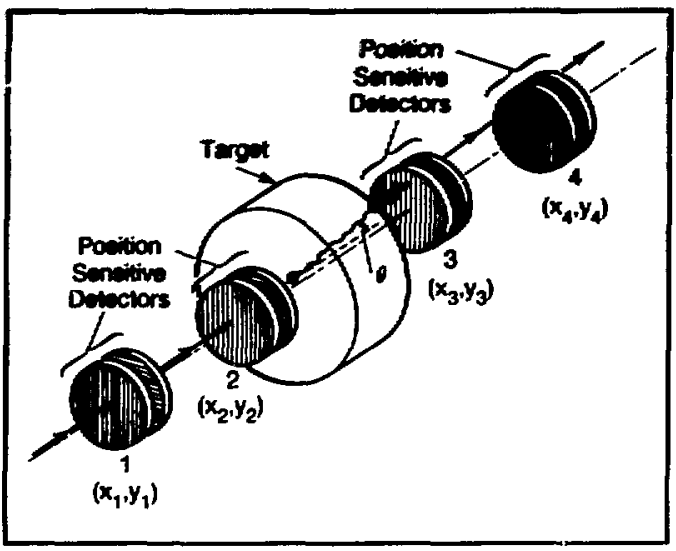

Fig. 1. Schematic diagram of experimental method to measure heavy-ion multiple scattering. Position-sensitive detectors 1 and 2 measure the incoming particle trajectory, detectors 3 and 4 the outgoing scattered particle trajectory. All detectors are fithium-drifted silicon. From the reconstructed particle trajectories, the target multiple scattering angle $\theta$ is determined. With the target removed, straight-through tracks serve to measure detector alignment and spatial resolution.

(XBL 8510-8550)

independent of beam collimators, absorbers, etc., placed upstream of it.

Each detector provides, on an event-by-event basis, measurement of particle energy loss in the detector. Fight separate detectors yield eight independent nieasurements of energy loss, and this information can be used to identify the incoming and outgoing particles.

Initial runs have been made with neon and iron beams in Cave II of the Biomed complex. Figure 2 shows the correlation in energy-loss measured by two adjacent detectors downstream of a $1 \mathrm{G}-\mathrm{cm}$ water target for incident $670-\mathrm{MeV} / \mathrm{A}$ neon ions. Events in the upper-right cluster correspond to primary beam particic;. Events in the lower clusters are those in which the primary beam particle has changed identity as it traversed the target. Events wherein the identity of the primary particle is preserved during the scattering process, i.e., elastic multiple scattering, ais selected in each measurement.

Figures 3a, b, $c$, and $d$ show rest'is for the scattering of $600-\mathrm{MeV} / \mathrm{A}$ Fe ions on a $1-\mathrm{cm}$ thick brass target. Shown are the profiles for the coordinates $\left(x_{3}, y_{3}\right)$ and $\left(x_{4}, y_{4}\right)$ for the downstream PSD's 3 and 4 . These profiles can be comparcd with measuremeits mate with the same beam on a 5 . cm thick lucite targel and with no target istraight throughs). Table I shows preliminary tesults for the

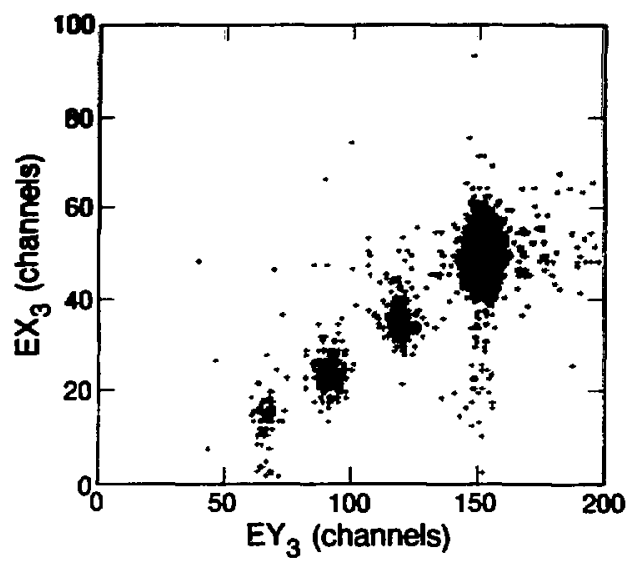

Fig. 2. Correlation in energy loss measured by two adjacent detectors downstream of a $10-\mathrm{cm}$ water target for incident 670-MeV/A neon ions. Events in the upper right hand cluster correspond to primary beam particles and were selected as the outgoing particles for this experiment. Events in the other clusters correspond to projectile fragments.

(XBL 8510-8548)

full width at half-maximum of the emerging beam. For each coordinate dimension, the width increases monotonically, as is expected. Further, the sensitivity to measuring the difference in the amount of scattering from different materials is exhibited. We see that a difference in width of $5 \%$ from Lucite to brass can be detected.

High precision differential measurements of multiple scattering are planned to permit comparison with theoretical calculations of this fundamental electromagnetic process. For thin targets, we anticipate interesting interference effects with nuclear elastic scattering. In addition, for thick targets, we expect nuclear fragmentation processes to affect the angular distributions, especially for large angles.

\begin{tabular}{|c|c|c|c|}
\hline & No target & $\begin{array}{l}\text { Lescite } \\
(5 \mathrm{~cm})\end{array}$ & $\begin{array}{l}\text { Brass } \\
(1 \mathrm{~cm})\end{array}$ \\
\hline$x_{3}$ & 1.90 & 2.26 & 2.50 \\
\hline$v_{1}$ & i. .91 & 2.18 & 2.26 \\
\hline$x_{4}$ & 1.90 & 2.14 & 2.26 \\
\hline Yo & ins & 2.18 & 2.26 \\
\hline
\end{tabular}



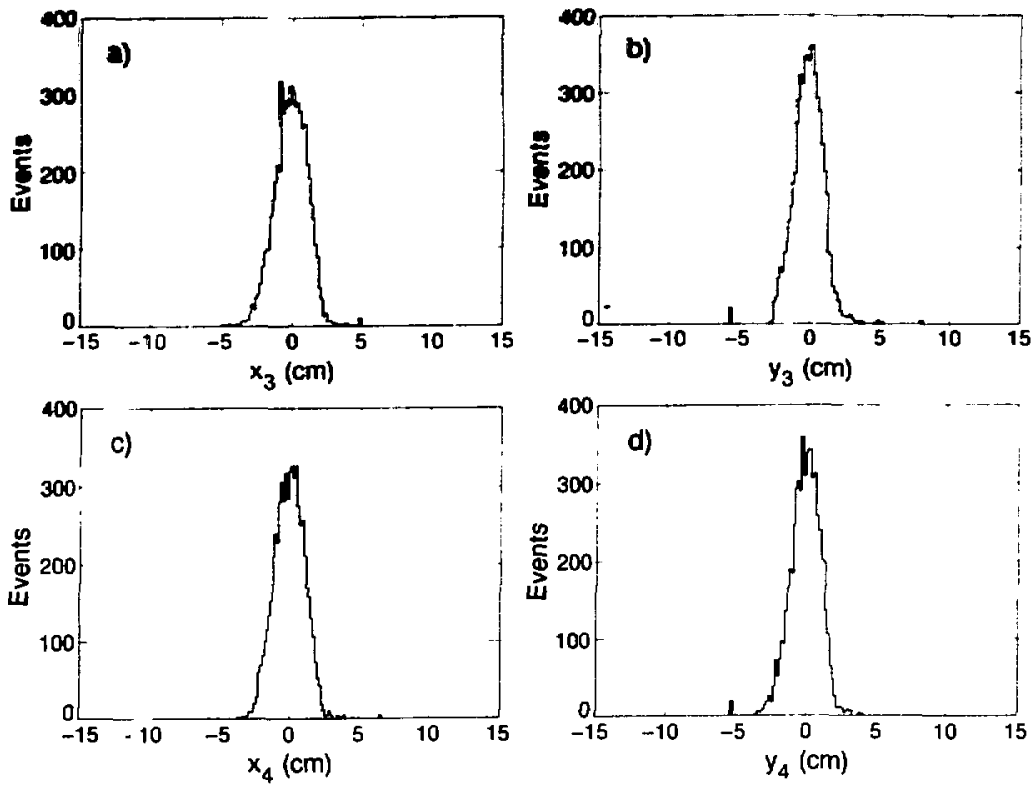

Fig. 3. Profiles for the $\left(x_{3}, y_{3}\right)$ and $\left(x_{4}, y_{4}\right)$ coordinates for the scattering of 600-MeV/A iron ions on a $1-\mathrm{cm}$ thick brass target.

(XBL 8510-8535)

\section{${ }^{16} \mathrm{O}$ AND ${ }^{56} \mathrm{Fe}$ EXCITATION FUNCTIONS}

John P. Wefel," T. Gregory Cuzik," Henry J. Crawford, ${ }^{\dagger}$ Walter Schimmerling, Peter J. Lindstrom, ${ }^{\dagger}$ Douglas E. Greiner, ${ }^{\dagger}$ and T. James M. Symons ${ }^{\dagger}$

The sources of radiation in space are the radiation trapped in the earth s magnetic field, solar particle radiation, and galactic cosmic rays. Man-made radiation may also be present. The cosmic radiation is fairly constant in time, both in its intensity and its composition. Solar flares have a cyclical activity; the particulate radiation dose cannot be predicled on a time scale grealer than hours or days. The radiation field to which a space worker is exposed in the course of a mission will also vary greatly, depending on the geomagnetic location,

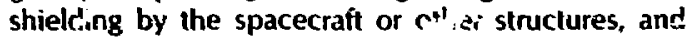
the exlent of extra-vehicular activities (EVA's).

Radiation dosimetry in space has a predictive function because the dose to space workers must

Toumion Stave Umensm.

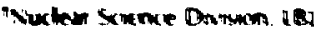

be estimated before they are exposed, but it also has an archival function because the actual exposures must be known after they have occurred. Measurements of these exposures are not likely to be sufficient because of the temporal and spatial variations involved and because of the limitations of personnel dosimeters in measuring radiation quality. Fr. this reason, space radiation dosimetry is likely to -ely increasingly on calculations that are based on fundamentai physical quantities, e.g. cross sections, cosmic-ray spectra, and so forth.

The energy spectra of the heavy elements in galactic cosmic rays increase from low energies to a broad maximum around 200-400 MeV/A, and then decrease with energy per nucleon approxinitely as $(E / A)^{-2.6}$. Thus, the estergies of galaclic cosmic ravs tall well into the range accessible at the Lawrence Berkeley Raborationy Bevalac in this enengy region, the ranges of theary nuclei in malter 
(15-35 $\mathrm{g} / \mathrm{cm}^{2}$ ) become comparable to their mean free palh for nuclear interactions. As a consequence, a significant fraction of the relativistic heavy-ion flux will undergo nuclear reactions in thick absorbers, e.g., shielding materials or tissue. When these reaclions can take place anywhere in a thick absorber, it is necessary to know these quantities as a function of particle energy for all particles present. In the present context, the first problem to be solved is the measurement of the probability for emission of a given fragment, at a given energy, into a given angle, i.e., inclusive differential cross sections, and of the probability for any reaction of an incident projectile leading to its disappearance, i.e., Iolal reaction cross sections.

Transport calculations of HZE particles depend fundamentally on a knowledge of inclusive fragmentation cross sections, i.e., the probability that the projectile suffers an interaction leading to a fragment (generally contained in a forward angle of $\sim 3^{\circ}$ ) and residual particles. There are also interesting problems in astrophysics that depend on such cross sections.

The measurement of these cross sections can be somewhat simplified by an experimental design that does not require detection of the residual particles (i.e., an inclusive experiment). A program to meastre excitation functions (differential inclusive cross sections as a function of projectile energy) using hydrogen as a target, has been initiated at $\mathrm{LBL}$ in collaboration with colleagues from Louisiana State University (LSU).

The experimental arrangement is shown schematically in Fig. 1 . The beam leaves the accelerator ring and is magnetically steered through a beam transport system to the target. A series of scintillation counters monitor the beam position and intensity. Different targets are mounted on a remotely controlled drive that can change targets without breaking the seal on the vacuum tank. The fragments from beam interactions in the target appear in the cave, spread out according to their emission angle from the interaction site. A particle identification telescope is mounted in the cave sich that it can be positioned within the main beam or at any desired angle on either side of the main beam. At large angles with respect to the beam, the high momentum tail of the momentum distribution can be examined in detail.

The particle identification telescope, showr in Fig. 2 , consists of a slack of solid-shate deteciors in which the particles ane brought to rest to delermine their alomic mass. The gekescope conusts of three

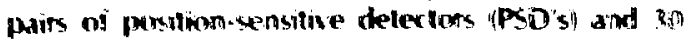

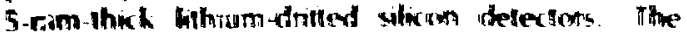
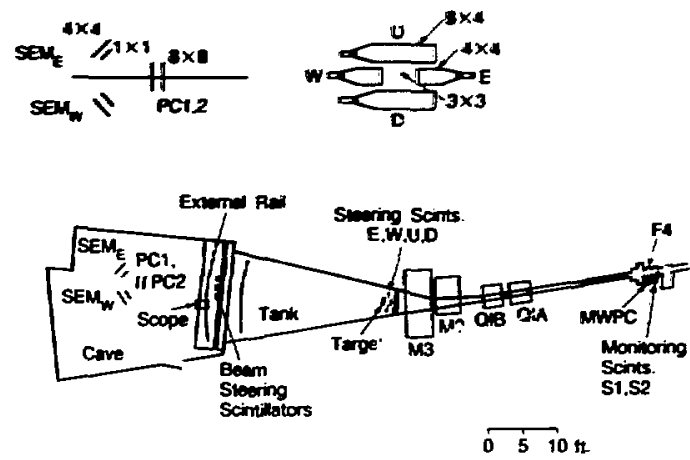

Fig. 1. Schematic diagram of the experiment arrangement for Experiment $683 \mathrm{H}$ showing the beam monitoring.

(XBL 8510-8534)

total thickness of the telescope is sufficient to bring to rest heavy fragments from the interaction of approximately $375-\mathrm{MeV} / \mathrm{A}{ }^{16} \mathrm{O}$ nuclei. The PSD's are employed to localize each event, in order to give fine positioning and to measure the trajectory through the stack to correct for events entering or leaving the sides of the telescope. All detectors are parallel to each other within $\pm 3^{\circ}$. An external pulser circuit into each preamplifier allows for individual calibration of the amplifier gains. The external pulser provides a monitor of each of the signal processing chains throughout the experiment.

The advantages of the arrangement shown in Fig. 1 are 1) minimum material aiong the beam line between target and telescope, 2) good spatial separation to measure the angular distribution of the fragments, 3) a well understood massdetermining technique, and 4) capability to use high beam rates for the large angles at which the telescope is outside of the main beam. The disadvantage is that the arrangement assumes a constant

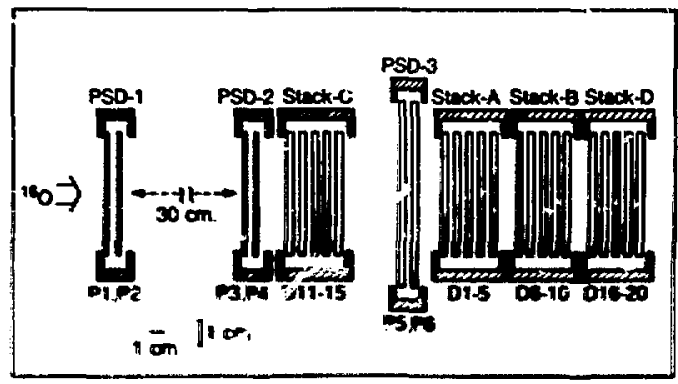

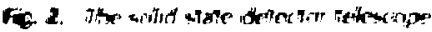


been kiction, ice, stability of the mypets atong the BAC une, and of magnets $M 2$ and $M 3$ in particular. We are working on methods to ensure accurole beam positioning.

An initial dala-1aking run was performed in Maxch 1984. The data analysis is still in progress, but preliminary results, discussed below, indicate that the experimental technique can give the needed high-quality data. Unfortunately, there were probtems with the beam delivery system and the magnet power supplies during the March run that limited the amount of data per beam hour that could be obtained. Solutions have been lound for these difficulties, and the beam time needed to acquire the data to meet cur objectives has been scheduled for April 1986.
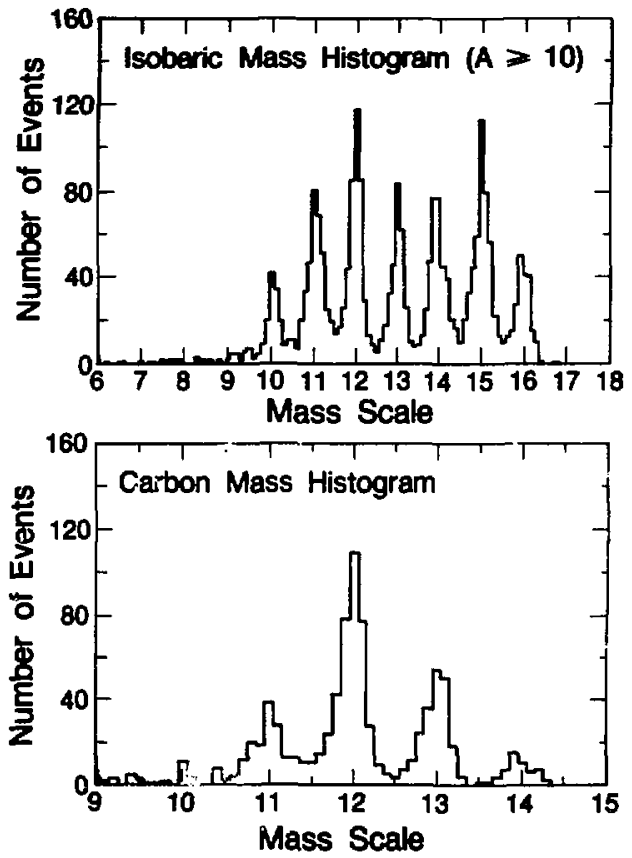

Fig. 3. Mass histogram obrained at $1.75^{\circ}$ to the beam direction. The iop portion shows the isobaric plot for $A \geqslant 10$ and the lower part shows the mass distribution for the elament carbon. (X8L 8510-85.3I)
Our first nun emploned a 225-NeV/A 1 th beam to study fragmentation in targets of cabon and potyethylene pius a blank larget holder. Runs mere aken at $0^{\circ}, 0.5^{\circ}, 0.75^{\circ}, 1.0^{\circ}, 1.75^{\circ}$ and $2.75^{\circ}$ to the beam direction. Figure 3 shows an example of the mass histograms derived from this initial data sel. The top portion shows the isobzic mass thistograms of $A \geqslant 10$ while the lower plot shows the resulis for carbon. Note that mass peaks for ${ }^{10} \mathrm{C}$ through ${ }^{14} \mathrm{C}$ are resolved but the abundance of ${ }^{10} \mathrm{C}$ is, as expected, quile low. From the ligure the isotopic mass resolution is of the order of 0.2 mass units. This should improve as erergy resolution and trajectory information is refined. Figure 4 shows preliminary results for the angular distribution of the carbon fragments produced by the incident oxygen beam in the carbon target.

\section{Preliminary Angular Distributions}

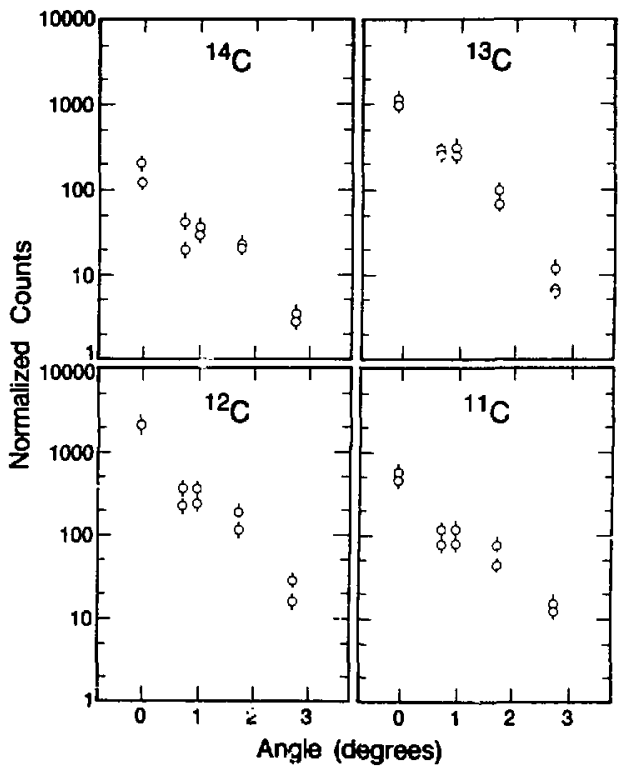

Fig. 4. Preliminan results for the angular distribution of carbon isotopes from oxigen fragmenting in a carbon target.

$(X B L 8510.8532)$ 


\title{
EEARET UNCRADE MMTIOVED DETECTION OF LOW LET PARTKCLES IN HIGHIY FACMENTED HEAVY ION EEAMS
}

\author{
Jorge Lacer," futius I. Almasi, and Cormelius A. Tobias
}

The BERKLET is a simple solid state detector telescope that was developed to measure the LET and the relative number of particles of different atomic numbers in heavy-ion beams under conditions of complex primary beam fragmentation.' It consists of a thin silicon radiation detector that measures the LET of individual incoming particles followed by a thick germanium detector where the particles stop and deposit their residual energy. The data from the BERKLET is displayed in a twodimensional histogram of LET and residual energy that, with some limitations, determines the atomic number of the particles in a beam. Track and dose-average LET values can be calculated for individual particles or for groups of particles, and particle velocities can be approximated.

We have recently made BERKLET measurements in neon-20 beams for the purpose of determining accurately the contribution to beam characteristics from low-LET particles in the fragmented beams. The frequency and LET range of this component of the beams could not be determined with the early design of the BERKLET. During the past year, we have been able to redesign the silicon detector in the telescope to allow for routine measurement of the low-LET part of the beam concurrent with measurement of complex high-LET components. $^{2}$

The original silicon detector consisted of a single wafer of $300-\mu \mathrm{m}$ thickness divided into two regions, a center "active" region, (Fig. 1, left) surrounded by a "guard" region that provided signals for the logic operations and that defined the area over which the measurement was being made. The edges of silicon detectors give erroneous results and have to be collimated electronically. The LET ratio of a silicon particle near its stopping point and that of a fast proton is of the order of 2500:1. The dynamic range of such measurements creates a difficulty. Even with a specially-designed event discriminator that would perform well under such a wide range of operating conditions, we had a problem with electronic interfierence between the two regions of the detector. When a heavy ion would traverse the guand region, a small signal would be induced in the "ectrve" region. That signal would

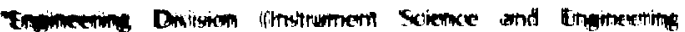

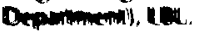

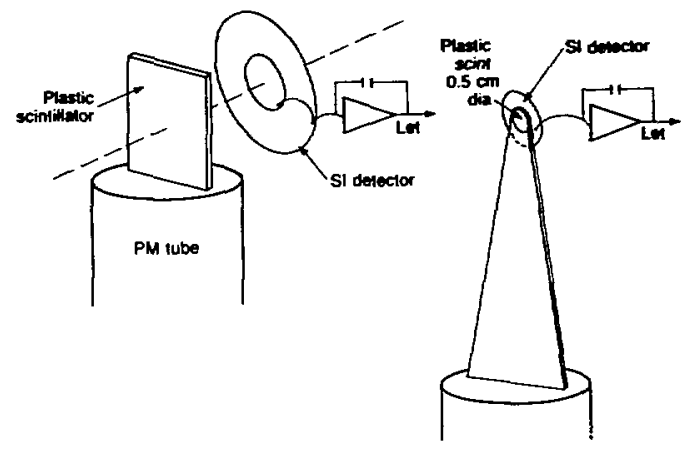

Fig. 1. Test design of new LET detector configuration and new final design.

(XBL 857-1124I)

appear to the electronic circuits as a light particle. There was no way, then, to determine whether a small signal was from a real particle or if it was an induced signal from a high-LET event in the guard region.

To correct the problem, we have devised a new first detector consisting of a thin smalldiameter plastic scintillator (Fig. 1, right) followed by a $300-\mu \mathrm{m}$ silicon detector, without a guard ring. By requiring a coincidence measurement from each of the two detectors, we define the active region of the LET detector without cross-interference, and we obtain an added advantage: the discriminator thresholds of both the plastic scintillator and of the silicon detector can be lowered until the respective noise sources are exposed. When we require a coincidence between the two detectors, the noise disappears for all practical purposes, and the signals of the very low-LET particles can be unambiguously discriminated.

A pieliminary experiment has been completed to lest the new design with our available equipment. A thin plastic scintillator was placed in front of the original silicon BERKLET detector, and in spite of the incorrect geometry, we have been able to analyze the results successfully. Figure 2 shows a comparison of Brass ionization measurements made in a neon-20 beam with either the standard ionization chambers (solid line) or the BERKLET (circkes). We studied the configuration of absorbers in the bean used for the cancer therapy bisls, without 
Therepy Neon

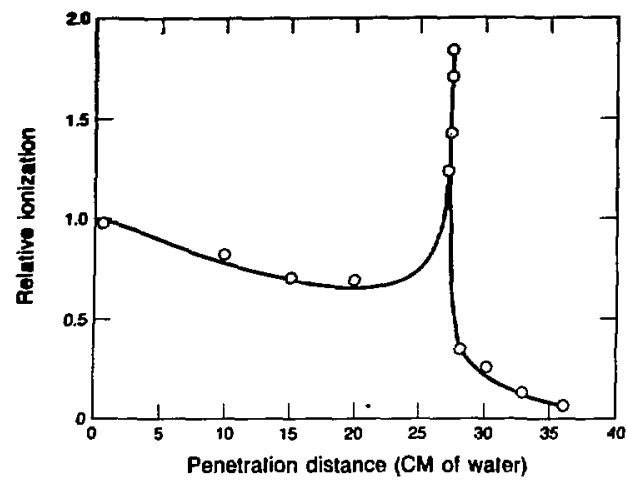

Fig. 2. Bragg curve comparison: ion chamber vs. BERKLET for therapy neon beam, without range modulation filter.

( $X B L$ 857-11238)

the range modulating ridge filter. The BERKLET results are obtained from the measured number of particles of a given atomic number and their average LET value, and include particles with LET values as low as $0.17 \mathrm{keV} / \mu \mathrm{m}$.

Figure 3 shows the relative number of particles in the beam as a function of the thickness of water absorber. The presence of large numbers of lowLET particles, ( $Z \leqslant 2$ in the figure) is confirmed, although their contribution to the total dose is small. Their presence causes little change to the calculation of the dose-average LET, but modifies considerably the track-average LET values. We expect that the completion of the modifications to the BERKLET will permit rapid optimization of clini-

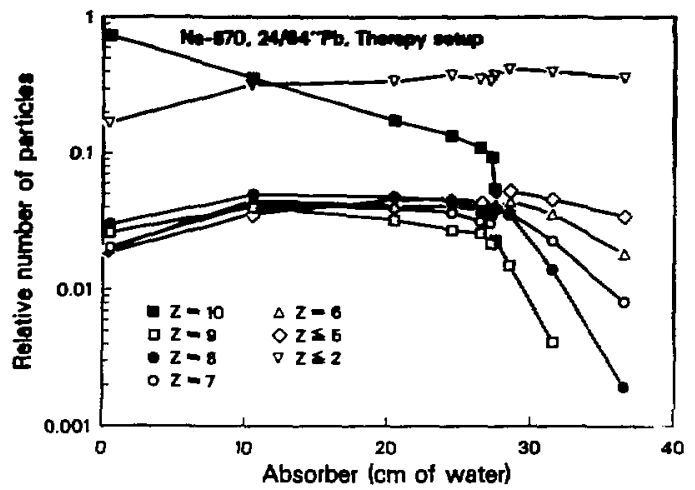

Fig. 3. Relative number of particles vs. $\mathrm{H}_{2} \mathrm{O}$ thickness depth oi penetration in water for the same neon beam of Fig. 2 .

(XCG 855-215)

cal heavy-ion beams and also will allow an analysis of particle-beam composition and track-structure effects on biological systems, which can be compared to time-of-flight measurements of particle distributions.

\section{REFERENCES}

1. Llacer, I., Tobias, C.A., and Holley, W.R. On-line characterization of heavy-ion beams with semiconductor detectors. Med. Phys. $11(3), 266-278$ (1984).

2. Llacer, J., Tobias, C.A., and Almasi, J.J. Low LET particles in heavy ion beams, Radiation Research Society Meeting, May 5-9, 1985, Los Angeles, California. (Abstract).

\section{CAREON FROTON EEAM COMPARISONS}

\section{Cornetius A. Tobias, Eleanor A. Aldkety, William T. Chn, Eernhard A. Ludewigh, and patios I. Almasi}

Based on research with prolon beams at Harvard ${ }^{1-3}$ and hetium beams al Betkeley, ${ }^{4-6}$ there has been a general recogaition of the value of highty localized particle besms for therapy. The treatment of ocular melenoma, juxtaspinal tumnos, pituitery and AV-milformations are examples. A commithe of the American Coliee of Reditiology has revitewied this matter recently." As a resulh, a number of laboratories are planning to propose the construc. tion of proton accelerators; however, we have not been able to find any divect comparisons of protions and hewry ions under stricthy identical conditions.

axed on theoretival considerations and on

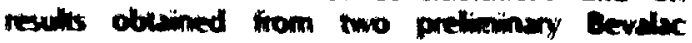


experiments, we have tentatively confirmed our hypothesis that carbon and neon beams are potentially better for beam localization than protons or helium ions of comparable range. When a small beam is used, multiple scatter broadens the beam as it penetrates an absorber. The scaltering for carbon beams is about $\sqrt{12}$ times less than for proton beams. The same can be said for the straggling. The end result is that the Bragg peak/plateau ratio is reduced for protons much more than for carbon, and the proton beam is physically broadened. Figure 1 , which is a plot of Bragg peaks from Bevalac carbon (308 MeV/u) and proton (155 MeV/u) beams extracted at energies that yield $15 \mathrm{~cm}$ penetration into tissue equivalent material, illustrates this point. The small circular data points were taken with a large $(2.0-\mathrm{cm}$ didineter $)$ beam downstream of a $1 . \mathrm{cm}$ collimator. The solid square symbols represent Bragg data taken with a small $(0.4-\mathrm{cm}$ diameter) beam downstream of a $1-\mathrm{cm}$ collimator. The carbon Bragg curves of the large and small beams are identical, while clear differences are shown between the large and small diameter proton beams. The Bragg peak of the smaller diameter proton beam is degraded in comparison to the larger diameter proton beam.

The same point is obvious from Fig. 2. Two linear arrays of polaroid beam spots are shown, the top set from a 20-rad (plateau dose) carbon exposure, the lower set from an 8-rad proton exposure. Water-filled tissue culture flasks were sandwiched between the polaroids during the exposure. The most extreme left polaroids are in the entrance plateau of the beam. The most extreme right polaroids are in the Bragg peak. At this depth, the carbon beam is still well-defined while the proton beam is not.

Further experiments are planned with Bevalac protons and carbon-ion beams to evaluate the importance of multiple scattering to the successful delivery of sharply-deinned and narrowly-collimated

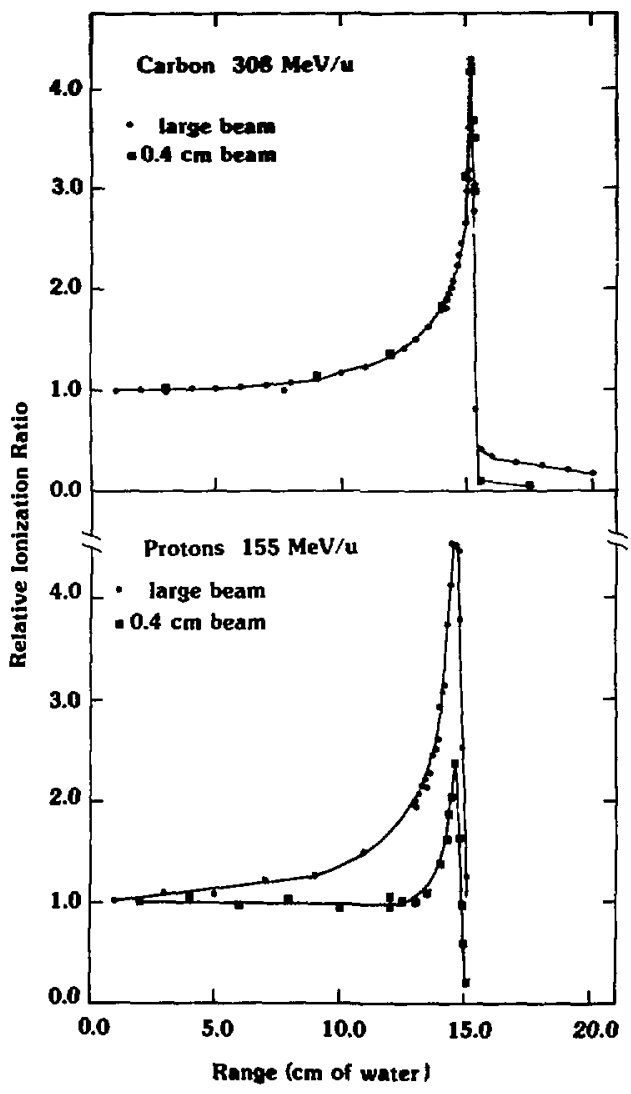

Fig. 1. Comparison of Bragg ionization curves from large beam (2.cm diameler) and narrowly collimaled $(0.4-\mathrm{cm}$ diameter) proton (155 MeV/u) and carbon ( $308 \mathrm{MeV} / \mathrm{u}$ ) Bevalac beams.

(XBL 853-3340)

dose distributions at clinically significant depths of penetration for the treatment of eye tumors and brain lesions. 


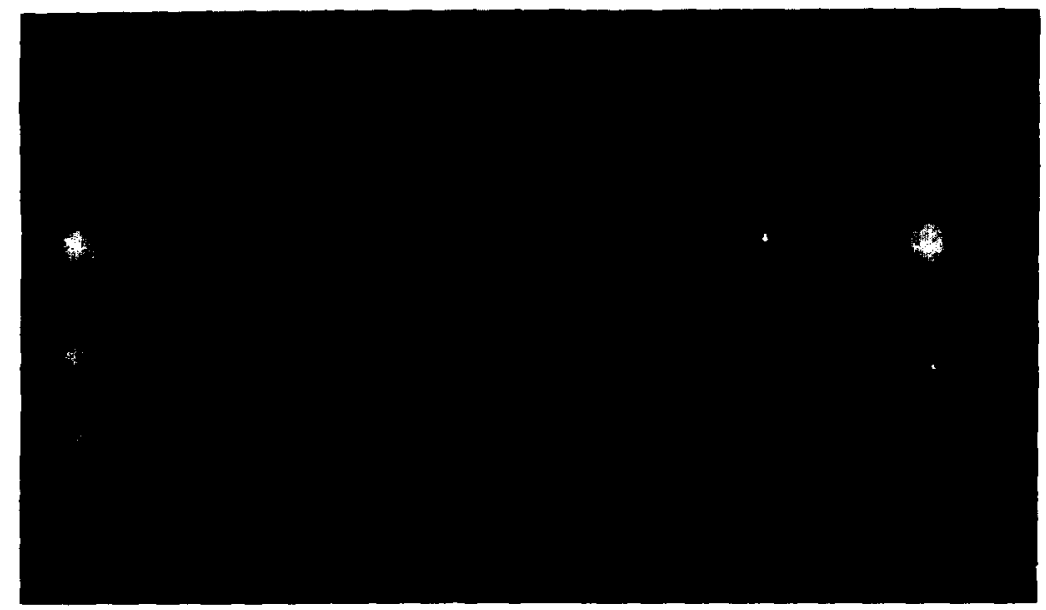

fig. 2. Polaroid beam spots from a 20-rad (plateau dose) carbon 308-MeV/L exposure and an 8-rad (plateau dose) proton $155-\mathrm{MeV} / \mathrm{u}$ exposure. The description of the experiment can be found in the text.

(XBB-858-6040)

\section{REFERENCES}

1. Munzenrider, J.E. Proton therapy at Harvard. Pion and Heavy-lon Radiotherapy, Preclinical and Clinical Studies (L.D. Skarsgard, Ed.) 363-372, July 1981.

2. Koehler, A.M. Use of protons in radiotherapy. in Proceedings of the Symposium on Pion and Proton Radiography, National Accelerator Laboratory, Batavia, Illinois, pp. 63-88 (1971).

3. Verhey, L.J., and Munzenrider, J.E. Proton beam therapy. Ann. Rev. Biophys. Bioeng. (in press).

4. Saunders, W.M., Castro, I.R., Quivey, J.M., Chen, G.T.Y., Lyman, L.T., and Collier, I.M.
Clinical experience with heavy-ion beams at Lawrence Berkeley Laboratory, Pion and Heavy-Ion Radiotherapy: Preclinical and Clinical Studies (L.D. Skarsgard, Ed.) 373-394 (July 1981)

5 Castro, J.R., and Quivey, J.M. Clinical expericnce and expectations with helium and heavy-ion irradiation. Int. \%. Radiat. Oncol. Biol. Phys. 3, 127-131 (1977).

6. Phillips, T.L., Fu, K.K., and Curtis, S.B. Tumor biology of helium and heavy ions. Int. /. Radiat. Oncol. Biol. Phys. 3, 109-113 (1977).

7. Cancer Treatment Symposia, Vol. 1 (Robert E. Wittes, Ed.) pp. 1-191 (1984).

\section{Molecular Studies}

\section{DETECTION OF RADATION INDUCED LESHONS AT THE MOLECULAR LEVE}

\section{Ruth 1. Roots, Cinofiranco Crosed, and Connelins A. Tobiss}

We have begun studies of radiation-induced changes in specific genes of mammalian cells, in particular with respect to genetic chanes in cells transformed by hinh-LET radiation. Aherstions in the prollooncesenes ase associatled with necpllastic crowith charackenistics. In momal cellis, cetting pro- tooncogencs function in DNA synthesis control, as for example the c-myc pene, while others may code for bene products that are componenis of growith fixtors of growith factor receptors.

To screen for enetic abnomilmities followions fadiation, ence location is compueded in normal and 
transformed cells. Secondly, gene expression levels are measured in normal and transformed cells.

Figure 1 shows the karyotype of male muntjac cells. There are three pairs of chromosomes plus the $Y$ chromosome. This cell line was chosen in preliminary work because of the low diploid chromosome number; primary cultures of normal diploid hamster embryo cells are now used. Chromosome spreads were made of cells harvested from mitotic selection. Following fixation of the cells, metaphase spreads were made, and the chromosomes were then G-banded.

We have chosen to study genetic changes in the $c$-myc protooncogene following radiationinduced cell transformation. Other protooncogenes may also be studied, but we have elected to begin our screening process with c-myc. The in-situ hybridization technique of the hybridization of a ${ }^{3} \mathrm{H}$-labeled $\mathrm{v}$-myc probe to chromosome spreads used in combination with chromosome banding can be used to identify the c-myc locus. This technique can, therefore, be used to identify gross abnormalities in a specific gene locus. It is expected that c-myc is present as a single copy. The in-situ hybridization technique that we are using is, however, capable of locating single-copy gene sequences. The value of the in-situ hybridization technique is that a gene locus can be assigned to a specific band on a specific chromosome.

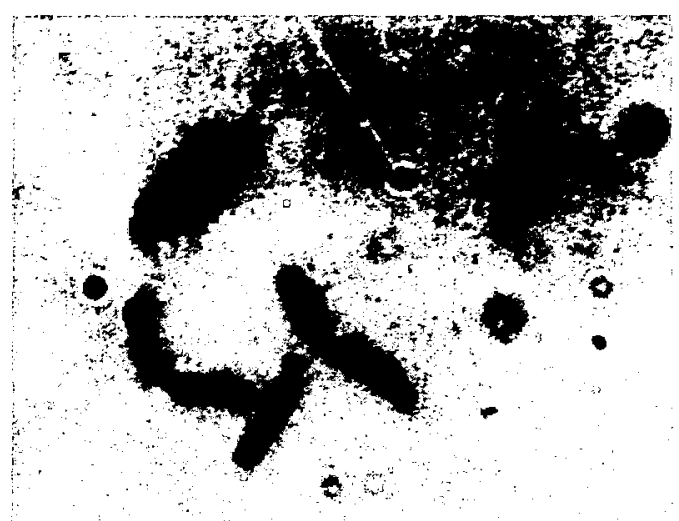

Fig. 1. Karyolype of the Muntiacus muntjak male deer cell $(2 N-7)$ showing $G$-banding patterns.

(XBB 854-2848)

The results of in-situ hybridization of ${ }^{3} \mathrm{H}$-labeled $v$-myc $(1.5 \mathrm{kbp})$ to unirradiated muntjac chromosome spreads are shown in Fig. $2 \mathrm{~A}$ and $2 \mathrm{~B}$. The autoradiogram Fig. $2 A$ was obtained from a oneweek's exposure to the emulsion, while Fig. $2 B$ is from a two-week's exposure. The v-myc hybridization appears to be on chromosome number 1 ; however, we have not yet made a systematic study of grain localization to identify the exact band position, and we regard these data as preliminary.

(A)

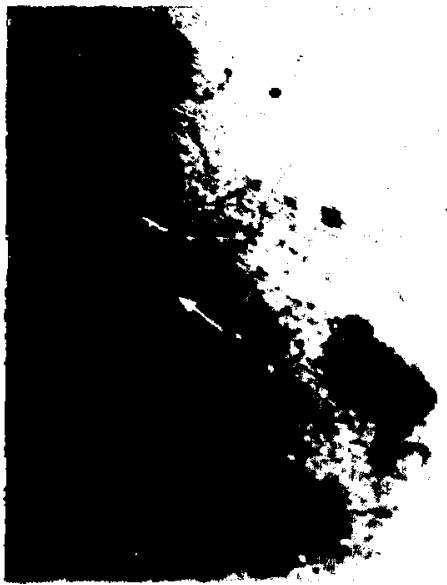

(B)

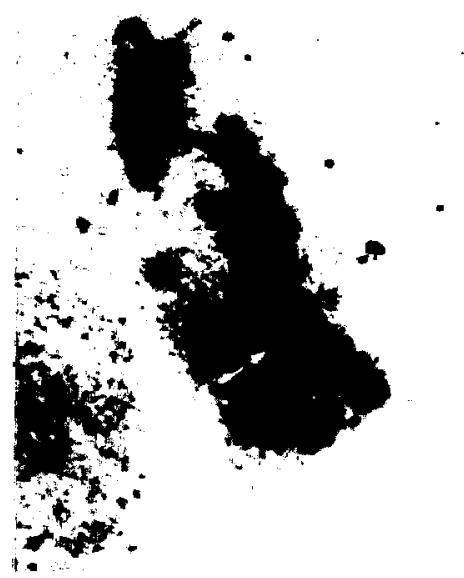

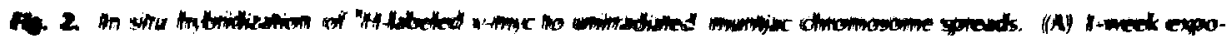

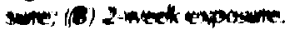




\section{MODUCTION OF STIAND RREAKS IN DNA BY WATER RADHCALS: A THEOAETICAL STUDY}

\section{Aloke Chatterjee, John L. Magee, Patrice Koehl, and William Holley}

Starting with the physical phenomena of energy deposition by ionizing radiation, we are attempting to develop an understanding of the mechanisms of radiation damage in living cells. Such an understanding is essential for 1) better protection against radiation as an environnental hazard, 2) an intelligent use of radiation as a diagnostic tool, and 3) treatment optimization for cancer patients undergoing radiation therapy.

The initial phase of energy deposition in a cell $\left(\sim 10^{-16} \mathrm{sec}\right)$, leads to a series of physicochemical $\left(10^{-16}-10^{-12} \mathrm{sec}\right)$, chemical $\left(\sim 10^{-12}-10^{-6}\right.$ sec), and biochemical $\left(10^{-9}-10^{-3} \mathrm{sec}\right)$ changes that eventually produce observable biological (seconds to years) damage. For a better understanding of the mechanisms of radiation damage these changes must be correlated both qualitatively and quantitatively and an effective research program must iriclude coordinated theoretical and experimental studies. Obviously the task is a difficult one and a major effort is needed to achieve this goal. We have initiated such a program with the participation of physicists, chemists, and biologists working in a collaboration.

The human cell is a highly organized and complex system, and although we understand a great deal about its response to various kinds of damage by ionizing radiation, there are many phenomena that still remain obscure. It was therefore decided that at the initial phase of our elfort a much simpler system should be used. This simpler system must, of course, be relevant and mimic the actual living cell in terms of radiation damage. Simian virus (SV40) DNA in dilute aqueous solution with Tris as a buffer seemed to meel our requirements and was chosen for the initial studies.

lust like DNA in a cell, the SV40 DNA molecule in aqueous solution can be damaged by two difierent mechanisms: indirect effect and direct effect. The indirect efiect generally means that radiation interacts with the surrounding water molecules, creation hydroxyl radicals that evenlually damae the DNA. In conitrast, damave due to the direct effect is caused by the direct deposition of enerby in the DNA molecule. We have made signivicant progress in understanding indirect effects with respect to SV10 DNA and this is the subject of this article. The drect eflect is under investigation and progress will soon be the subject of another report.

Although we have made a large number of experiments with different qualities of radiation using SV40 DNA solutions, our primary focus is on the theoretical development of this system. Experimental results are necessary for verification of the models introduced in the development. Understanding requires both theoretical and experimental aspects.

There are four main stages in our theoretical approach:

(1) Simulation of the SV40 DNA molecule through a computer code according to its stereochemical parameters obtained from $x$-ray diffraction studies.

(2) Location of the hydroxyl $(\cdot \mathrm{OH})$ radicals around the DNA as they are created in the tracks of the ionizing radiation.

(3) Following of each $\mathrm{OH}$ by simulation of its diffusive motion in the medium and calculation of its reactions.

(4) Use of computer graphics as an aid in the qualitative understanding of the diffusion and reaction processes mentioned above.

The calculation of the reactions of the $\mathrm{OH}$ radicals is the most important and difficult task. There are three reaction channels:

(1) Reaction with the other water decomposition products: $\cdot H, e_{2 q}^{-}, H_{3} \mathrm{O}^{+}$and other . OH's.

(2) Reaction with the Tris buffer.

(3) Reaction with the DNA molecule.

When an $\mathrm{OH}$ radical reacts with a DNA molecule, it can either react with the ribose phosphate backbone or with the bases. If it reacts with the ribose moiety a strand break results and if both strands are broken close to each other then there is a double strand break. Reactions of $\mathrm{OH}$ with the bases do not lead 10 strand breaks, bul other biological effects, such as mutation, base release, eic., are expected.

We have simulated the diffusion of OH by a Monte Catlo code that we developed. A resuh of our code is shown in fig. 1 tor a case where a $\mathrm{OH}$ 


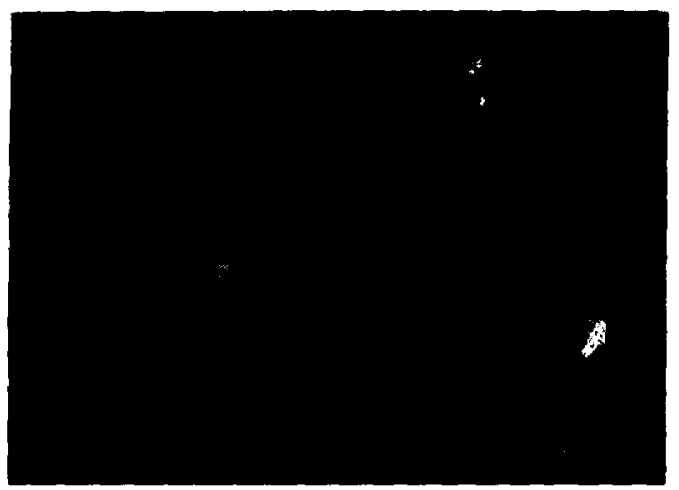

Fig. 1. Computer calculation of the diffusive molion of an $\mathrm{OH}$ radical that reacts with the ribose moiety of a DNA molecule. The radical is formed by the action of a $14-\mathrm{MeV}$ electron at the extrene leti of the figure and its subsequent motion is indicated. Radicals formed at the same position have an inimite variety of patterns of motion and trom many calculated paths we have selected one that lesds to reaction with the DNA. The reactive sites on the ribose moieties of the DNA are indicated by spheres: the reactive sites on the bases have much larger reaction radii and the overlapping reaction sphere are indicated by the paltern of small dots. Other possible reactions of the OH radical are with other water radicals $(\mathrm{E},-, \mathrm{m}, \mathrm{OH}, \mathrm{H})$ or with the butite. Actually for most of the many possible diffusive paths the radical moves away from the DNA and reacts with a butter molecule (Tris).

(CBB 857-5204) interacted with a sugar molecule on the DNA backbone. This particular radical could have gone in any direction with equal probability. There is no rule that it must go toward the DNA. The part of the SV40 DNA molecule represented in the same figure is in its B form and its stereochemical parameters have been obtained from $x$-ray diffraction patterns.

Through a large number of trials in our VAX computer we have obtained some meaningful average quantities that could be checked with experimental measurements. For example, we have been able to calculate the D37 (dose required to produce damage in $63 \%$ of the DNA molecules) for single-strand breaks for a 14-MeV electron beam, $500-\mathrm{MeV} / \mathrm{n}$ and $10-\mathrm{MeV} / \mathrm{n}$ neon beams. When compared with experiments, the agreement is quite reasonable and gives us a confidence in our approach. For $14-\mathrm{MeV}$ electron irradiation, it has been determined that about $80 \%$ of $\mathrm{OH}$ radicals attack the bases and about $20 \%$ of them interact with the sugar molecules.

The next stage is to introduce more complexities by adding histones and other proteins along with the DNA. We also expect to bring in enzymes that have the capability to repair strand breaks as found in celis. When we understand to a reasonable degree the processes involved in repair chemistry we hope to be ready to deal with the complexities of a cell.

\section{A STEREOCHEMICAL MODEL FOR THE REACIIONS OF DNA WITH THE HYDROXYL RADICAL}

William R. Holley, Patrice A. Koehl, Aloke Chatterjee, and John L. Magee

\section{INTRODUCTION}

lonizing radiation creales damage in DNA molecules in aqueous solution by two mechanisms: either the DNA itseli absorbs the energy and suifers the danage directly (direct eliect), or the molecules oi waler atsonb the energy and the radiation producis iormed neact with the DNA (inditecl erfect). For dilute anueous solutions of vitial DNA the damage is mainly indinect, and the OH radicall is getherally betiened to be the mojor combitibution to the proxtuction of slitand brasks on the DNA.

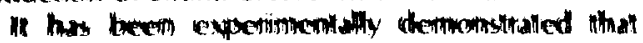

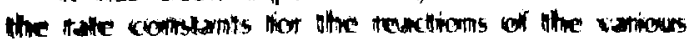

radical species with DNA are, in general, much lower than those for reactions will the isolated monomer unit. in order to understand the mechanisms of this effect, we have studied separately the action of $\mathrm{OH}$ on DNA and on isolated bases ficm a theorelical point of wiew.

The system we have chosen for sludy is dilute aqueeors solutions of Simian Vitus $\$ 0$ ONA. Tris bulfer is used to muinilain proper DNA conforma. thom and as a scavenger of OM radicals. This sys-

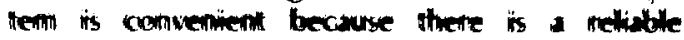
minethad for experiminemul determinuation of the 
strand breaks occurring after irradiation, and the average migration distance $(150 \mathrm{~A})$ for $\mathrm{OH}$ radicals in low Tris buffer concentration $\left(10^{-2}\right.$ mole $\left.~^{-1}\right)$ is in the domain of interest for a mammalian cell (90 A).

In the theoretical investigations discussed here the diffusion of $\mathrm{OH}$ and its reactions with a sugar or a base are assumed to follow the Smoluchowski theory. Two particles $A$ and $B$ diffusing in a solution are considered as having reacted with unit probability if they come within a distance $R_{A B}$, called the "reaction radius," of each other. We attribute to each sensitive site or: the DNA a reaction radius that can be calculated from known values for the rate constants.

\section{CALCULATIONS}

Using the stereochemical parameters of the DNA, obtained from crystallographic data, a 3D representation of the sensitive sites of the DNA is constructed. Nucleotides are built using the coordinates of each atom, and 2,000 different base pairs are constructed as shown in Figure 1. $\mathrm{OH}$ radicals formed by the radiolysis of the water are uniformly created in 3D three-dimensional space around this molecule, and their diffusion is simulated by a Monte Carlo technique. Each radical is followed separately till it reacts, either with another radical or with a Tris molecule, or with the DNA. We keep a record of how many radicals have reacted with a sugar moiety and how many have reacted with a base. The reaction probability $P_{r}$ is then calculated as the ratio of successful events to the total number of events, and from $P_{r}$ the average number of reactions per kilorad and the corresponding $D_{37}$ value are calculated.

The case of the isolated ribose or base has also been considered. It is simpler because there is only one reaction radius, but the overall calculation procedure remains the same. A typical base is simulated as a ceniral point sumounded by a sphere whose reaction radius has been determined to be 4 A irom experimentally obsened rate constants. The diffusion of the radicals and their reactions are studied with the procenlure described above.

In the theoretical analysis of the ditfusion ptocess it is well known that there is an extended region of influence wound a particle beyond the "reaction radius" that afifects the bocal concentration of interacting purtiches. A fime-dequendetis trate

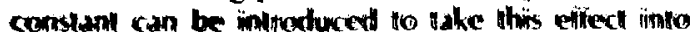

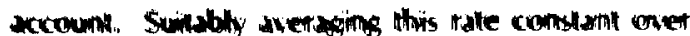

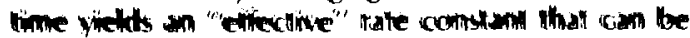

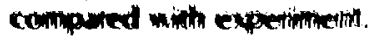

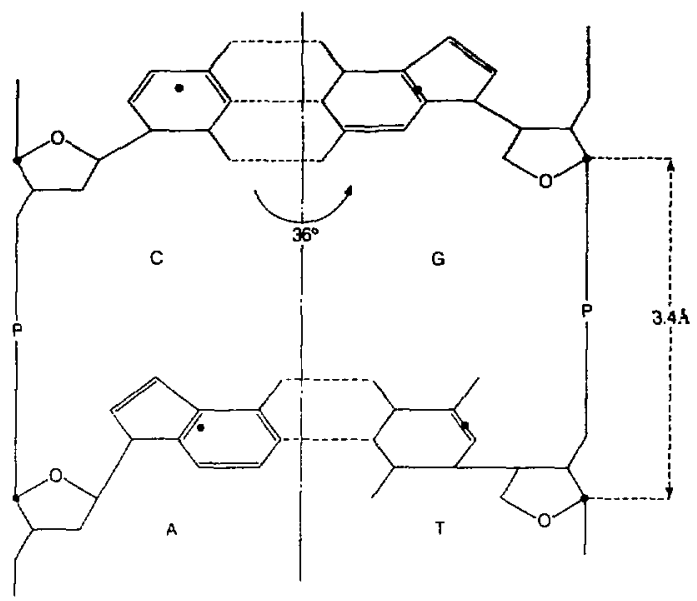

Fig. 1. Two consecutive base pairs of a DNA molecule taken from a stereochemical model are presented here. The guanine cytosine stack has been rotated through $36^{\circ}$ and projected on the plane combining adenine-thymine. The reaction centers that we use have been indicated by dots.

(XBL 858-3355)

\section{RESULTS}

Table 1 summarizes the values found for the time-averaged rate constant of $\mathrm{OH}$-base reactions for different buffer concentrations. For the isolated monomer all these values are around $6 \times 10^{9} \mid$ mole $^{-1} \mathrm{~s}^{-1}$ as expected. The variation of the effective rate constant $\mathrm{k}_{\mathrm{OH}-\text { base }}$ with buffer concentration is explained by the effects of the time-dependent rate constant; the higher the buffer concentration the shorter the average $\mathrm{OH}$ lifetime and the more important are the enhanced contributions of the time-dependent rate constant at short times to the elfeclive rate constant.

Table I. Fifective ante constants for $O H$-Base and ios OH-DNA reuctions.

\begin{tabular}{|c|c|c|}
\hline$\underset{\left.\text { (nnote }\left\|^{-\pi}\right\|_{1}\right)}{\mathbf{C}_{3}}$ & Konll-ame & $\begin{array}{l}\text { Kow-DNN Base } \\
\left.\text { if moke } y^{-1}\right)\end{array}$ \\
\hline$\pi 0^{-3}$ & $5.9 \times 11019$ & $1.6 \times \operatorname{MOC}^{14}$ \\
\hline $5 \times \ln ^{-1}$ & $60 \times 10 \times 10$ & $44 \times 100^{4}$ \\
\hline $\operatorname{nim} 0^{-2}$ & $0.10 \times n \times 0^{9}$ & $5.2 \times 110^{6}$ \\
\hline $2 \times n 0^{-2}$ & $6.2 \times \pi\left(0^{4}\right.$ & $5.9 \times 19 \mathbb{O}^{+1}$ \\
\hline $4 \times 119^{-2}$ & $16.5 \times \pi 0^{4}$ & $700 \times \pi 0^{n}$ \\
\hline$B \times \pi a^{-2}$ & 16: $\times \pi 1^{10}$ & $40 \times 110^{6}$ \\
\hline
\end{tabular}


A time-varying rate constant theory for reactions with DNA has not yet been formulated, but in terms of an effective time-averaged rate constant we find the values for $k_{O H-D N A}$ per base summarized in the last column of Table 1 . These results show a strong dependence on scavenger concentration, again reflecting the effects of an extended region around the DNA where the local concentration of interacting radicals is affected by the DNA molecule itself. Here the effects are even more pronounced than in the isolated monomer case because the interacting region is much larger, due to the combined effects of nearby reaction sites.
Another feature to be noted is that the effective rate constants per base from the DNA calculation are reduced by a factor of $\mathbf{1 0}$ or more compared to the values assumed for the individual reaction sites or obtained in the isolated monomer calculation. The reason for this reduction is that each reaction site in the polymer is shielded by nearby sites that intercept some of the $\mathrm{OH}$ radicals preventing their subsequent reaction with the site in question. The overall interaction rate is thereby reduced and the corresponding reaction rates are lowered in agreement with experimental results.

\title{
Cellular and Tumor Radiobiology
}

\section{NEOPLASTIC CELL TRANSFORMATION BY HEAVY ION RADIATION}

\author{
Tracy Chui-hsu Yang, Laurie M. Craise, Mantong Mei, and Cornelius A. Tobias
}

The major robjective of the present project is to understand the potential carcinogenic effects of cosmic rays and the mechanisms of radiationinduced cell transformation. During the past several years, we have studied the relative biological effectiveness of heavy ions with various atomic numbers and linear energy transfer (LET) on neoplastic cell transformation and the repair of Isansformation lesions induced by heavy ions in mammalian cells.' All these studies, however, were done with high dose rate. For risk assessment, it is extremely important to have dala on the low-doserate effect of neavy ions. Recently we initiater some dose-rate studies with high-LET iron parlicles and obsenved an enhancement effect of low dose rate.

For $x$ and gamma rays, many biological eifecis, e.g. cell killing, are dose-rate dependent. The effects of low dose rate are usually smaller than those of high dose rate. The effect of gamma rays on cell transiormation has also been found to be dose-rate dependent. For a given dose, the transformation litequency of kow dose rale is kower than that of high dose tale." In contrast to kow-LET ractiation, there are some dala lo stown thut kow-

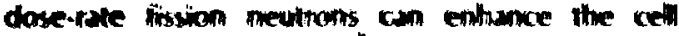

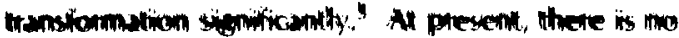

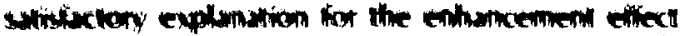

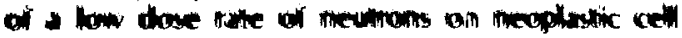

transformation. Because fission neutrons have a mixed-LET spectrum, it is unknown how the enhancing effect depends on LET and what its mechanisms are. The capability of the Bevalac in accelerating heavy ions with various charges to different energies provides an opportunity to study the dependence of enhancement effect on LET. With confluent cultures of the $\mathrm{C}_{3} \mathrm{H} 10 \mathrm{~T} 1 / 2$ cell line, we have recently initiated some studies on the lowdose-rate effect of high-LET particles on cell Iransformation. Figure 1 shows the results obtained for cell killing. There was no significant difference between cells irradiated with low- and high-doserate iron particles (600 MeV/u; $\sim 200 \mathrm{keV} / \mu \mathrm{m}$ ). For cell Iransformation, however, confluent cells irradiated with 2-rad/min iron particles gave a significantly higher transformation frequency than those irradiated wilh $100-\mathrm{rad} / \mathrm{min}$ iron beam, as shown in Fig. 2. The enhancement eflect was greater at lower doses than at higher doses. For example, the ransformation frequency for 50 rad of low dose tate iron particles was about the same as that for 100 rad of high dose tale. The Itansifomation frequency of dose $175 \mathrm{kad}$ of low dose rale was, however, no higher than that for 250 rad of high dose Miale.

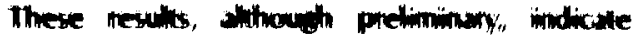

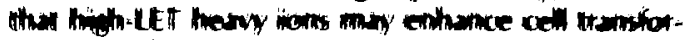

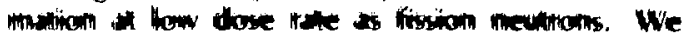




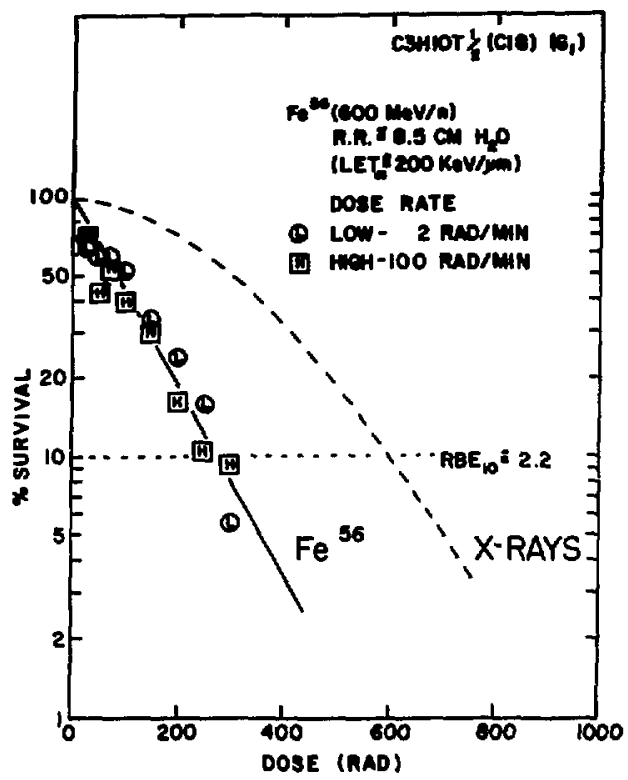

Fig. 1. Dose response curves for confluent C3H10TI/2 cells irradiated with 2-rad/min or 100-rad/min iron particles 1600 $\mathrm{MeV} / \mathrm{u} ; 200 \mathrm{keV} / \mu \mathrm{m}$.

(XBL 859-3858)

are continuing to investigate the low-dose-rate effects of heavy ions with various LETs on cell transformation and will study the role of repair and track structure in the enhancement effect. Information collected from these studies should help us in understanding the fundamental mechanisms of the low-dose-rate effect $n$ " $^{\prime}$ - aizing radiation.

\section{REFERENCES}

1. Yang, T.C., Craise, L.M., Mei, M., and C.A. Tobias. Neoplastic cell iransformation by heavy charged particles. Radiat. Res. 104 (1985) (in press).

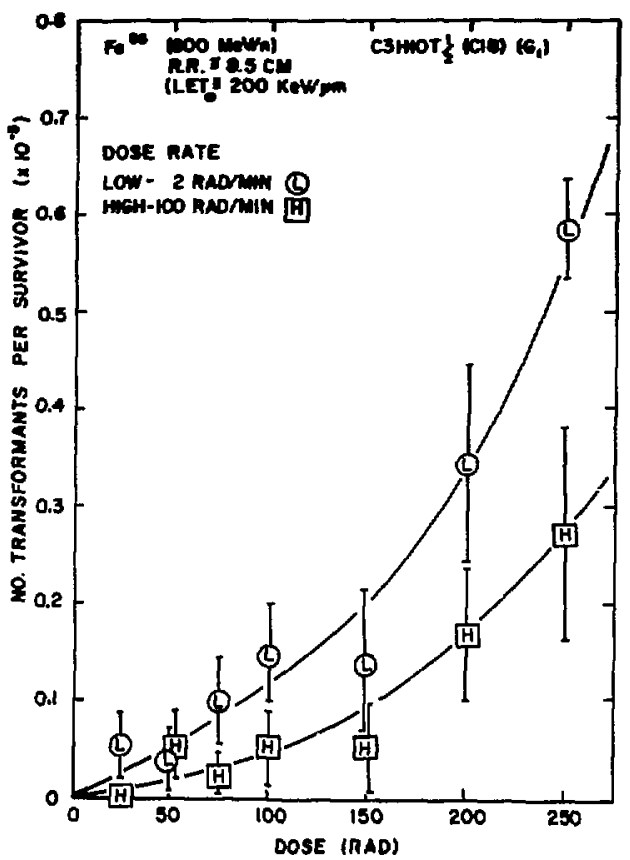

Fis. 2. An enhancement effect of a low dose rate of iron particles on cell iransformation. Coniluent $\mathrm{C} 3$ HIOT1 $/ 2$ cells irradiated with a low dose rate (2-rad/min) showed it significantly higher transfornation trequency than those exposed to high dose-rate iron particles $(100 \mathrm{rad} / \mathrm{min})$.

(XBL 859-3859)

2. Han, A., Hill, C.K., and Elkind, M.M., Repair of neoplastic transformalion damage following protracted exposure to ${ }^{60} \mathrm{Co}$ gamma rays. $B r$. J. Cancer 49. Suppl. 4, 91-96 (1984).

3. Hill, C.K. Buonagurs, F.M., Myers, C.P., Han A., and Elkind, M.M. Fission-spectrum neutrons at reduced dose rales enhance neoplastic Iransformation. Nalure 298, 67 (1982).

\section{CHEMCAL MODNACATION OF NEOMASTC CQU TMANSFOMNATHON BY HEAVY ION Bantion}

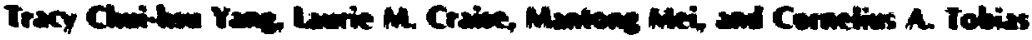

The obective of thes proiect is to obting quinti.

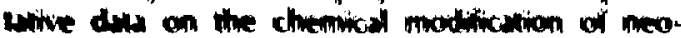

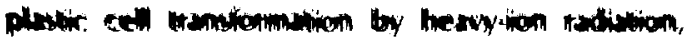

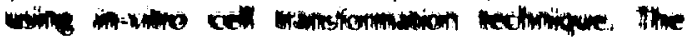

spexific aims ane il) to thes the potential effects of vatious chernicats on the expression of cell thanshormution, and 2) wo systematically comect information on the mecthunismos of expte: 
of cell transformation by ionizing radiation. Our recent experimental studies , with DMSO, 5azacytidine, and dexamethasone suggest that DMSO can effectively suppress the neoplastic cell transformation by high-LET radiation and that some nonmutagenic changes in DNA may be important in modifying the expression and progression of radiation-induced cell transformation.

Experimental observations on the modulation of radiation cell transtormation indicate that the process of neoplastic cell transformation is a complicated one and includes at least two different states: induction and expression. The induction is a relatively short one and may be complete after one cell division. The expression stage, however, will usually take several weeks and can be modulated by various physical and chemical agents. In our laboratory we have observed and reported earlier that fungizone (amphytericin B), an antibiotic tor fungus, and dimethyl suifoxide (DMSO) can decrease the frequency of cell transformation by $x$ rays. ${ }^{1.2}$ Even many days after x-irradiation, the presence of a low concentration of DMSO can significantly inhibit the neoplastic cell transformation by radiation. It was unknown, however, whether DMSO would have a similar effect on cells irradiated with high-LET radiation. Recently we did cell transformation experiments with energetic iron particles $(300 \mathrm{MeV} / \mathrm{u} ; \sim 500 \mathrm{keV} / \mu \mathrm{m})$ and found a significant decrease of cell transformation frequency in cells irradiated and treated with $0.5 \%$ DMSO for 1 week, starting 10 days after irradiation (Fig. 1). DMSO appears, therefore, very effective in suppressing the cell transformation by low- and high-LET radiation.

How DMSO suppresses the expression of radiation-induced cell transformation is an interesting question. There are several possible mechanisms: 1) altering the cytoplasmic membrane structure and functions, 2) acting on DNA to induce cell ditferentiation, and 3) reducing cellular free radicals that may pronsole the expression of cell transformalion. For a belter understanding of the mechanisms, we have investigated the potential efiecls of various themicals on neoplastic cell transionnation. These chersicals ane known to aci specifically on certain cellular onganelles or macromolecules. The experimental resulls from studives with DMSO. 5 . azacytidine, and dexamethasone are shown in Fing.

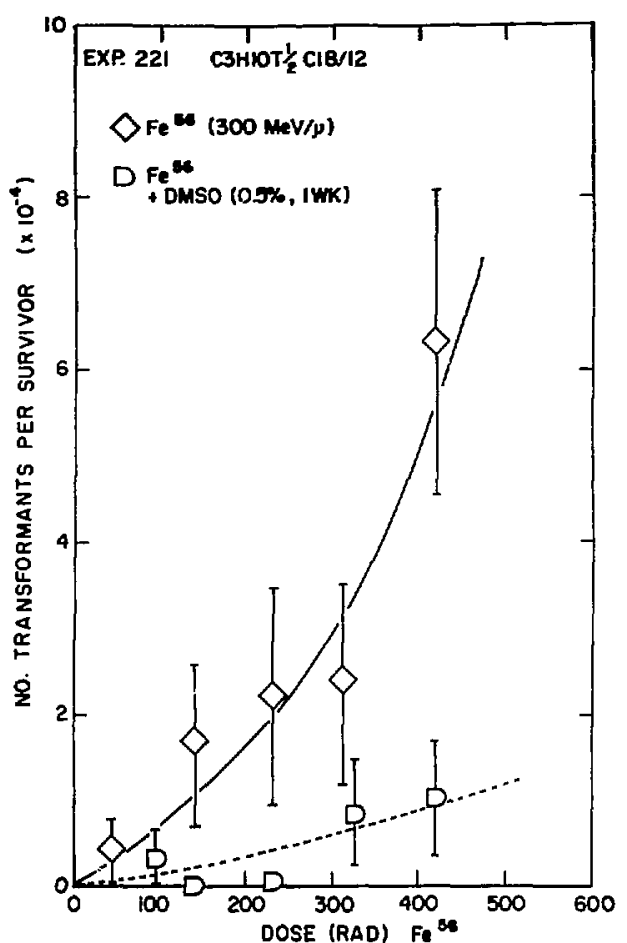

Fis. 1. Effect of $0.5 \%$ DMSO on transiormation of cells irradialed wilh ${ }^{56} \mathrm{Fe}$.

(XBL 846-2450)

2. Both DMSO and 5-azacytidine caused a significant decrease of Iransformation frequency. The dexamethasone, however, showed no effect on the cell transformacion by silicon ions $(320 \mathrm{MeV} / \mathrm{u}$; $\sim 88 \mathrm{keV} / \mu \mathrm{m})$. It has been reported that the possible targets for 5-azacylidine and dexamethasone are DNA and cell membrane receptors, respectively. ${ }^{3.4}$ The efiect of DMSO and 5-azacytidine on the progression of cell transiormation is interesting because neither chenical has been found to be mulagenic at the concentrations we used. Our results indicate that some nonmutagenic changes in the cellular DNA can intertere with the expressicn and progression process. The mechanism of progression and expression of Iransiomation, however, may be quite complicated; further sludies ane needed for a complete understanding of these processes. 


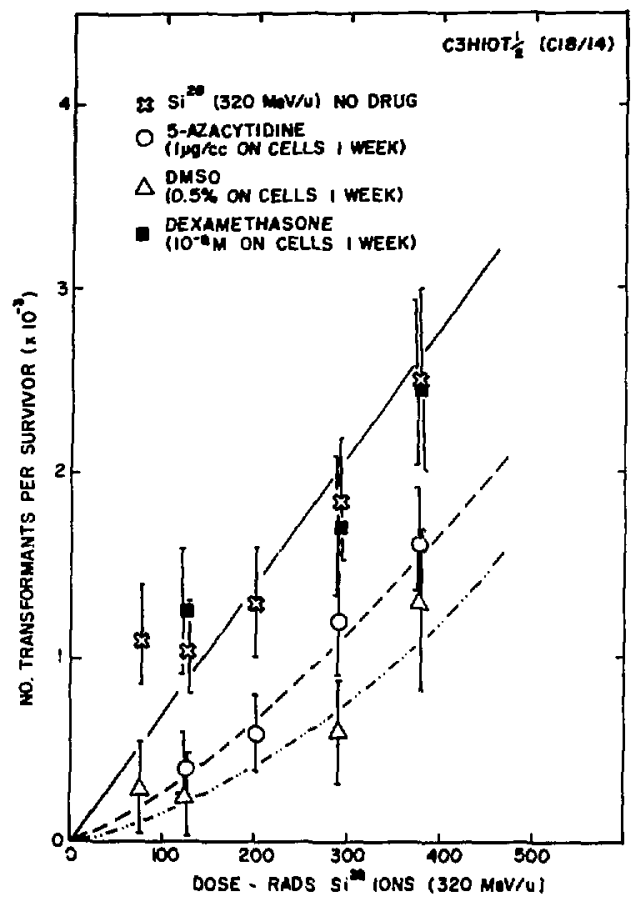

Fig. 2. Eifect of various drugs on transformation of ${ }^{28} \mathrm{Si}$ irradiated $\mathrm{C} 3 \mathrm{HIOT}(1 / 2) \mathrm{cel} / \mathrm{s}$.

\section{REFERENCES}

1. Yang, T.C., and Tobias, C.A. Neoplastic cell transformation by energetic heasy ions and its modification with chemical agents. $A d v$. Space Res. 4, 207-218 (1984).

2. Yang, T.C. and Tobias, C.A. Mechanisms of radiation-induced neoplastic cell transformation. Proceedings of Berkeley Conference in Honor of Jerzy Neyman and Jack Kiefer. Vol. 1. L.M. LeCam and R. A. Olshen, Eds. Wadsworth, Inc., pp. 343-366 (1985).

3. Chiu, P., and Blau, M.M. 5-Azacytidine permits gene activation in a previously noninducible cell type. Cell 401, 417-424 (1985).

4. Roberts, A. S., Anzano, M. A., Lamb, L. C., Smith, J.M., and Sporn, B.M. Antagonistic actions of retinoic acid and dexamethasone on anchorage-independent growth and epidermal growth factor binding of normal rat kidney cells. Cancer Res. 44, 1635-1641 (1984).

\title{
CELL AGE DEPENDENT VARIATIONS IN OXIDATIVE PROTECTIVE ENZYMES
}

\author{
Eleanor A. Blakely, Polly Y. Chang, Leora Lommel, and Cornelius A. Tobias
}

Oxidalive stress caused by reactive oxygen internediales (ROI) cam be produced in mammalian cells by ionizing radiation as well is by several radiomimetic asents (e.g., peroxides, etc.). Mammalian cells possess enzynes capable of enzymalically detoxinying both oxygen radical species and hythogen peroxide, resulting in a decrease in ROI and a restomation of the homeostatic milieu of the cell. Most mamamaliam cells exhibil distranct cell-

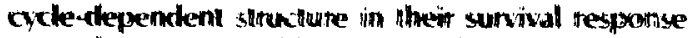
to oxülative stress, allthough a moleccular mechanis-

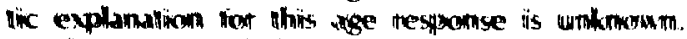

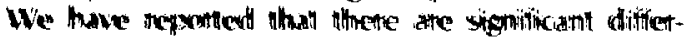

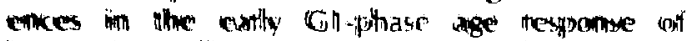

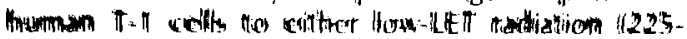

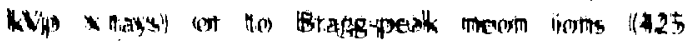

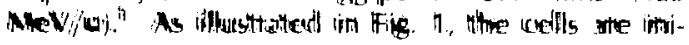

tially sensitive $10 \times$ rays in early $G_{y}$-phase. but then become increasing resistanl until approximately $\mathbf{4 . 5}$ hours posinitosis whon they again becme radiusensitive. In comtrast, the survival response of the synchronized cell populations at equivalent cell killing dflet Bragg-peak neon ions indicates an initial natlioresistance that decteases conlinuously as the cells enler $\$$ - phthase.

We atre all ampling to comrelate activity levels of antinoxindath enzymes belone and alter heavy-ion exposutes witt cellulat radiosensitivally. In prelimimary treasilutity experimenls with human $T_{+}-1$ cells

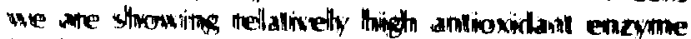

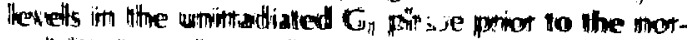

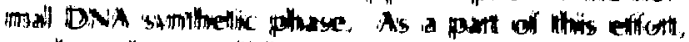

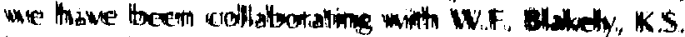

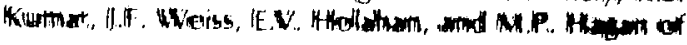




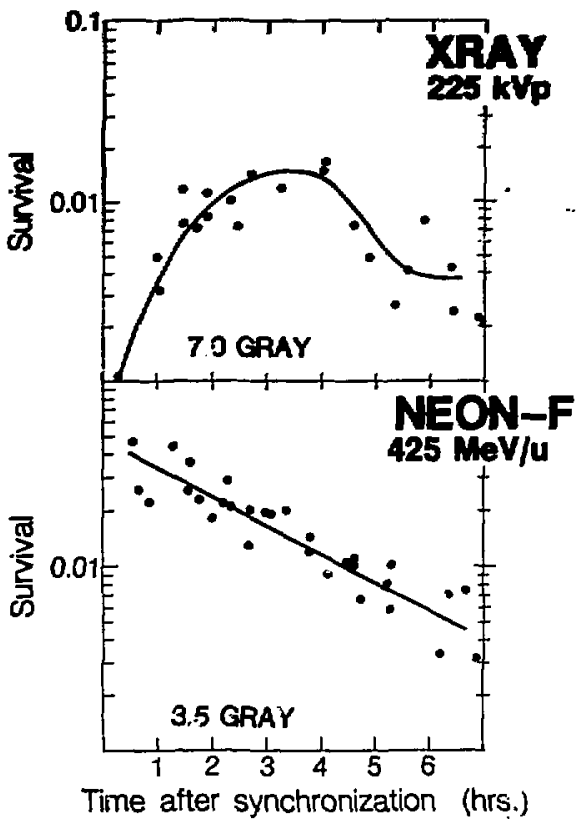

Fig. 1. Composite plot of agc response experiments with synchronized human $T-1$ cell populations exposed to either 7 Gray $x$ ray or 3.5 Gray neon ions.

(XBL 858-3349)

the Armed Forces Radiobiology Research Institute (AFRRI). Endogenous cellular levels of three antioxidant enzymes are being measured at various times in the unirradiated human $T_{-1}$ cell division cycle. The enzymes measured were: catalase (CAT), superoxide dismittase (SCD), and glutathione peroxidase (GSHPX).

Unlike the case in Chinese hamster V7a cells ${ }^{2}$ the early data with the synchronized human cell show that in very early $G_{1}$ phase (e.g., approximately 1.5 hours after mitolic selection) there are significant peaks in the le:r!s (U/mg cell protein) of both CAT and SOD (see Fig. 2). Boih enzymes show increases as the unirradiated cells progressed from milosis into $G$, phase while the levels of GSHPX measured in duplicale samples are somewhat more variable than was the case for the other two enzymes. These measurements are currently being replicated for confirmation before progressing to radiation studies. This work will primarily be completed with : tays and the Brags peak of the 425-MeV/o neon beam as a representative heavy ion, since we have well characterized the response of the $\mathrm{T}-1$ cell to damage from these radiations.

The human cell data have prompled as more

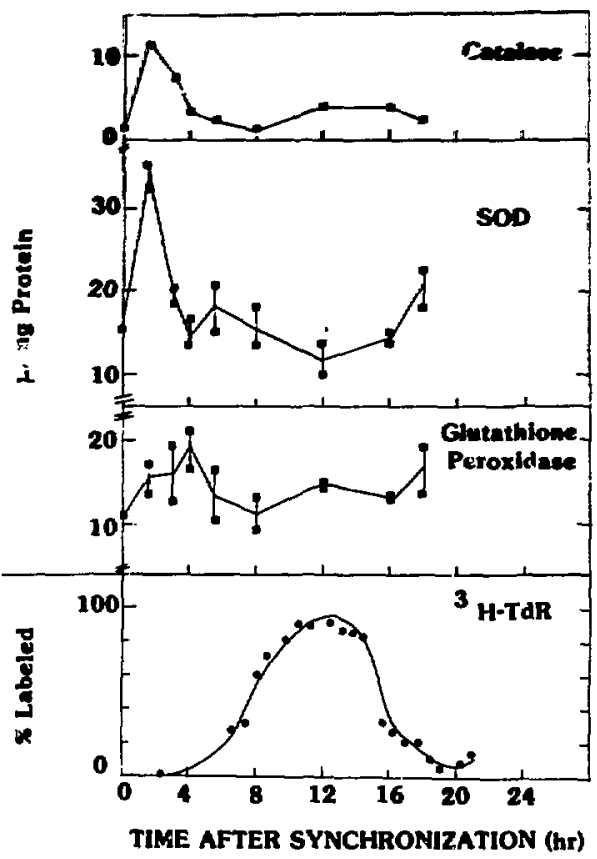

Fig. 2. Antioxidant enzyme levels measured at various ages in the unirradiated, synchronized human $T-1$ cell cycle. For reference, the bottom panel shows the incorporation of tritiated thymidine to identify cell cycle parameters. The three enzymes measured include: catalase, superoxide dismutase, and glutathione peroxidase.

(XBL 8412-5128A)

detailed analysis of enzyme levels during the -1 hour of $G_{1}$ phase in synchronous V79 cells, and will permit a comparison of enzyme activity levels in human and rodent cell lines. ${ }^{3}$ The findings are important to an understanding of the intrinsic cellular defense mechanisms that respond to an oxidative challenge because of the possibility of distinct cell-cycle population effects.

\section{REFERENCES}

1. Blakely, E.A., Chang, P.Y., and Lommel, L. Cell-cycle-dependent recovery from heavy-ion damage in $\mathrm{G}_{1}$-phase cells. Radiat. Res., 104: Supplement 8 S145-S157 (1985).

2. 'ikely, W.F., Kumar, K.S., Holahan, E.V., Hagan, M.P., Weiss, J.F., Jacocks, H.M. Intrinsic cellular mechanisms of resistance to oxidalive damage: ase response. Radiation Research Society Meeting, Los Angeles, CA (1985). Abstract. 
3. Blakely, W.F., Kumar, K.S., Blakely, E.A., Holahan, E.V., Hagan, M.P., Weiss, J.F., Chang P.Y., Sancho, A., Lommel, L., Hollies, C.L., and Tobias, C.A. Age response for oxi- dative profective enzymes: comparison of a human and rodent cell line. Radiation Research Society Meeting, Las Vegas, NV (1986). Abstract.

\section{RESPONSE OF AEROBIC AND HYPOXIC HUMAN LUNG SQUAMOUS CARCINOMA CELLS TO NEON IONS}

\section{Eleanor A. Blakely, Hiroshi Ohara, Polly Y. Chang, and Leora Lommel}

During the past year we began a collaborative study of the response of human squamous carcinoma cells to high-energy $(425 \mathrm{MeV} / \mathrm{u})$ neon-ion beams. The cell line was established in Japan in 1983 from a surgical specimen taken from a tumor mass.

Using techniques developed in our laboratory, monolayers of the asynchronous lung-tumor cells were grown in glass petri dishes, irradiated with either $150-k V p \times$ rays or Bragg-peak neon ions under controlled conditions of full aeration or hypoxia, and then trypsinized and plated to evaluate clonogenic potential.

The survival results of the experiment are presented in Fig. 1. Computer-assisted fits of the data have been made with the linear-quadratic model of cellular inactivation. The calculation of the aerobic RBE at $10 \%$ survival is $1.8 \pm 0.03$, and the $x$-ray oxygen enhancement ratio (OER) is $3.0 \pm$ 0.2 , while the neon OER value is $1.3 \pm 0.04$. These data with the human lung carcinoma cells confirm the enhanced cell killing and reduced oxygen effect previously reported for human T-1 fibroblasts at an LET of $183 \mathrm{keV} / \mu \mathrm{m} .^{\prime}$ In addition to the tumor-cell studies, experiments to measure the induction of chromosome aberrations in human blood cells in the plateau and distal end of an extended Bragg peak of a $425-\mathrm{MeV} / \mathrm{u}$ neon beam have been completed and are being analyzed. The results of both sets of experiments reported here will contribute to an evaluation of the clinical

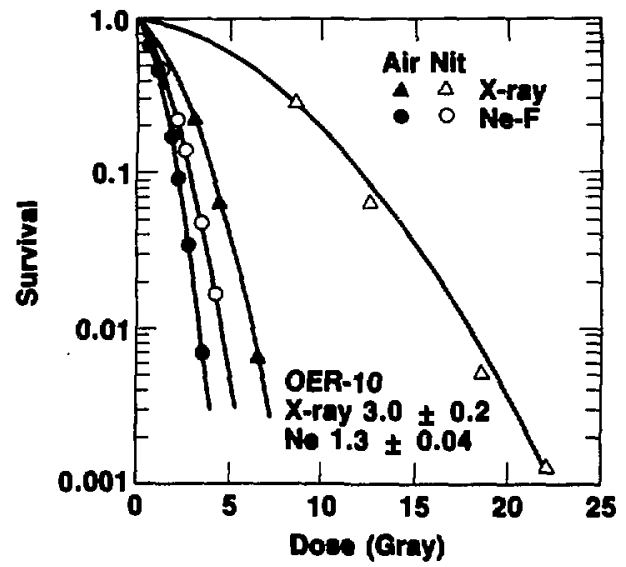

Fig. 1. Cellular survival in air or nitrogen of human lung squamous carcinoma cells exposed to $150-\mathrm{kV} p \times$ rays or Braggpeak 425-MeV/u neon ions.

(XBL 854-J1020)

potential of heavy-ion beams for the design of a medical accelerator in Japan.

\section{REFERENCES}

1. Blakely, E.A., Tobias, C.A., Yang, T.C.H., Smith, K.C., and Lyman, I.T. Inactivation of human kidney cells by high energy monoenergetic heavy-ion beams. Radiat. Res. 80 . 122-160 (1979). 


\title{
MULTHLE CHROMATIN RREAKS MODUCED BY NEON IONS
}

\author{
Edwin H. Goodwin, Eleanor A. Bakely, and Cornelius A. Tobias
}

There is a possibility that multiple chromatin breaks will occur within a cell nucleus from the passage of a single high-LET particle, but this has been difficult to examine in interphase nuclei under a light microscope because the chromatin is too diffuse to be seen. We have used a technique known as PCC, or premature chromosome condensation, to visualize interphase chromatin fragments and to score radiation-induced chromosome breaks without having to wait until the chromatin condenses at metaphase. Briefly, irradiated cells were fused to unirradiated mitotic $\mathrm{CHO}$ cells using polyethylene glycol in a modified version of a protocol developed by Pantelias and Maillie. ${ }^{2}$ As the cytoplasms merge, factors from the mitotic cell act upon the interphase chromatin causing it to condense "prematurely." These chromosome-like bodies, often called PCC fragments, can be seen in an ordinary microscope after fixation and staining by a modification of the procedure of Cornforth and Bedford. ${ }^{3}$ The unique value of the PCC technique is that it can be used to detect chromatin breaks in individual interphase cells within 30 minutes after irradiation.

Plateau-phase Chinese hamster ovary $\mathrm{CHO}$ TSH1 cells were exposed to single doses (30 to 240 $\mathrm{rad})$ of either $150-\mathrm{kVp} \times$ rays or neon ions accelerated at the Berkeley Bevalac. The particles had an initial energy of $425 \mathrm{MeV} / \mathrm{u}$ and a mean LET of $183 \mathrm{keV} / \mu \mathrm{m}$ near the Bragg ionization peak where the cell cultures were placed. Only those cells that were in the $G_{1} / G_{0}$ phase of the cell division cycle at the time of irradiation were scored. The $\mathrm{CHO}$-TSH1 cell line has a modal chromosome number of 21. The unirradiated controls, as expected, had an average of 21 PCC fragments per cell, while irradiated cells showed a dosedependent increase in fragment number with one chromosome break occurring for each 17 rad of neon-ion dose or 20 rad of $x$-ray dose. X-rayinduced iragments per cell had a Poisson distribution. The observed frequency of neon-jon-induced iragments was not Poisson, but rather showed multiple peaks and valleys." The anomalous distribution can be explained by assuming that a single heavy ion passing through the cell nucleus is capable of producing several chromatin breaks (see Fig. 1).
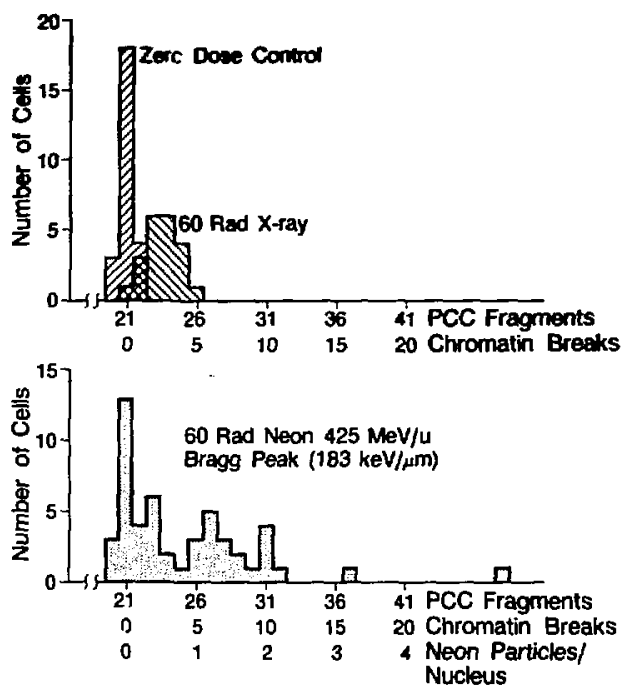

Fig. 1. Distribution of interphase chromatin fragments in Chinese hamster ovary CHO.TSH1 cells. (Upper panel) Distribution of fragments in control, unirradiated cells with a mean number of 21, and the distribution of additional fragments after 60 rad of $x$ rays. (Lower panel) Distribution of fragments in cells irradiated with Bragg-peak neon ions. The number of chromatin breaks and the number of neon traversals per cell have been calculated.

(XBL 8511-8599)

When a single heavy ion crosses a cell nucleus, it may produce DNA strand breaks and chromatin scissions wherever the ionizing track structure overlaps chromatin structures. The multiple yield of such lesions depends on the radial distribution of deposited energy and on the microstructure of the distribution of DNA in the cell nucleus, both of which place an upper limit on the number of lesions that can be produced by a single particle. The number of lesions per nucleus per particle can be described by a binomial distribution. The number of particles crossing a cell nucleus can be described by Poisson statistics. Combined, they yield the Neyman A distribution. Scoring chromatin breaks in CHO cells exposed to neon ions at 183 keV/am, we have obtained results compalible with the Neyman A distribution. The maximum number of suitable crossings was found to be 5 per particle 
per cell nucleus. The repair-misrepair model (RMR) has earlier suggested the occurrence of multiple DNA lesions in single heavy-ion tracks, and the current studies are in agreement with the RMR model as well as the deep-sieve model used to interpret the radiobiology of very heavy accelerated nuclei. ${ }^{5}$

\section{REFERENCES}

1. Johnson, R.T., and Rao, P.N. Nature 226, 717 (1970).
2. Pantelias, C.E., and Maillie, H.D. Somafic Cell Genetics 9, 5, 533 (1983).

3. Cornforth, M.N., and Bedford, J.S. Chromosome 88, 315 (1983).

4. Goodwin, E., Blakely, E.A., and Tobias, C.A. Multiple chromatin breaks produced by single heavy ions. Abstract, Radiation Research Society Meeting, Las Vegas, NV (1986).

5. Tobias, C.A., Goodwin, E.H., and Blakely, E.A. Theoretical distributions of DNA lesions in heavy-ion tracks. Abstract, Radiation Research Society Meeting, Las Vegas, NV (1986).

\section{EFFECT OF INHIBITION OF PROTEIN SYNTHESIS ON THE DEVELOPMENT OF THERMOTOLERANCE}

Polly Y. Chang, Eleanor A. Blakely, and Ileana Gonzalez-Flores"

The response of mammalian cells to nonlethal doses of elevated temperatures is modified by the development of a transient resistance to subsequent heat challenges. ${ }^{1}$ The mechanism of this thermotolerant response is unknown but may be linked to the synthesis of a family of proteins called heatshock proteins. ${ }^{2,3}$

We became interested in studying the response of a protein synthetic mutant cell line to hyperthermia. ${ }^{4}$ Published investigations of the role of protein synthesis in the development of thermotolerance to heat killing have used either chemical inhibitors or environmental factors to modulate synthetic activity. We, however, have chosen to use a temperature-sensitive mutant line, CHO-TSH $1,{ }^{5}$ which shuts down protein synthesis at nonpermissive temperatures of $40^{\circ} \mathrm{C}$ and above by the inactivation of its cytoplasmic nonmitochondrial leucyltransfer RNA (I-RNA) synlhetase enzyme. The parent cell line, CHO-SC1, was used as the control for these experiments, Exponentially growing, asynchronous CHO-TSH1 and CHO-SC1 cell populations were treated for limes up to 8 hours at $41.5^{\circ} \mathrm{C}, 42^{\circ} \mathrm{C}$, and $42.5^{\circ} \mathrm{C}$ (Fig. 1a). The wild-type cells showed the development of tolerance to heat killing at $41.5^{\circ} \mathrm{C}, 42^{\circ} \mathrm{C}$, and possibly at $42.5^{\circ} \mathrm{C}$, although the survival level at which tolerance developed at $42.5^{\circ} \mathrm{C}$ was too low to be statistically signiticant. The CHO-TSHI mutant cell showed ro tolerance at any of those temperatures (Fig- Ib).

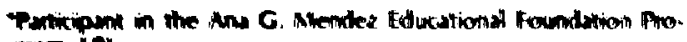
gram, ide.
We measured"the rate of total protein synthesis in both cell lines in pulse-labeling experiments with ${ }^{3} \mathrm{H}$-leucine under the conditions of our experiment. Results indicated that the rate of synthesis dropped precipitously within the initial hour of exposure to $42^{\circ} \mathrm{C}$ and remained low during the 3 hours of $42^{\circ} \mathrm{C}$ treatment (Fig, 2). When each cell line was returned to $35^{\circ} \mathrm{C}$ after the 3 -hour treatment at $42^{\circ} \mathrm{C}$, protein synthesis immediately resumed and eventually returned to control levels after 7 hours at $35^{\circ} \mathrm{C}$.

We have also looked at the effect of resumption of protein synthesis on cellular survival by using split-temperature treatments. The cells were exposed to $42^{\circ} \mathrm{C}$ for 3 hours and then returned to the permissive lemperature $\left(35^{\circ} \mathrm{C}\right)$ for 20 hours. The cells were then subjected to additional time at $42^{\circ} \mathrm{C}$ (Fig. 3). The wild-type cell response to the split-lemperature treatment is identical to that obtained with the single heat trealment, indicating that incubation at $35^{\circ} \mathrm{C}$ between $42^{\circ} \mathrm{C}$ heat treatmenis is not necessary for the development of tolerance. Inteiestingly enough, the Iemperaturesensitive mutant cell appears also to have developed a small amount of tolerance under splitdose conditions. When the incubation lime at $35^{\circ} \mathrm{C}$ belween split-42 $\mathrm{C}$ exposures was increased to 44 hours, we obsenved a decay of tolerance in both cell lines.

In summary, we have demonstrated loss of thermotolerance in a temperature-sensitive protein synthetic mutant. We hope to extend this work 

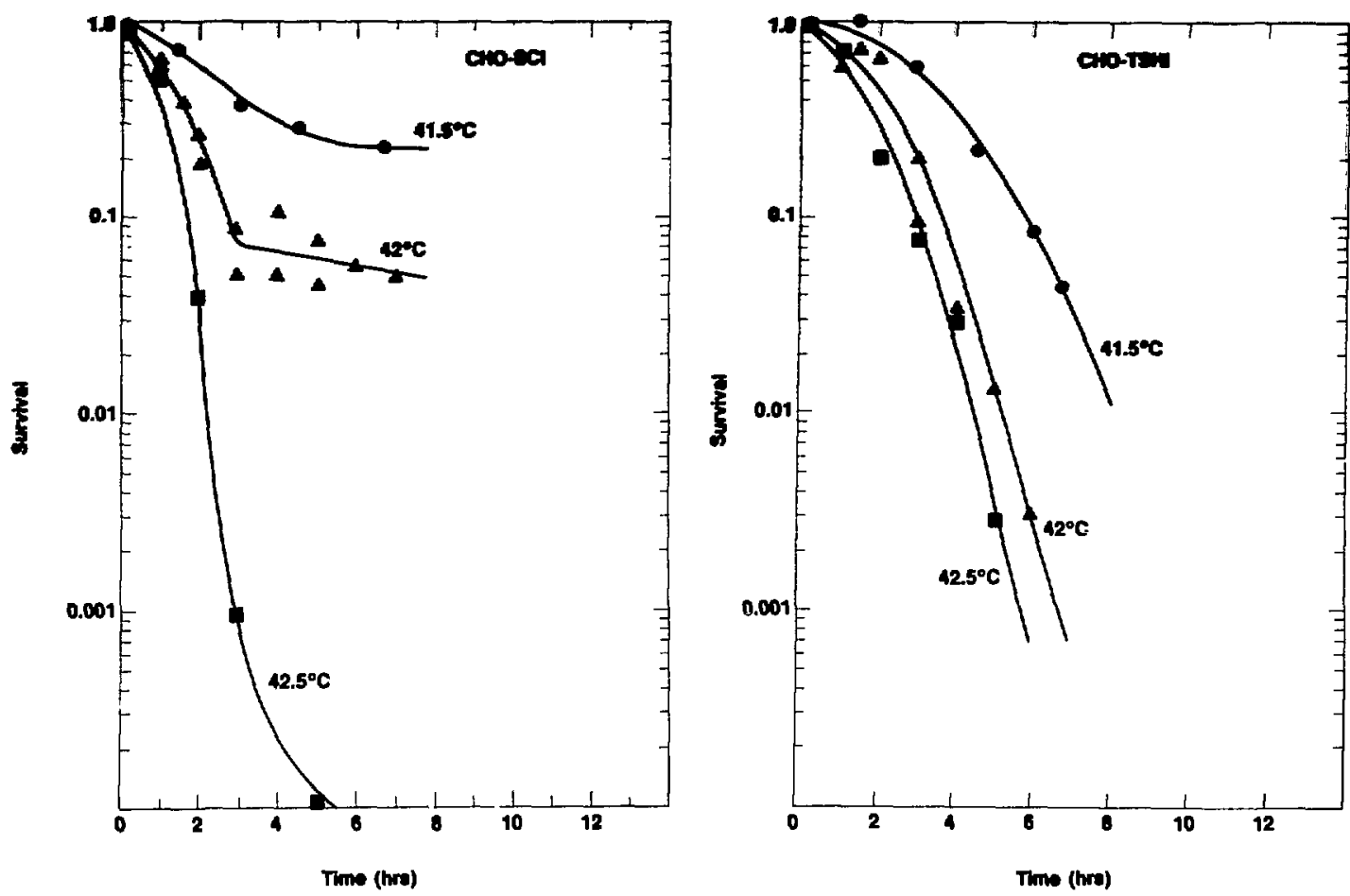

Fig. 1. CHO-SCl (wild-type) cells show thermotolerance at $41.5^{\circ} \mathrm{C}$ and $42^{\circ} \mathrm{C}$. At $42.5^{\circ} \mathrm{C}$ these cells show tolerance but at a lower level of survival than could be accurately measured. CHO.TSHI (remperature-sensitive mutant) cells show no thermotolerance at $41.5^{\circ} \mathrm{C}, 42^{\circ} \mathrm{C}$ and $42.5^{\circ} \mathrm{C}, 42^{\circ} \mathrm{C}$ survival experiments were done with and without pust-heat-treatment trypsinization and show no differences in survival fractions.

(XBL 854-11072A)

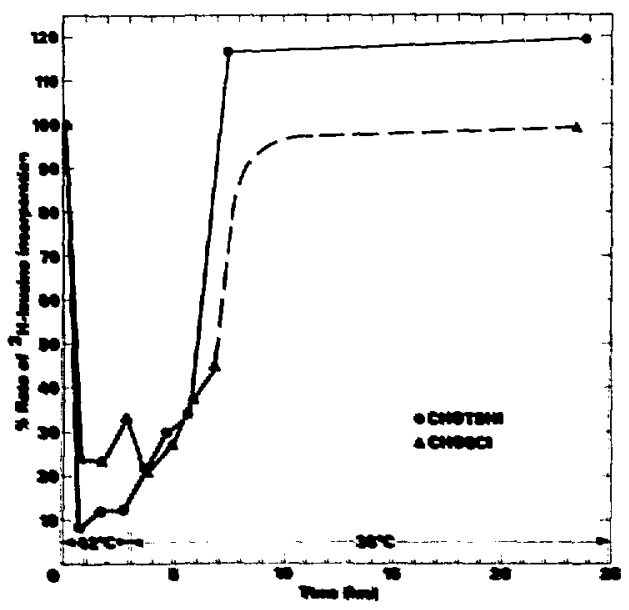

F. 2. Cells are tresled for $3 \mathrm{hr}$ at $12^{\circ} \mathrm{C}$ and retumed to $35^{\circ} \mathrm{C}$ for 23 hr. Protein synthesis, as measured by 'fflewcine upiske, is shut down inmedivity wher both cell tppes mere exposed to

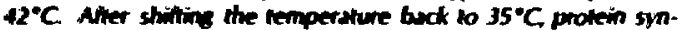
thesis is immedistely resumed, eventurly resching control levets when 7 he $335^{\circ} \mathrm{C}$

(x) $054-2721)$ 

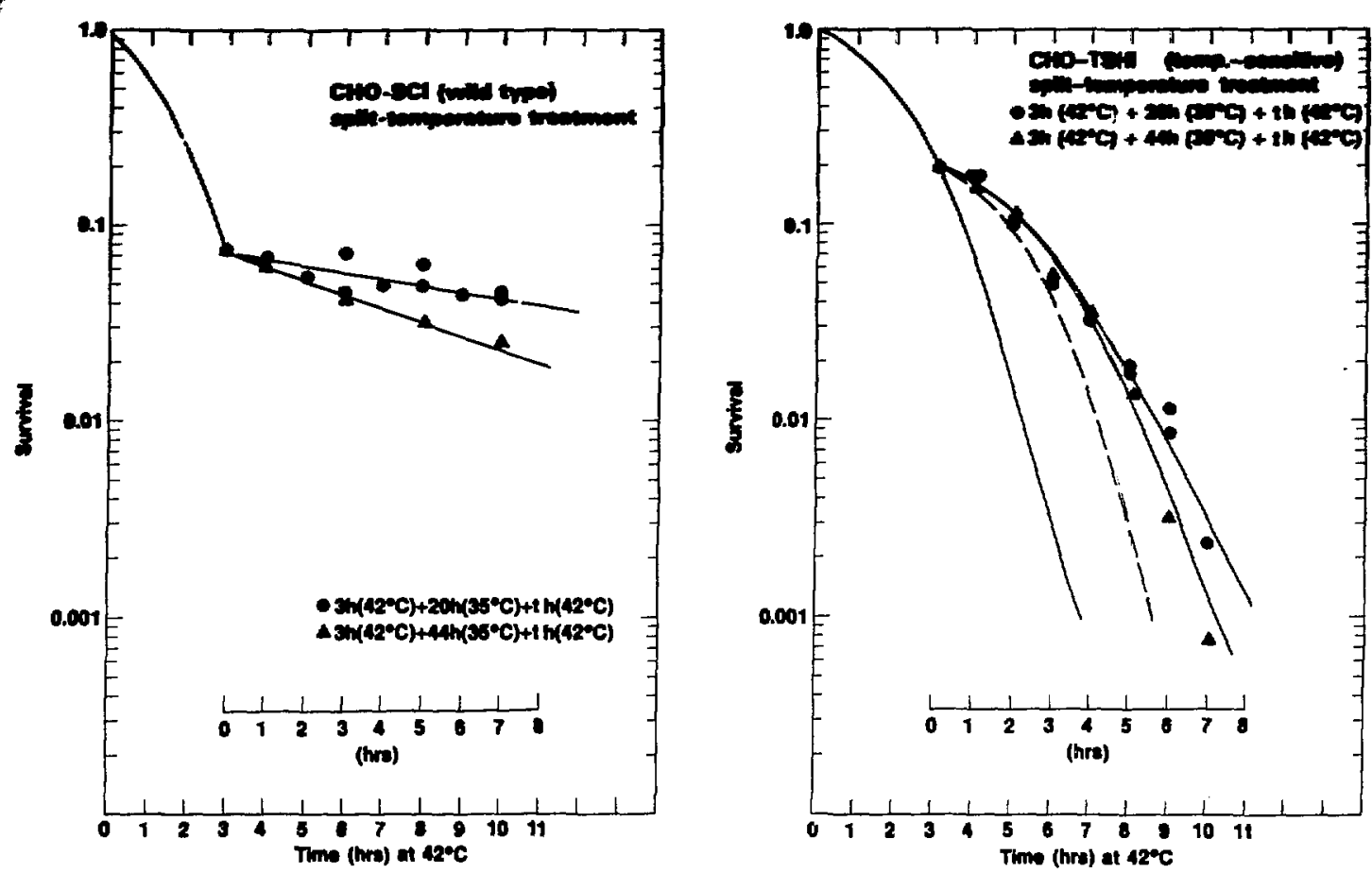

Fig. 3. Split-temperature studies are done where cells are exposed to $42^{\circ} \mathrm{C}$ for $3 \mathrm{hr}$, returned to $35^{\circ} \mathrm{C}$ for $20 \mathrm{ht}$ or $44 \mathrm{hr}$, and then exposed to a second $42^{\circ} \mathrm{C}$ dose for the times indicated on the abscissa. CHO-SC1, when allowed to recover for $20 \mathrm{hr}$, demonstrate a tolerant curve identical to that of the single cemperature treatment. After $44 \mathrm{hr}$ of incubation at $35^{\circ} \mathrm{C}$ between doses, a slight decay in tolerance is observed. CHO-TSHt did not show thermofolerance to the extent that the wild-type cells did, but survival data is obviously higher than if complete recovery from sublethal damage had taken place las shown by dotted line). With $44 \mathrm{hr}$ of incubation at $35^{\circ} \mathrm{C}$ between doses of $42^{\circ} \mathrm{C}$, a slight decay in tolerance is also observed.

(XBL 854-11074A)

furthe: by examining the difference in response of tolerant versus nontolerant cells to rediation.

\section{REFERENCES}

1. Henle, K.J., and Dethlefsen, L.A. Heat fractionation and thermotolerance: $A$ review. Cancer Research 38, 1843-1851 (1978).

2. Li, G.C., Petersen, N.S., and Mitchell, H.K. Induced thermotolerance and heat shock protein synthesis in Chinese hamster ovary cells. Int. I. Radiat. Oncol. Biol. Phys. 8, 63-67 (1982).
3. Hahn, G.M., and Li, G.C. Thermotolerance and heat shock proteins in mammalian cells. Radiat. Res. 92, 452-457 (1982).

4. Chang, P.Y., Gibbs, G.F., and Blakely, E.A. Loss of thermotolerance in a temperaturesensitive protein synthesis mutant. Radiation Research Society Meeting. Los Angeles, May 1985. (Abstract)

5. Thompson, L.H., Harkins, J.L., and Stanners, C.P. PNAS 70, 3094-3098 (1973). 


\title{
CEI CYCLE KINETICS AND IN VNO MCRONUCLE INDUCTION IN IAT BHAROMMYOSARCOMA TUMONS USING A MONOCLONAL ANTIOODY TO RrdURd AND CEll SOnTmic
}

\author{
Michael Nituse, S.M. Javed Afzal, Retsy C. Carr, Kristina S. Kavanau, \\ Thomas S. Tenforde, and Stanley B. Curtis
}

Redistribution of tumor cells within the cell cycle after irradiation is an important factor affecting the speed of tumor regrowth. Therefore measurement of the movement of both the total and the clonogenic population of tumor cells in vivo through the cell cycle as a function of time after irradiation is of special interest. Blocks at certain stages of the cycle, recruitment of resting cells into the proliferating compartment, and variations in the length of the G1-, S- and G2-phases are important effects induced in the tumor cell population by irradiation. Knowledge of the relative importance of such effects is critical to the identification of the dynamic processes that determine how the tumor as a whole responds to irradiation.

Flow cytometric techniques as well as labeling techniques with radioactive DNA precursors are widely used to study cell cycle kinetics of cell populations in tumors. Besides technical problems, the main disadvantage of the FLM (fraction of labeled mitoses) or $\mathrm{Ll}$ (labeling index) methods is that, in many tumors, only a fraction of the cells progress through the mitotic cycle. A similar problem occurs when the flow cytometric technique of DNA distribution analysis is applied in tumors. It is not possible to discriminate between cycling and noncycling cells.

A new flow cytometric technique was recently developed that reduces some limitations of earlier methods used for measurements of cell kinetics.' The method involves the use of a fluorescent monoclonal antibody to bromodeoxyuridine (BrdUrd) that attaches to BrdUrd incorporated into cells during S-phase in addition to the DNA stain propidium iodide which is used to estimate cellular DNA content. This technique allows the study of the progression of cells labeled during S-phase with BrdUnd and, in addition, discriminates between ONA synthesizing and nonsynthesizing cells in the case where both have a DNA content characteristic of S-phase cells.

The aim of the experiments reponted here was to investigate the applicability of this BrdUnd/ONA technique to a rat ihabdomyosarcoma lumor system growing in wivo and to study radiation-induced charges in the progression of cells through the cell cycle. Detrils of this technique are described else. where. ${ }^{2}$ in addition, the induction of micronuclei in tumor cells irradiated in vivo with $x$ rays or peak neon ions was studied. Micronuclei found in interphase cells after irradiation represent genetic material that is lost from the genome of the cells during mitosis. ${ }^{3}$ The formation of micronuclei that can mainly be ascribed to acentric chromosome or chromatid fragments occurs orly after cells go through one or more cell divisions. Cycling cells in the tumors were therefore continuously labeled with BrdUrd, and micronuclei induction was measured only in tetraploid cycling tumor cells using the flow cytometric cell sorting technique.

THE RHABDOMYOSARCOMA TUMOR: DIPLOID HOST CELLS AND TETRAPLOID TUMOR CELLS

To analyze the cell cycle kinetics of the rhabdomyosarcoma tumors (subline R2C5 of the rhabdomyosarcoma $R-1$ tumor which was implanted subcutaneously into syngeneic WAG/Rij rats) using incorporation of BrdUrd, the composition of the various cell populations in this tumor was studied with DNA distribution analysis. It was known from observations of metaphase chromosomes that the tumor cells growing in vitro have a tetraploid DNA content. A possible contamination of the dissociated tumor cell suspension from tumors in vivo by diploid host cells could be easily measured using DNA distribution analysis. Figure 1 A (insert) shows a typical DNA distribution of the dissociated cells from this tumor. Using diploid lymphocytes from the spleen of the tumor-bearing rats, which were also stained and measured urder the same conditions, it could be shown that the first peak in the DNA distribution in Fig. IA represented diploid host cells in G1-phase (2c DNA content). The telraploid tumor cells in G1-phase had 4c DNA content. Fig. IA shows the proportion of tetraptoid tumor cells, $\mathrm{N}_{\mathrm{T}} / \mathrm{N}_{\text {rots }}$, in growing tumors as a function of lumor weight. Very small tumors were found to contain only about $8 \%$ of the tetraploid tumor cells. The fraction of setraploid tumor cells increased to a maximum of about $20 \%$ of the total cell number in large tumors. A possible contamination of the letraplaind G1-peak by diploid G2-cells has been neglected. However, using incorporation 


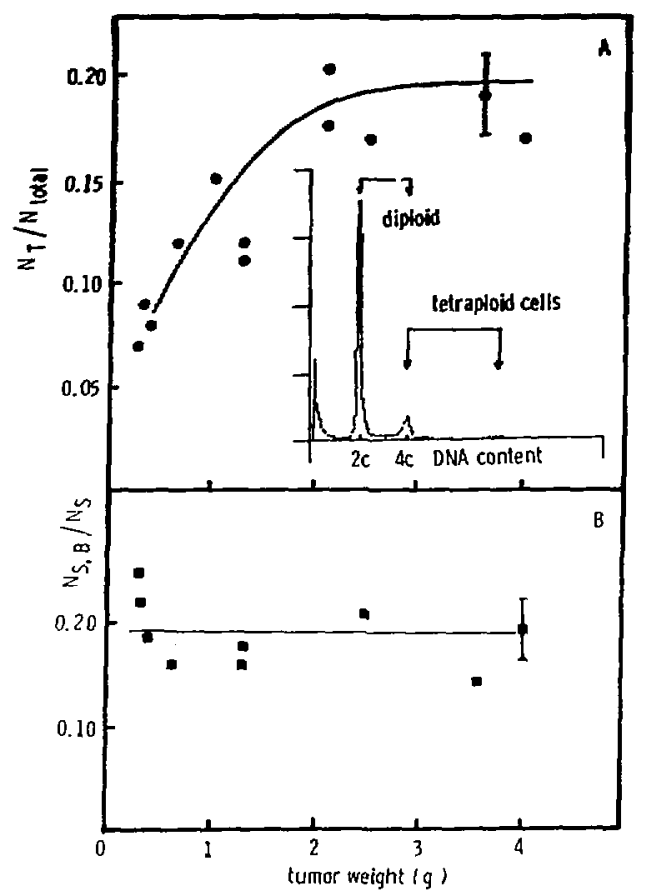

Fig. 1. (A) Fraction of tetraploid fumor cells $N_{T} / N_{\text {Total }}$ in dissociated suspensions of rat rhabdomyosarcoma cells as a function of tumor weight. Insert: DNA distribution of the dissociated cells of the lumor showing peaks for diploid host cells and tetraploid lumor cells. (B) Fraction of tetraploid tumor cells in 5-phase $\left(N_{S .8} / N_{\mathrm{s}}\right)$ that have incorporated BrdUrd at 2 hours afier an intraperitoneal injection of BrdUrd $1160 \mathrm{mg} / \mathrm{kg}$ body weight) as a function of fumor weight.

(XBL 8510-8542)

of BrdUrd in tumor cells growing in vitro ${ }^{2}$ the fraction of diploid G2-cells within the tetraploid G1peak could be estimated. A maximum of $20 \%$ of cells in the tetraploid $\mathrm{G} 1$-peak were actually diploid G2-cells. The reason for the high contamination of the tetraploid tumor cells by diploid host cells and the characteristics of these host cells are unknown at present. Probably the good vascularization of this tumor line was responsible for the observed effect. The results show that a study of cell cycle kinetics and of micronuclei induction in these tumors were hampered by the contamination with host cells. In the experiments described below. only the behavior of tetraploid tumor cells is discussed.

\section{PULSE LABELING AND CONTINUOUS LABELING OF TUMOR CELLS WITH Brd!Jrd}

For pulse labeling experiments with BrdUrd, tumor-bearing animals were injected intraperitoneally with $160 \mathrm{mg}$ BrdUrd per kilogram body weight. For continuous labeling experiments a "mini osmotic pump" (Model 2001, Alzet Co., Pako Alto, CA) was used. The pump, containing $200 \mu l$ of $0.3 \mathrm{~mol} / \mathrm{l}$ bromodeoxycytidine was implanted subcutaneously into the animal's back. Bromodeoxycytidine is converted intracellularly into BrdUrd in the organism. The advantage of bromodeoxycytidine is that it can be dissolved at a much higher concentration than that of BrdUrd, thus allowing a higher level of BrdUrd in the organism compared to that produced by a single or by repeated injections of BrdUrd.

Figure 2 (insert) shows a typical BrdUrd/DNA scatter plot of the tetraploid tumor cells (the diploid host cells were cut off for clarity) two hours after injecting $160 \mathrm{mg}$ BrdUrd per $\mathrm{kg}$ body weight. The cells were examined in the LBL FACS IV flow cytometer after staining the DNA of the cells with propidium iodide, and then defining the BrdUrd content of the cells using a monoclonal antibody to BrdUrd and a second fluorescein-conjugated goat anti-mouse antibody. The data were accumulated to form a bivariate $64 \times 64$ channel distribution (scatter plot) of DNA (red fluorescence from propidium iodide, abscissa) and BrdUrd (green fluorescence from fluorescein, ordinate) among the cells of the population.

From scatter plots such as the one shown in Fig. 2, the fraction of cells in S-phase that could incorporate BrdUrd after a single injection of BrdUrd $\left(N_{s, B} / N_{s}\right)$ was calculated. Figure $7 B$ shows this fraction as a function of tumor weight in unirradiated control tumors. Only about $20 \%$ of the Sphase cells were labeled with BrdUrd 2 hours after BrdUrd injection, and this percentage was independent of the tumor size. Figure 2 shows the fraction of labeled S-phase cells as a function of time after injecting BrdUrd. Four hours after injection, $N_{s, B} / N_{s}$ reached a plateau at a value of 0.25 . If tumors were irradiated with $20 \mathrm{~Gy}$ of $225-\mathrm{kVp} x$ rays, the fraction of labeled S-phase cells was even lower, probably because of a radiation-induced retardation of DNA-synthesis. This effect was also observed in experiments performed in vitro. Through observations of the scatter plots, we found 


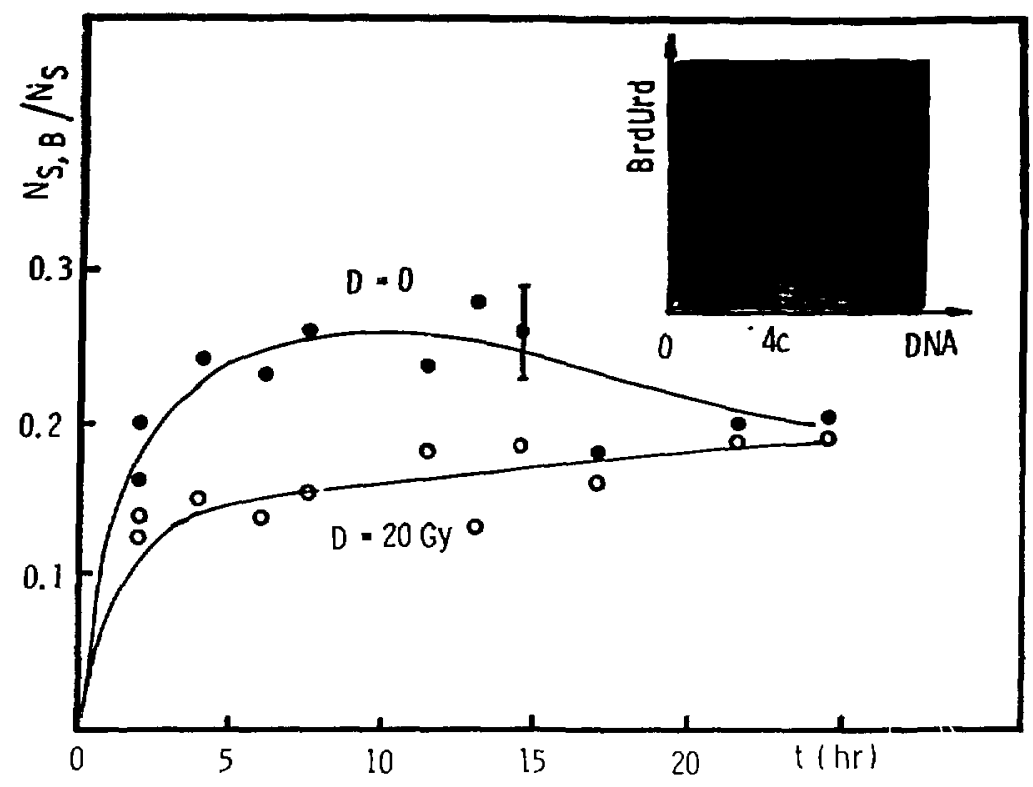

Fig. 2. Fraction of tetraploid tumor cells in S-phase $\left(N_{S . Q} / N_{S}\right)$ that have incorporated BrdUrd as a function of time after an intraperitoneal injection of BrdURD $(160 \mathrm{mg} / \mathrm{kg}$ bodyweight). Closed circles are unirradiated tumors; open circles are irradiated tumors $(20 \mathrm{~Gy}$ of $225-\mathrm{kVp} \times$ rays). The inserted scatter plot shows DNA and BrdUrd content of the tetraploid tumor cells 2 hours after injection of BrdUrd.

(XBL 8510-8543)

that, over 2 to 10 hours, the labeled cohort of Sphase cells moved into $(\mathrm{G} 2+\mathrm{M})$-phase. The fraction of labeled S-cells decreased at later times because some of these cells divided.

The rather low fraction of labeled S-phase cells in tumors is in contrast to similar experiments with these tumor cells growing in vitro which showed that all S-phase cells could incorporate BrdUrd after a 1-hour pulse of BrdUrd. Probably those cells in the in vivo tumor were not labeled because they were either resting or slowly synthesizing DNA. Some cells in S-phase may also not be labeled as a result of limitations in the transport of BrdUrd via blood vessels or through diffusion. The main disadvantage of the pulse labeling technique with BrdUrd in this rat rhabdomyosarcoma lumor is that it is not possible to distinguish whether cells in Sphase do not incorporate BrdUrd because they do not perform DNA synthesis or because there is no Brdund available to them. Therefore, experiments studying continuous labeling of tumor cells using an osmotic pump were performed.

Figure $3 A$ demonstrates the combined fraction of labeled $S$ - and $(G 2+M)$-phase cells as a function of time after implantation of the pump. A continuous increase of labeled cells as a function of time was observed. The fact that only $60 \%$ of S- and $(G 2+M)$-phase cells were found to be labeled even after 50 hours demonstrates that a rather large fraction of cells in this tumor is not cycling. These cells are probably resting cells or very slowly dividing cells in the tumor. Unfortunately, the fraction of resting $\mathrm{G1}$-phase cells could not be measured by this technique because of the overlap with a much higher fraction of diploid host cells in $(\mathrm{G} 2+\mathrm{M})$ phase that were also able to incorporate BrdUrd. ${ }^{2}$

In irradiated tumors (10 and $20 \mathrm{~Gy}$ of $225-\mathrm{kVp}$ $x$ rays, Fig. 3) the fraction of labeled $S$ - and $(G 2+M)$-phase cells reached a higher plateau than that of the controls. This effect is presumably caused by the radiation-induced G2-block in those cells of the tumor that were able to progress into the cell cycle. Figure $3 C$ shows the fraction of labeled $(G 2+M)$-phase cells for control tumors and irradiated lumors (20 Gy). Between 30 and 50 hours after irtadiation, about $85 \%$ of 62-phase cells became labeled in the irradiated tumors whereas onty $70 \%$ of these cells were labeled in the control 

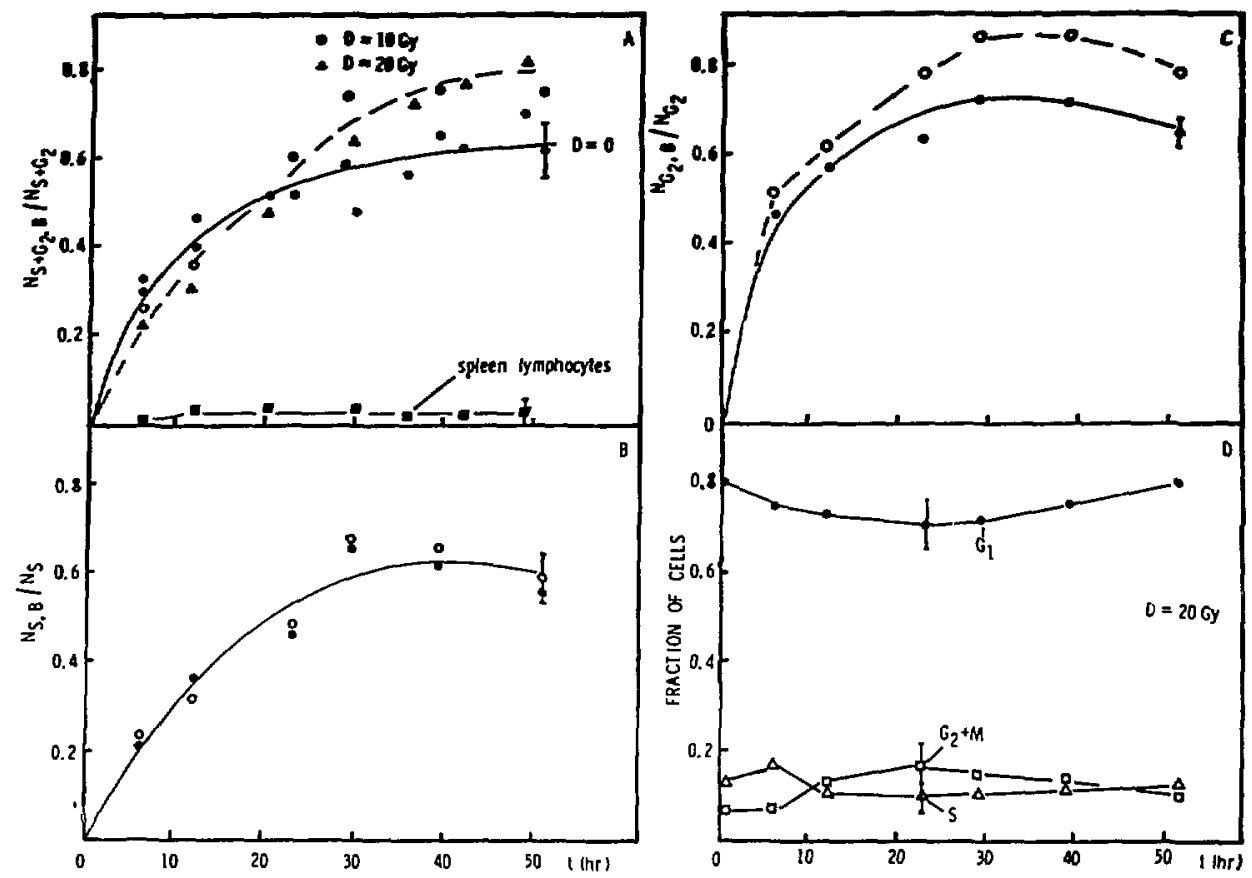

Fig. 3. (A) Fraction of cells in $(S+G 2)$-phase $\left(N_{S+C 2, b} / N_{s+C 2}\right)$ of tetraploid tumor cells that have incorporated Brd'Urd as a function of time alter implanting an osmotic pump. Closed circles are unirradiated control tumors; open circles and triangles are irradiated tumors. Closed squares represent fraction of cells in (S+G2)-phase in spleen Iymphocytes of the same animals. (B) Fraction of tumor cells in S-phase $\left(N_{S_{S},} / N_{S}\right)$ that have incorporated BrolUrd, as a function of time after implanting an osmotic pump. (C) Fraction of cells in $(G 2+M)$-phase $\left(N_{G 2, B} / N_{G z}\right)$ that have incorporated BrdUrd as a function of time after implanting an osmofic pump. (D) Fraction of tetraploid tumor cells measured by DNA distribution analysis in $G 1$-(closed circles), S. (open triangles) and (G2+M)-phase (open squares) as a function of time after irradiation with $20 \mathrm{~Gy}$ of $225-\mathrm{kVp} \times$ rays.

(XBL 8510.8544)

tumors. Whereas in the control tumors the labeled G2-phase cells divide, the labeled cycling G.:-phase cells in irradiated tumors are arrested in G2-phase for a certain time interval. Using DNA distribution analysis alone, the radiation-induced $\mathrm{G} 2$-block cannot be easily observed in this tumor because a large fraction of cells either do not progress into the cell cycle or are only progressing very slowly (Fig. 3D).

\section{MICRONUCLEI INDUCTION IN CYCLING IN VIVO TUMOR CELLS}

The experiments discussed above show that it is possible to discriminate between cycling and noncycline cells in solid tumors after continuous incorporation of BrdUrd by analyzing the dissociated cells in a flow cytometer usting a monoclonal antibody to Brdurd. The same rechnique in combinution with cell sorting was usted to study micronu-
Clei induction in irradiated tumors in vivo. Because micronuclei can only be observed in cells that have gone through at least one cell division after irradiation, cycling tumor cells were sorted according to their tetraploid DNA content and high BrdUrd content at $\mathbf{5 2}$ hours after irradiation, when most of the cycling cells had divided. Figure 4 (insert) shows BrdUrd/DNA scatter plots of cells in the tumor without BrdUrd (left panel showing control data to demonsirate the position of unlabeled cells) and after continuous incorporation of BrdUrd using an osmotic pump (right panel, where the sorted cells are indicated by the square). The cells were sorted with the High Speed Cell Sorter of the Lawrence Livermore National Laboratory the help of Dr. Joe Gray is cratefully acknowied bed). The fraction of micronucleated cells $N_{\text {man }} N$ in these sorted cells was measured in a fluorescence microscope. Fitswe 4 shows $\mathbb{N}_{\text {mand }} / \mathbb{N}$ as a function of dose for $\times$ rays 


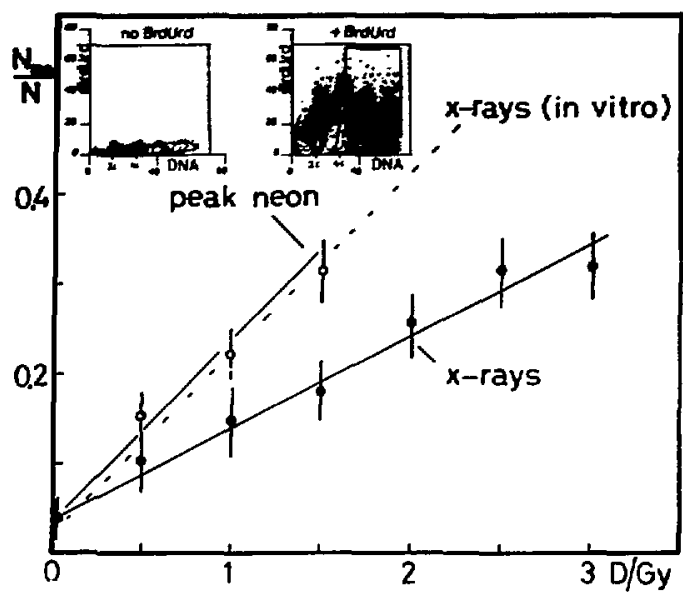

Fig. 4. Fraction of micronucleated cells $\left(N_{m n} / N\right)$ as function of dose in cells that were sorted from dissaciated tumor celis according to tetraploid DNA content and high Brdurd content (cycling tetraploid tumor cells). Closed circles are tumors irradiated with $x$ rays. Open circles are lumors irradiated with neon ions in the distal region of a $4-\mathrm{cm}$ spread peak. Dolled line represents exponentially growing cells irradiated in vitro. Insert: BrdUrd/DNA scatter plots of dissociated tumor cells. Lefi: no BrdUrd treatment, right: 52 hours after implanting the osmolic pump. The cells within the rechangular region denote those selected for micronucleus determination.

(XBL 8510-8545)

and in the distal region of a 4-cm spread peak of a neon-ion beam accelerated at the Bevalac (initial energy of $557 \mathrm{MeV} / \mathrm{u}$ ). A linear increase with dose was observed, similar to previous observations on micronuclei induction in exponentially growing in vitro cells. ${ }^{3}$ Comparing the in vivo $x$-ray results with those from in vitro experiments (dotted line) it can be seen that cycling tetraptoid tumor cells growing in vivo are less sensitive compared to in vitro cells. This is in accordance with cell survival data that also show a lower radiation sensitivity of tumors irradiated in vivo. The RBE value for neon ions in the distal portion of a $4-\mathrm{cm}$ spread peak is $2.2 \pm 0.5$, a value that is similar to that measured with in vitro cells. ${ }^{3}$

In conclusion, it has been shown that by using the BrdUrd/DNA technique in combination with cell sorting it was possible to study micronuclei induction in vivo in irradiated tumors, although this rhabdomyosarcoma tumor line had a large fraction of diploid host celis and contained both cycling and noncycling cells.

\section{REFERENCES}

1. Dolbeare, F., Gratzner, H., Pallavicini, M.G., and Gray, J.W. Flow cytometric measurement of total DNA content and incorporated bromodeoxyuridine. Proc. Natl. Acad. Sci. USA 80, 5573 (1983).

2. Nüsse, M., Afzal, S.M.J., Carr, B., and Kavanau, K. Cell cycle kinetic measurements in an irradiated rat rhabdomyosarcoma using a monoclonal antibody to bromodeoxyuridine. Cytometry 6, 611-619 (1985).

3. Nüsse, M., and Curtis, S.B. A test for micronuclei induction in rat rhabdomyosarcoma tumors after irradiation with $x$ rays and heavy charged particles. Biology and Medicine Division Annual Report 1983-1984, p. 114. Lawrence Berkeley Laboratory report LBL-18393 (1985).

\title{
TUMOR RADIOBIOLOGY STUDIES WITH HEAVY CHARGED PARTICLE BEAMS
}

\author{
Stanley B. Curlis, Thomas S. Tenforde, and S.M. Javed Afzal
}

The response of a rat rhabdomyosarcoma tumor systen; 10 irradiation with heavy charged particles is being evaluated irom experiments conducted both in vivo and in vitro. The radiobiological end points sturdied include tumor volume response, cellular sunvival aiter tumor irtarialion in situ, and cellkinelic parameters measured by llow cytomeiny. The prianany emphasis of our research during the past yo ar has beem in the following aneas: il repair of po'entially lethal damage, 2) dumot tepopulation kinetics following fraclionated doses o, neon ions, and 3) phase-specific cell survival following in situ $\mathrm{x}$-irradiation.

\section{REPAIR OF POTENTIALLY LETHAL DAMAGE}

Rat thabdomyosarcoma tumor cells grown as monolayer cultures exhibit substantial recovery from potentially lethal damage (PLD), but no repair can be measured in the in vino tumors. As 
opposed to the rapid plating technique used in vitro, the in vivo experiments involve a lengthy procedure of tumor excision, dissociation and plating, during which a substantial amount of PLD repair could occur and thereby go undetected. Using the well known PLD repair inhibitor, $\beta$ arabinofuranosyladenine $(\beta$-ara- $A)$, we have therefore been using an alternate approach to study PLD repair in vivo.

PLD repair was measured for tumors irradiated in situ with either $225-\mathrm{kVp} \times$ rays or $557-\mathrm{MeV} / \mathrm{u}$ neon ions in the distal position of a $4-\mathrm{cm}$ extended peak. Tumors implanted subcutaneously in syngeneic WAG/Rij rats were irradiated with $5.8 \mathrm{~Gy}$ of peak neon ions and 14.5 Gy of $x$ rays. These doses were chosen to produce about the same initial survival (0.06). Addition of $50 \mu \mathrm{M} \beta$-ara-A to the tumors immediately after $x$-irradiation and excision reduced the ultimate survival from 0.056 to
0.018, yielding a PLD inhibition factor of 3.11 (Table 1 and Fig. 1). The delayed exposure of tumor cells to $\beta$-ara-A after 1 or $2 \mathrm{hr}$ of incubation in medium lacking the inhibitor resulted in a progressive decrease of the inhibition factor to values of 1.60 and 1.47, respectively (Table 1 and Fig. 1). Addition of $\beta$-ara-A after $3 \mathrm{hr}$ of incubation in $\beta$ ara-A free medium did not inhibit the repair. When the tumors were excised at 3,6 or $12 \mathrm{hr}$ postirradiation and immediately exposed to $\beta$-ara-A for $3 \mathrm{hr}$ at $37^{\circ} \mathrm{C}$, no effect of the inhibitor on the ultimate cell survival was observed (Table 1 and Fig. 1).

Following 5.8 Gy of peak neon-ion irradiation, significantly less PLD repair was observed as compared to 14.5 Gy of $x$-irradiation (Table 1 and Fig. 1). Addition of $50 \mu \mathrm{M} \beta$-ara-A to the tumors immediately after neon irradiation reduced the ultimate survival from 0.067 to 0.032 , yielding a PLD

Table 1. PLD repair in neon and $x$-irradiated rhabdomyosarcoma tumors in the presence and absence of $\beta$-ara-A, $A^{\mathrm{a}, b}$

\begin{tabular}{|c|c|c|c|c|}
\hline \multirow[b]{2}{*}{ Tumor treatment after irradiation } & \multicolumn{2}{|c|}{$5.8 \mathrm{~Gy}$ of peak neon ions } & \multicolumn{2}{|c|}{$14.5 \mathrm{~Gy}$ of $x$ rays } \\
\hline & Surviving fraction & $\begin{array}{l}\text { PLD repair } \\
\text { Inhibition factor }\end{array}$ & Surviving fraction & $\begin{array}{c}\text { PLD repair } \\
\text { Inhibition factor }\end{array}$ \\
\hline Immediate excision and no $\beta$-ara-A & 0.067 & - & 0.056 & - \\
\hline $\begin{array}{l}\text { Immediate excision plus immediate } \\
\text { addition of } 50 \mu \mathrm{M} \beta \text {-ara- } \mathrm{A} \text { for } 4 \mathrm{hr} \\
\text { at } 37^{\circ} \mathrm{C}\end{array}$ & 0.032 & 2.09 & 0.018 & 3.11 \\
\hline $\begin{array}{l}\text { Immedtate excision plus addition of } \\
\beta \text {-ara-A after } 1 \mathrm{hr}\end{array}$ & 0.044 & 1.52 & 0.035 & 1.60 \\
\hline $\begin{array}{l}\text { Immediate excision plus addition of } \\
\boldsymbol{\beta} \text {-ara-A after } 2 \mathbf{h r}\end{array}$ & 0,050 & 1.34 & 0.038 & 1.47 \\
\hline $\begin{array}{l}\text { Immediate excision plus addition of } \\
\beta \text {-ara-A after } 3 \mathrm{hr}\end{array}$ & 0.064 & - & 0.060 & - \\
\hline $\begin{array}{l}\text { Excision } 3 \text { hr postirradiation: } \\
\text { (i) no added } p \text {-ara-A } \\
\text { (ii) immediate addition of } 50 \mu \mathrm{M} \\
\phi-a r a-A \text { for } 3 \mathrm{hr} \text { al } 35^{\circ} \mathrm{C}\end{array}$ & $\begin{array}{l}0.060 \\
0.052\end{array}$ & - & $\begin{array}{l}0.062 \\
0.055\end{array}$ & - \\
\hline $\begin{array}{l}\text { Excision } 6 \text { hr postirtadiation: } \\
\text { (i) no added para-A } \\
\text { (ii) immediate addition of ara-A }\end{array}$ & $\begin{array}{l}0.072 \\
0.074\end{array}$ & - & $\begin{array}{l}0.066 \\
0.062\end{array}$ & - \\
\hline $\begin{array}{l}\text { Excision } 12 \text { hr postintadiation: } \\
\text { (i) no added d-ard-h } \\
\text { (i) immediate addition of } x-\text { wan } A\end{array}$ & $\begin{array}{l}0.064 \\
0.070\end{array}$ & - & $\begin{array}{l}0.052 \\
0.054\end{array}$ & - \\
\hline
\end{tabular}

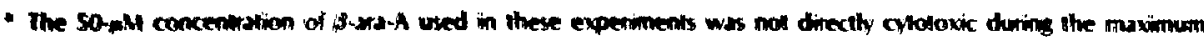
incubstion petiod of $41+4$ i $37^{\circ} \mathrm{C}$.

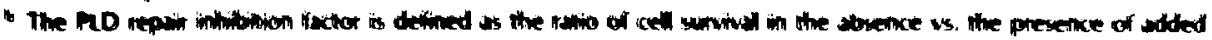
$\rightarrow \rightarrow+4=$. 


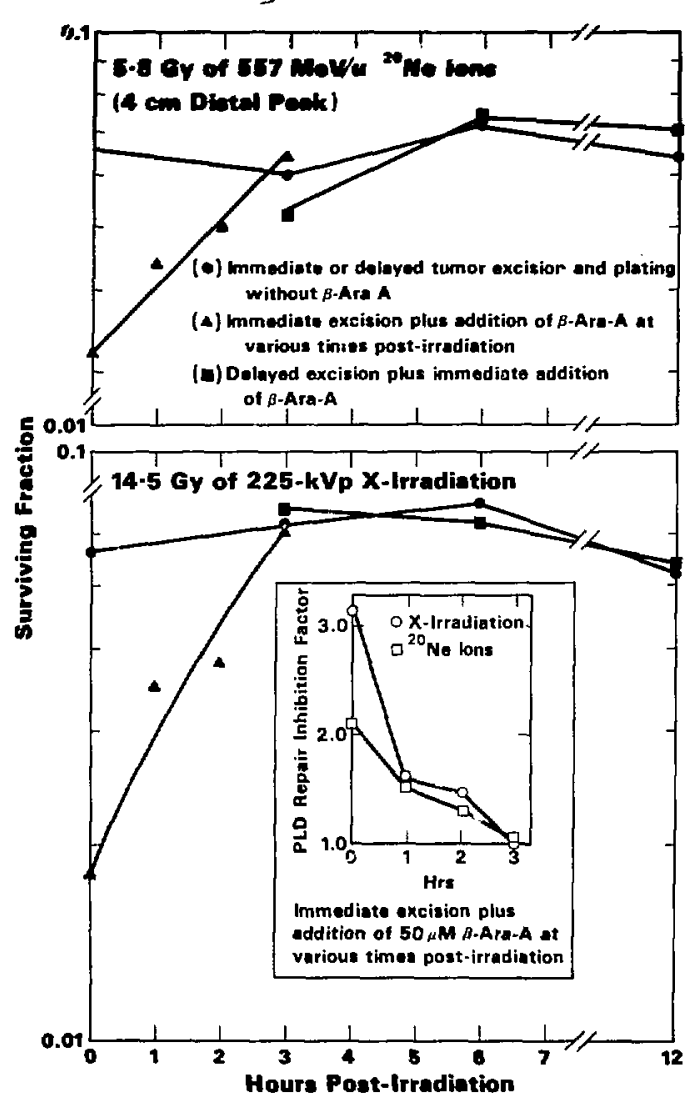

Fig. 1. PLO repair in rhabdomyosarcoma tumors irradiated in silu with $5.8 \mathrm{~Gy}$ of peak neon ions (upper panel) or with 14.5 Gy of $225 \mathrm{kVp} \times$ rays (lower panel) as a function of time

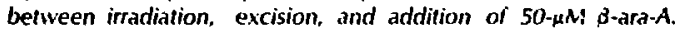
The $50, \mu M$ concentration of $\beta$-ara- $A$ used in these experiments was not direclly cytotoxic during the maximum incubation period of $3 \mathrm{hr}$ of $37^{\circ} \mathrm{C}$. The PLD repair inhibition tactor is delined as the ratio of cell survival in the absence ws. presence of added p-erri-A,

(XBL $851-8140)$

repair inhibition lactor of 2.09. However, as observed atter $\mathrm{x}$-irradiation, delayed exposure of tumor cells $10 \boldsymbol{\beta}$-ara-A for 1 or $2 \mathrm{hr}$ resulted in a progressive decrease of the PLD repair inhibition factor to values oi 1.52 and 1.34 , respectively.

We interpret these results as indicaling that thabdomyosarcoma tumors do repair a considerable amount of PLO in vivo following both kow and high-LET radiation. Using the excision assay procedure, however. this repait cannot be detected in the absence of $p$-ata-A because it is alheaty com. plete by the time the lumors ane disscociated and plated into tissue culture medium for the assay of colony-forming ability.

\section{Tumor Repupulation Kinetics Following Fractionated Doses of Neon Ions}

In these experiments, measurements were made of the time course of survival after fractionated irradiation. Groups of rhabdomyosarcoma tumors were irradiated in situ with daily fractionated doses ( 4 fractions in 3 days) of $557-\mathrm{MeV} / \mathrm{u}$ neon ions in the distal position of a 4-cm extended peak. Based on our earlier studies of tumor volume response following fractionated doses of neon ions, doses of 1.75 Gy per fraction of neon ions were administered to achieve a cell survival of approximately $2-3 \%$ by the end of a four-fraction schedule. Cell survival was assayed daily during irradiation and then daily up to 6 days postirradiation and every second day afterwards (up to 20 days postirradiation) in order to ascertain the cellular reginwth kinetics of tumors following the fractionated radiation treatment. Groups of 3 to 4 tumors were excised and assayed for cell survival by the excision assay technique at each time point. The results are presented in Fig. 2. As observed after a single 7-Gy dose of peak neon-ion irradiation, a significant decrease in cell survival was noted 3 days following the completion of irradiation. The rate of cellular repopulation and the radiation-induced tumor growth delay (calculated as the difference in the average time for irradiated and control tumors to reach a volume twice that at irradiation) was also the same following single dose and four-fraction radiation schedules.

\section{Phase-Specific Cell Survival Following in Vivo $X$ - Imadiation}

The survival of in vivo $\mathrm{R} 2 \mathrm{C5}$ tumor :ells in different cell-cycle phases was analy?ed by flow cytometry and cell sorting procedures following a 10-Gy dose of 225-kVp $x$ rays. Cell-kinetic parameters were delermined from the D'sA fluorescerice histograms of enzymatically dissociated tumor cells stained with a noncytotoxic $5 \mu \mathrm{M}$ concentration of Hoechst 33342 stain. Survival data for unirradiated control cells and for cells from trimors that were excised immediately following $x$-irradi:tion are shown in Fig. 3A and 3B. The fractional survival of GI and $(\mathrm{G} 2+\mathrm{M})$ cells were comparable to the overall sumvival of the entive tumor cell population following a 10-Gy $x$-tay dose (S.F. $=0.26)$. How. ever, the S-phare cells had a survival level that was $17 \%$ and $23 \%$ lomer than the survinual of the G1 


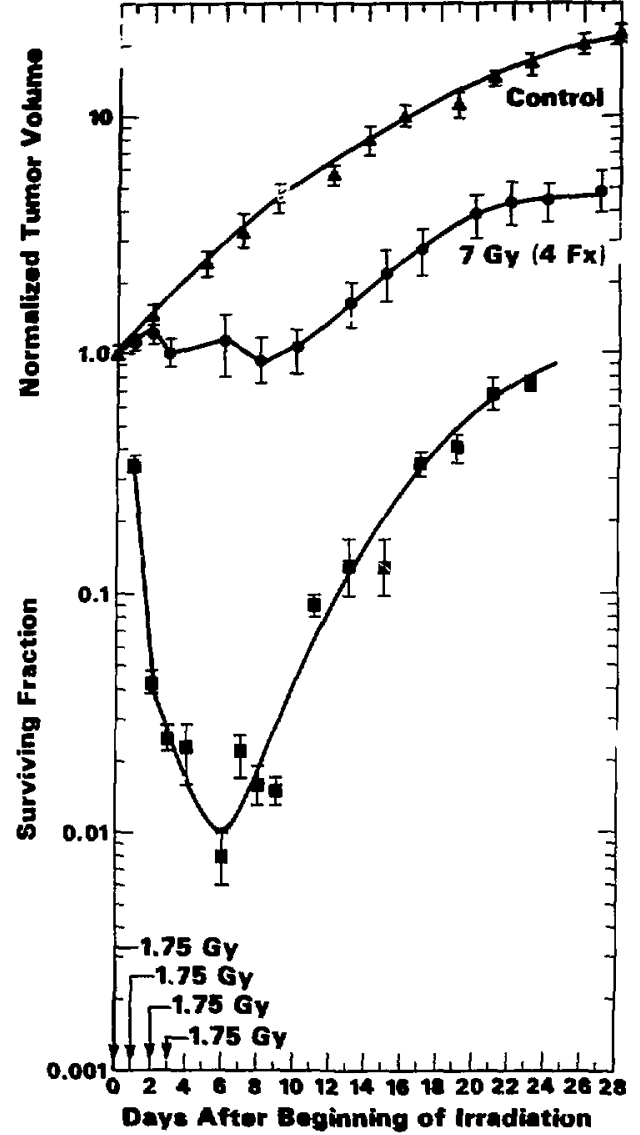

Fis. 2. Average tumor volumes are plotted as a runction of time for control rhabdomyosarcoma tumors and for tumors exposed to daily tractionated dosns of $1.75 \mathrm{~Gy}+4$ fractions in 3 dajs) of $f-c m$ extended-peak neon ions (upper panel). All lumor volumes were normatized to unity on the tirst day of imadiation. Surviting inction of lumor cells as a function of lime during and alter exposure to daily traclionated doses of $1.75 \mathrm{G}_{\mathrm{y}}(\mathrm{t}$ iractions in 3 days) of $\mathrm{H}$-cm extended peak neon ions is plolled in the lowir pariel. Groups of 310 t lumons were ISslyed at euch time soint. Error birs represent I I SEAI.

(XBL 85,8139 )

and $(G 2+M)$ cell populations, respeclively. Using the sunvival dala shown in Fig. $3 B$ and the fractions of turnor cells in difierent cell-cycle phases determined rrom the DNA iluorescence profile, it was calculated that the relative percentages of surviving lumor cclls that were in the $G \|_{3}, S$, and $(G 2 * M)$ cell-cycle phases at the time of intaliation were 78.28 .7 and $13.1 \% \%$, tespectively.

Tumor cell kincyics and phase-specific cell sumvival were andiged by thow cyometity and cell
(A)
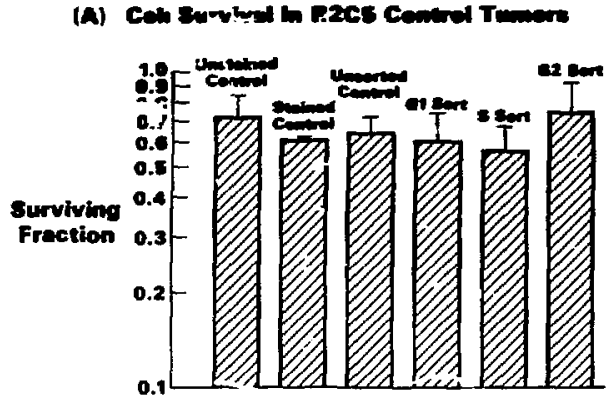

(B) Cell Survival Ir. R2C5 Tumare Exciesd Immodiately Foi:Jwing Irradietion In Situ With $10 \mathrm{~Gy}$ e? 225-kVp X-Aays

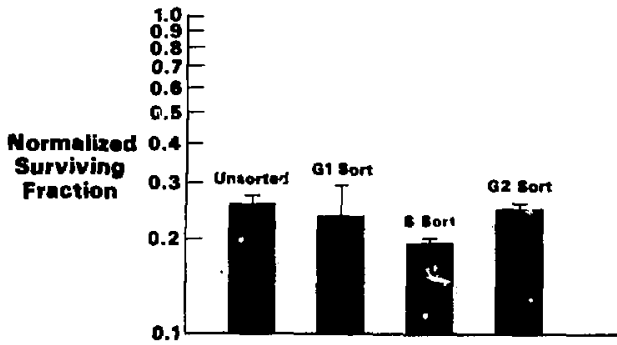

fig. 3. Phase-specific rall survival data are presented for unirradiated $\mathrm{R}^{\mathrm{C}} 5$ fur:sot $\mathrm{s}$ (panel $A$ ) and for tumors that received a $10-G y$ dose of $2 \cdot ;-k V p \times$ ays (panel $B$ ). The er:zymatically dissociated cells were stained wilh Hoechst $3.3342(5 \mu \mathrm{M}, 2 \mathrm{hr}$, $37^{\circ} \mathrm{C}$ and sorten on the basis of DNA fuorescence using a uv laser intensity of $150 \mathrm{mWW}$. The survival fractions of $x$-irradiated cells in each phase of the cell cycle were nornalized to the sur. vival of control cells in the corresponding cell-cycle phase. No statistically significam decrease in cell survival occurred in the control tumors as a result of the staining or cell sorting pro. cedures. In the irradiated tumor cell populations, the unsorted cells passed through the laser bean exhibited a $40.2 \%$ lower survival than the stdined cells that were not subjected to Row cytometry. The phase-specific survival of the irradiated rumor cells was renormalized to correct for the laser cytotoxicity.

$(X B L 857-8142)$

sorling procedures at $0,:, 2.5,4$ and 8 days following irradialion in situ wilh $10 \mathrm{~Gy}$ of $225-\mathrm{kVp} x$ rays. As showr in Fig. 4A. the pe cent of host iliploie? cells within the tumors incr:-sed from asproximately 70 \% to $85 \%$ of the cotal cell population at 2.5 days pustirradiation. The higher percentage of diploid bost cells relative to tetraploid tumor cells was also observed at + and 8 days postirtatialion. Figure $A B$ shows the relative distributions 0, Gi, 5 and $(G 2+M)$ cells in irradiated lumors al imes ranging from 0 to 8 duys following a $10 \mathrm{~Gy}$ dose of $x$ rays. Al I day postinradiation there was a turolold increase in the petcentige of $\mathrm{C}_{2}$ ceits (from 12.0 to 


\section{(A) Ralevine Percentinges of Diploid Host Cols and Tetraploin R2C5 Tumor Cels}

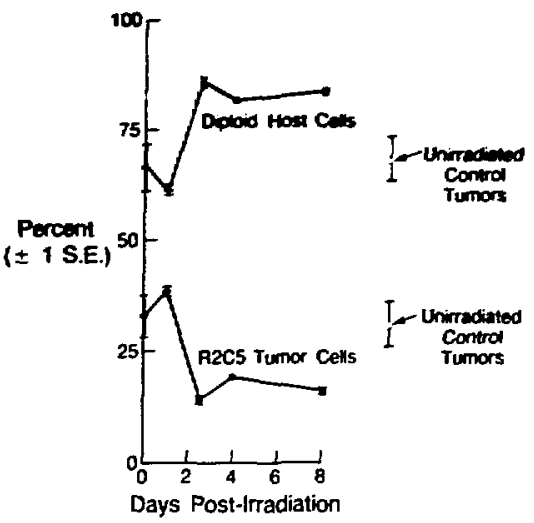

(B) Cell Cycle Distribution of R2C5 Tumor Cells Following X-Irradiation In Situ

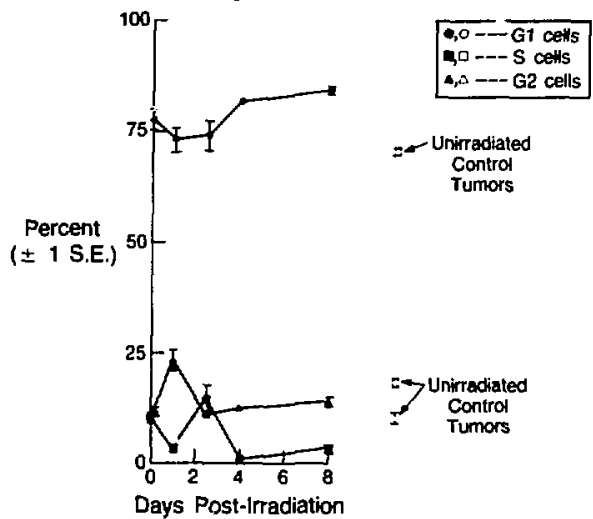

Fig. 4. The relative percentage of diploid host cells and tetraploid tumor cells (panel A) and the cycle distribution of the tumor cell population (panel B) are shown during the period from 0 to 8 days following a $10-G y$ dose of $225-k V p \times$ r. $y$ s.

(XBL 859-8474)

$23.5 \% 1$ as a result of the radiation-induced G2 block. Corresponding decreases in both the $G 1$ and $S$ cell populations were oinserved at i day following irradiation. At $\mathbf{2 . 5}$ days postirradiation the relative percentage of $\mathrm{G} 2$ cells decreased to $11.3 \%$. while the S-phase population showed a transient increase $1014.6 \%$ and the G1-phase population remained arinstant. This Iransient increase in $S$ phase cells may reflect the traversal of a cohon of cells into cycle following release irom the postinadiation G2 block. Between 2.5 and 8 days postirra. diation the relative percentage of $\mathrm{G} 2$ cells remained nearty constant, while the percent of $G 1$ and 5 celis increased and decreased, respectively.

The cell survival fractions in unsorted and sorted fumor cell populations al 0 to 8 days following a 10-Gy dose of $x$ rays are plotted in Fig. 5 . Tumor cells stained with 5 mM Hoechst 33342 and passed through the FACS N unit without sorting showed a significant 25 -fold drop in survival at 2.5 days postirradiation, similar to that previously observed in cellular repopulation sludies following a $20-G y$ dose of $x$ rays.

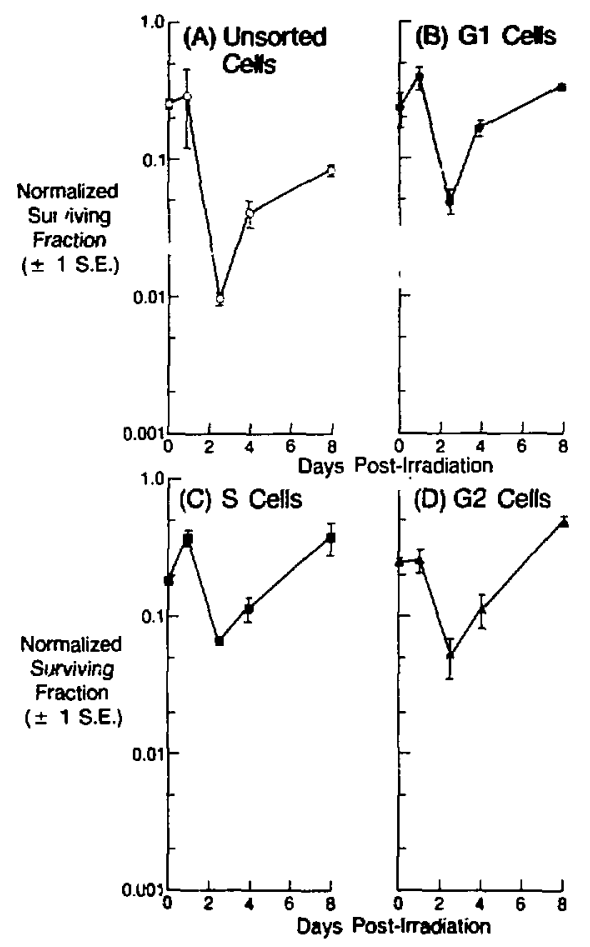

Fig. 5. Survival data are presented for unsorted (panel $A$ ) and sorted $G 1$. $S$, and $G 2+M$ populations (panels $B, C, D$ ) of $R 2 C 5$ fumors at $a$ to 8 days following x-irradiation in site. The enzymatically dissociated cells were slained with Hoechst 33342 (5 aAl, $2 \mathrm{hr}, 37^{\circ} \mathrm{Cl}$ and sorted on the basis of DNA iluorescence using a uv laser intensify of $150 \mathrm{~mW}$. The survival fractions of v-irradialed cells in each phase of the cell ocle were normalized using sunvial datu for unirratiated cells in the corresponding rell-cix'e phase. In the irradiated tumor cell populations at the tive lime points sfudied, the unsorted cells passed through the laser beam exhibiled a survival thal was 17.0\% $\pm 7.5 \%$ (S.E.) haves. on the average. Ihan the sunvial of Hoechststained cells that were not subjected to fom cytometny. For each incisiofus experimemt, the phase-specific survinal of the inadiater tumor cet's was renomalized to conted for the laser

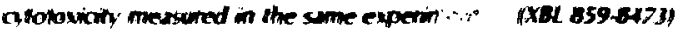


The sonted tumor cell populations in GI, $S$ and (G2+M) phases of the cycle exhibited B-, 6-, and 5-fold decreases, respectivety, in their survival at 2.5 days relative to 1 day postimadiation. At 8 days postirradiation the survival of the unsorted tumor cells was only one-third of the value measured immediately following irradiation (S.F. $=0.26 \pm$ 0.02), whereas sorted cells in the $G 1, S$ and $(G 2+M)$ phases had respective survival levels that were 41,97 , and $98 \%$ greater than the values determined immediately after $x$-irradiation. The unsorted cells thus exhibited a greater dip in survival and a slower rebound at 2.5 to 8 days postirradiation than the sorted tumor cell populations. Because an unsorted cell population contains a very large number of diploid host cells relative to sorted tumor cell populations, the possibility exists that the large dip in survival of unsorted cells may be mediated partly or wholly by the cytotoxicity of host cells that infiltrate the tumor beginning at 2 to 3 days following irradiation (see Fig. 4A).

Using the data presented in Fig. 4B on cellcycle distributions and the data shown in Fig. 5B, $5 C$ and $5 D$ on the survival of $G 1, S$, and $(G 2+M)$ tumor cells, calculations were made of the percen- tage of surviving cells in each phase of the cell cycle at 0 to 8 days following a 10-Gy dose of $225-k V p \times$ rays. These resulis are presented in Table 2. Throughout the 8-day postirradiation period, tumor cells in the G1 cycle phase constiluted 70 to $90 \%$ of the surviving ceil population. Fewer than $20 \%$ of the surviving tumor cells were measured in either the $S$ or the $(G 2+M)$ phases at any time up to 8 days following irradiation.

Table 2. Percent of surviving tumor cells in each cycle phase af $O$ to 8 days following irradiation in situ with $10 \mathrm{~Gy}$ of $225-k V p \times$ rays.

\begin{tabular}{cccc}
$\begin{array}{c}\text { Days } \\
\text { postirradiation }\end{array}$ & $\begin{array}{c}\text { Percent G1 } \\
\pm \text { S.E. }\end{array}$ & $\begin{array}{c}\text { Percent S } \\
\pm \text { S.E. }\end{array}$ & $\begin{array}{c}\text { Percent (G2+M) } \\
\pm \text { S.E. }\end{array}$ \\
\hline 0 & $78.2 \pm 21.4$ & $8.7 \pm 0.2$ & $13.1 \pm 0.5$ \\
1 & $79.4 \pm 15.8$ & $3.7 \pm 0.5$ & $16.9 \pm 3.1$ \\
2.5 & $69.6 \pm 16.4$ & $18.6 \pm 0.6$ & $11.8 \pm 3.8$ \\
4 & $90.4 \pm 12.8$ & $0.5 \pm 0.1$ & $9.0 \pm 2.5$ \\
8 & $76.8 \pm 3.2$ & $3.4 \pm 0.9$ & $19.8 \pm 1.4$ \\
\hline
\end{tabular}

\section{COMPARATIVE ANALYSIS OF MODELS DESCRIBING GLUCOSE UPIAKE IN THE BRAIN}

Hugo A. Massaldi

Several alternatives arise when the problem of building a model for glucose exchange between cerebral capillaries and tissue is considered. Among them, a version of the lumped. compartment model has been receiving increased use for describing the uptake and breakdown of glucose and glucose analogues in the brain, by means of imaging techniques. ${ }^{1-4}$ Although the inherent simplicity of this model justifies its use, few attempts have been made to challenge its assumptions ${ }^{5}$ or $e^{\text {valuate its methodology. }}{ }^{6}$ This report presents the preliminary analysis of an altempt to contribute in this respect. It has been intended to put the lumped model into a more general context, by pointing out the scope. limitations and implicit assuniptions of this and other available or new modeis that can be proposed with the same purpose. The different expressions and mechanisms used to present the glucose transport step constitute an essertial-and not less controversial-aspect of the modeling problem.
Although these elements may influence the model organization and assumptions to some extent, for the present purposes it is sufficient to adopt the simple notation of rate constants, as used in classical pharmacokinetics. A more thorough discussion of this problem is presented elsewhere. ${ }^{7.8}$

\section{MAIN RELATED MODELS}

\section{Capillary Exchange, Axial Gradient in Vascular Space}

Figure la shows a scheme of this model, which incorporates an axial flow and varying concentration along the capillary length as essential components. The assumptions with respect to the tissue compartment may vary widely and give rise to different solutions. A system of the type shown in Fig. 1a has been considered and solved",10 under various conditions, mainly in connection with the multiple. indicator technique and early washout curves. The 


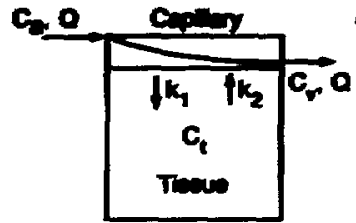

(a)

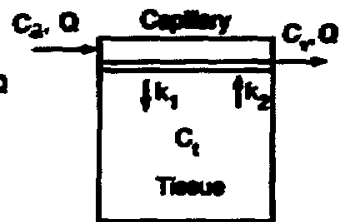

(b)
F. 1. Comparative analysis of models. a) Distributed model of capillary exchange, showing concentration gradient along capillary. b) Well-stirred model of capillary exchange, with uniform concentration in vascular compartment. (XBL 8511-8583)

situation described is perhaps the most real one for solute exchange in a capillary that does not have interconnections with other elements of the capillary bed. Its usefulness, however, and the benefits of this more detailed description, are limited to short-time experiments where the injection and sampling sites are rather close to the organ under study, since no consideration is given to the dynamics of flow.

\section{Well-Stirred Vascular Compartment}

Solutions to this model have been obtained. ${ }^{11,12}$ Figure $\mathbf{l b}$ illustrates that the well-stirred approximation implies the assumption of instantaneous mixing of the entrance solute within the compartment, which is assumed to operate at the exit concentration. Jacquez ${ }^{5}$ has recently challenged this assumption in favor of a distributed capillary model, of the type discussed above. However, his results do not show a substantial improvement and, probably because of the low extraction fraction of glucose and glucose analogues, the well-mixing assumption may not constitute an important drawback of the model. It should be mentioned, however, that the ignorance of the dynamics of flow, which depend on recirculation effects and the choice of the injection/sampling sites, still remains in this version, and therefore, it may not be adequate to represent the data of runs longer than a first-pass experiment.

\section{Lumped Coripartment Model}

This is the type of model used in compartmental analysis and classical pharmacokinetics to describe tracer distribution, obtain plasma disappearance curves, or estimate clearance rates for drugs in different regions of the organism. A scheme of this model and related parameters is shown in Fig. 2a. Again, its major limitation is the lack of allowrance for the dymamics of flow, which are explicitly ignored, both in the model scheme

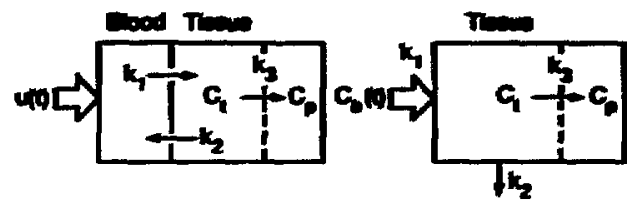

(4)

b)

Fic. 2. Comparalive analysis of modets. a) Lumpedcompartment model for exchange between btood and lissue. b) Compartment model scheme used in positron emission tomography (PET) of glucose analogues.

(XBL a5II-a569)

and in writing the balance equations. The wellknown solution to this model for a bolus injection in the blood compartment has the form of a summation of two exponential terms, and can be found in many classical textbooks (cf., Refs. 13,14).

The lumped model also makes use of the wellmaxing condition, by implicitly assuming that, upon injection, the solute instantaneously distributes uniformly in the blood compartment. With these approximations, the model may be adequate for fitling data and for simulating the behavior of an injected solute within a rather large vascular volume over time periods longer than those for the capillary distributed models. However, the situation is different when the precise estimation and correct interpretation of the kinetic constants are required. In this respect, and concerning its application to hepatic transport kinetics, it has been shown ${ }^{15}$ that the lumped model systematically underestimates the values of constants $k_{1}$ and $k_{2}$, and particularly, that the values of $k_{1}$, obtained from the earlier time data, may be incorrect by as much as $50-70 \%$.

\section{The PET Model}

The conventional lumped model, discussed above, not only ignores the general dynamics of flow and recirculation, but it also neglects the flow term in the balance for the vascular compartment, $Q\left(C_{2}-C_{v}\right)$. The PET model, shown in Fig. $2 b$, is a varient of the lumped model that is designed to cope with the flow problem by estimating and treating the concentration function in the brain capillary region as the input function to the tissue.

Thus, regarding the question of flow effects, this model represents an improvement with respect to the classical lumped model. However, several problems: associated with the precise determination of the actual input function in the brain capillan region, still remain." These make uncertain the 
eximation of the transport constants $k_{1}$ and $k_{2}$, particalaty from dita taken at the shorter tines, when the impulse input function changes more repidily.

\section{Step input/"Constant Infusion" Madel}

Another varient of the lumped model can be proposed to circumvent the problem of flow effects. It essentially consists of the same scheme as that of Fig. $2 \mathrm{~b}$, except that the input function, $C_{b}(1)$, is experimentally constructed so that it approaches a step function, as in the constant infusion experiment. The basis for this is the observation that, even in the PET model, where the solute is initially injected as a bolus, a quasi-constant slightly decreasing level is rapidly approached after the pass of the initial wave. Bischoff ${ }^{16}$ has also pointed out this finding for the brain vascular region, in a simulation study of solute distribution in the organism. The required constancy in $C_{b}$ may therefore be accomplished by a slow initial injection and one or more complementary, also slow injections of solute conveniently separated in time, all administered in the peripheral circulation.

The solution to this model, for a step-input function $C_{b}(t)=D$, is, for the total activity of labeled solutes in the tissue:

$$
\begin{aligned}
& \frac{C_{1}+C_{p}}{D}=\frac{k_{1}}{k_{2}+k_{3}} \\
& \left.\left\{k_{3} t+\frac{k_{2}}{k_{2}+k_{3}} \mid 1-\exp \left(-\left(k_{2}+k_{3}\right) t\right)\right\}\right\}
\end{aligned}
$$

Several curves corresponding to Eq. (1), for various values of the parameters, are shown in Fig. 3. The trends of the uptake activity curves are similar to those observed in experimental studies ${ }^{3,4}$ where a bolus is injected in the peripheral circulation. This can be considered as an indication that the step input condition, assumed by the proposed model, may represent a good approximation to the actual situation.

\section{CONCLUSIONS}

Despite strong limitations and apparently gross over-simplifications, the possible variants of the lumped model remain as convenient, and probably the best alternatives to describe glucose tracer distribution in the brain by means of noninvasive imaging techniques.

The final answer to the problem of model adequacy and parameter eslimation has to be provided by a comprehensive model that takes into account

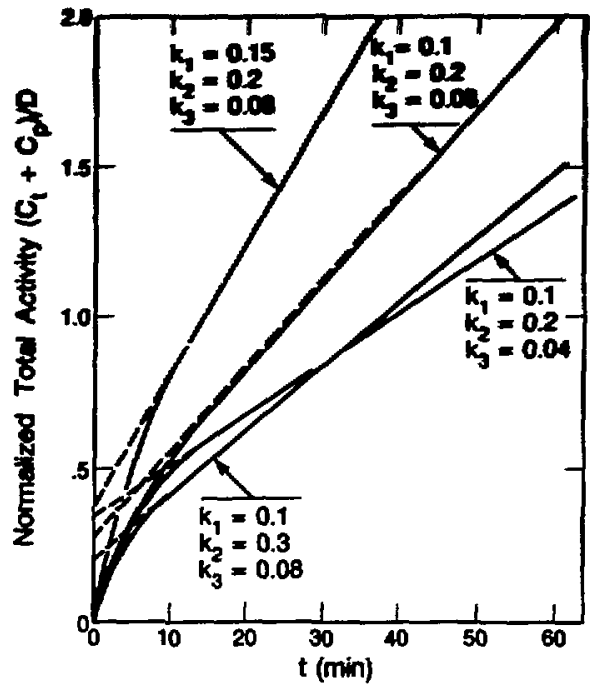

Fig. 3. Uptake curves of normalized total activity as a function of time, from "constant infusion" model. Eq. (1).

$(X B L 8511-8568)$

the mentioned effects of flow dynamics, recirculation, and delay in a consistent manner. Although a model like this will probably not be amenable to direct use with the present methodology, its results would allow the parameters of the simpler models discussed here to be estimated and contrasted by simulation. Work is in progress along this line.

\section{REFERENCES}

1. Sokoloff, L., Reivich, M., Kennedy, C., Desrosiers, M.H., Patlak, C.S., Pettigrew, K.D. Sakurada, O., and Shinohara, M. The $\left({ }^{14} \mathrm{C}\right.$ deoxyglucose method for the measurement of local cerebral glucose utilization: theory, procedure and normal values in the conscious and anesthesized albino rat. J. Neurochem. 28, 897-916 (1977).

2. Phelps, M.E., Huang, S.C., Hoffman, E.J., Selin., C., Sokoloff, L., an!l Kuhl, D.E. Tomographic measurement of local cerebral glucose metabolic rate in humans with $\left.\left.\right|^{18} \mathrm{~F}\right]$-Fluoro-2deoxi-D-glucose: validation of method. Ann. of Neurol. 6, 371-388 (1979).

3. Knittel, B. Kinetic analysis of dynamic PET data, Master's thesis, UC Berkeley, 1983.

4. Heiss, W.D., Pawik, G., Herholz, K., Wagrier, R., Goldner, H., and Wienhand, K. Regional kinetic constants and cerebral metabolic rate 
for glucose in nomal human volunleers deter. mined by dynamic positron emission tomography of $\left.\right|^{18}$ F\}-2Fluoro-2-deoxy-D-glucose. I. Cereb. Błood Flow Metabol. 4, 212-223 (1984).

5. Jacquez, J. Red blood cell as glucose carrier: significance for placental and cerebral glucose transfer. Am. 1. Physiol. 246, R289-R298 (1984).

6. Budinger, T.F., Huesman, R.H., Knittel, B., Friedland, R.P., and Derenzo, S. Physiological modeling of dynamic measuremenls of melabolism using positron emission tomography. In The Metabolism of the Human Brain Studied with Positron Emission Tomography, $T$. Greitz et al. Eds., New York, Raven Press, 1985.

7. Massaldi, H.A. Channel model and parameters of the glucose transporter. Nature, submitted for publication. Nov. 5, 1985.

8. Massaldi, H.A. Scope, limitations and applicability of models describing glucose uptake in the brain. A simulation study. Am. J. of Physiol., to be submitted for publication.

9. Levitt, D.G. Evaluation of the early extraction method of determining capillary permeability by theoretical capillary and organ models. Circ. Res. 27, 81-95 (1970).
10. Groresky, C.A., Ziegler, W.H., and Bach, G.G. Capillary exchange modeling barriet-limited and flow-limited distribution. Circ Res. 27. 739-764 (1970).

11. Middleman, S. Transport Phenomena in the Cardiovascular System. Wiley-Interscience. New York, pp. 193-194 (1972).

12. Huang, S.H., Phelps, M.E., Hotíman, E.J., and Kuhl, D.E. A theoretical study of quantitative flow measurements with constant infusion of short-lived isotopes. Phys. Med. Biol. 24, 1151-1161 (1979).

13. Atkins, G.L., Multicompartment Models for Biological Systems, Methiem and Co., London (1969).

14. Gibaldi, M., and Perrier, D. Pharmacokinefics, Dekker, New York, (1975).

15. Forker, E.L., and Luxon, B.A. Hepatic transport kinetics and plasma disappearance curves: distributed model vs. conventional approach. Am. J. Physiol. 235, E638-E660 (1978).

16. Bischoff, K.B. Applications of a mathematical model for drug distribution in mammals. In Chemical Engineering in Medicine and Biology, D. Hershey, Ed., Plenum, New York (1967)

\section{HOLISTIC BIOPHYSICS OF RED BLOOD CELL MEMBRANE SYSTEMS}

\section{Howard C. Mel, Gary V. Richieri, Hugo A. Massaldi, and Robert Bridwell}

Biological membranes may be viewed as a next step upward from the molecular level in organizational complexity, providing a foundation-setting for macrolevel properties, and for behavior of twodimensional structures. Such properties include semipermeability, porosity, stretchability, tear resistance, insulating ability, and other thermal, electrical, mechanical, osmotic and geometrical qualities. The holistic nature of this project resides in the fact that many of these features can be related to each other and to the lower level molecular properties of ionic permeability and transport, as well as to the higher level physiology of the whole animal-most notably that associated with the circulation of the whole blood. Our experimental and theoretical work, then, is aimed not only at leaming new facts about membranes of whole-cell systerns but also at elucidating underlying mechanisms "...emphasizing the organic or functional relation between parts and whole..." (a dictionary definition of holism).

We report this year on advances in both experimental and theoretical domains. First, the wholecell and membrane electrical resistivity and dielectric breakdown properties are exploited to lead to an improved measure of the electrical resistivity of the cytoplasm of the cell-an entree to information on the normal or abnormal state of hemoglobin. Secondly, new results are presented relevant to thermal responses of the membrane surface and the recently discovered phenomenon of "membrane stretch."1,2 We conclude with a summary of a new theoretical model-study that can predict osmotic fragility properties from cell size distributions determined by resistive pulse spectroscopy (RPS). 


\section{MEMARANE BREAKDOWN AND CYTOPLASMIC RESISTINTTY}

A relatively simple improvement in the electrical components of the instrumentation for resistive putse spectroscopy (RPS), now allows precise measurement of the electric field strength at which the red blowd cell membrane will begin to pass current, the so-called membrane breakdown potential. We can now also measure, to a much higher degree of accuracy than previously, the internal cytoplasmic resistivity of red blood cells (and presumably other cell types as well). The procedure is as follows. Red cells, in dilute suspension, are examined under both low and high electric field strength conditions. An example of the resulting data is presented in Fig. 1 (the circles are actual data from normal blood). A low applied electric field results in a line such as (a), while a high field leads to lines such as (b), (c) or (d), depending upon the internal resistivities of the cells. The membrane breakdown potential is seen simply as the crossover point between the low and high electric field lines.

The determination of cytoplasmic resistivity is somewhat more complicated. It requires the following equations:

$$
\begin{gathered}
\rho_{\text {cyto }}=\rho_{\text {medium }} \cdot W \\
w=1+\frac{f_{E} \times M_{r}}{1-M_{r}}
\end{gathered}
$$

where $\rho_{\text {cyto }}=$ cytoplasmic resistivity, $\rho_{\text {medium }}=$ medium resistivity, $\mathrm{f}_{\mathrm{E}}=$ shape factor of cells (varies from 1.0 to 1.5, and for normal isotonic red cells equals about 1.2$), M_{r}=$ ratio of slope of the high electric field line to that of the low field line.

The technique to determine the shape factor has recently been published. ${ }^{3}$

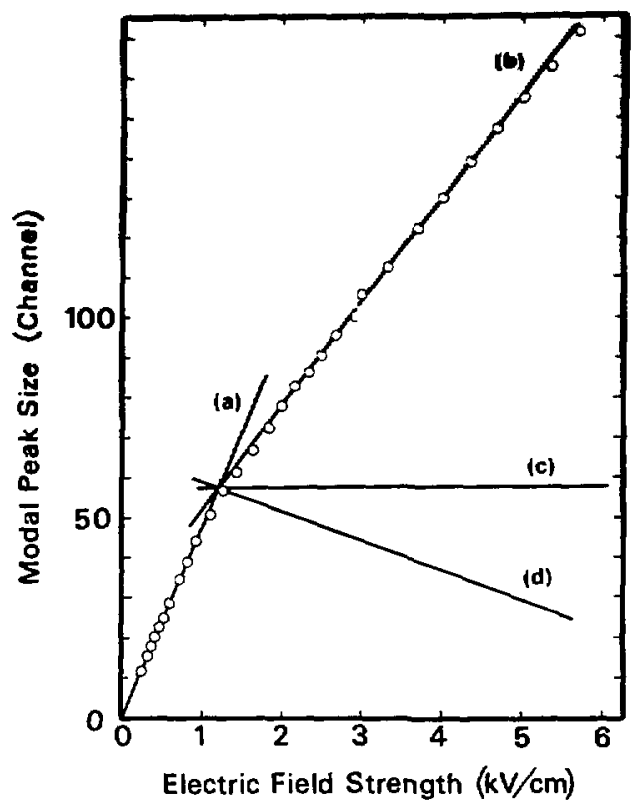

Fig. 1. Relationships between modal peak size (in channels) and applied field strength for various conditions of membrane and cytoplasmic resistivity. The circles represent experimental data for normal red blood cells in isotonic medium.

(XBL $8511-11474)$

The above approach has now been applied to several different kinds of blood cell samples. The values for membrane-breakdown potential and cytoplasmic-resistivity for 8-15 normal (AA), 5-11 heterozygous sickle cell trait (AS), and 15-20 homozygous sickle cells (SS) are included in Table 1. For these samples the membrane-breakdown values do not differ significantly amongst the three groups. However, the cytoplasmic-resistivity

Table 1. The average and standard error of the multiple cellular paramelers measured by the

\begin{tabular}{|c|c|c|c|c|c|c|c|c|c|c|}
\hline \multicolumn{3}{|c|}{ Volume } & \multirow{2}{*}{$\begin{array}{c}\text { Surface } \\
\text { area } \\
\left(\mu \mathrm{m}^{2}\right)\end{array}$} & \multirow[b]{2}{*}{$\begin{array}{l}\text { Shape } \\
\text { iactor }\end{array}$} & \multirow{2}{*}{\multicolumn{2}{|c|}{ Deformability }} & \multicolumn{2}{|c|}{ Frability } & \multirow{2}{*}{$\begin{array}{c}\text { Membrane } \\
\text { breakdown } \\
\text { (V/an) }\end{array}$} & \multirow{2}{*}{$\begin{array}{l}\text { Cyloplasmic } \\
\text { resistivity } \\
\text { (ohm-cm) }\end{array}$} \\
\hline $\begin{array}{l}\text { Modal } \\
\left(\mathrm{mm}^{3}\right)\end{array}$ & $\begin{array}{l}\text { Mean } \\
\left(\mathrm{km}^{\mathrm{s}}\right)\end{array}$ & c.v. & & & & & $\begin{array}{c}50 \% \\
(\mathrm{mOsm})\end{array}$ & Slope & & \\
\hline A $\begin{array}{r}89.8 \\
\pm 0.88\end{array}$ & $\begin{array}{c}93.5 \\
1.03\end{array}$ & $\begin{array}{c}16.0 \\
0.46\end{array}$ & $\begin{array}{r}129.2 \\
1.39\end{array}$ & $\begin{array}{l}1.201 \\
0.002\end{array}$ & $\begin{array}{l}1.08 \\
0.02\end{array}$ & $\begin{array}{c}11.2 \\
0.31\end{array}$ & $\begin{array}{r}127.8 \\
1.11\end{array}$ & $\begin{array}{l}5.0 \\
0.15\end{array}$ & $\begin{array}{r}1151 \\
32.5\end{array}$ & $\begin{array}{r}147.6 \\
2.12\end{array}$ \\
\hline AS $\begin{array}{r}85.5 \\
+2.23\end{array}$ & $\begin{array}{c}88.9 \\
3 .+1\end{array}$ & $\begin{array}{c}16.3 \\
0.45\end{array}$ & $\begin{array}{r}125.0 \\
2.62\end{array}$ & $\begin{array}{l}1.199 \\
0.002\end{array}$ & $\begin{array}{l}1.04 \\
0.02\end{array}$ & $\begin{array}{c}11.5 \\
0.42\end{array}$ & $\begin{array}{r}115.4 \\
1.42\end{array}$ & $\begin{array}{l}4.3 \\
0.33\end{array}$ & $\begin{array}{c}1190 \\
10.7\end{array}$ & $\begin{array}{r}148.7 \\
1.79\end{array}$ \\
\hline S5 $\begin{array}{r}87.6 \\
\pm 1.63\end{array}$ & $\begin{array}{c}91.6 \\
1.90\end{array}$ & $\begin{array}{r}23.4 \\
0.76\end{array}$ & $\begin{array}{r}11+4.3 \\
2.36\end{array}$ & $\begin{array}{l}1.160 \\
0.004\end{array}$ & $\begin{array}{l}0.65 \\
0.09\end{array}$ & $\begin{array}{l}0.3 \\
0.69\end{array}$ & $\begin{array}{c}9.5 \\
1.99\end{array}$ & $\begin{array}{l}2.0 \\
0.11\end{array}$ & $\begin{array}{r}1154 \\
33.0\end{array}$ & $\begin{array}{r}166.6 \\
7.49\end{array}$ \\
\hline
\end{tabular}
RPS lechnique for normal, sickle cell trail, and sickle red blood cells. 
values, and the population heterogeneity in this parameter are sionificantly higher for the SS cells.

Different information is available when the data of Fiz 1, the unnormalized raw datn, are plotted in another way. If they are replotted normalized to line (a) (i.e., taking each point along line (a) equal to 100\%) we obtain Fig. 2 wherein the dashed lines correspond to lines (a) and (b) from Fig. 1. In this figure the breakdown potential appears to occur at a much lower value as compared to the single point of intersection in Fig. $1(800 \mathrm{~V} / \mathrm{cm}$ compared to $1235 \mathrm{~V} / \mathrm{cm}$ ). This difference is ascribed to the heterogeneity of the cell population, heterogeneity either in size or in membrane breakdown characteristics, which leads to a lower breakdown potential than that applicable to the population average.

\section{SICKLE CELL TRAIT AND SICKLE CELIS}

Expanding upon the data presented last year, ${ }^{1}$ we present in Table 1 the values for eleven parameters measured by the RPS technique for the same three classes of normal, sickle trait, and sickle cells. New parameters provided are the true modal and mean cell volumes, surface area, shape factor, membrane breakdown potential, and cytoplasmic resistivity.

Small differences exist for certain of the properties, and large, significant differences for others. For example, these latter are found in the osmotic fragility for the AS samples, and in all but the

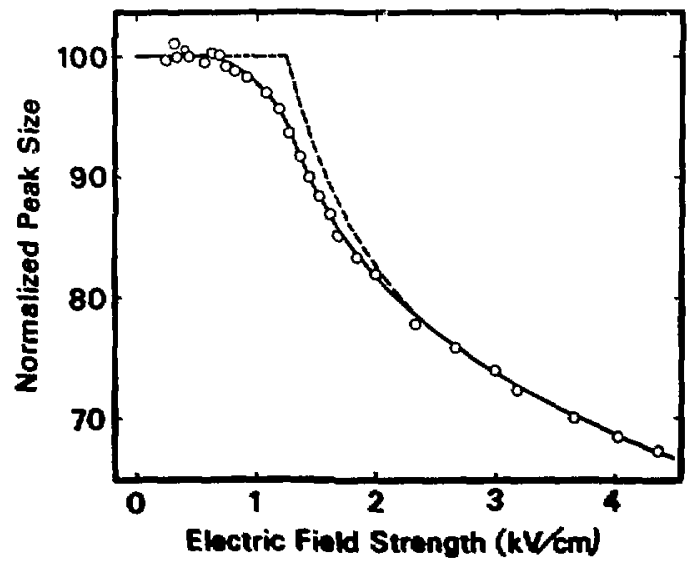

F. 2. Dala of fo. 1, recalculated and noimslized no: $100 \%=$ the pre-brealdoun appurent size at exch field strength (comesponating to imfinite membrane resistinity). The diched bines are the watues calculated for the solid bines (a) and (b) of f.t. 1. mean and modal sizes and the membrane breakdown potential for the SS samples. In our previous report' we showed how a multiparancter-index approach could provide an ahernative kind of holistic evaluator for purposes of comparing cell populations characterized by arrays of properties such as these. Refinement of this approach to take account of the new (or revised) measurements, and the added sample type (AS) is currently under scrutiny.

\section{TEMPERATURE AND OSMOTIC RESPONSES OF CELL VOLUME AND MEMBRANE SURFACE}

We previously reported on the phenomenon of "membrane stretch" and related temperaturedependent volume responses of ghost membranes.' These were produced at various osmotic pressures and temperatures (so as to assure formation of about $95 \%$ ghosts) in order to test the stability and reversibility of such membrane-surface area changes. Subsequent to theï formation, two-way transfers were then carried out between higher and/or lower temperatures. Results suggested that the reversibility was not complete for ghosts produced at $0^{\circ} \mathrm{C}$, but was almost complete for those produced at $40^{\circ} \mathrm{C}$.

A new question arose out of these findings, namely the extent to which the results depended upon the specific osmotic pressures at which the cells were hemolysed and measured. New experiments were therefore carried out wherein the ghosts were first resuspended in a series of intermediate osmotic pressures (175, 200, $250 \mathrm{mOsm})$, and then subjected to temperature changes. The results (Fig. 3) indicated that these ghost membranes behaved very much like intact-cell membranes, displaying almost complete reversibility in all samples. That is, the ghost volumes were equally able to respond reversibly to changes of temperature and of osmotic pressure.

An additional series of experiments was designed to sort out the separate (but interacting) effects of osmotic pressure and temperature on membrane integrity and volume responsiveness. Ghosts were produced to the $95 \%$ level at the requisite, respective osmotic pressures and temperatures. After 30 to $60 \mathrm{~min}$ samples were centrifuged, then all resuspended to the common conditions of $300 \mathrm{mOsm}$ and $25^{\circ} \mathrm{C}$. Following this equilibration they were resuspended (at $25^{\circ} \mathrm{C}$ ) in a series of osmotic media ranging from 100 to 300 mosm, and their volumes were measured.

The preliminary results (Fig. 4) sugigest that for the chosts criginally made at $0^{\circ}$ and $25^{\circ} \mathrm{C}$, theis past history has bittle effect on their ability to 


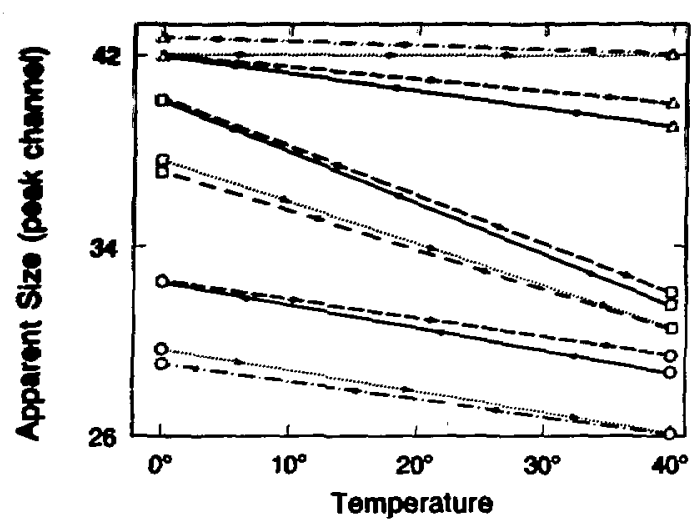

Fit. 3. Temperature reversibility of ghost volume changes. (Triangle, restored to $175 \mathrm{mOsm}$; square, restored to 200 mOsm; and circle, restored to 250 mOsm.) (XBL 8517-11477)

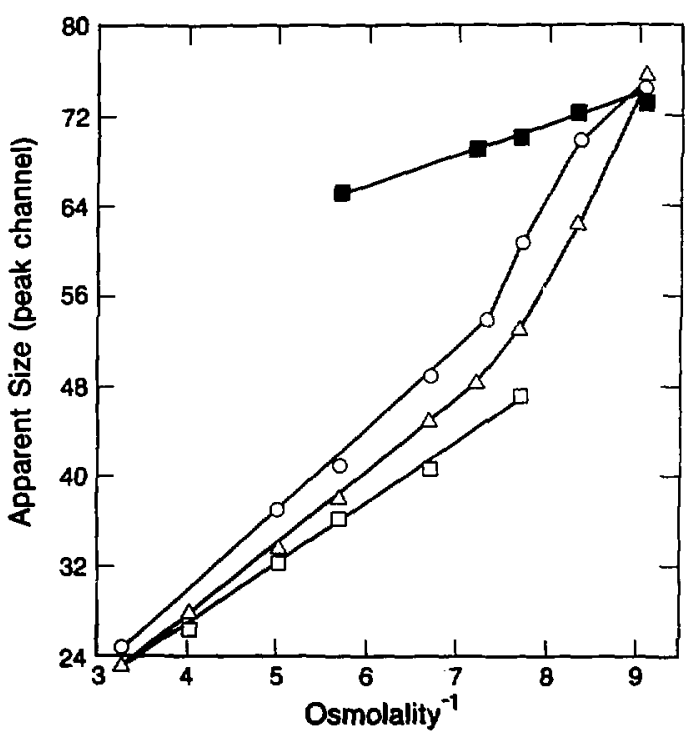

Fi. 4. Volume osmotic responses at $25^{n} \mathrm{C}$ for restored ghosts made under different conditions (1) triangles, made at $0^{\circ} \mathrm{C}, 130$ mOsm; circles made at $25^{\circ} \mathrm{C}, 120$ mOsm; and squares, made af $\left.10^{\circ} \mathrm{C}, 100 \mathrm{mosm}\right)$.

(XBL $8511-11470)$

respund osmotically-it is only the final condition that determines the results. However, for the $40^{\circ} \mathrm{C}$ ghosts an unexpected new phenomenon appeared: a double population-one responding well osmotically and the other (larger in volume) responding very little, indicating two classes of membrane stale. A unifying explanation for these new results is being sount in our current investigations.

\section{MEMBRANE FNLURE BY OSMOTKC FRACUITY}

A model that predicts the osmolic frestity curve of a red-cell population has been developed by combining the classic equation of Ponder with experimental size-distribution information of cell populations, as determined by resistive pube spectroscopy. Two of the parameters involved, namely a normalized osmotic volume correction, B, and a swelling index, $k$, are determined from the expermental, average properties of the population.

The equation derived for the equilibrium-value critical osmotic pressure (the condition where the membrane will first fail osmotically) as a function of cell size is:

$$
\pi_{i}^{*}=\frac{1-B / V_{i}^{*}}{k\left(V_{i}^{*}\right)^{n}-B / V_{i}^{*}}
$$

where $V_{i}^{*}=V_{i s o, i} / \hat{V}_{\text {iso }}$ is the ratio of a variable cell volume to the median cell volume at isotonicity, and the other parameters are:

$$
B=\frac{b}{\hat{V}_{\text {iso }}}
$$

(normalized cell volume correction for the average $\hat{\mathrm{V}}_{\text {iso }}$ population)

$\mathrm{b}=$ nonosmotically active cell volume;

$k=V_{c} / \hat{V}_{\text {iso }}$ (swelling index for the average population)

$\mathrm{n}_{\mathrm{e}}=$ surface area distribution index. The $\mathrm{n}_{\mathrm{e}}$ value was theoretically found to be linked to $B$ and $k$ by the following expression:

$$
n_{e}=-\log k / \log B
$$

Figure 5 shows the envelope of cumulative relative size distributions, percent frequency vs. $V_{i}^{*}$, of blood samples obtained from ten healthy donors. The data all fall within the two limiting broken lines, with average values represented by the full continuous line.

Figure 6 shows fragility curves, simulated according to Eq. (1), using the average size distribution shown in Fig. 5. It can be observed that, for increasing values of the swelling index, $k$, or the volume correction parameter, $B$, the curves are shifted to the left, i.e., to the region of less fragile populations. The values of the parameter $n_{e}$, calcubied from Eq. (2), are also indicated in Fig. 6 for each curve. For the curve with $B=0.48$, the effect of using values of $n$ above and below $n_{e}$ is 10 


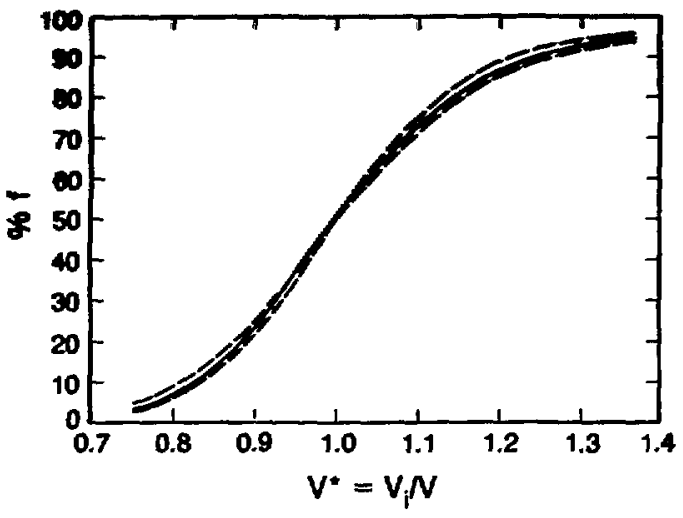

fi. 5. The average (solid curve) and range-envelope (dashed curves) of cumulative frequency size distributions of 10 normal blood-cell samples.

(XBL 8511-11473)

change only the slope of the curve; it does not alter the location of the $50 \%$ fragility point.

Figure 7 shows five sets of RPS fragility data, three of which belong to the healthy-donor samples, whose size distributions belong to those in Fig. 5. The remaining two data sets correspond to the hemoglobin 55 blood samples from donors with sickle cell disease, whose cell size distributions (not shown) were found to be much broader than the normal ones. The solid lines in Fig. 7 are the result of fitting the data with Eq. (1), by using the parameters indicated for each curve along with the corresponding size distributions. The only adjustable parameter is the index $n$, whose experimental values are also indicated in this figure.

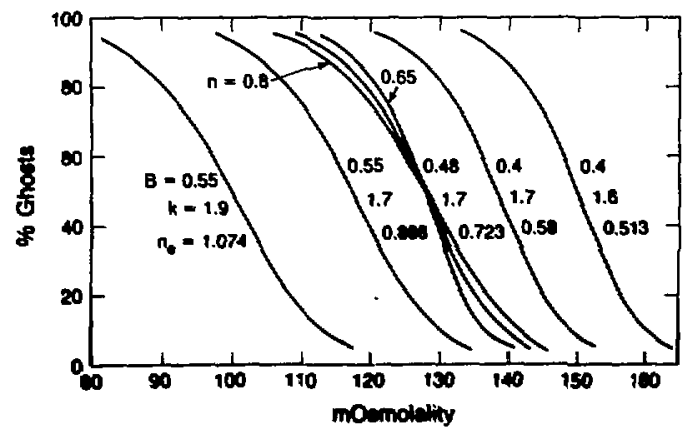

F. C. Dependence of theoretical fraitity curves on $b, k$ and n.

(XoL as 1)-11469)

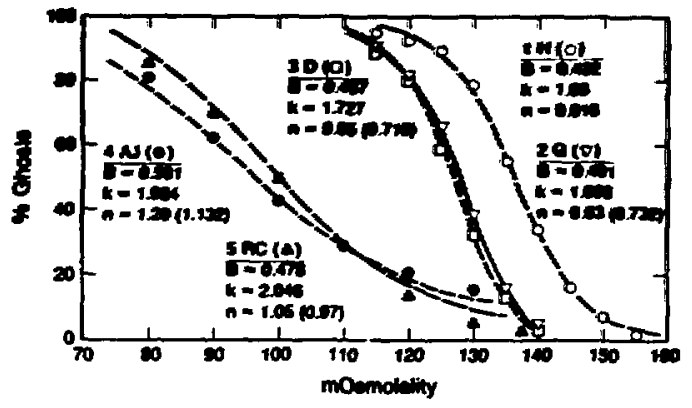

F3. 7. The actual fragility data of five samples are motched by theoretical fragility curves with specific values of $B, k$ and $n_{e}$ or ก.

(XBL 8511-11476)

We interpret these results as providing good support to the use of the present model, based on the experimental size distribution of a given cell sample. Another interesting consequence of the present model is that the distribution of excess surface areas follows closely the distribution of cell sizes for normal cells. This renders an essentially constant value for the surface-to-volume ratio of all cells, in agreement with experimental observations reported in the literature. ${ }^{4}$

\section{REFERENCES}

1. Mel, H.C., Richieri, G.V., Massaldi, $H_{\text {., }}$ and Bridwell, R. Red blood cell biophysics: osmotic fragility and related membrane phenomena. Biology and Medicine Division Annual Report 1983-1984, Lawrence Berkeley Laboratory report LBL-18393, p. 139 (1985).

2. Richieri, G.V., and Mel, H.C. Temperature effects on osmotic fragility, and the erythrocyte membrane. Biochim. Biophys. Acta 813, 41-50 (1985).

3. Richieri, G.V., Akeson, S.P., and Mel, H.C. Measurement of biophysical properties of red blood cells by resistive pulse spectroscopy: volume, shape, surface area, and deformability. I. Biochem. Biophys. Methods 11, 117-131 (1985).

4. Linderkamp, O., and Meiselman, H.J. Geometric, osmotic, and membrane mechanical properties of density-separated human red cells. Blood 59, 1121-1127 (1982). 


\section{Tiasue Effects of Heavy Charged Particle Ceams}

\section{LATE RADATION DAMACE IN THE MOUSE KDARY}

\section{Elward L Apen, Patricia Powers-liains, Kristion S. Kavanau, and Randy J. DeCuzman}

Late radiation damage to the kidney is a doselimiting factor for human cancer therapy when the treatment field is in the upper abdomenal region. The Berkeley Bevalac beams are being used for cancer radiotherapy, and information about the time-course and severity of renal damage following heavy-ion irradiation is of clinical and radiobiological importance.

We are studying the late effects in the mouse kidney after irradiation with single and fractionated doses of $x$ rays or heavy charged particles. The repair capacity of the renal functional unit (glomerulus and tubule) is examined with respect to the number of dose fractions and the dose per fraction. Keeping the length of time constant between the first and last fraction, i.e., 25 days, the mice are given $1,2,4,8$, or 16 fractions of radiation. This regimen enables us to conform to the current radiotherapy schedule of 4 consecutive days a week.

The kidney offers the opportunity for the separate examination of at least three functional elements, each of which is thought to have limited capacity for proliferation and repair. These sytems are 1) the functional secretory and absorptive epithelial cells of the post-glomerular reabsorption apparatus (the tubule), in which one can examine the functional status of both secretory and absorptive activity, 2) the glomerular apparatus in which ultrafiltration of the plasma is the functional endpoint as measured ultimately in the filtration rate and, 3) the rate of perfusion of the kidney by plasma presenting itself for filtration in the glomerular apparatus. The latter two are, of course, closely interrelated because in any given set of circumstances, reduced plasma flow to the glomerular apparatus (in the presence of a nurmally functioning glomerulus), will lead to a reduced filtered volume. On the other hand, alteration of the "filterability" of the glomerular tuft may produce reduction of the glomerular filtration rate while renal glomenular perfusion remains unakered.

Recent work by Williams and Denekamp,' Stewart et $2 . .^{2,3}$ and Apen and Stcwart have provided some methods to assess the functional status of the irradiated mouse kidney. The methods that have been fully developed include those for the measurement of glomerular filtration rate and tubular secretory function. In addition, hematocrit values will be a measure of the anemia of renal insufficiency. The functional tests are run at 8- to 10-week intervals during the year following bilateral kidney irradiation.

The method to estimate the glomerular filtration rate is based on the rate of clearance of ${ }^{51} \mathrm{Cr}$ labeled EDTA from the plasma. This label acts as does inulin in that it is filtered at the glomerular surface and is neither secreted nor reabsorbed in the tubules. Therefore, its rate of disappearance from plasma is an estimate of the glomerular filtration rate (GFR). The method is used clinically in humans and also works well, with minimum adjustment, in the mouse. Williams and Denekamp? describe the method in detail. Twenty microcuries of ${ }^{51} \mathrm{Cr}$-EDTA are injected into a mouse, and 40 minutes later a blood sample is taken from the retro-orbital sinus. The radioactivity in $20 \mu \mathrm{l}$ of plasma is counted. High residual ${ }^{51} \mathrm{Cr}$ levels in the plasma indicate a decreased GFR. Figure 1 shows the plasma activity of ${ }^{51} \mathrm{Cr}$-EDTA at times after an IP injection into a nonirradiated mouse.

We are using a modification of the urinary frequency apparatus developed by Stewart et al..$^{5}$ to assess tubular function. Mice are individually housed in small chambers with wire floors. Voided urine falls onto absorbent paper that is continuously drawn beneath the cages. The number of urine spots in 24 hours correlates with total urine output. The time course of the change in urination frequency as a function of dose is recorded.

We have begun to study the feasibility of using 131-hippuran to measure the effective renal plasma flow (ERPF) in mice. Hippuran is actively secreted into the tubular fluid and is removed during a single passage through the kidney. The basic method is similar to the SiCT-EDTA clearance test, and from the ratio of the EDTA/hippuran clearance we will devise a parameter proportional to the fittration fraction that represents the flomerular function independent of the perfusion rate. The renal 


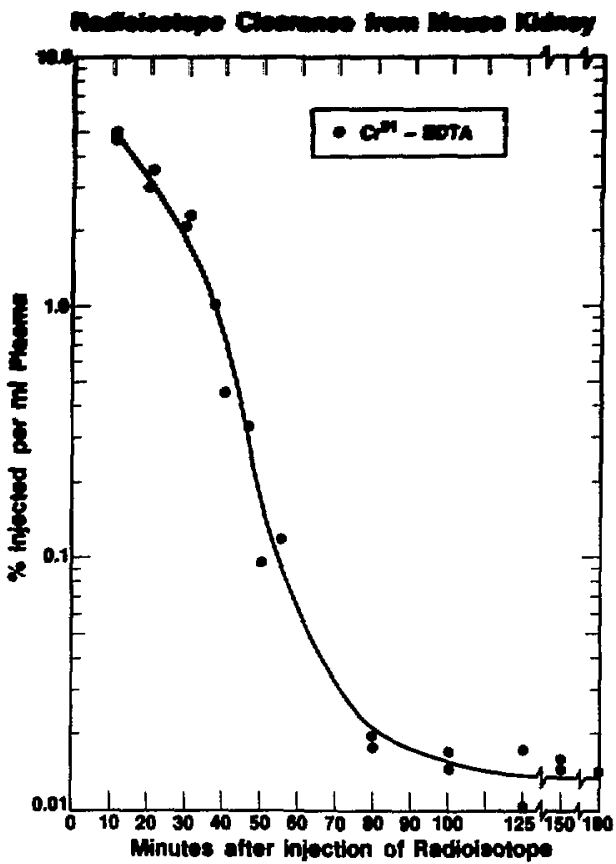

Fig. 1. The clearance curve of ${ }^{51} \mathrm{Cr}-$ EDTA (New England Nuclear) injected into $L A F_{1}$ mice.

(XBL 857-8399A)

plasma flow itself is an important measure of the vascular state of the kidney.

Alpen and Stewart ${ }^{4}$ observed that an anemia similar to the anernia of acute renal insufficiency in man develops as a result of radiation nephropathy in the mouse. The simple end point of the peripheral hematocrit was shown to be even more sensitive than the ${ }^{51} \mathrm{C}_{\mathrm{r}-\mathrm{EDTA}}$ clearance in detecting early radiation nephropathy. The functional significance of this end point remains to be explained. It is known that the anemia of acute renal insufficiency in man has at least two components: an increase in red cell hemolysis, possibly but not certainly in the kidney, and a decrease in the production of erythropoietin, the red cell regulating hormone that is mostly produced in the extragiomerular tuft in the kidney. The anemic changes have special significance because the effects probably arise from changes outside of the vascular or giomerular endothelium. We want to demonstrate the time relationship between the endothelial changes of glomerular damage and the onsat of anemia.

One of the factors in late radiation damage to the kidney is fibrosis, which misy contributc to renal
Filure." Fibrosis is characterized by excessive connective tissue proliferation, of which collowen is the main component. Using the hydroxyproline assay. Meistrich et al. increases in total collagen content in mouse kidneys can be detected by 9 months after $x$-imadiation. The concentration of hydroxyproline, an amino acid specific to collagen, may be a sensitive measure of late kidney damage. We are using a modification of the Woessner method" to measure the hydroxyproline concentration at 1 year after single and fractionated doses of irradiation.

Using the results of these experiments, we will compare the radiation response to $x$ rays with that to the high-energy Bevalac beams. A given biological response is produced by different doses for each type of radiation, and these differences are expressed as the relative biological effectiveness, or RBE. The RBE values for kidney, a late-responding tissue, may be higher than. the RBE for acuteresponding tissues, such as intestine. The fractionation experiments will also provide RBEs for the amount of repair between doses. Several preliminary experiments are in progress: 1 and 4 fractions of $670-\mathrm{MeV}$ neon, 1, 2 and 4 fractions of $670-\mathrm{MeV}$ silicon and 1 fraction helium. The 1, 2, 4, 8 and 16 fraction $x$-ray experiment is completed. Figure 2 compares the dose response curves for ${ }^{51} \mathrm{Cr}$-EDTA and 131 -hippuran activity, urinary frequency and plasmacrit (100-hematocrit). Each of these systems appears to be modified in a very similar way with respect to dose fractionation. As the number of fractions is increased, the total dose needed to obtain the same response level (i.e., 12 urine spots per 24 hours) is greater.

In Fig. 3 the reciprocal total $x$-ray dose required for an isoeffect level is plotted as a linear function of dose per fraction. The estimate of the ratio of the $y$ intercept $(\alpha)$ to the slope of the line $(\beta)$, the $\alpha / \beta$ ratio, is a relative measure of cell killing. This $\alpha / \beta$ ratio is derived from the linear-quadratic model equation: In (response) $=n\left(\alpha d+\beta d^{2}\right)$, where $d$ is the dose per fraction, and $n$ is the number of fractions. The $\alpha / \beta$ ratio is used to rclate changes in biological effectiveness to the dose per fraction. This can be a guide to radiotherapists in choosing the optimum size of dose per fraction. The $\alpha / \beta$ ratios for the kidney function responses (Fig. 2) are between 4.0 and 4.65 Gray: the $\alpha / \beta$ ratio for wet kidney weight is 5.1. When the heavy-ion irradiation experiments are compleled, we will compare those $\alpha / \beta$ ratios to those of $x$ rays to assess the fractionation effects on kidney lissue of both types of radiation. 


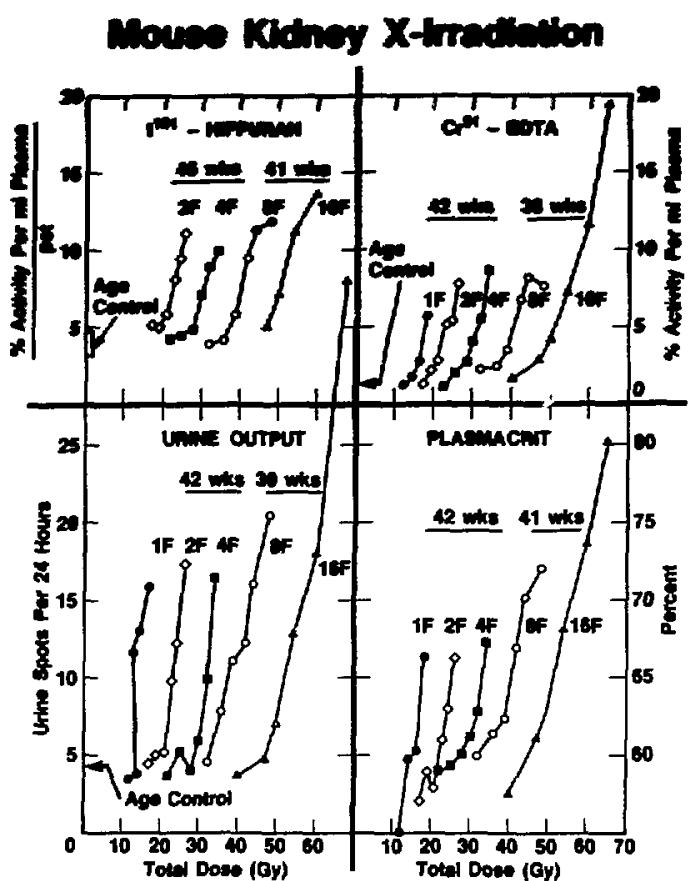

Fig. 2. Dose response curves for plasmacrits and three kidney function tests in mice. The curves are labeled with the fraction number and the number of weeks after the first dose that the test was done.

(XBL 857-8393A)

\section{REFERENCES}

1. Williams, M.V., and Denekamp, J. Sequential functional testing of radiation-induced renal damage in the mouse. Radiat. Res. 94, 305-317 (1983).

2. Stewart, F.A., Soranson, J.A., Alpen, E.L., Williams, M.V., and Denekamp, I. Radiationinduced renal damage. The effect of hyperfractionation. Radiat. Res. 98, 407-420 (1984).

3. Stewart, F.A., Soranson, I.A., Haughan, R., Alpen, E.L., and Denekamp, J. The RBE for renal damage after irradiation with $3 \mathrm{MeV}$ neutrons. Br. I. Radiol. 57, 1009-1021 (1984).

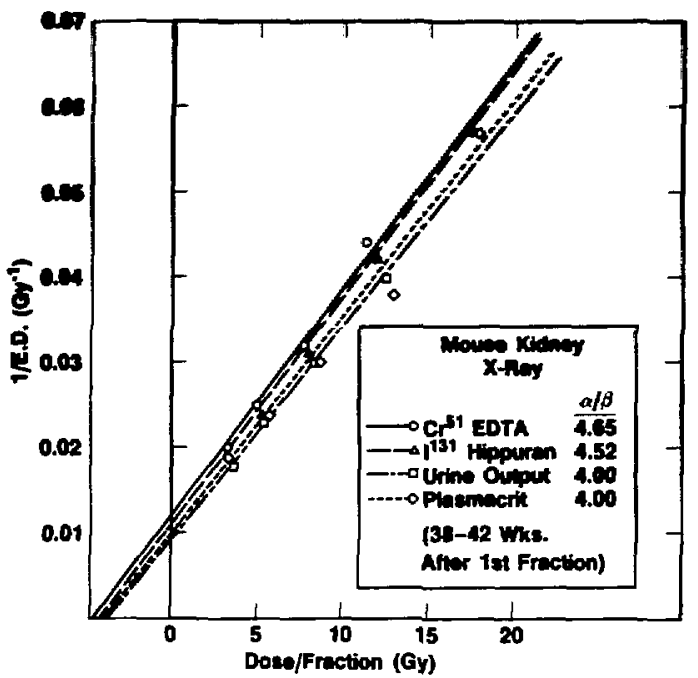

Fig. 3. The reciprocal total $x$-ray dose required for an isoeffect level versus the dose per fraction of $x$ rays. $\alpha$ is the value of the $Y$ intercept, and $\beta$ is the slope of the line. The data points represent isoeffect levels from $1,2,4,8$ and 16 fractions of $x$ rays.

(XBL B57-8394)

4. Alpen, E.L. and Stewart, F.A. Radiation nephritis and anemia. $B r$. J. Radiol. 57, 185-186 (1984).

5. Stewart, F.A., Michael, B.D., and Denekamp, 1. Late radiation damage in the mouse bladder as measured by -increased urinary frequency. Radiat. Res. 75, 649-659 (1978).

6. Chauser, B.M., Hudson, F.R., and Law, M.P. Renal function in the rat following irradiation. Radiat. Res. 67, 86-97 (1976).

7. Meistrich, M.L., Williams, M.V., Soranson, J., Fowler, J.F., and Denekamp, J. Increased collagen and fluid content of mouse kidneys at 9 months after single or fractionated $x$ irradiation. Radiat. Res. 99, 185-201 (1984).

8. Woessner, J.F. The delermination of hydroxyproline in tissue and protein samples containing small iroportions of this imino acid. Arch. Biochem. Biophys. 93, 440-447 (1961). 
Hanum MAOATION OF THE RAT SMNA COM

\section{Adrian Rodrigere, Edward L. Apen, Iandy J. DeCusman, and John C. Friolean}

The spinal cord is a relatively radioresistant nonproliferative tissue that is of interest because it is often within the treatment volume during heavyion radiotherapy. The spinal cord exhibits longterm or late effects from radiation damage that we are studying with respect to repair after dose fractionation. Fractionation studies of spinal cord will allow us to test the hypothesis that RBE values for late effects are much higher than RBE values for acute responses of tumors and proliferating tissue. These studies will also provide RBE values for repair. This information will provide a basis for choosing the appropriate ion species, energy, and tolerance dose for radiotherapy of tumors that are adjacent to the spinal cord. Studies with neutrons as a model of nigh-LET radiation indicate that more than four dose fractions do not provide added protection by repair processes. If this relationship holds for heavy ions, we can expect the RBE to increase with large fraction numbers.

In the present study with helium ions, we have repeated and expanded earlier studies by Leith et al. ${ }^{1}$ The helium ions will be used as the reference/low-LET radiation for comparison with heavy-ion responses in future studies.

The helium experiments include single dose, and 2,4 , and 8 fractions, given within 4 weeks ( 25 days). The dose schemes are designed to follow therapy treatment protocols: irradiations 4 days per week for 4 weeks, with a 3-day break each Friday through Monday.

White male CD-rats (approximately 10 weeks old and weighing $200-250 \mathrm{~g}$ ) from the Charles River Breeding Laboratories, Wilmington, $M A$, were used. The rats were irradiated in the thoracolumbar region of the spinal cord (after anesthesia with methoxyflurane, an inhalation anesthetic, and placement in restraining holders). They were positioned in the plateau region of ionization, and an $11 \times 16 \mathrm{~mm}$ semielliptical field was used for spinal cord exposure. The animals are maintained for 300 days and observed on a weekly basis for development of paralysis. After the animals begin to show decrements in hindlimb reflexes and leg strength, they are observed Iwice a week. The scoring system is the same as that used by leith and is shown in Table 1. Radiation-induced myelopalhy is considered to have occurred if the animal reaches a scone of S-2 or greater.
Table 1. Grading system for assessment of radiation-induced myelopathy.

\begin{tabular}{|c|c|}
\hline Grade & Description of gross impairment \\
\hline S-O & No difference from conlrols \\
\hline S-1 & $\begin{array}{l}\text { Delay in eliciting hindlimb-kick response upon } \\
\text { pressure to limbs }\end{array}$ \\
\hline S-2 & $\begin{array}{l}\text { Definite but mild impairment in hindlimb-kick } \\
\text { response with obvious curling of toes }\end{array}$ \\
\hline S-3 & $\begin{array}{l}\text { Impairment of kick moderately severe, and rats walk } \\
\text { with slight hunchback, or flatfooted }\end{array}$ \\
\hline S-4 & Severe impairment, marked hunchback, rats walk on toes \\
\hline S-5 & $\begin{array}{l}\text { Limb(s) completely useless, total paralysis (either } \\
\text { uni- or bilateral) }\end{array}$ \\
\hline
\end{tabular}

\section{RESULTS AND DISCUSSION}

Irradiated rats begin to exhibit loss in hindlimb reflexes and performance within 12 to 20 weeks postirradiation. The latency of onset of paralysis is dose dependent as is the mean severity score for each dose group. Figure 1 shows the helium irradiation results as percent paralysis versus total dose for single doses and 2, 4, and 8 fractions. With increasing numbers of fractions, the total dose required to reach a particular isoeffect $\quad 50 \%$ paralysis) is greater, indicating that there has been

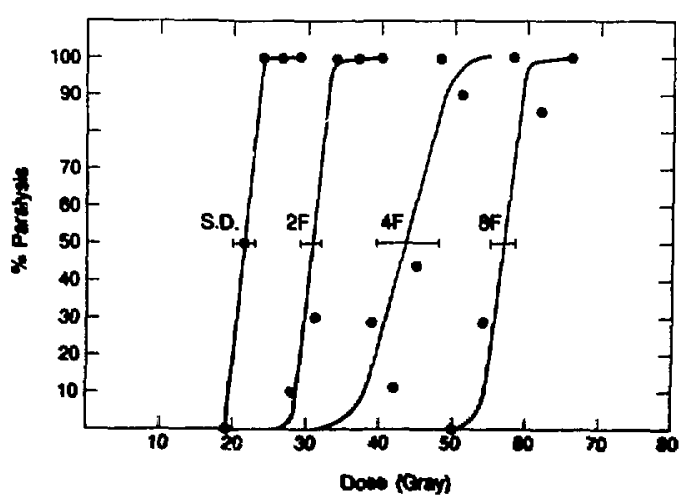

F. 1. Helium imadiation (plalewu) with single doses and 2, 1 , and a fractions detivered over 1 weats (25 dys). The EO 50 for puratsis is indicaled with $95 \%$ contidence limins.

ACL 857+11236) 
substantial repair. The EDso doses for the single dose and the 2, 4, and 8 fractions are 22.3, 30.6, 43.6, and 56.9 gray, respectively. The single dose paralysis data is comparable to previous results of Leith et al.' with helium irradiation and gives an RBE of 1.08 .

The isoeffect doses ( $E D_{50}$ ) were $e$ \%aluated with the use of the multifraction linear-quadratic model: In (Ejeffect $)=n\left(\boldsymbol{\alpha} d+\boldsymbol{\beta} \boldsymbol{d}^{2}\right)$, where $d$ is the size of the dose per fraction, and $n$ is the number of dose fractions. We can obtain the $\alpha / \beta$ ratio from the multifraction isoeffect data, which is a relative measure of cell killing in a tissue via the linear (single event) and quadratic (double event) modes and can be used as a parameter describing the response of a tissue to dose fractionation. The linearquadratic model can be transformed to

$$
\frac{:}{\mathrm{nd}}=\frac{\alpha}{\mathrm{E}}+\mathbf{d} \frac{\beta}{\mathrm{E}}
$$

and the data plcited as in Fig. 2. The slope of the line is an estimate of $\beta(\beta / E)$, and the intercept is an estimate of $\alpha(\alpha / E)$. The $\alpha / \beta$ value obtained for the spinal cord data is 2.43 . This value is comparable to published tissue $\alpha / \beta$ values and is within the range for $x$-ray spinal cord data of $5.4 .^{1,2}$ In comparison, fractionated neutron (15 MeV/amu) data ${ }^{3}$ hase an $\alpha / \beta$ value of 77.4 . There is little repair of neutron damage, and the high value indicates a large $\alpha$ component of linear cell inactivation. The repair capacity of a tissue after fractionation can be assessed by plotting the value of $\Delta \mathrm{Dr}$ against dose per fraction, as is shown in Fig. $3 . \Delta D r$ is the increase in dose per fraction needed for an isoeffect when the number of dose fractions is increased from $n$ to $n$ ':

$$
\Delta \mathrm{Dr}=\frac{\mathrm{Dn^{ \prime }}-\underline{\mathrm{Dn}}}{\mathrm{n}^{\prime}}
$$

This parameter may be of valur: to the radiotherapist in determining the dose per fraction and fraction number beyond which there is no further advantage or sparing, since there is no further repair (i.e., increase in dose per fraction). In Fig. 3 helium plateau radiation, $x$ ravs, and 15-MeV/amu neutrons are compared. For helium and $x$ rays the intercept on the $x$ axis is approximately the same $(1.5-1.6 \mathrm{~Gy})$, and for neutrons it is about $4 \mathrm{~Gy}$. There is no advantage in going to lower doses per fraction with neutron radiation because there is less repi than for $x$-ray and helium radiation.

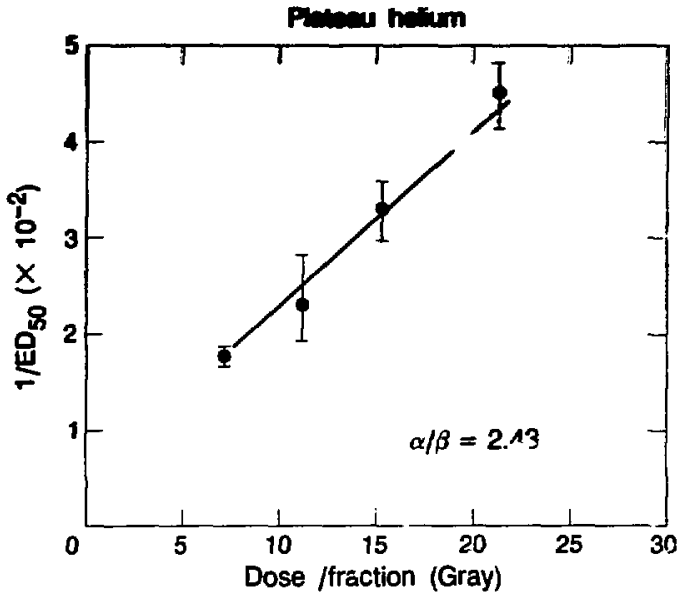

Fig. 2. Isoeffest plot $\left(1 / E D_{50}\right)$ versus dose per fraction of helium radiation.

(XBL $857-1$ (234)

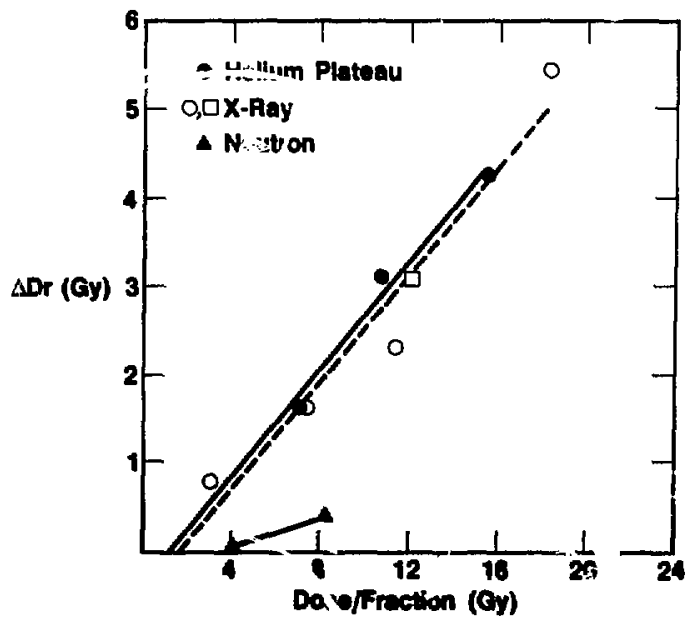

fi. 3. Thoraco-lumbar spinal cord repair ( $(\mathrm{U} r$ ) as a function of dose per fraction whon the number of iraclions is increased from $n$ to $n$.

$$
S D_{t}=\frac{D_{n}^{*}-D_{n}}{n^{\prime \prime}}
$$

$D_{i n}$ and $D_{n}$ are iscerfiect doses.

(XAL 857-8403) 


\section{REFERENCES}

1. Leith, I.T., Lewirsky, B.L., Woodkuff, K.H., Schilling W.A., and Lyman, J.T. Tnierance of the spinal cord of rats to irradiation with cyclotron accelerated helium ions. Cancer 35. 1692 (1975).

2. White, A. and Hornsey, S. Radiation damage to the rat spinal cond: The effect of single and fractionated doses of $x$ rays. Br. J. Radiol. $5 \pi$. 515-523 (1978).

3. Van der Koget, A.J., and Barendsen, G.W. Late effects of spinal cord irradiation with 300 kV $x$ rays and 15 MeV neutrons. Br. J. Radiol. 47, 393-389 \{1974\}.

\section{INACTIYATION OF MOUSE MARROW STEM CELLS BY HEAVY CHARGED PARTICLES}

\section{E. John Airsworth, Lynn J. Mahlmann, and John C. Prioleau}

A fundamental question of biophysics concerns relat:. aships between the physics and chemistry of ene zy deposition and biological effect. Charged particles provide an excellent tool to explore these relationships because different patterns of energy deposition may be achieved by varying atomic number, the energy of the particle, or both. We previously explored relationships between linear energy transfer (LET) and inactivation of bone marrow colony-forming units (CFU-S) where the experiments were performed using, primarily, spread Bragg curves similar to those used in radiation therapy.' Under those conditions extensive fragmentation is produced by the brass spiral ridge filters, and the range of LET's to which cells are exposed is large. The purpose of the present study was to extend our work on relationships between LET and relative biological effectiveness (RBE), using the plateau portion of the Bragg curve because primary particle fragmentation is minimized, LET can be estimated more confidently, and the physics of energy deposition can be expressed in quantities of either dose or particle fluence. Because of the complex nature of energy depositions by charged particles, including the possibility of recombination of free radicals near the particle trajectory as well as a broad specirum of delta ray energies, charged particle dose reflecting total energy deposition may be a less than whotily appropriate means by which to express the physical characteristics of charged particles. Inferences concerning the effect per particle can be derived confidently when the LET distribution is small, and, as would be predicted on phrsical grounds, interpretation of results on biological effects of different particles is influenced significantly depending upin whether dose or fluence is used. The present w'ork extends our earlier studies on CFU-S insestiation' and on cataract production," and complements ongoing studies on Harderian gland carcinogenesis ${ }^{3}$ and life-span shortening in mice. $^{4}$

The methods of irradiation and measurement of CFU-S survival have been described in detail elsewhere.' In brief, hybrid CB6FI mice were given total-body exposure to various charged particle beams in the plateau portion of the Bragg curve, marro: $N$ was harvested between 30 and 90 minutes following irradiation, marrow-cell suspensions were prepared, and appropriate aliquots were injected into supralethally gamma-irradiated recjpients. Recipient mice were sacrificed 8-10 days following irradiation; spleens excised and fixed in alcohol, acetic acid, and formalin (AAF); and surface colonies greater than $0.5 \mathrm{~mm}$ were counted with the aid of a dissection microscope. Survival curves and associated statistical parameters were computed by standard methods.

Figure 1 represents a summary of our current and past results. The lower curve describes the relationship between RBE at $10 \%$ survival and LET. $\mathrm{RBE}_{10}$ is defined as the ratio of doses, in cGy, that produces $10 \%$ survival. The triangular symbols indicate data collected using the plateau portion of the Bragg curve in recent experiments and th: :ircles indicate estimates from previously published measurements made using spread Bragg peaks. The curve drawn through these points is eye fitted and probably adequately describes RBE-LET relationships for inactivation of CFU-S. Note that the RBE achieves a maximum value of about 1.8 in the range of 80-100 keV per micron and declines at lower and higher LETs. The one point that departs significantly from the curve is for spread-peak argon particles where LET was estimated at approximately $600 \mathrm{keV} / \mathrm{mm}$. The RBE of about 1.1 was the result of a computed extrapolation number $n$ of 0.7, so 


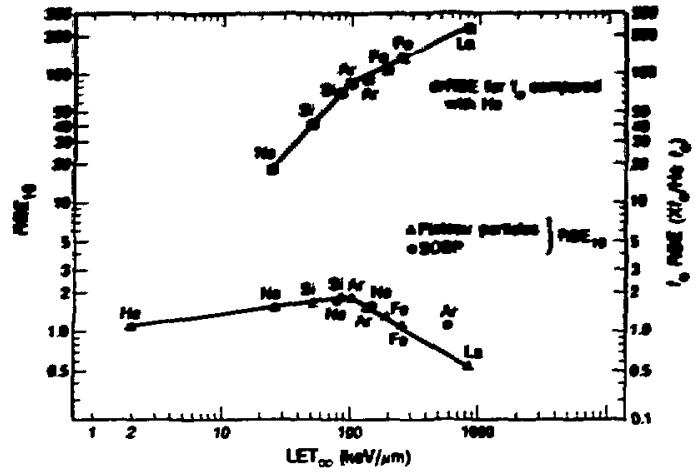

Fit. 1. Relative biological effectiveness (RBE) of charged particles for in.ctivation of mouse narrow colony-forming units (CFU-S). The lower curve indicates RBE at 10\% survival, compared with ${ }^{60} \mathrm{Co}$ gamma radiation (left ordinate): the upper curve indicates RBE based on particle fluence, compared with helium, to reduce survival by $1 / e$ (right ordinale). CFU-S inactivation measurements are based on replicate experiments.

(XBL 8517-8567)

the RBE value of 1.1 is probably spuriously high; based on $D_{0}$ estimates of $122 \mathrm{cGy}$ for gamma rays and $151 \mathrm{CGy}$ for argon particles, the RBE would be 0.8 , and provide a better fit to the curve.

The upper curve in Fig. 1 shows $f_{0}$ RBE where $f_{0}$ is defined as the particle fluence that reduces survival by $1 / e$. The relationship between $D_{0}$ and $f_{0}$ is that $D_{0}$ is in units of dose in cGy and $f_{0}$ is based on particle fluence. The $f_{0}$ RBE indicates the relative effectiveness per particle for inactivation in comparison with helium particles. Helium particles are the lowest-LET particle for which we have collected data and for which we can confidently convert from dose to fluence. It is quite clear that estimates of RBE based on dose and/or fluence are quite different. In the case of lanthanum the lower curve in Fig. 1 shows the RBE, in terms of dose, was approximately 0.5 , a value appreciably less than for helium; whereas, in terms of effectiveness per particle for inactivation, lanthanum is approximately 200 times more effective than helium. We have also estimated the number of particle traversals for helium and lanthanum in a nuclear area of approximately $50 \mu \mathrm{m}$, and preliminary calculations show that at the $f_{0}$ value the number of traversals is of the order of 0.6 for lanthanum and approximately 140 for helium.

Figure 2 further summarizes our results in terms of both inactivation cross section - and $t_{0}$ in relation to LET. We have followed the lead of Dr. Gerhard Kraft at GSI in evaluating the results based on inactivation of cross suction." and we are greatly indebled to him and to 5.8. Cutis of our Laborecory in consection with the andysis of these rexilss in kerms of a and $f$ in the cross-section andyis we have assumed the nucler diameter of a CFU-S to be approximately 8 microns, a value we inifer from the muclear size of a small mouse marrow Iymphocyte. The curve describing - with LET relates to the left ordinate and shows that cross section increases linearty, possibly with a slope slightly greater than 1.0, up to a LET of approximately $100 \mathrm{keV} / \mathrm{\mu m}$, but at higher levets of LET a diminished rate of increase occurs. The dotted line represents an extrapolation of the curve over the LET range of 2 to $100 \mathrm{keV} / \mu \mathrm{m}$, and the difference between the dotted curve and the curve fitted to the points between 100 and $1000 \mathrm{keV}$ per micron indicates the extent to which energy is wasted at energy deposition rates in excess of $100 \mathrm{keV} / \mathrm{\mu m}$. The descending curve in Fig. 2 relates to the right ordinate and indicates how $f_{0}$ decreases with increasing LET. For example, with helium at approximately $2 \mathrm{keV} / \mathrm{\mu m}$, the $\mathrm{f}_{0}$ is approximately 280 particles $\times 10^{6} / \mathrm{cm}^{2}$, and for lathanum, the comparable value is $1.3 \times 10^{6} / \mathrm{cm}^{2}$. The quotient of these two values indicares the RBE of approximately 200 plotted in the upper curve in Fig. 1.

The relationship between the present results obtained CFU-S and results obtained by Dr. Kraft and his co-workers at GSI is a subject of a manuscript in progress.

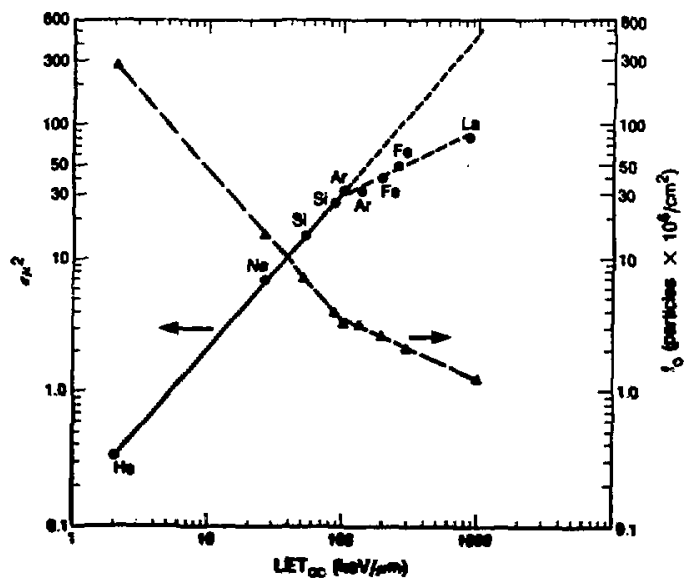

F. 2. Insctivation cross section (o) and purticle fluence (fol to reduce sunival of mouse murrow CFU.S by $1 / \mathrm{e}$. The exometric cross section of the nove minow CFU.S is extimined at bout

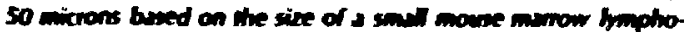
owe.

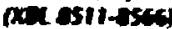




\section{REFERENCES}

1. Ainsworth, E.J., Kelly. LS., Mahmann, LJ., Schooley, I.C." Thomas, R.H., Howard, I., and Alpen, E.L. Response of cotony-formingunits-spleen to heavy charged particles. Radiat. Res. 96, 180-197 (1983).

2. Jose, J.G., and Ainsworth, E.J. Cataract production by heavy charged argon, neon, and carbon particles. Radiat. Res. 94, 513-528 (1983).

3. Alpen, E.L., Powers-Risius, P., Fry, R.J., and Ainsworth, E.J. Harderian gland carcinogenesis from high LET, high $Z$, charged particles. In Pion and Heavy Ion Radiotherapy:
Pre-Cinical and Clinical Studies. (C.D. Sonsgaud, Ed.). Elsevier Esomedial, New York, Amsterdam, Oxfond, pp. 335-341 (1933).

4. Ainsworth, EJ., Prioleau, I.C., and Mahlmann, L.). Life shortening effects of heary charod particles: A status report. Biology and Medicine Division Armual Report 1901-1902. Lawrence Berkeley Laboratory report LBL14986, p. 72 (1982).

5. Kraft, G., Blakely, E.A., Hieber, L. KraftWeyrather, W., Miltenburger, H.G., Muller. W., Schuber, M., Tobias, C.A., and Wulf, $H$. HZE effects on mammalian cells. Adv. Space Res. 4, No. 10, pp. 219-226 (1384).

\title{
ACUTE RADIATION LETHALITY IN MICE EXPOSED TO HEAVY CHARGED PARTICLES
}

\author{
E. John Ainsworth, John C. Prioleau, and Lynn J. Mahlmann
}

Relationships between relative biological effectiveness (RBE) and linear energy transfer (LET) are a fundamental aspect of the Biomedical Program at the Bevalac. Such relationships have been determined using various in-vivo and in-vitro model systems, and in general, the peak RBE appears to occur at a lower dose-averaged LET for in-vivo systems than is the case for the various in-vitro systems studied. ${ }^{1}$ The response of mouse bone marrow stem cells (CFU-S) is among the several in-vivo systems studied, and we have determined RBE-LET relationships using charged particle beams modified by ridge filters, such as are used in radiation therapy, as well as under conditions in which mice are exposed to high-energy particles in the plateau portion of the Brags curve., ${ }^{2,3}$ Because we had demonstrated previously that sensitivity to the hematopoietic syndrome, reflected by acute lethality within $\mathbf{3 0}$ days, could be predicted based on inactivation of mouse marrow CFU-S, ${ }^{4}$ a small program was undertaken to determine if the same relationship would hold for mice exposed to heavy charged particles. We are unaware of any previous measurements of acute radiation lethality made with heavy charged particles, but information is avallable for helium particles (Chong. 1971-see footnote to Table 1). The results from our acute lethality measurements may be compared with our previous marrow and activation data as well as with the responses of intestinal crypt survival reported by Alpen et al. 5

Mice were given total body exposures to either ${ }^{60} \mathrm{Co}$ gamma radiation or to various heavy charged particles. Our initial studies on CFU-S inactivation, as well as our acute lethality measurements, were based on LAF $_{1}$ mice, but because of availability problems, the studies were extended with $\mathrm{CBGF}_{1}$ mice, the latter strain being used also in our lifespan studies. ${ }^{6}$ The animals used in these studies ranged in age from approximately 3 to 6 months, and our general method of procedure was to extend our $\mathrm{LD}_{50}$ studies when the age of our mice exceeded the realms that were suitable for our life-span studies, and/or under circumstance when Bevalac beam time became available due to cancellation of another experiment or optimized use of beam time that provided a few extra hours for such short-notice experiments. The results were obtained between 1978 and 1982 by exposing as few as 8 to 12 animals per day under the circumstances where Bevalac beam time became available. The exposure configurations for heavy charged particles or gamma rays are the same as were described for studies on inactivation of CFU. S. ${ }^{2}$ Most of the results were collected using spread Brass peaks, but in 1983 we extended the study to include animals that were exposed to higher enerby aroon particles in the platea portion of the Brage cunve. Mice were exposed a various times of $\mathrm{dal}$ or nimbt, housed 4-5 per cres, and deaths checked 


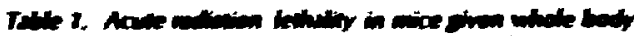

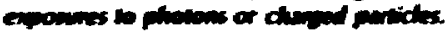

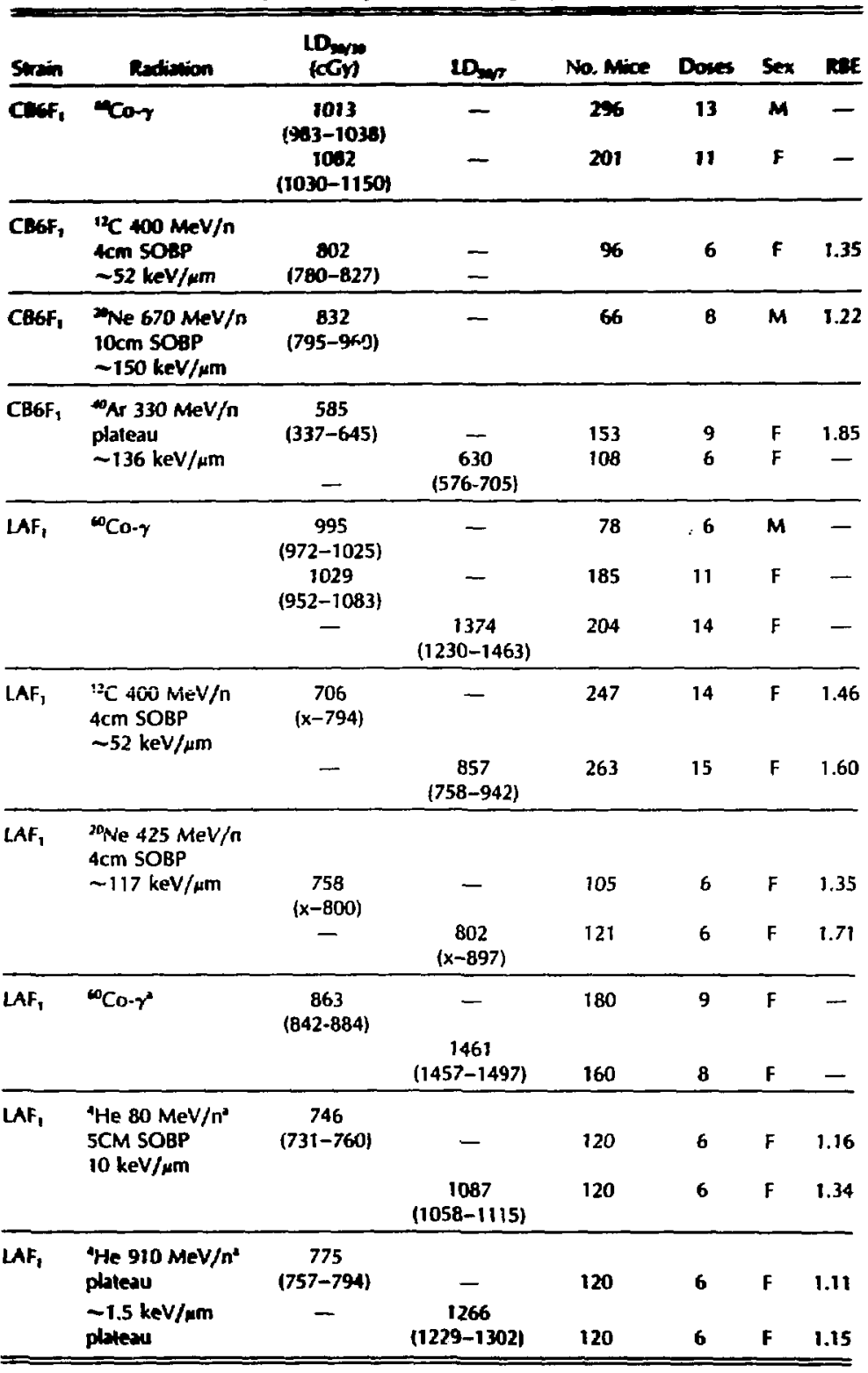

- C.Y.-L. Chone "In.Vivo Rediobiological Studies of a 910 MeV Hetium lon Deam," subminled in partisi satisfaction of the requirements for the degee of Doctor of Philosophy in Meoncal Physics, University of Caliomia, Derkeley, 1971 (121-314, 1971). 
dify for at lesst 30 days. Dingnostic bacteriology was performed on heart blood, spieen, and liver of some animals that died, especially within the first week after irradiation. The purpose of the bacteriology was to determine the extent to which Pseudomones aeruginosa might contribute to the threat of lethality or to the overall estimation of LD 50 . Mice were maintained on acidified tap water (pH 2.5) and P. aeruginosa was not detected bacteriologically; the most frequent organism found as a systemic invader in our postmortem cultures was a swarming Proteus species. Dose rates for charged particles were typically several hundred rad per minute, and for ${ }^{60} \mathrm{Co}$ gamma radiation, range from 20-40 rad per minute.

Table 1 is a summary of all the acute lethality data collected. Also included are old data collected by Claude Y-L. Chong and documented only in his $1971 \mathrm{Ph} . \mathrm{D}$. thesis. The dose ranges selected were such that $L D_{50 / 30}$ could be estimated, and in a few instances sufficient mortality occurred before 7 days that $\mathrm{LD}_{50 / 7}$ could also be estimated. The presentation of complete information on mean survival time is beyond the scope of this report, but suffice it to say that the frequency of deaths before 8-9 days was typically higher among animals exposed to charged particles than was the case for animals exposed to gamma rays. This fact made it possible to estimate $\mathrm{LD}_{50 / 7}$ adequately in some cases. The gamma radiation response of the two strains used showed no significant differences between strains or between sexes based on $L_{50 / 30}$. Based on $L_{50 / 30}$ the RBE's and $L A F_{1}$ are slightly higher than for $C_{B 6 F}$ mice, but the differences are probably not significant. Note that the lower $95 \%$ confidence limits for $\mathrm{LAF}_{1}$ mice exposed to neon or carbon particles could not be estimated confidently because too few points provided low levels of mortality. The RBEs based $\mathrm{LD}_{50 / 30}$ for carbon and neon are generally consistent with the RBEs of about 1.4 for these two particles based on the ratios of $D_{0}$ for photons $/ D_{0}$ for charged particles. The $L D_{50 / 30}$ for high eneigy argon particles was significantly lower than for neon or carbon particles, and the $L D_{50}$ is probably significantly elevated in comparison with the other two particles. This would indicate that the $L_{50 / 30}$ response has a generally similar pattern in terms of RBE-LET relationships as does the CFU. $S$ in activation response, since in both cases the maximum RBE occurs at a LET of the order of 80-100 keV/mm. Although RBEs based on LDsor? are of appreciable interest and can be compared with the previoushy published data by Apen el al.,", adequate estimates were made in only two cases for animals exposed to hexy changed particles. In the LAF 1 mouse the RBE for LDsayg appears to be in the range of 1.6-1.7. White the trend is that the RBE for LDsoy is higher than the RBE for LDsorye. the difference in $\mathbf{L D}_{50 / 30}$ is not statistically sigificant in those cases where an adequate comparison can be made, mainly, for $L^{A} F_{1}$ mice exposed to carbon particles and for CB6F1 mice exposed to plateau-argon particles. No estimate of RBE for $\mathrm{LD}_{50 / 7}$ can be made confidently in CB6F1 mice because no measurement was made for gamma radiation. Assuming that the measurement made in female $L A F_{1}$ mice or $L D_{50 / 7}$ would be applicable to the $C_{B 6 F_{1}}$, the RBE would be approximately 2.0 for plateau argon particles based on $\mathrm{LD}_{50 / 7}$. If such an $\mathrm{LD}_{50 / 7}$ estimate is valid, maximum values of $\mathrm{RBE}$ based on $L D_{50 / 30}$ and $L D_{50 / 7}$ are inferred from the present results at a LET of the order of 100 $\mathrm{keV} / \mu \mathrm{m}$. Based on the $\mathrm{LD}_{50 / 6}$ for low-energy helium particles the RBE is 1.3 , and 1.1 for highenergy helium, both values being significantly above 1.0 .

Comparison of the present results with earlier data obtained with fission spectrum neutrons is informative. Even with the maximally effective argon particles, the estimates of RBE for $L_{50 / 30}$ and $\mathrm{LD}_{50 / 7}$, namely about 1.9 and 2.2 , are below values published previously for fission spectrum neutrons where the comparable estimates are the order of 2.1 and 2.5 respectively. ${ }^{2}$ With carbon and neon particles at higher or lower LETs than $100 \mathrm{keV} / \mu \mathrm{m}$, the RBE estimates for $\mathrm{LD}_{50 / 30}$ and $\mathrm{LD}_{50 / 7}$ are appreciably lower than for fission spectrum neutrons.

Because no other data on acute lethality responses on animals given total-body exposure to charged particles are available, a useful purpose is served by documenting the present results. However, it should be duly noted that these results were collected over a period of several years, involved animals from many different shipments from a commercial supplier, and the bacteriological sample was small in scale. While the proximity of the $L D_{50 / 7}$ to the $L D_{50 / 30}$ is well known for animals exposed to high-LET neutrons, the number of deaths before 8 or 9 days observed in our animals exposed to charged particles indicates that more complete bacteriology should be done if such studies were to be repeated in the future. Although no Pseudomones aeruginosa was detected in these animals, it is well known that other intestinal organisms can produce early death, and more information is needed in this regand to confidently provide meaningful estimates for $\mathrm{LD}_{\mathbf{5 0} / 30}$ and $\mathbf{L D}_{50 / 2}$ after total boty exposure to heary charged particles. 


\section{REFEAENCES}

1. EA Bukely, No, F.Q.H., Curtis, S.B., and Tobies, CA. Heavy Ion RadiobiolooyCelludar Study (J.T. Lett, Ed.). Advances in Radiobiology 80, 295-383 (1984).

2. Ainsworth, E.J., Kelly, L.S., Mahlmann, L.J., Schooley, J.C., Thomas, R.H., Howard, J., and Apen, E.L. Response of colony-forming units-spleen to heavy charged particles. Radiat. Res 96, 180-197 (1983).

3. Ainsworth, E.J., Mahlmann, L.J., and Prioleau, J.C. Inactivation of mouse marrow stem cells by hervy charged particles, in this annul report.

4. Ainsworth, EJ., and Larsen, R. Colomyforming units and sunvival of irradiated mice with AET or endotoxin. Radiat. Res. 40, 149-176 (1969).

5. Apen, E.L., Powers-Risius, P., and McDonald, $M$. Survival of intestinal crypt cells after exposure to high- $Z$, high-energy charged particles. Radiat. Res. 83, 677-687 (1980).

6. Ainsworth, E.j., Prioleau, J.C., and Mahlmann, L.j. Skyhook Project Progress Report. In this annual report.

\section{SKYHOOK PROJECT: PROGRESS REPORT}

E. John Ainsworth, John C. Prioleau, and Lynn J. Mahlmann

Radiation carcinogenesis remains a fundamental problem in biophysics. Much is known regarding cellular inactivation based on proliferative death as a function of radiation quality or linear energy transfer (LET), but far less is known concerning the LET dependence for injuries that persist in surviving cells that are responsible for neoplastic transformation, mutagenesis, or other alterations in functional capabilities of surviving cells. While in-vitro studies of neoplastic transformation are extremely informative and provide information not readily obtainable from studies with intact experimental animals, ${ }^{1}$ prediction of in-vivo responses from in-vitro results remains a significant challenge. in the realm of external radiation studies with experimental animals, photons and fission-spectrum neutrons have been studied most extensively in comparisons of high- and low-LET radiation. ${ }^{2,3}$ We have initiated two types of animal experiments to complement and extend the existing data base comparing low- and high-LET radiations, and our studies utilize the Harderian gland model and range-finding or pilot stud:es on life-span shortening after exposure to heavy charged particles characterized by different LETs. Results from our animal studies have yielded new information concerning relationships between LET and carcinosenic effects and our animal studies are of themselves complementery. The Handerian dand model yields information on in-vivo transformation under conditions of lumor promotion that result from excess pituitary hor- mones, while the life-span studies, involving no extraneous promotion, indicate interactions between tumor induction and expression. Comparison of tumorisenic results on the Harderian gland and life shortening responses are expected to be highly informative.

The purpose of the Skyhook Project was to test the hypothesis that LET was an adequate predictor for life shortening and that low-energy charged particles characterized by a LET approximately the same as that for fission neutrons would produce quantitatively similar life-shortenins results after single or multifractionated doses. Another purpose was to evaluate life shortening, using comparatively high doses, over a LET range from less than 1 to approximately $200 \mathrm{keV} / \mu \mathrm{m}$. Pursuant to these goals, CB6F, mice were given total-body exposures to various charged particles at the Lawrence Berkeley Laboratory Bevalac, placed in a geriatric ward under highly controlled environmental conditions, and the irradiated and control populations were permitted to live out their normal life spans. The entry of various experimental groups into the Skyhook Project was described previously." A point to be emphasized is that the Skyhook Project is considered a pilot experiment that will yield sig. nificant results on single and fractionated doses of various charged particles under circumstances where the doses administered were relatively high. When these studies were initiated, it was obvious that the effectiveness of the various particles was 
unknown, and the experiments were designed anseming the LET would hive predictive vilue. The unimave objective was to produce a data base that would permin the vilimate design of a minimum number of low-dose studies where the goal is to determine the slope of the dose-response curve over a range of low doses that are relevant for radiation protection in terrestrial as well as space radiation emvironments.

Table 1 summarizes the present mortality status of the various groups in the Skyhook Project. The columns indicate the array of single total-body doses administered and the fractions represent the number of animals surviving in relation to the total number of animals irradiated. The columns to the left indicate single-dose experiments, and the three columns to the right indicate fractionated weekly doses given over 24 weeks. When mortality is complete among the various groups, the Skyhook Project will contribute significant new information on the following points: 1) life-span shortening as a function LET after single doses where the LET range explored is from 1 to approximately $200 \mathrm{keV} / \mu \mathrm{m}$; 2) the extent of sparing when doses of low-energy carbon particles are given as a single dose or in 24 weeldy fractions; and 3) the extent of spurine when hidh-enerdy neon particles ane given as a sinde dose or in 22-24 weetly fractions. Resuins of our studies with low-eneroy carbon particles and eamma rays were reported earliess and showr. 1) carbon is appreciably less effective than our fission-spectrum neutrons, although both are characterized by a LET of the order of 70-80 $\mathrm{keV} / \mathrm{\mu m}$; 2) dose fractionation produced little or no sparing effect on life shortening at those doses where the degree of life shortening produced by carbon particles was appreciable, and 3) the absence of a significant sparing effect does not occur only under circumstances where RBE or lifespan shortening is high.

New information has become available during the last year concerning RBE-LET relationships as mortality has been completed in test populations exposed to high-energy plateau ${ }^{20} \mathrm{Ne}$ particles and Jow-energy ${ }^{20} \mathrm{Ne}$ particles where the estimated dose-averaged LETs are approximately 30 and 130 $\mathrm{keV} / \mu \mathrm{m}$, respectively. Tables 2 and 3 and Figs. 1 and 2 summarize the life-shortening results from these groups, together with preliminary results based on incomplete mortality among animals

Table 1. Skyhook groups: Doses and current mortality."

\begin{tabular}{|c|c|c|c|c|c|c|c|c|c|}
\hline \multirow[b]{2}{*}{ Gamma } & \multicolumn{4}{|c|}{ Single dose } & \multirow[b]{2}{*}{${ }^{20}$ Si Plat. } & \multirow[b]{2}{*}{${ }^{56} \mathrm{Fe}$ Plat. } & \multicolumn{3}{|c|}{24 fractionation doses } \\
\hline & ${ }^{12} \mathrm{C}$ SOBP & ${ }^{20} \mathrm{Ne}$ SOBP & ${ }^{20}$ Ne Plat. & ${ }^{40} \mathrm{Ar}$ Plat. & & & Gamma & ${ }^{12} \mathrm{C}$ SOBP & ${ }^{20}$ Ne Plat. \\
\hline $\begin{array}{l}1430 / 83^{d} \\
20040 / 88 \\
2680 / 52 \\
35026 / 73 \\
45044 / 50 \\
4170 / 59 \\
50017 / 44 \\
5690 / 40 \\
60041 / 50 \\
70025 / 47 \\
7450 / 4 \\
7080 / 43\end{array}$ & $\begin{array}{ll}40 & 0 / 78 \\
80 & 0 / 49 \\
120 & 0 / 42 \\
160 & 0 / 46 \\
240 & 0 / 44 \\
320 & 0 / 44 \\
440 & 0 / 24\end{array}$ & $\begin{array}{ll}40 & 0 / 79 \\
80 & 0 / 46 \\
120 & 0 / 40 \\
160 & 0 / 48 \\
240 & 0 / 47 \\
320 & 0 / 47\end{array}$ & $\begin{array}{l}400 / 88 \\
800 / 48 \\
1200 / 47 \\
1600 / 47 \\
2400 / 40 \\
32035 / 135 \\
4000 / 48 \\
45024 / 94 \\
5000 / 47 \\
6007 / 131 \\
7500 / 7\end{array}$ & $\begin{array}{l}4018 / 122 \\
802 / 48 \\
1201 / 69 \\
1601 / 26 \\
2401 / 52 \\
3600 / 6\end{array}$ & $\begin{array}{l}401 / 79 \\
800 / 63 \\
1200 / 47 \\
1600 / 61 \\
2400 / 41 \\
3200 / 52 \\
4000 / 5 \\
45044 / 50\end{array}$ & $\begin{array}{l}4050 / 115 \\
8033 / 110 \\
16019 / 75\end{array}$ & $\begin{array}{l}17.30 / 69 \\
20.849 / 70 \\
23.70 / 45 \\
31.338 / 68 \\
35.00 / 43 \\
41.739 / 70 \\
54.231 / 69 \\
60.00 / 38 \\
79.00 / 44\end{array}$ & $\begin{array}{l}1.60 / 74 \\
3.30 / 37 \\
5.00 / 39 \\
6.60 / 39 \\
10.00 / 27 \\
13.30 / 46\end{array}$ & $\begin{array}{ll}17 & 26 / 70 \\
22 & 17 / 70 \\
31 & 5 / 70 \\
42 & 2 / 69\end{array}$ \\
\hline
\end{tabular}

\begin{tabular}{|c|c|c|c|c|c|c|c|c|c|}
\hline $195 / 539$ & $0 / 327$ & $0 / 307$ & $66 / 732$ & $23 / 323$ & $45 / 399$ & $102 / 300$ & $157 / 516$ & $0 / 262$ & $50 / 279$ \\
\hline
\end{tabular}

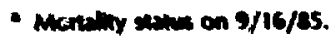

- MAS inclicans mem ane sumival.

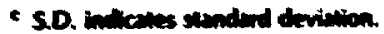

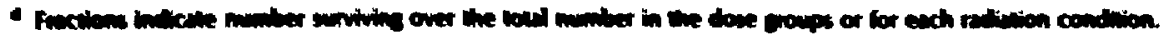




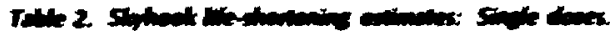

\begin{tabular}{|c|c|c|c|c|c|c|c|c|c|c|c|c|c|}
\hline \multicolumn{3}{|c|}{ An } & \multicolumn{4}{|c|}{ stivont } & \multicolumn{3}{|c|}{ "nent } & \multicolumn{4}{|c|}{ Cunont } \\
\hline Dove & MST & LS. & Dose & mas & \pm 5.0. & LS. & Dose & MST & LS & Dose & Mns & \pm 5.0 . & L.S. \\
\hline $\begin{array}{r}\mathbf{4 0} \\
60 \\
\mathbf{1 2 0} \\
160 \\
240\end{array}$ & $\begin{array}{l}003 \\
775 \\
746 \\
772 \\
736\end{array}$ & $\begin{array}{r}\overline{11} \\
46 \\
21 \\
57 \\
320\end{array}$ & $\begin{array}{r}40 \\
00 \\
120 \\
160 \\
240 \\
693\end{array}$ & $\begin{array}{l}700 \\
775 \\
703 \\
725 \\
649 \\
183\end{array}$ & $\begin{array}{l}201 \\
183 \\
177 \\
187 \\
220 \\
100\end{array}$ & $\begin{array}{r}13 \\
17 \\
90 \\
67 \\
144\end{array}$ & $\frac{40}{100}$ & $\begin{array}{l}830 \\
\frac{818}{811}\end{array}$ & $\bar{z}$ & $\begin{array}{r}40 \\
10 \\
120 \\
160 \\
240 \\
320 \\
440\end{array}$ & $\begin{array}{l}773 \\
734 \\
752 \\
696 \\
675 \\
695 \\
591\end{array}$ & $\begin{array}{l}170 \\
205 \\
204 \\
276 \\
192 \\
176 \\
175\end{array}$ & $\begin{array}{r}\overrightarrow{42} \\
25 \\
80 \\
101 \\
81 \\
186\end{array}$ \\
\hline \multicolumn{4}{|c|}{ Gamma } & \multicolumn{4}{|c|}{ Neon Plateau" } & \multicolumn{4}{|c|}{ Neon SOBPt } & & \\
\hline Dose & MAS & \pm S.D. & L.S. & Dose & MAS & \pm 5.0 . & L.5. & Dose & MAS & \pm S.D. & L.S. & & \\
\hline & & & & $\begin{array}{l}40 \\
80\end{array}$ & $\begin{array}{l}773 \\
714\end{array}$ & $\begin{array}{l}192 \\
203\end{array}$ & $\begin{array}{l}19 \\
79\end{array}$ & $\begin{array}{l}40 \\
80\end{array}$ & $\begin{array}{l}766 \\
802\end{array}$ & $\begin{array}{l}197 \\
194\end{array}$ & 27 & & \\
\hline & & & & 120 & 687 & 172 & 105 & 120 & 739 & 184 & 54 & & \\
\hline 143 & 751 & 175 & 25 & 160 & 753 & 194 & 40 & 160 & 716 & 184 & 77 & & \\
\hline & & & & 240 & 732 & 187 & 61 & 240 & 689 & 165 & 103 & & \\
\hline 268 & 703 & 191 & 74 & & & & & 320 & 686 & 186 & 107 & & \\
\hline 417 & 670 & 185 & 107 & 400 & 626 & 204 & 151 & & & & & & \\
\hline 569 & 668 & 201 & 108 & 500 & 585 & 243 & 191 & & & & & & \\
\hline 788 & 527 & 201 & 250 & & & & & & & & & & \\
\hline
\end{tabular}

MST $=$ median survival time.

MAS = mean after survival.

L.S. $=$ days lost.

= compared with controls of 776 days; survival $N=211$.

$\dagger=$ controls of 793 days; survival $\mathrm{N}=168$.

Table 3. Skyhook life-shortening table: Fractionated doses.

\begin{tabular}{|c|c|c|c|c|c|c|c|c|c|c|}
\hline \multicolumn{4}{|c|}{${ }^{12} \mathrm{C}$ SOBP" } & \multicolumn{4}{|c|}{ Gamma* } & \multicolumn{3}{|c|}{ Neon plateau"* } \\
\hline Dose(s) & MAS & \pm S.D. & L.S. & Dose(s) & MAS & \pm S.D. & L.S. & Dose(s) & MST & L.S. \\
\hline 0 & 776 & 184 & - & 0 & 776 & & - & 0 & 793 & - \\
\hline $1.6(40)$ & 790 & 186 & - & $17.3(415)$ & 730 & 186 & 46 & $17.0(408)$ & 704 & 89 \\
\hline $3.3(80)$ & 787 & 151 & - & $23.7(569)$ & 735 & 170 & 42 & $22.0(528)$ & 630 & 163 \\
\hline $5.0\{120\}$ & 703 & 211 & 74 & $35.0(840\}$ & 676 & 136 & rot & $31.0(744)$ & 474 & 319 \\
\hline $6.6(160)$ & 806 & 179 & - & $60.0(1440)$ & 569 & 185 & 207 & $42.0(1008)$ & 355 & 437 \\
\hline $10.0(240)$ & 712 & 204 & 64 & $79.0(1896)$ & 529 & 176 & 248 & & & \\
\hline $13.3(320)$ & 667 & 194 & 109 & & & & & & & \\
\hline
\end{tabular}

2/sed controts $\mathrm{N}-211$.

- Used controts $N=168$.

MST $=(50 \%)$ median survival time.

MAS = mean after survival.

exposed to high-energy silicon and iron particles. While rigorous statistical evaluation of the results is not yet complete, brge and consistent differences between the various eroups is not apparent. This may mean that the curve that describes relation. ships between RBE and LET for life shortening is relatively flat over the LET range of approximately 30-130 keV/ $\mathrm{mm}$, as has been described previoushy for the response of mouse marrow stem cells (CFU-S). While the scalter is great and firm con- 


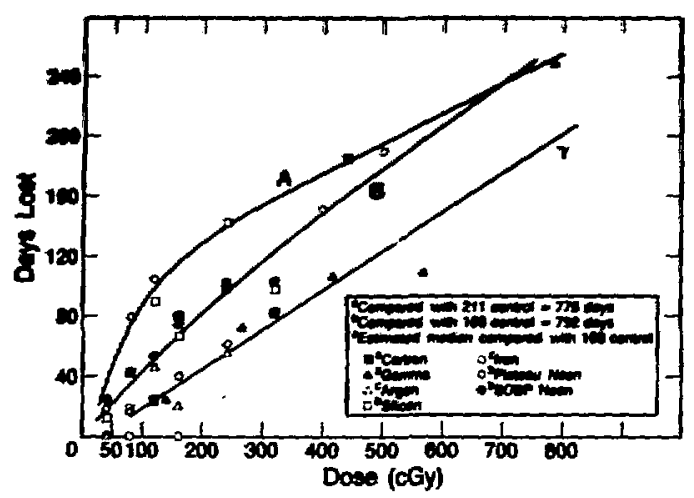

Fit. 1. Days of life span lost after single doses of photons or heavy charged particles. Estimates of days lost in male B6CF, mice given single doses of fission neutrons from the Janus reactor are as follows: 20 cGy-59 days, 80 cGy-124 days, 120 cGy-159 days, 240 cGy-216 days." Heavy changed particles appear less effective than fission neutrons.

(XBL 8511-8579)

clusions about curve shapes cannot be drawn now, the trend is that life-shortening effects of highenergy neon and silicon particles tends to be higher than for stopping carbon or neon particles, perhaps as a result of fragmentation produced by ridge filters. The curves drawn through the single disc results in Fig. 1 represent possible fits to the data where RBE for 100 days of life shortening could be in the range of 2 to 4 . In the case of 24 weekly fractionated doses, the results with carbon are inadequate for curve fittir: because RBE was overestimated. The speculative curve drawn parallel to interim results for stopping carbon indicates a possible fit to provide an RBE of no more than 4 at 100 days of life shortening. With high-energy neon, the curve eye-fitted to the points provides an RBE estimate of about 2 for 100 days of life shortening. Preliminary results with two other particle species have become available recently, based on early replicates, involving small sample sizes in which mortality is complete.

Figure 3 compares cumulative mortality curves for mice given $160 \mathrm{cG}$; of argon or iron particles. At this dose level it is likely that significant life shortening is produced, and these results provide no evidence that iron is more effective than argon for production of life shortening where the estimated LETs are approximately 130 and 200 $\mathrm{keV} / \mathrm{mm}$, respectively. These preliminary results are consistent with the interpretation that iron could be less effective than argon, indicating saturation of effects at an LET of approximalety $200 \mathrm{keV} / \mathrm{mm}$ based on life shortening. Should this prove to be

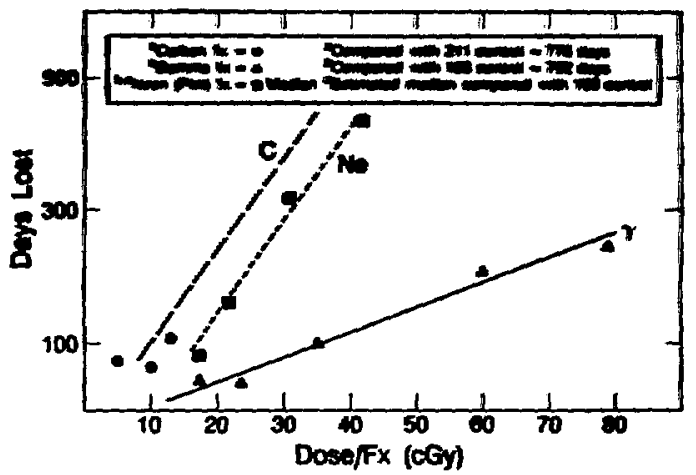

Fi. 2. Days of life span after 24 weekby fractionaled doses of stopping carbon particles, high-energy neon particles, or photons. The data for high-energy neon are based on estimated median survival lime and are considered preliminary. Estimales of days lost in male B6CF, mice exposed to 24 weekly fractionated doses of fission neutrons from the lanus reactor are as follows: 3.3 cGy-183 days, 5.0 cGy-262 days, $10 \mathrm{cGy}-316$ days, 13.3 cGy-417 days." Carbon and neon particles appear less effective than fission neutrons.

(XBL 851 1-8578)

the case when mortality is complete in the iron and argon groups, the results would be different than those obtained using the Harderian gland model system. Caution is prudent at this stage, and it is important to note that all the comparisons among the particles described here are based on dose rather than fluence. Were fluence used, and assuming equal life shortening for the various particles described, the relationship would be that the life-shortening effect, on a per particle basis, would increase with atomic number.

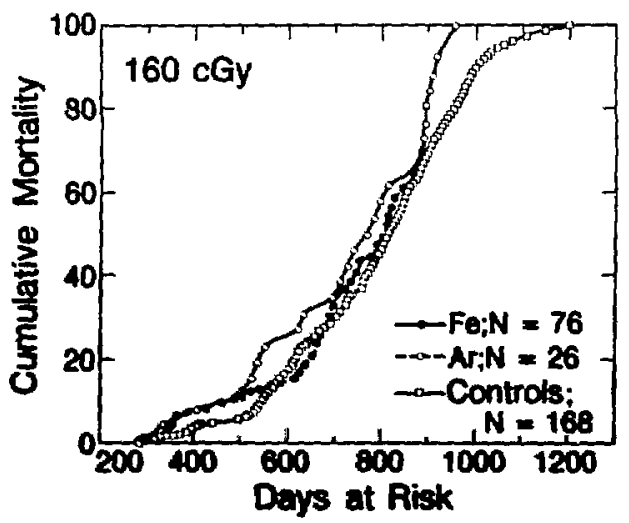

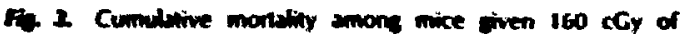

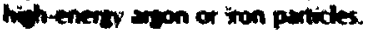


A fundmental isse in radiation rialk asesement concerns the efinects of protracted or low-rale exposures. All of our studies conducted so far hive imvolved fractionated or sinde doses administered at comparatively high rates, namely, in the range of several hundred rad per minute for sinde doses and in the range of 2-10 rad per minute for fractionated doses. In contrast to the results described above for fractionated doses of low-energy carbon particles, where little or no sparing effect was detected, a marked sparing effect has been detected for fractionated doses of high-energy neon particles characterized by an LET of approximately $30 \mathrm{keV} / \mathrm{m}$. The hypothesis tested in the neon experiment was that the sparing effect of fractionation would be as high for neon particles as for cobalt-60 gamma radiation, but results in Fig. 2 require rejection of this hypothesis. The lifeshortening effect of fractionated neon doses is appreciably greater than for cobalt-60 gamma radiation. The dose fractionation data are consistent with the hypothesis that the RBE is higher for carbon than for neon, where the LETs are about $70-80$ and $30 \mathrm{keV} / \mu \mathrm{m}$, respectively.

The definition of the most scientifically productive extension of the Skyhook Project is currently under assessment. Mortality will be complete in all groups currently under study in approximately two years, including all unirradiated control animals. Because of the fundamental importance of fractionated or low dose rates for radiation risk assessment purposes, the highest priority should be given to extension of our long life in term fractionation studies. The objective would be to evaluate RBELET relationships under conditions of long-term dose fractionation and testing the hypothesis that under those conditions, RBE will vary over a LET range of approximately $20-150 \mathrm{keV} / \mu \mathrm{m}$. If RBELET relationships for inhibition of repair or damage to critical repair systems vary independently from RBE-LET relationships of processes that contribute to life shortening after single exposures, this fact should be revealed by expanding our current dose-fractionation studies. The following expansions of our dose fractionation studies are under consideration: 1) eväluation of sparing effects at an LET below $30 \mathrm{keV} / \mathrm{mm}$ with which we currently have experience, for which purpose we are considering using high-energy carbon particles in the plateau portion of the Brags curve, where the LET is of the order of $10 \mathrm{keV} / \mathrm{mm}$, or high-energy helium or protons; and 2) long-term dose-fractionation studies that should be performed at at LET in the range of $130 \mathrm{keV} / \mathrm{mm}$, in which we will propose to use high-eneray aroon particles. By addition of these dose-fractionmion grous and some small supplementition of the current sincledose studies with the same particles, many fill estimates of sparins effects under dose-fractionation conditions could be obtained. The dose-fractionation studies proposed would directly complement in-vivo studies of neoplastic transformation under conditions of short-lerm fractionated exposures planned by our colleague T. Yang, thus providing further direct comparisons between in-vivo and in-vitro results directly relevant to the question of radiation carcinogenesis.

The Skyhook Project will achieve its original goal of providing at least qualitative estimates of RBE for life shortening after single or fractionated doses over a broad range of dose-averaged LETs. This information from our pilot studies will place us in a position to design the most critical experiments to define RBE over a range of low doses, a range directly relevant to radiation risk assessment. Since the goal of our pilot life-span studies is being achieved, we plan to terminate those studies when mortality is complete and initiate new studies on leukemogenesis using the mouse model system for myeloid leukemia. While the mechanism of mouse and human leukemia may not be the same, myeloid leukemia is a disease of considerable interest in terms of radiation hazards to man, and we would propose to use the mouse model to explore the effects of dose fractionation over a selected range of dose-averaged LETs. The most critical question concerns the extent to which dose fractionation produces sparing or even enhancement of leukemic risk, and our proposed studies would focus on this question. Another advantage of the murine leukemia model is that tumor expression is promoted by hornone injection, much as is the situation with our Harderian gland model system, and animals are sacrificed at $600-800$ days so that the information loss is minimal and considerable savings accrue in animal maintenance costs. Although the experiments to be conducted are not yet specified, we propose to initiate pilot studies with photons and at least a single heavy charged particle in the near future to determine the feasibility of using this model more extensively in lowdose studies. Because of our interest in radiation hazards in a space environment, we feel that it is important also to explore the extent to which environmental factors in a space environment could influence susceptibility to or expansion of myeloid leukemia. Hemodynamic changes are well known under those circumstances, and the likelihood appeas great that bone marrow cell kinetics will be in infiluenced sianificantly. Earlier results have 
demonstraved clearly that the life-time incidence of leckemia in mice is infivenced by the microbial emvironment, and by inference, by bone marrow cell kinetics.? It is our strong feeling that these matters should be explored more fully in order to ensure that leukemic risks in the space environment will not be seriously underestimated. Pursuant to this goal, we plan to initiate comprehensive studies on alterations in marrow cell kinetics and in determine the extent to which such alterations influence leukemogenic risk following photon irradiation.

\section{REFERENCES}

1. Yang, T.C., Craig, L.M., Mei, M., and Tobias, C.A. Neoplastic cell transformation by heavy ion radiation. In this annual report.

2. Ainsworth, E.J., Fry, F.J.M., Williamson, F.S., Brennan, P.C., Stearner, S.P., Yang, V.V., Crouse, D.A., Rust, J.H., and Bovak, T.B. Dose-effect relationships for life shortening, tumorigenesis and systemic injuries in mice irradiated with fission neutron or ${ }^{60} \mathrm{Co}$ gamma radiation. In Proc. Wth international Congress, International Radiation Protection Ass'n., Vol. 4, pp. 1143-1151 (1977).

3. Ullrich, R.L. Tumor induction in BALB/c mice after fractionated or protracled exposures to fission-spectrum neutrons, Radiat. Res. 97, 587-597 (1984).
4. Ninsworth, E, Prioleau, J.C. and Mathourm, L.). Life-shortening effects of heary changed particles: A status report. Biology and Medicine Division Arnual Report, Liwrence Berkeley Laboratory report LBL-14986 (1983).

5. Ainsworth, E.J., Prioleau, I.C., and Mahlmann, L.J. Life span studies on mice exposed to heavy charged particles: A Status Report, Biology and Aledicine Division Annual Report, Lawrence Berkeley Laboratory report LBL16840 (1984).

6. Ainsworth, E.J., Life span studies in mice exposed to heavy charged particles: Objectives and experimental design. In Proceedings of the 7th international Congress of Radiation Research, Amsterdam, July 1983.

7. Walburg, H.E., Jr., Cosgrove, G.E., and Upton, A.C. Influence of microbiological environment on development of myeloid leukemia in $x$-irradiated RFM mice. International J. of Cancer 1, 595-572 1968 .

8. Thomson, I.F., Williamson, F.S., Grahn, D., and Ainsworth, E.j. Life shortening in mice exposed to fission neutrons and $\gamma$ rays. 1 . Single and short-term fractionated exposures. Radiation Research 86, 559-572 (1981).

\section{Heavy lon Therapy and Related Physics and Biology}

\section{HEAVY CHARGED PARTICLE RADIOTHERAPY TRIAL}

Joseph R. Castro, Theodore L. Phillips, David E. Linstadt, J. Michael Collier, Samuel Pitluck," William T. Chu," Sheri D. Henderson, Tim R. Renner, Robert E. Walton, ${ }^{\ddagger}$ Jacquelyn J. Iler, Marilyn A. Fowler, Marc L. Kessier, and Monica M. Reimers

During the past year, patients have continued to receive helium, neon, and silicon heavycharged-particle irradiation as part of Phase $1 / 11 / 111$ trials of the following sites: 


\section{STUDY}

Phase II Studies of dose localization (base skull, juxtaspinal tumors, eic.)

Phase I-II Locally advanced carcinoma of the lung

Phase I-II Locally advanced carcinoma of the prostate

Phase 1-II Selected, advanced head and neck tumors

Phase I-II Locally advanced carcinoma of the pancreas

Phase 1-II Malignant glioma of the brain

Phase 1-ll Locally advanced, miscellaneous tumors

(biliary, gastric, soft tissue sarcoma, etc.)

Phase I RBE studies (skin and subcutaneous metastases)

Phase II Locally advanced carcinoma of the esophagus

Phase II Uveal melanoma

Phase III Uveal melanoma
PROTOCOL

NCOG 0R81/RTOG 79-11.

NCOG ORB1/RTOG 79-11

NCOG OR81/RTOG 79-11

NCOG ORB1/RTOG 79-11

NCOG OR81/RTOG 79-11

NCOG OR81/RTOG 79-11

NCOG OR81/RTOG 79-11

NCOG OR81/RTOG 79-11

NCOG 3E81/RTOG 79-08

NCOG $7081 /$ RTOG 79-09

NCOG 7085
The Phase 1-II studies in NCOG 7081 (eye), 3E81 (esophagus) and 0R81 (all sites) have been closed as of September 30, 1985. A total of 550 patients were accrued in these protocols.

Successor Phase II and III protocols for lung, prostate, eye, soft-tissue and bone sarcoma, skull and juxtaspinal tumors, Phase I silicon, and pancreas have been opened or are being developed. The results of the previous studies are briefly summarized here.

\section{DOSE LOCALIZATION STUDIES}

Through mid-1985, a total of 49 patients received heavy-charged-particle irradiation for chordoma, chondrosarcoma, meningioma, or neurilemmoma of the base of skull or juxtaspinal area. The mean tumor dose was 68 Gray-equivalent, ranging from 26 to 80 . Control within the irradiated area was obtained in 35 of $49(70 \%)$. The median follow up in all 49 patients is 21 months, with a range from 3-90 months. Serious complications were seen in a small number of patients, with cranial neve injury in two, transyerse myelitis in one, and brain necrosis in three patients.

In 12 patients with tumors of other histologies and/or sites, including lumors of paranasal sinuses, retroperitoneum, soft tissue and miscellaneous other sites, heavy charged particles were also used to deliver a higher lumor dose than possible with standard intadiation techniques. In this group, $21 / 42$ (50\%) have had local tumor control, also a good resuly considecing the extent and the range of tumar types treated. We believe that there are a number of sives in addivion to the ruxtespind//base of stull numoss that will show lone rem benellik from treatment with heavy charged particles.

\section{UVEAL MELANOMA}

The results in helium irradiation of uveal melanoma continue to be excellent. A tumor control rate of $95 \%$ with follow up from 3 to 90 months is seen (median: 28 months). Two hundred and five patients were accumulated in the Phase I-II study. Three dose levels of 60,70 and 80 Gray-equivalents (GyE) given in five fractions over 7-10 days were utilized. A small group of selected patients with tumors arising in the macula were treated to a lower dose of $50 \mathrm{GyE}$ in an attempt to control these tumors and preserve some vision, as an alternative therapy to enucleation. Considerable experience has been gained from this study in terms of treatment techniques, doses, results, and side effects of treatment. As with protons, the local control results are very good with a low level (5\%) of metastatic disease. At all dose levels we see about $15 \%$ of patients with neovascularity in the eve, often leading to difficult-to-treat glaucoma. Other complications occur less often, including perforated sclera, severe keratitis, painful or dry eye, with the result that 9/205 patients required enucleation for complications, in addition to five for tumor progression. Lens opacification has occurred in at least 25/205 patients, with several hoving lad successful lens extractions. Overall, about $55 \%$ of patients have retained vision of 20/400 of belter, more often in patients with smill tumons and, tow lumors more than $3 \mathrm{~mm}$ from the toner or optic disc. For the randomized Phase III study (NCOG 7Oai) we hrve setected 70 GyE in five fractions over $7-12$ dxys as the optind 
current helium technique. This is being compared to iodine-125 plaque therapy for appropriately sized and located tumors that can be treated by either technique.

\section{CARCINOMA OF THE LUNG}

The Phase |-II trial of advanced unresectable nonsmall-cell cancer of the lung has accrued 12 patients with primary untreated lung tumors. Treatment techniques and doses have been refined to permit acceptable toxicity, the chief being radiation pneumonitis, which has been seen at a moderately severe level in two patients. The tumor doses have ranged from 60 to $76 \mathrm{GyE}$ and survival from 2-15 months; four patients appear to have local control. A Phase III study (NCOC 2N84) comparing low-LET $x$ rays with heavy charged particles has been opened. The goal of the study is to determine whether the more precise dose distribution of neon and its high LET characteristics will provide higher local control and survinal than standard radiotherapy.

\section{CARCINOMA OF THE PROSTATE}

We have begun a Pl:ase III study (NCOG 4P85) for locally advanced carcinoma of the prostate based partly on the fact that the results in neutron and proton therapy of these tumors suggest that the local control rate and si rvival can be improved in patients with advanced tumors. This study tests whether the improved dose localization and high
LET of neon particles allow a higher and more effective dose to the prostate with better local control and survival as as evidenced by posttreatment biopsy and long term follow up.

\section{OTHER SITES}

The results of treatment of other sites have suggested the following sites for additionally planned protocol studies:

1. RTOG 10-52: A collaborative protocol with the $\mathrm{MGH}-$-4arvard group for the treatment of base-of-skull and cervical chordomachondrosarcoma with charged particles has been submitted as an intergroup study with RTOG.

2. NCOG/RTOG: Phase 1-ll trials with silicon ions will be designed to study normal tissue effects and tumor response in skin and subcutaneous metastatic nodules, and in enlarged unresectable lymphatic masses.

3. NCOG/RTOG: A Phase II trial of neon heavy charged particles for sites other than cervical and base-of-skull will be designed for patients with sarcoma of bone or soft tissue.

4. NCOG/RTOG: A new Phase I and II trial of combined chemotherapy and heavy-chargedparticle neon irradiation will be designed for locally advanced carcinoma of the pancreas, employing sequential infusions of methotrexare and 5-fluorouracil.

\section{ESTIMATING RADIATION THERAPY COMPICATION PROBABILITIES}

\section{John T. Lyman}

Treatment planning optimization involves selection of an acceptable plan that controls the local and regional spread of cancer without causing normal tissue complications." Clearly to accomplish this task it is desirable o have a means to estimate the complication probability of a given plan. While there are a number of fiactors that contribute to the probability of complication, it is well established thal dose, volume, and fractionation tre impontun factors. ${ }^{2-5}$ To ablain adequake initomation zbout the inradialled walume of difiterent nombl structures and the done divelivered no them in is necessury to calculave dese-distribu ons for the entine inadiuned

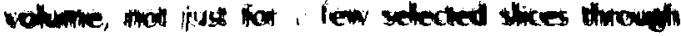

the volume.

This type of planning requires that a large number of depth-dose distributis $\Omega$ data be analyzed for a single treatment plan. Dose-volume histograms for individual normal structures are a good augmentation to the dose distributions because they provide a summany of the dose-distribution from a seties of CT stices for the individual normal stmuctures.

Minimizing the dose to the normal structures shwans imolves soine trade-offs. To spare one tissuce, in $\mathrm{mm}$ be necessiny to inctexse the dose to another, therefore compromises munt the mude wh

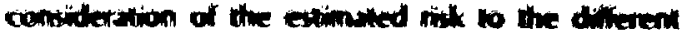


tissues. Frequently a decision must be made as to which is the better plan; to irradiate a smaller volume of an organ to a higher dose or to irradiate a larger volume to a smaller dose. The problem of identifying the more desirable of two dose distributions and the balancing the risk to the different organs is frequently a difficult task since there are no suitable data available to predict the consequences of using either plan. The desired data to aid this decision would be a library of dose-volume histograms with the associated complication probabilities for each normal structure. Lacking this data, if one is to effectively ise the dose-volume histograms, some method must be devised to rank the histograms. Since the histograms may have dissimilar shapes, a subjective visual inspection may not be adequate. The integral dose is not adequate since it does not address the volume dependence of the complication probability. A ranking that is based on clinical relevance would be better than one based on a subjective scale

An alternative to a library of complications correlated with dose-volume histograms is to use some method to identify different histograms that have the same complication probabilites. The development of such a method would then allow the identification of an equivalent histogram for which the tolerance dose data is available. Such a method of estimating the complication probability is being developed, based on the concept of the tolerance dose. ${ }^{6}$ The tolerance dose is a function of the portion of the normal structure irradiated. The probability of complication is a function of the d'sse and the tolerance dose and is reasonably represented by a sigmoid curve. Guidelines for tolerance dose for different normal structures are obtained from the literature. ${ }^{6-8}$ The values given are generally assumed to relate to the uniform partial volu. e irradiation of the structure with a conventional fractionation schiedule ( 1.8 to 2.0 Gy per fraction and five fractions per week). When sophisticated treatment planning is done (threedimensional cakculations or a series of twodimensional cakculations using adjacent slices of CT data 2.19 in is very apporent that most of the normal structures receive a non-uniform intadiation. Therefore the guidelines are not easily applied since they presumably apply to a difterent radiation distribution.

An alforithm has been whitien that can be used vo oblain an interpolaned extimate of the complica.

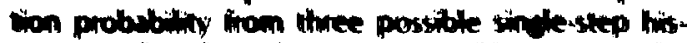
ceroms tha bound a two-step histortam. The

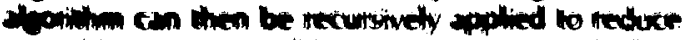

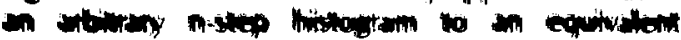

single-step histogram with the same estimaled complication probability.

This method has been adopied by the Working Group for the Evaluation of Particle Treatment Planning Intercomparison as the preferred method for the evaluation of normal tissue effects.

Results of using this ranking method are as follows: 1) For simple cases, approximated by a uniform partial volune irradiation, the method predicts a complication probability consistent with the tolerance dose data. 2) For alternate treatment plans that have similarly shaped histograms or integrai dose-volume histograms that do not infersect, the algorithm gives the best ranking to the histogram that represents to smallest volume and lowest dose to the structure. 3) For alternate treatment plans with dissimilarly shaped histograms (integral dosevolume histograms have at least one intersection), the best ranking is more difficult to ascertain visually because of the volume dependence of the tolerance dose which must be considered. Not all tissues have the same volume dependence, but the alogrithm will consistently use the assigned volume dependence, even though this model may not hold true for a human.

Two studies, ${ }^{11,12}$ that compared treatment complications with dose-volume histograms for heavy charged-particle treatment plans in a limited number of cases have been used as a preliminary test of the algorithm for the estimate of the complication probability. For the two different normal tissues studied, in all cases where a complication has been observed, there was a high estimate for the complication probability. When no complications were reported, the estimates were for a low complication probability in over $90 \%$ of the cases. These studies have been too limited to identify different shaped histograms that may lead to imilar complication probabilities.

Further work is required to establish which tissues are suitable for this analysis and the validity of the numeric value of the estimates of the complication probability, which is affecled by the normal tissue tolerance data used by the algorithm.

\section{REFERENCES}

1. Stewrat, R.R., and Gibbs, F.A. Prevention of radiation injuny: Predictabilithy and preventabivly of complication of recliation. injury. Ann. Bew. Mhed. 33, 385-395 (1902).

2. Brenthen, G.W. Dose fractionstion, dose

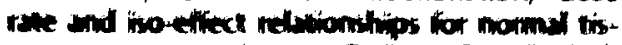

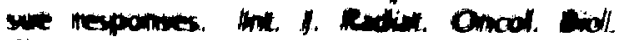

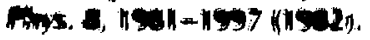


3. Cohen, L The weve factor in redivition oncologr. int. J. Radiat. Oncol Eiol. Mrys. B. 1771-1774 (1932).

4. Schubheiss, T.E, Orton, C.G., and Peck, RA. Models in radiodherapy: Volume effects. Med. Proys. 10, 410-415 (1983).

5. Withers, H.R., Thames, H.D., and Peters, LJ. Dose fractionation and volume effects in normal tissues and lumors. Cancer Ireatment Symposia 1, 75-83 (1984).

6. Rubin, P., and Casarett, G.W. A direction for clinical radiation pathology: The tolerance dose. In Frontiers of Radiation, Vol 6, I.M. Vaeth, Ed., pp. 1-16, University Park Press, Baltimore, MD (1972).

7. Rubin, P., Cooper, R.A., anc: Phillips, T.L. Eds., Radiation Biology and Radiation Pathology Syllabus, Set R.T.1: Radiation Oncology, pp. 2-7, Am. College of Radiology, Chicago (1975).

8. Cohen, L. Biophysical Modeling in Radiation
Oncolory. CAC Rese, Doca Renon (1903).

9. Coinein, M. The ustity of compened comoserpity in radiotion therepy. An extimate of outcome. lot. J. Radiat. Oncol. Biol. Pths 5. 1799-1007 (1979).

10 Goivein, M. and Abrams, M. Mukidimensional treatment planning: I. Delineation of anatormy. Int. f. Radiat. Oncol. Biol. Phys. 9, 777-787 (1983).

11. Zink, S.R., Castro, J.R., Chen, G.T.Y., Collier, J.M., Lyman, I.T., and Saunders, W.M. Treatment planning study compares heavy ion radiotherapy with photons for carcinoma of the esophagus. Int. J. Radiat. Oncol. Biol. Phys. (submitted for publication, 1985).

12. Austin-Seymour, M.M., Chen, G.T.Y., Castro, J.R., Saunders, W.M., Pitluck, S., Woodruff, K.H., and Kessler, M. Dose volume histogram analysis of liver radiation tolerance. Int. J. Radiat. Oncol. Biol. Phys. (accepted for publication, 1985).

\section{CLINICAL AND CELLULAR RADIOBIOLOGICAL STUDIES OF SILICON ION BEAMS}

Eleanor A. Blakely, Joseph R. Castro, Mary M. Austin-Seymour, George T.Y. Chen, Leora Lommel, Michael Yezzi, Polly Y. Chang, and Cornelius A. Tobjas

This is a report of our continuing studies to characterize the poteniial therapeutic significance of accelerated particle beams of silicon produced af the Berkeley Bevalac. We have previously reported radiobiological data in vitro that indicated that these heavy-ion beams can be used to deliver improved depth-dose distributions and enhanced biological effectiveness to tumors at depth.' As a part of Phase I studies involving treatment of two patients with metastatic lung nodules using silicon beams, we have undertaken an evaluation of the acute reaction of skin exposed incidental to the treatment of the lung disease. Parallel biological measurements of cellular survival were made. Our work, therefore, had three major objectives:

First, to measure the single-dose survival response of human fibroblasts in vitro to silicon or helium particle beams under conditions identical to those in which human skin was irradiated incidental to the treatment of lung nodules.

Second, to conduct Phase I skin studies with silicon particle beams in order to determine clinical relative biological effectiveness (RBE) vahues relative to helium ion beams for use in future Phase II-III clinical trials.

And, third, to attempt to correlate the relative acute response of human skin to a fractionated course of treatment with silicon or helium ions, with the single dose survival response of human fibroblasts in vitro.

Figure 1 illustrates the dual treatment fields used for the first patient. This is a 64-year-old man with multiple, slowly growing lung nodules that appeared about 10 years after resection of a renal cell carcinoma. Silicon and helium ions were selected for palliative irradiation of the metastatic nodules identified in the lower left lung. As shown here, a left lateral port was used for treatment, with the silicon beam having an initial extraction energy of $670 \mathrm{MeV} / \mathrm{u}$ to a depth in the patient of approximately $14 \mathrm{~cm}$, which was required to cover the target volume. A silicon dose of 12.0 physical Gray was selected to irradiate the lung nodules, and this dose was administered in six fractions over nine days. Ion chamber measurements indicated that the dose to the surface of the skin was 11.7 physi- 

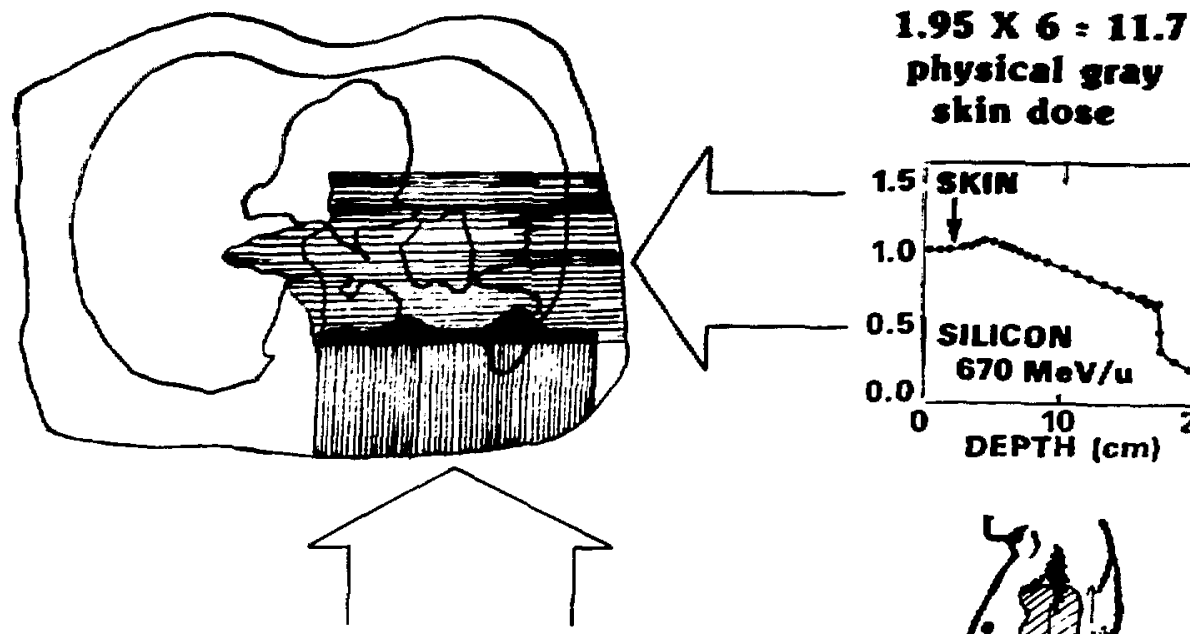

\section{$3.1 \times 6=18.6$ \\ physical gray \\ skin dose}
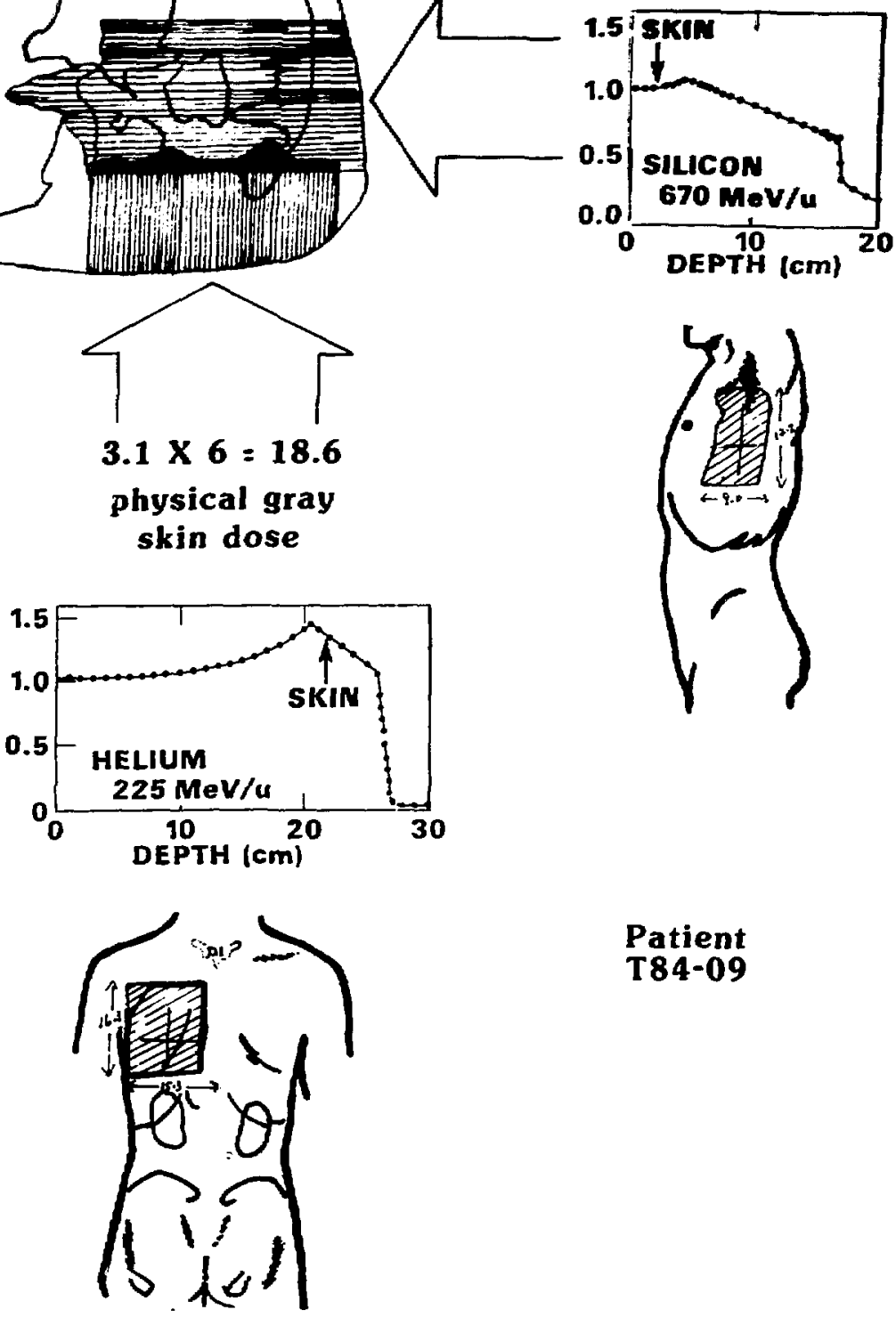

Patient

T 84-09

F. 3. Schematic representation of particte treatment plan for palliafive treatment of lung modules. Tub purts were used. each with a difikent purticke beum. A kefi tateral port was trealed wilh the silicon beam, and a keft possievio field was mealed with the helium beam. The absorbed dose fenets to the skin suriaces flhap nere inadianed incidenut to the sheutmentil are ginen. 
cal Groy. The area of stin exposed was approximincly $150 \mathrm{~cm}^{2}$.

The shin was localed in the plavew of the sijcon 12-cm extended Brase peak, and particlesensitive plastic detectors exposed at the surface of the skin, analyzed by Eugene Benton of the Universiny of San Francisco, indicated that the doseaverage LET was $46 \mathrm{keV} / \mathrm{km}$. The other area of hung nodules was treated with 225-MeV/u helium ions produced at the Berkeley 184-Inch Cyclotion. In the left posterior field used to treat these nodules, the superficial skin received a total of $\mathbf{1 8 . 6}$ physical Gray in six fractions, again delivered over nine days. The area of skin exposed to helium ions was approximately $250 \mathrm{~cm}^{2}$, somewhat more than the area of skin exposed to silicon ions. The helium treatment penetrated approximately $4.6 \mathrm{~cm}$ deep, and, therefore, the silicon field within the lung overlapped only to a small degree, as indicated. Neither skin surface received more than one particle species.

At the time this patient was treated, two biological experiments were set up for each ion field. We used two biological methods to measure the cell killing effects of the beams with exponentially growing human T-1 cells for these experiments. A small cylindrical glass chamber was set up with the appropriate amount of material upstream to duplicate the density of the three-dimensional compensation used in the treatment plan. The center of the $1.5-\mathrm{cm}$ chamber was, therefore, straddling the approximate location of the skin surface. A magnetic bar kept the cells in a stirred suspension. Cell samples were taken out before beginning the exposures and after a sequential set of doses in order to generate a complete dose-survival response. In addition, cells were grown in a monolayer attached to the inside of tissue culture flasks that were arranged in a linear array. Just prior to irradiation, the flasks that were completely filled with medium. Some flasks, filled with water, were used as blanks in order to distribute the biological sampling to areas of major interest. We call this configuration the submarine. The cells are later trypsinized and plated for survival.

Figure 2 summarizes the biological results for the first patient. The upper left panel represents the helium Bragg curves. Only the deepest range of the beam was used, with the tumor lying in the distal region, and the skin ending up in the proximal peak area. The physical dose distribution has been deliberately stoped down, due to the greater effectiveness of the particles with higher LET in the distal peak. And, in fact, the submarine sunvival

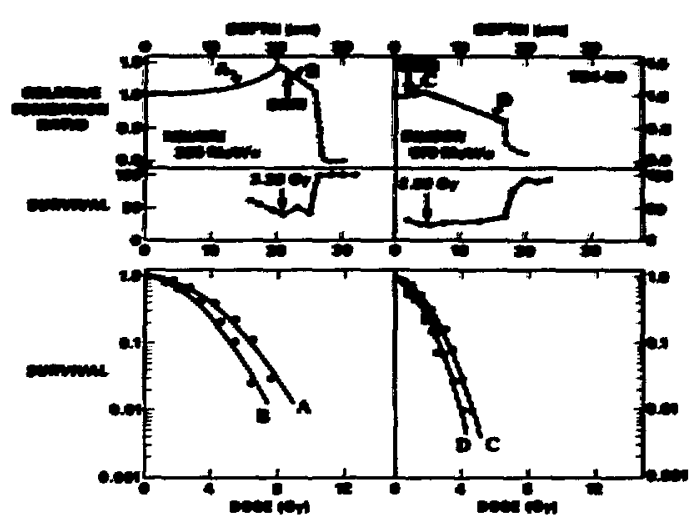

Fis. 2. Bragg curves and survival measurements for the patient treatmenl plan described in Fig. I. See text for further detais.

(XEL 819-7965)

experiment for this setup, which is plotted below the Bragg curve, indicates the relatively flat isoeffectiveness that can be achieved after a single proximal dose of $3.23 \mathrm{~Gy}$. In the lowest panel are plotted the whole-dose curves from the glass chamber. Curve $A$ was measured in the early plateau of the Bragg curve and is rather resistant. Curve $B$ was meast'red very close to the location of the skin and shows more effectiveness. The LET here has been measured by Luxton and Fessenden of Stanford to be approximately $10 \mathrm{keV} / \mu \mathrm{m},{ }^{2}$ On the right upper panel is a similar set of data for the silicon beam. Again, the physical slope of the dose appears to be well designed for uniform cell killing at a dose fraction of $2 \mathrm{~Gy}$ to the proximal peak. The $\mathrm{C}$ curve indicates survival effects at the plateau of the silicon Bragg curve. As expected, there is greater killing for $C$ at $46 \mathrm{keV} / \mu \mathrm{m}$ than for the helium curve B. Still greater killing is possible at position $D$, as indicated for the tumor mass. The location of the skin in the plateau of the silicon beam, or in the peak of the helium beam, was purely a function of the most appropriate treatment plan for the tumor.

There are difficulties associated with determining skin RBE values with particle beams. Even in Phase I trials, it is difficult to find patients in whom, for example, multiple nodules would permit a dose response study. Therefore, accuracy at determining a skin RBE with a new modality is highly dependent on a fairfy accurate estimate of an equivalent-dose level of the test radiation to the control radiation. If there is an equal skin effect, then one can be confident that the predicted RBE is relatively accu rate. However, it there is less skin reaction at the 
lext dose selected, then the true rexponse lies on a more resistant dose-eliect curve, which would yield a lower RAE but which is highty dependent on one's knowing the shape of the dose-response curve. Of course, the opposite could atso be true, and a more severe lest radiation reaction would lie on a more sensitive curve with a greater RBE. Uniortunately, we were only able to score the skin reactions on this patient out to three weeks, because the extent of the patient's disease required acditional therapy, and this could not be done with silicon. Approximately equal skin reactions were scored at three weeks posttreatment between the upper silicon fields and the lower helium fields, with possibly a little more reaction for helium, which was the larger of the two fields.

Palliative irradiation of the lung nociules of a 40-year-old woman with recurrent lung nodules following initial resection for bronchoalveolar carcinoma allowed the opportunity to compare skin reactions to silicon- and helium-ion irradiation in Phase I trials. Two nonoverlapping fields were selected. As shown in Fig. 3, the upper field was treaved with 15.8 physical Cray of Bronspeal sicon ions in 10 fractions over 16 dys. The twor in each case was in the distal rejon of the Bren peat. The skin surface, therefore, ended up in the proximal-peak region. Particle sensitive plastic. analyzed by Benton, indicated that the LET at the level of the skin surface was $84 \mathrm{keV} / \mathrm{mm}$. The lower field was treated with a total dose of 26.8 physical Gray of helium ions. The helium field for this case and aiso for the first patient ended up being in the proximal to midpeak region of the 6 cm helium extended Bragg peak. Treatment plans were prepared for the helium and for the silicon fields.

Bioiogical measurements made for this patient are depicted in Fig. 4. The (F) curve at the helium skin location is almost identical to the helium (B) curve from the first patient. The (H) curve representing the silicon skin exposure shows a greater reaction. After three weeks, the skin reactions in the two fields were approximately equal with perhaps the silicon field showing a little more color. However, at two months the skin reaction
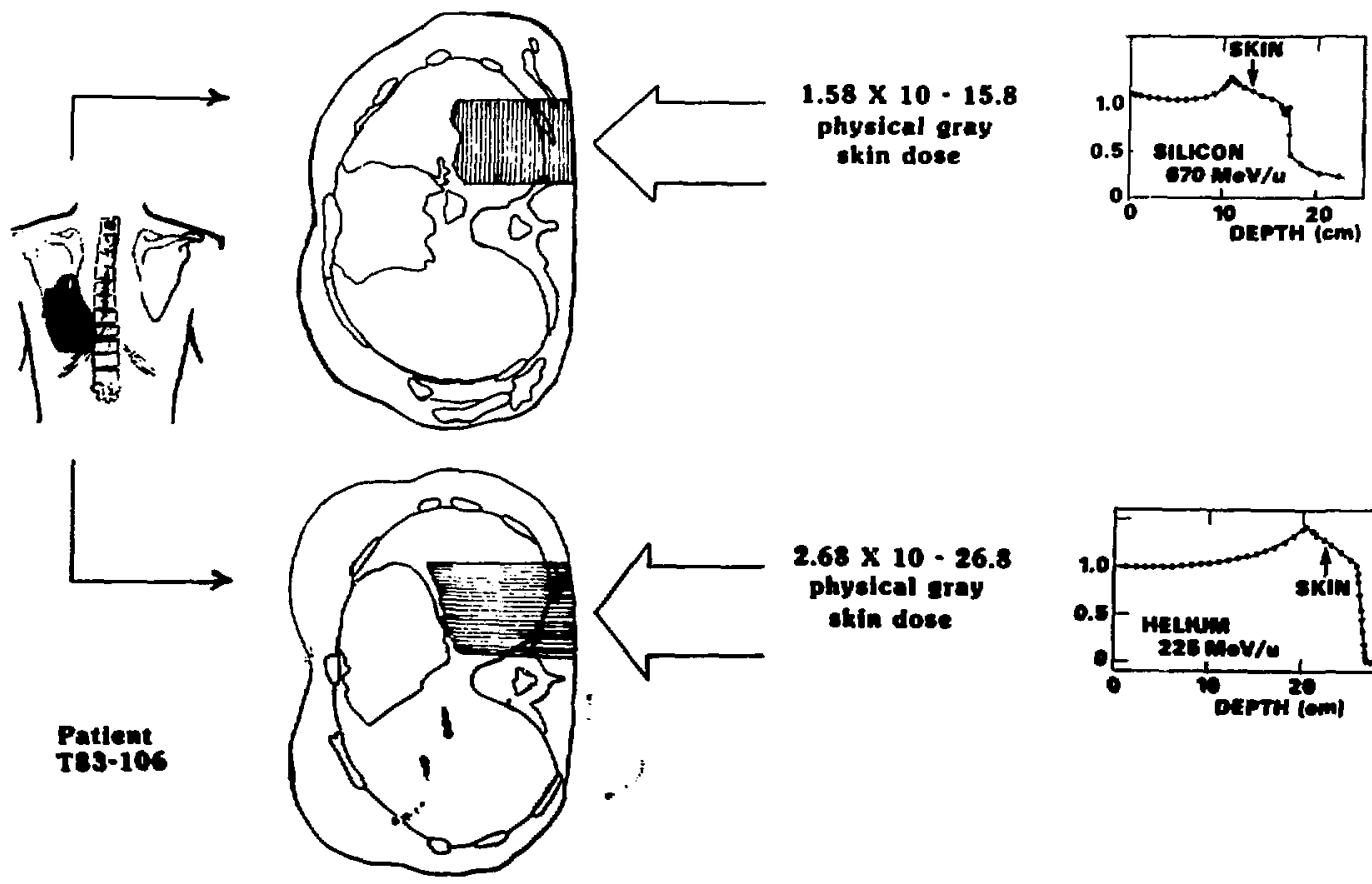
$2.68 \times 10-26.8$ physical sray skin dose

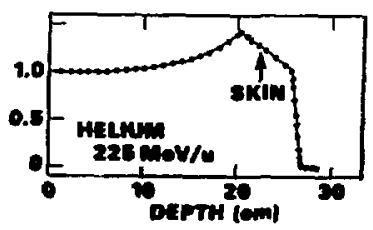

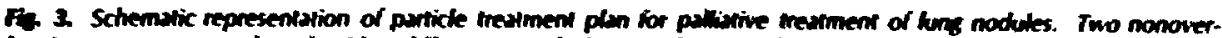

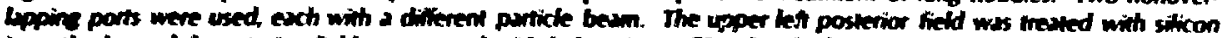

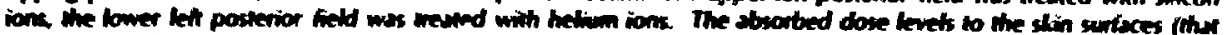
were incudiend incidencal to the mevimenll we civen.

axis $112+5117$ 


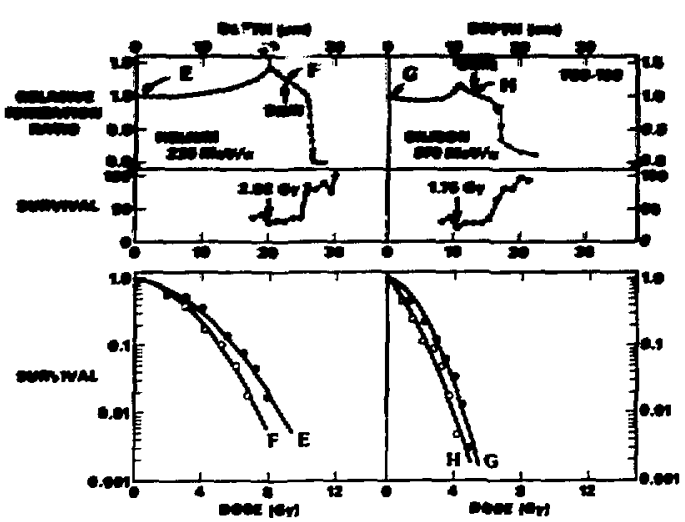

fig. 4. Bragg curves and survival measurements for the patiem treatment plan described in Fig. 3. See text for further details.

(XBL 849-7964)

in the silicon field developed a significantly greater reaction. Figure 5 presents the actual LET spectra for the first patient in the top panel and for the second patient in the bottom panel. In the silicon Bragg peak, the LET distribution is broader and has the higher peak value of $84 \mathrm{keV} / \mu \mathrm{m}$.

Table 1 summarizes the cellular in-vitro RBE values at $50 \%$ survival calculated for the ratio of doses of the two radiations to which the skin was exposed for each patient. This means the reference radiation is the helium Bragg peak radiation. For the first patient irradiated with plateau silicon ions, the RBE was $1.6 \pm 0.2$. For the second patient irradiated with Bragg peak silicon ions, the RBE was $3.2 \pm 0.4$. Allhough our skin RBE data are inadequate to permit a quantitative comparison with the biological data in vitro, there appears to be at least qualitative agreement between the two systems. We have, therefore, reached two tentative conclusions from this study:

First, the preliminary skin reaction observed in a patient exposed to Bragg peak silicon ions indicates that the reaction has an enhanced severity and an allered sequence relative to helium ions. Under ideal conditions silicon Bragg peak exposures should be limited to the lumor and should not include skin in the field. We have shown that effects that are usually seen one or two years after low-LET radiation may appear as early as sixty days after high-LET radiation. We are in the process of examining these effects in animal systems and have more experiments planned.

And, secondly, more radiobiological measurements made in parallel wilh clinical silicon Phase I trials are needed to evaluate the predictive value of in-vitro work for acute skin reactions.
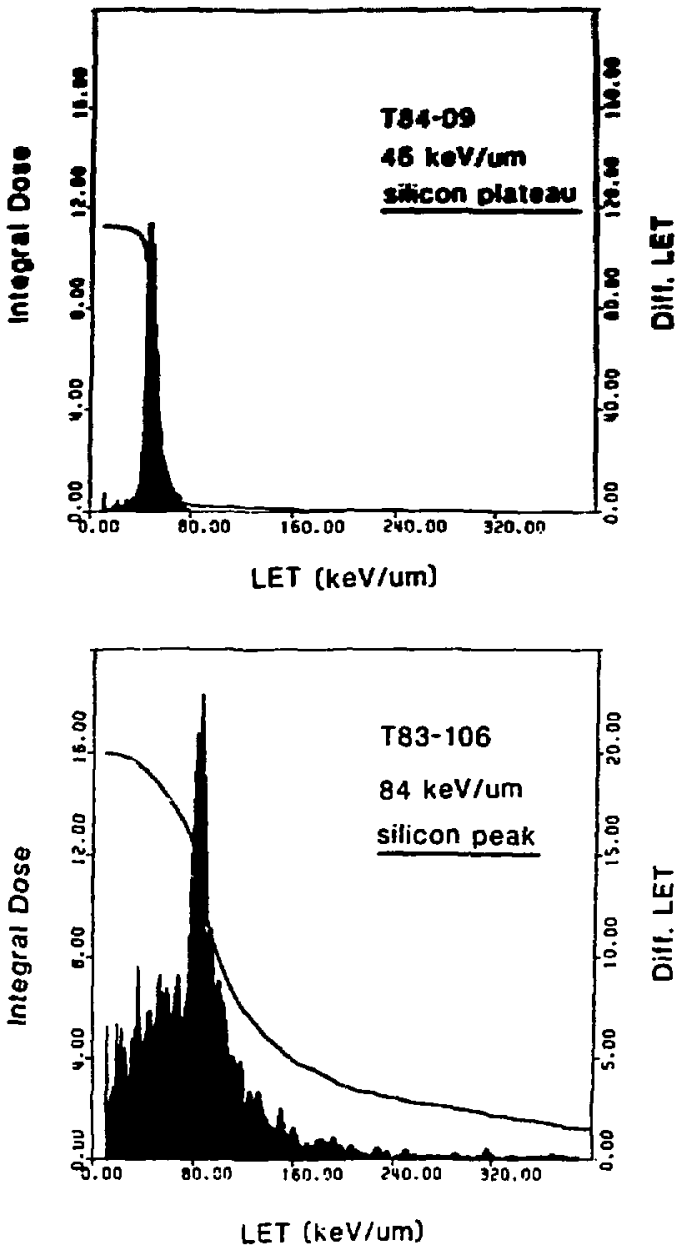

Fig. 5. Linear-energy-transfer (LET) distributions for silicon ion exposure of skin. (Upper panel) Silicon ions in the plateau of the Brage curve from Fig. I. (Lower panel) Silicon Bragg peak field from Fig. 3.

(XBL 8512-4950)

Table 1, Relative silicun RBE values.

\begin{tabular}{ll} 
Helium peak & $\begin{array}{c}\text { Cells in vitro } \\
\text { at } 50 \% \text { survival }\end{array}$ \\
\hline sificon plateau & $1.6 \pm 0.2$ \\
Hethim peat & $3.2 \pm 0.4$ \\
\hline
\end{tabular}




\section{REFERENCES}

1. Blakely, E.A., Ngo, F.Q.H., Curtis, S.B., and Tobias, C.A. Heavy-ion radiobiology: Cellular studies. Adv. Radiat. Biol. 11, 295-309 (1984).

2. Luxton, G., Fessenden, P., and Hotfman, W. Radial. Res. 79, 256-272 (1979).

\title{
maNNING CANCER TREATMENT WITH RADIOACTIVE BEAMS
}

\author{
Aloke Chatterjee, loseph R. Castro, Edward L. Alpen, Jorge Llacer, \\ George T.Y. Chen, and William T. Chu
}

When a heavy charged particle penetrates a medium, it deposjts a very small dose along most of its path except at the end just before its stopping point. The dose in this region peaks dramatically, this phenomenon being called the Bragg peak. In radiation therapy with heavy charged particles the goal is to superimpose the Bragg peak on the tumor volume.

The availability of high-energy radioactive beams at the Bevalac, such as ${ }^{11} \mathrm{C}$ and ${ }^{19} \mathrm{Ne}$, has provided a new dimension in optimizing cancer treatment with heavy charged particles. The diagnostic information available with these beams may preclude a large amount of uncertainty in localizing Bragg peaks on tumor volumes. It is well recognized that a slight error in placing the Bragg peak may result in severe underdosing of the tumor region and overdosing of nearby critical organs.

For precise localization of the Bragg peak, one requires an experimentally measured value of the water-equivalent thickness (thickness in a patient measured by using water as a standard) between the point of entry (of the beam particles) in a patient and the target (tumor) volume. Between these two end points there can be unknown amounts of bone, tissue, sinus, air, etc. and hence one may not be able to control precisely the incident energy of a heavy particle for the Bragg peak to coincide with the location of the lumor. In a technique that uses high-energy radioactive beams and a sensitive positron camera, the desired accuracy can be achieved through measurements in the actual treatment position, just before and immediately after the therapy.

In this technique energetic radioactive particles such as "C or "Ne arie used. These particles decay by enitting positrons, which then annihilate with the medium electrons to produce two gamma rays, separated by $180^{\circ}$. These gamma rays can then be detected in coincidence mode by a positron camera to locate their origin in space.

By adjusting the energy of the radioactive particles ( ${ }^{19} \mathrm{Ne}$ has been used most commonly) they can be stopped at the location of the tumor as verified by the positron camera. This method provides a direct measure of the required water-equivalent thickness. Radioactive particles have penetration properties that are very similar to those of the heavy charged particles used in the Bragg-peak therapy. The value of the water-equivalent thickness measured by a radioactive beam is then directly applicable for treatment planning with heavy charged particles.

Through numerous studies and measurements in human phantoms, it has been realized that for the success of the technique, the following requirements for a radioactive beam are extremely important: 1) accepted minimum flux is $10^{7}$ particles per pulse, 2) the energy spread must be as little as possible, and 3) at the location of the isocenter, the beam should be focused as tightly as possible. With respect to these requirements, some progress has been made in the last two years, as described below.

Radioactive beams are composed of secondary particles that are produced as a result of nuclear fragmentations of a suitable primary beam. For example, ${ }^{19} \mathrm{Ne}$ is produced from the accelerated stable isolope ${ }^{20} \mathrm{Ne}$ by getting rid of a neutron in a nuclear collision. Similarly, ${ }^{11} \mathrm{C}$ is produced from ${ }^{12} \mathrm{C}$.

Accelerated heavy ions from the Bevalac are brought to a focus and made to traverse a low- $Z$ target material (usually 2.5 - or $3.8-\mathrm{cm}$-thick Be). Through nuclear reactions in the larget, the projectike ion has a probability of being stripped of a neutron and emerging as a radioxctive ion. The nuclear reactions take place over the entire thick- 
ness of the taget with an energy spread due to the difference of the eneray loss rates of the primary and the secondary particles through the larget material. For example, in the reaction of 100 MeV/nucleon $20 \mathrm{Ne}$ - 19 Ne through a $2.5-\mathrm{cm}$-thick Be target, ${ }^{19} \mathrm{Ne}$, created near the entrance and exit of the target, will show a range difference of about $1.4 \mathrm{~mm}$ of water. A larger energy spread is introduced by the contribution of Fermi momentum (due to motion of nucleons inside a nucleus) of particles in nuclei involved in the nuclear reactions. Taking into account a Fermi momentum of approximately $100 \mathrm{MeV} / \mathrm{c}$, the resulting energy spread in the ${ }^{19} \mathrm{Ne}$ beam is estimated to introduce a spread of stopping ranges of the beam of approximately $1.5 \mathrm{~cm}$ in water. The measured Bragg peaks of ${ }^{20} \mathrm{Ne}$ and ${ }^{19} \mathrm{Ne}$ show the increase of about $2.3 \mathrm{~cm}$ for ${ }^{19} \mathrm{Ne}$ over that of ${ }^{20} \mathrm{Ne}$. The energy spread of the radioactive beam through the charged-particle transport system of the Bevalac is manifested in the experimental area as lateral dispersion of the beam, which contributes to a lower dose rate in a given irradiation volume. If the particles with different momentum were refocused, the stopping region becomes elongated. To make a spatially narrow radioactive beam with a sharp range, first the momentum spread of the beam must be reduced, and the resulting "monochromatized" beam must be refocused.

For reducing the momentum spread, a wedge made of lucite with a thickness variation from 0 to $1 \mathrm{in}$. over the base distance of 2 in. is placed in the ${ }^{19} \mathrm{Ne}$ beam between two bending magnets, upstream of a wire chamber. Since the higherenergy portion of the ${ }^{20} \mathrm{Ne}$ beam goes through the wider section of the wedge, the emerging beam has a much smaller energy spread. Such a procedure has been extremely successful in reducing the spread in the momentum of a secondary radioactive beam, and with ${ }^{19} \mathrm{Ne}$ it has now been possible to obtain $1-\mathrm{cm}$-size beam at the isocenter. In order to provide a greater flexibility, a large dial has been placed in the vacuum line of the Bevatron ring that contains wedges of different dimensions. For example, we have now four wedges having different wedge angles of $10.9^{\circ}, 14.1^{\circ}, 17.4^{\circ}$, and $25 . \%^{\circ}$. Depending upon the primary beam characteristics, we can select any one of the wedges and get a relatively suitable (tighter focus and reduced energy spread) radioactive beam for diagnostic applications. In order to improve the flux of the radioactive particles we have added a 20-ft-long vacuum pipe inside the therapy cave and directly along the cptical bench. The pipe connects to the ring of the Bevation and is aenerelly operaned at $10^{6}$ torr. By adopting this procedure the scativering of the radioactive particles has been reduced sinificantly, helping us achieve a greater hux of the secondary beam.

These improvements have made the radianctive beam adequate for diagnostic procedures in clinical therapy of cancer patients. In Fig 1 an arrangement of the positron camera with a patient's head between the banks of detectors is shown for localizing ${ }^{19} \mathrm{Ne}$ beam on a tumor volume in the brain. To date we have verified the water-equivalent thicknesses for Bragg-peak localization in three cancer patients, two of whom had brain tumors and the third a tumor in the thorax adjacent to the spinal cord. There was a very good agreement between the $C T$ data and the measurements made with ${ }^{19} \mathrm{Ne}$ except in the two brain tumor patients. The measurement in the third patient involved beam transmission through lung and hard bone as well as soft tissue. The patient's original treatment setup was in a seated position. However, the positron camera required the patient to be in a horizontal (supine) position. For compatiblity with the previous treatment setup, the patient's arms were raised over his head during measurement with the positron camera. In addition to this measurement, the patient was then rescanned in the supine posi-

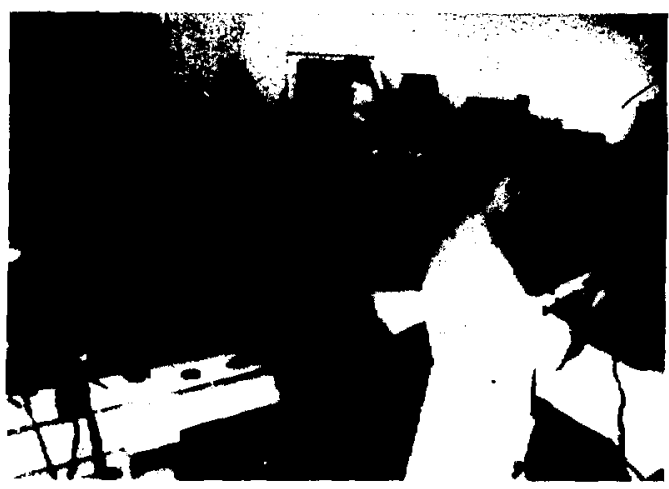

F. 1. A palient with a brain rumor is posinioned on a ineat. ment lable. Throuth x-ray imaing (vertical and horizontal) the tomor has been located at the isocenter la very stable refenence point). The two banks of the positnon camera are placed in such a way that the patien's had is immobitized bermeen them. When a radiaxtive bean is stopped at the isocenter as determined by the cawera, the exact watereguvalent thickness for t heavy purticle bem no penewave can be dewermined. This

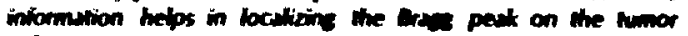
valume. 
tion and the waler-equivalent path lengih determined from the CT scan was found to be greater than the measurement by the radioactive beam by $5 \mathrm{~mm}$. Such a difference, if verified further, can be serious, and must be integrated in the treatment planning.
More patients have been scheduled for further comparison of the divenostic procedures using the CT scanner and the radioactive beam. It is hoped that such procedures will prove of great utitity ar it form a part of routine clinical procedures in the future.

\title{
ISOSURYIYAL TESTING OF BAR RIDGE FILTERS FOR WOBBLED NEON ION BEAMS
}

\author{
Stanley B. Curtis, Adrian Rodriguex, Tracy C-h. Yang, and Eleanor A. Blakely
}

An extensive series of experiments has been performed to test new bar filters that were designed in collaboration with the Heavy-lon Radiobiology Related to Oncology Program Project. The filters were constructed to produce constant cell survival across the spread Bragg peak region in preparation for phase III therapeutic trials with the magnetically "wobbled" neon-ion beam. Three different filter designs (designated 3E1, 3E2 and 3E3) were studied. Each design produced a different slope in the spread peak region of the Bragg ionization curve. The three design Clirves calculated by $C$.A. Tobias for an 8-cm spread-peak region are shown in Fig. 1. Initially, three different thicknesses for the filters were chosen $(4,8$, and $12 \mathrm{~cm})$ for each of two of the designs (designated 3E2-4, 3E2-8, 3E2-12, 3E3$4,3 E 3-8$, and $3 E 3-12)$, and the filters were tested

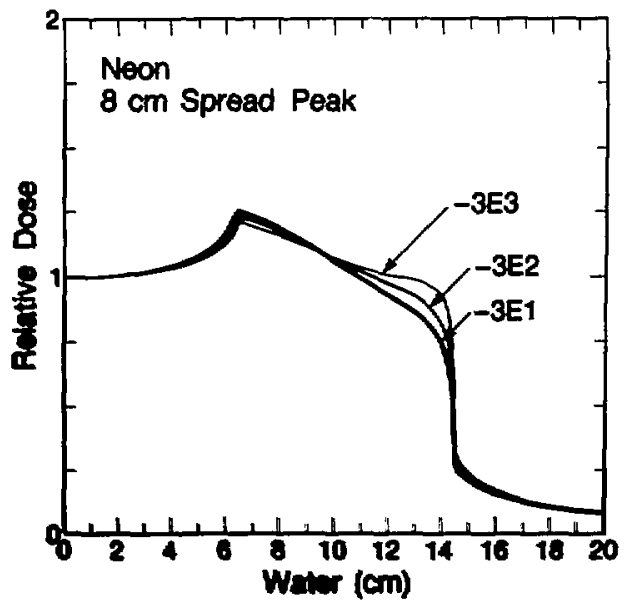

7. Desin curves for Ean preadpeal reions for

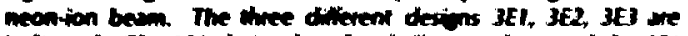

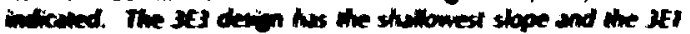

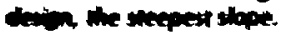

for iso-cell survival in neon-ion beams of two different energies (585 and $456 \mathrm{KieV} / \mathrm{amu}$ ).

Cell survival curves were obtained by standard techniques in the plateau, proximal, distal, and tail portions of the Bragg ionization curve. The data were analyzed by a computer-assisted leastsquares-fit to the linear quadratic $(\alpha, \beta)$ model of cellular inactivation. The best-fit parameters to the data provided RBE estimates for the proximal and distal spread Bragg peak regions for each of the filters tested. Isoeffectiveness within the spread Bragg peak was analyzed by comparing survival as a function of the dose delivered in the plateau entrance region of ionization. Peak-to-plateau dose ratios were obtained from the Bragg ionization curves of each filter and ion energy and used to convert the doses delivered in the Bragg peak to plateau doses. Examples for selected filters and energies are shown in Fig. 2. The survival curves are linear quadratic least-square-fits to the survival data in the proximal and distal spread Bragg peak regions, plotted here as a function of plateau dose. Thus, a superposition of the data points for the proximal and distal positions on such a plot ir:dicates isosurvival for that filter design.

From analysis of the data, it was decided to construct a series of filters at $1-\mathrm{cm}$ increments from 4 to $14 \mathrm{~cm}$ using the 3E3 design. The general conclusion of this analysis was that the design producing the flattest slope (3E3) produced the most constant cell survival across the peak region in the clinically important dose range.

Survival data obtained in the proximal-peak position of the Bragg curves for the already completed 3E3 filters have been useful in developing RBE tables for the upcoming phase III trials. Proximal peak RBEs are used along with other data, including cell survival measurements as a function of depth atter a sinde exposure, to determine the physical doses to be delivered to the pitients. 

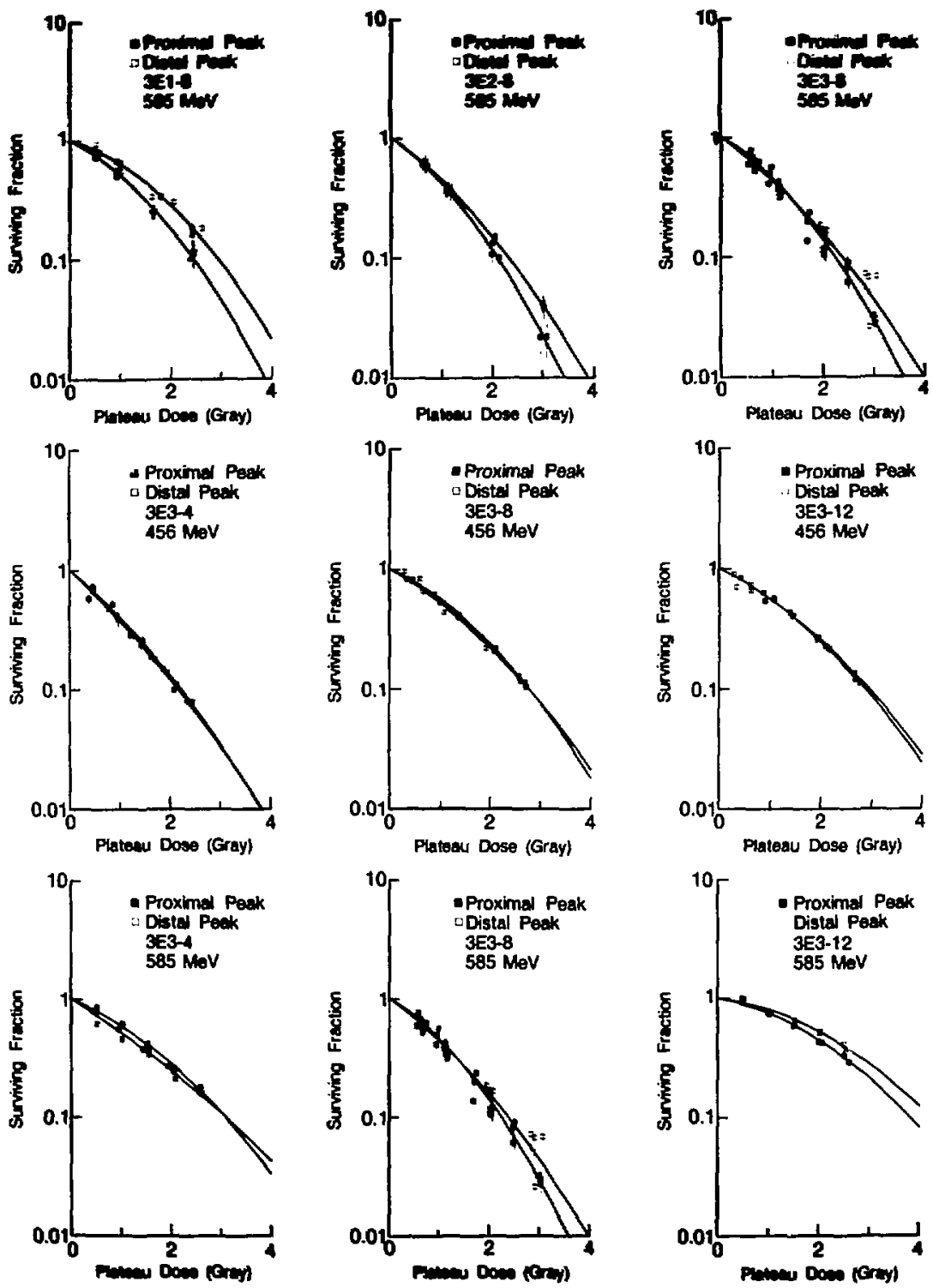

Fi. 2. Comparisons of survival of human $I I$ cells in the proximal and distal regions of the spread bras pest as a iunction of the dose in the plateau. Filler desiens are compared thal differ in the degree of slope in the spread Dras peals (3E1. JE2, and 3E3) and in their widths (4. a and $12 \mathrm{~cm}$ ). Fillers were kested nith neon-ion beams

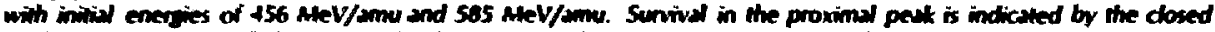

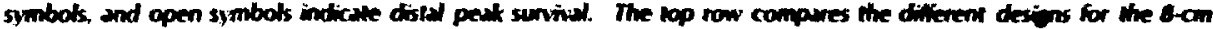

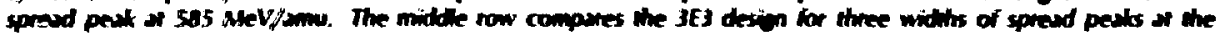

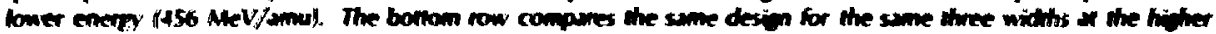
energy (BSes AteV//2mu). 


\title{
RADIONOLOCKCAL STUDES FOR HaUM ION THEAPY Of UVEAL MELANOMA
}

\author{
Eleaner A. Dalkety, fohn T. Lymam, Geore T.Y. Chen, Joceph R. Castro, \\ Pelly Y. Chame, Leora Lommel, Frederick Yealer," George j. Hampton," \\ Nina C. Wowe, and Shari-lyn K. Eaba*
}

A review of the literature reporting relative biological effectiveness (RBE) of helium ions shows that there is considerable variation in values reported by several authors from different institutions (for a summary see Ref. 1). RBE values at the beam entrance or dose plateau of the Bragg curve when calculated relative to $225 \mathrm{kVp} \times$ rays are, in general, very close to unity. Average RBE values in the Bragg peak region are around 1.3 but, depending on the cell system and the width of the extended Bragg peak, range from 1.0 to 2.5 .

This report summarizes part of the radiobiology that has been done at Berkeley's 184-Inch Cyclotron with helium-ion beams, which are currently being used to successfully treat uveal melanoma. ${ }^{2}$ There were two main objectives to this study. The first was to confirm the adequacy of ridged filter design to achieve uniform cell killing across narrowly collimated and narrowly extended Bragg peaks of only a few centimeters in depth of range, which primarily involved measuring the RBE in vitro by two independent types of experiments. The second objective was to compare the physical and biological usefulness of a 308-MeV/u Bevalacaccelerated carbon beam for its potential application to the treatment of small localized volumes near critical structures.

Figure 1 demonstrates how the unmodified sharp Bragg peak, which is illustrated at the extreme right of the upper panel, can be broadened to wider and wider dose distributions with a rotating variable absorber that spreads the stopping particles over broader ranges. The dose has been deliberately sloped io be greater in the proximal peak and smaller in the distal peak where the particles ara more effective. The end result is to achieve isoeffective killiris across the width of the filter. Must large brain or abdominal tumors require the i2-, 8-, and $4 . \mathrm{cm}$ filters (also shown in the upper panel of Fig. 1). The special eve fitters are shown in the lower panel of the same figure. The withts of these fitters by comparison are much sm-lier, covering only 1.5 to $2.6 \mathrm{~cm}$. The variable ab. Aber fiters used to produce the smill beams are made of stacked luxite sheets. They rolate on a central axis, siving them the appearance of i pro.

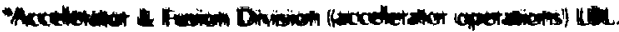

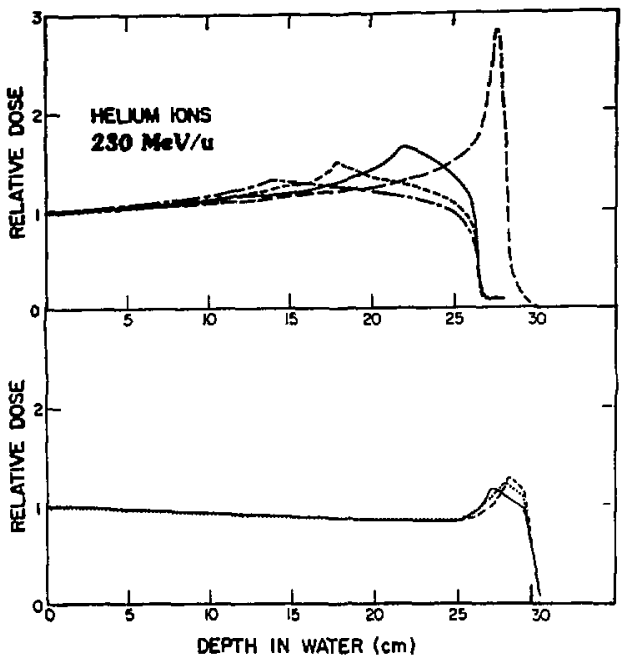

Fig. 1. 230.MeV/u helium Bragg ionization curves. (Top panel) unmodified, $4-\mathrm{cm}, 8 . \mathrm{cm}$, and $12 \mathrm{~cm}$ extended peaks. (Bottom panel) $1.5-\mathrm{cm}, 2.0-\mathrm{cm}$, and $2.6-\mathrm{cm}$ eye flters.

(XBL 859-4055)

pellor. The beam is aligned so that it passes through the center of each vane.

Two biological methods were used to measure the cell killing effects of these beams. Exponentially-growing human T-1 cells were plated into either $25 \mathrm{~cm}^{2}$ tissue culture flasks or in a circular area approximately $2 \mathrm{~cm}$ in diameter at the center of $35-\mathrm{mm}$ plastic petri dishes. After the cells attached to the bottom of the petri dish, they were covered with $1 \mathrm{ml}$ of culture medium. The cells in the petri dishes were prepared approximately 24 hours before irradiation, the cells in the flasks only approximately 5-6 hours before exposure. The Rask experiments were not irypsinized after exposure and thereb, provided two kinds of information. When the monolayer of irradiated cells was stained with methylene blue eleven days Later the pattern of cell killing permitted a check on the colinnation and depth of penetration of the beam, and knowing the number of cells plated, the multiplicity of the cell's at the time of exposure and the plation efficiency of conitol cultures, it was also possible to core survinian colonies within the $2.5 \mathrm{~cm}$ dimmeter ses inadiend. In the petiri dish expeniment, the 
cells are inserted into a special aluminum chamber that assures reproducible alignment in the beam. After exposure, the cells are trypsinized, resuspended, counted, plated, and incubated at $37^{\circ} \mathrm{C}$ for eleven days. Colony forming ability was scored by staining the cultures with $1 \%$ methylene blue and by counting surviving clones having at least 100 cells.

The petri dishes were exposed to a range of heavy-ion doses to generate a complete doseresponse survival curve for an accurate calculation of RBE at several depths of range. The tissue culture flasks were irradiated at a physical dose of 8 $G y$ to the proximal peak in pairs behind variable amounts of polyethylene tissue-equivalent absorber. The flasks were filled with tissue culture medium at the time of exposure, and, thus, $1.8 \mathrm{~cm}$ of waterequivalent material was present between each exposed cellular monolayer. Depending on the width of the eye filter, six to eight pairs of flasks were irradiated to measure cell killing across the several-centimeter depth of penetration for each filter. The proximal-peak dose of 8 Gy was selected because it was similar to the dose fraction size to be used in the clinic.

Figure 2 shows an enlargement of the three eye filters of $1.5,2.0$ and $2.6 \mathrm{~cm}$. Arrows on the curves designate the proximal and distal peak positions where survival curves were measured with the cells growing in the petri dishes. Survival data as a function of depth from two replicate experiments are plotted in the middle panel. The two data points plotted for the $1.5-\mathrm{cm}$ filter were taken from the whole-survival curves measured with that beam. Within the limitations of the biological scatter, there

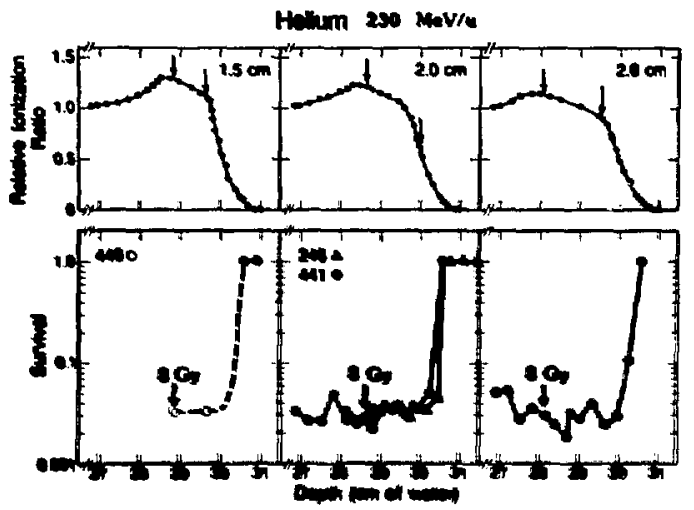

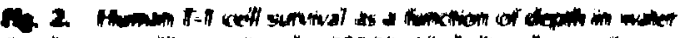

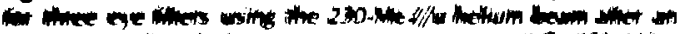

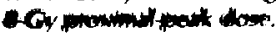

appears to be isosurvival across the three filters with this system.

For reference, survival was measured in the entrance of the beam in each experiment for an RBE calculation using cells from the same population used for each experiment on the same day. The data were computer-fit to a linear-quadratic expression and the RBE values as a function of dose level were calculated by a program written by Norman Albright. ${ }^{3}$ Figure 3 summarizes the survival results from the largest and smallest eye filters. The helium plateau data show a radiosensitivity that is intermediate between $x$-ray and gamma-ray survival, which makes cell survival in the beam entrance a good low-LET radiation reference for RBE calculations. Cell killing is slightly greater in the proximal peak of the $1.5-\mathrm{cm}$ filter compared to the $2.6-\mathrm{cm}$ filter. The survival curves measured in the distal peak positions of the filters were virtually identical. The distal peak position of the helium beam with an estimated average LET value of approximately $10 \mathrm{keV} / \mu \mathrm{m}$ is most effective for cell killing. At a position only a few centimeters upstream, the killing effectiveness is slightly less. The proximal-peak RBE value in the $1.5-\mathrm{cm}$ beam is $1.4 \pm 0.14$, whereas the $2.6-\mathrm{cm}$ beam value is 1.3 \pm 0.12 . The distal-peak RBE value for both filters is $\mathbf{1 . 6} \pm \mathbf{0 . 1 2}$. Most eye tumors require the larger filter. For simplicity of practical use in the clinic: the single RBE value of 1.3 in the proximal peak is cited as the helium-ion RBE value. Higher RBE

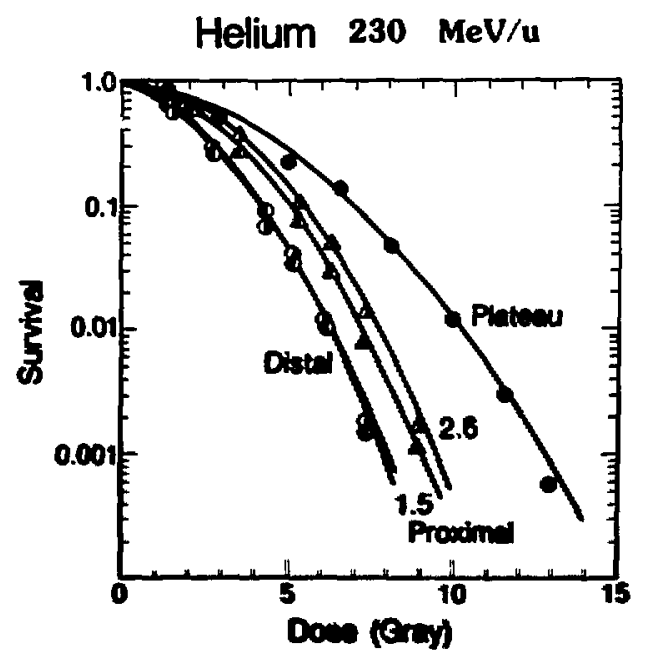

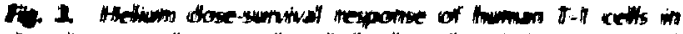

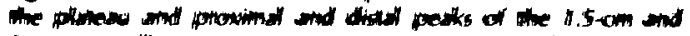
2.60im ene thents. (act absure) 
values are measured in the distal peak, but the downward stope of the Bragg curve in the distal peak is designed to ensure uniform cell killing across the filter at the desired dose level.

In summary, we have drawn two conclusions from this work:

1. The RBE measured in vitro in the $230 \mathrm{MeV} / \mathrm{a}$ helium-ion extended Bragg peak is 1.3-1.6.

2. Isoeffective biological response can be achieved with variable absorber filters that produce narrowly collimated Bragg peaks extended only a few $\mathrm{cm}$ for treatment of uveal melanoma.

\section{REFERENCES}

1. Raju, M.R. Heavy-particle Radiotherapy, Academic Press, New York (1980).

2. Blakely, E.A., Lyman, J.T., Chen, G.T.Y., CasIro, J.R., Chang, P.Y., and Lommel, L. Radiobiological studies for helium-ion therapy of uveal melanoma. Presented at the meeting of the American Sociely of Therapeutic Radiologists, Miami Beach, Florida, 1985. Int. J. Radiat. Oncol. Biol. Phys. 11, Suppl. 1, 134 (1985).

3. Albright, N.W. Computer programs for the analysis of cell survival data. Abstract presented at the Radiation Research Society, Los Angeles, California, 1985. 


\section{SECTION 5. LIPOPROTEIN AND STRUCTURAL BIOLOGY}

\section{INTRODUCTION}

The Lipoprotein and Structural Biology Group was formerly known as the Structural Biophysics Group. The new group title provides a more coherent description of the activities of investigators in the group; a major research effort is in lipoprotein metabolism and its relat:onship to cardiovascular disease, and another is on structural-functional correlates of biologically important macromolecules. In both areas, members of the group are internationally recognized in their respective areas of research.

Lipoprotein research has been ongoing in the Biology and Medicine Division for more than 30 years. The lipoprotein research group, under the leadership of Alex Nichols, has recently been awarded a program project grant to continue their innovative studies on plasma lipoproteins. The investigators in lipoprotein metabolism have been instrumental in developing and applying new techniques that measure and define lipoprotein levels and subclasses and provide new insights into the role of lipoproteins in health and disease. The summaries in this section indicate the breadth of research in lipoproteins from their importance in the newborn to the genetic basis of lipoprotein patterns in adults.

The internationalism of lipoprotein research is exemplified by the report of Alex Nichols, who has been carrying out a collaboration with investigators in Milan, Italy. These investigators have been examining lipoproteins in several families who have an unusual genetic defect in which a single amino acid substitution is found in the major protein (apolipoprotein Al) transported by high-density lipoproteins (HDL). Patients with the defect have unusually low levels of plasma HDL. Nichols and his colleagues were able to show that this defect is associated with a shifi in HOL subpopulations to smallet denser particles than those of the normal adult. Identification of these unust II HOLs will provide a stepping stone for further studies on regulation of lipoprotein metabolism in cases where HDL subpo. pulations are abnomall.

In the part few years, Ronald Krauss and his associales have noted that aduh $\mathrm{COL}$ fell into jeveral distincll subpopultations or particles based on size and demsity. Some subjects rended to have

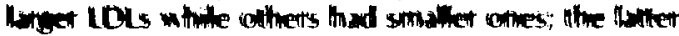

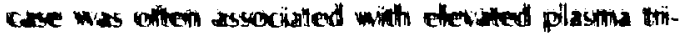

glycerides. This led Ronald Krauss and Melissa Austin to ask the question whether the HDL pattern or phenotype is genetically controlled. Although the reported studies are ongoing, the results are very intriguing at.d suggest that indeed LDL-subpopulation distribution may be genetically controlled and that a single gene locus with two alleles may be involved. The genetic regulation of human lipoproteins is a new and developing area in the Lipoprotein Research Group and promises to be an important area for the future.

Analysis of HDL and LDL-subclass distribution using high resolution nondenaturing gradient gels was developed by Alex Nichols and Ronald Krauss. This technology is now used throughout the lipoprotein research community but has lacked a direct method for quantitation of lipoprotein subclasses. Resolution of this important methodological problem has been undertaken by Frank Lindgren who, as the present report indicates, has initiated a program to quantitate gradient-gel data. His approach using analytic ultracentrifugation as a measure of mass for discrete subclasses and relating these data to chromogenicity in the gel looks promising and is extremely important to the field. The precision of analytic ultracentrifugation mass and density measurements for lipoproteins such as LDL is dependent on precise determination of the partial specific volume and hydration of these particles. In this report, Talwinder Kahlon and Frank Lindgren and their colleagues have shown that LDL measurements are very sensitive to the solvent background and that all physical parameters must be controlled before LDL molecular weight can be measured.

In a continuing elifort to understand developmental aspects of lipoprotein metabolisn!. Trudy Forte and colleagues investigated apolipoprotein E (apoE) distribution and plasma concentration in human umbilical cord blood. ApoE is recognized by cell receptors that also recognize LDL apoB; hence apof may potentialty regulate cholesterol metsbolism in cells. Apot concentration in the nemberm was almost twoliold higher than that of adults even though the nemboin plasma cholesteral level was onty one thind that in aduts. Apot in the

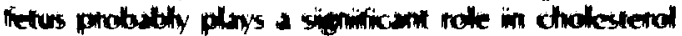

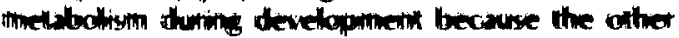


major cholesterol-transporting lipoprotein, LDL, is low in the fetus.

Members of the Lipoprotein and Structural Biology Group who are investigating molecular aspects of biological structures are in the forefront of their field. If one were to express a unifying theme in this area of research it is that each investigator is seeking to unravel the three-dimensional structures of macromolecules and to relate these structures to the regulation of biological processes.

Studies on the structure of biologically important molecules often includes the development of unique techniques and approaches for visualization and preservation of structural information. Kenneth Dowring and Robert Glaeser developed a new appioach for enhancing contrust and image information in high-resolution transmission electron microscopy. Ordinarily high-resolution transmission electron microscopy is accompanied by degradation of the image during electron irradiation. They reasoned that this was due to bending and lateral motion in the sample brought about by beam irradiation. To circumvent the problem they have developed a sophisticated computer-controlled system that takes a series of photographs of a sample irradiated by a very small electron-beam spot size. The composite image shows remarkable contrast without sacrificing structure. This Downing and Glaeser work heralds the way of the future for generating high-quality images of biological molecules for analysis of three-dimensional structure.

Bing Jap and Seok-Hwan Kong have initiated studies on the secondary structure of halorhodopsin (hR). This retinal protein functions as a light-driven electrogenic chloride pump in the bacteria $H$. halobium. Jap and Kong found that this transmembrane protein is similar in molecular weight and amino acid composition to that of the light-driven proton pump of bacteriorhodopsin. Circular dichroism indicates that both proteins have similar secondary struclure; howe: er, they lack immunological crossreactivity. From the compositional studies they have calculated that the $h R$ protein contains five helices that transverse the membrane. In the future, they plan to unravel the three-dimensional structure of $h R$ to see whether this protein has structural features in common with other ion tran. sport proteins such as bacterionthodopsin. Another maior breakthrough for Bing Iap was the isolation and crystalliation of PhoE, tansmembrane pro- tein that forms pores regulating phosphate diffusion in $E$. coli outer membranes. The PhoE protein is atypical of most integral membrane proteins in that it consists mainly of anti-parallel pleated strands instead of helical segments. Reconstitution of large highly symmetric crystals of PhoE by Jap will permit him to carry out three-dimensional structural analysis of this unusual biological macromolecule.

Thomas Hayes and his associates are well known for their development of a mechanism for examining frozen hydrated tissue in the scanning electron microscope. This technology has profoundly affected our conception of lung structure as outlined in the present report by Jacob Bastacky, Hayes, and their colleagues. Examining lung tissue in the frozen-hydrated state prevents critical point Jrying artifacts and reveals that the liquid suiffactant interface in the lung alveoli is continuous. Until this elegant demonstration of surfactant intactness, it was thought that the alveolar aqueous surfactant layer contained pores or regions of discontinuities.

Not all studies on the macromolecular structure of biologically important molecules depend on electron microscopic visualization. This point is very aptly made in the innovative differential polarizaiion imaging microscope developed and described by Marcos Maestie. With this microscope he is able to see differences in hemoglobin structure such as that of normal versus sickled hemoglobin. This new form of imaging has the potential for providing information on both concentration and spatial orientation of biological polymers.

A rapidly developing area in the field of macromolecular structure and interaction is that of antibody-antigen interactions. In the present report, Jack Owicki and Aaron Kantor describe a sophisticated artificial model system with which to study the molecular basis of agglutination. Phospholipid liposomes carrying an antigenic hapten (fluorescein) were produced and interacted with different monoclonal antibodies to the hapten. Their studies thus far reveal that there is little correlation between agelutination ability of the various monoclonal antibodies and affinity of antibody for antigen. Such a phenomenon may be related to conformational ikexibility or geometric distribution of antigen on ricodel membranes. It is definitely a promising new concept to be explored in future studives. 


\section{Lipoproteins}

\section{THE ALAMLNO HDL PARTICLES}

\section{Alex V. Nichois, Guido Franceschini," Cesare R. Sirtori," and Elaine 1. Gong}

Interest in the structure and function of human high density lipoproteins (HDL) derives in major part from epidemiologic observations of their inverse correlation with the risk of arterial disease. $^{1.2}$ Based on such observations, an increased incidence of premature disease would be expected in individuals with very low levels of HDL. Surprisingly, several rare familial disorders characterized by decreased levels of HDL do not show the presence of premature vascular lesions. ${ }^{3,4}$ One such disorder appears to be the apolipoprotein variant designated $\mathrm{Al}_{\text {Milano }}$ or $\mathrm{Al}_{\mathrm{M}}$, which is generally associated with reduced plasma levels of $\mathrm{HDL}$ and elevated levels of triglyceride. ${ }^{5}$ The mutant apolipoprotein Al is characterized by a single cysteine-for-arginine replacement at position $173 .^{6}$ The apolipoprotein is capable of forming intermolecular disulfide bonds that can produce dimers and mixed disulfide complexes. Since Al is the major apolipoprotein component of normal $\mathrm{HDL}$ and thereby plays a crucial role in determining both the lipid-binding capacity and the ultimate particle size properties of HD! considerable change in HDI. particle properties and distribution might be expected from participation of $\mathrm{Al}_{\mathrm{M}}$ dimers and mixed disulfide complexes in HDL structure.

Franceschini et al. ${ }^{7}$ have shown that HDL from affected carriers (designated $\mathrm{Al}_{\mathrm{M}}^{+}$) are characterized by a predominance of $\mathrm{HDL}_{3}(\mathrm{~d} 1.125-1.20 \mathrm{~g} / \mathrm{ml})$ which are enriched in triglyceride and low in cholesterol content. By means of chemical crosslinking, at least three apparent $\mathrm{HDL}_{3}$ subspecies with differential apolipoprotein composition of the protein moiety were identified by these investigators.

In the present investigation, the particle polydispersity of HDL in plasma of ten $\mathrm{Al}_{\mathrm{Mi}}^{+}$and six $\mathrm{Al}_{\mathrm{M}}^{-}$(nonaffected kindred) was evaluated (Table 1$)$. For determination of HDL particle size distribution. gradient gel electrophoresis (protein stain) was periormed on the ultracentriugal $d \leqslant 1.20 \mathrm{~g} / \mathrm{ml}$ fraction isolated from plasma of all of the above subjects. ${ }^{8}$ For determination of the flotation rate distri-

Center Enrica Groxs Pankey Lnnersity of Milan, 20129 silas. nak. bution of $\mathrm{HDL}$, the same ullracentrifugal iraction isolated from a smaller subgroup (six $\mathrm{Al}_{\mathrm{M}}^{+}$and four $\mathrm{Al}_{\bar{M}}^{-}$. see Table 1) of the above subjects was analyzed by anaiylic ultracentrifugation. ${ }^{9}$ Total HDL and HDL-subclass levels determined by analytic ultracentrifugation are listed in Table 1. Total plasma $\mathrm{HDL}$ of the group of $\mathrm{Al}_{M}^{+}$subjects ranged from 18 to $238 \mathrm{mg} / \mathrm{dl}$ and showed a predominance of $\mathrm{HDL}_{3}$ as gauged by the pattern area within the flotation interval of $F_{1.26}^{o} 0-3.5$. $\mathrm{HDL}$ material in the flotation interval of $F_{1.20}^{o} 3.5-9.0$, corresponding to the $\mathrm{HDL}_{2}$ subclass, was observed in $\mathrm{Al}_{\mathrm{M}}^{+}$with higher plasma $\mathrm{HDL}-\mathrm{C}$ levels. Plasma concentrations of $\mathrm{HDL}_{2}$ in these subjects ranged from 0 to 55 $\mathrm{mg} / \mathrm{dl}$. The analytic ultracentrifugal results were consistent with the rate zonal ultracentrifugal data reported by Franceschini et al. ${ }^{7}$

As indicated above, the particle size distributions of $\mathrm{HDL}$ of $\mathrm{Al}_{M}^{+}$and $\mathrm{Al}_{M}^{-}$were determined directly by gradient gel electrophoresis. By means of this technique, we previously described the occurrence in normal human plasma of at leasi five major subpopulations: three within the $\mathrm{HDL}_{3}$ subclass $\left(\left(\mathrm{HDL}_{3 \mathrm{a}}\right)_{\mathrm{gge}},\left(\mathrm{HDL}_{3 \mathrm{~b}}\right)_{\mathrm{gge}}\right.$, and $\left.\left(\mathrm{HDL}_{3 \mathrm{c}}\right)_{\mathrm{gge}}\right)$ and two within the $\mathrm{HDL}_{2}$ subclass $\left(\left(\mathrm{HDL}_{2 \mathrm{~b}}\right)_{\mathrm{gge}}\right.$ and $\left.\left(\mathrm{HDL}_{2 \mathrm{a}}\right)_{\mathrm{gge}}\right)^{10}$ Peak maxima of the above subpopulations fall within specific particle size intervals that define the subpopulations: 12.9-9.7 $\mathrm{nm}$, $\left(\mathrm{HDL}_{2 \mathrm{~b}}\right)_{\mathrm{gge}} ; 9.7-8.8 \mathrm{~nm},\left(\mathrm{HDL}_{2 \mathrm{a}}\right)_{\mathrm{gge}} ; 8.8-8.2 \mathrm{~nm}$,

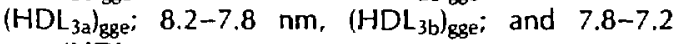
$\mathrm{nm},\left(\mathrm{HDL}_{3 \mathrm{c}}\right)_{\mathrm{gge}}$.

Gradient gel electrophoresis patterns of $\mathrm{Al}_{\mathrm{M}}^{+}$ HDL were characterized by two major peaks with maxima located primarily within the particle size intervals of the $\left(\mathrm{HDL}_{3 a}\right)_{\mathrm{Bge}}$ and $\left(\mathrm{HDL}_{3 b}\right)_{\mathrm{gge}}$ subpopulations (Fig. 1). Compared to $\mathrm{Al}_{M}^{-} \mathrm{HDL}$ (Fig. 2), the $\mathrm{Al}_{\mathrm{M}}^{-} \mathrm{HDL}$ were unique in consistently exhibiting a distinct peak within the $\left(\mathrm{HDL}_{36}\right)_{\mathrm{gee}}$ interval. Peaks with maxima in the $\left(\mathrm{HDL}_{2 \mathrm{i}}\right)_{\text {gee }}$ interval, which in normal subjects contains the larger species of the $\mathrm{HOL}_{2}$ subclass, were either minor or nondetectable. Patterns of $\mathrm{Al}_{\mathrm{M}}^{-} \mathrm{HOL}$ were similar to palterns previously reported for normal subjects. ${ }^{\circ}$ They were characterized by a major peak with maximum in the $\| \mathrm{HDL}_{33}$ interval and by peaks of varying 


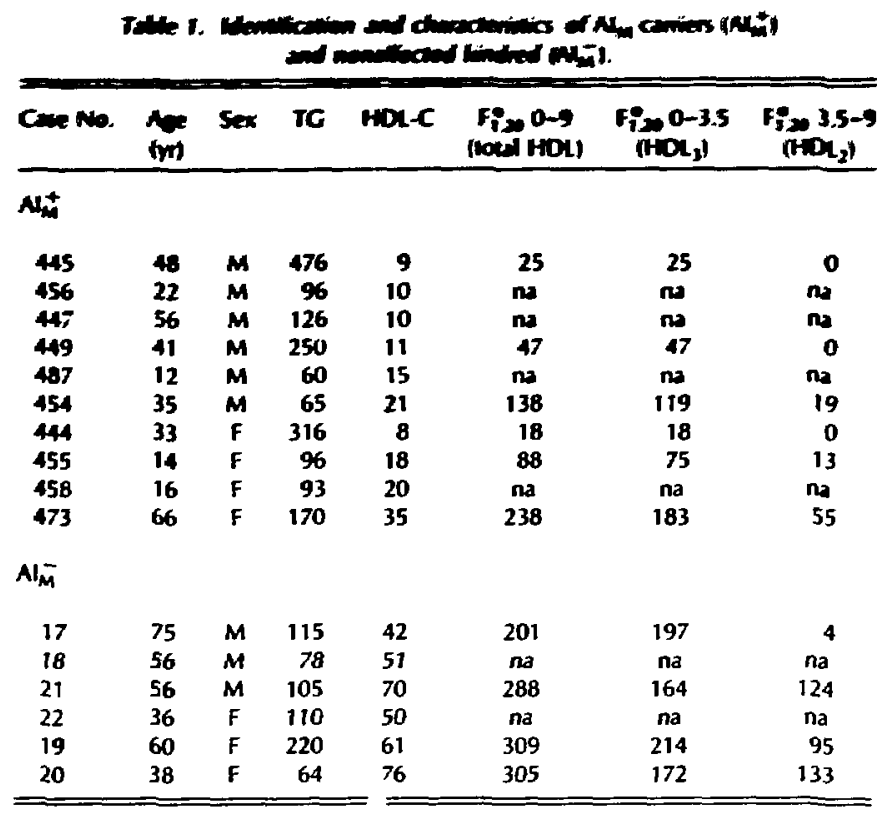

Abbreviations: TG, triglyceride; HDL-C, HDL cholesterol; $F_{1.20}^{0} 0-9,0-3.5$, and 3.5-9: flotation rate intervals measured by analytic ultracentriugation; na, not analyzed by analytic ultracentrifugation. All values in $\mathrm{mg} / \mathrm{dl}$.

amplitude in the $\left(H D L_{2 b}\right)_{g g e}$ interval. The latter peaks, when present, were correlated with the presence of material in the $\mathrm{HDL}_{2}$ flotation interval $F_{7.20}^{o} 3.5-9.0$ in corresponding analytic ultracentrifugal patterns. In some patterns of control subjects, a shoulder on the major $\left(\mathrm{HDL}_{32}\right)_{\text {gege }}$ peak was noted, indicating the occurrence of material within the $\left(\mathrm{HDL}_{3 b}\right)_{\mathrm{gge}}$ interval.

In $\mathrm{HDL}$ patterns of all $\mathrm{Al}_{\mathrm{M}}^{+}$, the mean particle sizes of the major components, with peaks in the $\left(\mathrm{HOL}_{3 \mathrm{a}}\right)_{\mathrm{se}}$ and $\left(\mathrm{HOL}_{3 b}\right)_{\text {coe }}$ intervals (sizes at peak maxima: $8.55 \pm 0.09$ and $7.97 \pm 0.02 \mathrm{~nm}$, respectively). were sinilar to those $(8.44 \pm 0.07$ and $7.88 \pm 0.02 \mathrm{~nm}$, respectively) observed in $\mathrm{Al}_{\bar{M}}$. The mean particle size of the major component in the $\left(H D L_{2 b}\right)_{5 e}$ interval, however, was smaller $(9.85 \pm 0.02$ vs. $10.34 \pm 0.29 \mathrm{~nm})$ in the $\mathrm{Al}_{M}^{+}$compared with that in the $\mathrm{Al}_{\bar{M}}^{-}$. Thus, the parficle sizes of the major $\mathrm{HDL}_{3}$ subpopulations in $\mathrm{Al}_{\mathrm{M}^{+}}^{+}$ were not significantly different from sizes of their counterparts in $\mathrm{Al}_{\mathrm{M}}^{-}$. It should be noted that the mean particle sizes of the major components of $H D L$ in $A_{\bar{m}}^{-}$compared closely with those of a lange number $(n=1911$ of subjects studied by us in the U.SA.
$\mathrm{Al}_{\mathrm{M}}^{+} \mathrm{HDL}$ exhibited three characteristic gradient gel electrophoresis patterns (patterns I, II, and III, Fig. 1) that reflected the relative contributions of the two major peaks. The main distinguishing feature among the three patterns was the relative content of the smaller and larger HDL subpopulations that had peak maxima in the $\left(H D L_{3 b}\right)_{8 g e}$ and $\left(\mathrm{HDL}_{3 a}\right)_{\mathrm{gge}}$ intervals, respectively. Pattern I had the highest relative content of smaller subspecies, and pattern III had the highest relative content of larger species. In addition, it should be noted that the group of $\mathrm{Al}_{M}^{+}$with pattern I exhibited the lowest mean HDL-C value (l: HDL-C, $9.3 \pm 1.5 \mathrm{mg} / \mathrm{dl}$ ) compared with that of $\mathrm{Al}_{\mathrm{M}}^{+}$with patterns II and III (II: HDL-C, $14.6 \pm 4.6 \mathrm{mg} / \mathrm{dl}$, and III: HDL-C, $28.0 \pm 9.9 \mathrm{mg} / \mathrm{dl}$ ). Thus, it appears that as HDL-C levels are reduced, the particle size pattern becomes relatively enriched in the smaller $H D L$ subpopulations. Such progressive relative enrichment of HDL patterns with species of smaller particle size as a function of decreasing plasma concenIration of HDL-C was also observed in normal subjects. The correlation coefficient relating the percent of the lotal HDL pattern area in the particle size interval of the small subpopulation (HDL $\mathbf{H a l}_{2}$ 


\section{PATTERA}
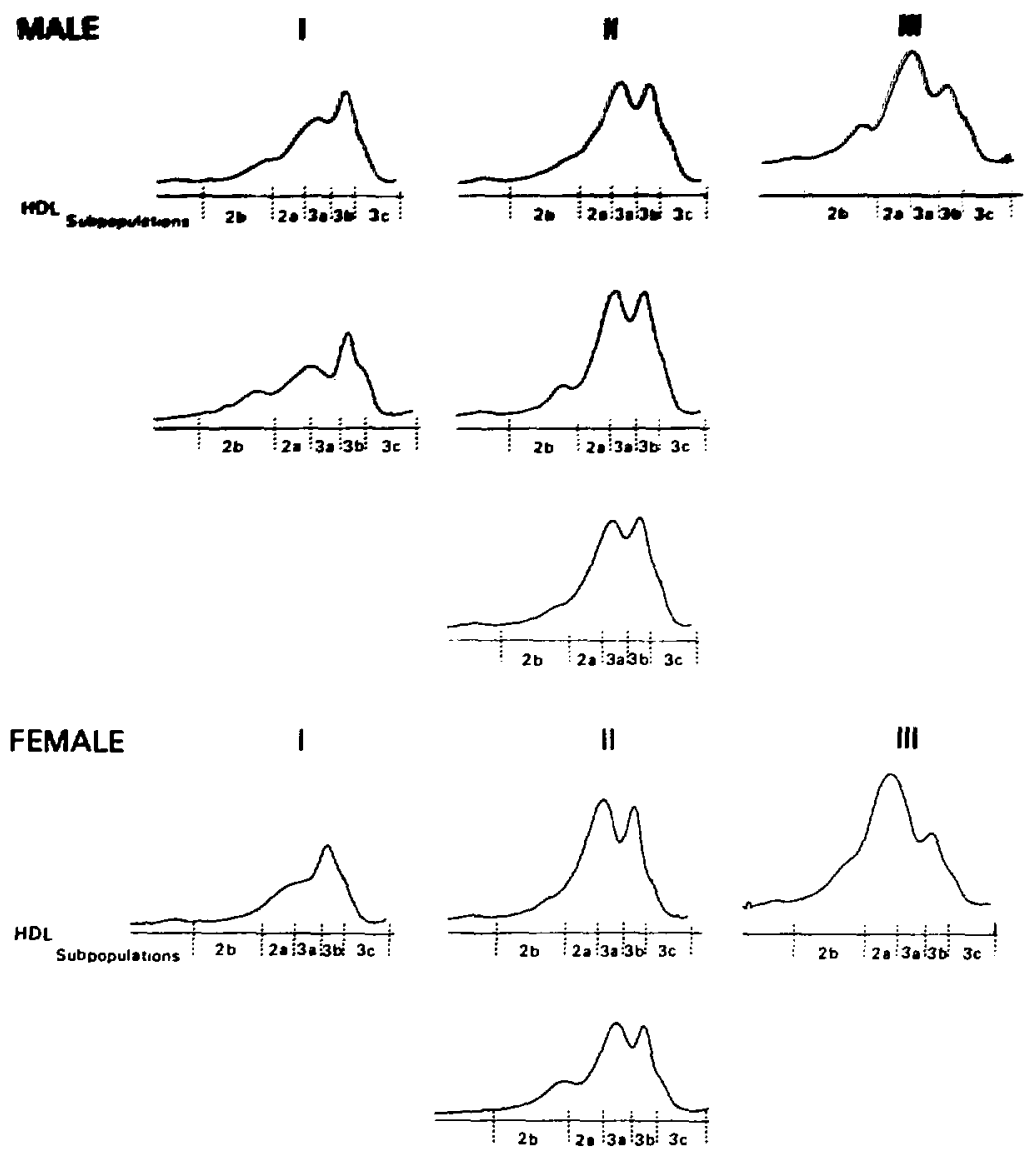

Fig. 1. Gradient gel electrophoresis patterns of male and female $\mathrm{Al}_{M}^{+}$.. Patterns are obtained by densitometry of protein-stained gradient gels $(4-30 \%$ polyacrylamidel." Intervals corresponding to particle size ranges of HDL subpopulations in normal human plasma are indicated below pallerns. See text for numerical values of particle size intervals corresponding to the HDL subpopulations. Numbers appearing at lower left of each pattern designale $\mathrm{Al}_{\mathrm{A}}^{+}$described in Table $\mathrm{l}$.

(XBL 859-3975)

vs. HDL-C was $-0.79(p<0.001)$ for normal male adults (age range: $35-59 \mathrm{yr} ; \mathbf{n}=91$ ). ${ }^{11}$ The correlation coefficient between the same parameters for an admiltedly small sample of ten $\mathrm{Al}_{M}^{+}$subjects was -0.90 ( $p<0.001$ ). The mean HDL.C plasma levels in the above ten $\mathrm{Al}_{M}^{+}$subjects was $15.7 \pm 8.3$ $\mathrm{mg} / \mathrm{dl}$, while the mean level in the 91 normal male subjects was $44.0 \pm 11.6 \mathrm{mg} / \mathrm{dl}$.

Furthermore, it is well established that low levels of HDL-C are frequently associated with elevated plasma triglyceride levels and this also appeared to be the case for $\mathrm{Al}_{M}^{+}$. Thus, for 33 $\mathrm{Al}_{M}^{+}{ }^{12}$ the correlation coefficient for plasma triglyceride level vs. HDL-C was $-0.30(p=0.05)$; for 91 normal males cited above, the correlation coefficient for the same variables was -0.49 (p < 0.001). When values of plasma triglyceride (TG) and HDL-C were plotted for the ten $A \mathrm{I}_{\mathrm{M}}^{+}$and six $\mathrm{Al}_{\bar{M}}^{-}$whose $\mathrm{HDL}$ were analyzed by electrophoresis, the group of $\mathrm{Al}_{\mathrm{N}}^{*}$ with pattern 1 exhibited the lowest mean HDL.C value and the highest mean TG value, relative to those of $\mathrm{Al}_{\mathrm{M}}^{+}$with patterns ॥ 
muse
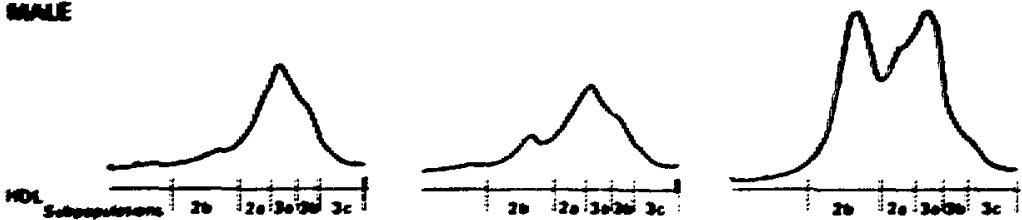

FEMale
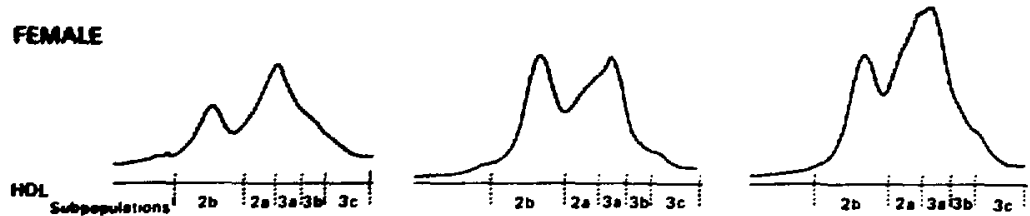

Fiz. 2. Gradient gel electrophoresis patterns of male and female $\mathrm{Al}_{\mathrm{M}}^{-}$(see caption of Fig. I and Table 11 .

(XBL 859.3976)

and III (Fig. 3). These data suggest that the pattern I distribution, in which the relative content of the smaller $\left(\mathrm{HDL}_{3 \mathrm{~b}}\right)_{\mathrm{gge}}$ subpopulation was increased, reflected the presence of both elevated plasma TG

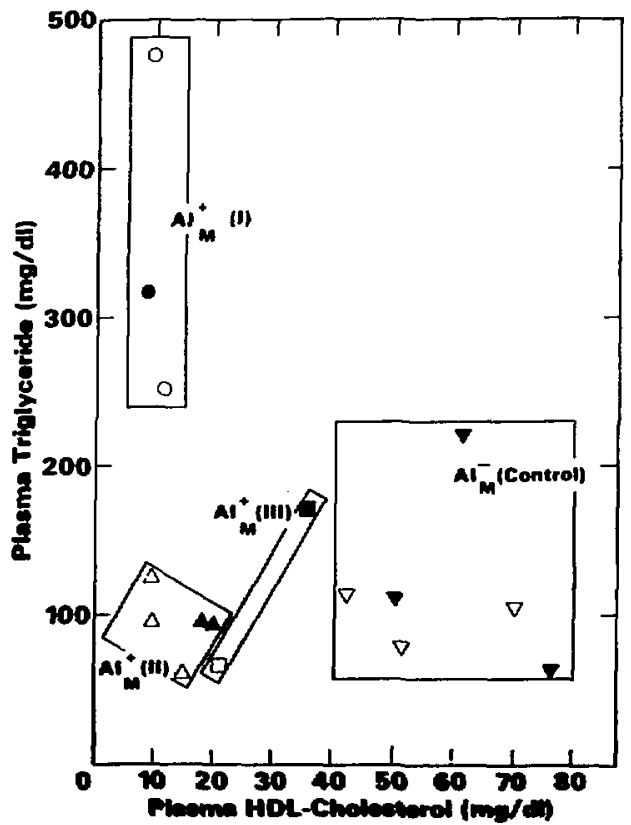

F. 3. Plot of trighreride and HDL-chotesterol levets in plasma

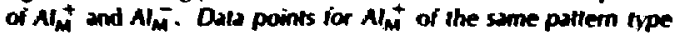
II II, and III and for $\mathrm{A}_{\mathrm{M}}^{-}$control ane enclased by solid fines. Sex of subjects is indicaled. Subjects in this foure are those described in Iable I and in For. 1 and 2. levels and very low levels of HDL-C. The observation of the relative enrichment of HDL with smaller sized particles in the presence of hypertriglyceridemia $(222-2500 \mathrm{mg} / \mathrm{dl})$ and reduced $\mathrm{HDL}-\mathrm{C}$ levels $(22-30 \mathrm{mg} / \mathrm{dl})$ has been previously reported by Eisenberg et al. ${ }^{13}$

In conclusion, our investigation of HDL particles in $\mathrm{Al}_{M}^{+}$indicates the following: 1) the $\mathrm{Al}_{M}^{+} \mathrm{HDL}$ particle size distribution consists mainly of two major components, one broad peak (mean size, $8.55 \mathrm{~nm})$ in the $\left(\mathrm{HDL}_{2 a+3 a}\right)_{g g e}$ interval and one sharp peak (mean size, $7.97 \mathrm{~nm}$ ) in the $\left(\mathrm{HDL}_{3 b}\right)_{\text {gge }}$ interval; 2) the mean particle sizes of the major $\mathrm{HDL}_{3}$ components are similar to those observed within the same HDL subpopulation intervals in $\mathrm{Al}_{M}^{-}$and normal subjects; 3) three pattern types of $\mathrm{HDL}$ distribution can be identified in $\mathrm{Al}_{M}^{+}$that reflect the relative contribution of the two major HDL components; 4) the pattern type with greatest relative build-up of smaller $H D L$ particles is observed in a group of $\mathrm{Al}_{M}^{+}$with highest mean TC and lowest mean HDL-C values; and 5) the statistical relationship between plasma TG and HDL-C levels in $\mathrm{Al}_{M}^{+}$is inverse as observed in normal and hypertriglyceridemic subjects.

\section{REFERENCES}

1. Miller, G.J., and Miller, N.E. Plasma highdensity lipoprotein concentration and development of ischaemic heart disease, Lancet 1, 16 (1975).

2. Gordon, T., Castelli, W.P., Hjortland, M.C., Kannel, W.B., and Dawber, T.R. High density lipoprotein as a protective factor against 
coronary heat dixease; the Framingham study, Am. I. Med. 62:707 (1977).

3. Assmam, G., Herbert, P.N., Fredrickson, D.S. and Forte, T. Isolation and characterization of an abnormal high density lipopnotein in Tangier disease, I. Clin. Imvest. 60, 242 (1977).

4. Carkon, LA. Fish eye disease: a new familial condition with massive corneal opacities and dyslipoproteinemia, Eur. I. Clin. Invest. 12, 41 (1982).

5. Franceschini, G., Sintori, C.R., Capurso, A., Weisgraber, A.H. and Mahley, R.W. A-I Milano apoprotein: decreased high density lipoprotein cholesterol levels with significant lipoprotein modifications and without clinical atherosclerosis in an Italian family, J. Clin. Invest. 66, 892 (1980).

6. Weisgraber, K.H., Rall, S.C., Bersot, T.P., Mahley, R.W., Franceschini, G., and Sirtori, C.R. Apolipoprotein $\mathrm{Al}_{\text {Milano: }}$ detection of normal $\mathrm{Al}$ in affected subjects and evidence for a cysteine for arginine substitution in the variant AI, l. Biol. Chem. 258, 2508 (1983).

7. Franceschini, G., Frosi, T.G., Manzoni, C., Gianfranceschi, G., and Sirtori, C.R. High density lipoprotein-3 heterogeneity in subjects with the apo-Al Milano variant, 1. Biol. Chem. 257, 9926 (1982).

8. Nichols, A.V., Blanche, P.J., and Gong, E.L.

\section{GENETIC STUDIES OF LDL SUBCLASSES}

\section{Melissa A. Austin and Ronald M. Krauss}

The clustering of cardiovascular disease in families has been observed in many studies and may be due, at least in part, to genetic abnormalities of lipid and lipoprotein metabolism. Among the plasma lipoproteins, those of low density (LDL) and intermediate density (IDL) have been related directly to the development or coronary heart disease, while levels of high-density lipoproteins (HDL) are inversely related to cardiovascular disease risk. Genetic abnormalities leading to high-risk lipoproteiti levels have been identified to date in only a small number of disease entities, such as the LDL-recepior defect responsible for hypercholesterolemia in $0.2 \%$ of the population.

Over the last several years, work in our laboratory has established the existence of considerable heteroseneity within $I D L$ and $I D L$ and the
Gradient od electnophoresis of humen plasma high density lipoproteins. In Handbook of Electrophoresis, Vol. III, LA Lewis, ed., CRC Press, Boca Raton, Florida (1983).

9. Lindgren, F.T., lensen, LC., and Haich, F.T. The isolation and quantitative anahysis of serum lipoproteins. In Blood Lipids and Lipoproteins, G.J. Nelson, ed. Interscience, New York (1972).

10. Blanche, P.J., Gong, E.L., Forte, T.M., and Nichols, A.V. Characterization of human high-density lipoproteins by gradient gel electrophoresis, Biochim. Biophys. Acta 665, 408 (1981).

11. Lindgren, F.T., Nichols, A.V., Wood, P.D., Adamson, G.L. Austin, A.M., Glines, L.A., Martin, V., and Krauss, R.M., in preparation.

12. Franceschini, G., Sirtori, C.R., Gianfranceschi, G., Menotti, A., Cerrone, A., Orsini, G., and Gualandri, V. A-I Milano apoprotein: identification of the complete kindred and evidence of a dominant genetic transmission, Amer. J. Human Cenetics, in press (1985).

13. Eisenberg, S., Gavish, D., Oschry, Y., Fainaru, M., and Deckelbaum, R.J. Abnormalities in very low, low, and high density lipoproteins in hypertriglyceridemia, /. Clin. Invest. 74, 470 (1984).

existence of a number of distinct subpopulations within these classes. It is possible that previously unrecognized genetic traits could affect levels of particular LDL or IDL subclasses that are preferentially involved in the development of cardiovascular disease. To assess the genetic contribution to levels of LDL subclasses, we have initiated studies of normal kindreds and have used mathematical modeling techniques to identify phenotypes among the family members.

To date, we have screened 17 nuclear families from 8 large Mormon kindreds living in the Bay area. Mormons were chosen because they often have large families with good geneological recurds. A total of 78 family members have participated in the study by supplying a blood sample for lipoprotein analyses and completing a me fical interview. 
One of the mod sensitive means of identefying LOL subdases is padient at electrophoresis (CCE). This procedure separates lipoprotein particles by sixe, and examples of the resuling curves for low-density lipoproiein (LDL) are shown in Fit 1. To identify subclasses within the LOL ranse based on these data, we have developed a mathematical modeling technique using nontinear regression. The component curves identified by this technique are indicated by dotted lines and comespond to subclasses of LDL particles. The sum of the component curves approximates the total LDL gradient gel scan. The peak particle diameters of the curves are then calculated using calibration data from protein standards. The use of the mathematical modeling has allowed us to identify quantitatively two distinct LDL GGE patterns, $A$ and $B$, and we have proposed that these patterns actually represent genetic phenotypes. Pattern $A$ is more common, and is characterized by a major peak of large LDL subclasses, and a minor peak of smaller LDL subclasses. In contrast, pattern B, present in about $10-15 \%$ of individuals studied to

\section{LDL GGE SCAN SAMPLE 8517}

\section{PATTERN A}

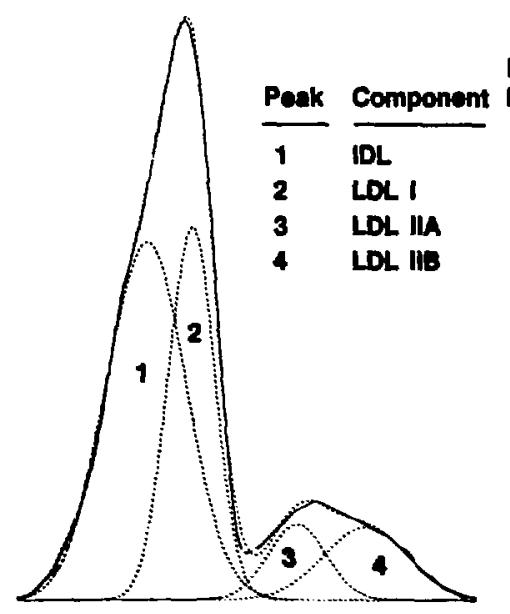

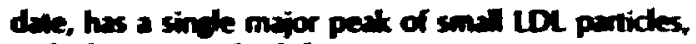
with strewing to the leti.

The evidence fom the bunties studied so for is consisent with a single locus, two allele, cenctic pohymorphism controming the LDL subclass pitterns. One of the most intriguing findings is that there appeass to be a threshoid effect at about age 40. That is, individuals with a "B" allele at the proposed locus may only express the $B$ phenotype once they have reached age 40. An example of a pedigree for a family carrying this trait is shown in Fig. 2. The parents, sample numbers 8628 and 8627 , are both 70 years of age and their children range from age 44 to 29 . The two youngest sons, sample numbers 8714 and 9005 , are identical twins. The father in this family has pattern $A$, as do two of the children with sample numbers 8715 and 8904 . However, the mother has pattern $B$ and has apparently passed it on to her oldest son, sample number 8709 . The twins have patterns somewhat intermediate between the usual $A$ and $B$ patterns. They may carry the $B$ allele, but do not express the $B$ phenotype because they are under age 40 .

\section{LDL GGE SCAN SAMPLE 8518 PATTERN B}

\begin{tabular}{lc}
$\begin{array}{l}\text { Peak } \\
\text { Diam (A) }\end{array}$ & $\begin{array}{l}\text { Percent } \\
\text { Ares }\end{array}$ \\
\cline { 1 - 1 } 273 & 50.4 \\
270 & 30.9 \\
262 & 7.8 \\
257 & 10.9
\end{tabular}

Fi. I. Gradient get efer ro, "resis scans of low densihy boporoteins. Two paterns, A and B, have been idenlified in the genetic studies. Solio ..ires are the actud gradien gel dats. The dotted componemt curves were identified by mathematical modeling using non-tinear regression and correspond to LDU subclasses. The pesk purticle diameters of the component curves were delermined from caltbration dis from protein standards, and the relafine srea under each cune was cakculted. 


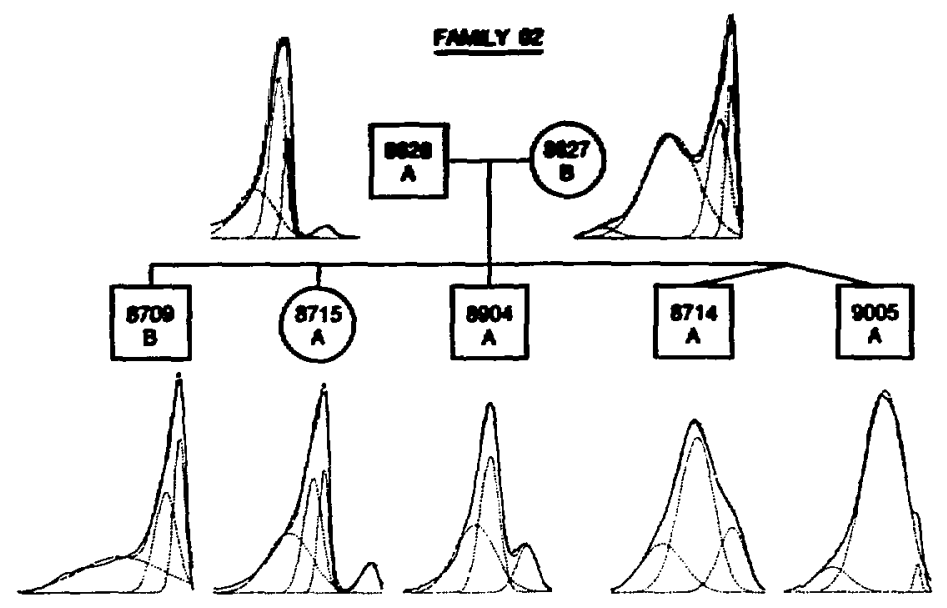

Fig. 2. Pedigree of an informative family in the genetic study. The sample number and phenotype of each subject are given, and the LDL GGE scan with component curves from the mathematical modeling is shown.

(XBL 8510-8539)

In addition to the genetic results, interrelationships between these phenolypes based on LDL and other lipids and apoproteins have been found. After statistical adjustment for age, sex, and obesity as needed, subjects with phenotype $B$ were found to have relatively increased triglyceride and apoprotein B levels. $\mathrm{HDL}$ cholesterol and $\mathrm{HDL}_{2}$ mass were also significantly lower in subjects with phenotype B compared to those with phenotype A. These differences are all in directions that suggest a higher risk of coronary disease in phenotype $B$ than in phenotype $A$.

This initial study of LDL GGE subclass patterns in families has provided preliminary evidence that LDL subclasses are genetically controlled. The phenotype characterized by a predominance of small LDL particles may predispose subjects to elevated triglyceride and apo B levels and decreased $\mathrm{HDL}$ levels. As part of our upcoming program project, 150 to 200 additional families will be studied, and possible dietary and environmental influences on the phenotypes will be considered. If the genetic hypothesis is confirmed, we will have identified a relatively common genetic trait that may predispose the development of coronary heart disease.

\title{
INTERCORRELATIONS OF SUBCLASSES OF LDL AND HDL BY GRADIENT GEL ELECTROPHORESIS (GGF! AND ANALYTIC ULTRACENTRIFUGATION (ANUC)
}

\author{
Frank T. Lindgren, Alex V. Nichols, Peter D. Wood," Gerald L. Adamson, \\ Melissa A. Austin, Laura A. Glines, Vera Martin, and Ronald M. Krauss
}

The relationships between $L D L$ and $H D L$ subclasses as determined by gradient gel electrophoresis and analylic ultracentrifugation were determined in a normal human male population of 120 , ages 35 to 39 years.
The $R_{f}$ inlervals relative to albumin on Pharmacia 4/30 gradient gels between $R_{f} \quad 0.45-0.63$, $0.63-0.71,0.71-0.78$, and $0.78-0.84$, defined as $H L_{2 b}, H L_{2 a}, H D L_{34}$ and $\mathrm{HDL}_{3 b}$ respectively, were highly comel ted to AnUC iniervals F $4-9$, 3-4, 2-3, and 0-2 respectively. Mean correlation coefficienis were 0.84 (all $p<0.0001$ ). Similarly, 
CGE subclasses of $L D L$ with $R_{4}$ intervals relative to apolemitin on Pharmacia $2 / 16$ gradient gets between $R_{4} \quad 0.22-0.30,0.30-0.36,0.36-0.38$, $0.38-0.41,0.41-0.45$, and $0.45-0.49$ cror usponded to $S_{f} 10-20, S_{f} 8-10, S_{f} 6-8, S_{f} 4-6, S_{f} 2-4$, and $S_{f}$ 0-2 respectively, as measured by AnUC. Mean correlation coefficients were 0.62 (all $p<0.0001$ ).

At the time this study were done, LDL and HDL cholesterol values had been determined on 91 of the 120 plasma samples. Total lipoprotein LDL and $\mathrm{HDL}$ mass in $\mathrm{mg} / \mathrm{dl}$ were calculated on each sample $(\mathrm{LDL}$ cholesterol $=\mathbf{0 . 3 3 3} \times$ total $\mathrm{LDL} ; \mathrm{HDL}$ cholesterol $=0.168 \times$ total HDLI. These calculated values, when compared to lipoprotein mass values from the AnUC, gave correlations of $r=$ $0.90, p<0.0001$ (LDL) and $r=0.85, p<0.0001$ (HDL).

More exact calculation of GGE subclass concentration requires evaluation of selative dye uptake per unit mass of each subclass (Coomassie Blue stain). The initial densitometer scan of each sample on GGE is saved in data files on the PDP-11 34-A computer disc, and may be recalled for further analysis when desired.

\section{PARTIAL SPECIFIC VOLUME AND PREFERENTIAL HYDRATION OF LOW DENSITY LIPOPROTEIN SUBFRACTIONS}

Talwinder S. Kahlon, Gerald L. Adamson, Laura A. Glines, Joseph R. Orr, and Frank T. Lindgren

Partial specific volume $(\mathrm{V})$ and preferential hydration of low-density lipoprotein (LDL) subfractions need to be determined in order to characterize these highly atherogenic components of the human plasma lipoprotein spectra. Molecular weights of LDL subfractions have been determined by sedimentation equilibrium, ${ }^{1}$ using an estimated $\bar{v}$, as given by $\bar{v}=1 / \rho$, where $\rho$ is the corresponding background gradient density of isolation. However, for accurate molecular weight determinations of LDL subfractions by sedimentation equilibrium valid determination of $\bar{v}$ is essential. Preferential hydration of LDL has not been thoroughly investigated; however, no values of preferential hydration have been reported. ${ }^{2}$

We have determined $\bar{v}$ for five LDL subfractions ( $n=5-7$ ) and evaluated preferential hydration ( $n=2$ ) for LDL subfraction 3 in normolipoproteinemic subjects in order to characterize these highly atherogenic components of the human plasma lipoprotein spectra. Mean values for $\bar{v}$ were $0.9757 \pm 0.0019 \mathrm{ml} / \mathrm{g} ; 0.9701 \pm 0.0007 \mathrm{ml} / \mathrm{g}$; $0.9674 \pm 0.0016 \mathrm{ml} / \mathrm{g} ; 0.9616 \pm 0.0016 \mathrm{ml} / \mathrm{g} ;$ and $0.9550 \pm 0.0025 \mathrm{ml} / \mathrm{g}$ for subfractions $1,2,3,4$, and 5, respectively. However, molecular densities (o) oblained from $p=1 / \sqrt{v}$ for respective $L D L$ subfractions were 1.0249, 1.0308, 1.0337, 1.0399, and $1.0471 \mathrm{~g} / \mathrm{ml}$, respectively. The preferential hydration of lipoprotein subitaction 3 in $\mathrm{NaCl} \cdot \mathrm{H}_{2} \mathrm{O}$ solution was 2.9 to $4.8 \mathrm{w} \%$, whereas values were much lower $(0.3-0.6 \mathrm{w} \%)$ in $0.196 \mathrm{M} \mathrm{NaCl} \cdot \mathrm{Xm}$
$\mathrm{NaBr}-\mathrm{H}_{2} \mathrm{O}$ solvent system (Fig. 1). It was observed that the linearity of $\eta \mathrm{F}^{\circ}$ vs. $\rho$ may not be valid for solvents $\mathrm{NaCl}-\mathrm{NaBr}-\mathrm{H}_{2} \mathrm{O}$ of density as high as $1.4744 \mathrm{~g} / \mathrm{ml}$. Thus, flotation velocity data using extreme salt concentrations $(1.4744 \mathrm{~g} / \mathrm{ml}$ and higher) may be viewed with caution.

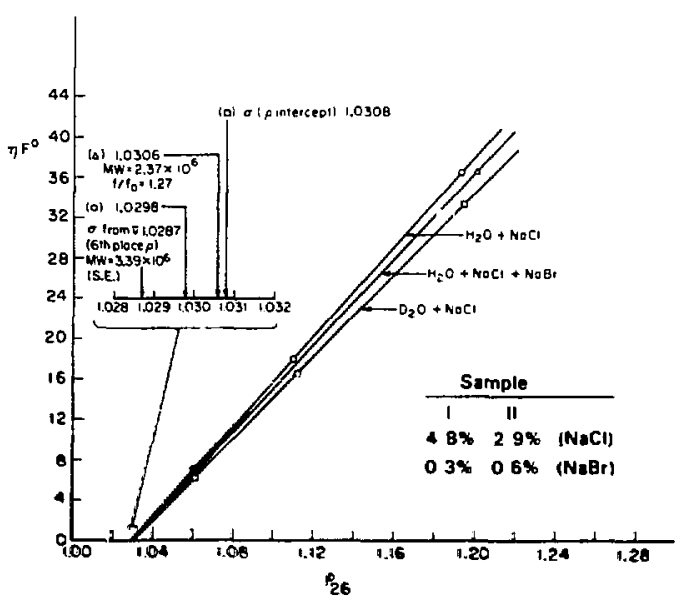

F. 1. F' vs plots, LDL subtiaction 3, sumple II. Preferen. vial mitration is given by $\Gamma=\nabla_{w-1 D}-\bar{v}-10 L / 1$

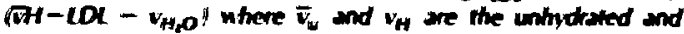
Indined partis specific tollumes of LOL subfiaction 3, obuined

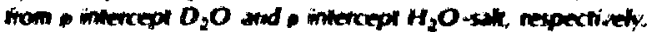

(xit b32-35is 


\title{
REFERENCES
}

1. Kahlon, T.S., Adamson, G.L., Shen, M.M.S., and Lindgren, F.T. Lipids IT, 323-330 (1982).
2. Fisher, W.R., Granade, M.E., and Mauldin, J.L. Biochem. 10, 1622-1629 (1971).

\section{SERUM LIPD AND LIPOFROTEIN CONCENTRATIONS FOLLOWING EXPOSURE TO OZONE}

\author{
William J. Vaughan, Gerald L. Adamson, Frank T. Lindgren, and John C. Schooley
}

The effects of exposure to ozone $\left(\mathrm{O}_{3}\right)$ on concentrations of serum lipids and lipoproteins were investigated. Male and female guinea pigs were exposed to $\mathrm{O}_{3}$ at $1 \mathrm{ppm}$ for two weeks. Serum concentrations of cholesterol, triglycerides, lowdensity (LDL) and very-low-density (VLDL) lipoproteins were elevated after $\mathrm{O}_{3}$ exposure, particularly in the males. During $\mathrm{O}_{3}$ exposure the food intake per day decreased (for a constant body weight), suggesting that metabolic rate and possibly basal metabolic rate was lower. Lung wet weights increased during $\mathrm{O}_{3}$ exposure by $87 \%$ for males and $45 \%$ for females. When individual lungweight/body-weight ratios were correlated with cholesterol and $L D L$ values from the same animal, a high correlation is found for males $(r=0.81, P<$ 0.05 ), suggesting that there may be a relationship between lipoprotein elevations and lung damage for males. Because elevated concentrations of lipids and lipoproteins in humans increase the risk of coronary heart disease (CHD), the lipoprotein results suggest that an epidemiological study of the incidence of $\mathrm{CHD}$ with metropolitan $\mathrm{O}_{3}$ levels may be warranted.

\section{APOLIPOPROTEIN (APO) E I.EVELS AND DISTRIBUTION IN HUMAN CORD BLOOD}

Trudy M. Forte, Paul A. Davis," and Conrad B. Blum ${ }^{\dagger}$

Apo $E$ is a protein of 35,000 molecular weight that plays an important role in the catabolism of lipoproteins, particularly those of dietary origin (chylomicron remnants). Apo $E$ is a ligand recognized by both the apo B-E and apo $E$ receptors on cells. Apo E-containing particles are internalized through the receptor mechanism, thus delivering cholesterol to cells. In adult plasma a majority of apo $E$ is associated with very low density lipoproteins (VLDL), and the remainder is associated with high densily lipoproteins (HDL). There is one inherited lipoprotein abnormality in which this patlern is drastically altered. This abnormality is abetalipoproteinemia, in which plasma of afflicted subjecis contains no chylomicrons, VLDL, or low density lipoproteins (LDL). but does contain HDL.

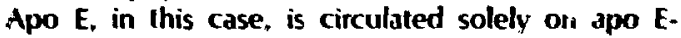

Depatment of Merticine, Umersily of Cationis, Dawis

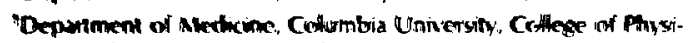
ciuns and surgeoms, Now rooth. rich HDL, which may functionally substitute for the absent apo B and thus transport cholesterol to cells via the receptor mechanism.

The lipid and lipoprotein patterns of newborn infants in some respects are similar to those in abetalipoproteinemia. In the newborn, plasma cholesterol is low, and VLDL and LDL moieties are extremely low. This observation suggested that apo $E$ transport in the newborn may not be primarily in the less dense lipoproteins but rather in HDL. This question was examined in cord-blood samples.

Plasma concentrations of apo $E$ and lipids (cholesterol, triglyceride, HDL-cholesterol, and LL-cholesterol) in 95 cord blood samples and in 49 adult colasma samples are shown in Table 1. The apo E level in cord blood is $60 \%$ greater than aduit plasma, while the newborn cholesterol level is only $40 \%$ that of the adult. LDL in the cord blood is extremely low and represents less than $25 \%$ that of adult values. 
Table 1. Fixma concentrations (Mean i S.O.) of apo E, cholesterol, Iristyceride HOL cholesterd, and $\mathrm{LO} \mathrm{C}$ cholesterol.

\begin{tabular}{lrr}
\hline & $\begin{array}{c}\text { Normal } \\
\text { neonates } \\
\text { (n }=95)\end{array}$ & $\begin{array}{c}\text { Adults } \\
(\mathrm{n}=49)\end{array}$ \\
\hline Apo E (mg/dl) & $58.1 \pm 26.5$ & $35.8 \pm 10.4$ \\
Cholesterol $(\mathrm{mg} / \mathrm{dl})$ & $72.1 \pm 21.3$ & $185.1 \pm 33.0$ \\
Triglyceride $(\mathrm{mg} / \mathrm{dl})$ & $37.8 \pm 16.9$ & $97.6 \pm 36.1$ \\
HDL-cholesterol $(\mathrm{mg} / \mathrm{dl})$ & $39.7 \pm 12.4$ & $54.7 \pm 11.2$ \\
LDL-cholesterol $(\mathrm{mg} / \mathrm{dl})$ & $24.8 \pm 13.6$ & $110.4 \pm 29.7$ \\
\hline \hline
\end{tabular}

To determine the distribution of apo $E$ relative to cholesterol, plasma samples (cord blood and adult) were applied to $6 \%$ agarose columns and fractions eluted with phosphate buffer. The elution profiles are shown in Fig. 1. Cord blood apo E (Fig. 1A) shows a very pronounced peak in fractions 60-80, which is the recovery region for HDL. Adults, on the other hand, have a distinct bimodal distribution, with larger amounts of apo $E$ in the larger-sized, triglyceride-rich region (fractions $40-60)$ as well as in the HDL region. Quantitation
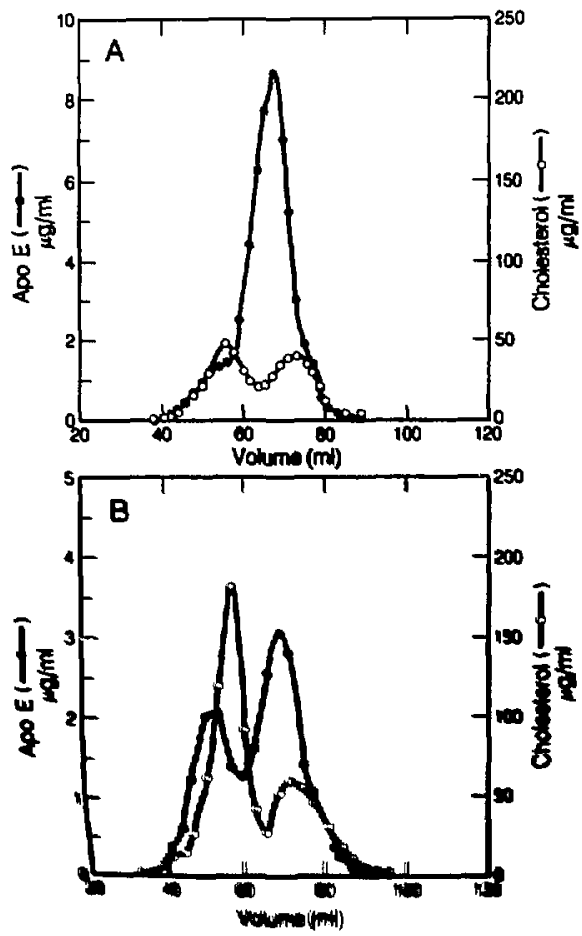

of apo $E$ in the major column fractions (Table 2) reveals that only $13 \%$ of total apo $E$ in cord blood is associated with the less dense, larger-sized particles, which are also rich in triglyceride, while $87 \%$ is in the HDL fraction. In contrast, adult plasma contains a major fraction of its apo $E$ in the triglyceride-rich fraction. (Table 2).

The results of the culumn fractionation for cord blood apo $E$ are similar to those seen in subjects with abetalipoproteinemia, ${ }^{1}$ which is consistent with our observation that, in cord blood, VLDL levels are extremely low and LDL levels are also considerably reduced. These two classes of lipoproteins carry apo $B$, which is recognized by the apo B-E receptor and is responsible for the major transport of cholesterol into cells in the adult. However, since apo $B$ levels are depressed in the fetus, it is probable that apo $E$ plays a more significant role in cholesterol transport in the developing human. Since most of the apo $E$ in the neonate is in the HDL fraction, it is this fraction that is an important supplier of cholesterol to cells and tissues in the rapidly growing fetus.

Table 2. Lipoprotein fractionation of apo $E$ in cord blood and adult human plasma by agarose column chromatography.

\begin{tabular}{lcccc}
\hline & $\begin{array}{c}\text { Triglyceride-rich } \\
\text { lipoproteins }\end{array}$ & \multicolumn{3}{c}{$\begin{array}{l}\text { High density } \\
\text { lipoprateins }\end{array}$} \\
\hline$\%$ & $\mu \mathrm{g} / \mathrm{ml}$ & $\%$ & $\mu \mathrm{g} / \mathrm{ml}$ \\
\hline Cord blood" & 13 & 7.4 & 87 & 49.5 \\
Adult blood & 42 & 15.0 & 58 & 20.3 \\
\hline
\end{tabular}

- Data for cord blood plasma are the mean of two experiments, each using pooled plasma. Data for normal adult plasma are the means obtained from $\mathbf{3 0}$ individuals.

\section{REFERENCES}

1. Blum, C.B., Deckelbaum, R.J., Witte, L.D., Tall, A.R., and Cornicelli, 1. I. Clin. Invest. 70. 1157-1169 (1982).

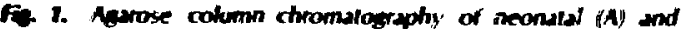
adul (O) humen plasms. Chotesterol and yoo $E$ concentrations were meusured in each fination. The firs pest of the

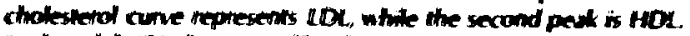

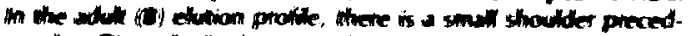

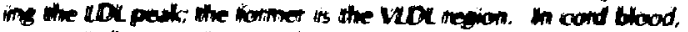

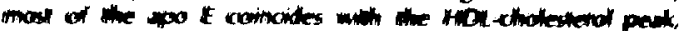

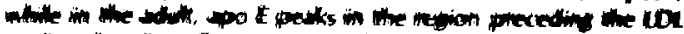

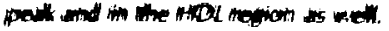




\title{
FONMATKON OF PHOSPHOLPD RKCH HDL WITH UNUSUAL PHYSICAL FROPERTIES: POSSIAE MODEL FOR INTERSTITIAL FLUID HDL
}

\author{
Trudy M. Forte, Robert W. Nordhausen, C. Luming Ren, and Alex V. Nichols
}

We have previously shown that sheep lung lymph' and human lymphedema interstitial fluid ${ }^{2}$ contain a unique high density lipoprotein (HDL) particle that forms square-packing arrays during negative-slaining electron microscopy. In contrast, plasma HDL contains spherical, hexagonal-packing particles. The unusual square-packing particles isolated from interstitial and/or lymph fluid are characterized by a relative increase in both phospholipid and free cholesterol moieties. Overall, the particles are larger and more buoyant than their plasma counterpart. To test the hypothesis that incorporation of excess phospholipid into HDL may be responsible for the altered physical properties of the particles, an in-vitro model system was developed. This consisted of bovine $\mathrm{HDL}_{2}$ incubated with dimyristoylphosphatidylcholine (DMPC). HDLs were incubated with increasing amounts of DMPC and the products were examined by gradient gel electrophoresis and electron microscopy.

Figure 1 shows the changes in particle size as determined by gradient gel electrophoresis following incubation of $\mathrm{HDL}_{2}$ with $\mathrm{HDI}$. phospholipid to DMPC mole ratios of $1: 0.5,1: 1$, and $1: 2$. The major peak at $12.5 \mathrm{~nm}$ in control HDL (A) progressively becomes larger as indicated by a shift to smaller $R_{f}$ values. A second component of smaller size $(9.4 \mathrm{~nm})$ is present at the 1:0.5 ratio and increases with increasing DMPC. This second component is constant in particle size as indicated in Table 1, which shows particle diameters for the various peaks. The electron microscopic structures of HDL-DMPC incubation products are seen in Fig. 2A-D. With increasing DMPC, there is an increase in particle diameter and an alteration in the shape of particles. The change in HDL packing is mosl pronounced at a ratio of 1:2 where particles iorm exlensive square-packing arrays (Fig. 2D). For comparison. HDLs with similar struclural properties bul isolated from mative biological sources are also shown. Lange HDLs imon a patient with abetalipoproleinemia have a lendency to form squarepacking arrays. This structural anomaly is even more pronounced in the sansple of sheep lung

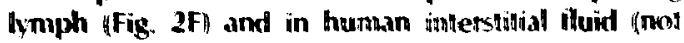
shown H,

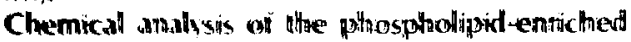

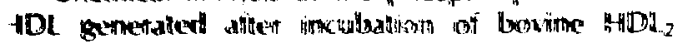

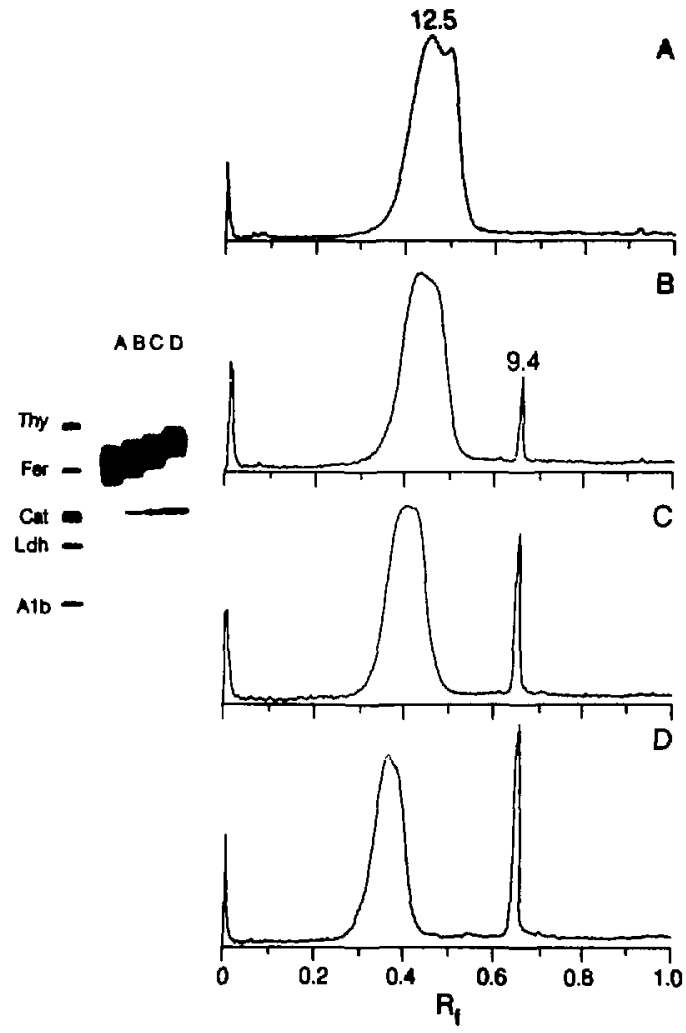

Fig. 1. Gradiem gel (Coomassie-stuined get in insef) and corresponding scans of fraction I-HOL incubated with increasing amounts of DMPC. (A) HDL-control; (B) HDL-phespholipid IDMPC. 1:0.5: ICI HDL-phospholipid/OMPC. 1:I: (D) HDLphospholipid/DMIPC, I:2. In the presence of increasing DMPC. the HDL pesh $112.5 \mathrm{~nm}$ io control) shifls to langer diameters; concomitanth. a product of smaller diameter is also generated. The numbers own the peats andicate partic le diameter in $\mathrm{nm}$. (XBB 849.7226

with DMIPC at a ratio of 1:2 revealed that the persent of phospholipid in the particle doubled. On the other hand, the percent protein in the particle decreased to almost $50 \%$ of the control value. Analysis of the protein moiety revealed that the major protein of bovine HOL was apo AL. Based on the composition of the particles and their estitmated moluecullat weighths, we calcutaled that the 
Table 1. Effect of DMPC incubation on bovine fraction I-HDL particle size distribution as determined by gradient GEL electrophoresis. Fraction I-HDL was incubated at $37^{\circ} \mathrm{C}, 12-16 \mathrm{hr}$, with increasing amounts of DMPC.

Values represent mean \pm S.D. for four determinations.

\begin{tabular}{ccc}
\hline $\begin{array}{c}\text { Mole Ratio } \\
\text { HDL-phospholipid:DMPC }\end{array}$ & $\begin{array}{c}\text { Large Particle } \\
\mathrm{nm} \pm \text { S.D. }\end{array}$ & $\begin{array}{c}\text { Small Particle } \\
\mathrm{nm} \pm \text { S.D. }\end{array}$ \\
\hline 0 & $12.3 \pm 0.1$ & - \\
$1: 1.05$ & $12.9 \pm 0.08$ & $9.5 \pm 0.1$ \\
$1: 1$ & $13.7 \pm 0.1$ & $9.4 \pm 0.08$ \\
$1: 2$ & $14.6 \pm 0.08^{2}$ & $9.4 \pm 0.08$ \\
\hline
\end{tabular}

- These particles form square-packing arrays upon negativestaining electron microscopy.

control HDL possesses three molecules of apo Al. The major larger-sized product formed after incubation at the HDL-phospholipid to DMPC ratio of 1:2, however, contained only two molecules of apo Ai. This suggests that during incubation DMPC is incorporated into the HDL particle until a critical phospholipid to protein ratio is reached; when this ratio is exceeded, apo Al is displaced from the surface. The resulting phospholipid lipid-rich but proteinpoor particle is stable. Two factors may be responsible for the appearance of square-packing arrays during negative staining:

1) the particles may be spherical, but the distribution and conformation of surface molecules, especially apoprotein, are altered so that they are favorably disposed to foster particle-to-particle interaction. Because of the nature of the particle-to-particle interactions, particles are organized into square lattices.

2) Free-standing particles may be square (or cuboidal! and, at appropriate concentrations furing negalive staining, form square arays. Square shapes of free-slanding human interstitial-fluid HDL have recently been seen in our laboratory (work in progress); hence, this possibility cannot be excluded.

The ability of HDL to incorporate a lange amount of polar lipids, (phospholipid and tree cholesterol) into its surface is probably of functional importance. HDL entering the intersinta! space may remove excess phospholipid and cholesterol from peripheral lissues and hence play an important
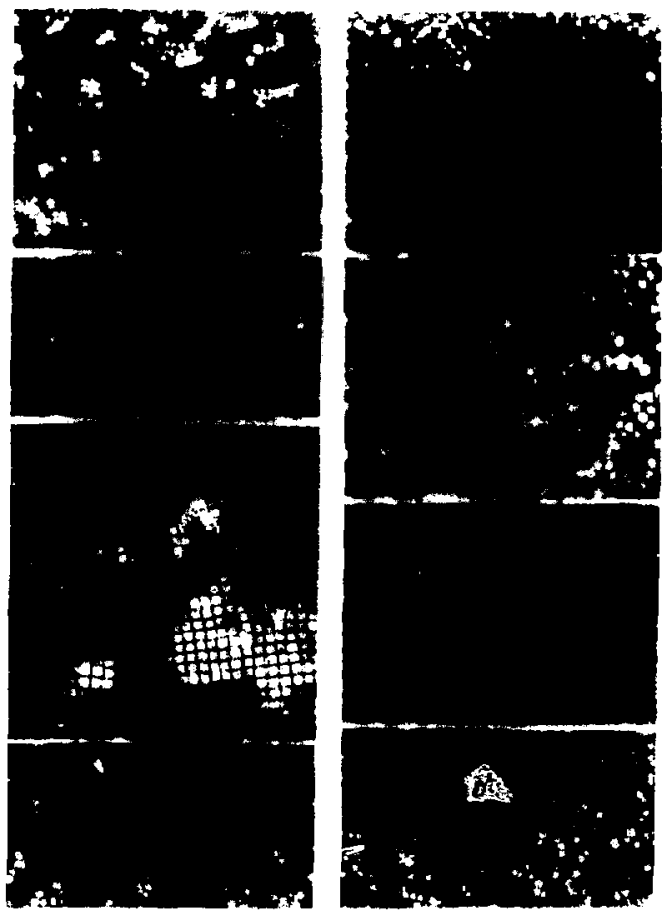

Fig. 2. Electron micrographs of negative/ stained fractions. (A) HDL incubated without DMPC. (B) Product of HDL incubated with DMPC (ratio 1:0.5); note occasional rectangular or square particles (arrow). (C) Product of HDL incubated with DMPC (ratio 1:1); a few sijuare-packing particles are apparent (arrow). (D) Large molecular-weight profuct of HDL incubated with DMPC (ratio 1:2); note characterist. square-packing particles. (E) HDL particles of $d \leqslant 1.063 \mathrm{~g} / \mathrm{m}$ l isolated from the plasnia of a palient with abetaljpoprofeinentia; particle size 12.8 $\pm 0.7 \mathrm{~nm}$ on a side Note similarity of square-packing geometny to that produced in (D). I Square-packing particles, $d=1.057-1.063 \mathrm{~g} / \mathrm{ml}$ fraction, iso e $^{2} d$ from sheep lung $/ \mathrm{ymph}$; particle size is $14.9 \pm 2.1 \mathrm{~nm}$, a side. Bars represent 100 nm.

(XBB 853-1951)

role in reverse choleslerol transport.

\section{REFERENCES}

1. Forte, T.M., C oss, C.E., Gunther, R.A., and Kramer. G.C. 1. Lipid Res. 24, 1358-1367 (1984).

2. Forte, $\pi . M$. Reichl, D.R., Hong, I.L., and Ruda, D.N. Attetroscterosis 4, 564a (1984). 


\title{
Structural Biology
}

\section{DIFFERENTIAL POLARIZATION IMAGING MICROSCOPY}

\author{
Marcos F. Maestre, William Mickols," Ignacio Tinoco, Ir.," and \\ Stephen H. Embury ${ }^{\dagger}$
}

In this report we describe the development of a differential polarization imaging microscope. The technique is shown to be a logical extension of the research on the interaction of circularly polarized light with structures whose dimensions are large with respect to the wavelength of light. ${ }^{1}$ The polarized light technique used initially to study these biological structures was circular dichroism. Circular dichroism is the difference in the extinction coefficient when a sample is illuminated by two different forms of circularly polarized light:

$$
\Delta t=\epsilon_{L}-\epsilon_{R}
$$

where $\epsilon_{L}$ and $\epsilon_{R}$ are the specific extinction due to left and right circularly polarized light respectively. Receritly this technique has been extended to the measurement of single microscopic particles by the development of a circular dichroism (CD) micrnscope. $^{2}$

The CD microscope has been used to study the circular dichroism and circular intensity differential scattering (CIDS) spectra of Zhinese Hamster tissue culture cells as a function of the life cycle of the cells. ${ }^{3}$ There are distinct spectra associated with the different states in the life cycle if the cell, and correlations exist with the amount of DNA in the cell as monitored by absorbance at $260 \mathrm{~nm}$ in the CD microscope.

In the CD microscope, the objective lens in the instrument only serves to collect the $t_{i}$. Ismitted light through the microscopic object plus some iraction of the scattered light, depending on the aperture of the lens. The collected ligit is nol used to form an image but insfead is measured by a standard photomuliiplier detector to give spectral curves. In the next section we describe the insirumenl that produces diffetential himages cblained inom incident illumination of specilic polarization.

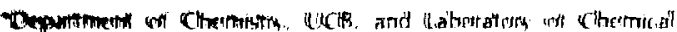

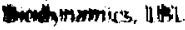

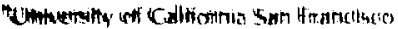

\section{THE DIFFERENTIAL IMAGING MICROSCOPE}

If an imaging system (a lens or a microscope) is placed between the chiral sample and the detector, an image can be formed (Fig. 1). For a chiral sample the image produced by illumination with one polarization form of light can be different from the image produced when the illumination is of another polarization form. The difference between these two images when using circularly polarized light is called the circular differential image. With the proper detection system and associated electronics, we can use the imaging properties of the microscope to form a differential image with a spatial distribution of information. The information in the image is now a point by point measurement of the

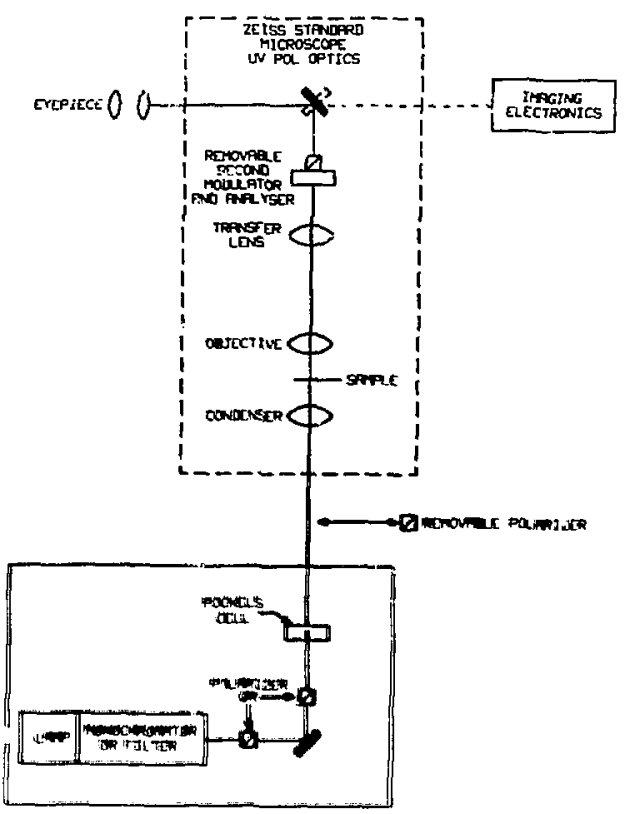

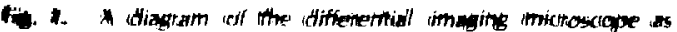

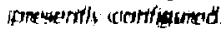


$\boldsymbol{A}$

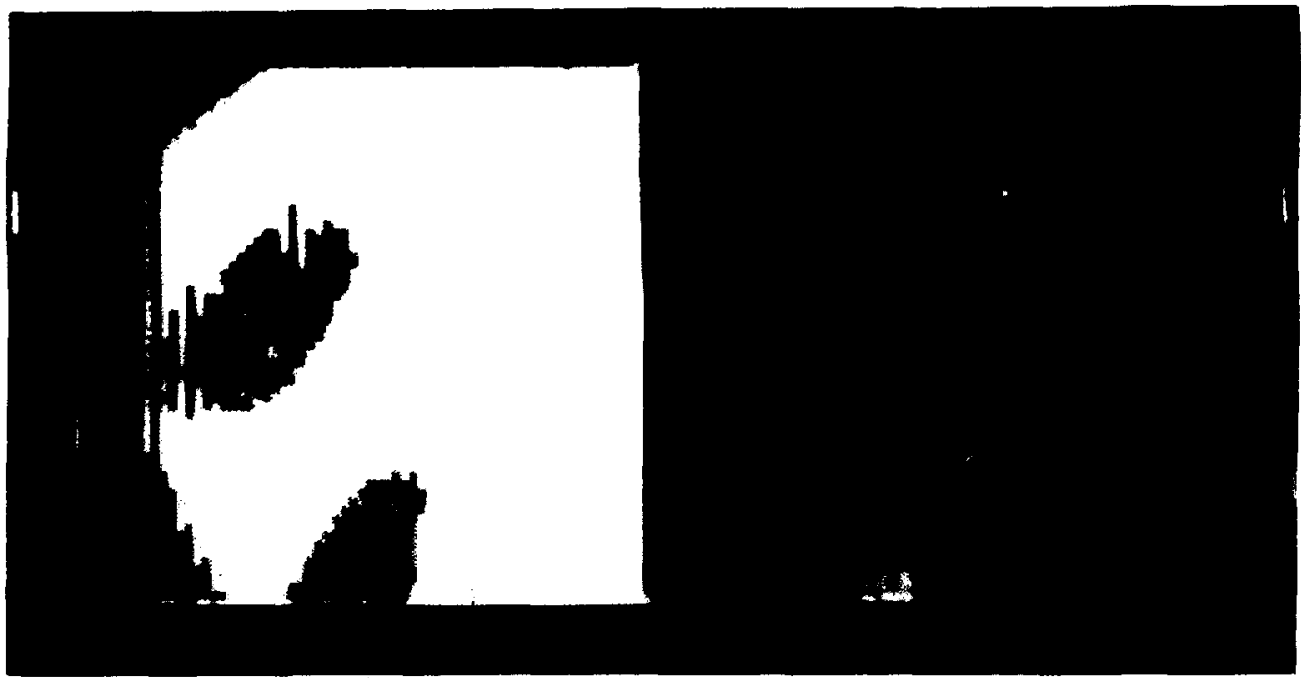

B

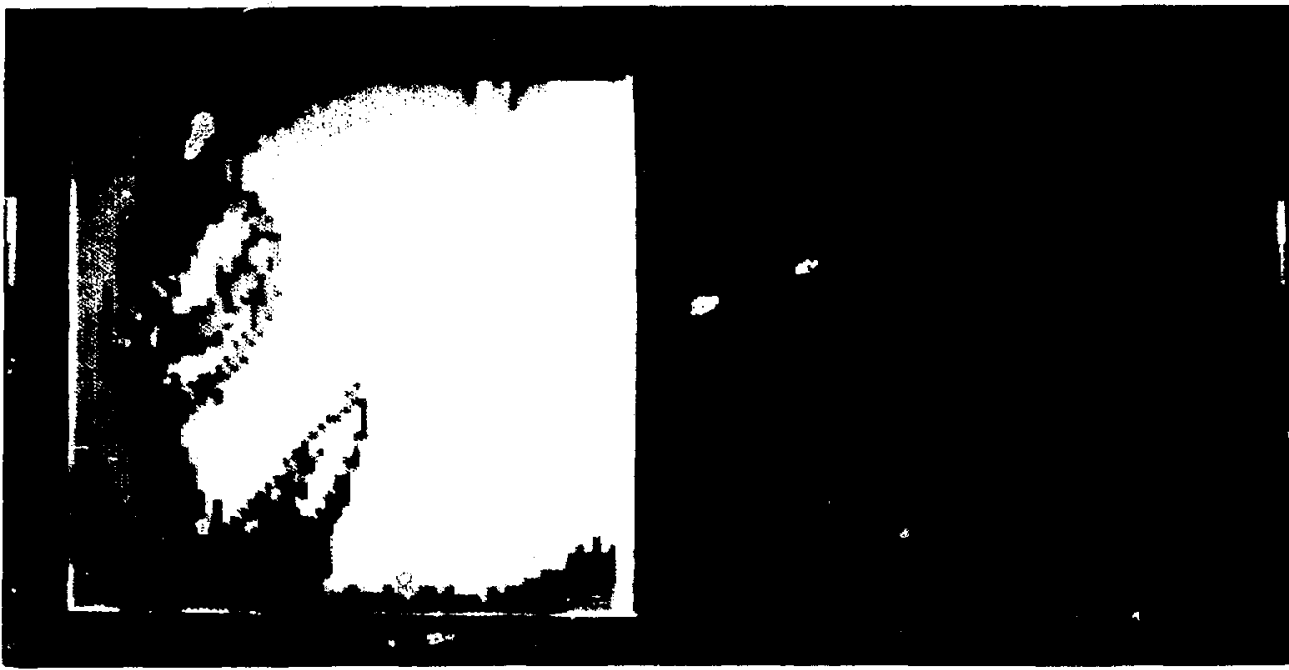

Fig. 2. These photographs are black and white coppies of color conterd images of rixed deoxygenaled red btaod cells trom a palient with sickle cell anemia. The helt hand image is the intensily image. Mlasimum-intensity is while ftop of the felt hand code barl. minimum intensity is black (botlom oi left conte bart. The right hand image is the linear dichrousm imuge. The maximum positive value is the top of the right hand code bar, langest negative is the bontom ni the right hand corte bar. The top cell is an itreversibly sickled cell, and the botion cell is a reversibly sichled cell. Figure at was laken on the hemoglobin absotasnce band at $115 \mathrm{~nm}$ aswd shows a lange amount of aligned

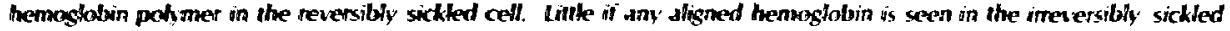

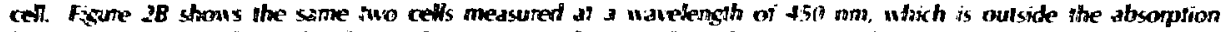

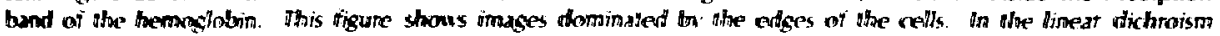

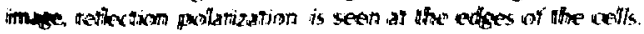

(f(A) $\times B$ C 848-65.3\%, (B) XAC 548-6535) 
cincula dichnoism, in the case of transmmined ting momurement, of circular differentis scatiering conmbution 10 the imare cross-section in the case of dik-field mimination." The spatial nesolution of the neasurement is linited by the lens resolving power. pixel geometry in the detector, and available light intensity.

The information contained in the circular differential image will not be the same information as that of the image produced using unpolarized light. In the Satter case the optical contrast that distinguishes one ieature of the sample from another is provided by differences in the absorption and in the index of refraction of the various parts of the sample. in a circular differential image the contrasi is provided by differences in the interaction of different parts of the samipie with left and right circularly polarized lighi.

Preliminary studies (with a differential imaging microscope using linearly polarized light) on the po:';mer formation of hemoglobin $S$ in the sickling of intact red blood cells have shown that the technique works, as can be seen in Fig. 2.5 Two images are presented in each of the figures, the left image represents the total intensity, and the sight represents the differential image. This direct comparison of the images allows the identification of regions within cels containing aligned ctromophores. Information on the spatial distribution and corrcentration of the pohmer has been obtained, on both sickled and imeversibly sidked cells. The lechnique shoms pomise as a new way of measuring the spatial distribution of chirality and orientaticn inside microscopic biological materials This information can then be used to construct possible models of the biological organization.

\section{REFERENCES}

1. Tinoco, 1., Jr., Bustamante, C., and Maestre. M.F. Ann. Rev. Biophys. Bioeng 9, 107-4' (1980).

2. Maestre, M.F., and Katz, J. Biopolymers 21. 1899 (1982).

3. Maestre, M.F., Salzman, G.C., Tobey, R.A., and Bustamante, C. Biochemistry 24, 5152-5157 (1985).

4. Keller, D., Bustamante, C., Maestre, M. $\overline{\mathrm{r}}$. and Tinoco, I., Jr., Proc. Nat. Acad. USA 82, 401-405 (1985).

5. Mickols, W., Maestre, M.F., Tinoco, 1., Ir., and Embury, S. Proc. Nat. Acad. Sci. USA 82, 6527-6531 (1985).

\section{ABSORPIION FLATTENING IN THE CIRCULAR DICHROISM SPECIRA OF SMALL MEMBRANE FRAGMENTS}

Robert M. Glaeser and Bing K. Jap

The inhomogeneous distribution of chromophore occurring in a particulate suspension can result in a reduction in the apparent molar ellipticity recorded in circular dichroism (CD) spectra. The possibility of such a systematic error has often been a matter of concern when CD spectra of cell membrane proteins are recorded. The recent publication of $\mathrm{CD}$ spectra for bacleriorhodopsin in native membranes, in sonicated membranes, and in detergent-solublized form ${ }^{1}$ gives a unique opportunity to apply the theoretical analysis of Cordon and Holzwarth ${ }^{2}$ so as to provide a definitive answer to the question of whether absorption flattening is significant for membrane particles. We show here that the data of Mlao ar:d Vallace can be combined with the theoretical anai'sis of Gordon and Holewarth to rule vut significant absorption flatten- ing effects over the range $200-240 \mathrm{~nm}$ for submicrometer-sized membranes. In addition, the results show that absorption flattening can be disregarded even at $190 \mathrm{~nm}$ for membranous material in the size range below $100 \mathrm{~nm}$. The demonstration that there are no major flattening effects in the $C D$ spectra of bacteriorhodcpsin, particularly in the region of $200-240 \mathrm{~nm}$, means that the experimental spectra are incompatible with the proposal that this transmembrane protein contains seven transmembrane helices.

The CD spectra between 200 and $240 \mathrm{~nm}$ presented by Mao and Wallace for native purple membrane, sonicated purple membrane, and bR solubilized in octyl glucoside are nearly identical, as can be seen from the published curves as they are redrawn in Fig. 1. The ellipticity of the native 


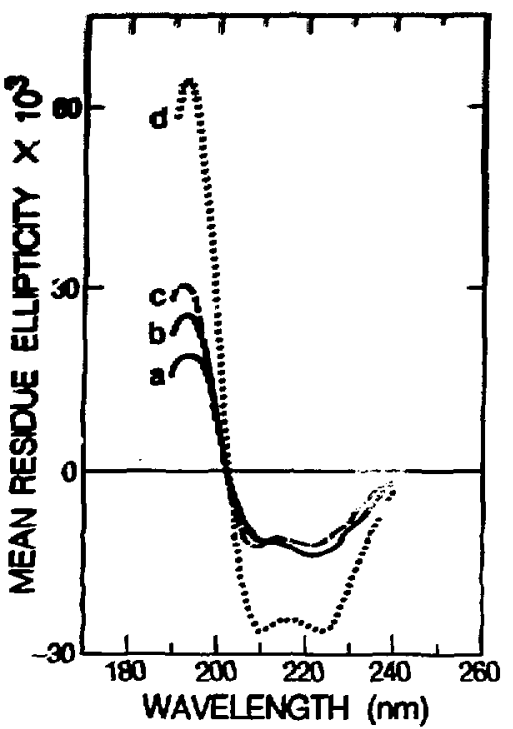

Fig. 1. CD spectra of bacterionadopsin redrawn in simplified form from Mao and Wallace.' Curves (a) and (b) represent the spectra obtained with native purple membrane, sonicated purple membrane and $b R$ solubilized in octylglucoside; these three spectra are very similar between $200 \mathrm{~nm}$ and $240 \mathrm{~nm}$ and are shown here as a single curve, but the curve for native membrane, (a), is significantly lower at $190 \mathrm{~nm}$ than the average value of the curves for sonicated purple membrane or octylglucoside solubilized $b R$, shown as (b). Curve $(c)$ is the sioctrum of $b R$ incorporated into SUVs, and it stows small differences from $(b)$ over the whole range of wavelengths. Curve (d) is the theorefical spectrum calculated by Mao and Wallace for a protein containing $80 \% \alpha$-helix, $10 \% \beta$-turn, and 10\% random coil.

(XBL 8411-8061)

membrane patches at $190 \mathrm{~nm}$ is about 0.75 times that of the other types of specimen preparation, indicating that absorption flattening is significart for intact purple membrane sheets at the shorter wavelengths. The ratio of "true" solution absorbance to the average number of particles per opt:cal path [cf. Gordon and :Ho! zwarth, Ref. 2] has its most extreme value at $: 90 \mathrm{~nm}$, and thus the absorption-flattening effect cannot be any greater at longer wavelengths than it is at $190 \mathrm{~nm}$.

The experimental data presented by Mao and Wallace are therefore inconsistent with their suggestion that there is a significant absorptionflattening effect in the $C D$ spectra over the range 200-240 nm. The data of Mao and Wallace do show, however, that there can be a significant absorption-flattening effect at $190 \mathrm{~nm}$ for native membranes. The latter effect can be avoided by the use of sonicated membrane pieces or by the

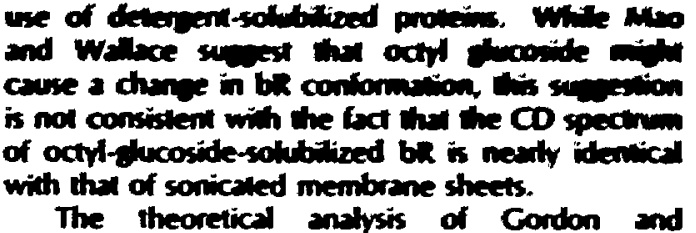
Holzwerth can be used to show qumbitatively that the spectra obtained by Mao and Wallace with native and sonicated purple membranes are not consistent with there being a significant amount of absorption flattening at $221 \mathrm{~mm}$. Mao and Wallace calculate that the mean residue ellipticity of $\mathbf{b R}$ at $221 \mathrm{~nm}$ would be about -28000 if the helix conrtent were as high as $80 \%$. The measured ellipticity of sonicated purple membrane patches is only -14000 , however, which would require that the $C D$ be flattened by a factor of $Q_{B}$ (notation of Gorton and Holzwarth) equal to 0.5 . This degree of flattening corresponds for the model of spherical shells to a value of $A_{\text {sol }} / q m=0.5$, where, in the notation of Gordon and Holzwarth, $A_{\text {sol }}$ is the absorbance of an ideal solution of protein and $\mathrm{qm}$ is the average number of absorbing particles per centimeter. The value of $\mathrm{qm}$ must increase as $\mathrm{N}^{1 / 3}$ where $N$ is the total number of particles per cubic centimeter. $N$, in turn, must be inversely proportional to the area of the membrane patches because the total amount of membrane area is conserved upon sonication. Taking the size of native membranes to be, conservatively, no smaller than $2500 \AA$ and the size of the sonicated membranes to be $250 \AA$ (as reported by Mao and Wallace), the value of $A_{\text {sol }} / \mathrm{qm}$ of the native membrane suspension ought to ve equal to 2.3. According to Fig. 3 of Gordon and Holzwarth, $Q_{B}$ would then be less than 0.1 for the native membranes, i.e., 5 times smaller than for the sonicated membranes. Experimentally, the molar ellipticity of native purple miembranes is hardly distinguishable from that of sonicated membranes at $221 \mathrm{~nm}$, implying that there must be very little absorption flattening in either case at that wavelength.

That membrane patches in the submicrometer size range should have very little absorption flattening is further supported by the fact that Gordon and Holzwarth give an empirical estimate of $Q_{B}=$ 0.7 for red blood cell ghosts at $221 \mathrm{~nm}$. This value corresponds to $A_{\text {sol }} / q m=0.21$. Assuming that the ghosts have a size equal to $5 \mu \mathrm{m}$, one finds that $A_{\text {sol }} / \mathbf{q m}$ should be no larger than 0.045 for vesicles that are smaller than $0.5 \mathrm{~mm}$. The corresponding value of $Q_{\text {e }}$ for submicrometer pieces of membrans is only aboul 0.9 . 
We believe the the aroments presented bove show that abyorption fatiening has no significiont efiect a $221 \mathrm{~nm}$ in any of the spectra presenied by Mao and Wallace. The data of Mao and Wallace confirm earlier estimates ${ }^{34}$ that the helix convent of bR represents only about $50 \%$ of the peptide chain. If the helix content is as little as $50 \%$, then there can only be a maximum of five transmembrane a-helices as opposed to the seven helices predicted from electron microscopy. We will not repeat here the evidence that has been presented ${ }^{4.5}$ in support of a structural model that would contain five helices and four strands of $\beta$ sheet. We believe that it is only important to stress two points: 1) the experimental CD spectra of $b R$ differ greatly in amplitude from the theoretical spectrum of a protein containing seven helices, each of which is $30 \mathrm{~A}$ or greater in length; and 2) light scattering and absorption flattening do not produce experimentily spowicant distortions of the CO spectors of native membranes. pumiculatly in the region 200-2 20 $\mathrm{nm}$, and therefore they are undble to explain the discrepancy between experimental results and the seven-helix modet.

\section{REFERENCES}

1. Mao, D., and Wallace, B.A. Biochemistry 23, 2667-2673 (1984).

2. Gordon, D.f., and Holzwarth, G. Arch. Biochem. Biophys. 14?, 481-488 (1971).

3. Becher, B., and Cassim, I.Y. Biophys. I. 16, 1183-1200 (1976).

4. Jap, B.K., Maestre, M.F., Hayward, S.B., and Glaeser, R.M. Biophys. J. 43, 81-89 (1983).

5. Glaeser, R.M., and Jap, B.K. Biophys. J. 45 , 95-97 (1983).

\section{CONTRAST IMPROVEMENT WITH SMALL SPOT ILLUMINATION FOR HIGH RESOLUTION ELECTRON MICROSCOPY OF BEAM SENSITIVE SPECIMENS}

Kenneth H. Downing and Robert M. Glaeser

The contrast observed in images of beamsensitive crystalline specimens is found to be significantly less than one would predict based on observations of electron diffraction patterns of the specimens. ${ }^{1}$ Factors such as finite coherence, inelastic scattering, and the limited MTF of the photographic emulsion account for some of the decrease in contrast. It appears, however, that most of the loss in signal is caused by motion of the specimen during exposure to the electron bean. The introduction of point and other defects in the crystal, resulting from radiation damage, causes bending and lateral motion that degrade the contrast in the image. We have therefore sought to determine whether the beam-induced specimen motion can be reduced by reducing the area of the specimen that is illuminated at any one time. Not only should the total stress be decreased in proportion to the area that is illuminated, but the constraint against motion should be increased by the smaller perimeter of the illuminated area.

Our electron microscope installation at LBL provides a unique opportunity for testin; this hypothesis. Using a field-emission-gun (FEG) illumination system, we can record images with a beam diameter of $400-2000 \AA$ while retaining the high spatial coherence required for good phase contrast. Under computer control, a series of specimen areas can be irradiated in order to expose a large fraction of the pholographic film. We have tested this scheme experimentally by recording images of the 3.7-4.2 $\AA$ lattice of monolayer crystals of paraffin $\left(\mathrm{C}_{44} \mathrm{H}_{90}\right)$. These images show contrast that is consistently higher, by factors of 2-5, than obtained with a beam of normal diameter of 1-3 $\mu \mathrm{m}$.

Figure 1 is an electron micrograph of a paraffin crystai. This micrograph was obtained by stepping a beam of diameter less than $1000 \AA$ through a 5 $X 5$ array, exposing a number of discrete areas within the crystal. The paraffin lattice is resolved in each of the exposed areas. Within the small illuminated areas, we often find a significant variation in the contrast of the lattice image, as illustrated in Fig. 2. In order to investigate this effect further, an optical bench was used to obtain optical dark-field images of large areas of electron micrographs. Figure 3 shows three independent darkfield images obtained from the micrograph in Fig. 1. Variations in intensity within each of the illuminated 


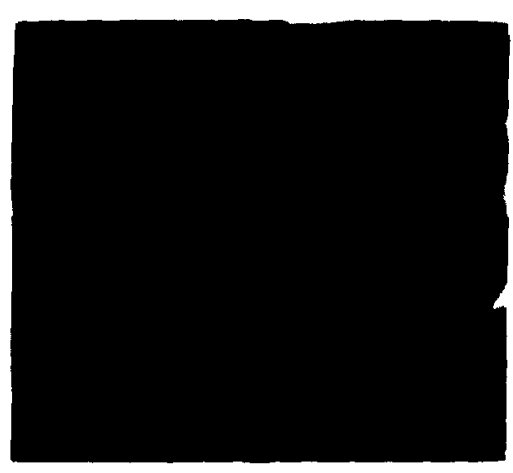

Fi. 1. Micrograph of parafin constal. Deam was stepped through $5 \times 5$ array of positions under computer controt, down vertical columns starting at left. Bar $=7000 \mathrm{~A}$. Beam shape is due to residual astigmatism of illumination system.

(XBB 853-2151A)

areas, corresponding to variations in contrast in the micrograph, are caused by changes in the angle of illumination within the small areas. This problem can be minimized by the use of small illumination apertures.

The improvement in image contrast provided by this technique should be immediately applicable in the solution of atomic structures by electron crystallography. Already, the structure of purple membrane from Halobacterium haloisium has been

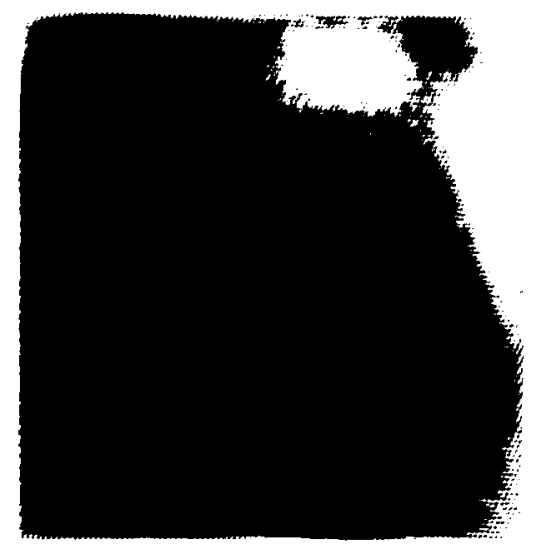

Fit. 2. Compuler-fihered image of small section of one of the images as in Fig. 1 . A $7 \times 7$ element box was masked anound each diffraction spot in the $512 \times 512$-element Fourier transform of the micrograph, and this image obtained by inverse Fourier iransform.

(XBB a50-8716)

determined in projection to a resolution of $3.5 \Lambda_{,}^{2}$ by applying advanced computational processing to conventional electron micrographs obtained at LBL, EMBL (Heidelberg), and the Fritz-Haber Institute (Berlin). The use of images recorded with the small-spot illumination system should greatly speed up the extension of this work to the determination of the three-dimensional structure.
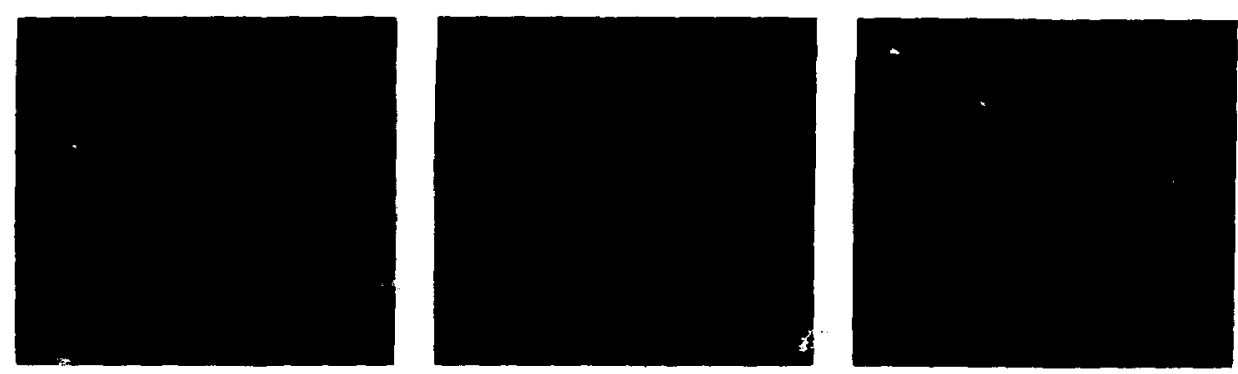

Fi. 3. Dark field images of micrograph in Fig. 1 obtained on optical bench. (a), (b), and (c) correspond to three diffraction spot pairs. (XBB 853-21518) 


\section{Merentances}

1. Henderson, R, and Cheser, R.M. Quantitative analysis of image contrast in electron micrographs of beam-sensitive coystals. Uthramicroscopy, IE, 139 (1985).

\section{SECONDARY STRUCTURE OF HALORHODOSFIN}

\section{Fing K. lap and Seok-fiwan Kong}

Halorhodopsin (hR) is one of three retinalcontaining proteins found in Halobacterium halobium. The biological function of $h R$ is to serve as a light-driven electrogenic chloride pump, transporting chloride ions inward across the membrane. Halorhodopsin has many similarities and dissimilarities when compared to bacteriorhodopsin (bR), a light-driven proton pump. Both proteins contain a retinal moiety that is apparently in a similar environment, as suggested by Raman spectroscopy, indicating that structures surrounding the retinal in both of these proteins are very similar. On SDS ge! electrophoresis, $h R$ shows a slightly greater mobility than that of $b R$, suggesting that $h R$ has a similar molecular mass (i.e., 26000 daltons). The amino acid compositions of $h R$ and $b R$ are quite similar when residues are grouped as being hydrophobic, neutral, and polar. Proteolytic fragments of $h R$ and bR are aiso similar when the proteins are partially digested with Staphylocorcus aureus V8 protease. However, $h R$ also shows distinct spectroscopic and photochemical properties. For example, in contrast to the case of $b R$, she photocycle intermediates and kinetics, as well as the posiiion of $h R$ absorption maximum, are affected by the presence of chloride ions. There is evidence that $h R$ has a distinct chloride binding site, involving a sulfhydryl group. Furthermore, $h R$ and $b R$ lack immunological crossreactivity, suggesting a significant difference in the primary sequences of $b R$ and $h R$.

We have obtained ultraviolet circular dichroism (CD) spectra of $b R$ and $h R$ solubilized in a detergent solution containing $15 \mathrm{mM}$ octylglucoside and $20 \mathrm{mM}$ sodium phosphate $(\mathrm{pH} 7)$ as shown in Fig. 1. The spectra are very similar in magnitude and in their delailed shapes, suggesting that $h R$ and $b R$ have very similar secondary structure contents. The .D specira of $h R$ in buffer containing $3 \mathrm{M} \mathrm{NaCl}$ and $20 \mathrm{mM}$ sodium phosphate $(\mathrm{pH}$ 7) were also measured to see if any conformational change was
2. Henderson, R, Baldwin, I.R, Downing, K.H., Lepack, I., Zemin, F. Structure of purple membrane from Habbacterium hrobiums: Recording, evaluation, and measunement of electron micrographs at 3.5 A resclution. Uhramicroscopy (in press 1986).

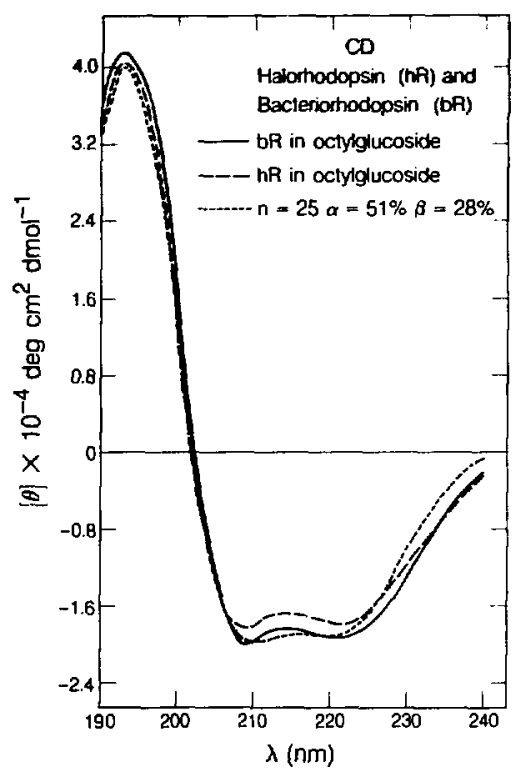

Fig. 1. The $C D$ spectra of $h R$ and $b R$ solubilized in a detergent solution containing $15 \mathrm{mM}$ octylghcoside and $20 \mathrm{mM}$ sodium phosphate $i \mathrm{pH} 7)$. The theoretical curve was obtained from a least-square best fit between the $h F$ spectrum and basis funcfions for helical chain lengths of 25 iesidues (Ref. 1). The spectra of $h R$ and $b R$ show a substantial similarity, indicating that their secondary struclural contents are similar.

(XE! 856-8341A)

induced by an increase in ionic strength. The spectra of $h R$ with and without $3 \mathrm{M} \mathrm{NaCl}$ are virtually identical, indicating that low ionic strength and the absence of chloride ions have no significant effect on the secondary structure of $h R$. Least-square curve fitting of the $C D$ spectrum of $h R$ in octylglucoside, using the basis functions of Chen et al. yieks an a helical content of $51 \%$ and $\phi$ struclure 
convent of 20\%, asuming that the helices in hR hove on swerese leneth of 25 nesidues. The theoretical curve obtinied from the lesst-square fitins is in acceptible apreement with experimental dat (Fiz. 1). Assuming that the molecular masses of hR and bR are indeed simitar, the number of helical strands in hR that can transverse across a membrane of $45 \AA$ thickness is no more than five, similar to that of $6 R .^{2}$

It is intriguing to know whether there is a simibarity in the pumping mechanism between $h R$ and $b R$, since $h R$ pumps chloride ions and $b R$ pumps protons. It is not yet known whether the chemical species being transported by $b R$ is protons $\left(\mathrm{H}^{+}\right)$, hydroxyls $\left(\mathrm{OH}^{-}\right)$, or $\mathrm{H}_{3} \mathrm{O}^{+}$, etc. If bR indeed transports hydroxyl or $\mathrm{H}_{3} \mathrm{O}^{+}$through a channel, it is then quite possible that the molecular mechanisms of operation are quite similar for $b R$ and for $h R$. The current evidence that the ultraviolet $C D$ spectra of these two proteins are very similar is consistent with--but does not prove-that the folding of these two proveins is similer. If the similarity in the three-dimensional moleculer structures of these proveins does indeed exist, these proveins may share a common mechanissm of ion transport. 11 is. therefore, important to determine she threedimensional molecular structures of hR directly by diffraction techniques. Crystallization of hR into wo-dimensional ordered arrays fo: subsequent electron crystallographic structure delermination is currently in progress.

\section{REFERENCES}

1. Chen. Y.H., Yang, T.J., and Chan, K.H. Determination of the helix and $\beta$ form of proteins in aqueous solution by circular dichroism. Biochemistry 13, 3350-3359 (1974).

2. Jap, B.K., Maestre, M.F., Hayward, S.B. and Glaeser, R.M. Peptide-chain secondary structure of bacteriorhodopsin. Biophys. 1. 43, 81-89 (1983).

\section{STRUCTURAL ANAiYSIS OF PhoE PORIN, AN OUTER MEMBRANE PROTEIN FROM ESCHERICHIA COLI}

Bing K. Jap

The outer membrane of gram-negacive bacteria such as Escherichia coli and Salmonella typhimurium generally plays at least two different roles in biological processes. It acts as a molecular sieve for hydrophilic solutes, and it serves as a part or all of the receptor for bacteriophages. The outer membrane contains 3 high density of porins, which are pore-forming proteins, that are specific for passive diffusion of small molecules. Furthermore, the closing and opening of these pores appear to be subject to biochemical regulation.

The outer membrane contains a variety of porins of similar mulecular - veight and similar amino acid composition, which nevertheless have substantially different chemical specificities for restricting free diffusion of aqueous solutes, and which show different palterns of gene expression under different rhysiological conditions. The predominant porin in wide-type $E$. coli is called OmpF. A similar member is called OmpC; it has a somewhat larger molecular weight but smaller pore diameter (as measured from diffusion studies). Under growth condition of high osmotic strength, express,on of OmpF is turned off and that of OmpC is turned on.
Another protein of the pore-forming family, PhoE porin, is preferentially expressed when cells are starved for phosphate. PhoE porins show some selectivity of the type of molecules that can diffuse through the pores.

The primary and secondary structures of a number of pore-forming proteins have some surprising features. The primary sequences show a uniform distribution of polar and hydrophobic residues along the entire peptide chain. Hydropathic profiles of the proteins show no stretches of hydrophobic segments long enough to transverse the lipid bilayer as helical rods similar to those found in many other integral membrane proteins. Even more surprising are the secondary structure data of OmpF porin, which exhibit a high content of $\beta$ structure with no evidence for $\alpha$ helical domains. Furthermore, recent results from total attenuated reflection infrared spectroscopy and from largeangle diffuse $x$-ray diffraction studies indicate that anti-parallel $\beta$-pleated strands span the membrane with a mean length of $10-12$ residues oriente approximately normal to the membrane plane. In view of this information it has become inlriguing to 
know how these proveins are folded in the molrophobic interior of the membrane, and how they are secreted across the inner membrane and then inserted into the outer membrane.

Our proteolyic digestion study of PhoE proteins indicates that there are only a small number of proreolytic sites accessible for papain digestion. Digestion of Phot with panain for 2 hours gives two new bands as observed by SDS-PAGE (Fig. 1). The apparent molecular weight of each of these bands is aboul 18,000 daltons. These results are consistent with the conclusion drawn from the studies

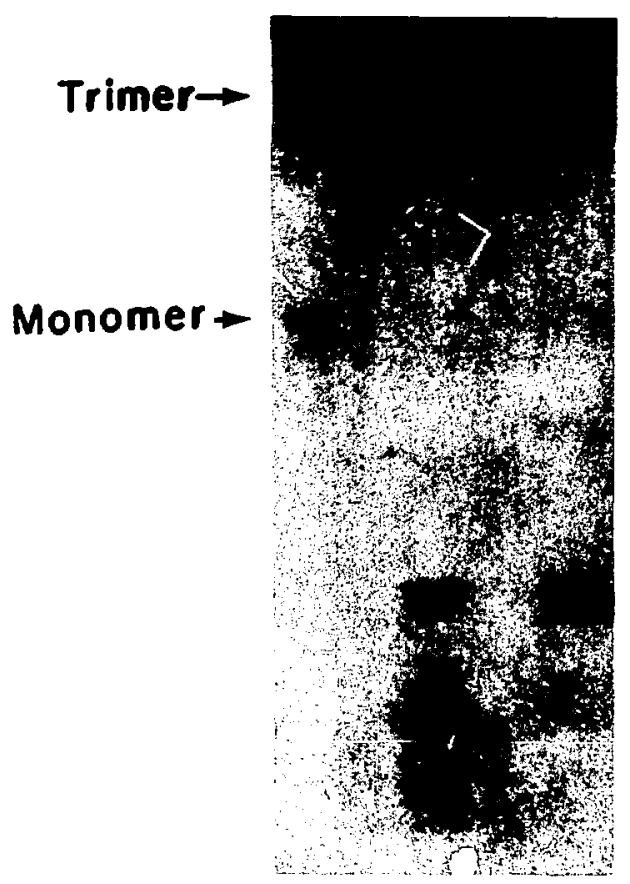

Fig. 1. Coomassie brilliant blue stained 15\% polyacrylanide/SDS gel of PhoE before and after digestion with papain. Lane 1 shows a mixture of monomer and trimer of Phot protein. Suniples of Phof were digested with papain for 2 hours and were then applied to the gel with and without boiling in the SDS sample buffer (lanes 5 and 4, respectively). Before electrophoresis, the papain was inactivated. Two bands with approximate mass of 18,000 dattons were observed when the jample was boiled in sumple buifer. When papain treatment was extended to 2 days, additional bands with apparent masses of 15.000 and 10,000 dallens appeared fitmes 3 and 2 with and without boiling, respectively).

(XBB 846-4591) of hybrid ene products, which indicale that the region locaied between rexidue 142 and 267 is surface exposed.' Prolonoed dinestion with papin (2 dxy) produces two additional bands in the cal, sugaesting that there are additional exposed siles that nevertheless are not easily accessible to papain. Interestingty, the cleaved Phot protein normally remains as 3 single unit that migates at a rate identical to that of the intact trimer in SDSPAGE. The cleaved peptides, lik ? the intact monomers, are observed only when the specimen is boiled in the sample buffer before electrophoresis. This shows the ur.usual stability of the porin trimer, indicating that the cooperative involvement of many residues may play an important role in the formation of trimers. Similar observations have also been reported for bacteriophage $\lambda$ receptor (maltoporin) by the Rochenbusch group. ${ }^{2}$

Our initial attempt to reconstitute PhoE protein from detergent solution into two-dimensional crystalline sheets was not very successful. Only a small percentage of the protein formed sheet-like patches, and the patch sizes obtained were rather small. The negatively stained images of these patches did show crystalline order as judged from their optical diffraction. However, images of the crystalline patches occasionally showed a different space group and a different lattice constant. This kind of polymorphism has also been observed for crystalline OmpF. ${ }^{3}$ Improvement over the initial attempts was made by using a slow dialysis procedure in the removal of detergent and by making sure that the proteins were completely free of lipopolysaccharide. To obtain the required level of purity in the protein, we have used an additional column-chromatography step in our purification procedure.

The reconstituted PhoE crystalline patches now have an average diameter larger then $1 \mu \mathrm{m}$. A typical image of a negatively stained PhoE patch is shown in Fig. 2 together with its optical diffractogram. The optical diffraction pattern shows a rectangular lattice, with lattice corstants, $a=72 \mathrm{~A}$ and $b=125 \AA$. The images of PhoE show that the crystals are superior to those of OmpC. The coherent crystalline order of PhoE appears to extend to the entire patch, while that of OmpC is limited to small domains. Furthermere, our PhoF crystals show a single rectangular crystal form while those of OmpC show polymorphism: "Work is currently in progress to determine the three-dimensional structure of PhoE porin by electron crystallographic techniques. 


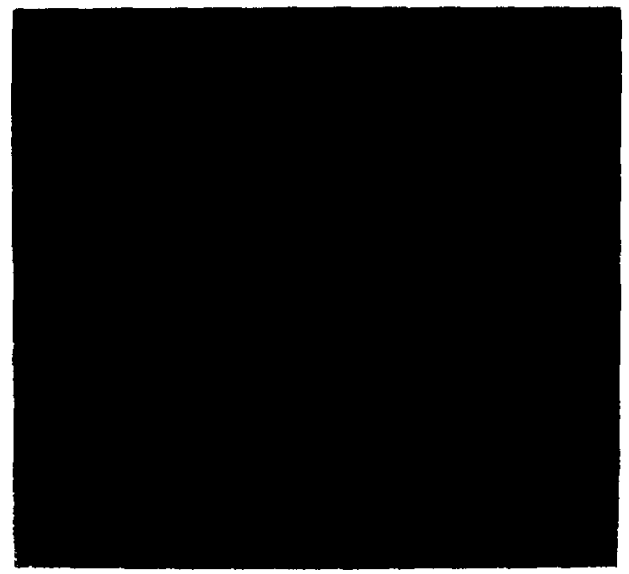

Fi. 2 A typical image of a reconstiluted PhoE porin negatively stained with uranyl acetate and its optical diffraction pattern.

(XBB 859-7383)

\section{REFERENCES}

1. Tommassen, J., van der Ley, P., van Zer, M. and Adterbers, M. Localization of functional domains in $E$. col' K-12 ovter membrane porins, EMBO /. 4, 1583-1587 (1985).

2. Schenkman, S., Tsugita, A., Schwart2, M. and Rosenbusch, J.P. Topology of phage $\lambda$ receptor protein, J. Biol. Chem. 259, 7570-7576 (1984).

3. Dorset, D.L., Engel, A., Haner, M., Massalski, A., and Rosenbusch, J.P. Two-dimensional crystal packing of matrix porin. A channel forming protein in Escherichia coli outer membranes. J. Mol. Biol. 165, 701-710 (1983).

4. Chang, C.-F., Mizushima, S., and Glaeser, R.M. Projected structure of the pore-forming OmpC protein from Escherichia coli outer membrane. Biophys. J. 47, 629-639 (1985).

\title{
LOW TEMPERATURE SCANNING ELECTRON MICROSCOPY OF FROZEN HYDRATED LUNG
}

\author{
Jacob Bastacky, Gregory R. Hook, Gregory L. Finch, and Thomas L. Hayes
}

Water makes up most of the tissue portion of the lung, the remainder of the lung being filled with air. Each cell is $50-80 \%$ water, and these cells are organized into a system of airways, that are covered with an aqueous layer. Water makes up approximately $98 \%$ of the mucus in the larger airways, and the surface lining layer of the air sacs in the small airways is hypothesized to be mostly water.

Since electron beams require a vacuum for propagation, electron microscopy has required the removal of this water from the lung prior to examination. Biolcsical samples, in general, have had to be dried for examination in the scanning electron microscope (SEM), by either air drying, treeze drying, or most commonly, critical point drying. These procedures are effective in preventing water vapor from contaminating the vacuum of the microscope and in reducing surface-tension effects, but they still cause samples to shrink. In a recent study' we found that lung lissue shrank almost $20 \%$ linear dimension with critical point drying or to about $50 \%$ of its original volume.

II is possibte, using technology develojed at LBL, to completely avoid drying the sample and to preserve the vacuum in the microscope by freezing the lung, transferring it to the microscope, and keeping it frozen at approximately $-180^{\circ} \mathrm{C}$ during examination. This reduces the vapor pressure of water to negligible levels that do not interfere with the electron beam.

There are a number of advantages to low temperature scanning electron microscope (LTSEM) analysis: 1) Fixation by freezing can be faster than the several seconds it takes for intracellular motion to stop with a chemical fixative such as glutaraldehyde. Freezing can stop action in a small fraction of a second in very thin pieces of tissue. 2) Freezing can eliminate the shrinkage of bioiogical samples associated with critical point drying $(20 \%$ linear dimension) and freeze drying (7\%). , Cells that change configuration during dehydration and drying due to elution of solvent-extractable cor. ponents (such as lipids) are better maintained by freezing since no cell constituents are lost 4) Cells such as intraluminal macrophages washed out of the lung or translocated within the lung by aqueous fixalives are preserved in silu by freezing. 5) Mucus and surfactant are preserved in situ with their native air/liquid interface. 6) Freezing allows 
inhaled particles embedded in mucus or surtactank or phapocyosed by macrophanes to be kept in place. 7) $x$-ray micramalysis can be quantialive without loss of extractable components or masking by heavy metal fixative/stains. B) Electron-beam damage to the specimen is reduced by keeping the sample cold during examination. 9) Freezing is a one-step specimen preparation process, quicker and with fewer procedural variables than the usual alternative of combined chemical fixation, dehydration, and critical point drying.

Freeze-fixation and LTSEM also have certain disadvantages: Ice crystals form during freezing. distorting cells and displacing ions; handling of samples at $-196{ }^{\circ} \mathrm{C}$ requires special tools and procedures to avoid warming and surface contamination with condensed ice; and a specialized SEM stage is required. However, these disadvantages are ameliorated in that micrometer-sized crystals do not interfere with the structural information we need at the alveolar level, procedures to avoid ice contamination are routine in cryobiology, and several LTSEM stages are now commercially available.

Since water is preserved as ice in frozenhydrated samples one can examine cells with their cytoplasm and organelles still-hydrated and the surface of airways with its aqueous blanket intact. The important interface between air and the liquid lining airways and alveoli can be imaged en face at magnifications up to $10,000 X$ in the frozenhydrated state in the LTSEM. This is the first imaging technique that has made this possible (see Fig. 1). We will use this technique to resolve the current controversy as to whether the aqueous layer in the alveoli is continuous or discontinuous. The alveoli are tiny $(1 / 4 \mathrm{~mm})$ spherical outpouchings along the last few millimeters of the airway where gas exchange orcurs. Surface-tension forces are ir.. portant determinants of respiratory mechanics at the alveolar level, and these forces are reflected by the configuration of the liquid surface of the alveoli. We hypothesize that the aqueous lining layer is continuous, and that there are no dry areas on the alveolar wall. This continuity would allow surface forces to distribute over the surface of the alveolus and this hypothesis would be consistent with the physical model of the alveolus as an expandable major portion of a sphere. The liquid layer lining the alveolus is lopped by a layer of surfuce-active maverial that, bee a detergent, lowers the surface lersion of the atveoli and thus reduces their tendency to collapse. Clinically, the liquid in the atreoli is important in a number of diseases. For example, the premature baby's inability to make surfactant causes respiratory distress syndrome, the major cause of hospitalization and death in intensive care nurseries in the United States. Major advances in therapy for this problem have been made. Current efforts to introduce artificial surfactant into the alveoli require an understanding of the normal configuration of the liquid lining the alveoli.

The major instrument used in our research studies is a scanning electron microscope with a special cold stage and a cryo-specimen preparation chamber as an integral part of the microscope. It was designed by James Pawley, of our laboratory, and John T. Norton, of the AMRay Corporation,* and was built by the AMRay Corporation. The prototype is in use in our group at the Donner Laboratory, and the instrument is now commercially available (AMRay 1000A SEM with biochamber). The cold stage operates with a joule-Thomson refrigerator that cools to $-190^{\circ} \mathrm{C}$ utilizing 1200 psi dry nitrogen gas. The stage has full $360^{\circ}$ rotation, $0-45^{\circ}$ tilt and $\pm 1{ }^{\circ} \mathrm{C}$ temperature control. The vacuum of the specimen preparation chamber attached to the microscope is continuous with that of the microscope, which allows us to fracture a clean, fresh surface and coat for electrical conduction under vacuum, and then to examine the surface with the low-magnification stereo-light microscope. All the necessary manipulations to prepare a frozen sample for microscopy can be performed within the vacuum of the microscope, and the lung can be routinely examined in the microscope in the frozen-tiydrated state.

We have demonstrated that the LTSEM can be used to study the shape of alveoli in the hydrated state, the patency of the pores of Kohn in the alveolar wall, the shrinkage and distortion of the lung with drying, the configuration of the mucus layer in the airways, and the thickness of the pleural space, all matters of import in the functioning human lung.

AMRay Corporation, 160 Middlesex Tumpike, Bedlord, MA 01730 


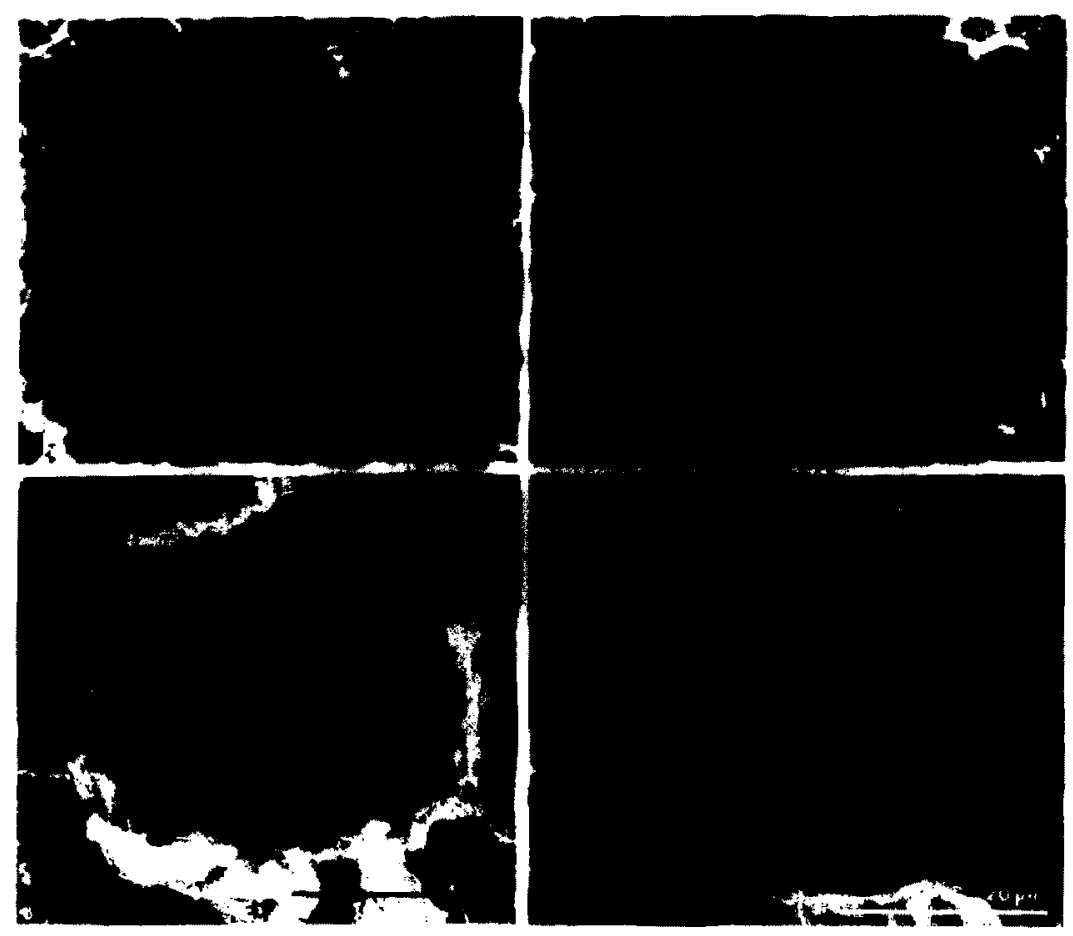

Fig. 1. Comparison of scanning electron microscopy of dried lung $(a, c)$ and low temperature scanning electron microscopy (LTSEM) of frozen-hydrated lung (b,d) from the mouse. The frozen-hydrated lung has retained all of its water as ice during photography at $-190^{\circ} \mathrm{C}$ in the LTSEM. The open pores (of Kohn) in the alveolar wall seen in conventional critical point dried lungs are not evident in alveoli frozen with their liquid in place. Note also the worm-like capillaries bulging from the surface of the critical point dried alveolus as compared to the rounded contour of the frozen-hydrated alveolus. The alveolar wall is typically folded in critical point dried lungs possibly due to the almost $20 \%$ shrinkage that accompanies critical point drying. The bar in each picture represents $20 \mu \mathrm{m}$. Variation in $x$ and y magnification of figs. $1 a$ and 16 is less than 1\%; deviation from orthogonality is also less than $1 \%$ so that alveolar shape can be directly compared in the frozen-hydrated and critical point dried states in Figs. 1 a and $i b$.

(XBB 850-1013I)

\section{REFERENCE}

1. Bastacky, I., Hayes, T.L., and Gelinas, R.P. Quantitation of shrinkage during preparation for scanning electron microscopy: huınan lung. Scanning 7, 134-i40 (1985). 
MMUNive RECOCAHTION OF MEMINANES

\section{Aron B. Kantor and fohn C. Owicki}

Recent work in our laboratory has concentrated on the molecular mechanisms of interactions involving biological surfaces. Of particular interest to us is the immune syslem, many of whose functions are mediated by specific ligand-receptor recognition interactions at the cell membrane.

One example is the binding of antibodies to antigen-bearing membranes, which can have at least two effects. First, the mere presence of bound antibodies marks the antigen-bearing cell as a candidate for destruction. Second, multivalent antibodies may cross-link the antigenic cells, which agglutination may itself inactivate the cells or may make them more susceptible to destruction by phagocytes.

Prior to this year we have primarily studied a model system for the binding of antibodies to isolated cells. We have now begun to investigate aggulination in the same model system. The rest of this report will describe the model system and some of our preliminary observations on molecular mechanisms of aggulation.

\section{MODEL SYSTEM}

The system consists of phospholipid vesicles (liposomes) rendered antigenic by the attachment of a hapten (fluorescein) and monoclonal antibodies (MABs) directed against the hapten. The possible antibody-hapten interactions are detailed schematically in Fig. 1.

The liposomes are composed of phosphatidylcholines, sometimes with cholesterol, and are 50-100 $\mathrm{nm}$ in diameter. We have synthesized' a haptenated peptidyl-phospholipid of the form fluorescein-tryglycine-phosphatidylethanolamine, which is incorporated into the lipusomes iypically at 0.1 to 1 mole \% of total phospholipid. The tripeptide spacer projects the hapten out from the membrane far enough for antibodies to bind. The MABs, murine immunoglubulin C's (lgG's) of varying isolype and affinity for hapten, have been obtained from E.W. Voss, Ir.

Fluorescein was chosen as a hapten because its brilliant fluorescence is stistantially quenched upon antibody binding. This permits the convenient Pluorometric assay of the birding kinetics and equilibrium down to subnanrmolar hapten concentrations. Since the hapten iluorescence is sensilive to its entironment, alto permil. analys:

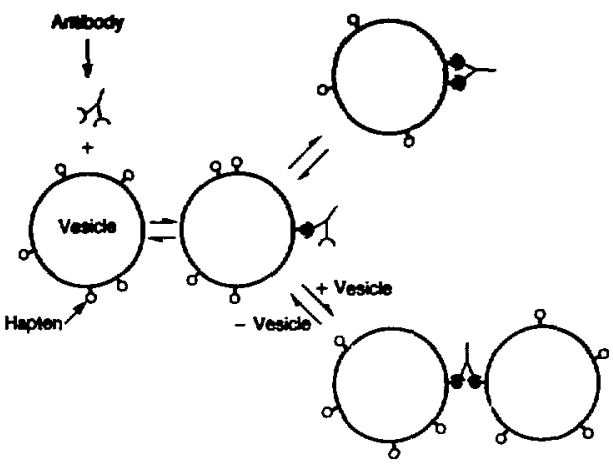

Fig. 1. Schematic representation of the interactions of bivalent antibodies with vesicles (liposomes) bearing monovalent haptens. An antibody in solution first binds monovalently to the liposome (center). Further (bivalent) binding can be either intravesicle (top right) or intervesicle (bottom right). A competition exists between the two modes of bivalent binding, and only intervesicle binding produces aggregation. (XBL 8510-8513)

of the state of the hapten prior to antibody binding, e.g., the absence of complications like haptenhapten aggregation. Previous studies have established the basic behavior of the hapten in the liposomal membrane ${ }^{2}$ and characterized the binding of antibodies to these membranes. ${ }^{3}$

\section{STUDIES OF MEMBRANE AGGREGATION}

The aggregation of particles, either by nonspecific colloidal forces or specific ligand-receptor interactions, has long been studied by biological and physical scientists. Experimental studies have been largely phenomenological, and the process is complicated enough that theorists have been forced to make greal simplifying approximations.

Our model system promises to be of fundamental use in this field for two reasons. First, the behavior of thi' components of the system is well defined, understood, and controllable. For example. the haptens diffusn freely on the liposomal surface and their lateral density is readily controlled and varied. Second, by monitoring fluorescence quenching, it is pissible to measure the number of ligand-receptor bonds as well as the amount of agsregation.

Figure 2 shows the results of an initial experir.ent on the ability of different concentrations of 


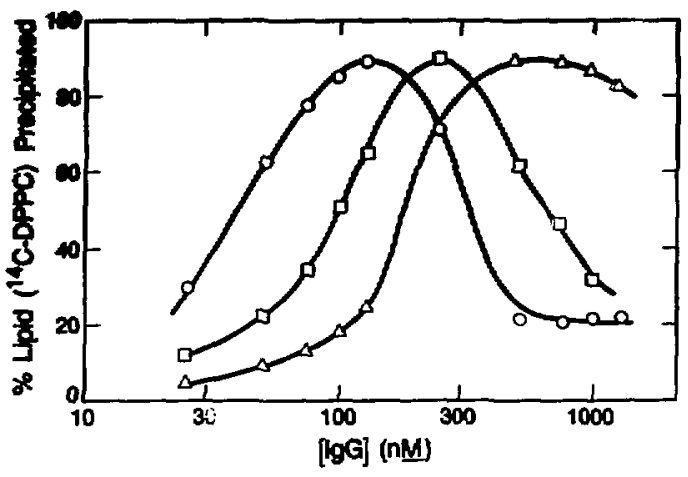

Fis. 2. Precipitation of antigenic liposomes by varying concentrations of antibody, MAB 6-19-1 ( $\left./ g G_{1}\right)$. The liposomes were labeled with ${ }^{14} \mathrm{C}$-phospholipid and were centrifugally precipitated $(12,000 \times \mathrm{g}, 5 \mathrm{~min})$ after two hours of incubation with antibodies at $25^{\circ} \mathrm{C}$. The bulk lipid concentration of the liposomes was dipalmitoyl-phosphatidylcholine: cholesterol $2: 1$ on a molar basis. The three sets of data represent different mole fractions of hapten-bearing phospholipid in the liposomes: circles, 2\%; squares, 1\%; triangles, $0.5 \%$. The three-dimensional concentration of hapten was the same (1670 nM) in all cases, so the concentration of liposomes was highest in the $0.5 \%$ har,ten preparation. The observed biphasic response resembles the classical immunological precipitin reaction.

(XBL 8510-8511)

antibodies to precipitate vesicles. Results are given for three vesicle preparatior $s$ with different lateral densities of hapten. In sach case there is an optimum concentration of antibody: too little, and not enough is bound to cross-link; too much, and not enough free hapten is left to permit cross-links when antibody-coated liposomes encounter one another. This picture is qualitatively in accord with the classical precipitin reaction.

Figure 3 demonstrates that antibodies differ in their abilities to aggregate antigenic structures. Analysis of antibody binding to the vesicles (by fluorescence quenching) suggests that this does not reflect differences in antibody binding. The more efficient aggregator, MAB 6-19-1 $\left(\mathrm{lg}_{1}\right)$, in this case has a lower affinity for hapten than the other anti-

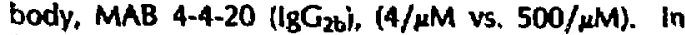
fact, we generally observe surprisingly litle correlation of aggregating ability with affinity. We believe that the variability in aggregation refects differences in the competition between intra-and interliposome bivalent binding (see Fig. 1). These difference in Ium must refiect differences in properties such as the conformational ilexibility and the

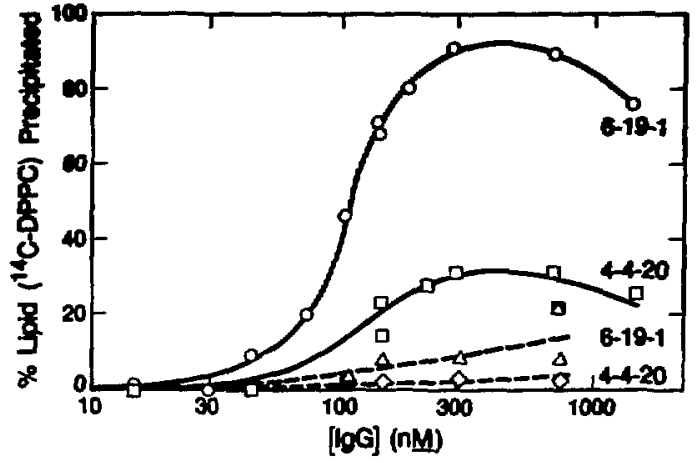

Fig. 3. Differences in the ability of two monoclonal antibodies to precipitate liposomes under conditions similar to those in Fig. 2, but with a 10 -min centrifugation. The two antibodies are $M A B$ 6-19-1 $\left(l_{g} G_{1}\right)$ and 4-4-20 $\left(l_{g} G_{2 b}\right)$. Solid curves, 1 mole 96 hapten; dashed curves, 0.1 mole \%; hapten concentration 1670 $n M$ in both cases. See text for a discussion of the differences between the behavior of the antibodies.

(XBL 8510-8512)

geometric disposition of hapten binding sites on the IgG molecules.

We are presently pursuing the molecular mechanisms involved in the aggregation phenomena.

\section{ACKNOWLEDGEMENTS}

We gratefully acknowiedge useful discussions with M. Carlson, K.-D. Lee, A. Petrossian, and S. Stanton. A.B.K. is an N.I.H. Piedoctoral Trainee. This research was supported by N.I.í. grant Al19605 to J.C.O.

\section{REFERENCES}

1. Petrossian, A., Kantor, A.B., and Owicki, I.C. Synthesis and characterization of a highly fluorescent peptidyl-phosphatidylethanolamine. I. Lipid Res. 26, 767-773 (1985).

2. Stanton, S.G., Kantor, A.B., Petrossian, A., and Owicki, J.C. Location and dynamics of a memirane-bound fluorescent hapten: a spectroscopic study. Biochim. Biophys. Acta 776, 228-236 (1984).

3. Petrossian, A., arnd Owicki, J.C. Interaction of antibodies with, liposomes bearing fluorescent haptens. Bicchim. Biophys. Acta 776. 212-227 (1984). 


\section{SECTION 6. CELLULAR AND MOLECULAR BIOLOGY}

\section{INTRODUCTION}

The major research emphasis in the Cellular and Molecular Biology Group continues to be the study of all aspects of carcinogenesis and mutagenesis. An important development during the past year has been the initiation of a more integrated, multi-investigator approach to fundamental research in cancer biology at the biochemical, molecular, and cellular levels. The Cellular and Molecular Biology Group consists of individual scientists with related research interests: increasing our understanding of carcinogen action by examining DNA damage, repair, and recombination events, cellular transformation, and gene expression in cancerous and normal cells. This multidisciplinary approach encompasses use of a wide variety of experimental systems (purified enzymes, bacteriophage, bacteria, yeast, mammalian, and human cells) and of experimental techniques (genetics, protein chemistry, molecular biology, immunology, biochemistry, and tissue culture). The presence of this depth and breadth of background, when focused on a particular area of investigation (such as carcinogenesis and mutagenesis), can provide a unique integrated and coordinated assault on a research problem. Such a focused approach is particularly appropriate for a national laboratory that can bring together scientists from several disciplines to work interdependently on a common project. Our goal is to take advantage of the multidisciplinary nature of our group by fostering specific collaborations among the members. The development of a deliberately organized interactive network of investigators should produce research of nigher quality and greater imagination because the pooled expertise will facilitate transfer of information and techriques among the different exp rimental systems.

The increased understanding of carcinogen action, which is the central question of the research of this group. is an important aspect of the energyrelated research supported by DOE at LBL. The by-products of energy production, e.g., radiation and chemical pollutants, have serious biomedical implications, one of which is the initiation. promo. tion, and progtession of cancer, A multi-investigalor pposaxh is purticularly viluable for a disedsu like

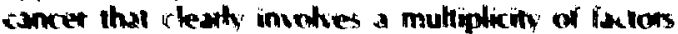
related to numeious uteas on scretace. Funthermore. these factors act in a series of interlocking stages that are not definable in terms of a single scientific discipline.

The response of the Cellular and Molecular Biology Group to the multidisciplinary aspects of cancer research is to set up interacting groups that do not necessarily conform to traditional scientific disciplines but are appropriate for studying specific cellular and molecular aspects of cancer. This approach has evolved the following four collaborative groups: DNA Replication/Recombination, DNA Repair, Carcinogenesis, and Differentiation. The descriptions of current research are presented in the order of the four collaborative groupings, except that each investigator's contributions are presented together to retain the integrity of individual programs. Because most investigators have research projects and/or collaborations in more than one subgoup, group barriers melt in this presentation, leaving a continuum of research programs from the more molecular to the more cellular aspects of carcinogenesis/mutagenesis. This is as it should be in integrated, multi-investigator research efforts. These reports are by no means comprehensive of all the research going on in the group but act as samples of current investigative approaches.

In the area of DNA replication and recombination, Junko Hosoda has utilized a phage system for isolation and investigation of DNA binding proteins. Here, she and her coworkers use mutations in two specific phage genes to clearly demonstrate the importance of protein-protein interactions in DNA replication. By collaborations with Michael Esposito, Hosoda has expanded her studies to include eukaryotic systems, namely yeast. In their initial studies, they have used a clever double column technique 10 resolve several DNA binding proteins fron a series of yeast mutants. These proteins have specific demonstrable enzymatic activity. Libby Holbrook, a posidoctoral scholar in Esposito's group, is responsible for characterizing cae of these enzymes, a resolvase. The Esposito/Hosoda collaboration is clearty making sood progress loward their staled god of last year: to use purified pro. leins to reconstifute recombination and replicition ststems in atto from defined components. Dimi.

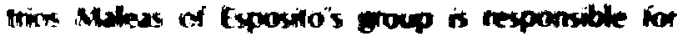


chasacterization of specific recombination defective mutants of yeast. These basic genetic studies will continue to yield new grist for the Hosoda protein purification mill. Robert Mortimer's group is also involved in DNA studies in yeast. They describe here their compilation of established genetic maps for yeast and a genealogy of yeast strains currently in use. Keeping such a compilation up to date and accurate is absolutely essential to high quality research in yeast systems. Mortimer also maintīins a Yeast Genetic Stock Center as a service to investigators throughout the world.

In the area of DNA repair, Mortimer and his associates have been using clones of specific genes involved in DNA repair to test whether expression of these genes is inducible by DNA damage. Recently, they have applied a new technique (orthogonal field alternating gel electrophoresis) for isolating large strands of DNA, including whole chromosomes, to DNA repair studies. This technique has a tremendous potential in conjunction with specific yeast mutants for investigating DNA damage and genes involved in DNA repair. Priscilla Cooper has used $E$. coli as a model system to study the DNA repair processes that promote survival. Cooper has developed the hypothesis that damage in the vicinity of the DNA replication fork poses a specin problem but repair-competent cells can effectively repair such lesions. To test this hypothesis she and Vincent Ling developed a twodimensional gel system to isolate replication forks from the main body of DNA. The success of this approach has been verified by transmission electron microscopy. The stage is now set for the testing of her hypothesis. Tony Leadon, who joined LBL and our group only a few months ago, is concentrating his research attention on repair of specific DNA sequences in mammalian cells. This overall experimental strategy is to simplify the analysis by using defined DNA sequences and specific, sensitive probes for DNA damage so that the processing of damage can be anal;zed at the molecular level. In conjunction, he has developed several immunoz;says for measuring DNA damage and repair that employ monoclonal antibodies to specific base modifications. Using these methodologies in primate cells in cullure he has bien able to measure DNA damage and its repair withill specific sequences. He has iound that DNA damage is preferentially repaires is the integraled and actively Iranscribed chime ". plasmid, pSV2-gpt. compared with the bulk oi the genome while tepair is delicient in the heletuclsomatic alpha DNA sequences in the same monker cells. He is evlending these

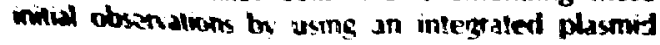

that contains an inducible promoter so that nepair can be sludied in " "zme gene when it is expressed at a basal nd afier induction. He is also examining . repair of damage in endogenous genes to determine to what extent the efficiency of repair is dependent on the transcriptional activity of the gene. We know that Tony Leadon will be an asset to the Division and his avidity for collaboration will promote the interactions desired by the group members.

In the area of carcinogenesis, the name of Bea Singer is well known on the Berkeley campus. She joined our group last spring and continues her fine work on chemically defining all of the modifications in DNA resulting from direct-acting carcinogens. Her current interest is the mechanism(s) for repairspecific DNA lesions and the importance of each lesion in mutagenesis. Goth-Goldstein reports on studies with direct-acting carcinogens in cells deficient or proficient in repair of DNA damage. Her goal is to investigate at the genetic level the mechanism(s) for induction of resistance to DNA damage. Although studies at the level of DNA are of paramount importance in understanding how an inheritable change in DNA results in cancer, another important aspect is attempting to evaluate the potential health risk of environmental carcinogens to the population as a whole. To this end Lois Gold. Bruce Ames and their associates have developed a numerical index of carcinogenis potency and application to an ever increasing data base has, to date, verified its value in risk assessment.

Carcinogenesis, of course, is more than modification of DNA. It has many stages; for example, initiation ind promotion, all with many facets. Experimel.,s by Mina Bissell, in conjunction with Anthony Howlett and Bets?y Cullen, demonstrate that viral infection, the first step in virally induced transformation, is dependent upon the developmental stage of the host cell. Using the sars: viral system, Bissell and her coworkers ha'e provocative evidence that the promotional stages of viral carcinogenesis, whether induced by wounding or chemical promoters, all involve inflammatory reactions.

As indicated by Bissell's first report and many to follow, there is an increasing interest in the relaiionship between normal cellular differentiation and developm $=n t$ and carcinogenesis. In erder to understand how aberrations in: modulation of differentiation it into the carcinogenic fuzzle, we must first define how normal differentiation is regu. lated in a given cell npe. Bissell and associate present their nork in this regaro in the murine mamman eputhelial cell. From thes nork examin. 
ing expression of genes related to milk proteins and to a specific protein, transferrin, it is clear the extracellular matrix composition and cell shape are factors in normal modulation of differentiation. The investigation of transferrin expression may have special significance in that this protein has been related to one of the proto-oncogenes. Collagen is the differentiated product of fibroblasts, the cells being studied by Richard Schwarz and Nancy Owens with respect to differentiation and the carcinogenic process. Their research has provided evidence for a unique means of controlling cellular activity. By an as yet incompletely understood mechanism, collagen gene expression is modulated by secretion of the collagen molecule. In efforts directed toward understanding the relationship between differentiation and carcinogenesis in the human mammary epithelial cells, Gordon Parry's group has developed a series of monoclonal antibodies to cell surface proteins. They are using these antibodies to address such questions as their role in cell polarity, their expression in malignancy and the implications of this expression in tumor biology and clinical medicine. This same approach is now being applied to the normal, tumor-derived and transformed human mammary cells being grown by
Martha Stampfer. Stampfer's group reported last year on the first successful attempt to induce transformation in human epithelial cells in culture with a chemical carcinogen. This year Stampfer and Bartley describe distinct differences in growth control in normal and transformed cells. In conjunction with Gerri Levine, these investigators present their efforts to define modulation of differentiation in human mammary epithelial cell. The results of these studies will provide a basis for dissecting the role of perturbations of differentiation in the development of transformed and malignant cells. The final paper from our group presents Glenn Hall's efforts to apply a molecular approach to identify subspecies of bees. Such identification is basic to setting up an effective eradication program for the impending invasion of African bees.

The Cellular and Molecular Biology Group has had a scientifically fruitful year. Collaborations and interactions have been formalized and valuable new associates have been assimilated. The group members have recognized and acted upon the unique contribution that program development possible in a national laboratory system can make in the field of carcinogenesis and mutagenesis.

\title{
BACTERIOPHAGE T4 GENE 32 PROTEIN AFFINITY COLUMN CHROMATOGRAPHY
}

\author{
Junko Hosoda, Herbert W. Moise, Maren Bell, and Midori Hosobuchi
}

We have previously shown that a large number of T4 early proteins bind to columns made from gene-32 protein covalently bound to agarose. This protein-protein interaction, for a number of proteins that are involved with replication-recombination, involves the $A$ region (a 51-residue stretch of the peptide chain including the carboxy terminus) of gene-32 protein. Experiments done with ame1, an amber peplide of gene-32 prolein lacking 15-20 residues from the $A$ region, have demonstrated that most of the proteins that had affinity for the gene32 protein columns also had affinily for the ame1 columns. In some cases the binding aifinity was reduced to the ariel column; in other cases, e.g.. gene-61 protein, the binding aifinity remained the same. The gene-32 amel mutant, ischated by $T$. Minagawa irom a pseudo reverlant of $\$ 9 \mathrm{isC9}$, is nol a kethal mulation. When grown in E. coli $\mathbf{B}^{1}$, it has a slightly reduced burst size, incheased un. sensitivity: and a slightlly reduced recombimalion efficiency when compared to wild-type phage (T4D). These defects are more pronounced when ame1 is grown in E. coli B40 su1 hosts (Table 1).

We found, however, that when a mutation in gene 61 (amE219) was added to gene-32 ame1 and the double mutant was grown in a sul host, the combined mutations become lethal-the relative plating efficiency dropped to $<3.7 \times 10^{-3}$ as shown in Table 1 . The gene-61 product is an RNA primase essential for slarting DNA replication in the reverse direction from the replication fork movement. Figure 1 shows that the rate of DNA synthesis in E. coli B40 sul infected with the double mutant was greatly reduced when compared to iniection with the single mutant in Bene 61, which showed the normal rate of DNA synthesis, or to infection with gene-32 amel, which showed a slightly reduced rale.

To determine if thene was an alteralion in the inferaction between amel and gene-61 protein in 
Table $\mathrm{r}$.

\begin{tabular}{|c|c|c|c|}
\hline Host & Phage & $\begin{array}{c}\text { Relative plations } \\
\text { efficiency }\end{array}$ & Burs! size \\
\hline$B^{E}$ & $a m^{+}$ & & 172.0 \\
\hline B40sul & $\mathrm{am}^{+}$ & 1.00 & 180.0 \\
\hline$B^{E}$ & 32 ame1 & & 37.2 \\
\hline B40sut & 32 ame1 & 0.45 & 10.8 \\
\hline $\mathbf{B}^{\mathbf{E}}$ & $61 \mathrm{amE} 219 \times 2$ & & 2.8 \\
\hline B40sul & $61 \mathrm{amE} 219$ & 1.00 & 36.0 \\
\hline $\mathbf{B}^{E}$ & 32 ame -61 amE219 $\times 2$ & & 2.0 \\
\hline B40su 1 & 32 ame1-61 amE219 $\times 2$ & $>3.7 \times 10^{-3}$ & 0.0 \\
\hline
\end{tabular}

the sul suppressed cells, columns were prepared by covalently coupling either ame $1 / B^{E}$ (ame1 grown in $E$. coli $\mathrm{B}^{\mathrm{E}}$ ) or ame1/su1 (ame1 grown in $E$. coli B40 su1) to agarose. ${ }^{14} \mathrm{C}$-labeled extracts from either T4D or 61 amE219 grown in B40 su1 were applied to the columns in buffer containing $0.05 \mathrm{M}$ $\mathrm{NaCl}$ and $0.01 \mathrm{M} \mathrm{MgCl}$. Bound proteins were eluted with $\mathrm{NaCl}$; and fractions were analyzed by two-dimensional gel electrophoresis. Gene-61 pro-

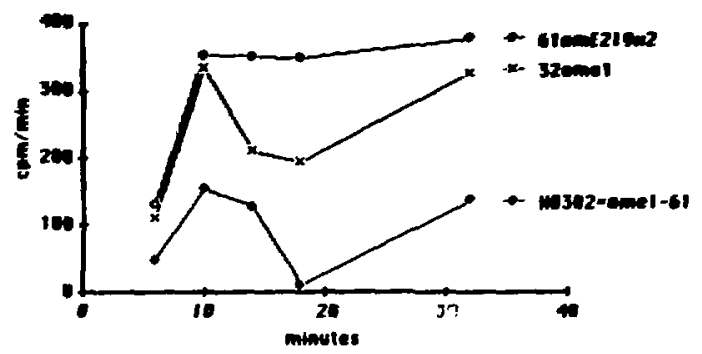

Fig. 1. TdR incorporation rates in E. Coli..40/suI.

(XBL 8511-8571)

tein from $T 4 D$ eluted from the ame $1 / B^{E}$ and ame1/su1 columns at $0.6 \mathrm{M} \mathrm{NaCl}$, which is the same concentration needed to elute gene-61 protein from a gene-32 protein column. From the ame1/sul column the T4D gene-61 protein eluted with $0.6 \mathrm{M} \mathrm{NaCl}$. However, the gent-61 protein from 61 amE219 did not elute with an $\mathrm{NaCl}$ concentration tried (including $2.0 \mathrm{M}$ ). $T_{i}$,; suggesis that the lethality and inhibition of DNA :eplication seen with the suppressed double mutant is caused by the irreversible binding of gene- 61 protein/su1 to ame1.

\section{FRACTIONATION OF DNA METABOLIC PROTEINS OF SACCHAROMYCES CEREVISIAE BY DNA CELLULOSE CHROMATOGRAPHY: SSB-1, SS-DNA DEPENDENT ATPASE, DNA POLYMERASE, DNA PRIMASE, TOPOISOMERASE I, AND RESOLVASE}

Junko Hosoda, Libby L. Holbrook, Herbert W. Moise, Kathleen A. Bjornstad, Dimitrios T. Maleas, Midori Hosobuchi, Maren sell, and Michael S. Esposito

We are continuing a collaborative program to characterize the DNA binding proteins of wild-type yeast strains and mutants exhibiting defects in DNA repair and recombination. Last year we analysed the single stranded DNA (SS-DNA) cellulose binding proteins of a recombination proficient yeast strain (LBLI) and cnisogenic rec mutants derived from it, and demonstrated by mo-dimensional get electiophoresis and fluorography that several rec mulants exhibit deficits in discrete DNA binding proleins." In the present study we have employed a double stranded DNA (OS.DNA) celiulose column coupled to a SS-DNA cellubose column to separate the bindine prokeins that have affinity bo double stranded DNh from those specific for sinule shanded DNh. The upper DS.DNA celmulose column and lower SS-DNA cellulose column were connected in series. Proteins which have relatively strong affinity for DS-DNA remain on the DS-DNA cellulose column. Those which have litlle or no affinily for DS-DNA, but bind to SSDNA, are collected on the SS-ONA cellulose column. The two columns were separated and proteins bound to each column were eluted employing a $\mathrm{NaCl} g$ ra. dient. Our aim was to determine whether DNA cellulose columns would, in fact, bind a variety of DNA metabolic activities and achieve sufficient fiactionation of them to warrant use of DNA collu. bose column chromatospatyy as a method for screening mulanks for enzyimatic detects. To thi'

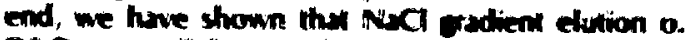
OSDNA cellulose columns incetionstet single 
stranded DNA dependent ATPase and topoisomerase I, that $\mathrm{NaCl}$ gradient elution of 55 DNA cellulose columns fractionates DNA polymerase, primase and SSB-1, and that a previously unreported resolvase activity of mitotic yeast cells binds to both DS- and SS-ONA cellulose columns. The SSB-1 protein was further purified to near homogeneity.

\section{DNA CELLULOSE CHROMATOGRAPHY}

For this study we have employed a haploid strain BJ2168 (MATa prb1 pep4 prcl. constructed by Dr. Elizabeth $W$. Jones of Carnegie-Mellon University) that exhibits minimal proteolytic activity due to mutations in the genes governing proteinase B, proteinase $C$, and processing of precursors of vacuolar hydrolases. The use of this strain together with inhibitors of proteolytic enzymes minimizes degradation of DNA metabolic enzymes and proteins by endogenous proteases. Proteins were extracted by methods similar to those of LaBonne and Dumas ${ }^{2}$ and applied to a DS-DNA cellulose column connected in series to a denatured SS-DNA cellulose column. The columns were uncoupled and eluted separately. One-dimensional sodium dodecyl sulfate polyacrylamide gradient gel electrophoresis (1D SDS-PAGE) was employed to identify fractions from the SS-DNA cellulose column containing the yeast single stranded DNA specific binding protein SSB-1, which was then purified to near homogeneity by hydroxylapatite chromatography. The remaining fractions from DS- and SS-DNA cellulose columns were pooled, concentrated, and assayed for single stranded DNA dependent ATPase, DNA polymerase, DIVA primase, resolvase, and topoisomerase I activities.

\section{SS-DNA DEPENDENT ATPASE ACTIVITY}

Enzymes and proteins exhibiting SS-DNA dependent ATPase activity comprise a large group of species that play key roles in DNA replication, reyair, and recombination. Column fractions were sulveycd for both SS-DNA dependent and SS-DNA independent ATPase aclivilies (Fig. 1). Fraction ds. 5 (cluted with $0.32-0.34 \mathrm{M} \mathrm{NaCl}$ of the DS-DNA cellulose column exhibited the highest level of $5 S$. DNA dependent ATPase activity. DS-DNA cellulose column fractions exhibiled litte SS-DNA independent ATPase axtivily. By contrast, iractions ss-2 (eluled with 0.17-0.19 M NaCl) and 55.6 (etured with 0.30-0.33 Mi) from the SS-DNA celluhose column contuined high levets of SS-DNA inciependent AIPase axtruily.

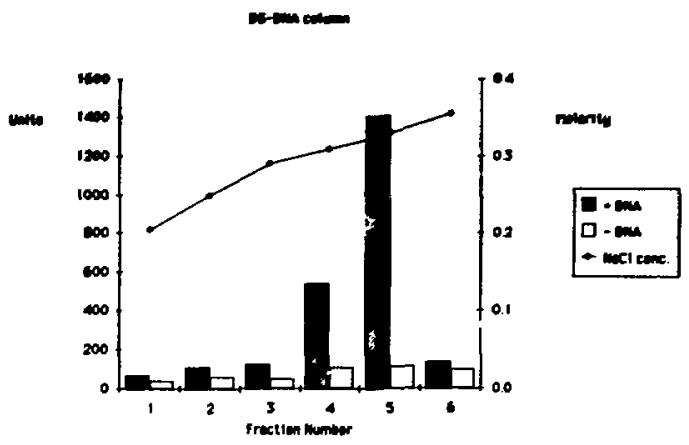

Fi. 1. SS-DNA dependent ATPase and SS-DNA independent ATPase activities of DS-DNA cellulose pooled column fractions. The ATPase activity was monitored by the conversion of $\left[^{3} \mathrm{H}\right.$ $A T P(30,000 \mathrm{cpm} / \mathrm{nmole})$ into $\left[{ }^{3} \mathrm{H} \mid-A D P\right.$ which were separated by thin laver chromatography on Cellulose PEI with $1 \mathrm{M}$ formic acid and $0.5 \mathrm{M} \mathrm{LiCl}$. One unit of activity corresponds to the conversion of $1 \mathrm{nM}$ ATP into ADP in 45 minutes at $37^{\circ} \mathrm{C}$. To assay ATPase in the presence of DNA, $10 \mu \mathrm{g} / \mathrm{ml}$ of fd-viraf DNA was added.

(XBL 8510-4462)

DNA POLYMERASE ACTIVITY AND DNA PRIMASE ACTIVITY

Saccharomyces cerevisiae has at least two DNA polymerases, I and II. DNA polymerase I is a true DNA replicase representing about $90 \%$ of the tutal activity present in exponential phase yeast cells ${ }^{3}$ while DNA polymerase II, a minor activity, exhibits proofreading 3 '-exonuclease activity thus resembling prokaryotic DNA polymerases. We surveyed fractions from both the DS-DNA cellulose and SSDNA cellulose columns for polymerase activity employing both activated calf thymus DNA and poly(dA-dT)/poly(dA-dT) as primer-templates. We did not observe significant levels of activity in fractions from the DS-DNA cellulose column. How. ever, pooled fractions ss-3 and ss-6 from the SSDNA cellulose column exhibited peak levels of DNA polymerase activity (Fig. 2). The observed synthesis is due to DNA polymerase activity and nol terminal transferase activity.

A DNA primase activity copurifies wilh DNA polymerase I activily in a siructural complex that may partially dissociate during DNA-cellulose chromatography." We therefore assayed fractions 55.2 and 55.6 for DNA primase activity using the single-slranded closed circular fd-DNA as template in the presence ard absence of a ribonucleotide triphosphate mixture (DNA polymetase cannot utilize closed circular single stranded M-DNA as a kem. plble for DNA synithesis in the absence of primase acinity or nibonucleotide triphosphates). The 


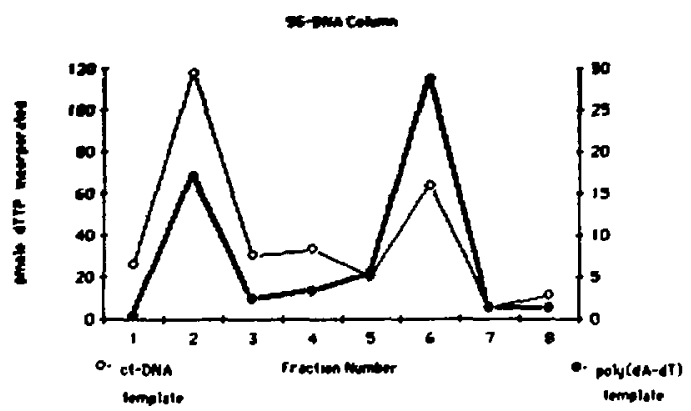

Fit. 2. DNA polymerase activity of pooled S5-DNA cellulose column fractions. The polymerase reaction was monitored by incorporation of $\left.\right|^{3} H$-dTTP into acid precipitable material. Either $150 \mathrm{\mu g} / \mathrm{ml}$ of activated calf thymus DNA or $0.5 A_{2 \text { conm }}$ unit $/ \mathrm{ml}$ of poly $(d A-d T) / p o l y(d A-d T)$ was used as a template.

(XBL 8510-4463)

results shown in Table 1 demonstrate that fraction ss-2 contains DNA primase activity.

\section{SINGLE STRANDED DNA SPECIFIC DNA BINDING PROTEINS}

Several investigators have reported the isolation of single stranded DNA specific DNA binding proteins of Saccharomyces cerevisiae that stimulate in vitro DNA synthesis by homologous DNA polymerase I. We followed the SSB-1 isolation procedure of LaBonne and Dumas ${ }^{2}$ because it yielded the largest protein. Two-dimensional gel electrophoresis showed that our SSB-1 protein is an acidic protein (pl $5.5 \pm 0.2$ ) with a molecular weight of 40,000 daltons, which agrees with the value given by LaBonne and Dumas. ${ }^{2}$

Preliminary experiments indicate that our SSB-1 preparation stimulates DNA polymerase activities in both the ss-2 and ss-6 fractions, but the optimal SSB-1/DNA ratio is apparently different for each. The activity in the ss-2 fraction, which contains primase activity, requires a higher SSB-1/DNA ratio than the one in the ss- 6 fraction (Table 2).

\begin{tabular}{|c|c|c|c|c|}
\hline $\begin{array}{l}\text { Fraction } \\
\text { number }\end{array}$ & $\begin{array}{l}\text { INTP's } \\
\text { Present + } \\
\text { Absent - }\end{array}$ & $\begin{array}{c}\text { Time of } \\
\text { incubation } \\
\text { (min) }\end{array}$ & $\begin{array}{l}\text { Percent of } \\
\text { template } \\
\text { replicated }\end{array}$ & $\begin{array}{l}\text { Petcent of } \\
\text { total replication } \\
\text { dependent upon } \\
\text { primase aclivity }\end{array}$ \\
\hline \multirow[t]{3}{*}{$s s-2$} & + & 5 & 1.7 & 88.2 \\
\hline & + & 15 & 8.4 & 85.7 \\
\hline & + & 30 & 14.1 & 80.2 \\
\hline \multirow[t]{3}{*}{$5 s-2$} & - & 5 & 0.2 & \\
\hline & - & 15 & 1.2 & \\
\hline & - & 30 & 2.8 & \\
\hline \multirow[t]{3}{*}{ ss-6 } & + & 5 & 0.2 & 0.0 \\
\hline & + & 15 & 0.6 & 0.0 \\
\hline & + & 30 & 0.4 & 0.0 \\
\hline \multirow[t]{3}{*}{ ss-6 } & - & 5 & 0.1 & \\
\hline & - & 15 & 0.6 & \\
\hline & - & 30 & 0.9 & \\
\hline
\end{tabular}

'The primase assay conditions were essentially the same as the polymerase assay except that fd-viral DNA $(5 \mu \mathrm{g} / \mathrm{ml})$ was used as the template in place of activated calf-thymus DNA. The assay was done in pairs: one member received $200 \mu \mathrm{M}$ each of CTP, GTP and UTP (rNPTs) and the other received none.

\section{RESOLVASE AND TOPOISOMERASE | ACTIVITIES}

The fractions obtained from chromatography of strain B/2168 on DS- and SS-DNA cellulose were also assayed for resolvase and topoisomerase 1 activities using the p8R322 dimer as a substrate (see next report by L.L. Holbrook et al.). Topoisomerase $I$ binds to DS-DNA cellulose and elutes in fractions ds-4, ds-5, and ds-6 (0.3- $0.37 \mathrm{M}$ $\mathrm{NaCl}$ ) and fractions ss-2 to $55-8(0.17-0.38 \mathrm{M} \mathrm{VaCl})$ and to a lesser degree ds-2 and ds-3 $(0.22-0.30 \mathrm{M}$ $\mathrm{NaCi}$, contain an activity that cleaves the supercoiled dimer, to linear dimer, and therefore this activity can be identified as the resolvase. A restriction-type reaction that is sequence-dependent and would give linear monomer products is not observed. (See L.l. Holbrook et al., next report.) 
Table 2. Stimulation of DNA polymerase activity by SSB-1:

\begin{tabular}{|c|c|c|c|c|c|}
\hline $\begin{array}{l}\text { Pooled } \\
\text { fraction } \\
\text { number }\end{array}$ & $\begin{array}{c}\text { SSB-1 } \\
\text { concentration } \\
\mu \mathrm{g} / \mathrm{ml}\end{array}$ & $\begin{array}{c}\text { SSB-1/DNA } \\
\text { ratio } \\
(w / w)\end{array}$ & $\begin{array}{c}\text { Incubation } \\
\text { time } \\
\text { (min) }\end{array}$ & $\begin{array}{c}\text { c.p.m. } \\
\text { incorporated } \\
\text { per } 8 \mu l\end{array}$ & $\begin{array}{c}\text { Percent } \\
\text { Stimulation } \\
\text { by SSB-1 }\end{array}$ \\
\hline \multirow[t]{4}{*}{ ss-2 } & 0.0 & 0.0 & $\begin{array}{l}15 \\
30\end{array}$ & $\begin{array}{l}165 \\
519\end{array}$ & \\
\hline & 5.1 & 0.5 & $\begin{array}{l}15 \\
30\end{array}$ & $\begin{array}{l}177 \\
741\end{array}$ & $\begin{array}{r}7.6 \\
42.8\end{array}$ \\
\hline & 10.2 & 1.0 & $\begin{array}{l}15 \\
30\end{array}$ & $\begin{array}{l}228 \\
717\end{array}$ & $\begin{array}{l}38.6 \\
38.3\end{array}$ \\
\hline & 20.4 & 2.0 & $\begin{array}{l}15 \\
30\end{array}$ & $\begin{array}{l}318 \\
933\end{array}$ & $\begin{array}{l}93.3 \\
80.0\end{array}$ \\
\hline \multirow[t]{4}{*}{$55-6$} & 0.00 & 0.00 & $\begin{array}{l}15 \\
30\end{array}$ & $\begin{array}{r}847 \\
2063\end{array}$ & \\
\hline & 5.1 & 0.5 & $\begin{array}{l}15 \\
30\end{array}$ & $\begin{array}{l}1422 \\
3877\end{array}$ & $\begin{array}{l}67.8 \\
87.9\end{array}$ \\
\hline & 10.2 & 1.0 & $\begin{array}{l}15 \\
30\end{array}$ & $\begin{array}{l}1540 \\
3573\end{array}$ & $\begin{array}{l}81.8 \\
73.2\end{array}$ \\
\hline & 20.4 & 2.0 & $\begin{array}{l}15 \\
30\end{array}$ & $\begin{array}{l}2026 \\
3033\end{array}$ & $\begin{array}{r}139.3 \\
62.6\end{array}$ \\
\hline
\end{tabular}

2The assay procedure was the same as the polymerase assay with the polyidAdT)/poly(dA-dT) template except that the polymer template concentration was reduced to $0.25 A_{260 \mathrm{~nm}}$ unit $/ \mathrm{ml}$ (approximately $10 \mu \mathrm{g} / \mathrm{ml}$ ). All ingredients except S5B-1 were first mixed on ice and then SSB-1 was added. The reaction tube was then placed in a $30^{\circ} \mathrm{C}$ bath to start the reaction.

\section{REFERENCES}

1. Esposito, M.S., Hosoda, J., Golin, J., Moise, H., Bjornstad, K., and Malear D. Recombination in Saccharomyces cerevisiae: REC-gene mutants and dNA-binding proteins, Cold Spring Harbor Symp. Quant. Biol. 49, 41 (1984).

2. LaBonne, S.G., and Dumas, L.8. Isolation of a yeast single-strand deoxyribonucleic acid bind- ing protein that specifically stimulates yeast DNA polymerase 1, Biochemistry 22, 3214 (1983).

3. Chang, L.M.S. DNA polymerase from Baker's veast, J. Biol. Chem. 252, 1873 (1977).

4. Singh, $H_{\text {., }}$ and Dumas, L.B. A DNA primase that copurifies with the major DNA polymerase from yeast Saccharomyces cerevisiae. I. Biol. Chem. 259, 7936 (1984).

\title{
IN WIRO RESOLUTION OF HOLLIDAY JUNCTIONS BY CELL-FREE PROTEIN EXTRACTS OF SACCHAROAYCES CEREVISIAE: RESOLVASE ACTIVITY OF HAPLOID AND DIMLOID CELLS
}

\author{
Libby Litzemkercer Holbrook, Kathleen A. Ejornstad, Dimitrios T. Maleas, and \\ Nichael S. Esposito
}

A cenira: fealure of molecular models of eenelic recombination is the formation and resolution of Holliday junctions." Our previous studies of the properties of spontaneous milotic recombins. fion in Sxchuromyces cerevisive led lo the predic.

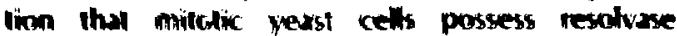

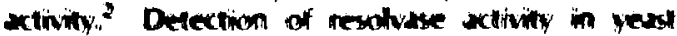

exiracts has been facilitated by the previous studies of Mizuuchi el al. ${ }^{3}$ and Lilley and Kemper, which demonstrated that the resolvase (endonuclease VII) controlled by gene 49 of $T+$ cleaves Holliday junc. tions formed by crucirorm extrusion at sites of inverted nepeals. In our invitial studies, milotic cell populations of both haphoind and diphoind recombina. 
tion proficient strains were surveyed for resolvase activity toward plasmids containing cruciforms.

Plasmids ColE1 and pBR322 contain inverted repeats of 13 and 11 bases respectively, ${ }^{5,6}$ which can assume cruciform structures as demonstrated by $\mathrm{S1}$ nuclease sensitivily. ${ }^{7,8}$ These plasmids were used to screen yeast extracts for resolvase activity. The assay is based on conversion of the supercoiled form to the linear form by cleaving at the site of cruciform extrusion as diagrammed in Fig. 1. Once the cruciform is resolved, the plasmid is no longer a substrate for resolvase.

The $\mathrm{Rec}^{+}$haploid strains ${ }^{9}$ NLBL1 (MAT $\alpha$ ade5 met73-c cyh2' trp5 LEU1 ade6 cly8 his7-1 tyri-2 lys2-2 ade2-1 ura3-1 CAN1 ${ }^{5}$ ) and NLBL3 (MATa ADE5 met13-d CYH2 ${ }^{5}$ TRP5 leu1 ADE6 CLY8 his7-2 tyr1-1 lys2-1 ade2-1 ura3-1 can71) as well as the diploid NLBL1 X NLBL3 express resolvase activity toward either substrate. A time course of the reaction on ColE1 catalyzed by proteins in the NLBL1 extract is shown in Fig. 2. Production of linear plasmid is observed upon incubation from 15 to 90 minutes. The supercoiled form disappears at 15 to 30 minutes. The open circular form is also observed which presumably is not a substrate for resolvase since it no longer contains a cruciform structure. At longer incubation times the substrate is degraded (by nonspecific nucleases also present in the crude preparation). As is the case for endonuclease VII of $T 4,{ }^{10}$ yeast resolvase activity is dependent on the presence of $\mathrm{MgCl}_{2}$ and does not require ATP. The yeast activity is also inhibited by concentrations of $\mathrm{NaCl}$ or $\mathrm{KCl}$ greater than $50 \mathrm{mM}$ (not shown).

While assaying crude extracts, an ATPindependent topoisomerase $\mathrm{I}$ activity was detected. This is most likely that previously described by Goto et al." since both are ATP-independent and resistant to coumermycin.

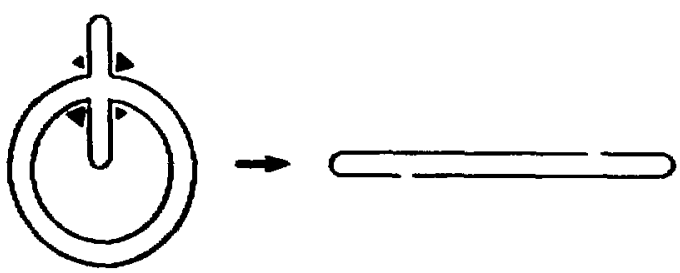

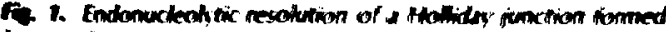
by cruofitom exwusion of imented nepest sequences conumined in a spercoived plesind. Resolhuse chenes tither the mo im.

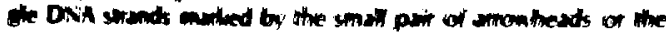

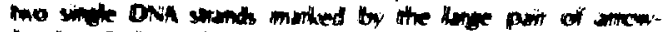

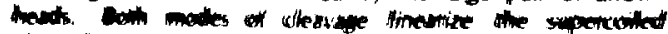
phiment.

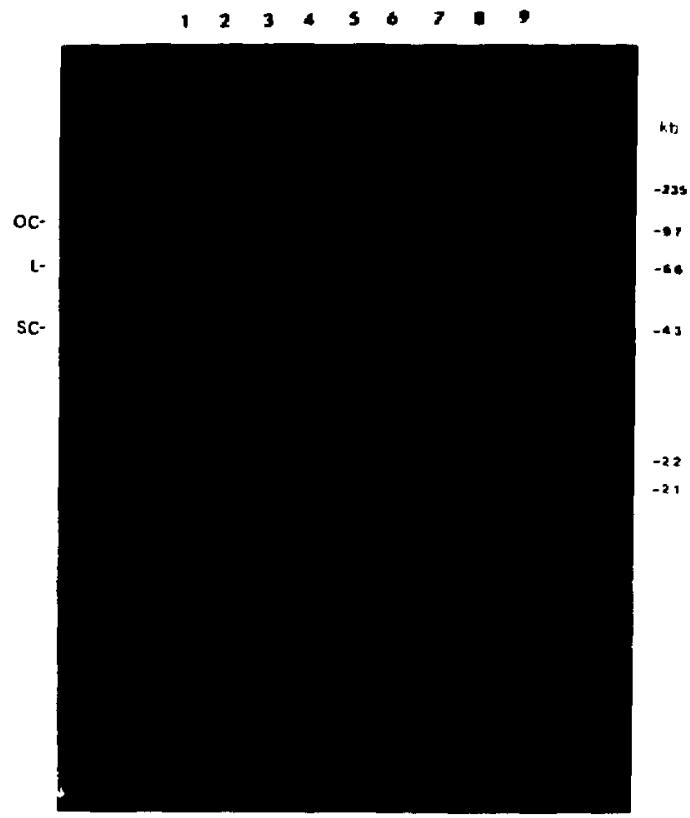

Fig. 2. Time course of resolvase reaction calalyzed by extracts of NLBL1. Cells were grown at $24^{\circ} \mathrm{C}$. Resolvase was assayed in $2 M$ ammonium sulfate pellets prepared by a modification of a published procedure ${ }^{15}$ using ColEl DNA $(6.6 \mathrm{~kb})$ in the reaction described in the legend to Fig. 3 and incubating for the follow. ing minutes: 0 (lane 3), 15 (lane 4), 30 (lane 5), 60 (lane 6), 90 (lane 7), and 120 (lane 8). Lane 1-COlE1 DNA. Lane 2 - linear ColE1 DNA produced by ECOR1 restriction of the plasmid. Lane 9 - linear standards generated by Hindlll digestion of lambda DNA. Sizes are indicated in kilobases. $O C$ : open circular DNA. L: linear DNA. SC: supercoiled DNA. (XBB 850.8642)

The supercoiled dimer form of pBR322 was used to dislinguish the resolvase activity from a restriction enzyme. Whereas both resolvase and a restriction enzyme that cuts the DNA once will produce linear DNA from a monomeric plasmid, they will produce fragments of different sizes from a dimeric plasmid. Resolvase recognizes a structural feature whose presence is dependem upon supercoiling (crucilorm) and introduces one endonucleolytic scission at a Holliday junclion thereby producing a linear dimer DNA product. A restriction enzyme recognizes a specific sequence and therefore will make two cuts in the dimer plasmid to give the linear monomer product. These species are easily distinguished by agarose gel electrophoresis.

Four nec mulanis (rect90, rect113, rect67, tect6) iscolaled in this Lbonatory" ${ }^{12}$ (Tabte 1) mere screened for both reschase and topoisommerase I 
Table 1. Properties of recombination mutants isolated in strain CBL1.:

\begin{tabular}{|c|c|c|c|c|c|}
\hline & \multicolumn{2}{|c|}{ Median frequencies per $10^{3}$ c.f.u." } & \multirow[b]{2}{*}{$\begin{array}{l}\text { Total } \\
\text { Cyh' }\end{array}$} & \multirow[b]{2}{*}{$\begin{array}{c}\text { Resolvase } \\
\text { activitye }\end{array}$} & \multirow[b]{2}{*}{$\begin{array}{c}\text { In vitro } \\
\text { recombination } \\
\end{array}$} \\
\hline & $\begin{array}{l}\text { Leu'Trp'Cyh' } \\
\text { inler. rec. }\end{array}$ & 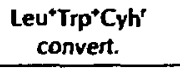 & & & \\
\hline $\operatorname{rec} 754^{e}$ & 0.00 & 0.00 & 0.82 & & - \\
\hline rec 780 & 0.00 & 0.00 & 0.00 & & \\
\hline rec416 & 0.00 & 0.00 & 0.00 & & \\
\hline rec $490^{\circ}$ & 0.00 & 0.00 & 0.00 & + & - \\
\hline $\operatorname{rec} 413^{t}$ & 0.00 & 1.12 & 2.50 & + & - \\
\hline $\operatorname{rec} 336^{\circ}$ & 0.00 & 1.88 & 6.85 & & - \\
\hline rec $467^{i}$ & 0.00 & 3.02 & 4.71 & + & + \\
\hline reci41 & 0.11 & 5.20 & 30.69 & & \\
\hline rec100 & 0.17 & 0.42 & 1.23 & & + \\
\hline rec234 & 0.37 & 0.00 & 0.62 & & ++ \\
\hline rec139 & 1.10 & 0.00 & 263.33 & & \\
\hline rec201 & 1.18 & 0.16 & 14.29 & & \\
\hline rec146 & 8.80 & 0.57 & 27.87 & & +++ \\
\hline rec 276 & 10.74 & 1.12 & 157.77 & & +++ \\
\hline rec952 & 12.69 & 1.94 & 25.40 & & \\
\hline rec395 & 16.90 & 1.60 & 123.20 & & \\
\hline rec193 & 23.73 & 2.20 & 123.60 & & +++ \\
\hline rec409 & 54.48 & 5.36 & 48.20 & & \\
\hline $\operatorname{rec} 46^{\circ}$ & 243.48 & 19.95 & 1715.17 & + & +++ \\
\hline rec199: (cdc9) & 369.84 & 36.41 & 2025.00 & & + \\
\hline LBL1 & 1.34 & 0.72 & 15.46 & + & + \\
\hline
\end{tabular}

'Spontaneous milotic gene conversion of $\mathrm{CYH}^{2}$ to $\mathrm{cyhz}$ in the chromosome VII disomic strain LBL1 (MATa ade5/ADE5 mel13-c/met13-c cyh2/CYH2s trp5/TRP5 leut/LEU1 ade6/ADE6 cly8/CLY8 his7-1 (yr1-2 lys2-2 ade2-1 ura3-1 CANI') and mutants derived from it results in red $\mathrm{Leu}^{+} \mathrm{Trp}^{+}$cycloheximide resistant $\left(\mathrm{Cyh}^{\prime}\right)$ segregants. Spontaneous intergenic recombination in the interval CYH2 - TRPS results in white Leu* Trp ${ }^{*}$ Cyh' segregants. The ensemble of conversional, exchange, and chromosomal loss events contributes to total Cyh' resistant segregariss. 219 Median frequencies are based upon analyses of 25 colonies of the control Rec ${ }^{*}$ strain LBLI and a minimum of five colonies of each mutant. ${ }^{12}$ All of the mutants characterized with respect to dominance versus recessivity exhibit meiotic defects when studied in diploid MATa/MATa cells. The recessive mutations complement one another. The recombinational defecls of rec 490 and rec 413 are temperature sensitive; rec 336 and rec 199 (cdc9) are conditional lethals.

bc.f.u. = colony forming units.

'Resolvase activity was measured as described in Fig. 3. The rec413 and recr490 strains were grown at both permissive and restrictive lemperatures for recombination. Rec haploids and diptoids grown al $24^{\circ} .29^{\circ}$, and $36^{\circ} \mathrm{C}$ exhibil resolvase activily.

Assayed by in vitro plasmidic recombination (L.S. Syminglon and R. Kolodnet, personal com). munication). ${ }^{14}$

Recessive mutalion.

Dominant or semidominant mulation.

activities. The temperature sensitive mulants (rec490, rect13) were grown at both the permissive and the restrictive temperatures for recombination. All mutanis assayed expressed both resolvase ard topoisomerase I activitives, implying that their recombinational defects are not due to mutalions in the siructural or regulatory genes controlling these activilies. Assays on crude exiracts prepared firom strains helerozygous and homozygous for the rect6 mutalion (see report by D.T. Maleas et al.) as well as the Rec ${ }^{4}$ diphoid are shown in Fig. 3. All crode extracts contain nesolvase activity thet catalyzes 


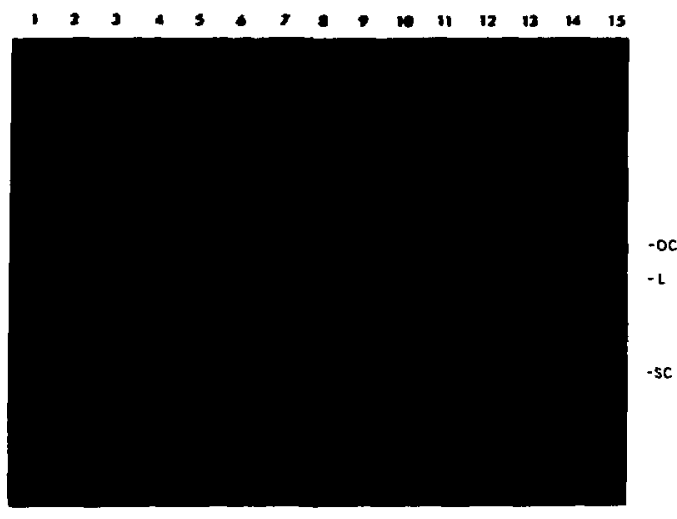

Fig. 3. Resolvase and topoisomerase $/$ assays of diploids containing the rec46 mutation. Lanes 1 through $8-2 M$ ammonium sulfate pellets. Lanes 9 phrough 14 - $3 M$ ammonium sulfate pellets. Pairs were assayed as described below for 1 and 2 hours respectively. Lanes 1,2,9, and $10-$ Rec $^{*}$ diploid. Lanes $3,4,11,12$ - rec 46 heterozygote. Lanes 5 and 6 - a second rec46 heterozygote. Lanes 7, 8, 13, 14 - rec46 homozygote. Lane 15 - $p$ BR322 dimer plasmid $(8.7 \mathrm{~kb})$. Mitotic cell populations of control and rec mutant strains were grown at $29^{\circ} \mathrm{C}$ in $100 \mathrm{ml}$ YPD cultures supplemented with $100 \mathrm{mg} / \mathrm{liter}$ adenine sulfate and harvested at a density of $5 \times 10^{7} \mathrm{cel} / \mathrm{s} / \mathrm{ml}$. The cell pellet was resuspended in $50 \mathrm{mM}$ Tris- $\mathrm{HCl}, \mathrm{pH} 7.51 \mathrm{mM}$ EDTA, $10 \%$ $(w / v)$ sucrose and stored at $-70^{\circ} \mathrm{C}$. The thawed cells were treated at $37^{\circ} \mathrm{C}$ for 30 minutes with $0.5-\mathrm{mg} / \mathrm{ml}$ zymolyase, 5 $\mathrm{mM}$ 2-mercaptoethanol, $1 \mathrm{mM}$ PMSF, and $2 \mu \mathrm{g} / \mathrm{ml}$ Pepstatin A. The cells were then treated on ice for 60 mimutes with $0.4 \mathrm{M}$ $\mathrm{KCl}, 0.1 \mathrm{mM}$ protamine sulfate, and $10 \%$ streptomycin sulfate, briefly sonicated, and cleared by centrifugation. The supernate was successively brought to $2 \mathrm{M}$ and then $3 \mathrm{M}$ ammonium sulfate by the addition of $3.8 \mathrm{M}$ ammonium sulfate, $p H 7$. The precipitated proteins in each fraction were collected by centrifugation and stored at $-70^{\circ} \mathrm{C}$. A protein peller was resuspended in $20 \mathrm{mM}$ Tris $\mathrm{HCl}, \mathrm{pH}$ 7.5, $0.1 \mathrm{mM}$ EOTA, 10\% (V/v) glycerol. Resolvase activity was detected by the conversion of supercoiled to linear DNA in a $10 \mu$ ? reaction mix consisting of $20 \mathrm{mM}$ Tris$\mathrm{HCl}, \mathrm{pH} 7.51 \mathrm{mM}$ dithiothrejtal, $10 \mathrm{mM} \mathrm{MgCl}_{2}, 0.25 \mathrm{mg} / \mathrm{ml}$ bovine serum albumin, 0.1 Ag p8R322 supercoiled DNA and yeast proteins. Alter incubation for $1-2$ hours af $30^{\circ} \mathrm{C}$, the reaction was stopped by the addition of $20 \mathrm{mM}$ EDTA. DNA species were separated by electrophoresis through $0.8 \%$ agurose in TAE ( $10 \mathrm{mM}$ Tris-HCl, $20 \mathrm{mM}$ acelate, $0.5 \mathrm{mM} E D \mathrm{TA}_{\text {, }}$ pH 8.0) bufifer and visualized by ethidium bromide liuorescence under uv iransillunination. Topoisomerase I acrivity was assayed in the same manner by delecting the formation of repoisomers from supercoiled DNA OC: open cinculy DNA.

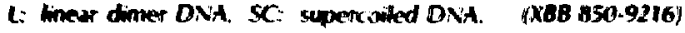

production of linear dimet from supercoiled PBR322 dimer DNA 2Mi ammonium suliale fraction, lanes 1-8) and lopoisometase I activily that relaxes the supercoiled DNA to a ladket of lopoiso-

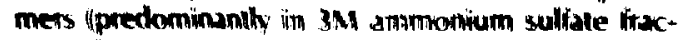
tion. Lones 9-14t). No thandis corresponding to linear monomer or smaller products were observed, suggesting the absence of a restriction reaction.

The fractions obtained from DNA cellulose chromatography (see report by J. Hosoda et al.) of a protease deficient strain (B)2168, MATa prb1 pep 4 prc1) were also assayed for resolvase and topoisomerase $!$ activities (Figs. 4 and 5) using the pBR322 supercoiled dimer as a substrate. Topoisomerase 1 bound to native DNA cellulose and eluted at $0.3-0.37 \mathrm{M} \mathrm{NaCl}$ (fractions ds-4, ds-5, and ds-6, Fig. 4). Resolvase bound predominantly to single-stranded DNA cellulose under the conditions used and eluted at $0.17-0.38 \mathrm{M} \mathrm{NaCl}$ (fractions ss-2 to ss-8, Fig. 5). Some activity was also observed to elute from native DNA cellulose at $0.22-0.30 \mathrm{M} \mathrm{NaCl}$.

The resolvase protein(s) that we have identified will be purified to homogeneity and used to obtain mutations in the resolvase gene(s). We wish to inquire whether mitotic and meiotic yeast cells

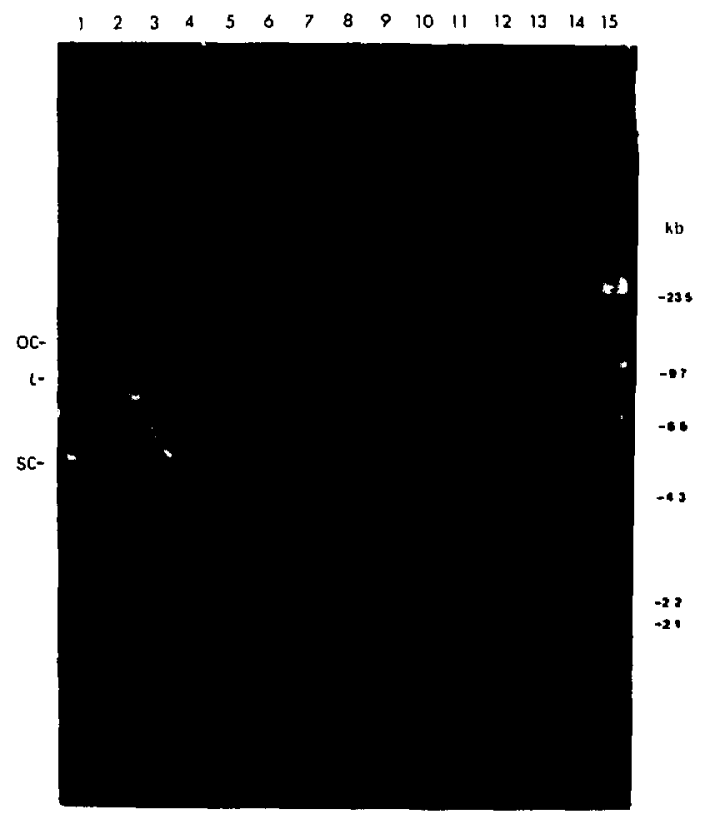

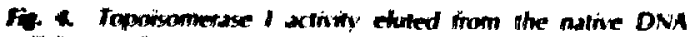

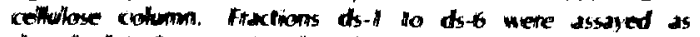

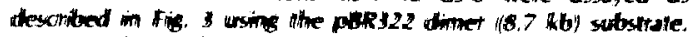

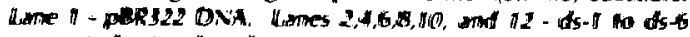

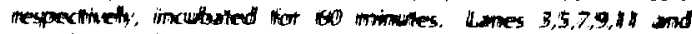

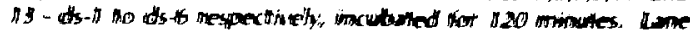

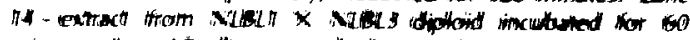

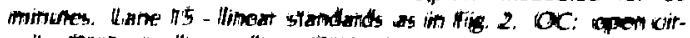

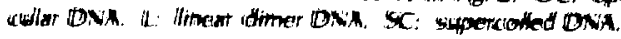




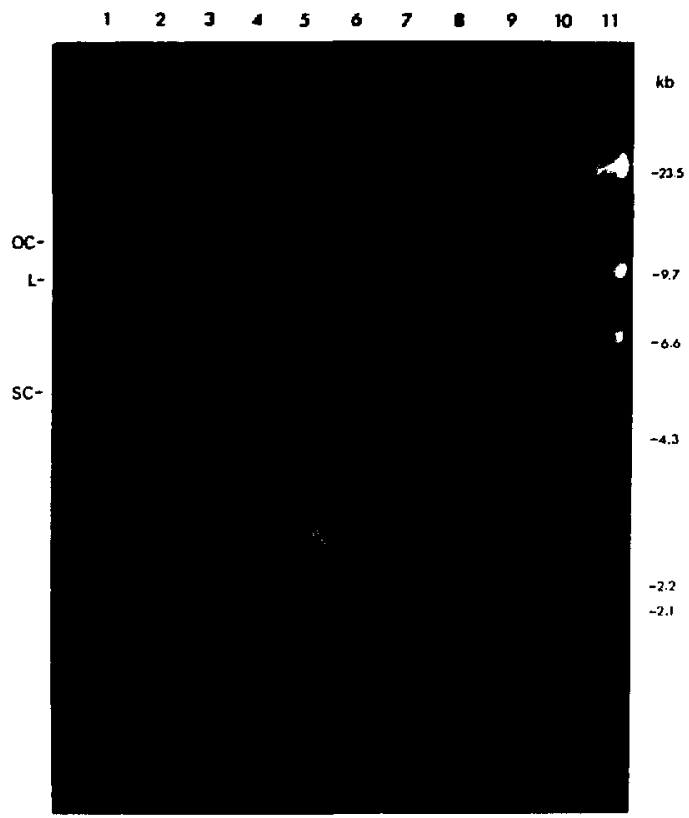

Fig. 5. Resolvase activity eluted from the single-stranded DNA cellulose column. Fractions 55.1 to 55.8 were assayed as described in fig. 3 using the pBR.322 dimer $(8.7 \mathrm{~kb})$ and incubating for 60 minutes. Lane 1 - no yeast proteins added. Lanes 2 through $9-5 s .1$ to $5 s .8$ respectively. Lame 10 as in Lane 14 of Fig. 4. Lane 11 - linear standards as in Fig. 2. OC: open circular DNA. L: linear dimer DNA. SC: supercuiled DNA.

(XBB 850-8641)

defective in resolvase activity are viable, and to determine their recombinational phenolypes both in vivo and in vitro. In the case of mitotic diplcid cells we will ascertain whether resolvase defective cells (if viable) exhibit the hyperrecombination phenotype predicted by the prereplicational model of mitolic recombination ${ }^{13}$ or the hyporecombination phenotype predicted by alternative models. ${ }^{2}$

\section{REFERENCES}

1. Holliday, R.A. Mechanism for gene conversion in fungi. Genel. Res. 5, 282-304 (1964).

2. Espeosilo. M. $S_{n}$ and Wagslaif, I. Mechanisms oi matotic recombination, in The slolecular Brology of the Veast Sacharonnces: Lije Cucte and anheratunce., Sirathem, I.N., lones.
E.W., and Broach, J.R., Eds., Cold Spring Harbor Laboratory, Cold Spring Harbor, New York, pp. 341-370 (1981).

3. Mizuuchi, K., Kemper, B., Hays, J., and Weisberg, R.A. T4 endonuclease VII cleaves Holliday structures. Cell 29, 357-365 (1982).

4. Lilley, D.M.J., and Kemper, B. Cruciformresolvase interactions in supercoiled DNA. Cell 36, 413-422 (1984).

5. Oka, A., Nomura, N., Morita, M., Sugisaki, H., Sugimoto, K., and Takanami, M. Nucleotide sequence of small ColE1 derivatives: Structure of the regions essential for autonomous replication and colicin E1 immunity. Mol. Gen. Genet. 172, 151-159 (1979).

6. Sutcliffe, J.G. Complete nucleotide sequence of the E. coli plasmid pBR322. Cold Spring Harbor Symposia of Quantitative Biology 42, 77-90 (1978).

7. Lilley, D.M.J. The inverted repeat as a recognizable structural feature in supercoiled DNA molecules. Biochemistry 77, 6468-6472 (1980).

8. Panayotatos, N., and Wells, R.D. Cruciform structures in supercoiled DNA. Nature 289, 466-470 (1981).

9. Esposito, M.S., Maleas, D.T., Bjornstad, K.A., and Bruschi, C.V. Simultaneous detection of changes in chromosome number, gene conversion and intergenic recombination during mitosis of Saccharomyces cerevisiae: Spontaneous and ultraviolet light induced events. Current Genet. 6, 5-11 (1982).

10. Kemper, B., and Garabaett, M. Studies of T4-head maturation. I. Purification and characterization of gene-49-controlled endonuclease. Eur. J. Biochem. 115, 123-131 (1981).

11. Goto. T., Laipis, P., and Wang, I.C. The purification and characlerization of DNA topoisomerases I and II of the yeast Saccharomyces cerevisiae. J. Biol. Chem. 259, 10422-10429 (1984).

12. Esposito, M.S., Hosoda, J., Golin, J., Moise, H., Bjornstad, K., and Maleas, D. Recombination in Saccharomyces cerevisiae: REC-gene mutanis and DNA-binding proteins. Cold Spring Harbor Symposia on Quantilalive Bio:ogy $19,41-48$ (1984).

13. Esposito, M.S. Evidence that spontaneous mitolic tecombination occurs at the two- 
strand stage. Proc. Natl. Acad. Science USA 75, 4436-4440 (1978).

14. Symington, L.S., Morrison, P.T., and Kolodner, R. Genetic recombination catalyzed by cellfree extracts of Saccharomyces cerevisiae. Cold Spring Harbor Symposia on Quantitative
Biołogy 49, 805-814 (1984).

15. Symington, L.S., Fogarty, L.M., and Kolodner, R. Genetic recombination of homologous plasmids catalyzed by cell-free extracts of Saccharomyces cerevisiae. Cell 35, 805-813 (1983).

\section{THE REC46 GENE OF SACCHAROMYCES CEREVISIAE CONTROLS MITOTIC CHROMOSOMAL STABILITY, RECOMBINATION AND SPORULATION: CELL-TYPE AND LIFE CYCLE STAGE SPECIFIC EXPRESSION OF THE reC46-1 MUTATION}

Dimitrios T. Maleas, Kathleen A. Bjornstad, Libby L. Holbrook, and Michael S. Esposito

Studies of chromosomal recombination during mitosis and meiosis of Saccharomyces cerevisiae have demonstrated that recombination at these two distinct stages of the yeast life cycle proceeds by mechanisms that appear similar bu involve discrete mitosis-specific and meiosis-specific properties. We are employing rec mutants as a genetic tool to identify the partial reactions comprising recombination and the involvement of individual REC gene products in mitotic and meiotic recombination. The sequence of molecular events that results in genetic recombination in eukaryotes is presently ill-defined. Genetic characterization of REC gene mutants and biochemical analyses of them for discrete defects in DNA metabolic proteins and enzymes (in collaboration with the laboratory of Junko Hosoda) are beginning to remedy this gap in our knowledge.

in this report we summarize the genetic properties of the rec46-1 mutation.

\section{PROPERTIES OF THE REC46-1 MUTANT}

We have isolated a broad spectrum of ultraviolet light-induced hyperrecombination and hyporecombination mutants affecting spontaneous mitotic gene conversion and intergenic recombination of markers on chromosome $V I I$ in an $n+1$ MATa strain (LBL1, see Fig. I and Table 1) disomic for this chromosome.",2 Several of the rec mulations isolated, including rect6-1, exhibit analogous phenotypes with respect 10 in-witro recombination between DNA plasmids catahyzed by mitotic celltree extuxts. The rect6-1 mutation was chosen for fwther andisis since its hyperrecombinationd phenokpe both in wive and in vituo is amones the mosk exturine cherved to dye. in the experi- ments described below we have characterized rec46-1 strains for mitotic chromosomal stability, mutability, intragenic recombination, intergenic recombination, sporulation, and $\mathrm{HO}$ directed interconversion of mating-type alleles. The genetic analysis of rec46-1 demonstrates it to be a recessive mutation that enhances mitotic chromosomal loss and mitotic recombination. The hyperrecombinational activity of rec46.1 has been observed in genetic intervals tested on three chromosomes (II, $V$, and VII). MATa and MATa HO rec46-1 haploids are proficient in $\mathrm{HO}$ directed interconversion of mating type alleles and their survival is not detectably affected by presence of the HO gene. MATa/MATa rec46-1/rec46-1 diploids are sporulation deficient and lose colony forming ability following exposure to sporulation medium, indicating that the REC46 gene is essential for normal meiosis, $^{3}$

The rec46-1 mutation was recovered among survivors of ultraviolet light mutagenesis $165 \%$ survival) of the $L B L 1 n+1$ hyperhaploid strain disomic for chromosome VII. ${ }^{1,2}$ The LBL1 strain (Fig. 1) forms red colonies on both chemically defined and complex glucose nutrient media owing to the ade2

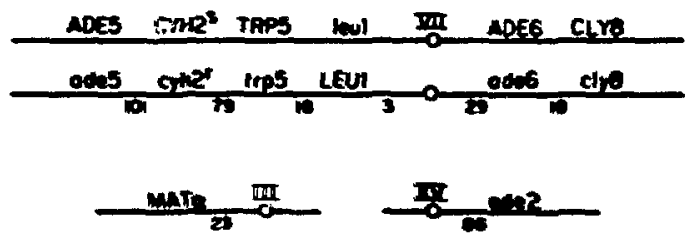

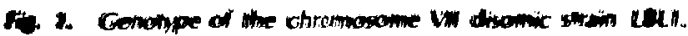

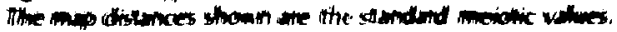

(1042 24+575) 


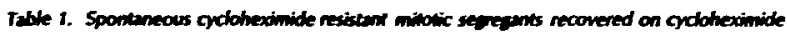

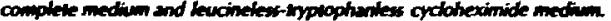

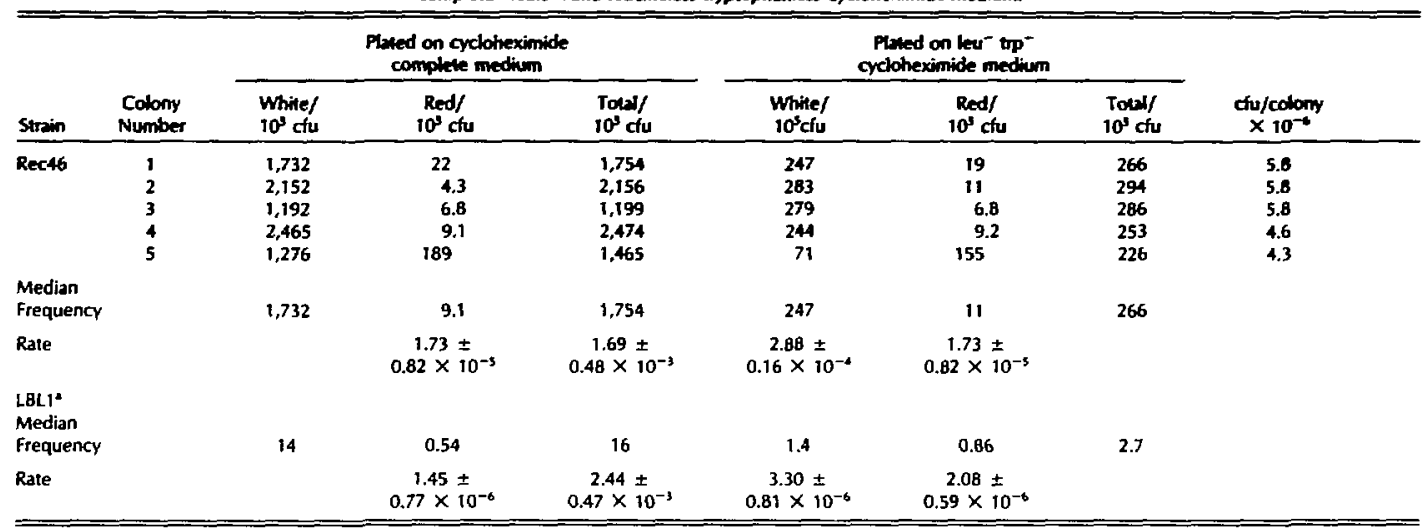

'The values shown for the parental Rec' strain, LBL1, are based upon previously published analyses of $252.5 \mathrm{~mm}$ colonies grown an synthetic complete mejiurn at $24^{\circ} \mathrm{C}$ (Rel. 4 ).

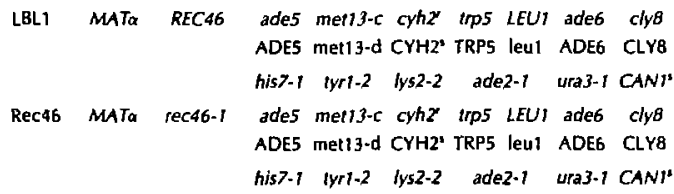

mutation that confers adenine auxotrophy and accumulation of a cell-limited red pigment. The chromosome VII disomic pair is heterozygous at six loci distributed on both sides of the centromere (Fig. 1). The trans arrangement of markers at the LEU1 and TRP5 loci ensures maintenance of the disomic cundition since rare haploid segregants (resulting from chromosomal loss or mitotic nondisjunction) are unable to grow on omission media lacking both leucine and Iryptophan. Gene conver-

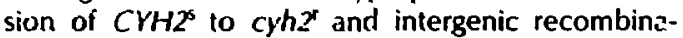
tion in the interval $\mathrm{CYH} 2-\mathrm{TRP} 5$ can be monitored selectively and simultaneously by plating cells on leucineless-tryptophanless cycloheximide medium. Gene conversion of $\mathrm{CYH}^{5}$ to $\mathrm{Cyh}^{\prime}$ yields red cycloheximide resistant $c y h \geq / c y h x$ disomic recombinants helerozygous at the other five chromosome VII loci and occurs at a rate of $1.7 \pm 0.70 \times$ $10^{-6} /$ cell division in strain LBLI, Intergenic recombination in the inlerval CYH2-TRP5 resulis in white cyclohe aimide resistant $c y h / / c y h z$ recombinanis that are also homozygous for the ade5 mutation. They form white colonies because the ade5 mutalion blocks ned pigment formation. Such segregants are heteroaygous at the four memaining chuomosome VII makker koci and arise at a rate of

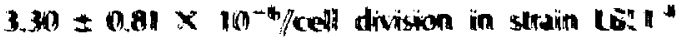
The ensemble of mintodic extents nesulting cyctolwexi- mide resistant segregants including restitution of haploidy, intergenic recombination between the CYH2 locus and the centromere of chromosome VII, and conversion of $\mathrm{CYHZ}^{\mathrm{s}}$ to $\mathrm{Cyh}^{\mathrm{r}}$ can be recovered by plating cells on cycloheximide synthetic complete medium. Genetic analysis of cycloheximide resistant segregants recovered in this manner from strain LBLI demonstrates that cycloheximide resistant restituted haploids exhibiting no delectable recombination on chromosome VII comprise $62 \%$ of ail cycloheximide resistant segregants and arise at a rate of $1.37 \times 10^{-5} /$ cell division."

The rec46-1 disomic slrain yields cycioheximide resistant segregants at a rate per cell division that is approximately 50 limes greater than that of the parental LBLI sirais. Conversien of $\mathrm{CYHZ}$ to $\mathrm{CyhZ}$ occurs at a rate approximalely 10 times greater than in LBLI. Intergenic recombination in the interval CYH2-TRP5 occurs at a rale approximately 100 times grealer than in LBLI (Table I).

A sample of 100 white segregants from cycloheximide complete medium were characte:ized to delermine the fraction of cycloheximide resistanl segregants that had lost the COHT bearing chmomosome, i.e., ades cyhz ups LEUt ade6 dyo restituled haploids. The whine segregants were tesked for from th at $36^{\circ} \mathrm{C}$ on YPO medium to 
detect segregation of the iemperature sensitive lethal cly8, growth on tryptophanless and leucineless media, and were mated with MATa ade5 and ade6 tester strains to monitor segregation of ade5 and ade6. A total of 86/100 tested exhibited restitution of haploidy. These data indicate that restitution of haploidy in the rec46-1 disomic strain occurs at a rate per chromosome per cell division of $1.45 \times 10^{-3}$ (i.e., $1.69 \pm 0.48 \times 10^{-3} \times 0.86$ ) which is 100 times greater than that of the LBL1 strain $\left(1.37 \times 10^{-5}\right.$ per chromosome per division).

\section{MITOTIC RECOMBINATION IN rec46-1/rec46-1} MATa/MAT $\alpha$ HYBRIDS

REC46/REC46, REC46/rec46-1/rec46- 1 and rec46-1/rec46-1 hybrids ( $R \times 38, R \times 26$ and $R \times 64$, respectively, see Table 1) having chromosome VII genotypes that allow one to distinguish red $\mathrm{Cyh}^{\mathrm{r}}$ gene convertants from while $\mathrm{Leu}^{+} \mathrm{Trp}^{+} \mathrm{Cyh}^{r}$ intergenic recombinants arising from recombination in the $C Y H 2-T R P 5$ interval were analyzed further. The hybrids employed are also heteroallelic at LYS2 and HIST (chromosome II) and were employed to determine whether the rec46-1 mutation enhances intragenic recombination of heteroalleles resulting in prototrophy. The mitotic rates/cell division of events resulting in red $\mathrm{Leu}^{+} \mathrm{Trp}^{+} \mathrm{Cyh}^{r}$ gene conver- tants, while $\mathrm{Leu}^{+} \mathrm{Trp}^{+} \mathrm{Cyh}^{r}$ intergenic recomvinants, Can resistant segregants, $\mathrm{LYSs}^{+}$prototrophs, and $\mathrm{His}^{+}$prototrophs are shown in Table 2. Red $\mathrm{Leu}^{+} \mathrm{Trp}^{+} \mathrm{Cyh}^{\mathrm{r}}$ gene convertants and white $\mathrm{Leu}^{+}$ $\mathrm{Trp}^{+} \mathrm{Cyh}^{r}$ intergenic recombinants occur in MATa/MAT $\alpha$ rec46-1/rec46-1 diploids (RX64) at rates that are only five-fold greater than those of MATa/MATa REC46/REC46 diploids (RX38). Can' segregants and total $C y h^{r}$ resistant segregants in MATa/MATo rec46-1/rec46-1 diploids (RX64) occur at rates that are ten-fold higher than those of MATa/MAT $\alpha$ REC46/REC46 diploids (RX38).

$\mathrm{Lys}^{+}$and $\mathrm{His}^{+}$prototrophs are recovered 10 to 15 times more frequently from the rec46-1/rec46-1 hybrid (RX64) than from the REC46/REC46 (RX38) and REC46/rec46-1 (RX26) hybrids (Table 2), indicating that the rec46.1 mutation stimulates intragenic recombination resulting in prototrophy. We analyzed the lysine prototrophs recovered from each of the three hybrids to determine whether the rec46-1 mutation confers mutator activity. The ade2-1, 1ys2-1 and 1ys2-2 alleles are ochre nonsense mutations that are suppressed by inminant forward mutations, sup ${ }^{+}$to SUP ochre. The SUP mutants contain anticodon mutations of tyrosyl tRNA genes that cause :yrosine to be inserted at the position of $m \cdot$ RNA uchre codons. Diploid ade21/ade2-1 cells heterozygols for such SUP muta-

Table 2. Spontaneous mitotic gene conversion, intragenic recombination and intergenic recombination in REC46/REC46, REC46/rec46-1, and rec46-1/rec46-1, MATa/MATa hybrids."

\begin{tabular}{|c|c|c|c|c|c|c|c|}
\hline Genotype" & Hybrid & $\begin{array}{l}\text { Median No. } \\
\text { Red Leu'Trp } \\
\text { Cyh } / 10^{6} \text { clu }\end{array}$ & $\begin{array}{c}\text { Median No. } \\
\text { White Leu+Trp* } \\
\text { Cyh'/106 cfu }\end{array}$ & $\begin{array}{c}\text { Median No. } \\
\text { Lys\% } \\
10^{7} \mathrm{cfu}\end{array}$ & $\begin{array}{c}\text { Median No. } \\
\text { His } / \\
10^{7} \mathrm{cfu}\end{array}$ & $\begin{array}{c}\text { Median No. } \\
\text { Total Cyhr/ } \\
10^{5} \mathrm{cfu}\end{array}$ & $\begin{array}{c}\text { Median No. } \\
\text { Can'/ } \\
10^{5} \mathrm{cfu}\end{array}$ \\
\hline & Rate & $2.14 \pm 0.80 \times 10^{-6}$ & $7.46 \pm 2.36 \times 10^{-6}$ & $9.56 \pm 3.78 \times 10^{-8}$ & $1.24 \pm 0.47 \times 10^{-7}$ & $1.83 \pm 0.50 \times 10^{-3}$ & $5.94 \pm 1.38 \times 10^{-5}$ \\
\hline $\begin{array}{l}\text { REC46 } \\
r e c 46-1\end{array}$ & RK26 & 10.7 & 81.9 & 13.0 & 15.0 & 24.7 & 30.0 \\
\hline \multirow{2}{*}{$\begin{array}{l}\text { rect6-1 } \\
\text { rect6-1 }\end{array}$} & RX64 & 70.9 & 640.5 & $102.0^{\circ}$ & 165.0 & 99.2 & $\$ 73.2$ \\
\hline & Rate & $7.83 \pm 2.76 \times 10^{-6}$ & $7.63 \pm 1.72 \times 10^{-8}$ & $1.34 \pm 0.06 \times 10^{-6}$ & $2.14 \pm 0.09 \times 10^{-6}$ & $1.11 \pm 0.24 \times 10^{-1}$ & $4.10=1.39 \times 10^{-4}$ \\
\hline
\end{tabular}

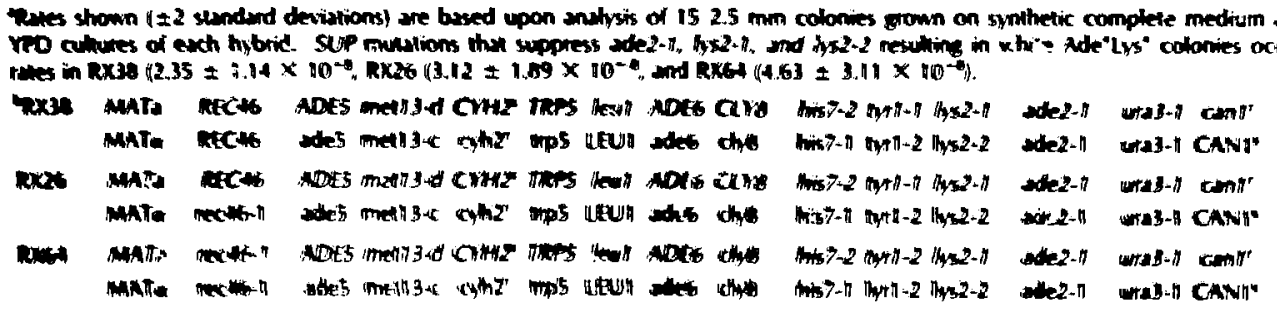


tions fail to accumulate the red pigment normally accumulated by ade2-1 strains and grow slowly on adenineless medium. The very same suppressors fully restore lysine prototrophy in lys2-1/lys2-2 beleroallelic diploids, and are readily detected as white Lys ${ }^{*}$ colonies that exhibit partial restoration to adenine prototron'hy. Suppressor mutations of this type account for approximalely $20 \%$ of the events resulting in $\mathrm{Lys}^{+}$segregants of the lys21/lys2-2 heteroallelic control MATa/MATa REC46/REC46 jybrid (Table 2). The rec461/rec46-1 diploid does not exhibit milator activity for this class of mutations.

\section{SPORULATION DEFICIENCY OF rec46-1/rec46-1 DIPLOIDS}

Diploids homozygous for the rec46-1 mutation exhibit very low levels of sporulation when compared with coisogenic REC46/REC46 and REC46/rec46-1 controls (Table 3). Exposure of rec46-1/rec46-1 dip!oids to sporulation medium eventuaily results in death of both nonsporulated and sporulated cells. These results indicate that the REC46 gene is essential for sporulation (meiosis and ascospore formation).
HOMOTHALLIC INTERCONVERSION OF MATING TYPE ALLELES BY reC46-1 HO MEIOTIC SEGREGANTS

The MATa rec46-1 haploid test strain, a heterothallic ho haploid, was mated with ascospores of a homothallic ( $H O / H O)$ diploid strain. The resultant hybrid was sporulated at $24^{\circ} \mathrm{C}$ and tetrads were analyzed to monitor segregation of the rec46-1 mutation and $\mathrm{HO}$ directed interconversion of mating type alleles among rec46-1 $\mathrm{HO}$ meiotic segregants. HO ascosporal colonies consisting primarily of $M A T a / M A T \alpha ~ H O / H O$ diploids were recugnized by their failure to mate with heterothallic haploid MATa and MATa test strains.

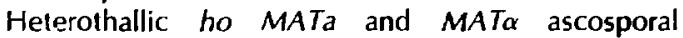
colonies were recognized by their ability to mate with heterothallic test strains of opposite mating type. A total of 53 tetrads in which all four spores survived were analyzed. There were two MATa/MATa HO/HO diploidized ascosporal colonies per tetrad: 43 were $M A T a / M A T \alpha$, rec461/rec46-1, HO/HO and 63 were MATa/MATa, REC 46/REC 46, HO/HO. The rec46-1 mutation thus does not block $H O$ directed interconversion of mating type alleles.

Table 3. Spcrulation deficiency of rec46-1/rec46-1 hybrids.

\begin{tabular}{|c|c|c|c|c|c|}
\hline Hybrid & Genotype & $\begin{array}{l}\% \text { Four and three- } \\
\text { spored asci }\end{array}$ & $\begin{array}{l}\text { "\% Two and one- } \\
\text { spored asci }\end{array}$ & $\begin{array}{c}\% \text { Non- } \\
\text { sporulated cells }\end{array}$ & $\begin{array}{l}\text { Total } \\
1 \% \text { asci }\end{array}$ \\
\hline$R \times 38$ & $\begin{array}{l}\text { REC46 } \\
\text { REC46 }\end{array}$ & 72.2 & 19.5 & 8.3 & 91.7 \\
\hline$R \times 62$ & $\begin{array}{l}\text { REC46 } \\
\text { rec } 46-1\end{array}$ & 72.8 & 16.6 & 10.6 & 89.4 \\
\hline$R \times 66$ & $\begin{array}{l}\text { REC } 46 \\
\text { rec } 46-1\end{array}$ & 87.4 & 5.8 & 6.8 & 93.2 \\
\hline$R \times 67$ & $\begin{array}{l}\text { REC } 46 \\
\text { ee } 46-1\end{array}$ & 76.1 & 19.4 & 4.5 & 95.5 \\
\hline$R \times 6 B$ & $\begin{array}{l}\text { REC } 46 \\
\text { rector-1 }\end{array}$ & 80.5 & 12.5 & 7.0 & 93.0 \\
\hline RXGI & $\begin{array}{l}\text { rect6-1 } \\
\text { rec } 46-1\end{array}$ & 1.0 & 0.7 & 98.3 & 1.7 \\
\hline$R \times 63$ & $\begin{array}{l}\operatorname{rec}+6-1 \\
\operatorname{rac} t 6-1\end{array}$ & 0.5 & 0.9 & 98.6 & 1.4 \\
\hline RXot & $\begin{array}{l}\sec +6-1 \\
\operatorname{tec} 46-3\end{array}$ & 2.2 & 1.8 & 96.0 & 4.0 \\
\hline $2 \times 65$ & $\begin{array}{l}\sec \div 6-\pi \\
\sec +6-1\end{array}$ & 0.9 & 3.5 & $\% .2$ & 3.8 \\
\hline
\end{tabular}


The data presented above demonstrate that the REC 46 gene controls mitotic chromosomal stability, gene conversion, intragenic recombination, and intergenic recombination. The chromosomal instability and hyperrecombinational phenotype exhibited by rec46-1 strains suggests that the REC46 gene product modulates recombination and prevents accumulation of DNA damage leading to chromosomal loss. This interpretation of the fuiction of the REC46 gene product is consistent with the observation that rec46-1 strains exhibit enhanced sensitivity to killing by $x$ rays and ultraviolet light and are defective in meiosis. ${ }^{2}$

The phenotypes of MATa rec46-1 $n+1$ chromosome VII disomics and MATa/MATa rec461/rec46-1 diploids differ significantly. Both exhibit a five- to ten-fold enhancement of the rate of mitotic gene conversional events. In contrast, the MATa rec46-1 $n+1$ chromosome VII disomic exhibits a one-hundred-fold enhancement in the rates of intergenic recombination and restitution of haploidy while MATa/MATa rec46-1/rec46-1 diploids exhibit only a ten-fold enhancement of the rate of intergenic recombination and no evidence of mitotic chromosomal loss resulting in $2 n-1$ monosomic segregants.

Previous studies have demonstrated that $M A T a / M A T \alpha$ cells are more resistant to the killing effects of $x$ rays than are MATa/MATa and

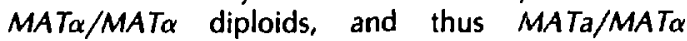
diploids may possess a pathway for repair of $\mathrm{x}$-ray damage that is not present in MATa and MATa haploids or in MATa/MATa and MAT $\alpha / M A T \alpha$ diploids. Operation of this pathway in MATa/MATa rec46-1/rec46-1 diploids may result in repair of the class of prerecombinational lesions, e.g., double strand breaks, that result in chroinosomal loss and high levels of intergenic recombina- tion in MATa rec46-1 $n+1$ chromosome VI disomics. Persisting prerecombinational lesions may account for the ten-fold enhancement in the spontaneous rate of gene conversion, intragenic recombination, and intergenic recombination exhibited by MATa/MATa rec46-1/rec46-1 hybrids. Tests of this hypothesis are in progress.

\section{REFERENCES}

1. Esposito, M.S. Molecular mechanisms of recombination in Saccharomyces cerevisiae; Testing mitotic and meiotic models by analysis of hypo-rec and hyper-rec mutations. In Controlling Events in Meiosis, C.W. Evans, H.G. Dickinson, Eds., Soc. Exptl. Biol. Symp., The Company of Biologists Ltd. Scarborough, North Yorkshire, 38, pp. 123 (1984).

2. Esposito, M.S., Hosoda, J., Golin J., Moise, H., Bjornstad, K., and Maleas, D. Recombination in Saccharomyces cerevisiae: REC gene mutants and DNA-binding proteins. Cold Spring Symp. Quant. Biol. 49, 41-48 (1984).

3. Esposito, M.S., Maleas, D.T., Bjornstad, K.A., and Holbrook, L.L. The REC46 gene of Saccharomyces cerevisiae controls mitotic chromosomal stability, recombination and sporulation: cell-type and life cycle stage-specific expression of the rec46-1 mutation. Curr. Genet. in press (1986).

4. Esposito, M.S., Maleas, D.T., Bjornstad, K.A., and Bruschi, C.V. Simultaneous detection of changes in chromosome number, gene conversion, and intergenic recombination during mitosis of Saccharomyces cerevisiae: Spontaneous and uitraviolet light induced events. Curr. Genet. 6, 5-11 (1982).

\section{THE GENETIC MAP OF YEAST AND THE GENEALOGY OF LADONATONY STIAINS}

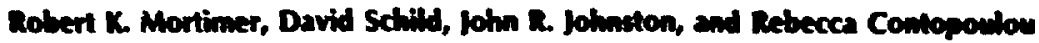

The reast Saccharomyces cerevisiae is currently widely used for biochemical, molecular and genetic research. Besides its itaditional importance in the baking and brewing indusiries, it is increasingly being used for industrial production of recombinam DNu products. The ease by which this yeast can be enetically and molecularty manimolated. cou. pled with its extensive genetic map, have made it panticularly suitable for use in both research and industry. During the past year we have been involved in several areas of yeast reseanch, the pals of which are to improve yeast as a ceneral organism for study. These wers include collating and publishing a new edition of the peast genetic 
map, developing a new mapping method for yeast, constructing a detailed genealogy of many commonly used laboratory strains, and maintaining the Yeast Genetic Stock Center.

Genetic maps of many organisms have been constructed and are frequently being updated as more genes are studied and mapped. The yeast Saccharomyces cerevisize has one of the most extensive maps with over 500 genes mapped. This map is useful in many ways, including 1) estimating the number of chromosomes, since they cannot be reproducibly counted by cytological means, 2) determining that most functionally related genes are not clustered, and 3) examining whether two mutations with different phenotypes are alleles of a pleiotropic gene. In the area of yeast recombinant DNA, genetic mapping has recently gained significance both in cloning genes or regions of the genome by chromosome walking from a closely mapped gene, and in determining that a particular gene has been cloned.

The new recently published genetic map of Saccharomyces cerevisiae describes the location of 568 genes distributed over 16 metacentric chromosomes plus a single gene, $K R B 1$, located on a seventeenth chromosome (Fig. 1). Since our last major review in 1980, 251 genes have been added to the genetic map. In addition, several linkages that had been established only by mitotic or aneuploid analyses have been confirmed by tetrad analysis. Only chromosomes VIII, IX, X, XI, XII, and $X V I$ remain with regions not confirmed by tetrad analysis. Assuming a minimum of $100 \mathrm{cM}$ for these regions, the total minimum length of the yeast map is now $4500 \mathrm{cM}$, which is $100 \mathrm{cM}$ less than our estimate made five years ago. This, and the fact that the total number of mapped genes has increased by $79 \%$ argues that the current lengths of the yeast chromosomes are close to their actual lengths.

Mapping in S. cerevisiae has proved more complex than in most genetically studied organisms because of the high level of meiotic recombination (> 100 crossover events per meiosis) and the large number of chromosomes. Several methods, have been deveioped to simplify mapping in yeast and recently several new methods based on chromo. some loss, mon-disjunction and chromosome transfer have been reported. We have developed a method based on chromosome loss in diploid strains homozygous for a mulation (rad52) invelved in DNA repair.

Sxcharomices cerevisive diploids homonygous to the rad52.1 mutation have previoushy been stomn in our laboratory to lose chromosomes mitotically. Spontaneous events and events following low levels of $x$-ray or methyl-methanesultionate treatment result in monosomic diploids, while higher levels of treatment result in near haploidization. This rad52-1 dependent chromosome loss has been used to develop a new mapping method ${ }^{2}$ which can be used to assign a previously unmapped gene to a chromosome. This mapping method and subsequent tetrad analyses have been used to locate hom 6 on chromosome $X$, ade4 on chromosome XIII, and $c d c 3 I$ on chromosome XV, and to demonstrate that met5, previously assigned to chromosome $V$, actually maps to chromosome $X$; the met $^{-}$marker on chromosome $V$ has been shown to be met6. GAL8O and SUP5, previously assigned to an unmapped fragment, have now been mapped to the right arm of chromosome XIII.

The recent explosion of interest in the genetics and molecular biology of the yeast Saccharomyces cerevisiae has resulted in rapid expansion of our knowledge of this organism. Several lines of evidence have recently shown various heterogeneities among strains in, for example, the distribution of restriction sites, in position and numbers of transposable elements, and in chromosome polymorphisms. To assess the relative contributions to these heterogeneities from recent events, as opposed to differences introduced in the development of the initial breeding stocks, we have developed a pedigree of some of the most common laboratory strains. ${ }^{3}$

We have constructed a genealogy of strain $\$ 28 B C$, from which many of the mutant and segregant strains currently used in studies on the genetics and molecular biology of Saccharomyces cerevisiae have been derived. We have determined that its six progenitor strains were EM93 (Mrak), EM126 (Mrak), NRRL-210 (Wickerhan) and the three baking strains Yeast Foam, FLD, and LK. We have estimated that approximately $88 \%$ of the gene pool of $\$ 288 \mathrm{C}$ is contributed by strain EM93. The principal ancestral genotypes were those of segregant strains EM93-1C and EM93-3B initially distributed by C.C. Lindegren to several laboratories. We have analyzed an isolate of a lypholized culture of strain EM93 and determined its Benotype as MATa/MATa SUC2/SUC2 gal2/GAL2 mal/mal mel/mel CUPI/Cupl FLOI/fiol. Strain EM93 is therefore the probable origin of genes SUC2, gal2, CUPI and thol of $528 B C$. We give details of the current avaiLability of several of the progenitor strains and propose that this genealogy should assist in elucidating the origins of several types of genetic and molecular heterogeneities in Succharomyces. 


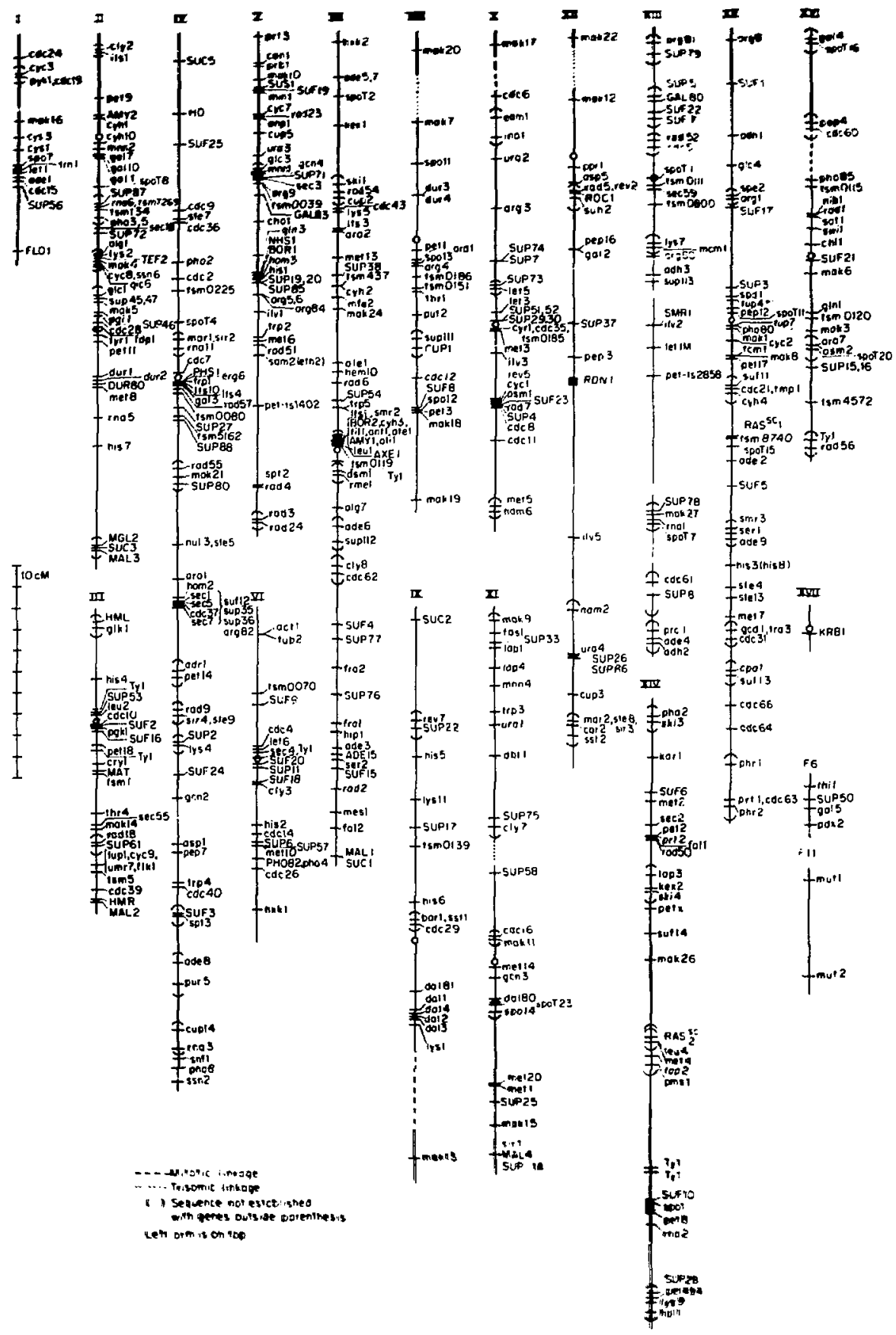

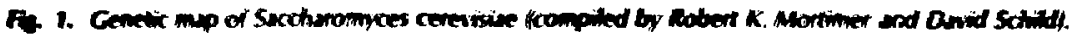


Our Lboratory maintains the Yeast Genetic Stock Center, which tests, stores, and sends out strains of Saccharomyces cerevisize. The current collection contains approximately 700 different strains, 100 of which have been added during the last year, and over $\mathbf{1 0 0 0}$ strains a year are sent out. These strains are distributed to both academic institutions $(\sim B 0 \%)$ and industries $(\sim 20 \%)$, throughout the world ( $\sim 40 \%$ to foreign countries). The fifth edition of the Yeast Genetic Stock Center Catalogue was issued in 1984.

\section{REFERENCES}

1. Mortimer, R.K., and Schild, D. Genetic map of Saccharomyces cerevisiae, Edition 9.

Microbiol. Rev. 49, 181-213 (1985).

2. Schild, D., and Mortimer, R.K. A mapping method for Saccharornyces cerevisiae using rad52-induced chromosome loss. Genetics 110, 569-589 (1985).

3. Mortimer, R,K., and Johnston, J.R. Genealogy of principal strains of the yeast genetic stock center. Genetics-in press.

4. Mortimer, R.K., and Contopoulou, R. Yeast Genetic Stock Center Catalogue, Fifth Edition (1984), Department of Biophysics and Medical Physics, UC Berkeley.

\section{ISOLATION AND MGLECULAR CHARACTERIZATION OF YEAST DNA REPAIR GENES AND THE USE OF A NEW GEL SYSTEM TO STUDY DNA REPAIR}

Robert Mortimer, John Game, David Schild, Mari Aker, Gary Cole, Rebecca

Contopoulou, Vincent Cook, Minh Dang, Leslie Kay, Susan Lovett, and Karen Sitney

\section{STUDY OF DNA REPAIR GENES}

We have previously reported the isolation and characterization of several genes involved in the recombinational repair of DNA damage in yeast. Mutations in these genes, RAD50 to RAD57, result in sensitivity to ionizing radiations, recombination deficiencies, and meiotic problems. These studies have shown that 1) the size of some of these genes are: RAD51 - $1.4 \mathrm{~kb}$, RAD52 - $1.6 \mathrm{~kb}$, RAD54 $2.8 \mathrm{~kb}, R A D 55-1.5 \mathrm{~kb}$. Disruptions of several of these genes have been isolated (RAD51, RAD52, RAD54, RAD55 and RAD57) and since these null alleles are viable, it demonstrates that none of these genes code for essential functions. The null alleles have also aliowed us to more accurately determine the phenotypes of mutations in these genes. For example, using a null allele $R A D 54$ we have recently shown that this gene is necessary for meiosis; previous studies using a leaky mutation in RAD54 indicated incorrectly that this gene played no major role during meiosis. Null alleles of RAD57 have been shown to have much stronger effects on DNA repair at $23^{\circ} \mathrm{C}$ than at $30^{\circ}$ or $36^{\circ} \mathrm{C}$. demonstrating that this gene is normally necessary for repair primarily at lower temperalures; similar results were reported last year for RADSS.

To characterize these genes better at the molecular level, we ane continuing to sequence the cloned genes. We have established the DNA sequence of RAD54 and have determined most of the sequences of RAD51 and RAD55. RAD52 has been sequenced by another group. We are examining the $5^{\prime}$ sequences of these and other repair genes for possible common promoter sequences.

Using the cloned genes we are now determining whether any of these genes are inducible. Using both quantitative Northern hybridization and studies of lacZ-fusions with these genes, we have determined that RAD54 is induced by DNA damage but that RAD52 is not inducible. The RAD54 gene has been found to be induced both by $x$ rays and uv light, but not by heat shock. RAD54 has also been found to be highly inducible by entry of rells into mejosis. Preliminary experiments indicate that RAD51 also is inducible and experiments are underway to confirm this and to determine which, if any, of the olher genes in this group are inducible.

In addition to the RAD50 to RAD57 genes, we have worked on another gene inyolved in $x$-ray repair in yeast that is in a different rad mutant series and is designaled RAD24. The RAD24 gene has been cloned and its map position on chromosome $V$ has been more precisely defined. The only known mutan in this gene confers uv- and $x$-ray 
sensitivity and blocks the cell's ability to undergo meiosis. The gene is very closely linked to another RAD gene (RADI) on chromosome $V$, and we have determined both the order of these two genes on the chromosome, and the genetic distance between them. Using Southern hybridization and genetic complementation tests we have confirmed that the two loci are separate and do not overlap. The original RAD24 clone has been subcloned and gene activity is conferred by an approximately $3-\mathrm{kb}$ fragment, which has itself been subcloned into an M13-related vector suitable for DNA sequencing. In addition, the plasmid-encoded RAD24 gene has been disrupted in vitro and will shortly be used to transform wild-type cells to make RAD24 deletion mutants for biological characterization.

\section{ORTHOGONAL FIELD ALTERNATING GEL ELECTROPHORESIS (OFAGE) FOR STUDYING DOUBLE STRAND BREAK REPAIR}

One of the major effects of ionizing radiation on the chromosomes of cells is the induction of DNA double strand breaks. These breaks result in chromosome aberrations and very frequently in cell death. The standard procedures for assaying double strand breaks have been through the use of neutral sucrose gradients and neutral elution procedures. These methods, while informative, yield only average information about the effects on the DNA complex of an organism. They record a decrease in a average molecular weight and, if repairs occur, a corresponding increase in molecular weight. Recently, a procedure has been developed for electrophoretically separating individual yeast chromosomes $(200-2000 \mathrm{~kb})$ based on alternating field orthogonal gel electropheresis, ${ }^{1}$ and we have adapted this new tecinnique for studying doublestrand break repair and chromosome rearrangements (Fig. 1). We have shown that a dose of $x$ rays $(48,000 \mathrm{rad})$ decreases the intensity of individual chromosome bands due to breakage; the relative decrease increases with chromosome size. The fragments produced by chromosome breakage appear as a broad smear in the 100kb-1000kb region. The distribution of fragments agrees very

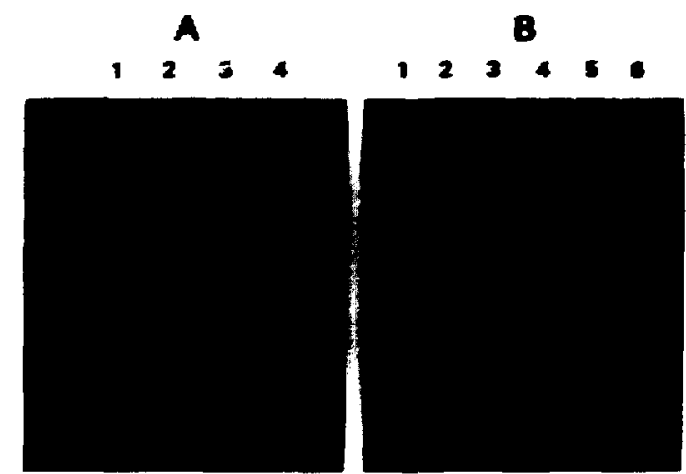

Fig. 1. (A) Effect of irradialing wild type diploid cells with 48 krad and allowing for repair at $30^{\circ} \mathrm{C}$; samples:

1. 0 krad, $1-0$.

2. $48 \mathrm{krad}, t=0$,

3. $48 \mathrm{krad}, \mathrm{t}=5 \mathrm{hr}$,

4. $0 \mathrm{krad}, \mathrm{t}=5 \mathrm{hr}$.

(B) Effect of irradiating rad54.3 mulant dipoid cells with 48 krad and allowing for repair at $23^{\circ} \mathrm{C}$ and at $36^{\circ} \mathrm{C}$; (rad54-3 mutants are sensitive $10 \times$ rays at $36^{\circ} \mathrm{C}$, but less so at $23^{\circ} \mathrm{C}$ ). Samples:

1. $0 \mathrm{krad}, \mathrm{t}=0$,

2. $48 \mathrm{krad}, t=0$,

3. $0 \mathrm{krad}, \mathrm{f}=5 \mathrm{hr}, 23^{\circ} \mathrm{C}$,

4. $48 \mathrm{krad}, \mathrm{t}=5 \mathrm{hr}, 23^{\circ} \mathrm{C}$.

5. $0 \mathrm{krad}, \mathrm{t}=5 \mathrm{hr}, 36^{\circ} \mathrm{C}$,

6. $48 \mathrm{krad}, t=5 \mathrm{hr}, 36^{\circ} \mathrm{C}$.

$(\times 81850-10247)$

well with target theory predictions about breakage of the different chromosomes. If a period of repair is allowed before assay, wild-type cells show a return to a near normal distribution of chromosome intensities. However, three $x$-ray sensitive mutants, $R A D 51, R A D 52$, and $R A D 54$, failed to repair the damage and, in fact, degraded the broken fragments. Wie are currentiy further adapting the OFAGE technique for studying double strand break repair in mammalian cells.

\section{REFERENCE}

1. Carle, G.F., and Olson, M.V. An electrophoretic karyotype for yeast. Proc. Natl. Acad. Sci. (USA) 82, 3756-3760 (1985). 


\section{ISOLATION Of DNA FACMENTS CONTANING REMLCATION FONKS BY TWO

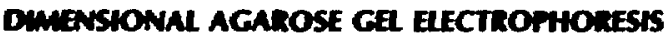

\section{Priscilla K. Cooper and Vincent Ling}

The fundamental goal of our research program is to understand on a molecular level the interaction between DNA repair processes that promote survival after damage and other processes that affect mutation resulting from such damage. In Escherichia coli, and probably in eucaryotic cells as well, the mutagenic or lethal consequences of any particular lesion in DNA depend on cellular processing of that lesion. Our particular interest is in those processes in $E$. coli that are facets of regulatory networks of genes induced by the damage itself, especially the SOS regulatory network, which is induced by a variety of DNA-damaging treatments that interfere with replication, and the adaptive response, which is induced by DNA damage from exposure to chemical alkylating agents.

Our previous work has identified and characterized on SOS-regulated inducible repair process, long patch excision repair, and suggested that it may be the primary process effecting resistance both to uv irradiation and to alkylation damage. We are currently investigating the nature of the small fraction of damage sites where long patch repair occurs as one approach to understanding the means by which inducible processes promote survival. Results of a variety of experiments have suggested that one requirement for the induced long patch process may be in repair of lesions introduced in the vicinity of DNA replication forks. In principle, such lesions would be expected to pose special problems for the constitutive excision repair process because of the unwinding and separation of the complementary strands. In order to test the hypothesis that the induced excision repair process is able to effectively repair such lesions, a method is required that would allow resolution of DNA fragments containing replication forks from the rest of the genome. We have recently developed such a technique, which is described here.

Two-dimensional agarose gel electrophoresis has previously been used to separate branched DNA molecules arising as intermediales in recombination from linear forms of DNA.' The separation is based on the iact that the mobility of branched siructures in agarose difiers from that of linear double-siranded molecules as a function of voltage and gel concentration. In principle this altered mobility shoukd be true for three-ammed replicalion iorks as well as for model $X$ siructures. Honeves,
The situation is more complex when analyzing genomic DNA for replication forks, since the forks will exist randomly throughout the genome, i.e., they will be contained in DNA fragments of a range of molecular weights. In addition, any given DNA fragment can be expecied to have varying amounts of fork structure depending on how far the replication fork has traversed through that segment of DNA at the time of isolation. A further complication is that replication forks from a variety of sources have been shown to possess varying degrees of single-stranded character. Since singlestranded DNA has higher mobility in agarose than native DNA but a branched structure should retard migration, the expected behavior of replicating DNA during electrophoresis is not entirely obvious from a priori considerations. Nevertheless, we have succeeded in adapting the two-dimensional gel technique to achieve resolution of nascent DNA as an arc, identified by the autoradiographic detection of a pulse label, distinct from the arc containing the bulk of the DNA.

The technique we are using is as follows. Exponentially growing cells are briefly labeled with a high specific activity of ${ }^{3} \mathrm{H}$-thymidine and either harvested immediately into ice-cold "stop mix" or filtered to remove the label and then grown for a period in unlabeled medium before harvesting. The cells are washed, lysed, digested with proteinase $K$, and the DNA is purified by phenol extraction followed by chloroform-isoamyl alcohol and ether extractions. The DNA is then restricted to completion with Eco RI and electrophoresed through a $0.3 \%$ or $0.7 \%$ agarose horizontal slab gel at $1 \mathrm{~V} / \mathrm{cm}$. The lanes are excised and each is set into a second dimension gel of $1.5 \%$ agarose with $0.5 \mu \mathrm{g} / \mathrm{ml}$ of ethidium bromide and electro. phoresed at $2 \mathrm{~V} / \mathrm{cm}$. After photographing, the gels are immersed in a fluorographic enhancer, dried, and auloradiograms are prepared from them.

Figure $I$ is a photograph of the ethidium fluorescence in the firsl and second dimension gels, with a larder of molecular weight markers run in the first dimension shown at the top of the photograph. As can be seen from the ethidium fluorescence in the second dimension gel, the bulk of the DNA migrates in a single arc. However, the autoradiugram of the DNA from cells harvested immediately after pulse labeling (Fig 2A) reveals that a 


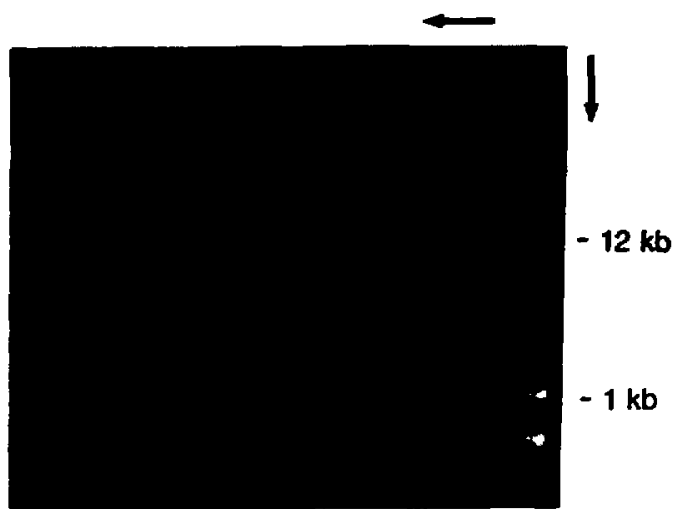

Fis. 1. Ethidium fluorescence of ECo RI restricted genomic DNA of E. coli electrophoresed in first and second dimension agarose gels as described in text. Top lane: size markers in the first dimension.

(XBB 850-9933)

large proportion of the radioactive label is contained in a second arc of DNA, invisible by ethidium fluorescence and therefore representing a very small proportion of the total genomic DNA. This second arc must be derived from replicating DNA, since interposing a period of growth in unlabeled medium between the pulse label and the harvest causes essentially all of the radioactive label to "chase" into the main arc (Fig. 2B). In fact,

(A)

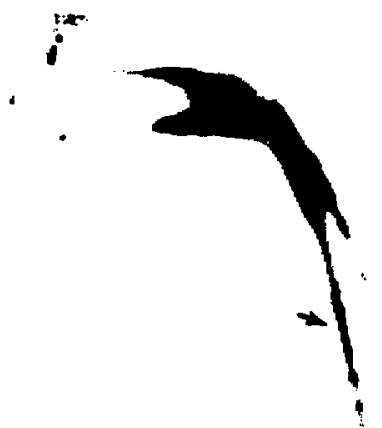

$$
2 \min 4 \cdot \pi
$$

even inadequate stopping conditions prevent visualization of the second arc, emphasizing its mascent nature. We have reproducibly oblained the second arc from a number of different DNA preparations and after restriction with a variely of different enzymes having six-base recognition sequences. and also after restriction with Dph I, which cuts at fully methylated GATC sequences. . In addition, various combinations of altering gel concentration and voltage between the two dimensions have allowed resolution of the nascent DNA.

Visual inspection of the relationship between the two arcs suggests that the nascent DNA has a higher mobility than the main arc. In fact, reconstruction experiments using heat-denatured restriction digests of labeled DNA have shown that the second arc migrates with single-stranded DNA, and digestion with $\mathrm{S} 1$ nuclease prior to electrophoresis removes the second arc. There are two possible interpretations of these findings: 1) the arc of nascent DNA is composed of single-stranded fragments arising from disruption of replication forks during purification; or 2) it includes replication forks having single-stranded regions, and the mobilicy of a large proportion of the material in it results from the combination of effects of branched structure and of single-strandedness. The apparent size distribution of the DNA in the second arc, which includes material migrating even more slowly than a single-stranded $23-\mathrm{kb}$ fragment, is very difficult to

(B)

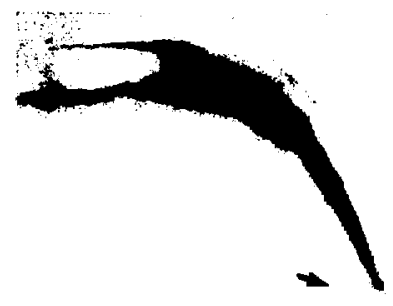

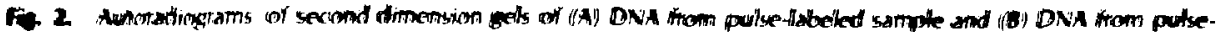

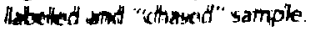


reconcile with the first interpretation. Indeed, as would be predicted if the second possibility were correct, after S1 digestion at least a portion of the label from the nascent DNA is transferred to the main arc rather than disappearing. Moreover, the second arc persists after digestion with a singlestrand exonuclease (exonuclease 1), as would be expected if at least some of the single-stranded regions are internal to double-stranded structures. We have also employed binding to nitrocellulose filters as a sensitive means of detecting small single-stranded regions in DNA to further assess the structure of the nascent DNA. While $20-25 \%$ of the pulse label in the restricted DNA sample is single-stranded by the criterion of sensitivity to digestion by $\mathrm{S} 1$ nuclease, we find that this relatively small fraction of the newly synthesized DNA that is single-stranded results in retention on nitrocellulose of $60-65 \%$ of the pulse label. This result confirms the interpretation that the S1-sensitive nascent DNA represents single-stranded regions covalently attached to predominantly double-stranded structures of newly synthesized DNA rather than consisting of single-stranded fragments.

Analysis by electron microscopy of the structure of replication forks in a variety of systems ranging from bacteriophage lambda to Drosophila cleavage nuclei has established the frequent occurrence of single-stranded connections both at branch points and on daughter arms but distal to the branch point. Types of structures observed are illustrated in the diagram of Fig. 3, taken from Kriegstein and Hogness. ${ }^{2}$ Structures $B$ and $C$, in which a singlestranded "whisker" protrudes from the fork, are

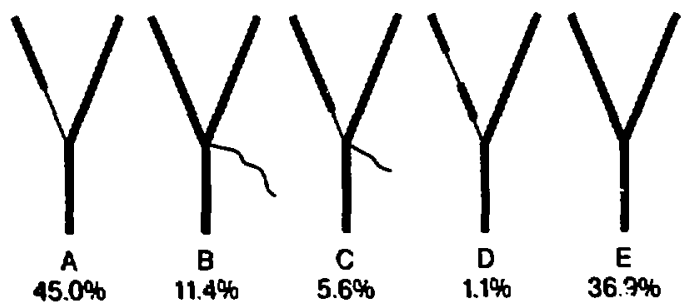

presumed to arise by branch migration of structure A. In order to allow EM verification of the structure of the nascent DNA that we are resolving electrophoretically, we have taken advantage of the preferential binding to nitrocellulose of the nascent DNA fraction to enrich our DNA restriction digest for pulse-labeled DNA. The restricted pulselabeled DNA that bound to nitrocellulose under high salt conditions was eluted, dialyzed, and concentraled, and a portion of it was analyzed by two-dimensional gel electrophoresis as described. After the enrichment procedure, all of the labeled DNA was found to migrate in the position of the arc of nascent DNA. Another portion of the enriched DNA was examined by electron microscopy, with restricted genomic DNA (i.e., not enriched for nascent structures by filter binding) serving as a contro! comparison. Figure 4 shows representative examples of the types of forked molecules seen with much higher frequency in the enriched sample than in the control; these are analogous to the structures diagrammed in Fig. 3.

Taken together, these results make it clear that the Iwo-dimensional gel system that we have developeci does resolve DNA replication forks from linear DNA fragments. We now plan to apply this technique to testing the hypothesis that induced long patch repair occurs in replicating DNA. The technique will also allow us to directly determine the fate after DNA damage of pre-existing replication forks in both SOS induced and noninduced cells, thus providing a test of the model that induction enhances postdamage replication ability by promoting release of blocked forks. When combined with enrichment of the starting DNA preparation for nascent structures followed by hybridization of the electrophoretically isolated replicating DNA with appropriate cloned probe DNAs, this technique will also allow us to detect the replication of specific genes under a variety of circumstances.

Fig. 3. Classes of replication forks observed in previous work in a variety of systems ranging from bacteriophage lambda to Drosophila. Frequencies are from Kriegstein and Hogness."

(XBL 8511-8594) 

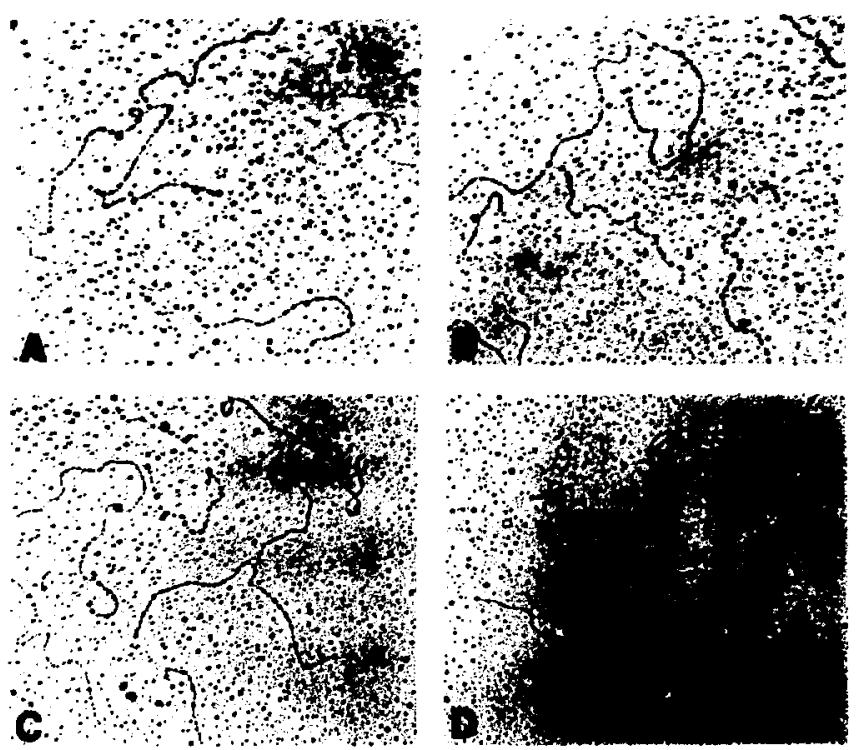

Fis. 4. Representative electron micrographs of replication forks observed in DNA migrating in the position of the pulse label in the autoradiogram of Fig. 2A. Structures shown are: (a) all or nearly all double-stranded; (b) singlestranded arm; (c) single-stranded connection at the fork; and (d) all double-stranded but with single-stranded "whisker."

(XBB 8512-9911A)

\section{REFERENCES}

1. Bell, L., and Byers, B. Anal. Biochem. 130: 527-535 (1983).
2. Kriegstein, $H_{\text {., }}$ and Hogness, D. Proc. Natl. Acad. Sci. USA 71, 135-139 (1974).

\section{ALKYLATION OF POLYNUCLEOTIDES IN VITRO AND IN VIVO}

\section{Bea A. Singer, Sylvia J. Spengler, Frank Chavez, and Heinz Fraenkel-Conrat}

The work done under this grant for the last 15 years has explored multiple aspects of how simple non-metabclically activated animal (and human) carcinogens exert their biological effects. We found that all nitrogens and oxygens can react, to varying extents, in model systems, ir human cells in culture, and in whole animals, 1,2 Subsequent work has investigated not only the formation of the 13 possible adducts and 16 possible phosphotriesters, but also their persistence, mechanisms of repai,. and mutagenic polential.
This report of work at LBL since April 1985 focuses on the effect of a series of $\mathrm{O}^{4}$. alkylthymidines on the extent and fidelity of replication using $E$. coli DNA polymerase I (Pol I) under conditions that enhance rather than diminish fidelity.

We originally proposed, based on data of DNA from ethyinitrosourea-treated rats, that $\mathrm{O}^{4}$-ethyl dT was likely to be an initiating event in carcinogenesis." More recently, work from the Swedberg Lboratony ${ }^{34}$ has indicated the persistence of both 
$\mathrm{O}^{6}$-methyl dT and $\mathrm{O}^{4}$-ethyl dT as implicated in the initiation of hepatocellular tumors from animals treated with similar carcinogens.

We synthesized the deoxynucleoside triphosphates with methyl, ethyl, or isotropyl groups on the $\mathrm{O}^{4}$ of thymidine. Using a poly(dA-dT) template as a model for B-form DNA, or activated DNA as a template, we found that all three alkyl-dTTPs could substitute for dTTP to a high extent when dTTP was present. Extensive synthesis occurred, although the rate was slower than when $\mathrm{AATP}$ and $\mathrm{dTTP}$ were used with Pol I alone. The synthesized poly(dAdT, $\mathrm{O}^{4}$-alkyl dT) polymers were then used as templates for misincorporation studies. In contrast to the level of misincorporation directed by Pol I from poly(dA-dT), which was $1 \mathrm{dG} / 80,000$ nucleotides synthesized, the polymers containing $\mathrm{O}^{4}$-alkyl dT caused misincorporation ranging from 1 dG/600 nucleotides (methyi) to $1 \mathrm{dG} / 5000$ nucleotides (isopropyl). ${ }^{5}$

The next question was how effective were the $\mathrm{O}^{4}$-alkyl dTTPs in the absence of any other source of DTTP. Both the rate of polymer elongation and the $K_{m} s$ were examined, and it was found that the size of the alkyl group played a major role. The rates at three temperatures and the $K_{m}$ data are shown in Figs. 1 and 2.

The temperature studies were undertaken to test whether the early termination of synthesis shown in Fig. 1(a) could be due to the formation of a frayed and ineffective $3^{\prime}-\mathrm{OH}$ primer terminus. If this were true, then lower temperatures of synthesis should favor stabilization of the terminus and allow synthesis to proceed further. This was, in fact, the case, since at $0^{\circ}-10^{\circ}$ the relative extent of synthesis with the $\mathrm{O}^{4}$-derivatives was significantly higher than at $30^{\circ}-40^{\circ}$. The high $K_{m}$ values for the alkyl triphosphates indicated that they would not complete well with dTTP. Thus, the effect of this type of adduct is likely to arise from formation of the specific adduct in DNA rather than incorporation of a carcinogen-modified dTTP from the nucleotide pool.

We have also construcled a model for the probable structure of DNA that permits the insertion of the modified dTs opposite dA while apparently maintaining the normal struclure and appropriate energy of the helix (Fig. 3). This propped-open struclure which allows formation of a single hydrogen bond is suggested to explain proton nmr data on $\mathrm{O}^{+}$exchange rates.

Another plan designed to test the effect of $\mathrm{O}^{4}$. Ikyl dTTPs in a biokgical system is a cooperaline project with Dr. L. Loeb in the Department of

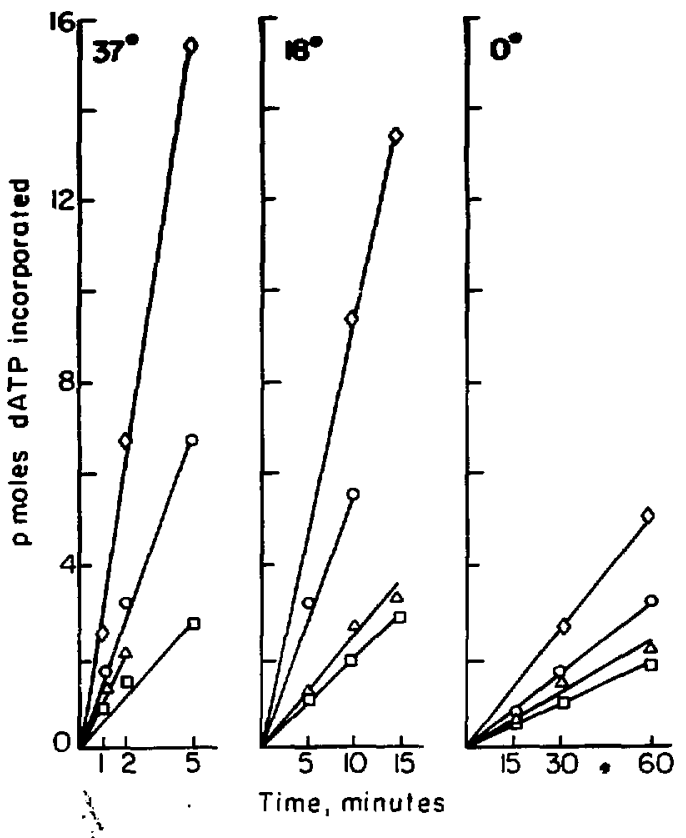

Fig. 1. Fate of incorporation of $O^{4}$-alkyl dTTPs during the linear portion of sy nhesis by Pol 1 at (a) $37^{\circ},(b) 18^{\circ}$, and (c) $0^{\circ} . \diamond, d T T P ; 0 . m^{4} d T T P ; \Delta, e^{4} d T T P ; \square, i p r^{4} d T T P$. The level of incorporation in the absence of an; dTTP has been substracted from these points.

(XBL 8510-8528)

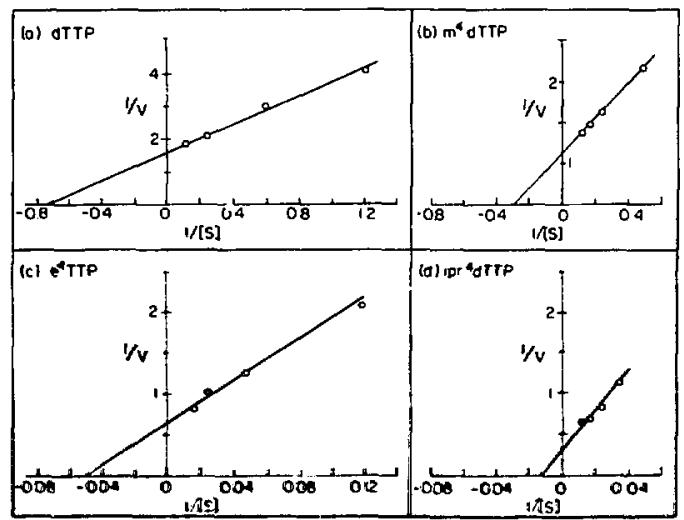

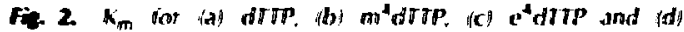

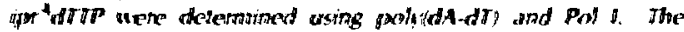

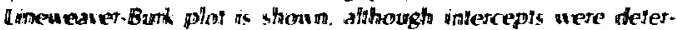
mined on the direct lineat plot at fisernthal and Comish-

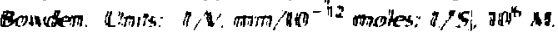

(1) 81258008529$)$ 
(a)

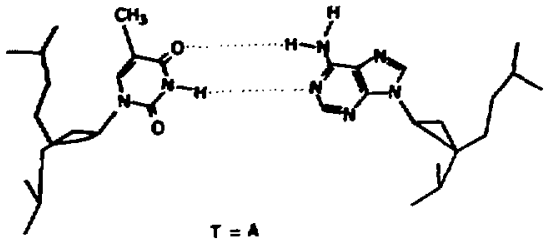

(b)

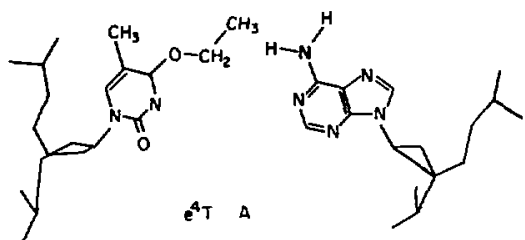

(c)

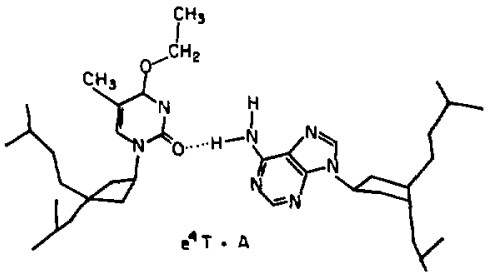

Fis. 3. Base-pairing of $d T$ or $O^{4}$-ethyl $d T$ with $d A$. (a) Watson-Crick T-A base pair: (b) $\mathrm{e}^{4} T$ (syn) and $A$ in the Watson-Crick positions; (c) $\mathrm{e}^{4} T$ (syn) and $A$ in an open-helix position in which one H-bond can be forned. The position (syn to N3) and the size of the ethul group are based on data of Birmbaum (p.c.).

(XBL 8510.8530)
Pathology, University of Washington. This work utilizes methods for site-directed insertion of the desired alkyl-dTTP within an amber codon of $\$$ X174 and subsequent biological assay for revertants in E. coli spheroplasts. . Preliminary data indicate that the kinetics of incorporation opposite dA of the $\mathrm{O}^{4}$ alkyl dTTPs follow the same dependence on size shown in the poly(dA-dT) experiments.

Similarly designed experiments that compare the properties of various $\mathrm{O}^{2}$-alkyl dTTPs are in progress. In an earlier report, we found the $O^{2}$-methyl dTTP would be substituted for dTTP, but ihe miscoding of the resulting polymer was lower than that observed for polymers containing $\mathrm{O}^{4}$-methyl dT. ${ }^{7}$

\section{REFERENCES}

1. Singer, B. Nature 264, 333-336 (1976).

2. Singer, B., Spengler, S.J., and Bodell, W.J. Carcinogenesis 2, 1060-1073 (1981).

3. Swenberg, J.A., Dyroff, M.C., Bedell, M.A., Popp, J.A., Huh, N., Kirstein, J., and Rajewsky, M.F. Proc. Natl. Acad. Sci. (USA) 81, 1692-1695 (1984).

4. Richardson, F.C., Dyroff, M.C., Boucheron, J.A., and Swenberg, J.A. Carcinogenesis 6, 625-629 (1985).

5. Singer, B., Spengler, S.J., Fraenkel-Conrat, H., and Kuśmierek, I.T. Proc. Natl. Acad. Sci. (USA) 82, in press (1986).

6. Mirau, P.A. and Kearns, D.R. Biopolymers 24, 711-724 (1985).

7. Singer, B., Sági, J., and Kuśmierek, I.T. Proc. Natt. Acad. Sci. (USA) 80, 4884-4888 (1983). 


\section{EFfECTS OF ALKYLATING CARCINOGENS ON HUMAN TUMOR CELS IN CULTURE}

\section{Regine Goth-Goldstein and Mildred Hughes}

Chemicals that can induce tumors at various sites in experimental animals are useful tools to examine the process of carcinogenesis. The initial interaction of a carcinogenic agent with cells can be studied in cell culture systems. It is primarily the reaction of a carcinogen with cellular DNA that results in the expression of biological damage such as cell killing, chromosomal aiterations, mutations, and malignant transformation. Cells possess, however, various defense mechanisms to cope with the damage induced by carcinogens, and the initial DNA damage can be modified by various DNA repair processes. Alkylating carcinogens like $\mathrm{N}$ methyl-N'-nitro-N-nitrosoguanidine (MNNG) are the best-studied carcinogens with regard to their reactivity with cellular components and particularly with DNA. They produce a wide variety of lesions in DNA. The biological relevance of some of these lesions could be identified in bacteria through studies with mutants that are defective in the repair of specific alkylated DNA bases. Thus, it could be concluded that alkylation of the N3 position of adenine or guanine produces a lethal lesion, whereas alkylation of exocyclic oxygens of DNA bases leads to mutagenic lesions with 06methylguanine (06-MG) being the major contributor to the mutagenic effects of methylating agents. In mammalian cells, the absence of defined mutants in DNA repair of alkylation damage hias hindered the evaluation of the biological itfect of alkylation lesions. A good correlation exists between the amounts of 06-MG icrmed by different agents and their mutagenicity or carcinogenicity. Further, the persistence of this lesion and of 04-alkylthymine in various tissues correlates well with tumor incidence in these tissues. It is less clear what DNA lesions contribute to cell killing. A group of human tumor cell strains fail to support the growth of MNNGdamaged adenovirus. Cells of this phenotype, which has been lermed Mer-, have other characteristics in commor, among them that they are hypersensitive to the cylotoxic effects of alkylating agents, and that they are unable to repair 06-MG. From this it has been concluded that in human cells O6-MG is not only a mutagenic lesion as in bacteria, but also a lethal lesion. The widely stu- died HeLa S3 cell line that originated from a human tumor, has the Mert phenotype, but a Mer - variant (HeLa MR) has arisen in one laboratory. This offers the possibility to study Mer- and Mer+ cells of otherwise similar genetic background. Our work in the last year has concentrated on comparing the response of HeLa Mert and Mervariants to alkylating agent.

HeLa MR cells are extremely sensitive to the cytotoxic effect of MNNG as measured by their colony-forming ability after a 1-hour exposure to MNNG. The ratio of the $D_{10}$ (the dose that reduces survival to $10 \%$ ) of the two variants is 30 (see Fig. 1, open symbols). We found previously that Chinese hamster ovary $(\mathrm{CHO})$ cells that survive a highly toxic dose of MNNG have a permanently increased resistance to the cytotoxic effects of this agent. We wanted to determine if this change in sensitivity is unique to the $\mathrm{CHO}$ cell line or if it is a general response, also occurring ini HeLa cells. The finding was surprising. When the two HeLa variants were treated with a highly' toxic dose of MNNG, the surviving population of HeLa S3 cells was equally or even slightly more sensitive to a second exposure of MNNG, whereas the surviving HeLa MR population was much more resistant to MNNC with a survival dose-response similar to that of HeLa S3 (see Fig. 1, closed svmbols). From a number of clones derived from single cells of the pretreated HeLa MR pcpulation, the majority had the Mer+ phenotype in respect to MNNG sensitivity. These clones were also more resistant to methylnitrosourea and ethylnitrosourea, but they were as sensitive as the untreated HeLa $M$ ? , cells to chloruethylnitrosourea (CNU), the parent compound of various chemotherapeutic alkylating agents.

The lethal lesions of CNU are DNA crosslinks which are formed from monofunctional DNAadducts. These precursors of DNA crosslinks can be repaired by the same enzyme that repairs 06MG. Mer+ cells that have this enzymatic activily can prevent the formation of DNA crosslinks, whereas in Mer-cells that are deficient in this repair enzyme, lelhal crosslinks are formed. The finding that preireated HeLa MR cells are seasitive 


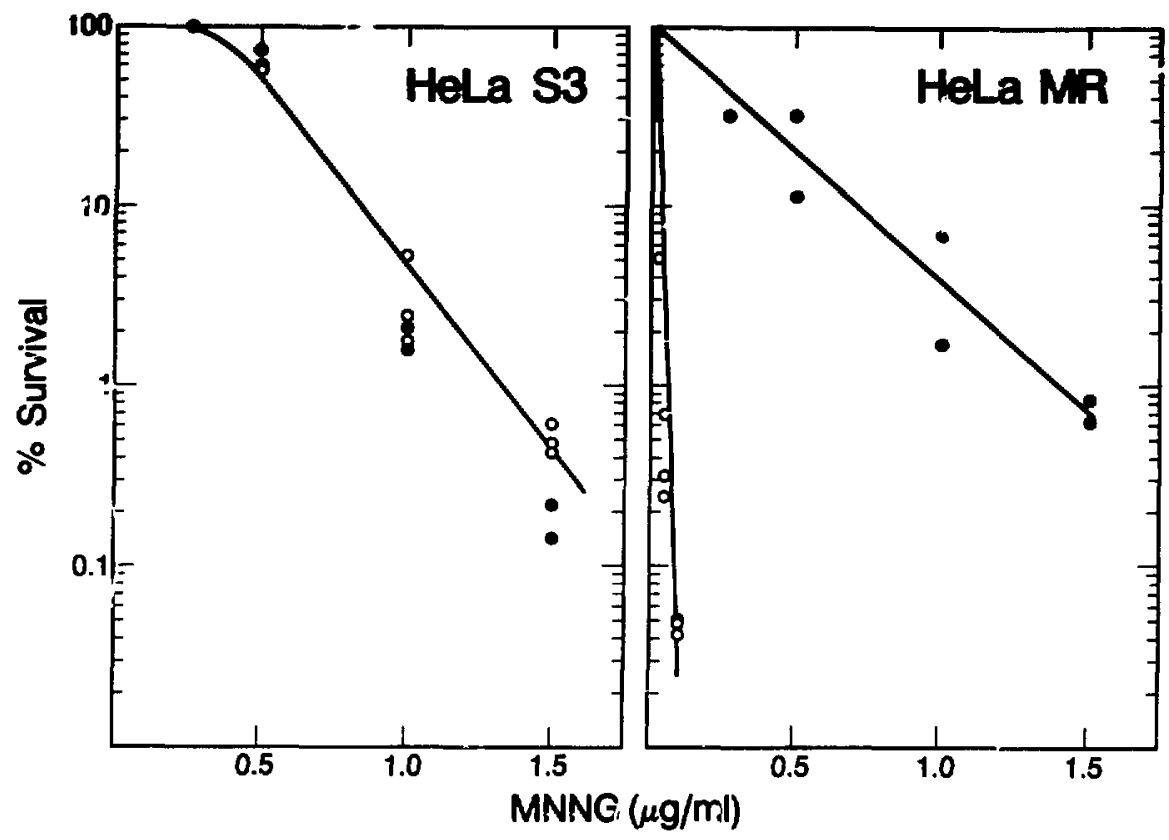

Fig. 1. Cell survival of HeLa 53 (left panel) and HeLa MR (ritht panel) after a 1-hr treatment with MNNG; 0 , survival of nonpretreated cells; $\cdot$, survival of cell population that survived a highly toxic dose of $M N N G(2 \mu \mathrm{g} / \mathrm{ml}$ in case of Hela $S 3,0.2 \mu \mathrm{g} / \mathrm{ml}$ in case of HelaMR) 3 weeks prior 10 second exposure.

(XBL $8511-8586)$

to CNU indicates that these cells are lacking the 06-MG repair enzyme. We could show that this is in fact the case. When DNA methylated with ${ }^{14} \mathrm{C}$ MNNG was incubated with cell extracts of the HeLa variants and the methylated DNA bases quantified by HPLC separation, 06-MG was removied only during incubation with HeLa S3 extract.

This shows that the two characteristics of the Mer- phenotype, hypersensitivity to alkylating agents and inability to repair 06-MG, can be uncoupled, and indicates that $06-\mathrm{M} 6$ is not a lethal lesion.

\section{CARCINOGENIC POTENCY}

Lois Swirsky Gold, Aruce N. Ames, Renae I. Magaw, Catherine Wright, Joan Schwalbe", Georganne Dackman, Mark Dlumenthal, and Thomas H. Slone

Eifons to use animal bioassays in the evaluation of the potential health sisk of chemicals to humans have beeri hampeted by the lack of a standardized

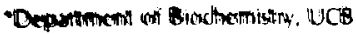

method of comparing experimentit results. Expersmental protocols as weil as the type of information: reponed in the literalure are quile diverse. Nior over, quanlitative estimales of carcinogenic potency. have not been made for large numbers of chenical 
carcinogens. Our Carcinogenic Potency Database is an attempt to quantify and standardize the animal bioassay literature and io organize it systematically.

\section{TO 50: A NUMERICAL INDEX OF CARCINOGENIC POTENCY}

As an index of carcinogenic potency, we have secommended the $J D_{50}$, or tumorigenic dose rate for $50 \%$ of the test animals. Briefly, the $\mathrm{TD}_{50}$ is defined as the chronic dose rate to induce tumors in half the test animals (in the absence of tumors in the control group). $\mathrm{TD}_{50}$ can be calculated for any single category of neoplasm or any combination of neoplasms. This numerical index permits comparisons of diverse lest results and improves past efforts to estimate carcinogenic potency in two ways. First, our calculation takes into accoulit whatever spontaneous tumor incidence occurs in control animals. Second, where data are available about the time of deatt, and tumor inciderice of each animal, we estimaie a $\mathrm{TD}_{50}$ using this information. This is important because animals given high doses of a chemical frequently die early owing to chemical toxicity rather than to tumors, and failure to account for this early mortality could lead to under-estimates of the true potency. A full statistical description of $T D_{50}$ is given in Sawyer et al. ${ }^{\prime}$ and Peto et al. ${ }^{2}$ We have found that the range of $T D_{50}$ is more than 10-million-fold. For male rats, the range in values is from $101 \mathrm{ng}$ for $2,3,7,8$ tetrachlorodibenz's-p-dioxin (TCDD) to $5.98 \mathrm{~g}$ for Food, Drug \& Cosmetic (FD\&C) Green No. 1.

\section{THE CARCINOGENIC POTENCY DATABASE}

The database of lest results reported in the literature prior to July 1981 was published in a plot format in 1984. 2.3 A supplement that includes experimental results published through December 1982 will appear in the same journal in 1986 . The two publisher databases together contain results of approximately 3,200 experiments on 835 chemicals.

The database is readily accessible for qualitative and quantitative analysis. All positive and negative experiments that fit a se! of standard criteria based on suitability ior estimation of potency have been included in the database, i.e., tests in which 1) exposure was chronic, 2) the route of administration was likely to result in the whole body being exposed, 3) a s-ale compound was administered to the aninals, and 4) a control group was used. Some chemicals for which there is otherwise sufficrent evidence e: carcinogenicity ase not included ii, ior example, the roule of adrministration was skin painting or subcutaneous injection, of if the dose level could not be measured in $\mathrm{mg} / \mathrm{kg} / \mathrm{dry}$.

We have included in the database information about a variety of factors that are important in interpreting bioassays, such as 1) the TD 50 and its statistical significance, 2) the species and strains that have been tested chronically, 31 the roule and duration of compound administration, 4| the tumor types, 5) the proportion of animals with specific types of tumors in dosed and control groups, 6) the shape of the dose-response curve, and 7/ the author's opinion about carcinogenicity.

During the past year we have been updating the database to include experimental results published through December 1983. We have also added several tests that were publisied in earlier years but that were only identified recently in our ongoing literature search.

\section{FRËQUENCY OF CARCINOGENIC 、 PONSE IN ANIMAL TESTS AND CO · ARISON OF RESULTS IN RATS AND MICE}

We are currently compiling a sum, ry of the database in terms of 1) the proportion of chemicals tested in long-term bioassays that have been determined to be carcinogens in at least one experiment, 2) the common site of tumor induction for these chemicals in different species, and 3) the distribution of the carcinogenic potency of these test agents. This summary has importance both as historical information and as specific background data for other analyses. Table 1 summarizes the prevalence of carcinogens among various groups of chemicals in the database; $49 \%$ of all chemicals were carcinogenic in at least one experiment, and this proportion varies slightiy among the subgroups of chemicals examined. Chemicals tested in rats and chemicals tested in mice usually have similar proportions of carcinogens. The proportion of chemicals tested in both rats and mice that was found to be cajcinogenic was generally higher than that found among chemicals tested in only one species.

In a related analysis we focus on the chemicals tested in both rals and mice, and investigate interspecies extrapolation in the following ways: 1) a comparison of the carcinogenic response of rats and mice to the same chemicals, 2) the imporlance of ti.e target siles in one species to the prediction of the carcinogenic response to the same chemical in the other species, and 3) the relationship between the carcinogenic potency of a chemical in one species and its carcinogenicity in the second. By examiring how well tests in rats and mice predict one another we ho, "o gain insight into 


\begin{tabular}{|c|c|c|c|}
\hline \multirow[b]{2}{*}{ Set of chemicats" } & \multicolumn{3}{|c|}{ Arimal species kesled } \\
\hline & Any species & Ras & Mice \\
\hline \multicolumn{4}{|l|}{ 1. Al Chemicats in Database } \\
\hline $\begin{array}{l}\text { All chemicals } \\
\text { NCI/NrP bioassay chemicals" } \\
\text { Lilerature chemicals }\end{array}$ & $\begin{array}{r}379 / 765(49 \%) \\
89 / 186(47 \%) \\
307 / 632(49 \%)\end{array}$ & $\begin{array}{r}252 / 538(47 \%) \\
62 / 100(34 \%) \\
198 / 387(51 \%)\end{array}$ & $\begin{array}{r}225 / 524(43 \%) \\
71 / 100(39 \%) \\
163 / 361(43 \%)\end{array}$ \\
\hline \multicolumn{4}{|l|}{$\begin{array}{l}\text { I. Chemicals Tested in } \\
\text { Both Rats and Mice }\end{array}$} \\
\hline $\begin{array}{l}\text { All chemicals } \\
\text { NCI/NTP bioassay chemicals } \\
\text { Liferature chemicals }\end{array}$ & $\begin{array}{l}179 / 306(59 \%) \\
84 / 174(48 \%) \\
96 / 146(66 \%)\end{array}$ & $\begin{array}{r}132 / 306(43 \%) \\
59 / 174(34 \%) \\
74 / 146(51 \%)\end{array}$ & $\begin{array}{r}150 / 306(49 \%) \\
70 / 174(40 \%) \\
61 / 146(55 \%)\end{array}$ \\
\hline \multicolumn{4}{|l|}{$\begin{array}{l}\text { 111. Chemicals Tested in Both } \\
\text { Sexes of Rats and Mice }\end{array}$} \\
\hline $\begin{array}{l}\text { All chemicals } \\
\text { NCI/NTP bioassay chemicals } \\
\text { Literature chemicals }\end{array}$ & $\begin{array}{r}123 / 226(54 \%) \\
83 / 168(49 \%) \\
37 / 67(55 \%)\end{array}$ & $\begin{array}{r}89 / 226(39 \%) \\
58 / 168(35 \%) \\
30 / 67(45 \%)\end{array}$ & $\begin{array}{r}104 / 226(46 \%) \\
70 / 168(42 \%) \\
31 / 67(46 \%)\end{array}$ \\
\hline \multicolumn{4}{|c|}{$\begin{array}{l}\text { Results given as the number of carcinogens/number of chemicals tested, with } \\
\text { associated percentages. }\end{array}$} \\
\hline \multicolumn{4}{|c|}{$\begin{array}{l}\text { b NCl/NTP bioassays refer to experiments conducted by the National Cancer } \\
\text { Institute/National Toxicology Program. }\end{array}$} \\
\hline
\end{tabular}

the feasibility of interspecies extrapolation of bioassay results, which ultimately includes extrapolation between rodents and man.

\section{ASSOCIATION BETWEEN CARCINOGENIC POTENCY AND TUIMOR PATHOLOGY IN RODENT CARCINOGENESIS BIOASSAYS}

During the past year we have used the $\mathrm{NCl} / \mathrm{NTP}$ bioassays to investigate the association between carcinogenic potency and tumor pathology. Since the $\mathrm{TD}_{50}$ is only one measure of potency, it cannot fully summarize the results of animal cancer tests on a particular chemical. We have, therefore, investigated other measures of hazard, including 1) whether a chemical induces tumors of more than one type in a single-sex sp.cies group of test animal, 2) whether the tumors caused the death of the animal or whether they were discovered incidentally at necropsy after terminal sacrifice, and 3) whether metastases of the induced tumor occurred. The latter two characteristics are indicative of the malignancy and bioloby of the tumor. VJe show that there is no obvious relationship between carcinogenic potency as measured by $\mathrm{TD}_{50}$ and the particular target organ in which tumors were induced, i.e., for the chemicals that cause tumors at a particular site, the calculated $\mathrm{TD}_{50}$ values span a wide range.

We also find that the three hazard indicators described above are sometimes interrelated, but that the $T D_{50}$ values for each hazard vary considerably. In Table 2, we report the distribution of $T D_{50}$ values for chemicals that produce tumors at multiple sites within a single-sex species group, and indicate the groups in which tumors were induced at multiple sites. We observe that multiple-site carcinogens span a broad range of potency. The frequency distribution of potency values for multiplesite chemicals is similar to the distribution for other carcinogens. However, multiple-site carcinogens are significantly more likely than other chemicals to induce tumors in both rats and mice (71\% vs. 39\%) rather than in only one of the species.

A variety of chemical classes are represented among the multiple-site compounds. It is especially noteworthy that chlorinated compounds are underrepresented, since most of them induced only liver tumors. Of 17 chemicals containing only carbon. hydrogen, chlorine, and optionally oxysen, only 3 
Table 2. Carcinonenic povency $\left(T_{0}\right.$ w in ma/kg body w1/dy

of 40 chemicals thet induced lumors if mone then one tanget sife in a sex species group.

\begin{tabular}{|c|c|c|c|}
\hline \multirow[b]{2}{*}{ Chemical } & \multirow[b]{2}{*}{ ID $_{\mathbf{x O}^{\circ}}$} & \multicolumn{2}{|c|}{ Muniple sile groups" } \\
\hline & & $\begin{array}{c}\text { Most } \\
\text { potent }\end{array}$ & Other \\
\hline 23,7,8-tetrachlorodibenzo-p-dioxin & 256 (ng) & FM & \\
\hline 1.2-dibromo-3-chloropropane (inhalation) & 0.11 & MR & (FM,MM,FR) \\
\hline Lasiocarpine & 0.14 & $\mathbf{F R}$ & (MRI \\
\hline Thio-tepa & 0.15 & MR & (FR,MM) \\
\hline Procarbazine. $\mathrm{HCl}$ & 0.19 & FM & (MM,FR,MR) \\
\hline Phenesterin & 0.21 & $\mathbf{F M}$ & (MM) \\
\hline Acronycine & 0.50 & MR & $(F R)$ \\
\hline Estradiol mustard & 0.68 & FM & (MM) \\
\hline 1,2-dibromo-3-chloropropane (gavage) & 0.86 & $\mathbf{F R}$ & \\
\hline 1,2-dibromoethane (inhalation) & 1.10 & MR & $(F M, M M, F R)$ \\
\hline 1,2-dibromoethare (gavage) & 1.26 & $\mathbf{F R}$ & $(F M, M M, M R)$ \\
\hline 2,4-diaminotoluene & 1.43 & FR & \\
\hline Hydrazobenzene & 3.55 & $M R$ & (FR) \\
\hline Cupferron & 5.33 & MR & $(F M, F R)$ \\
\hline $4,4^{\prime}$-thiodianiline & 5.52 & $M R$ & (FM,MM,FR) \\
\hline 5-nitroacenaphthene & 5.98 & FR & (ME,MR) \\
\hline 1,2-Dichloroethane & 11.50 & MR & $(\mathrm{FM})$ \\
\hline 3-amino-9-ethylcarbazole mixture & 11.80 & MR & $(F R)$ \\
\hline Azobenzene & 19.20 & $M R$ & (FR) \\
\hline 2,4,5-trimethylaniline & 20.40 & FR & \\
\hline Dapsonec & 22.40 & MR & \\
\hline$o$-Toluidine. $\mathrm{HCl}$ & 23.30 & $M R$ & $(\mathbf{F R})$ \\
\hline 5-nitro-0-anisidine & 28.10 & MR & (FR) \\
\hline o-Anisidine, $\mathrm{HCl}$ & 31.90 & $M R$ & \\
\hline 1-amino-2-methylanthraquinone & 34.10 & MR & \\
\hline Selenium sulfide & 46.80 & $F M$ & \\
\hline t,1,2-trichloroethane & 47.60 & IM & \\
\hline 1.5-naphthalenediamine & 50.80 & $\mathbf{F R}$ & (FM) \\
\hline p-Cresidine & 69.00 & FM & (FR,MR) \\
\hline 2,4-diaminoanisole sulfate & 72.60 & $M R$ & $(\mathrm{FR})$ \\
\hline Trist 2,3-dibromopropyl)phosphate & 80.10 & FM & $(\mathrm{MM})$ \\
\hline Nitrofenc & 85.30 & MM & \\
\hline Aniline. $\mathrm{HCl}$ & 126.00 & $F R$ & \\
\hline 1.4-dioxane & 126.00 & $\mathrm{FR}$ & \\
\hline 5-chloro-0-toluidine & 134.00 & MM & $(\mathbf{F M})$ \\
\hline 4-chloro-o-phenylenedianine & 197.00 & MR & (FR) \\
\hline 5-nitro-o-toluidine & 242.00 & FM & $(M M)$ \\
\hline Trifluraline $e^{c}$ & 330.00 & FM & \\
\hline $\begin{array}{l}\text { Nitrilotriacetic acid, } \\
\text { Irisodium salt monohydrate }\end{array}$ & 511.00 & MR & (FR) \\
\hline 1, $3^{\prime}$-dimethoxybenzidine $-4,4^{\prime}$-dissocyanate & 742.00 & MR & (FR) \\
\hline Nitrilotriacetic acid & 1450.00 & FR & (MR) \\
\hline 2-amino.anthraquinone & 1490.00 & FM & \\
\hline
\end{tabular}

- The $1 D_{s_{0}}$ value fisted is for the combination of all tumors evaluated in the NCI/NTP Technical Reports as treatment-related. For chemicals causing tumors a multiple sites in more than one sex species group, the lowest composite TD $_{\mathrm{s}}$ value is listed followed by the appropriate sex species group. The other sex species groups are given in parenitheses. $\mathrm{TD}_{50}$ values are cakculated to three significant figures.

- FM = Temale Mouse, MM = Male Mouse, FR = Female Rat, MR = Male Rat.

- For these experiments, carcmogenicity at muliple sites nas evalualed using poroled controts, and no composite TD, was cakulated. The reported TD, salues are those cakuloted on the bass of the most potent individud tanget she 
(10\%) induced muliple tumors, compared to 37 of the 71 (52\%) other carcinogens.

\section{OTHER ANALYSES OF PROGRESS}

Several other analyses using the results of the Carcinogenic Potency Database are in progress. We have identified "near-replicate" carcinogenesis bioassays by selecting from the entire database those cases in which a single compound was tested more than once in a particular species, strain, and sex of rodent by the same route of administration, and have examined the extent of reproducibility of the results in these different tests. We are comparing several methods for summarizing the potency of a carcinogen when several experiments have been conducted and a number of different $\mathrm{TD}_{50}$ values have been estimated for this same chemical. In addition, we have developed a crude index of potential human hazard by comparing the estimated human exposure levels for a compound to the carcinogenic potency in laboratory animals; we are applying this index to occupational exposures in order to determine which compounds stand out and therefore should have high priorities for some sort of regulatory action. In earlier analyses we have examined the relationship in carcinogenic potency between rats and mice ${ }^{4}$ and com- pared two methods of estimation TDso-one based on summary incidence data and the other on lifetable data.

\section{REFERENCES}

1. Sawyer, C., Peto, R., Bernstein, L, and Pike, M.C. Calculation of carcinogenic pctency from long-term animal carcinogenesis experiments. Biometrics 40, 17-40 (1984).

2. Pelo, R., Pike, M.C., Bernstein, L., Gold, L.S., and Ames, B.N. The TD 50 : A proposed general convention for the numerical description of the carcinogenic potency of chemicals in chronic-exposure animal experiments. Environmental Health Perspectives 58, 1-8 (1984).

3. Gold, L.S., Sawyer, C.B., Magaw, R., Backman, G.M., de Veciana, M., Levinson, R., Hooper, N.K., Havender, W.R., Bernstein, L., Peto, R., Pike, M.C., and Ames, B.N. A carcinogenic potency database of the standardized results of animal bioassays. Environ. Health Perspect. 58, 9-319 (1984).

4. Bernstein, L., Gold, L.S., Ames, B.N., Pike, M.C., and Hoel, D.G. Some tautologous aspects of the comparison of carcinogenic potency in rats and mice. Fundam. Appl. Toxicol, to be published in Vol. 5 (1985).

TEMPORAL EXPRESSION OF ROUS SARCOMA VIRUS IN MICROINIECTED EMBRYONIC CHICK LIMBS

Anthony R. Howlett, Betsey Cullen, and Mina J. Bissell

Rous Sarcoma Virus (RSV), is an acutely oncogenic avian retrovirus that can induce the transformation of cultured chick embryo fibroblasts (CEF) and lead to the formation of rapidly growing sarcomatous tumor in neonate and adult birds. ${ }^{1,2}$ In contrast, the introduction of RSV into the limb buds of stage 21-24 shick embryos does not lead to the appearence of tumors within a similar time frame despite the fact that viral spread and proviral integration is generally apparent. ${ }^{3}$

The transforming capacity of RSV has been shown to be accounted for by the activity of a phosphokinase $\left(\mathrm{pp}^{\mathrm{xt}}\right.$ ), that can specifically phosphorylate tyrosine residues and thereby alter the pattem of phosphorylation of cellular proteins that may be involved in the maintenance of cell struc. ture and the control of cell proliferation. It is believed that the activities of the virus, as mediated by integrated src oncogene, are alone sufficient to drive the transformation process. although increasing evidence to the contrary has been accumulating over the years to suggest that the process may be more complex. Tumor promotors have been shown to induce the transformation of CEF cells that have been infected with temperature sensitive (ts) mutants of RSV and cultured at the nonpermissive temperature," and recently studies into the effects of wounding on tumor development in adult birds has strengthened the concept that additional factors that may be of physiological relevence may play role in the mulistase process of vird cancin cenesis.s The possibitity that additional host-related 
factors are required for the transformation of embryonic lissue infected in ovo is of considerable interest. We have undertaken further studies aimed at providing a greater understanding of the developmental regulation of virus-host interactions through an analysis of the process of viral spreading and viral-oncogene expression in the first semester of chick development.

Using a monoclonal antibody directed against a . viral structural protein (designated P196s), kindly provided by T. Pawson, we have determined the kinelics of viral infection and spread following inoculation with $10^{4}-10^{5}$ ffu of RSV into the upper right limb bud of day-4 embryos. P19 positive cells could be detected in coverslip cultures of dissociated limb buds fixed 2 hours after seeding. Using the 2-hour cultures as an approximate measure of viral infection in ovo, the spread of the virus in the embryo was characterized by a saltatory kinetic with two rapid bursis of infectious spread and intervening periods oi stasis (Table 1 and Fig. 1). Very low levels $(<1 \%)$ of viral P19gag positive cells were apparent from freshly dissociated limbs of 5-, 6and 7-day old embryos. A sharp rise in the number of infected cells was demonstrable nn day 8 to give a value of $12 \%$, which was approximately sustained until day 11, when a second sharp increase was observed.

The subsequent spread of virus in the dissociated limb bud cultures was assessed after 24 and 48 hours and after 7 days. Two contrasting patterns, were apparent (Fig. 2). The low levels of virus that were found in the early (5-, 6- and 7-day) embryos remained low through 48 hours in culture and rose to approximately $10 \%$ after 7 days in culture. A similar pattern of infectivity was demonstrated in day-7 embryo cultures up to 48 hours, whereafter

Table t. Kinetics of viral spread in ovo and in cullure.

\begin{tabular}{|c|c|c|c|c|c|}
\hline \multirow{3}{*}{$\begin{array}{l}\text { Embryo age } \\
\text { priot to limb } \\
\text { dissociation } \\
\text { (days) }\end{array}$} & \multirow{3}{*}{$\begin{array}{l}\text { Time elapsed } \\
\text { after RSV } \\
\text { inoculation } \\
\text { (days) }\end{array}$} & \multicolumn{4}{|c|}{$\begin{array}{c}\text { Percentage cells expressing } \\
\text { viral P19gdg }\end{array}$} \\
\hline & & \multicolumn{4}{|c|}{ Hours in culture } \\
\hline & & 2 & 24 & 48 & (7 days) \\
\hline 5 & $\mathbf{I}$ & $<1$ & 2 & $2 / 3$ & 10 \\
\hline 6 & 2 & $<1$ & $N / 0$ & $N / D$ & 13 \\
\hline 7 & 3 & $\mathbf{I}$ & $2 / 3$ & 4 & 68 \\
\hline B & 4 & 12 & 18 & 69 & 100 \\
\hline 9 & 5 & 12 & 21 & 86 & 300 \\
\hline 10 & 6 & $n^{2}$ & 69 & 98 & $\$ 00$ \\
\hline 11 & $\vec{r}$ & 50 & no & 100 & $n$ \\
\hline
\end{tabular}

Ancounat of Vird Spreed in owe

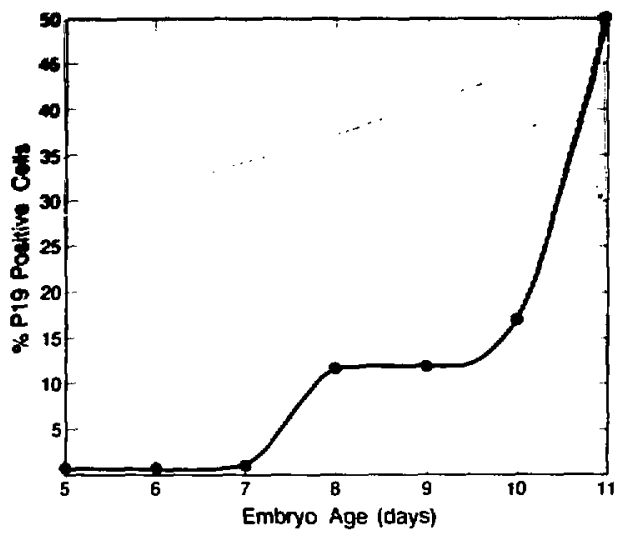

Fig. 1. Infectious spread of RSV in mo as determined by measurement of viral aftigen $P 19$ sag eipresston in dissociated day-5 through dav-11 embryos $(1-7$ days post intection).

(XBL 851()$-8509)$

the percent of $\mathrm{P} 19^{g a g}$ positive cells increased to $70 \%$ within 7 days. Limb bud cultures of days 8 , 9 , 10, and 11 were all found to be mass transformed by 7 days. The major difference in the kinetic of viral spreading between these day-8, -9, -10 and -11 cultures appeared to be the rapidity with which they approached the maximally infected level (Fig. 2).

In conclusion, it has been shown that the spreading of RSV in infected limb tissue follows two

Kinetics of Viral Spread in Culture

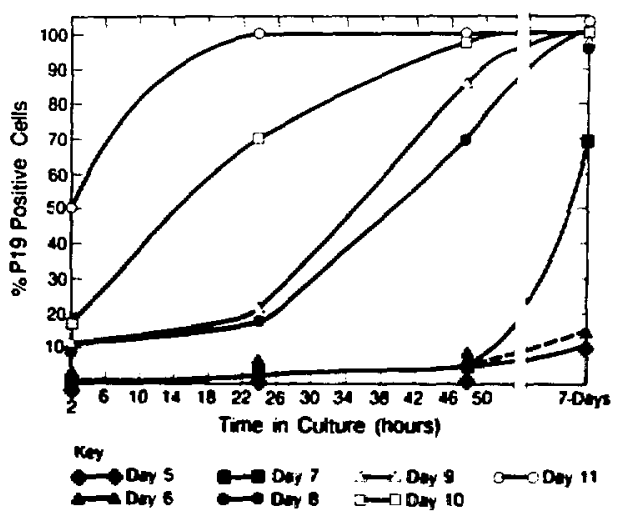

F. 2. Quanitation of the Iransmission of RSV in cultures of limb bud cells dissociated rom dy-5 through day-ll embnos 17-7 diss post infertionl.

(Not a510-8510) 
distinguishable palterns in ovo and in culture. The data would indicate that viral susceptability is developmentally regulated and may be lirked to the program of differentiation of the various component cells of the limb. Preliminary data of P19 localization in frozen sections of day-10 limbs (5 days post-infection) show that viral uptake is not ubiquitous. P19 staining was not apparent in epidermal structures or the cartilagenous component of the limb but was found in areas corresponding to muscle and fibrous connective tissue differentiation. We are now determining the nature of the cell types that are the initial targets of RSV and whether the early transmission of the virus in the limb buds occurs vertically along defined lineages in a developmentally related manner or horizontally by cross infection of other cell types that become susceptible as development proceeds.

\section{REFERENCES}

1. Purchase, H.G., and Burmester, B.R. In Diseases of Pouliny, Hofsted, Ed., lowa Stale University Press, pp. 502-568 (1972).

2. Hanafusa, H. In Comprehensive Virolosy, Vol. $1 C$, Fraenkel-Convat, $H$., and Wagner, $R$., Eds. Plenum Press, New York, pp. 401-482 (1977).

3. Dolberg, D.S., and Bissell, M.J. Nature 309, 552-556 (1984).

4. Bissell, M.J., Hatie, C., and Calvin, M. Proc. Natl. Acad. Sci. 76, 348-352 (1979).

5. Dolberg, D.S., Hollingsworth, R., Hertle, M., and Bissell, M.J. Science, in press (1985).

\section{TUMOR PROMOTERS CAUSE RSV-MEDIATED TUMORS ONLY WITH CONCOMITANT LOCAL IRRITATION}

Mark D. Hertle, David S. Dolberg, Robert E. Hollingsworth, and Mina J. Bissell

We have established previously that wounding is required for Rous Sarcoma Virus (RSV) to cause sarcomas in young chickens. ${ }^{1,2}$ Tumors were induced by the intramuscular injection of $10^{7}$ focus forming units (ffu) in one wing, which yielded the primary tumor within 7 days, and by clipping with a stainless steel clip in the other wing. Tumors due to circulating virus formed at the clip 1-2 days after the primary tumor. We then asked two further questions. Could the virus cross the epithelial layer of the gut, and in the absence of a primary tumor, could circulating virus cause a tumor if the bird were wounded? To answer these questions, we gave 5-day old chicks $5 \times 10^{6}$ ffu of RSV by mouth as an aerosol, and clipped the chicks in both wings as previously described. ${ }^{1.2}$ Tumors formed in about $20 \%$ of the chicks (7 out of 39 ). Chicks that received the virus but no clip did not develop tumors.

This aerosol experiment demonstrates that RSV is capable of effectively crossing the epithelial barrier of the lung and/or gut. Furthermore, the nonmalignant hemorrhagic lesions that frequently occur in these animals demonstrate that the endothelial barrier of the capillaries can be breached without subsequent tumor formation. Even if injury were to alter the compartmentalization of the virus, wounding and wound healing are, in addition, good candidates for a more active role in tumor formation for several reasons. It is suspected, for instance, that wounding is a first-stage promoter in chemical carcinogenesis ${ }^{3}$ and, in some animal models, tumors are found to develop at wounding sites ${ }^{4}$ regardless of the method of carcinogen application. Some human tumors are also found at sites of wounding, ${ }^{5}$ and the process of wound healing involves factors that have been implicated in the process of tumorigenesis and tumor promotion.

Since wounding has been proposed to have tumor promoting activities, we asked whether other knuwn tumor promoters such as phorbol esters might be able to substitute for the co-carcinogenic effect of wounding. In order to ask this question, however, we needed to first minimize the wounding involved in the delivery of the substances being tested. Our approach was to implant a $1-\mathrm{mm}$ 


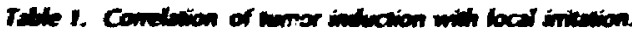

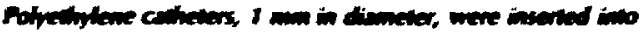

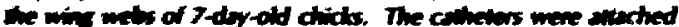

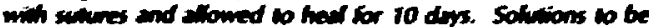
cesed were tren injected throush the catheter in $0.1+m$ l volumes every outer dy for a boed of three iniections. Virus was injected in the opposive wing at the fivine of the first colheser injection. The extent of the focal reaction was judsed by the relative amounts of swelline scabbing and tissue necrosis and is shown as -, no reaction; \pm , minimal reaction; and,$++t$, and +++ , incressing necrosis; a shash indicates that some animals showed a reaction and others did not. Tumors are indicated by the percentuge of animals with tumors in the wing containing the catheler, and $n$ is the number of chickens tested. TPA is 12-O-telradecanoylphorbal 13-acetale; PDD and 4xPDD are phorbol and 4-a phorbol 12,13-didecanoale, respectively.

\begin{tabular}{|c|c|c|c|}
\hline Treatment & $\mathbf{n}$ & $\begin{array}{l}\text { Extent of } \\
\text { local } \\
\text { reaction }\end{array}$ & $\begin{array}{c}\text { Tumors } \\
(\%)\end{array}$ \\
\hline $\begin{array}{l}\text { Medium containing }<10 \% \\
\text { methanol }\end{array}$ & 5 & - & 0 \\
\hline Methanol ${ }^{\mathbf{A}}$ & 6 & $++1-$ & 50 \\
\hline $\begin{array}{l}\text { TPA in medium containing } \\
\quad<10 \% \text { methanol }(0.1 \text { to } 0.5 \mu \mathrm{g})^{\mathrm{b}}\end{array}$ & 4 & - & $0^{c}$ \\
\hline TPA in methanol $(<0.2 \mu \mathrm{g})$ & B & + & 50 \\
\hline TPA in methanol (>0.2 $\mu \mathrm{g})$ & 4 & +++ & 100 \\
\hline $\begin{array}{l}\text { PDD and } 4 \alpha P D D \text { in medium } \\
\qquad<10 \% \text { methanol })(0.1 \text { to } 2 \mu \mathrm{g})\end{array}$ & 0 & - & 0 \\
\hline $\begin{array}{l}\text { PDD and } 4 \alpha \text { PDD in medium } \\
(<10 \% \text { methanol })(5 \mu \mathrm{g})\end{array}$ & 0 & $\pm /-$ & 0 \\
\hline PDD in methanal ( 0.2 to $1 \mu \mathrm{g})$ & 10 & ++ & 50 \\
\hline $4 \alpha$ PDD in methanol $(0.2$ to $1 \mu \mathrm{g})$ & 24 & ++ & 50 \\
\hline Mezerin in medium $(0.1$ to $5 \mu \mathrm{g})$ & 3 & - & 0 \\
\hline Mezerin in medium (10 $\mu \mathrm{g}$ ) & 3 & +++ & 67 \\
\hline $\begin{array}{l}\text { Mezerin in methanol (0.05 to } \\
0.5 \mu \mathrm{g})\end{array}$ & 6 & +++ & 83 \\
\hline
\end{tabular}

- Tumors associated with methanol occurred almost exclusively when there was a local reaction. When there were no reac. tions, there were no tumors.

- The amount refers to the total dose administered through the catheter in each application (total of 3 in the course of 6 days).

c In some cases, the cathelers were pulled out by chickens in the middle of the experiment, and these chickens are not included. polvethefyene tube, approximately $5 \mathrm{~cm}$ in length, into one wing of an eight-dry-old chick. The wing was allowed to heal for 10 days with the catheter in place. Various tumor promoters were then administered through the catheter while the opposite wing was injected with RSV in the usual manner. Tumor promoters dissolved in a minimum amount of solvent (less than 10\% methanod in water) caused tumors only at high concentrations (Table 1) and only when they were accompanied by necrosis and local inflammatory reactions. When the amount of methanol was increased, inflammation and necrosis became evident at the site of administration even with lower concentrations of the tumor promoters. Increasing the concentration of tumor promoters in the presence of high levels of solvent resulted in more prominent inflammation and necrosis with subsequent appearance of tumors (Table 1). Methanol (100\%) also caused tumors in $50 \%$ of chickens. When tumors occurred, they were also preceded by inflammation and edema. We conclude that inflammation, as well as mechanical puncture or laceration, can act as co-carcinogens in RSV-mediated tumor formation. Furthermore, at least in this system, irritation and local injury appear to be the mode by which tumor promoters allow the induction of tumors.

\section{REFERENCES}

1. Dolberg, D.S., Hollingsworth, R.E., and Bissell, M.J. Wounding and its role in RSV-mediated tumor formation. Annual Report 1983-1984, Biology \& Medicine Division, Lawrence Berkeley Laboratory report LBL-18393, p. 216 (1985).

2. Dolberg, D.S., Hollingsworth, R., Hertle, M., and Bissell, M.J. Wounding and its role in RSV-mediated tumor formation. Science 230, 676-678 (1985).

3. Slaga, T.J. Environ. Health Perspec. 50, 3-14 (1983).

4. Rous, R., and Kidd, J.G. J. Exp. Med. 73, 365 (1941).

5. Haddow, A. Adv. Cancer Res. 16, 181-234 (1972). 
Synthesis of mouse milk proteins in mammary gland epithelial cells is regulated not only by lactogenic hormones but also by the extracellular matrix (ECM) and its components. ${ }^{1.2}$ Previously, we have shown that the rate of milk protein synthesis and the level of $\boldsymbol{\beta}$-casein message are increased remarkably in primary mammary epithelial cells from the pregnant gland cultured on floating collagen gels when compared to the same cells cultured on a plastic surface. ${ }^{3,4}$ Recently, we have obtained a mouse mammmary gland cell line, COMMA-1-D (C-1-D) that has retained a degree of milk protein expression and hormonal response, ${ }^{5}$ allowing us to investigate more easily and economically the mechanisms by which hormones and ECM: modulate gene expression.

Milk protein gene expressions in the cell line are monitored at both transcriptional and translational levels. Quantitation of specific mRNA is performed by hybridization of purified cytoplasmic RNA to various ${ }^{32} \mathrm{P}$-labeled cDNA probes using RNA dotting method. Separation and specificity determination of milk proteins are carried out using SDS-poly-acrylamide gel electrophoresis followed by reaction to specific antibodies using Western transfer technique. ECM materials, either extracted fromi a mouse chondrosarcoma tumor (EHS) as a complex mixture or the purified individual components of ECM, are utilized as substrata on which cells are grown for comparison.

Mouse mammary gland cell line $C-1-D$ was grown on plastic or collagen substrata in the presence or absence of prolactin. Figure 1. shows the comparison of cytoplasmic RNA isolated from C-1$D$ cells grown on plastic (numbers 1 and 2), on attached gels (numbers 3 and 4 ) and on floating gel (numbers 5 and 6). Odd numbers are from cells grown in the absence of prolactin and even numbers are from cells grown in the presence of prolactin. Amounts of RNA dotted are based on an equal amount of DNA present in the cell. The second row and third row of dots are 2-fold and 4fold dilutions of the first dot. Results from this experiment indicate that C-1-D cells grown in the presence of prolactin are generally synthesizing 4io B-foid more $\beta$-casein mRNA than those grown in the absence of probactin. Regarding the effect of substralum, when prolactin is present, cells grown on floaling collagen get produce 8-fold more of

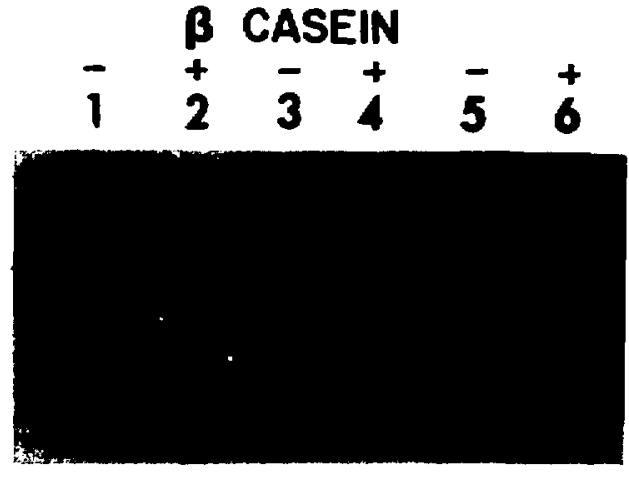

Fig. 1. Quantification of $\beta$-casein mRNA in COMMA-1-D cells. RNA was extracted and processed from cultures grown for 4 days on plastic $(1,2)$, on flat gels $(3,4)$ and on floating gels $(5,6)$. Cultures 2, 4, and 6 contained prolactin. All cultures contained insulin and cortisol. RNA equivalent to $15 \mu \mathrm{g}$ of DNA was dotted (top). Second and third dots are $1 / 2$ dilutions.

(XBB 857-5669)

casein message than those on the attached collagen gel and 16-fold more than those on the plastic surface. $\beta$-casein gene expression in the C-1-D cell, therefore, is modulated not only by hormones but also by the ECM. When milk protein synthesis was measured on polyacrylamide gels, $\beta$-casein synthesized on the floating collagen gel (number 3 ) amounted to least 5-fold more than on the plastic surface (number 4 ) as judged from the intensity of staining in the Western blot (see Fig. 2). These characteristics of C-1-D are similar to the cultures of primary mammary epithelial cells reported previously.

We extended our study of relation between milk protein gene expression and ECM to include other major components of ECM besides collagen (Cl), such as hyaluronic acid (HA), laminin (L), heparin (H), heparan sulfate (HS), and fibronectin (F). The complete basal lamina extract from the EHS tumor is also included for comparison. Figure 3 shows levels of $\beta$-casein message in C.1.D cells grown on either plastic dishes coated with various ECM components (fig. 3A) or grown on thick collagen gets coated with various ECM components (Fic. 3B). The Latter were floaled 2 days after seedint- Cytoplasmic RNA extracted from pregnant mammary aland (MG) and from C-1-D cels grown 
4

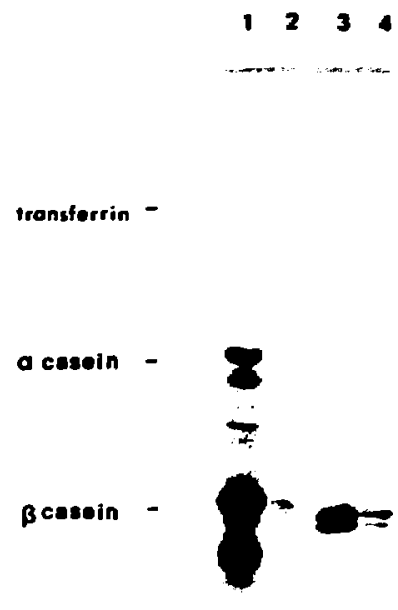

B

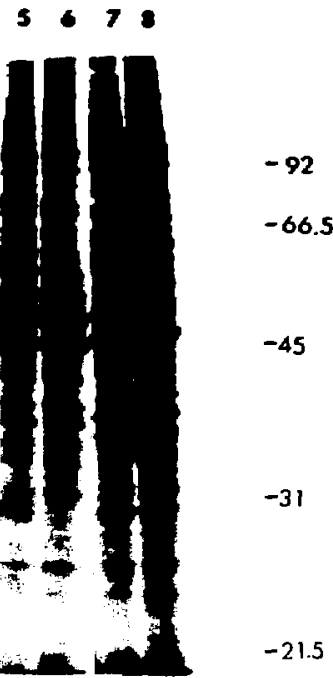

Fig. 2. A comparison of milk proteins and total protein synthesis in primary mouse mammary epithelial cells (PMME) and COMMA-1-D cultures: PMME $(1,2,5,6)$ and COMMA-1-D $(3,4,7,8)$ were grown on either plastic $(2,4,6,8)$ or floating gets $(1,3,5,7)$ in the presence of the three laclogenic hormones.

(XBB 856-6398)

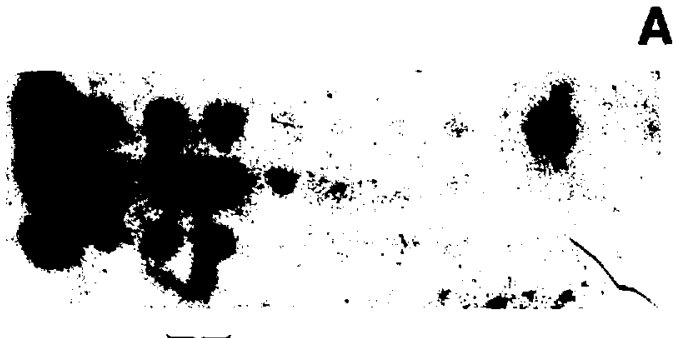

MG $C$ EHS H L HS H F $P$

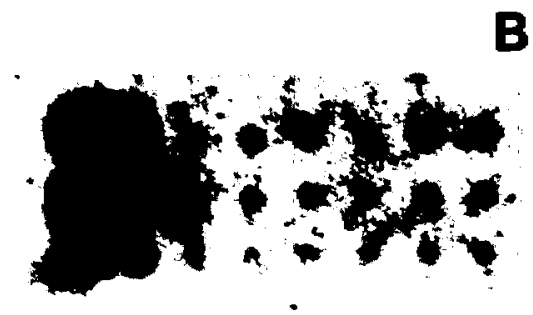

Mg Ens in $L$ H hs F CI on the plastic surface $(P)$ are used as controls in this experiment. In general, cells grown on .'. lagen gel (Fig. 3B) regardless of the coating material synthesize more $\beta$-casein message than those on the plastic surface (Fig. 3A). However, the most important finding of this experiment is that individual components such as laminin, hyaluronic acid, heparin, heparan sulfate or fibronectin do not produce any discernible effect on $\beta$-casein gene expression. In contrast, C-1-D cells grown on EHS complex produce a large amount of $\beta$-casein message, up to $70 \%$ of that produced in the pregnant mammary gland. Since, Etis complex probably contains all the major and minor components of ECM essential for differentiated gene expression, it

Fi. 3. B-casein mRNA levels in COMMTA-1-D cells grown on difierent substratid. (A) Cells grown on either plastic (P) or plasfic colled with difierent substrat.s. MIG is equivilent RNA iso. fated trom late preguant gland. ECM = exiracellular matrix, NMUAIG = "notmal" murine mamman gland cell line, $L=$ laminin, $F=$ tibmectin. HS = heparan suliate, $H=$ heparan, $C I=$ collagen I. HA $=$ hyaluronic acid. LAP $=$ whey didic protein. (B) Syster culfunes wete plased on rop of costed thick collagen pels. Two das athet piating. the gets were thatev and cells unere thanested ho dass later. Aliter collagenase thediment,

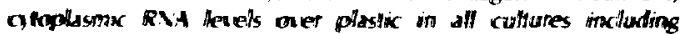
those gtoum om IAS-roaled collagen sets.

(nas 457.5667$)$ 
is not suprising to find that EHS is the best sut stratum for high expression of p-casein bene. On the other hand, the absence of measurable effect of individual ECM components on the differentialed ene expression does not preclude their involvement in this process. It is possible that either a concurrent action of two or more components of ECM is required for such activation or that a threedimensional structure of the right components is required. The mechanism by which differentiated gene expression is modulated by the ECM is undoubtedly very complex and is being investigated further in our laboratory.

in conclusion, we have found that mouse mammary gland cell line COMMA-1-D behaves similarly to the cultures of primary mouse mammary epithelial cells with regard to their specified gene expression in response to hormones and substrata. We also find that the mechanism of gene expression modulated by the ECM is complex and the individual major components of ECM elicit little or no effect on $\beta$-casein gene expression. We pro-

\section{CLONING OF MOUSE TRANSFERRIN CDNA}

Li-How Chen, Ming-Liang Li, and Mina J. Bissell

Transferrin (Tf), an iron-binding protein of molecular weight $80 \mathrm{kDa}$, is one of the major components of milk.' In our study of the regulation of mouse milk protein, we have shown that Tf is synthesized and secreted by mammary epithelial cells themselves in addition to being synthesized by the liver. Our data also indicate that there is a large increase of $\mathrm{Tf}$ in mouse mammary gland in pregnancy compared to the virgin state (Fig. 1).

Besides providing iron to the cells, new lines of evidence ${ }^{2,3}$ indicate that $\mathrm{Tf}$ may be involved in mediating proliferation and differentiation during developmental processes. An additional role as a growth factor for cultured cells has also been suggested for Tf. To study the regulation of Tf in mammary gland, we decided to clone the mouse Tf.

A mouse mammary fumor cDNA library was constructed using $\lambda$ Bt10 vector system $\left(2.5 \times 10^{5}\right.$ independent clones; the library was kindly provided by T. Fung and H. Varmus). A total of $1.0 \times 10^{6}$ phage was plated out and screened by a rat-partial If CDNA probe the rat clone kindly provided by pose that ECM activates gene expression in the manmary cells by a concurrent action of major components of ECM, by a change in cell shape, or by a minor, as yet unidentified component of the ECM.

\section{REFERENCES}

1. Emerman, I.T., and Pitelka, D.R. In Vitro 13, 316-328 (1977).

2. Bissell, M.J., Hall, G.H., and Parry, G. J. Theoret. Biol. 99, 31-68 (1982).

3. Lee, E.Y-H., Parry, G., and Bissell, M.J. J. Cell Biol. 98, 146-155 (1984).

4. Lee, E.Y-H., Lee, W.-H., Kaetzel, C.S., Parry, G., and Bissell, M.J. Proc. Natl. Acad. Sci. USA 82, 1419-1423 (1985).

5. Danielson, K.G., Oborn, C.J., Durban, E.M., Butel, J.S., and Medina, D. Proc. Natl. Acad. Sci. USA 81, 3756-3760 (1984).

M. Griswald). Positive clones were identified and further purified. The suitable cDNA inserts were then subcloned into plasmid Puc18. Three such clones were obtained. pMTf-5 contains the longest cDNA inserts $(2.2 \mathrm{~kb})$ of clones with one internal EcoR1 site; pMTf-01 and PMTF-03 each contain an adjacent EcoR1 fragment of pMTf-5 insert. Restriction digestion analysis was carried out and the respective maps of these three clones were generated as shown in Fig. 1.

A Northern blot experiment was performed to determine the size of Tf message. Cellular RNA isolated from mammary gland was fractionated on MOPS gel, fixed onto nitrocellulose paper and hybridized to pMTf-5 and PRTF. The rat cDNA clone was used for comparison. As shown in Fig. 2, a band of $2.4 \mathrm{~kb}$ was detected in each case. The size of these bands were close to the known size of human serum If message $(2.3 \mathrm{~kb})$. This result further confirms that If is synthesized by mouse mammary gland cells.

Using pMTf-5, we looked at the level of : message at different developmental stages of mouse 


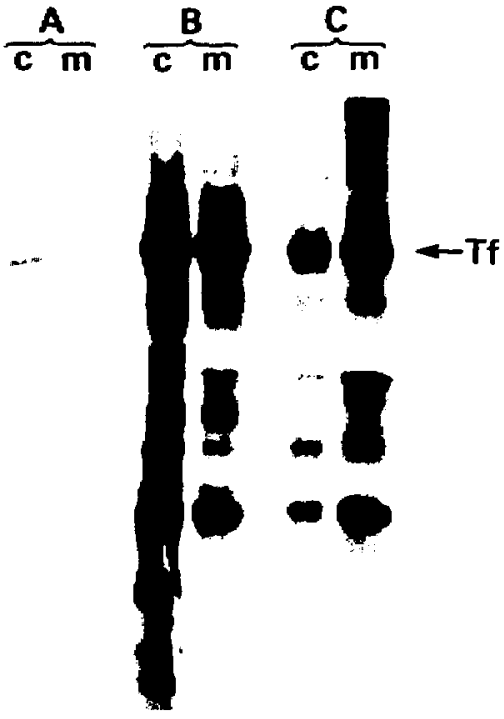

Fig. 1. Production and secretion of Tf in mouse mammary gland. Mammary gland from virgin (A), pregnant (B) and lactating $(C)$ mice were minced and incubated with ${ }^{35} S$-methionine for 3 hours. Cells $(\mathrm{c})$ and media $(\mathrm{m})$ were immunoprecipitated by a polvclonal antimouse milk serum and separated by SDS-PAGE. Equal amounts of samples (based on equal amounts of DNA) were applied to the gel. While virgin gland makes the transferrin, the level increases dramatically in pregnancy and lactation. (This experiment was performed by Eva Y.H. Lee when she was at the laboratory.)

(XBB 840-8546A)

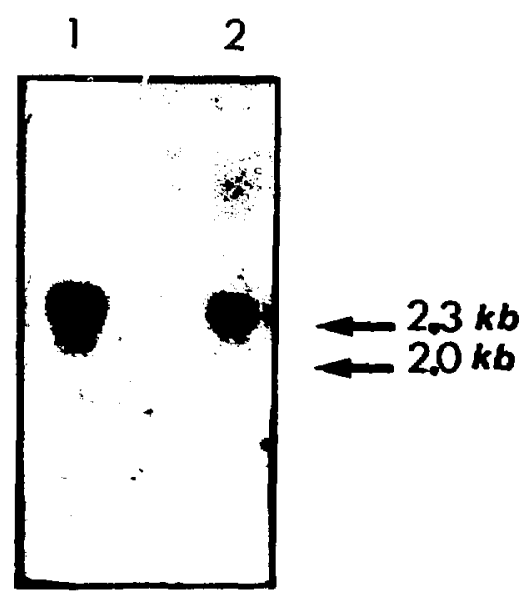

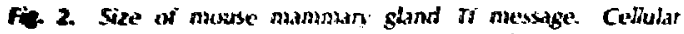
RNA irom mouse mammany gland probed with: If Miri-S, 2) oRti as described in the tevt.

qX8B 850.9906) mammary gland. Total cellular RNA were prepared from mammary gland, lung, and liver of virgin and lactating mice, and were dotted and probed with pMTf-5. The result (Fig. 3) indicated a 20-fold increase in the level of Tf message between virgin and lactating glands.

In summary, we have cloned mouse Tf CDNA and have used it to show TF mRNA is modulated at different stages of mouse mammary gland differentiation. We now have subcloned mouse Tf CDNA into $M-13$ and are sequencing the necessary clones to provide unambiguous proof of the identity of the clones.

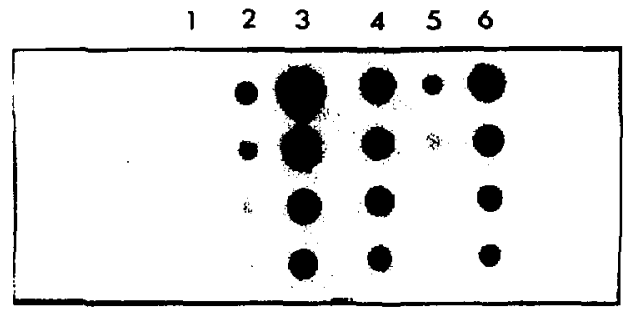

Fig. 3. Comparison of If message levels in various tissues. Virgin mice (1,2,3); lactating mice $(4,5,6)$. Cellular RNA from mammary gland (1 and 4), lung (2 and 5), liver (3 and 6). Amounts of RNA dotted were based on equal amounts of DNA of the tissue. Each lane represents a series of 2-fold dilutions.

(XBB 850-9907)

\section{REFERENCES}

1. Massan, P.L. and Hereman, J.F. Comp. Biochem. Physiol. 39B, 119-129 (1971).

2. Beach, R.L., Popiela, H., and Festaff, B.W. FEBS 156, 151-156 (1983).

3. Mescher, A.L., and Munaim, S.I. /. Exp. Zool. 230, 485-490 (1984). 


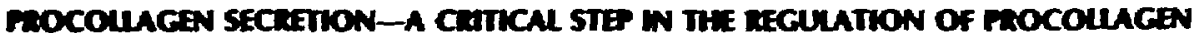 SMNHEXS}

\section{Nancy Omews and bichard I. Sclwwarz}

Regulation of protein synthesis in eucaryotic cells is generally thought to occur at the level of RNA transcription. Supfurt for this view comes from a comparison with procaryotes where some of the best examples of protein regulation are at the level of gene transcription. Additionally, since many eucaryotic genes are present in the genome as a single copy, high protein production from these genes would require amplification by the mechanism of accumulating mRNA transcripts. In some ways, regulation of procollagen synthesis in primary avian tendon (PAT) cells appears to fit this common model. On addition of ascorbate, an inducer of procollagen synthesis, procollagen mRNA levels rise by 6-fold, reflecting the similar increase in procollagen production. Such data would strongly support a transcriptional control model if it were not for kinetic studies.

Understanding the kinetics of an induction process can localize the rate-controlling step in a pathway. This step needs to be shifted early in the induction process so that it can then trigger other steps in the pathway to alter their rates. Increased transcription is kinetically the last step in the pathway to change after ascorbate induction and this all but rules out the possibility of it being the controlling step. ${ }^{1}$ The secretion rate of procollagen from the cell, on the other hand, changes rapidly $(<1 \mathrm{hr})$ with ascorbate (Fig. 1) and by the same 6fold increase seen later for translation rates and mRNA levels. ${ }^{2}$ For this and other reasons enumerated elsewhere, ${ }^{1,2}$ the rate of procollagen secretion is very likely the rate-controlling step.

This raises the important question of how procollagen is secreted from the cell. For the sake of clarity one can reduce this question to distinguishing between a path through the Golgi on its way to secreton' vesicles, or alternatively, by a more direct route from the endoplasmic reticulum to the plasma membrane. This question is not new; quite to the contrary, the mechanism of procollagen secretion has been actively debated for many years. ${ }^{2-4}$ PAT cells are an ideal sysiem for this type of study because, when fully induced by ascorbate, they produce half of their total protein synthesis as procollagen ${ }^{5}$ and secrete it rapidly from the cells with a half-life of $20 \mathrm{~min}$.' One might expect that a cell dominated by its need to secrete procollagen

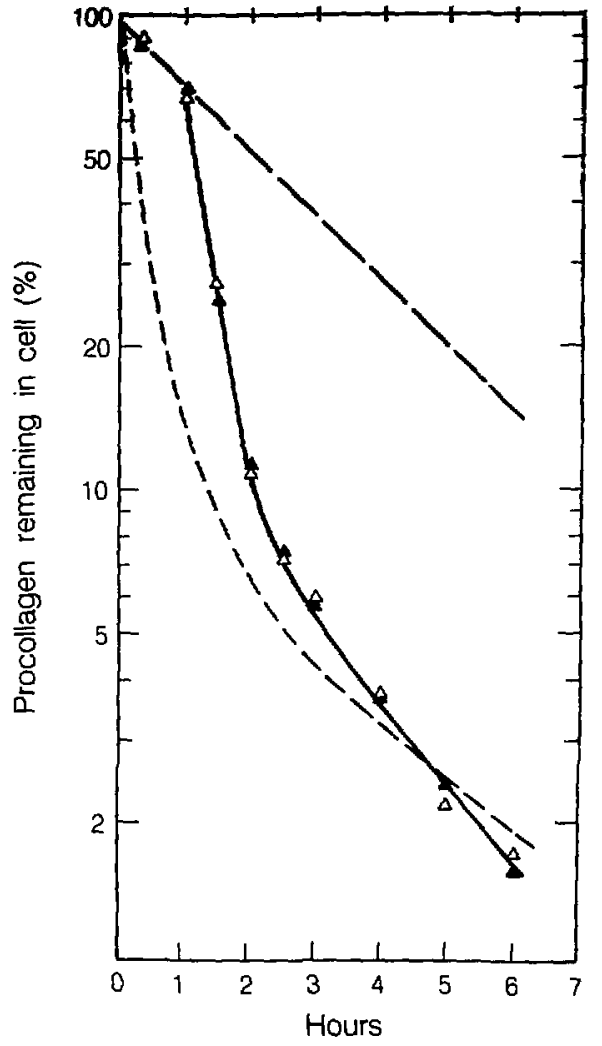

Fig. 1. Secretion of procollagen into the medium for PAT cells given ascorbate at 0 time (triangles). PAT cells were labeled with ' $\mathrm{H}$ proline for $2 \mathrm{hr}$ and then chased at $O$ time with $100 \times$ excess cold proline. The data for cells without ascorbate llong dash; 120-min half-life) and cells given ascorbate at the time of the pulse (short dash; 20-min half-life).

(XBL 8510-4390)

would readily reveal its secretory mechanisms. This is not the case. For instance, visualization of the Golgi apparatus in the electron microscope is difficult with fibroblasts. ${ }^{3}$ Alternatively, using specific enzymes one can track the migration of a secreted glycosylated molecule through the Golgi by changes in the sugar configuration occurring in that organelle: glycosylated groups on the secrefert pro. tein become resistant to the enzyme endoglycos: dase $H$. However, we have observed as have ofl. 
ers ${ }^{6}$ that procollagen remains sensitive to this enzyme even after being secreted. So transit through the Golgi can not be proven by this method.

Other researchers have taken advantage of the fact that secretion appears to be energy dependent and as such might show a strong temperature dependence. In other systems a change of only $5^{\circ} \mathrm{C}$ has slowed secretion rates by about 3-fold making it easier to trace the secretory pathway. Procollagen, however, is remarkably temperature insensitive (Table 1). This result raises questions about the type of secretory mechanism that would be so temperature independent.

Similarly, one might expect that if secretory vesicles are involved in procollagen secretion their transport would require a system capable of directed motion. A likely candidate for this would be the microtubules, which have been shown in other cell types to affect procollagen secretion. While an inhibitor of microtubules, cholchicine, does reduce procollagen secretion rates, the effect is not dramatic-from 20-min half-life inside the cell to $45 \mathrm{~min}$. Such a limited change is more likely to be a result of a secondary effect because loss of microtubules can alter multiple cellular processes.

Another approach has been to use electron microscopy and autoradiography. In this case cells were labeled with ${ }^{3} \mathrm{H}$ proline, then chased with cold proline. Assuming a vesicular transfer, one would expect to observe "hot spots" of multiple tritium tracks over the vesicles during the chase period that would be predicted to contain large amounts of labeled procollagen. No hot spots were observed (Fig. 2 and Ref. 2), but negative data are never conclusive.

In summary, procollagen secretion is an important step in the regulation of procollagen synthesis in tendon cells. The mechanism by which procollagen leaves the endoplasmic reticulum and crosses the plasma membrane is still uncertain. Attempts to establish either Golgi or vesicle involvement in the process have been negative. The possibility that anolher transport mechanism could be involved in procollagen secrelion remains an aller-
Table 1. Temperafure sensitivity of secretion hatf-lives $(t / 2)$.

\begin{tabular}{cccc}
\hline \hline & $\begin{array}{c}11 / 2 \\
\text { procollagen } \\
- \text { ascorbate }\end{array}$ & $\begin{array}{c}11 / 2 \\
\text { procollagen } \\
+ \text { 2scorbate }\end{array}$ & $\begin{array}{c}11 / 2 \\
\text { noncollagen }\end{array}$ \\
\hline 41 & 120 & $\sim$ & 70 \\
39 & 120 & 20 & 75 \\
32 & 120 & 20 & 140 \\
29 & $*$ & 36 &. \\
25 & & $*$ & - \\
\hline
\end{tabular}

Not secreted with first-order kinetics.

native explanation and one that we continue to explore.

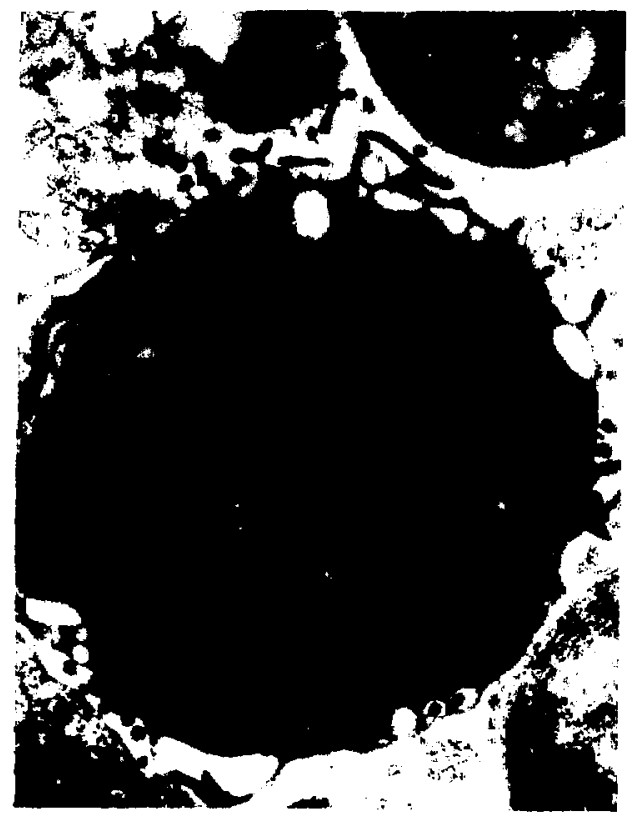

Fi. 2. Eleciron autoradiograph of PAT cells labeled for i hr and then chased with excess cold proline for $10 \mathrm{~min}$.

(XBB 850-8605) 


\section{REFERENCES}

1. Rowe, L.B., and Srhwarz, R.I. Mol. Cell. Biol. 3, 241 (1983).

2. Schwarz, R.I. i. Biol. Chem. 260, 3045 (1985).

3. Olsen, B.\}. In Cell Biology of Extracellular Matrix, Hay, E. Ed., Plenum Press, New York, pp. 139-177 (1 11$)$.

4. Pierce, G.B., Jones, A, Orfanakis, N.G., Nakane, P.K., and Lustig, L Differentiation 23, 60 (1982).

5. Schwarz, R.I., and Bissell, M.J. Proc. Natl. Acad. Sci. USA 74, 4453 (1977).

6. Clark, C.C. J. Biol. Chem. 254, 10798 (1979).

\title{
AN ANALYSIS OF THE AIVIIGENIC STRUCTURE AND MODE OF REGULATION OF A DIFFERENTIATION ANTIGEN ON THE SURFACE OF NORMA! AND MALIGNANT HUMAN MASMMARY EPITHELIAL CELLS
}

\author{
Lenny Moss, Betsey Cullen, and Gordon Parry
}

The composition and properties of the apical plasma membrane of mammary epithelial cells is modulated extensively according to the stage of cellular differentiation. In the lactating gland the membrane is specialized to deal with the scrretory activity of the cell, including the secretion of tat droplets into the milk. This is a process that involves budding of lipid droplets surrounded by apical plasma membrane from the cell surface into the milk. Several lactation-specific proteins and giycoproteins have been identified in this membrane, and monospecific and monoclonal antibodies have beer. prepared against some of these components. Studies utilizing such antibodies have revealed that manmmary carcinoma cells express significant quantitie's of some of these membrane antigens. One such antigen is a high-molecularweight mucin-like glycoprotein containing a large number of O-linked carbohydrate chains. Several groups have raised monoclonal antibodier against this molecule and have utilized them in a variety of diagnostic and clinical studies of tumorigenesis. This antigen appears to be expressed in a large number of mammary carcinomas as well as on the surface of carcunomas of ovarian and intestinal origin. In one study, monoclonal antihodies raised against this antigen were used with apparent success to treat a patient suffering from ovarian cancer.

While many clinical sludies of antigen expression have been reported, basic knowledge of the structure of this molecule and the mechanisms regulating its expression have not been examined. Prompted by the possible clinical significance of the antigen and by our major interest in understanding mechanisms regulating the expression of cell surface glycoproteins. we have undertaken a study of antigen expression in normal human lactating breast tissue and ir, a human mammary carcinoma cell line, 734B.

\section{IDE: TIFICATION OF THE MAJOR ANTIGENIC DOMAIISS OF THE MUCIN}

4. panel of monoclonal antibodies was generated against purified human milk fat globule membrane (the membrane that is derived from the apical surface of normal lactating epithelial cells), and monoclonal antibodies that recognized the high-molecular-weight mucin molecule were selected by screening anitbodies using Western blotting procedures. A total of 13 hybridomas were selected and propagaied on this basis. Consistent with what has been reported by others, the mucin was found in both the memiorane and in the soluble fraction of milk (the sirimmed milk fraction). Interestingly, when skimmed r.:'k components were resolved to SDS-polyacrylamide gel electrophoresis and the naiure of the binding components examined by Western bluiting (Fig. 1), it was found that some monoclonal antibodies bound to only the high-molecular-weight mucin bands while others bound to lower-inolecular-weight components as well as to the large species. Some of these lowermolecular-weight molecules were also found in the milk fat globule membrane. Based upon a large number of experiments, we were able to categorize the panel of antibodies into four classes according to the number of molecular species they identified and their apparent affinity for these resolved molecules. As the antibodies in each of these classes clearly had different specificities, it was apparent that there were at least four antigenic epi- 


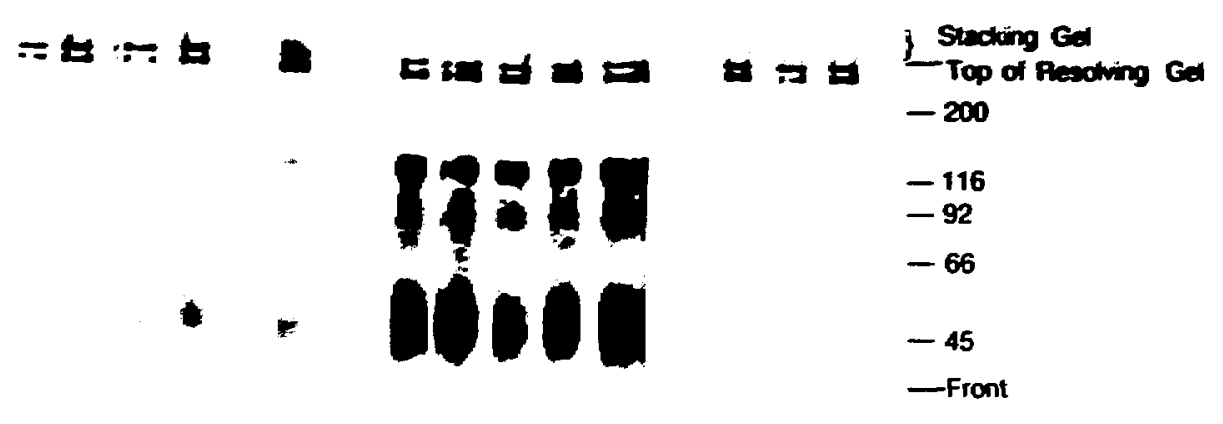

$\begin{array}{lllllllllllll}1 & 2 & 3 & 4 & 5 & 6 & 7 & 8 & 9 & 10 & 11 & 12 & 13\end{array}$

Fig. 1. Western blots of human skimmes milk proteins, blotted with monoclonal antibodies reactive against human milik fat globule membranes. All tracks of the gell contained the same sample of skimmed milk but were blotted with one of 13 separately isolated monoclonal antibodies. The samples are gouped into categories on the basis of the number of bands staising and the relative intensities with which they are stained.

(XBB 850-9098)

topes associated with the high-molecular-weight mucin. Additionally, as all 13 of the monoclonal anitbodies fell into one or other of these classes, it appeared that these four epitopes represented the major antigenic eptopes of the mucin. Competetive ELISA assays utilizing carbohydrate maierial derived from membranes by exhaustive proteolytic digestion revealed that these epitopes probably represented different carbohydrate structures.

\section{STRUCTURAL RELATIONSHIPS AMONG THE MAJOR EPITOPES}

Procedures were developed for immunoprecipitating the mucin from membranes and from skimmed milk. When immunoprecipitates were resolved by SDS-poly-acrylamide gel electrophoresis and then analyzed by Western blotting procedures utilizing anitbodies against different epitopes from those that were imnunsprecipitated, crossreactivity was observed. This implied that the four epitopes were associated with a single molecular species in the membranes and that there was not a family of mucin-lisie molecules tiat each expressed a different epitope.

Immunoprecipitation experiments also demonstrated that some of the lower-molecular-weight antibudy binding species. notably a component with a molecular weight of $140 \mathrm{kDa}$, was associated with the mucin in the membrane. When monoclonal antibodies that bound only to the highmolecular-weight mucin bands were used for munoprecipitation and the immunoprecipitates .ere then probed by W'stern blotting using a monoclonal antibody that recognized the $140-\mathrm{kDa}$ component as well, it was found that this was indeed associated with the high-molecular-weight species. Not all the lower-molecular-weight antibody-binding components behaved in this way, however, and it is not clear whether al! these are mucin-derived or whether the epitopes are expressed on other molecular species in addition to the mucin.

\section{EXPRESSION OF MUCIN LIKE ANTIGENS ON HUMAN MAMMARY CARCINOMA CELLS}

Immunofluorescence and immunoelectron microscopy revealed that all the monoclonal antibodies bound to 734B cell cultures. However, not all the cells were stained by the antibodies. The basis of this heterogeneous staining was probed further by deriving five clonal variants of the $734 \mathrm{~B}$ cultures and then determining the extent of heterogeneous staining. It was found that there was considerable heterogeneity, even in the clones, in that only a certain proportion of the cells were stained. This implied that antigen expression in individual cells was nol constant but was subject to variation in culture.

An additional interesting fealure was that some of the epitopes were expressed independently in the clonal populations. Thus, for example, as is shown in Tabie 1. $50 \%$ of the cells of cinne 1 stained with monoclonal anlibody $\mathrm{P} 2 \mathrm{Cl}$, but only 5\% stained with P2A1 and P3B1. In the case of clone 3 cultures, $50 \%$ of the cells stained with anlibody BC4. but tewer than $5 \%$ with P2C1. It is not 


\begin{tabular}{|c|c|c|c|c|c|c|c|}
\hline $\begin{array}{l}734 \\
\text { Cell } \\
\text { done }\end{array}$ & ma1 & P31 & $\begin{array}{c}P 2 C 1 \\
\text { ( } \% \text { Cells }\end{array}$ & eCA & $\mathbf{1 0 6}$ & EC10 & $\begin{array}{c}\text { Mix of } \\
216\end{array}$ \\
\hline 1 & 5 & 5 & 50 & 5 & 20 & 10 & 00 \\
\hline 2 & 5 & $=5$ & 20 & $<5$ & 20 & $<1$ & n.d. \\
\hline 3 & 40 & 20 & $<5$ & 50 & 40 & 20 & 80 \\
\hline 4 & 10 & $<5$ & 20 & 10 & 10 & 5 & 60 \\
\hline 5 & 25 & 20 & 60 & 10 & 10 & 5 & 70 \\
\hline $\begin{array}{l}\text { Uncloned } \\
\text { population }\end{array}$ & 65 & 60 & 30 & 70 & 70 & 60 & 70 \\
\hline
\end{tabular}

yet clear whether this is associated with the malig- nant phenotype of the cell or in is associaled with maintaining the cells in culture. Experiments are in progress, in collaboration with J. Bartley and $M$. Stampfer, that will illuminate this point.

These immunofluorescence experiments studying anlibody binding to clones of the 7348 cells also revealed that the number of epitopes recognized by the antibodies might be greatrr than fow. Three antibodies $\mathrm{BC} 4, \mathrm{BC} 6$, and $\mathrm{BC10}$, reacted similarly in Western blotting experiments, but reacted somewhat differently with clones of the 734B cells. We conclude that there may be up to six different epitopes composing this mucin. More detailed biochemical experiments will, of course, be necessary to clarify this point.

\section{THE ROLE OF TIGHT JUNCTIONS AND CELL SUBSTRATUM INTERACTIONS IN CONTROLLING MEMBRANE POLARITY IN CULTURED HUMAN MAMMARY EPITHELIAL CELLS}

\section{Gordon Parry, Lenny Moss, and Betsey Cullen}

The plasma membrane of epithelial cells is segregated into two distinct domains: an apical domain and a basolateral domain. Tight junctions form the boundary between the rwo domains. The polarity is established relative to the attachment substratum, which is usually the basement membrane in vivo or the culture dish in cell culture experiments (Fig. 1). A variety of experiments carried out by others using several different model syslems have suggested that both the attachment substratum and tight junctions play an important role in regulating membrane polarity. However, many conflicting sets of data have been published, particularly for experiments using virally irfected cells as a model system, and the real role of each of these factors remains to be clarified.

To this end we have used a monoclonal antibody raised against a differentiation antigen on apically derived milk fat globule membranes to study regulation of membrane polarity in cultured human mammary epithelial cells. Immunofluorescence procedures were used for most of these experiments, and these wer complemented whenever necessary by immuno-electronmicroscopy analysis.

The experiments monitored membrane polarity at different cell densities. The idea underlying this approach was that cell-substratum interactions would be constant and independent of cell density. while cell-cell interactions would be very different

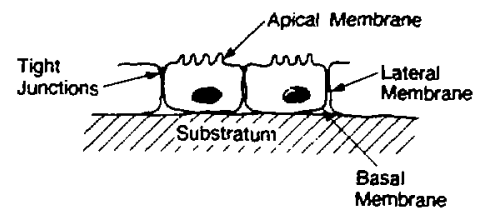

Fig. 1. Diagram illustrating the organization of the plasma membrane in epithelial cells.

(XBL 8511-8584)

at low and high cell densities. We found that the antigen was highly polarized at high cell density and was clearly detectable in the apical microvilli. At very low cell densities, however, it was not polarized and was found in the lateral membranes as well as the apical membranes. This result clearly demonstrated that cell-substratum interactions in themselves were not sufficient to establish membrane polarity, and that cell-cell interactions were very important. An interesting siluation was observed at intermediate cell densities when the cells formed small distinct islands of cells (see Fig. 2). Antibody staining of these islands revealed that the cells all exhibited distinct apical polarity, but cells on the edge of the istands exhibited distinct staining on the free Lateral membrane. As I' Lateral membrane did not contain a tight junctio. this observation was consistent with the view that 


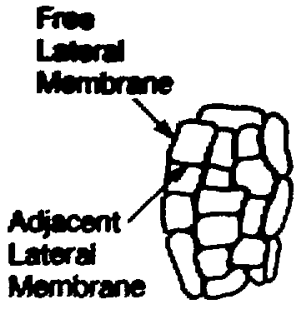

(a)

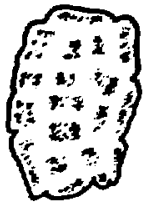

(b)

Fi. 2. (a) Diagram illustrating the organization of cells in a typical "island" of epithelial cells. Cells on the edge of the island have a free lateral surface, whereas cells in the center of the island are completely surrounded by other cells, and all lateral surfaces are in contact with other cells. (b) Diagram illustrating the distribution of fuorescently labeled antibodies bound to the surfaces of cells in an island such as that in (a): the antibody stains the face lateral membranes strongly, but not the lateral membranes of cells in the center of the island nor the adjacent lateral membranes of edge cells. (XBL 8511-8585)

in the absence of a tight junction the apical antigen was able to diffuse into the lateral zone. Notably, however, staining of the lateral membranes at the interface of adjacent cells was never detected. By using an antibody against $\mathrm{Na}^{+} / \mathrm{K}^{+}$, ATPase, a baso-

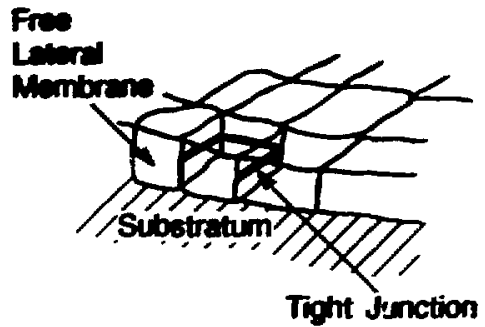

fiz. 3. Diagram illustrating the organization of the tight jumxtion in edge cells of a small island of epithelial cells.

(XBL 8511-8581)

lateral component of these cells, it was possible to show that access of the antibody to these regions, and indeed to all the internal cells of these small islands, was not a problem. As is illustrated in Fig. 3 , it should be possible for antigen to diffuse beneath the tight junctions in these edge cells and to label all the lateral surfaces, not just the free one. To account for this finding, it would appear that factors other than the tight junctions contribute to the development of membrane polarity. These factors clearly involve cell-cell interactions of an unknown nature, and experiments are currently underway to identify them.

\section{GROWTH CONTROL IN HUMAN MAMMARY EPITHELIAL CELLS}

Martha Stampfer and Jack Bartley

Our laboratory has developed culture systems for the growth of human mammary epithelial cells (HMEC). Mammary tissues from both normal and tumor-bearing breasts are readily available as discard material from reduction mammoplasties and mastectomies. These cells are of particular interest for the study of human cellular carcinogenesis and differentiation since 85 to $90 \%$ of all human cancers, and $99 \%$ of breasl cancers, originate from the epithelial cell type, and the breast epithelial cell in wivo is capable of many specialized, differentiated functions.

Two different media have been used in our laboratory for the growth of HMEC: MM, which contains the undefined elements of fetal calf serum and conditioned media as well ds several growh faclors and hormones, and MCDB170 (deveioped in Di. Richard Ham's Laberatony at the University of Colorado). which contains bovine pituitary extract
(BPE) as its only undefined element. Over 70 different HMEC specimens have been grown in MM. HMEC derived from nontumor tissues typically grow for 10-15 population doublings. The cells initially maintain a uniform, cobblestone epithelial appearance. At senescence, cellular morphology is still epithelioid, but more heterogeneous, with large vacuolated cells that can no longer divide. No cells have displayed an indefinite growth polential. We have now been able to examine growth in MCDB 170 of 23 cell specimens from 19 different individuals. In all cases, at second to fourth passage, the vast majority of the cell population terminally differentiales; growth ceases and the cell morphology appears myoepithelial. However, a small subpopulation that maintains the cobblestone epithelial morphology is capable of long-term active growth in culture-about 35 to 65 population doublings. Al senescence, the cell morphology remains 
epinthetial. In several cases HMEC from the same individual have been follomed to senescence 5 to 10 separale times. Each lime, cell growth consistently decreases around the same passage level (12 to 22, depending upon the individual) and no cells have acquired an indefinile growth potenlial. These results with human epithelial cells differ greatly from those seen with cells of rodent Grigin. where spontaneous transformation to immortality is common.

The growth of HMEC in both MM and MCDB170 is stimulated by insulin, hydrocortisone, and epidermal growh factor. Additionally some factor(s) in the BPE are also requiren! for growh in MCDB170. However, removal of these growth factors does not lead to an immediate cessation of division, or a $G_{o}$ growth arrest, as is often the situation for cells of fibroblastic origin. In collaboration with other laboratories, we have observed that TGF $\beta$ which can be growth stimulatory for fibroblastic cells, rapidly inhibits the growth of normal HMEC. In other systems, TGF $\beta$ has been shown to be an inhibitor of normal epithelial cell growth, .vhile tumor-derived cells may be unaffected. It may therefore prove a useful tool in the selection of variant transformed cells from a normal cell population.

We have demonstrated that the usual pattern of HMEC growth and senescence can be altered by the exposure of cells to the chemical carcinogen benzo(a)pyrene (BaP). HMEC from specimen 184, grown in $\mathrm{MM}$ and exposed to BaP in primary culture, displayed many cells capable of around twice the usual number of population doublings. From this extended life population, two immortal cell lines (184A1 and 184B5) have emerged. We have begun testing the effect of BaP on cells grown in MCDB170. Thus far two individuals have been examined; an increased growh potential con be seen, but we do not yet know if cells with an indefinite life span have appeared. However, we have obtained a new immortal cell line (18AWI) from celts that have never been exposed to BaP. Cells from specimen 184 were grown in MCDB170 for 8 passages, and then switched to MM. Although most cells senesced by passage 15 , one clonal patch has continued growing. We do not know what caused the transformation to immortality in this case.

The immortal cell lines, besides having aberrancies in the normal pathway to senescence, display other properties that distinguish them from their normal parental cells. They are all aneuploid; yet, unlike immortal cell lines derived from tumor lissues or tumor virus infection, they maintain a near diploid karyotype and show only a few chromosomal changes after 100 to 200 population doublings. They may thus be extremely useful for experimentation in which a relatively stable karyotype is desired. Neither 184A1 nor 18485 is tumorigenic in immunosuppressed mice. At low passage levels $184 \mathrm{~A} 1$ shows no, and 184B5 shows very little anchorage-independent growth. However, introduction of tumor virus genetic material can render both lines tumorigenic, and 184A1 anchorage independent. The possible role of known oncogenes in the transformation of these HMEC is currently being examined. Both $184 \mathrm{~A} 1$ and 18485 show some differences in growth factor requirements from their parent cell, and they also contain TGF $\beta$-resistant populations. These cell lines, as well as the normal long term cell cultures, should provide useful substitutes for examining the mechanisms and factors controlling growth and senescence of human epithelial cells in culture.

\section{EXPRESSION AND MODULATION OF DIFFERENTATION IN HUMAN MAMMARY EPITHELIAL. CELS}

Iack Bartley, Gerri Levine, and Martha Stampfer

All mammalian cell types undergo stages of maluration in which a stepwise process produces highly differentiated cells with special functions. Because disruptions in this progression may be related to carcinogenesis, a complete understanding of it lakes on special significance. We have been using the cuhure systerns developed in our labora- lory for growing human mammary epithelial cells (HMEC) to sludy regulation of differentiation in these cells.

Mammary epithelial cell maturation and differentiation is complicated by superimposition upon the maturation process of the hormonal influences sccompanying puberty, the estrous cycle, meno- 
pause, and those of pregancy, Lactation, and involution. Thus, unitke skin epithelial cells, the pathway of functional differentiation in mammary epithelial cells is not necessarily coincidental with that for terminal differentiation. Studies in vivo and in organ culture have helped to clarify the maturation stages in rodents, but the relationship belween these slages and systemic hormonal influences remains obscure. Even less is known about these processes in humans. Tissue culture systems offer the possibility for experimental examination of these questions. This potential, however, would be restricied, if, as has been suggested, cells placed in culture lose the ability to express tissue-specific traits. On the other hand, several studies in epithelial cell systems have indicated that cells in culture, while not displaying tissue-specific properties, have not lost their capacity for expression; e.g., rat mammary epithelial cells growing in monolayer cultures for up to 20 days will grow into normal gland when transplanted in vivo into abdominal fat pads cleared of their mammary elements, and rat tracheal epithelial cell lines and normal cells in culture for one week regain the ability to form mucociliary epithelium when placed on tracheal grafts in vivo. It is more likely that current culture conditions are either not sufficient to support expression of functional differentiation or are selecting for a stage characterized by proliferation. In this context, it is important to note that systems for studying mammary function in culture have been successful at maintaining the functions typical of prelactation and lactation, but not in inducing any of these functions in cells from quiescent glands. Additionally, given the complexity of mammary cell maturation and differentiation, cells from quiescent glands may be present in a stage of maturation not readily recognizable because our knowledge of mammary function is largely based on the highly differentiated cells present during lactation. With this concept in mind, wo are developing markers for evaluating differentiation in HMEC that are based on properties not necessarily linked to lactation.

HMEC grown in the MM vs. the MCDBi70 medium invariably undergo senescence after far fewer cell divisions. The selection and proliferation properties of the posi-selection cells in the MCDB170 serum-free medium might be due to their being less differentialed progenitor cells, while those in MM are more committed to differentiated function, thereby being unable to express long-term proliferation. This concept of a basic "fference in expression of differentialed function sy HMEC grown in each medium is supported by the pattern of glucose utilization and protein syn. thesis and secretion. HMEC gromn in MM have a distinctly different pattem of ghucose utilization from that of cells from the same specimen in MCDB170. Most notably, the syntheses of glycogen and lactate are increased. These increases correspond to changes observed in murine mammary epithelial cells during the Iransition from the virgin to the pregnant state. Because of the regulatory significance of glycogen synihesis in mammary epithelial cells in late pregnancy, it is Jikely that increased glycogen synthesis is a reliable indicator of the prelactational stage of the mammary epithelial cell. We therefore suggest that HMEC in MM are apparently in a metabolically differentiated state resembling cells present during midpregnancy, whereas HMEC in MCDB170 are metabolically less differentiated. This contention is supported by autoradiograms of electrophoretic gels demonstrating synthesis and secretion of a caseinrelated protein by cells in $M M$ but not in MCDB170.

To further test the possibility that postselection cells in MCDB170 are progenitor cells, we transferred cells grown for eight passages in MCDB170 to MM. Syntheses of glycogen, lactate, and milk proteins were examined 4 to 24 hours later (Tab!: 1). Control cells remained in fresh MCDB170. Syntheses of glycogen and lactate are increased at the earliest time point. In a second experiment, a xyloside was added to one set of cells to inhibit glycosylation of protein. This treatment did not affect glycogen and lactate synthesis.

We have partially purified $\beta$-casein, the major protein in human milk, to aid in the analysis of proteins secreted into the medium in these experiments. This preparation was used to produce polyclonal antibodies, and hybridomas are currently being screened for monoclonal antibody production. The polyclonal antibody preparation has been used for detection of milk proteins secreted into the medium. The proteins were precipitated from the media in replicate plates examined for glycogen and laclate synthesis. After separation by polyacrylamide gel electrophoresis, the proteins were transferred to nitrocellulose and probed with the polycional antibody for the presence of caseinrelated proteins (Western blot technique).

Comparison of proteins secreted by posiselection cells to those in human milk revealed that a protein produced by cells switched to MM Comigrated with a protein in human milk (Fig. 1). This protein was not recovered in medium from cells remaining in MCDB170, and its synthesis and/or secretion was severely and specifically inhibited by the addition of xyloside. The action of the 
Twbie 1. Effect of andirm on tucose merubolite pumern.

\begin{tabular}{|c|c|c|c|c|c|}
\hline \multicolumn{6}{|c|}{ Swilching from MCOB170 $10 \mathrm{MM}$} \\
\hline \multirow{3}{*}{$\begin{array}{l}\text { Time in 2nd } \\
\text { medium (hrs) }\end{array}$} & \multicolumn{5}{|c|}{ Ghucose incorporation (nm/ms protein): } \\
\hline & \multicolumn{2}{|c|}{ Glycogen } & \multicolumn{2}{|c|}{ Lactate } & \multirow{2}{*}{$\frac{\text { Lipid }}{\text { Ist Expt }}$} \\
\hline & Ist Expt. & 2nd Expl. & Ist Expl. & 2nd Expt. & \\
\hline $\mathbf{0}$ & 6.4 & 9.0 & 340 & 415 & 2.30 \\
\hline 4 & 14.1 & 18.3 & 995 & 489 & 3.95 \\
\hline $\mathbf{8}$ & 10.4 & 29.1 & 815 & 763 & 3.61 \\
\hline 24 & 141.6 & 25.6 & 407 & 717 & 2.02 \\
\hline $24+x y$ loside & - & 23.0 & & 836 & \\
\hline \multicolumn{6}{|c|}{ Switching from $M M$ to $M C D B 170$} \\
\hline 0 & 70.2 & & 1075 & & 3.29 \\
\hline 4 & 11.5 & & 498 & & 2.98 \\
\hline 8 & 16.2 & & 501 & & 2.34 \\
\hline 24 & 8.9 & & 365 & & 1.92 \\
\hline
\end{tabular}

Cells growing eighth passage from specimen $\mathrm{H} 161$ in medium MCDB170 for 8 passages were fed with $M M$ and $\mathrm{H16}_{1}$ cells in second passage in MM were shifted to MCDB170 at 0-time. At 3, 7 and $23 \mathrm{hr}$ later $\left[\mathrm{U}^{-14} \mathrm{C}\right.$ glucose was added and the incorporations into glycogen, lactate and lipid were determined $1 \mathrm{hr}$ later as described in the text. A replicate experiment going from $\mathrm{MCDB} 170$ to $\mathrm{MM}$ was repeated several months later. In this experiment a replicate MCDB170-MM plate was treated with xyloside for $24 \mathrm{hr}$. Replicate plates acting as controls were fed with the same type of fresh medium at 0 -time and incorporation of glucose into products determined at the same time intervals as the test plates.
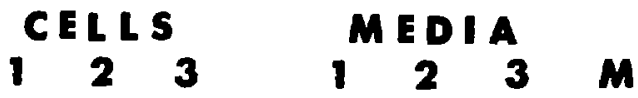

$\mathbf{t}$

$\mathbf{4 5}$
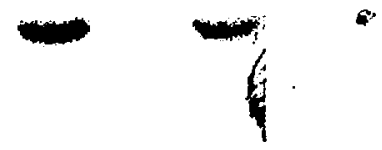

31

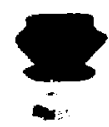

Fig. 1. IVestern blots using an antibody to human casein of pnoteins in cell lyoles and media from HMEC. The experiment is the same as experiment 2 in Table 1 . Lanes 1 and 3 represent resulls from cells grown in MM; those in Lne 3 receried x) foside. Lane 2 contains proleins from control cells remaining in MCOBIza. Lane M contains casein puritied irom human nolll. No positiveh reacting prokeins were found in any cell lysales or in medium from

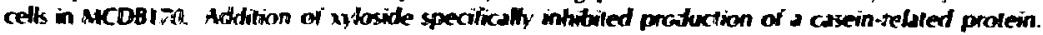


mploside in this case is likety due, in part, 10 inhibifion of seconetory vesicle formation which would, in turn, inhibit secretion of milk proteins. The specific protein in question is not $\beta$-casein but a caseinrelated protein that may arise from incomplete processing within the cell or from partial postsecretion degradation.

From these preliminary studies, it appears that MCDB170 supports growth of progenitor cells that can express some differentiated functions when placed in $M M$, and this stage appears to be comparable in many ways to rodent mammary epithelial cells from animals in early to mid-pregnancy.

In contrast to rodent systems, HMEC have not required artificial matrices, such as collagen gels, to express some differentiated functions. There are several possible explanations for this fact. First, we are dealing with cells at a physiological stage in which changes in cell shape (cuboidal to columner) and polarization are not rigorously required. Secondly, because HMEC apparently synthesize and/or accumulate sufficient matrix for long-term growth and for the specific modulations of phenotypic expression demonstrated here, it is possible that modulation of matrix formation can occur within 4 hours after the switch to medium. The source of matrix material might be different for cells in each medium. In the case of cells in $M M$, some matrix components might be synthesized, and other underfined factors from the medium minth be organized into matrix. In MCDB170, it is more likefy that HMEC are able lo form the mitrix necessary for growh because this medium has been optimized for growth of these cells. The experiments with xyloside are compatible with this explanation. Inhibition of matrix processing by xyloside could alter the cell's ability to synthesize and/or secrete protein.

The next steps in evaluating the functional potential of normal postselection cells are: 1) to continue development of markers for detecting stages of HMEC maturation, and 2) to attempt induction of the prelactational and lactational states. In light of the work in this field in other species, we anticipate that induction of the lactational state will likely require a prepared matrix to provide proper cell orientation and/or allow for changes in cell shape. A reduction in intracellular CAMP and specific changes in the complement of hormones and growth factors in the medium are also anticipated. The ability to grow HMEC under defined conditions presents an advantageous starting point for such studies.

The expert technical assistance of Linda Hayashi, Annie Pang, and Annette Drew in the conduct of these studies is gratefully acknowledged.

\section{EVALUATIOI: OF NORMAL AND TRANSFORMED HUMAN MAMMARY EPITHELIAL CELLS WITH MONOCLONAL ANTIBODIES TO CELL SURFACE ANTIGENS}

Martha Stampfer, Gordon Parry, and Jack Bartley

The composition of the cell surface molecules of mammary epithelial cells may vary with the state of cellular differentiation and as a result of malignant or immortal transformation. Monoclonal antibodies (MAb), capable of recognizing the cell surface antigens that vary under these conditions, can be valuable tools both for detecting cells at different stages of differentiation and transformation and for probing the underlying biochemical processes. We have utilized six MAb's raised against human milk-fat globule antigens (HMFGA) to determine if any of them can distinguish states of differentiaion or Iransformation in our human mammary epithelial cell (HMEC) cultures. Data rom other mammany epithelial systems have shown that some MFGA are found in greatest amounts in cells derived from lactating breasts, and also that tumor-derived cells may show increased amounts of specific HMFGA. Thus far, we have examined cells mainly by indirect immunofluorescence, which allows the cell surface properties of individual cells in the population to be visualized. Studies recently initiated will identify the proteins involved by gel electrophoresis and Western blots.

Our experiments thus far have vielded the following results:

1. The six different MAb's (designated 1-6) display four different patterns of binding.

2. Normal HMEC grown in MCDB170 show almost m' binding of MAb's 2 and 3, and, depending upon tho individual, 10 to $50 \%$ of cells display a punctale patiern of fluorescence with MAb's 1 
and 5. When transferred to MM, $100 \%$ of the cells are now punctate positive for MAb's 1 and 5, and some cells $(1-10 \%)$ are positive for MAb 2 . This result is consistent with the other data we have obtained, which suggests that MCDB170 maintains the cells in a less differentiated state than the MM medium.

3. Cells derived from mastectomy tissues, and atypical reduction mammoplasty specimens, may show increased expression of MAb's 1, 2, and 5 when grown in MCOB170 compared to normal tissues.

4. The two cell lines derived from normal tissues after treatment with benzo(a)pyrene had distinct patterns of HMFGA expression. The more differentiated line, 184B5, had increased expression $(\sim 100 \%)$ of MAb's 1 and 5 in MCDB170, as well as some expression of MAb's 2 and 3 (5-20\%). The less differentiated line, 184A1, had a decreased percentage of cells positive for MHb's 1 and 5, but a small ( $<5 \%$ ) number of cells positive for MAb's 2 and 3.

5. Treatment of cells with neuraminadase, to remove sialic acid residues, rendered all cells $100 \%$ positive for MAb's 1, 2, and 5, and had no effect on expression of MAb 3.

These results are consistent with the data of others that suggest that HMFGA may be markers of both differentiation and transformation. From these preliminary results, it is likely tiat MAb's 1 and 5 are more sensitive to states of differentiation, whereas MAb's 2 and 3 may be more indicative of cellular transformation.

\section{USE OF DNA RESTRICTION FRAGMENT LENGTH POLYMORPHISMS FOR THE IDENTIFICATION OF HONEYBEE RACES}

\section{H. Glenn Hall}

Honeybees of African descent (Apis mellifera scutellata, formerly A.m. adansonii), ${ }^{1}$ accidently released from experimental hives in Brazil 28 years ago, have populated most of South and Central America, now as far north as Honduras, where they have largely displaced the honeybees of European descent. ${ }^{2}$ The bees are a problem mainly because of their ferocious stinging. Isolated introductions of the Africanized bees have already occurred in this country, a notable case this past summer in California, ${ }^{3}$ which have been largely contained. However, based on the bees' migration rate through Central America, they are expected to arrive in the US en masse within five years. In 1972, the National Academy of Science Committee on the African Honey Bee correctly concluded that the African bees' entry into the United States was inevitable and would be an environmental danger to the population and catastrophic to the commercial beekeeping and pollination industries." By USDA estimates, the beekeeping industry will lose from $\$ 26$ to $\$ 58$ million annually. ${ }^{5}$ The same report calculates that $\$ 19$ billion worth of agricultural products are dependent upon honeybee pollination. Thus, the total loss as a result of the African bee could be immense.
Possible methods of control include quarantine and extermination, stock certification, and selective breeding for a gentle hybrid. All of these approaches are dependent upon a reliable identification method to distinguish the African bee from the European bee. Establishing a precise means of identification was a major recommendation by the National Academy Committee, but current methods are inadequate. Presently, the most effective method of identification of the African bee is through morphometric statistical analysis. ${ }^{6}$ However, the method is subjert to environmental influences and cannot reliabiy distinguish hybrids past one generation. Allozyme analysis is both uncertain and impractical as a means of identification because of the limited allozyme variability in bees.?

Until now, all efforts to identify bees have been limited to phenotype or, at best, allozyme analysis. This study is the first effort to directly utilize genetic material for identification and certification of bees. The identification is based on DNA fragments generated by restriction eirdonucleases. I have established a library of honeybee DNA probes and have begun their lesting against two lines of European bees. The initial results encourage further development of this method. 
Fragnents of honeybee nuclear DNA, generaved with Psl, were randomly inserted into the Pstl site of the $E$ coll plasmid p8R322." From the bacterial colonies transformed by the plasmids, those carnying inserts of honeybee DNA of about 4 Kb or greater and not containing repetition sequences were selected (fig. 11." DNA was isolated from two samples of honeybees: one designated as Italian (A.m.ligustica), and the other as Carniolan (A.m.camica). The DNA was digested with eight separate restriction enzymes, the fragments separated by agarose gel electrophoresis, blotted onto membranes, and hybridized with the denatured radioactively labeled probes. ${ }^{10}$ So far, 17 probes have been tested. Between the DNA of the Iwo samples, six additional fragments were found in the Carniolan line, some of which are shown in Fig. 2.

The European honeybees in this country, although designated as different races, were derived from a limited number of founding populations and probably have a similar hybrid genotype. Genetic differences between African and European bees are likely to be much greater, with a higher frequency of restriction fragment polymorphisms than found here among the European bees. Africanized samples have recently been obtained from Costa Rica and will soon be tested with the same probes. Restriction site differences at many, preferably widespread, loci can potenticity be found, 'o that after several generations and multiple recom!, inational events, the alleles found in hybrids will quan-

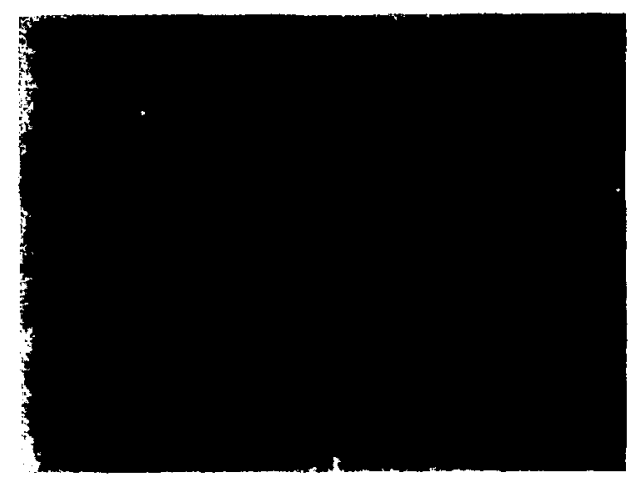

Fin. 1. Colonies, aheaty setected for conlaining plasmids with inserts. were hibridized to tokal labeled honeybee ONA. Because oi the high concentration of repetilise sequences and the very how concentration of singletopy sequences, colonies containing inserts of mepetitine DNA exhibit intense hytridizalion, whereas those with inserts of unique sequences do mol.

(XB8-850-10246)

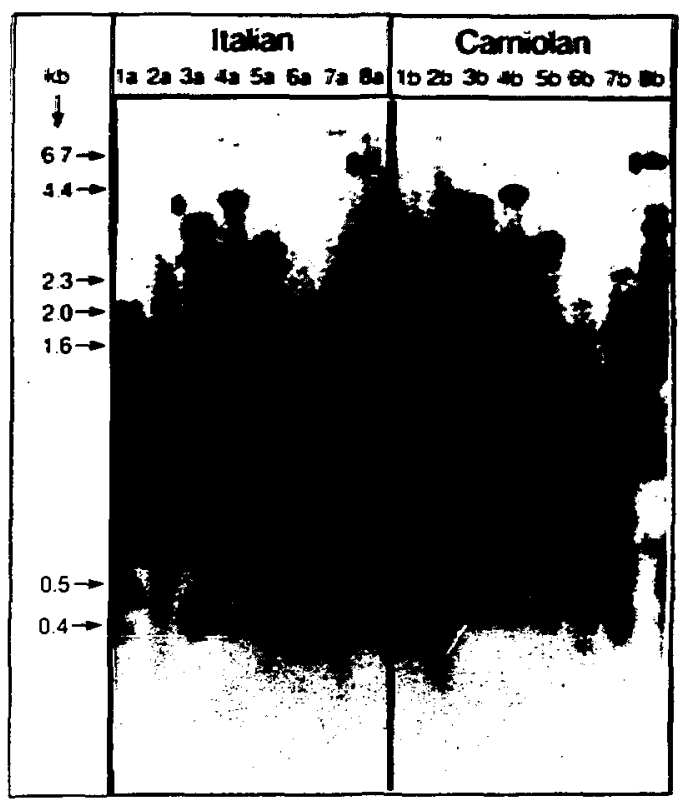

Fig. 2. Southern blot of tolal honeybee DNA hybridized to a mixture of four probes. The discrete bands demonstrate that the strategy for obtaining unique sequence probes was successful. Lanes ta through 8a each contain DNA from the Iralian line digested with a different restriction enzyme listed below. Lanes $1 b$ through $8 b$ each contain DNA from the Carniolan line digested with the corresponding restriction enzymes in lanes 1 a through 8a. Lambda phage DNA digested with HindIII and pBR322 digested with Hinfl were used as molecular weight standards. Arrows indicate differences in the fragments. Note that the lack of bands in lanes $3 a$ and 8 a are accompanied by an increase in the density of the next lower major bands compared to the corresponding bands in lanes $3 b$ and $8 b .1=M s p l, 2=$ Tål, $3=$ Alul, $4=$ Haell, $5=$ Hhal, $6=$ Mbol, $7=$ Hinfl, $8=$ Ncil.

(XBB-850-10249)

titatively reflect the degree of crossbreeding. Probe and enzyme combinations that reveal distinctive differences will first be sought and selected among a few samples of European and Africanized bees, and the selected probes will then be tested against many more samples. It must be determined which polymorphisms just represent variability within populations and those that show significant gene frequency differences among the populations.

Restriction fragment length polymorphisms characteristic of, and hopefully diagnostic for Africanized bees and their hybrids will provide is reliable means of identification that will be especially needed when major expensive decisions to exterminate or quarantine large numbers of colonies are to be made. Genotype identification by restriction 
frements wim be very applicable for certification of breeder stocks as non-African or as acceplable mbrids. Certification of stocks should aid selective breeding lor sentle African bees. Beyond the identification of Africanized bees, the results of this research as it develops further will enhance future studies of basic honeybee genetics, such as in determining gene linkage and following gene flow and variability in populations.

\section{REFERENCES}

1. Ruttner, F. African races of honeybees. Proc. 25th Int. Congress on Apiculture (1976).

2. Michener, C.D. The Brazilian bee problem. Ann. Rev. Entomol. 20, 399-416 (1975).

3. Cobey, S., Lawrence, T. Status of the Africanized bee find in California. Amer. Bee 1. 125, 607-711 (1985).

4. Final Report of the committee on the African honeybee. NAS-NRC, Washington, D.C. (1972).
5. McDowel, $R$. The Africanized honey bee in the United States. What will happen to the U.S. beekeeping industry? USOA Aricultural Economic Report No. 519 (1984).

6. Dahy, H.V., Balling S.S. Identification of Africanized honeybees in the westem hemisphere by discriminant analysis. J. Kansas Ent. Soc. 5J, 857-869 (1978).

7. Rinderer, T.E., Sylvester, H.A. Identification of Africanized bees. Amer. Bee J. 121, 512-516 (1981).

8. Maniatis, T., Fritsch, E.F., Sambrook, 1. Molecular Cloning. A Laboratory Manual. Cold Spring Harbor Laboratory (1982).

9. Grunstein, M., Hogness, D. Colony hybridization: a method for the isolation of cloned DNAs that contain a specific gene. Proc. Natl. Acad. Sci. 72, 3961-3965 (1975).

10. Southern, E. Gel electrophoresis of restriction fragments. Methods Enzymol. 68, 152-176 (1979). 


\section{ATrENDICES}

\section{APPENDIX A. List of Contracts and Grants Supporting Portions of Work Presented in This Annual Report}

\section{INVESTKGATOR}

\section{E.). Ainsworth}

E.J. Ainsworth

E.L. Alpen

E.L. Alpen

J.C. Bartley

G. Brecher

T.F. Budinger

T.F. Budinger

T.F. Budinger

T.F. Budinger

J.R. Castro

A. Chatterjee

G.K. Clemons

P.K. Cooper

S.B. Curtis

\section{CONTLACT OR GAANT}

NASA P.O. T3516-G

Life-Shortening Effects of HzE Particles on

Mice/Heavy Ion Cell Transformation

PHS Grant TW 00980

Killing Mouse Marrow Stem Cells by Heavy Atomic Nuclei

PHS Grant CA 30236

Advanced Design Research Heavy Ion Medical Accelerator

PHS Grant RR 05918

Biomedical Research Support Grant

PHS Grant CA 38889

Human Mammary Cells: Modulation of Differentiated States

PHS Grant AM 27454

Kinetics of Transfused Stem Cells in Normal Mice

PHS Grant HI. 25840

Cardiovascular Flow and Metabolism

PHS Grant AG 05890

Cerebral Blood Flow Patterns in Alzheimer's Disease

PHS Grant HL 07367

Quantitative Cardiovascular Research, Training Grant

IBM Instruments

P.O. 4521310

NMR Imaging Project

PHS Grant CA 19138

Treatment of Cancer w/Heavy Charged Particles

PHS Grant CA 27024

Bragg Peak Localization by Radioactive Beams

PHS Grant HL 22469

Radioassay of Erythropaietin

PHS Grant CA 32986

Inducible Responses to Carcinogenic DNA Damage

PHS Grant CA 17411

Response of Rat Tumo: Cells to Heavy lons 
P.W. Durbin

P.W. Durbin

S.N. Ebbe

M.S. Esposito

M.S. Esposito

T.M. Forte

R.M. Glaeser

R.M. Glaeser

L.S. Gold

R. Goth-Goldstein

R. Goth-Goldstein

H.G. Hall

J. Hosoda

R.H. Huesman

R.M. Krauss

S.A. Leadon

R.P. Liburdy

R.P. Liburdy

I.T. Lyman
NRC IAC 60-65-012

Development of Metabolic Modets for Mratine Eanth and Actinide Radionuclides

PHS Grant ES 02698

Biological Testing of New Actinide-Chelating Agents

PHS Grant AM 21355

Kinetics of Megakaryocyte and Platelet Turnover

PHS Grant GM 29002

Comparative Analysis of Mitolis and Meiolic

Recombination

PHS Grant ES 02756

Genetic Effects of Carcinogens in Mitosis and Meiosis

PHS Grant HL 07279

Lipoprotein Methodology, Structure and Function

Training Grant

PHS Grant GM 23325

Biological Structure Analysis by Electron Microscopy

PHS Grant RR 02246

Precision Scanning Microdensitometer Facility

PHS IAG 222-Y01-ES-10066

Quantitative Species Extrapolation in Carcinogenesis

PHS Grant ES 01916

Alkylating-Carcinogens Mutagenesis in Mammalian Celis

PHS Grant ES 03603

Inducible Resistance to Alkylating Carcinogens

PHS Grant HD 17892

Extracellular Matrix and Eptithelial Lumen Morphogenesis

PHS Grant GM 16841

Structure and Function of Helix Stabilizing Protein

PHS Grant CA 38086

Scatter Compensation in Emission Tomography

PHS Grant HL 33577

Plasma Lipoproteins in Coronary Artery Disease

PHS Grant CA 40453

DNA Repair in Specific Sequences of Mammalian Cells

ONR Contract NO0014-84-F-0186

Mechanisms of Microwave Interactions with Liposome Membranes

PHS Grant RR 02570

Radio Frequency Assisted HPLC

PHS IAG YOI-CM-20110

Evaluation of Treatment Planning for Particle Beam Radiotherapy 
I.T. Lyman

M.F. Maestre

R.K. Mortimer

A.V. Nichols

T.W. Sargent

T.W. Sargent

W. Schimmerling

W. Schimmerling

R.I. Schwarz

B.A. Singer

M.R. Stampfer

M.R. Stampfer

T.S. Tenforde

T.S. Teniorde

C.A. Tobias
PHS Grant CA 22286

MAPM B682-19

Charged Particle Beam Dosimetry Task Group: Dosimetry zotocol

PHS Grant Al 08427

Physical Structure of Viruses, Chromosomes and Cell Nuclei

PHS Grant GM 30990

Yeast RAD Genes in Repair, Recorrt ination and Meiosis

PHS Grant HL. 18574

Lipoprotein Methodology and Biomedical Applications

PHS Grant MH 36801

Transmethylation Kinetics in Schizophrenia

V.A. Medical Center, Mirtinez

V612P-1194

Cerebral Metabolic Indices of Dementia Pathophysiology

PHS Grant CA 23247

Physical Characteristics of Heavy Ion Beams

NASA P.O. L22395A

To Measure the Production of Neutrons by High Energy Heavy lons

PHS Grant CA 37958

Manipulation of the Differentiated Slate by Oncogenesis

PHS Grant CA 42736

Alky!dtion of Polynucleotides in vitro and in vivo

PHS Grant CA 24844

Characterization of Human Mammary Cells

PI IS Grant CA 30028 (R.Ham, Univ. Colorado)

P.O. $42 \measuredangle 881$

Defined Medium for Human Mammary Epithelial Cells

EPRI Contracl RP 799-21

Microprocessor-Controlled Personal Dosimeter for

Recording Low-Intensity Power Frequency Magnetic Fieic':-

EPRI Contract RP 2572-5

Assessment of Biological Effects Associated with Magnetic Fields from a Superconducting Magnet Energy

Storage System

PHS Grant CA 15184

Heavy Ion Radiobiology Related to Oncology 


\section{APPENDIX B: 1985 Publications}

\section{CONTRIBUTIONS TO JOURNALS}

Apen, E.L., Saunders, W., Chatterjee, A., Lacer, J., Chen, G.T.Y., and Scherer, J. A Comparison of water equivalent thickness measurements: C.T. method vs. heavy ion beam technique. Brit. J. Radiology 58, 542-548 (1985).

Bartley, J.C., and Stampfer, M.R. Factors influencing benzo(a)pyrene metabolism in human mammary epithelial cells in culture. Carcinogenesis 6, 1017-1022 (1985).

Bastacky, 1., Hayes, T.L., and Gelinas, R.P. Quantitation of shrinkage during preparation for scanning electron microscopy: human lung. Scanning $7,134-140$ (1985).

Bastacky, J., and Hayes, T.L. Safety in the scanning electron microscope laboratory. Scanning 7, 255-272 (1985).

Beer, M., Caspar, D.L.D., and Claeser, R.M. Introduction, Papers from EMSA Symposium on Electron Crystallography of Macromolecules. Ultramicroscopy 13, vii (1984).

Bialek, W., and Schweitzer, A. Quantum noise and the threshold of hearing. Phys. Rev. Lett. 54, 725-728 (1985).

Blum, C.B., Davis, P.A., and Forte, T.M. Elevated levels of apolipoprotein $E$ in human cord bloot plasma. J. Lipid Res. 26, 755-760 (1985).

Erown, M.S., Garcia, J.F., Thibbs, R.H., diid Dallman, P.R. Decreased response of plasma immunoreactive erythropoietin to "available oxygen" in anemia of prematurity. I. Pediatrics 105, 793-798 (1984).

Crown, MS., Phibles, R.H. Carcia, J.F., and Dallman, P.R. Posinalal changes in enythropoietin levels in untransfused premature initants. \&. Pediatrics 103, 612-617 (1983).

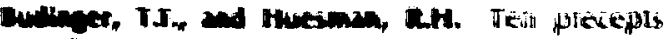
for quantiutive data acquistion and anshysit. Cinculution 72 (Suropl. Ni), 53 (19a5).
Bustamante, C., Maestre, M.F., and Keller, D. Simplified expressions for the circular intensity differential scattering of chiral aggregates. Biopolymer 12 1595-1612 (1985).

Calderon, I.L., Contopoulou, C.R., and Mortimer, R.K. Isolation of a DNA fragment that is expressed as an amber suppressor when present in high copy number in yeast. Gene 29, 69-76 (1984).

Carr, K.E., Hayes, T.L., McKoon, M., Bastacky, S.J., and Kamel, H.M.H. Etched surfaces of plastic embedded and frozen hydrated gastrointestine. /. Submicroscopic Cytology 16, 219-226 (1984).

Castro, J.R., Chen, G.T.Y., Pitluck, S., Cartigny, A., Phillips, T.L, Saunders, W.M., Collier, J.M., Woodruff, K.H., Friedman, $M_{\text {., and Austin- }}$ Seymour, $\mathbf{M}$. Helium charged-particle radiotherapy of locally advanced carcinoma of the esophagus, stomach, and biliary tract. Am. J. Clin. Oncol. (CCT) 6, 629-637 (1983).

Chandra, M., Miller, M.E., Garcia, J.F., Mossey, R.T., and MicVicar, $M$. Serum immunoreactive erythropoietin levels in patients with polycystic kidney disease as compared with other hemodialysis patients. Nephron 39, 26-29 (1985).

Chang, C.-F., Mizushima, S., and Glaeser, R.M. Projected structure of the pore-forming OmpC protein from Escherichia coli outer imembrane. Biophys. J. 47, 629-639 (1985).

Chang, C.-F., Ohno, T., and Clacser, R.M. The fatty acid monolayer technique for preparing frozen-hydrated specimens. I. Electron Microscopy Technique 2, 59-65 (1985).

Che, D.H., Smaiers, W.M., Castio, J.R., mine, AR., Slone, R.T., Crowion, JS., Eurridks, M.,

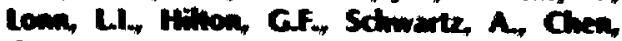
G.T.Y, lyou, IT, Coliex, IML, sin, M.,

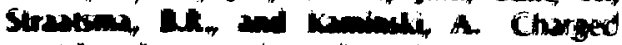
particle therapy for choroids mellanoma. Opthithalmollosy 90, 1219 (1583). 


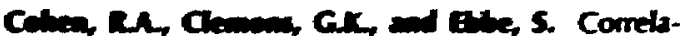
tion between bioassay and radioinmunoassay for erythropoietin in human serum and urine concentrates. Proc. Soc. Expll. Biol. Med. 179, 296-299 (1985).

Costelio, M.l., Vitanen, P., Carrasco, N., Foster, D.L, and Kabach, HA. Morphology of proteoliposomes reconstituted with purified lac carrier protein from Escherichia coli. J. Biol. Chem. 259, 15579-15586 (1985).

Dolberg. D.S., Hollingsworth, R., Hertle, M., and Bissell, M.J. Wounding and its role in RSVinduced tumor formation. Sc-ence 230, 676678 (1985).

Dukes, P.P., Ma, A., Clemons, G.K., and Meytes, D. Measurement of human erythroid burst promoting activity (BPA) by a specific cell culture assay. Exp. Hematol. 13, 59-66 (1985).

Durbin, P.W., and Schmidt, C.T. The U.S. Transuranium Registry report on the ${ }^{241}$ Am content of a whole body. Part V. Implications for metabolic modelling. Health Physics 49, 623661 (1985).

Ebbe, S., Adrados, C., and Phalen, E. Independence of megakaryocyte number and size in long term cultures of normal mouse marrow. Exp. Hematol. 13, 817--820 (1985).

Eckleman, W.C., Reba, R.C., Rzeszotarski, W.J., Gibson, R.E. Hill, T., Holman, B.L., Budinger, T.F., Conklin, J.J., Eng, R., and Grisson, M.P. External imaging of cerebral muscarinic acetylcholine receptors. Science 223, 291-293 (1984).

Finch, G., McNeil, K., Democko, C., Lai, C., Bastacky, I., Hayes, T.L., and Fisher, G. A technique permitting correlative microscopy (light microscopy, scanning electron microscopy, transmission electron microscopy and high voltage electron microscopy) of cultured alveolar macrophages. 1. Electron. Microsc. Tech. 2. 69-70 (1985).

Forte, TM. Ren, C.L., Nordiluasen, R.W., and Nichols, A.V. Formation of phospholipid-rich HDL: A model for square-packing lipoprotein particles found in interstitial fluid anc in abetalipoproteinemic plasma. Biochim. et Bioph, sice Acts 834, 386-395 (1985).

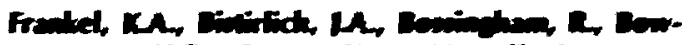

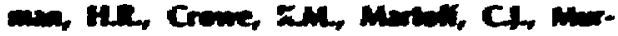

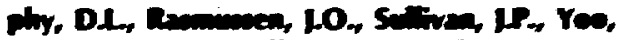

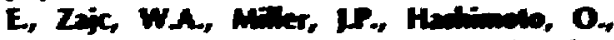
Koike, M., Peter, J., Benewson, W., Crawkey. GM., Kadry, E, Nolen, Jr., JA., and Quebert, 1. Pion production romar mid-rapidity in highenergy heavy-ion collisions. Phys. Rev. C 32. 975-982 (1985).

Friedland, R.P., Budinger, T.F., Koss, B., and Ober, B.A. Alzheimer's disease: Anterior-posterior and lateral hemispheric alterations in cortical glucose utilization. Neuroscience Lefters 53. 235-240 (1985).

Friedland, R.P., Prusiner, S.B., Jagust, W.J., Budinger, T.F., and Davis, R.L. Bitemporal hypometabolism in Creutzfeldt-jakob disease measured by positron emission tomography with ${ }^{18} \mathrm{~F}$-2-fluorodeoxyglucose. J. Comput. Assist. Tomog 8, 978-981 (1984).

Glaeser, R.M., Jubb, I.S., and Henderson, R. Structural comparison of native and deoxycholatetreated purple membrane. Biophys. J. 48 , 775-780 (1985).

Glaeser, R.M. Electron crystallograph: of biological macromolecules. Ann. Rev. Phys. Chem. 36, 243-275 (1985).

Goldberg, 1.J., Mazlen, R.G., Rubenstein, A., Gibson, J.C. Paterniti, Jr., J.R., Lindgren, F.T., and Brown, W.V. Plasma lipoprotein abnormalities associated with acquired hepatic triglyceride lipase deficiency. Metabolism 34, 832-835 (1985).

Goodman, J.W., Hall, E.A., Miller, K.L., Shinpock, S.G. Interleukin 3 promotes erythroid burst formation in "serum-free" cultures without detectable erythropoietin. Proc. Natl. Acad. Sci. USA 82, 3291-3295 (1985).

Greiner, D.E., Crawford, H., Lindstrom, P.I., Kidd, J.M., Olson, D.L., Schimmerling, W., and Symons, T.j.M. Uranium nuclear reactions at $900 \mathrm{MeV} /$ nucleon. Phys. Rev. C 31, 416-420 (1985).

Hamson, $W$. R., and Ainsworth, E.j. 16,36-dimethyl prostaglandin $E_{2}$ induces radioprotection in murine intestinal and hematopoietic stem cells. Radiation Res. JC.3. 196-203 (1985). 


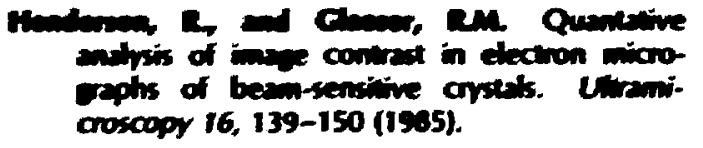

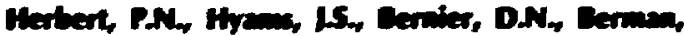

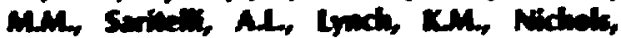
A.V., and Forte, TM Apolipoprotein 8-100 deficiency: intestinal steatosis despile apolipoprotein B-48 synthesis. J. Clin. Invest. 76, 403-412 (1985).

Itamy, L, and Aipen, EL. Two-dimensional diffusion limited system for cell growth. Cell Tissue Kinet. 18, 597-611 (1985).

Hoeg, JM. Segal, P., Grezt, R.E, Chang, Y.S., Lindgren, F.T., Adamson, C.L, Frank, M., Erickman, C., Erewser, H.S., Jr. Characterization of plasma lipids and lipoproteins in patients with beta 2 -glycoprotein I (apolipoprotein H) absence. Atherosclerosis 55, 25-34 (1985).

Jansson, LT., Perkio, M.V., Clemons, G.K., Retino, C.J., and Dallman, P. Erythropoietin concentration during the development and recovery from iron deficiency in the rat. Blood 65 , 959-963 (1985).

Kao, W. W-Y., Kao, C. W.-C., and Schwarz, R.I. Prolyl hydroxylase production can be uncoupled from the regulation of procollagen synthesis. Experimental Cell Research 157, 265-270 (1985).

Keller, D., Bustamante, C., Maestre, M.F., and Tinoco, 1., Jr. Model computations of differential scattering of circularly polarized light by dense mecromolecules. Biopolymers 24, 783-797 (1985).

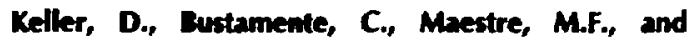
Tinoco, I., Jr. Imaging of opically active biological structures using circularly polarized light. Proc. Nätl. Acad. Sci. (USA) 82, 401-405 (1985).

Kelie, S., Hotwe, 1., and rineth, M.. Interaction of tumor promoters with epithelial cells in culture: an immunofluorescence study. Exp. Cell Res. 160, 259-274 (1985).

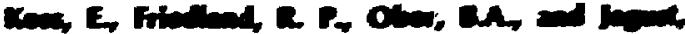
Wt Difevences in laterd hemispheric sommetries of ducose cotiverion between eidyand beve-onset Albeimer-type dementia. Am. f. Psychinty 142, 638-640 (1985).

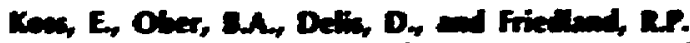
The Stroop color-word test: indicator of dementia severity. Internil. I. Neuroscience 24, 53-61 (1984).

Kraft, C., Hieter, L, Schuber, M, Muller, W., Craft-Weyrather, W., Wulf, H., Milienburzer, H., Elakely, E.A., and Tobias, C.A. HZE effects on mammalian cells (COSPAR: Life Sci. Space Res.) Adv. Space Res. 4, 219-226 (1984).

Lee, E., Y.-H.P., Lee, W-H., Kaetzel, C.S., Parry, G., Dissell, M.J. Interaction of mouse mammary epithelial cells with collagen substrata: regulation of casein gene expression and secretion. Proc. Natl. Acad. Sci. USA 82, 1419-1423 (1985).

Leven, R.M., Schick, P.K., and Budzynski, A.Z. Fibrinogen biosynthesis in isolated guinea pig megakaryocytes. Blood 65, 501-504 (1985).

Liburdy, R.P., and Magin, R.L. Microwaves stimulated drug release from liposomes. Radiation Research 103, 266-275 (1985).

Liburdy, R.P., and Vanek, Jr., P.F. Microwaves and the cell membrane. II. Temperature, plasnıa, and oxygen mediate microwave-induced membrane permeability in the erythrocyte. Radiation Research 102, 190-205 (1985).

Liburdy, R.P., and Wyant, A. Radiofrequency radiation and the immune system. III. In vitro effects on human immunoglobulin and on murine T- and B-lymphocytes. Int. Journal of Radiation Biology 46(1), 67-81 (1984).

Lloyd, R.D., Inwenger, F.W., Mays, C.W., Atherton, D.h., lowes, C.W., Taylor, G.N., Stevens, W., Durbin, R.W., lewer. N., Jones, E.S., Kappel, M.l., Raymond, K.N., and Weitl, F.L Removal of Pu and Am from beagtes and mice by 3,4,3-LKCAM(c) or 3,4,3-LKCAM(S). Radiation Res. 99, 106-128 (1984). 


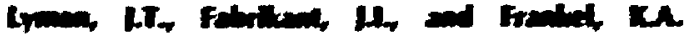
Chared-particle stereotactic radiosureny. Nud. instr. and Methods in Phys. Res. B10/11. 1107-1110 (1965).

McCam, J, Hown, L., and kullor, I. An evaluation of Safmonella (Ames) test data in the published literature: application of statistical procedures, and analysis of mutagenic potency. Mutation Research 134, 1-47 (1984).

Maestre, M.f., Salzman, G.C., Tobey, R.A., Rustamante, C. Circular dichroism studies on single Chinese hamster cells. Biochemistry 24, 5152-5157 (1985).

Mathis, C.A., Sargent III, T., and Shulgin, A.T. lodine-122-labeled amphetamine derivative with potential for PET brain blood flow studies. J. Nucl. Med. 26, 1295 (1985).

Mazoyer, B.M., Koos, M.S., and Huesman, R.H. Dead time correction and counting statistics for positron tomography. Phys. Med. Biol. 30, 385-399 (1985).

Mikols, W.C., Bustamante, C., Maestre, M.F., Tinoco, I. Ir., and Embury, S.M. Differential polarization microscopy: a new imaging technique. Biotechnology 3, 711-714 (1985).

Mickols, W., Maestre, M.F., Tinoco, I., Jr., and Embury, S.H. Visualization of oriented hemoglobin $\mathrm{S}$ in individual erythrocytes by differential extinction of polarized light. Proc. Natl. Acad. Sci. USA 82, 6527-6531 (1985).

Monteiro, P.J.M., Bastacky, S.J., and Hayes, T.L. Low-temperature scanning electron microscope analysis of the Portland cement paste early hydration. Cement and Concrete Research 15, 687-693 (1985).

Mortimer, R.K., and Schild, D. Genetic map of Saccharomyces cerevisiae, Edition 9. Microbiological Reviews 49, 181-213 (1985).

Nichols, A.V., Blanche, P.I., Cons, E.L, Shore, V.G., and Forte, T.M. Molecular pathways in the transformation of model discoidal lipoprotein complexes induced by lecithin: cholesterol acyltransferase. Biachim. Biophys. Acta. 834 285-300 (1985).

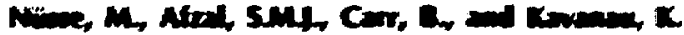
Cell cycle binetic mexsurements in an imadiated rat thabdomyosarcoma using a monoclonal antibody to bromadeoxyuridine. Cytometry 6, 611-619 (1905).

Olson, IE, Schimmertioc, Wo, Wimbor, A., Uhlumer F., and Tobias, CLl Peak-detect-and-hold circuit for instantaneous measurement of bioelectric signals. I. Electrophysiol. Tech. 12, 151-157 (1985).

Parry, G., Lee, E. Y.-H., Farson, D.A., Koval, M.H., Biscell, M.). Collagenous substrata regulate the nature and distribution of glycosaminogiycans produced by differentiated cultures of mouse mammary epithelial cells. Exp. Cell Res. 156, 487-499 (1985).

Pennathur-Das, R., Alpen, E., Vichinsky, E., and Lubin, B.H. Evidence for a heterogeneous response to erythropoietin in the $C F U_{E}$ pool of human bone marrow. Experimental Hematology 12, 31-37 (1984).

Pennathur-Das, R., Alpen, E., Vichinsky, E., Garcia, J., and Lubin, B.H. Evidence for the presence of $\mathrm{CFU}_{\mathrm{E}}$ with increased in vitro sensitivity to erythropoietin in sickle cell anemia. Blood 63, 1168-71, (1984).

Perez, C.F., Botchan, M.R., and Tobias, C.A. DNAmediated gene transfer efficiency is enhanced by ionizing and ultraviolet irradiation of rodent cells in vitro. 1. Kinetics of enhancement. Radiation Research 104, 200-213 (1985).

Phillips, M.H., Anderson, L.W., and Lin, C.C. Electron excitation cross sections for the metastable and resonant levels of $\mathrm{Ne}\left(2 \mathrm{p}^{5} 3 \mathrm{~s}\right)$. Phys. Rev. A 32, 2117-2127 (1985).

Roots, R., Chatterjee, A., Chang, P.Y., Lommel, L., and Blakely, E.A. Characterization of hydroxyl radical-induced damage after sparsely and densely ionizing irradiation. Int. \% Radiat. Biol. 47, 157-166 (1985).

Roots, R., Kraft, G., and Gosschalk, E. The formation of radiation-induced DNA breaks: The ratio of double-strand breaks to single-strand breaks. Imt. I. Radial. Oncol. Biol. Phys. 11. 259-265 (1985). 


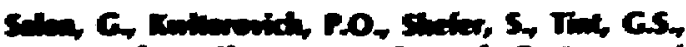

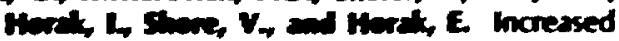
plasma cholestanol and 5 alpha-soturated plan sterol derivatives in subjects with sitosverolemia and xanthomatosis. I. Lipid Reseunch 26, 203-209 (1985).

Smanks, WM. Radiation oncology: The use of beams of photons or particles for the treatment of tumors. J. Radiation Physics and Chemistry 24, 357-364 (1984).

Smonders, W.M., Char, D.H., Quivey, IM., Castro, Ja., Chen, G.T.Y., Collier, J.M., Cartigny, A., Chakely, E.A., Lyman, I.T., Zink, S.R., and Tobias, $C_{A}$ A. Precision, high dose radiotherapy: Helium ion radiotherapy of uveal melanoma. Int. 1. Radiat. Oncol. Biol. Phys. $11,227-233$ (1985).

Saunders, W.M., Chen, G.T.Y., Austin-Seymour, M., Castrs, J.R., Collier, J.M., Gauger, G., Gutin, P., Pnillips, T.L., Pitluck, S., Walton, R.E., and Zink, S.R. Precision, high dose radiotherapy: II. Helium ion treatment of tumors adjacent to critical central nervous system structures. Int. J. Radiat. Oncol. Biol. Phys. 11, 1339-1347 (1985).

Schaefer, E.J., Ordovas, J.M., Law, S.W., Ghiselli, G., Kashyap, M.L., Srivastava, L.S., Heaton, W.H., Albers, J.J., Conner, W.R., Lindgren, F.T., Lemeshev, Y., Segrest, J.P., and Brewer, H.B. Ir. Familial apolipoprotein A-I and C-III deficiency, variant II. J. Lipid Res. 26, 1089-1101 (1985).

Schild, D., Mortimer, R.K. A mapping method for Saccharomyces cerevisiae using rad52-induced chromosome loss. Genetics 110, 569-589 (1985).

Schooley, J.C., Kullgren, B., and Fletcher, B.L. Growth of murine bone marrow adherent stromal cells in culture without hydrocortisune in a low oxygen environment. Interntl. J. Cell Cloning 3, 2-9 (19:35).

Schiwarz, R.I. Procollageri secretion meets the miximum requirements for the rate-controlling step in the ascorbate induction of procollagen synthesis. 1. Biol. Chem. 260. 3045-3049 (1985).

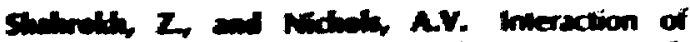
human-phasma low-density lipoproveins wath discoidal complexes of apolipoprovein A-1 and phosphatidylocholine, and characterization of the interaction products. Bioctiein. et Biophysica Acta 837, 296-304 (1985).

Swich, H.S., Lippman, M.E., Hiller, A.s., Stampler, M.R., and Hackett, A.J. Response to adriamycin of cullured normal and malignant human mammary epithelial cells. J. Natl. Cancer Inst. 74, 341-347 (1985).

Souten, R.L., Budinger, T.F., and Higgins, C.B. Magnetic resonance imaging of prosthetic heart valves. Radiology 154, 705-707 (1985).

Stampfer, M.R., and Bartley, I.C. Induction of transformation and continuous cell lines from normal human mammary epithelial cells after exposure to benzo(a)pyrene. Proc. Natl. Acad. Sci. USA: 82, 2394-2398 (1985).

Stockman, J.A., Graeber, A.E., Clark, D.A., McClellan, K., Garcia, I.F., and Kavey, R.E. Anemia of prematurity: Determinants of the erythropoietin response. 1. Pediatrics 105, 786-792 (1984).

Susa, J.B., Gruppuso, P.A., Widness, J.A., Domenech, M., Clemons, G.K., Sehgal, P., and Schwartz, R. Chronic hyperinsulinemia in the fetal rhesus monkey: effects of physiologic hyperinsulinemia on fetal substrates, hormones, and hepatic enzymes. Amer. I. Obst. Gyn. 150, 415-420 (1984).

Teng, B., Sniderman, A., Krauss, R.M., Kwiterovich, Ir., P.O., Milne, R.W., and Marcel, Y.L. Modulation of apolipoprotein B antigenic determinants in human low density lipoprotein subclasses. J. Biol. Chem. 260, 5067-5072 (1985).

Tinoco, I., Ir., Maestre, M.F., Bustamante, C., Keller, D. Use of circularly polarized light to s'udy macromolecules. Pure and Applied Chemistry 55, 1423-1428 (1984).

Tobias, $C_{\text {A A }}$. The future of heavy-ion science in biology and medicine. (Failla Memcrial Lecture, February 28, 1983, San Antonio, Texas). Radiation Research 103, 1-33 (1985). 


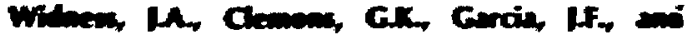
Schmartz, R. Plaswis immunoreactive enythropoietin in normal worsan studies sequentially during and after pregnancy. Amer. J. Obstet. Gynocol. 149, 646-650 (1984).

Writams, P.T., Hakkell, W.L, Vranizan, K.M., Chair, S.N., Kramss, R.M., Superko, H.R., Abbers, J.J., Frey-Hewitt, B., and Wood, P.D. Associations of resting heart rate with concentrations of lipoprolein subfractions in sedentary men. Circulation 71, 441-449 (1985).

Williams, P.T., Krauss, R.M., Wood, P.D., Albers, l.J., Dreon, D., and Ellsworth, N. Associations of diet and alcohol intake with high-density lipoprotein subclasses. Metabolism 34, 524-530 (1985).

Williamson, M.S., Game, J.C., and Fogel, S. Meiotic gene conversion mutants in Saccharomyces cerevisiae. I. Isolation and characterization of pms1-1 and pms1-2. Genetics 110, 609-646 (1985).

Wolman, S.R., Smith, H.S., Stampfer, M., and Hackett, A.J. Growth of diploid cells from breast cancers. Cancer Genetics and Cvtogen 16, 49-64 (1985).

Wrenn, M.E., Durbin, P.W., Howard, B., Lipsztein, J., Rundo, J., Still, E.T., and Willis, D.L. Metabolism of ingested uranium and radium. Health Physics 48, 601-635 (1985).

Yang, T.C., and Jobias, C.A. Neoplastic cell transformation by energetic heavy ions and its modification with chemical agents. Adv. Space Res. 4, 207-218 (1984).

Yang, T.C., and Tobias, C.A. Effects of heavy-ion radiation on the brain vascular system and embryonic development. Adv. Space Res. 4. 239-245, (1984).

Yano, Y., Eudinger, T.F., Mathis, C.A., Singh, M., Moore, D.H., Hunter, J.e Jones, R.M., and Ebbe, S.N. Gallium-68 chemistry for labeling platelels, proleins and lipoproteins. I. Labelled Compds. Radiopharm. XXI, 999-1001 (1984).

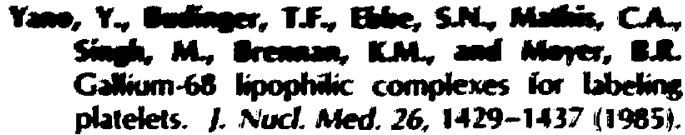

Ychas, IM., Afzal, SM.J., and Afzal, V. Variation in normal tissue response to WR-2721. Inlesntl. J. Radiat. Oncol. Biol. Phys. 10, 1537-1539 (1984).

\section{CONTRIBUTIONS TO BOOKS AND FROCEEDINGS}

Albers, J.J., Warnick, G.R., and Nichols, A.V. Laboratory measurements of HDL. Pages 381-414 in Clinical and Metabolic Aspecis of High Density Lipoproteins, N.E. Miller and G.J. Miller Eds. Elsevier Science Publications, B.V., Amsterdam, 1984.

Albright, N.W., and Tobias, C.A. Extension of the time-independent repair-misrepair model of cell survival to high-LET and multicomponent radiation. In Proceedings of the Berkeley Conference in Honor of Jerzy Neyman and Jack Kiefer, U.C. Berkeley, July 1-15, 1983, Volume 1, L. LeCam, Ed. Wadsworth International Publication, 1985.

Bissell, M.J., Lee, E. Y.-H.P., Li, M., Chen, L.-H., and Hall, H.G. The role of the matrix in regulation differentiation of endocrine sensitive cells. In Proceedings, The Second NIADDK Symposium on the Benign Proslatic Hyperplasia. U.S. Government Printing Office, 1985.

Budinger, T.F., Huesman, and R.H., Knittel, B., Friedland, R.P., and Derenzo, S.E. Physiological modeling of dynamic measurements of metabolism using positron emission tomography. Pages 165-183 in The Melabolism of the Human Brain Studied with Positron Emission Tomography, T. Greitz Eds. Raven Press, New York, 1985.

Chen, G.T.Y., Austin-Seymour, M., Castro, J.R., Collier, J.M., Lyman, J.T., Pitiuck, S., Saunders, W.M., and Zink, S.R. Dose volume histograms in treatment planning evaluation of carcinoma of the pancreas. Pages 264-268 in Proceedings Bth International Conference on Computers in Radiotherapy (Toronto, Canada, 1984). IEEE Computer Press (ISBN! 81860551-6), Silver Springs, Maryland, 1984. 


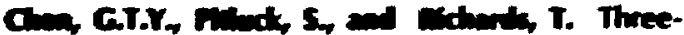

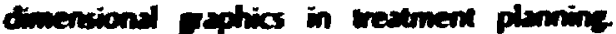
Panes 61-65 in Proceedines of Anoual Meetin of National Computer Graphics Association (NCGA), May 1904.

Che, W.T. Curtis, S.B., Llacer, J., lenwer, T.h., and Seremen, R.W. Wobbler facility for biomedical experiments at the Bevalac. IEEE Trans. Nucl. Sci. NS-32: 3321-3323, 1985.

Derenzo, S. E, Eudinger, T.F., and Huesman, R.H. Detectors for high resolution dynamic positron emission tomography. Pages 21-31 in The Metabolism of the Human Brain Studied with Positron Emission Tomography, T. Greitz, et al, Eds. Raven Press, New York, 1985.

Downing, K.H. and Glaeser, R.M. Small-spot illumination for high-resolution electron microscopy of beam-sensitive specimens. Pages 320-321 in Proceedings of the 43rd Annual Meeting of the Electron Microscopy of Society of America, G.W. Bailey, Ed. San Francisco Press, Inc., San Francisco, California, 1985.

Downing, K.H., and Glaeser, R.M. Simplified treatment of statistical error in electron crystallography. In Proceedings Ninth European Congress on Electron Microscopy (Budapest, 1984), 1985.

Esposito, M.S. Molecular mechanisms of recombination in Saccharomyces cerevisiae: Testing mitotic and meiotic models by analysis of hypo-rec and hyper-rec mutations. Pages 123-159 in Controlling Events in Meiosis, C.W. Evans and H.G. Dickinson, Ed. Soc. Exptl. Biol. Symposium 38: The Company of Biologists, Ltd., Scarborough, North Yorkshire, 1984.

Fabrikant, J.l., Lyman, I.T., and Hosobuchi, Y. Stereotactic heavy-ion Bragg peak radiosurgery for intracranial vascular disorders. Pages 1128-1132 in Neurosurgery, R.H. Wilkins, S.S. Rengcharry, Ed. McGraw-Hill, New York, 1985.

Frielland, RS., Erwn, A., and Eudinger, T.F. Parhological and positron emission tomographic correlations in Alzheimer's disease. (Lefter to the Editor) Lancet i:228, 1985. (January 26. 1905)

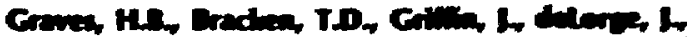

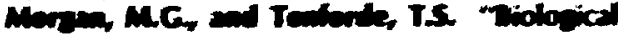
Effects of 60-Hz Power Tramsisssion lines" Report of the Florida Electric and Magnetic Fields Science Advisory Commission, 266 pp. March 1905.

Geari, T.G., Wefel, J.\$. Cramiond, HA. Greiner, D.E. Linditrom, P.f. Schimanertine, W., and Symomi, T.J.M. Implication of new measurements of ${ }^{16} \mathrm{O}+\mathrm{P} \rightarrow{ }^{12.13} \mathrm{C},{ }^{14.15} \mathrm{~N}$ for the abundances of $C, N$ isotopes at the cosmic ray source. Pages 80-83 in volume 2, 19th ACRC Conference Papers. NASA, Washington, D.C., 1985.

Mall, K., Wells, S., Keller, D., Samori, B., Maestre, M.F., Tinoco, I., Jr., and Bustamante, C. CIDS Measurements of planar and focal conic orientation tions of cholesteric liquid crystals. In Proceedings of the Workshop on Applications of Circularly Polarized Radiation, F. Allen and C. Bustamante, Eds. (University of New Mexico, May 18-20, 1984), Plenum Press, New York, 1985.

Kaback, H.R., Carrasco, N., Foster, D.L., Garcia, M.L., Goldkorn, T., Patel, L., and Vititanen, P. The lac carrier protein from Escherichia coli. in Electrogenic Transport: Fundamental Principles and Physiological Implications, M.P. Blaustein and M. Lieberman, Ed. Raven Press, New York, 1984.

Lamoreaux, P., Bebbington, W.P., Duguid, J.O., Durbin, P.W., LeGrand, H.E., Matuszek, J.M., Mendelhoff, J., Schneider, A., Sherby, O.D., and Sloss, L.L. The Management of Radioactive Waste at the Oak Ridge National Laboratory: A Technical Review. Panel on Oak Ridge Wastes, National Research Council. National Academy Press, Washington, D.C., 1985.

Liburdy, R.P., and Magin, R.I. Microwavestimulated drug release from liposomes. (Correspondence). Radiation Res. 103: 2ó6-275, 1985.

Lyman, fohn T. Heavy charged-particle beam dosimetry. Pages 267-280 in Advances in Dosimetry for Fas! Neutrons and Heavy Charged Paticles for Therapy Applicalions, Proceedinos IAEA Advisory Group Meeting, (Vienna, Austria June 14-18, 1982); WEA Vienna, 1964. 
Masere, Mf. Circular dichroism. Pines 291-311 in Optical Techniques in Bioloyical Research. D.L. Rousseau, Ed. Academic Press, New York, 1905.

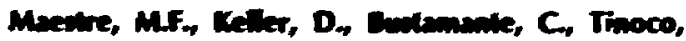
I. 1. Circular differential microscopy. In Proceedings of the Workshop on Applications of Circularly Polarized Radiation; F. Allen and C. Bustamante, Eds. (University of New Mexico, May 18-20, 1984). Plenum Press, New York, 1985.

Mortimer, R.K., and Contopoulou, $R$. Yeast Genetic Stock Center Catalogue, Fifth Edition, 1984 (55 pages)

Mortimer, R.K., and Schild, D. Genetic map of Saccharomyces cerevisiae. Pages 224-233 in Genetic Maps 1984, S.J. Obrien, Ed. Cold Spring Harbor Press, New York.

Mosteller, F., Fabrikant, J.I., Fry, R.J.M., Lagakos, S.W., Miller, A.B., Saenger, E.L., Shottenfeld, D., Scott, E.L. Van Ryzin, I.R., and Webster, E.W. Assigned share for radiation as a cause of cancer. In Review of Radioepidemiologic Tables Assigning Probabilities of Causation. National Academy of Sciencs - National Research Council, National Academy Press, Washington, D.C., 1985.

Resnick, O.A., Chow, T., Nitiss, I., and Game, J. Changes in the chromosomal DNA of yeast during meiosis in repair mutants and the possible role of a deoxyribonuclease. Pages 639-649 in Recombination at the DNA Level. Cold Spring Harbor Symposia on Quantitative Biology XLIX, Cold Spring Harbor Laboraiory, 1984.

Seeman, N.C., Maestre, M.F., Ma, R.I., and Kallembach, N.R. Physical characterization of a nucleic acid junction. Pages 99-108 in Molecular Basis of Cancer, R. Rein, Ed. Alan R. Liss, 1985.

Stampfer, M.R., and Barley, J.C. Development of human epithelial cell cullure systems and their use for studies of carcinogenesis and differentiation. In In Vitro Models for Cancer Research, Volume 3. M. Weber and L. Sekely.

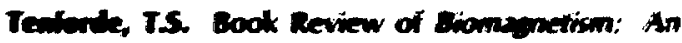

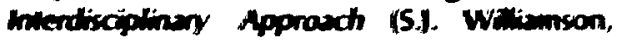
G.L. Romani, L. Kautiman, and I. Modera, Eds.]. IEEE Engineering in Med. and Biol. 4 42. 1905.

Tenforde, T.S. Mechanisms for biological effects of magnetic frelds. Pages 71-92 in Biological Effects and Dosimetry of Non-fonizing Radiation: Stat: and ELF Electromagnetic Fields. M. Grandolfo, S.M. Michaelson, A. Rindi, Eds. Plenum Press, New York, 1985.

Tenforde, T.S. Physical properties of high-voltage ELF eleclromagnetic fields and their interaction with living systems. Pages 195-213 ir Electric Energy Systems Research, Proceedings of Workshop held April 24-26, 1985. National Research Council Committee on Electric Energy Systems, National Academy Press, Washington, D.C., 1985.

Tenforde, T.S. Biological effects of stationary magnetic fields. Pages 93-127 in Biological Effects and Dosimetry of Non-lonizing Radiation: Static and ELF Electromagnetic Fields, M. Grandolfo, S.M. Michaelson, A. Rindi, Eds. Plenum Press, New York, 1985.

Tenforde, T.S. Biological effects of ELF magnetic fields. Pages 79-127 in Biological and Human Health Effects of Extremely Low Frequency Electromagnetic Fields. Report of the American Institute of Biological Sciences, Arlington, Virginia, March 1985.

Tenforde, T.S., Gaffey, C.T., and Raybourn, M.S. Influence of stationary magnetic fields on ionic conduction processes in biological systems. Pages 205-210 in Proceedings of the Sixth Symposium and Technical Exhibition on Electromagnetic Compatibility, T. Dvorak, Ed. Zurich, Switzerland, 1985.

Tobias, C.A., Albright, N.W., and Yang, T. C.-H. The roles of ionizing radiation in cell transformation. In Proceedings of the Berkeley Conference in Honor of Jerzy Neyman and jack Kiefer (U.C. Berkeley luly 1-15, 1983), volume 1. L. LeCam, Ed. Wadsworth International Publication, 1985. 


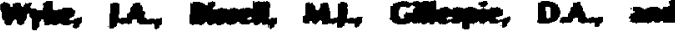
levand P. The molecular basis for phenotypic modulation in cells containing an integrated viral sic oncogene. In Hormones and Cell Regulation, Vohume 9 (Proceedings of the 9th European Symposium on Hormones and Cell Regulation), B. Hamprecht and J. Cumont, Eds. Elsevier, Amsterdam, 1985.

Yane T.C.H., and Tobias, C.A. Mechanisms of radiation-induced neoplastic cell transformation. Pages 343-371 in Proceedings of the Berkeley Conference in Honor of Jerzy Neyman and Jack Kiefer (U.C. Berkeley July 1-15, 1983) Volume 7 , L. LeCam, Ed. Wadsworth International Publication, 1985.

\section{LBL REPORTS ISSUED}

Albright, N.W. and Tobias, C.A. Extension of the time-independent repair-misrepair model of cell survival to high-LET and multicomponent radiation. LBL-17112, July 1983, 27 pp.

Bastacky, J., and Hayes, T.L. Scanning electron microscope laboratory safety. LBL-19441, April 1985, $48 \mathrm{FP}$.

Biology and Medicine Division, LBL. Heavy charged particles in research and medicine Abstracts/Program, presented at the Symposium on Heavy Charged Particles in Research and Medicine (Lawrence Berkeley Laboratory, Berkeley, CA, May 1-3, 1985) LBL-19352, May 1985, 51 pp.

Blakely, E.A., Chang, P.Y., and Lommel, L. Cellcycle-dependent recovery from heavy-ion damage in $G_{1}$-phase cells (Presented at the Symposium on Heavy Charged Particles in Research and Medicine, Berkeley, CA, May 1-3, 1985). LBL-20402, May 1985, 31 pp.

Endinger, T.F., Huesman, R.H., Knittel, B., Friedbind, R.P., and Derenzo, S.E. Physiological modeling of dymamic measurements of metabolism using positron emission tomography. LBL-18454, Ociobet 1964, 20 pp.

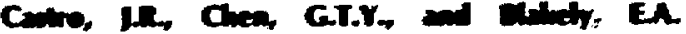
Current considerations in hervy chozed purticle radiotherapy: A clinical research trial of the University of Califomia, Lawrence Berkeley Laboratory, Northem Califomia Oncolosy Group and Radiation Therapy Oncolosy Group (Presented at the Symposium and Heavy Charged Particles in Research and Medicine, Berkeley. CA, May 1-3, 1985). LBL-20394, May 1985, 22p.

Chatteriee, A. Discussion of the Physics Session. (Presented at the Symposium on heavy Charged Particles in Research and Medicine, Berkeley, CA, May 1-3, 1985). LBL-20395, May 1985, 6 pp.

Chu, W.T., Curtis, S.B., Llacer, I., Renner, T.R., and Sorensen, R.W. Wobbler facility for biomedical experiments at the Bevalac. LBL-18953, 1985.

Clemons, G.K. Comparison of radioimmunoassay and bioassay of erythropoietin. LBL-19861, lune 1985, $23 \mathrm{pp}$.

Curtis, M.P. Cornelius A. Tobias, Symposium Honorary Chairman. (Presented at the Symposium on Heavy Charged Particles in Research and Medicine, LBL Berkeley, California, May 1-3, 1985). LBL-20390, May 1985, 4 pp.

Curtis, S.B. The LPL Model applied to low dose rates. LBL-18539, April 1985, 20 pp.

Curtis, S.B. Discussion of the Models of Biological Action Session (Presented at the Symposium on Heavy Charged Particles in Research and Medicine, Berkeley, May 1-3, 1985). LBL20397, May 1985, 15 pp.

Curtis, S.B., and Alpen, E.L. Introduction to the Symposium on Heavy Charged Particles in Research and Medicine (Presented at the Symposium on Heavy Charged Particles in Research and Medicine, Berkeley, CA, May 1-3, 1985), L8L-20398, May 1985, 3 pp.

Derenzo, S.E., and Rudinger, T.F. Advanced instrumentation for positron emission tomography. (Presented at the NATO Advanced Sludies Institute Series - Physics and Engineering of Medical Imaging, Maratea, Italy, September 23-October 5, 1984). LBL-19435, April 1985. 19 pp. 


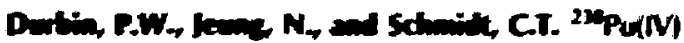
in monkeys: Overview of metsbolissm. LBL20022, July 1985, $96 \mathrm{pp}$.

Falviliant, I., lyman, J.T., and Frankel, K.A. Heavy charged-particle Bragg peak radiosurgery for iniracranial vascular disorders. (Presented at the Symposium on Heavy Charged Particles in Research and Medicine, Berkeley, CA, May 1-3, 1985). LBL-20393, May 1985, 28 pp.

Fry, R.J.M., Powers-Risius, P., Alpen, E.L., and Ainsworth, E.J. High-LET radiation carcinogenesis (Presented at the Symposium on Heavy Charged Particles in Research and Medicine, Berkeley, CA, May 1-3, 1985). LBL-20392, May 1985, 20 pp.

Hallowes, R., and Stampfer, $M$. Workshop on in vitro culture systems. LBL-19623, May 1985, $8 \mathrm{pp}$.

Hutchinson, M.S. Electrophoretic and biochemical studies of erythrocyte membrane structure. LBL-18458 (Ph.D. thesis), December 1984, 160 pp.

Lawton, M.S. Phase-encoded, rapid, multiple-echo (PERME) nuclear magnetic resonance imaging. LBL-20101, August 1985, 23 pp.

Lyman, I.T. Complication probability as assessed from dose-volume histograms. (Presented at the Symposium on Heavy Charged Particles in Research and Medicine; Berkeley, CA, May 1-3, 1985. LBL-19630, May 1985, 7 pp.

Maestre, M.F., Keller, D., Bustamante, C., and Tinoco, I., Ir. Circular differential microscopy. LBI -20169, August 1985, 17 pp.

Mathis, C.A., Jones, R.M., and Chasko, J.H. Overall radio-HPLC design. LBL-19433, December 1984, $45 \mathrm{pp}$.

Mazoyer, B.M., Huesman, R.H., Budinger, T.F., and Knittel, B.L. A study of factors that affect the precision of compartmental model parameter estimation using dynamic positron emission lomograpliy. LBL-19614, May 1985, $30 \mathrm{pp}$.

Perez, C.F. lonizing and ultraviolet radiation enhances the eificiency of DNA mediated gene transier in vilso. LBL-18303 (Ph.D. thesis), August 1984, $234 \mathrm{pp}$.

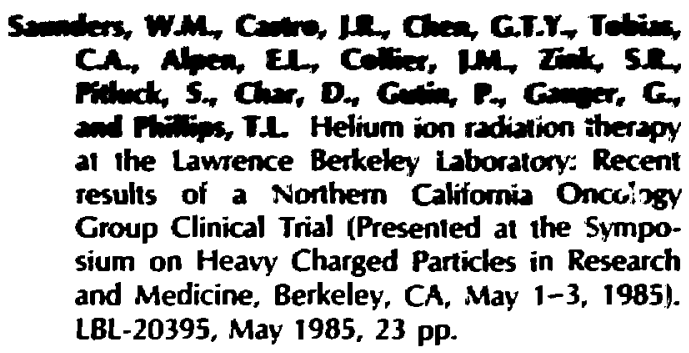

Tenforde, T.S. Biological effects of ELF magnetic fields. LBL-19157, January 1985, 62 pp.

Tenforde, T.S. Physical properties of high-voltage ELF electromagnetic fields and their interaction with living systems. LBL-19710, April 1985, 21 pp.

Tenforde, T.S. Magnetic field applications in modern technology and medicine. LBL-19711, May $1985,36 \mathrm{pp}$.

Tenforde, T.S., and Budinger, T.F. Biological effects and physical safety aspects of NMR imaging and in vivo spectroscopy. LBL-20053, August 1985, $57 \mathrm{pp}$.

Tenforde, T.S., Levy, L., and Veklerov, E. Monitoring of circadian waveforms in rodents exposed to high-intensity static magnetic fields. LBL18384, October 1984, 37 pp.

Tobias, C.A. Symposium Honorary Chairman (Presented at the Symposium on Heavy Charged Particles in Research and Medicine, Berkeley, CA, May 1-3, 1985). L8L-20390, May 1985, 4 pp.

Tobias, C.A. Summary of Symposium on Heavy Charged Particles in Research and Medicine. (Presented at the Symposium on Heavy Charged Particles in Research and Medicine, Berkeley, CA May 1-3, 1985). LBL-20399. May 1985, 22 pp.

Tobias, C.A. The repair-misrepair model in radiobiology: Comparison to other models. (Presented at the Symposium on Heavy Charged Particles in Research and Medicine, Berkeley, CA, May 1-3, 1985). L8L-20400, May 1985, 45 pp. 


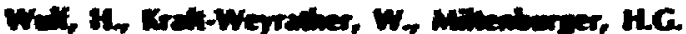
Mincil. EA, and Tolios, CA Heary-ion effects on mammation cells: inactivation measirement with different cell lines. (Presented at the Symposium on Heavy Charged Particles in Research and Medicine, Berkeley CA, May 1-3, 1985). LBL-20391, May 1985, 28 pp.

Yane T.C., Craise, LM., Mei, M.T., and Tobias, C.A. Neoplastic cell transformation by heavy charged particles. (Presented at the Symposium on Heavy Charged Particles in Research and Medicine, Berkeley, CA May 1-3, 1985). LBL-20401, May 1985, 23 pp.

Yano, Y., Budinger, T.F., Ebbe, S.N., Mathis, C.A., Moore, D.H., Singh, M., Brennan, K., Moyer, B.R., and Nichols, A. Gallium-68 Chemistry for labeling platelets, proteins and lipoproteins. LBL-18388, 1984.
Yeri, MA. The role of protein synthesis in the repair of sublethal x-ray damage in a mutant chinese hamster ovary cell line. LBL-19500 (Ph.D. thesis), April 1985, 156 pp.

\section{Patents}

Liburdy, R.P. Method for Determining Cell Membrane Dielectric Breakdown. U.S. Patent No. 4,472,506. Granted September 18, 1984. 


\section{APrENDIX C: Biology and Medicine Division Staff September 30, 1985}

The accomplishments of the Biology and Medicine Division are che in large measure to the capability and dedication of its staff. Listed below are those who have participated in the Division's programs during fiscal year 1985 as full- or part-time employees, consultants, and participating guests. The guest staff includes visiting scientists, postdoctoral trainees, resident physicians, graduate and undergraduate students, and summer research participants.

\section{DIVISION HEAD}

Edward L. Alpen

Thomas L. Hayes, Deputy

\section{DIVISION ADMINISTRATION STAFF}

Mary P. Curtis

Janice C. DeMoor

De A. Eggers

Michael B. Fizer

Wendell Hom

Allan W. Long

Georgia A. Peterson

Robert W. Springsteen

Baird Whaley

$t$ Herbert Wiener

Mary L. Worth

\section{DIVISION SCIENTIFIC STAFF}

S. Javed Afzal Judith Aggeler E. John Ainsworth

- Steve P. Akeson Julius J. Almasi

\# Bruce N. Ames Melissa A. Austin

John C. Bartley John B. Bassel S. Jacob Bastacky

- Astrid Baumgartner

¥ Alan I. Bearden Eugene V. Benton

Peter A. Berardo Leslie Bernstein Stephen E. Bicknese Mina 1. Bissell Eleanor A. Blakely

- Caridad Borras

5 George Brecher Kathieen M. Brennan Michsel F. Bnuno

15 Thomis F. Budinger Ruph suncher

leth Ciology and Medicine Division prior wo Seplember $20,1905$. "Retined during fincal vear nos5. aculy uc Corlukey.

rixenty UC Sin frentionco.

Heculny ue ounis.
- Gail E. Butterfield

- Barbara E. Cahoon Vincent P. Carabillo

5 Joseph R. Castro

- Lai-Man Chan

* Chung-Fu Chang Aloke Chatterjee

* George T.Y. Chen Gisela K. Clemons John P. Cody J. Michael Collier Priscilla K. Cooper

- Tom L. Crisswell Stanley B. Curtis

- Maya Das Stephen E. Derenzo

- David S. Dolberg Kenneth $\mathrm{H}$. Downing

- Werner K. Doyle

- D.M. Driscoll Patricia W. Durbin

5 Shirley N. Ebbe

- Herschell S. Emery

* Masahiro Endo Michael S. Esposito

5 Jacob l. Fabrikant

- Gregory L. Finch James Fontanesi Trudy M. Forte David L. Foster Heinz Fraenkel-Conrat Kenneth A. Frankel

¥ Michael Freeling

II Robert P. Friedland

- Gunther Gademann Comelius T. Gaffey John C. Game

5 Grant E. Gauger Peter S. Geisster Orsolya Genzel

- lack R. Gerson

† Robert M. Glaeser Lois S. Gold loseph D. Goldstein

- $\operatorname{lon}$ W. Goodman Rezine Goth-Goldsiein

- Martin H. Graham Dile E Greermuth

- Ciamiranco Grossi

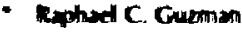

Alicthed P. Hingem

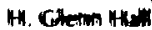


- Colin G. Hamison

- Vrim R. Havender John W. Holf́man Eurene V. Hotahan Libby L. Holbrook William R. Holley

5 Yoshio Hosobuchi Junko Hosoda Jerry. Howard Anthomy R. Howlett Mirko I. Hroval John P. Huberty Ronald H. Huesman

- Philippe Hugues

II William J. Jagust Bing $K$. Jap Lin C. Jensen John R. Johnston

Talwinder S. Kahlon Leon N. Kapp

- Jerry S. Kidd Patrice A. Koehl

- Betty Koss

5 Ronald $M$. Krauss

Michael La Belle

$\ddagger$ John H. Lawrence Steven A. Leadon

- Wen-Hwa Lee Robert M. Leven

- Francine C. Levin

- Jack Levin

- Robert H. Levinson Ming $\mathrm{Li}$ Robert P. Liburdy

- Jonathan G. Lieber Tz-Hong Lin Frank $T$. Lindgren David E. Linstadt Helen $F$. Londe Susan T. Lovelt Bernhard A. Ludewigt John T. Lyman

Marcos F. Maestre John L. Magee Andrew C. Magyarosy Neela B. Manley

- Edward K. Markell Vera Martin Huso A. Massaldi Chester A. Mathis Bemand M. Mazoyer Elisabelh Mazoyer loyce C. McCann

- Man-Tons Mei

t Howard C. Miel

- Kuthieen L. Miller

- Nolk K. Mitra Steplien M. Mondein

- Dnid H. Moose

1 Debert X. Montinarer

- Iolm P. vermone
Richard A. Mushlin Thomas A Auslinet

Yen H. Nguryen

\# Alex V. Nichols

- Hiroshi Ohara

- Rebecca C. Osbom

¥ William G. Owen

¥ John C. Owicki

Gordon Parry

- John J. Peloquin

- Carl F. Perez Mark H. Phillips

\& Theodore L. Phillips

Gian M. Ratto

- Michael S. Raybourn

- Todd L. Richards Gary V. Richieri Adrian Rodriguez Mark S. Roos Ruth 1. Roots

Thornton W. Sargent, III

- 乌 William M. Saunders David Schild Walter Schimmerling John C. Schooley

- Stanley Schuman

- Jeffrey M. Schwartz Richard I. Schwarz Zahra Shahrokh Alexinder T. Shulgin Paul $H$. Silverman Bea A. Singer

$\ddagger$ jerome R. Singer Thomas $\mathrm{H}$. Slone Sylvia I. Spengler Martha R Stampfer

- Susan G. Stantón Henry H. Stauffer Thomas Swain

- Susan F. Sweigert Thomas S. Tenforde

$¥$ Cornelius A. Tobias

- Conrad N. Trumbore Sylvanus A. Tyler

- Peler E. Vaik Karen 14. Vranizan

- Liang-Zhong Wang

- Klaus 1. Weber

5 Philip R. Weinstein Margarel R. While Paul T. Williams Kay H. Woodrutf Mervyn Wons

- Wennie H. Wu. Tracy C. Yans Yuldio Yamo

- Chinowar Yen

- Sandta R Zitok 
Annelle C. Drew

Bill $M$. Du Four

Linda D. Abe

James R. Abney

Frederick E. Abrams

Gerald L. Adamson

- Myungho Ahn

Mari Aker

Kari P. Baken

- David Baker

John R. Baker

Violet Barghe-Sharghi

Josephine L. Barr

George M. Basile

Maren Bell

Sindy $E$. Berger

- William H. Bingham

* Patricia Biscay

Kathleen A. Bjornstad

Patricia |. Blanche

- Mark G. Blumenthal

Nicolas R. Bolo

Yvonne C. Bopp

* Jochen Braun Mitch C. Brenner

Robert S. Bridwell

S. Kay Bristol

Gerald L. Brooks

- Erik T. Burns

Mary Cabbage

John L. Cahoon

- Denise Capra-Young

- Mark L. Carlson Mardel M. Carnahan Dorothy A. Carpenter

- Betsy C. Carr Albert C. Casselhoff Lida M. Caylor Rosemarie L. Celli

- Dominic Chan Polly Y. Chang Frank Chavez Li-How Chen Melody L-Y Cheng

- Larry I. Chevez

Dennis Chin

Gary M. Cole

C. Rebecca Contopoulou Vincent E. Cook

- Maria R. Costin

Laurie M. Craise

Freddie L. Crenshaw, Ir.

Christopher Cullander

Beisey L. Cullen

Kanu 8 . Dalal

Ninh N. Dan

Randy 1. Deguzmam

Gres T. De lory

Dorlene I. DeNunumcor

- Sursar M. Detilinge

Uhen Nima Dom

Ninetita A Dantsom

- Latumand T-. Divaltiong

- Mark F. Henteleff
- Katherine A. Dukes

- Dennis P. Duncan

Eva L. Edwards Julie A. Ellison Nina Engineer

David E. Erkenbrack

Diana E. Fajardo

Dennis Fantin

Deborah A. Farson

Sherry L. Fitzsimmons

- Brian L. Fletcher

- Ted Y. Fong

Myrtle L. Foster

Marilyn A. Fowler

Roscoe Frazier

* Carol l. Fujihara

Charlie M. Fuller

- Carla L. Fulton

- Roassana A. Garon

Lance A. Gee

John C. Gilbert

Christine Giotas

Queen E. Gipson

Brian ). Glassner

Laura A. Glines

Michael A. Glotzer

Tennessee W.-Y Gock

Elaine L. Gong

Edwin H. Goodwin

Sara P. Goolsby

- Eddy A. Gosschalk

- Robert A. Graf

- Joan M. Graham

Angela Habrek-Davidson

William A. Hare

- Ronald M. Harris

Andrew C. Hasenfeld

Carroll Hatier

Virginia C. Havens

Lilian E. Hawkins

Linda G. Hayashi

- lefírey V. Hernandez Mark D. Hente

Peter Hertz-Herskovits

Sharon A. Hibdon

- Kalhleen I. Hicks

Lynn R. Hlatky

Christine S. Hong

L aura I. Hom

Midori Mosobuchi

Mildred $x$. Huches

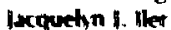

Donied Young-Bin lim

- Andela lngendary

Conla P. lluety

Nytum in. Jeung

- Penticia A. Hotmecom

- Nurra ham tones

Mecese M. Jathes 
- Jay S. Joseph James W. Judnick

David M. Kang

Aaron B. Kantor

Kristina S. Kavanau

- David E. Kellog Marc L. Kessler

- Susan B. Klein Paul'M. Kleinman Brian L. Knittel Seok-Hwan Kong Renee I. Kopa Lori E. Kopp Kari L. Koszdin Susanne E. KuehI Birgetta R. Kullgren Natalia Kusubov

- Christian Labadie Clifford E. Lai

- Mark T. Lasartemay

- Mark S. Lawton Ann Lee Kyung-Dall Lee Samuel Lee

- Yue-Hwa Pan Lee

- Barbara A. Leivonen Gerri A. Levine Lynette L. Levy Richard P. Levy

- John P-J Lin

- Vincent Ling Leora Lommel

- Peggy A. Lopipero lanice K. Louie

- Katherine A. Louie Janet S. Lowe Karen E. Lowe

- Helen Lu

- Beverly G. McCalla Tommy 1. McKey

- May F. McKoon Velma B. MCNeal

- Ian 5. Madfes Renae I. Magaw Lynn J. Mahlmann Dimitrios T. Maleas

- Missie I. Mantis

- William I. Meecharn Marc S. Mendonca

- Roy E. Minizer Barbura Modilinski Herbert W. Monve, III

- Maveen H. Morfíd Lermy Mloss

- Brim R Alover Kimbeaty Nuksthy

- Don L Alupiny Serves. Nicben the Nom trimence Andy Ningom

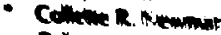

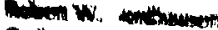

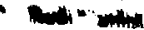


Exthen inilums

- Howerd a Wons

Heon thiong

Som J.5 Nion

- Vira vions

- Cinterme birnet

- Scesarillu
A'derict $x$ yome

- Tilame en ve

shiarshen bete

Jo intun $1 \mathrm{x}$ Nere

Michoel I Yezz: 


\section{Combrimees}

\section{Dension adrear commutree}

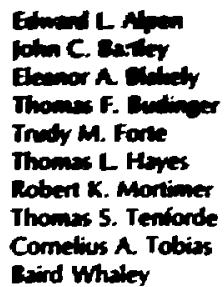

\section{DMSION STAFF COMMITTEE}

Shirley N. Ebbe, Chair Aloke Chatteriee Trudy M. Forte John T. Lyman

Tom W. Sargent

\section{EQUIPMENT COMMITTEE}

Tony W. Sargent, Chair John C. Bartley Aloke Chatterjec Shirley N. Ebbe Cometius T. Caffey Frank T. Upham

- Alan W. Lons

- Herbert Wiener

\section{SPACE COMMITTEE}

Thomas S. Tenforde, Chair Gisela K. Clemons Michuel 5. Esposito Tracy C. Yang Yukio Yano

- Allan W. Lons

\section{SALARY COAMITTEE}

Edward L. Apen, Chai John C. Bartley

Thoms F. Budinger

Trudy Forte

Thomas L Hayes

Thomas 5. Tenforde

Comelius A. Tobies

- Eaind Whaley
Humar Use commatres

\author{
Hewn H. Stmer, Che二 \\ Thomen F. Sucting \\ Ceone T.Y.Chen \\ Shiler N. Ebbe \\ Kathieen E. Handion \\ t Janice C. Dewloor
}

- Baind Whaley

\section{RADIOACTIVE DRUG RESEARCH COMMITTEE}

\author{
jacob 1. Fabrikan, Chair \\ Edward L. Apen \\ Aloke Chatteriee \\ Henry H. Stauffer \\ Paola 5. Timiras \\ Joseph D. Goldstein (Consultant) \\ $t$ Janice C. DeMoor
}

\section{ANIMAL WELFARE COMMITTEE}

John C. Bartley, Chair

Herman Bonasch

Kathlenn Brennan

Joan W. Goodman

John C. Schooley

- Robert W. Springsteen

$t$ Janice C. DeMoor

\section{DONNER LIBRARY COMMITTEE}

\section{Ronald M. Krauss, Chair}

Edward L. Bennett

Thomas F. Budinger

Stanley 8. Curtis

Thomas L. Hayes

Frank T. Lindgren

Howard C. Mel

- Dorothy F. Denney

- Gloria L. Haire

\section{DONNER SEMINAR COMMITTEE}

\author{
Stephen E. Derenzo, Chair \\ Michaed 5. Esposito \\ Staniey B. Curtis
}

